\title{
SEARCH FOR DARK MATTER PRODUCED IN ASSOCIATION WITH A STANDARD MODEL HIGGS BOSON DECAYING TO $B$-QUARKS WITH THE ATLAS DETECTOR
}

by

Anindya Ghosh
A thesis submitted in partial fulfillment
of the requirements for the
Doctor of Philosophy degree in Physics in the
Graduate College of
The University of Iowa

\author{
August 2021 \\ Thesis committee: \\ Usha Mallik, Thesis Supervisor \\ Philip E. Kaaret \\ Frederick N. Skiff \\ Vincent G.J. Rodgers \\ Spyridon Argyropoulos
}


Copyright by

Anindya Ghosh

2021

All rights reserved 


\section{ACKNOWLEDGEMENTS}

No big task can be accomplished alone, certainly not a $\mathrm{PhD}$ in experimental particle physics. I have been fortunate enough to get proper guidance throughout my journey as a graduate student. It has helped me to grow both academically and personally. First of all, I would like to thank my supervisor Professor Usha Mallik, who was kind enough to give me the opportunity to work in her group and collaborate with other students and researchers at CERN. She has been a constant support and guided me throughout my journey as a graduate student at the University Of Iowa in every aspect, beginning from guiding me in this field to providing insights to my thesis. Thank you very much for all your help and guidance.

I would also like to thank The University Of Iowa and the physics department for letting me be a part of this wonderful place. Thank you to my professors at the university with whom I have had helpful discussions during my early semesters as a grad student. I'm thankful to Professor Philip E. Kaaret, Professor Frederick N. Skiff, and Professor Vincent G.J. Rodgers, for being in my $\mathrm{PhD}$ thesis committee and providing inputs during my interactions with you. I would like to thank Jeanne Mullen, who has been always supportive and helped dealing with all the paper works during my time at The University Of Iowa.

I would like to thank Dr. Spyridon Argyropoulos, who has helped me in every way possible in my research. He has taught me many aspects of experimental particle physics, from coding to analyses in ATLAS. Thank you Spyros for providing valuable suggestions for the thesis. I have learned a lot from you and without your support the journey would have been much tougher.

I would also like to thank Marc, Somdutta, and Weitao who were kind enough to read my thesis and give valuable inputs. I would also like to thank other colleagues at CERN, without whom physics analyses in ATLAS would not be possible.

And lastly thank you to my parents who have always been supportive and encouraged me

whenever I needed them. A special thanks to my friends who have been of immense support during these years. 


\begin{abstract}
The Standard Model (SM) of particle physics is one of the biggest triumphs of modern physics. The SM has been immensely successful in explaining the new elementary particles and how they interact with each other. A plethora of experiments have been conducted to validate the SM predictions, and so far the SM predictions and experimental observations are in good agreement, the most recent evidence being the discovery of Higgs Boson at the LHC in 2012. However, SM alone is insufficient to answer many open questions in modern physics, such as the presence of dark matter (DM) and dark energy (DE) in our universe. Ordinary matter, observed so far by various experiments, accounts for only about $5 \%$ of the energy density of the universe, while a large fraction is in the form of DM $(\sim 27 \%)$ and DE $(\sim 68 \%)$. While the nature of DM is still unknown, one of the leading hypotheses suggests that it consists of Weakly Interacting Massive Particles (WIMP). All evidence point to the interaction between DM and SM to be very weak. DM searches are being pursued in collider experiments alongside direct and indirect detection experiments. In this thesis, a search for dark matter candidates produced in association with a Standard Model Higgs boson decaying to two $b$-jets is presented. The search uses a dataset of $p p$ collisions at $\sqrt{s}=13$ $\mathrm{TeV}$ corresponding to an integrated luminosity of $139 \mathrm{fb}^{-1}$, recorded by the ATLAS detector. The results are interpreted in the context of the Two-Higgs Doublet Model (2HDM) with an additional vector or pseudoscalar mediator. The $2 \mathrm{HDM}$ is connected to the so-called Higgs portal models, in which DM particles interact with the SM particles only through their couplings with the Higgs sector. The analysis did not discover any DM particles and constraints are put on the model parameters. Some parts of the benchmark DM model phase-space are excluded and improvements are observed compared to previous results. Another limitation of the SM is its inability to explain the accelerated expansion of the universe. One possible explanation in the context of a homogeneous and isotropic universe is the mysterious DE, which is a repulsive force that counteracts the gravitational pull. This thesis describes the search for DE in the $t \bar{t}+E_{\mathrm{T}}^{\text {miss }}$ final state with a dataset of $p p$ collisions at $\sqrt{s}=13 \mathrm{TeV}$ corresponding to the integrated luminosity of $36.1 \mathrm{fb}^{-1}$ recorded by
\end{abstract}


the ATLAS detector. The results are based on a reinterpretation of the search for supersymmetric partners of top-quark to constrain conformal couplings of DE to SM matter. No DE particles were discovered and exclusion constraints are put on the DE model parameters. 


\section{PUBLIC ABSTRACT}

From the discovery of the electron in 1897 by J.J Thomson to the Higgs boson in 2012 at the LHC, a plethora of elementary particles have been detected by various experiments. The Standard Model (SM) of particle physics has been enormously successful in describing all the observed elementary particles and explaining the interactions between them at the most fundamental scientific level. However, it is clear that SM alone is insufficient to answer many open questions in modern physics, such as the presence dark matter (DM) and dark energy (DE) in our universe. Ordinary matter, observed so far by various measurements, accounts for only about $5 \%$ of the energy density of the universe, while a large fraction is in the form of DM ( 27\%) and DE ( $68 \%)$. The SM alone is insufficient to explain the existence of DM or DE, hence Beyond Standard Model (BSM) theories are postulated to explain the dark sector. One such set of BSM models are the so-called Higgs portal models, where the DM interacts with the SM particles through the Higgs sector. Various types of experiments are conducted around the world to search for DM and DE, and one of them is the Large Hadron Collider (LHC) in Geneva, Switzerland. The LHC is a particle accelerator that collides high-energy protons and data from the $p p$ collisions are recorded by four large detectors, the largest being the ATLAS detector. This thesis describes the search for DM and DE with the $p p$ collision data recorded by the ATLAS detector and the results are interpreted in terms of certain DM and DE models. No DM or DE particles were discovered, hence constraints are put on the DM and DE model parameters. 


\section{TABLE OF CONTENTS}

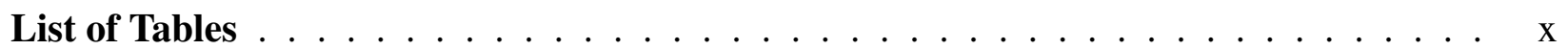

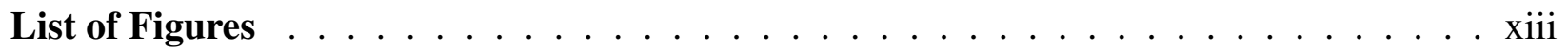

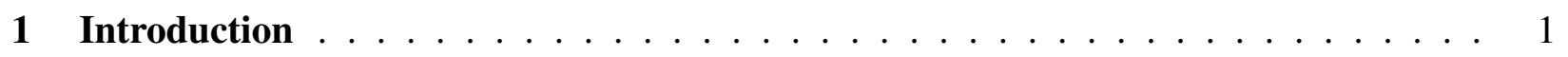

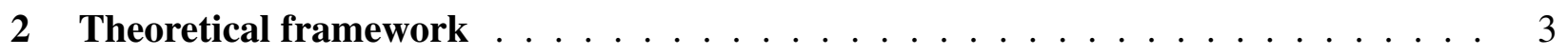

2.1 The Standard Model of particle physics $\ldots \ldots \ldots$. . . . . . . . .

2.1.1 Elementary particles and their interactions $\ldots \ldots \ldots \ldots$

2.1.2 Symmetry and Gauge fields $\ldots \ldots \ldots \ldots$

2.1.3 Electromagnetic force and Quantum Electrodynamics $\ldots \ldots \ldots$

2.1.4 Strong force and Quantum Chromodynamics . . . . . . . . . . . 9

2.1.5 Weak force and Electroweak unification . . . . . . . . . . . . 11

2.1.6 Electroweak Symmetry Breaking and Higgs Mechanism . . . . . . . . 14

2.2 The success and limitations of Standard Model $\ldots \ldots$. . . . . . . . . 18

2.3 Dark matter and dark energy $\ldots \ldots \ldots \ldots$

2.3.1 Evidence of dark matter $\ldots \ldots \ldots \ldots \ldots$

2.3.2 Dark matter candidates . . . . . . . . . . . . . . . . . . 21

2.3.3 Dark matter detection $\ldots \ldots \ldots \ldots \ldots$

2.3.4 Accelerating universe and dark energy . . . . . . . . . . . . . 24

2.4 The Two Higgs Doublet Model (2HDM) . . . . . . . . . . . . . . . . . 25

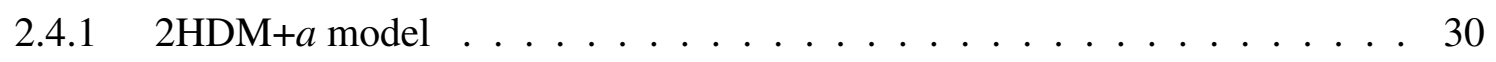

$2.4 .2 \quad Z^{\prime}-2 H D M$ model $\ldots \ldots \ldots \ldots \ldots \ldots$

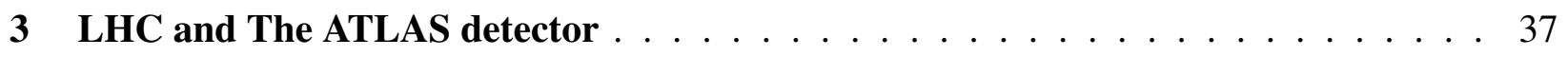

3.1 The Large Hadron Collider . . . . . . . . . . . . . . . . . . . 37

3.1 .1 Design of the $\mathrm{LHC} \ldots \ldots \ldots \ldots \ldots$ 
3.1 .2 Beam acceleration and injection . . . . . . . . . . 39

3.1 .3 LHC magnet system . . . . . . . . . . . . . . . 40 40

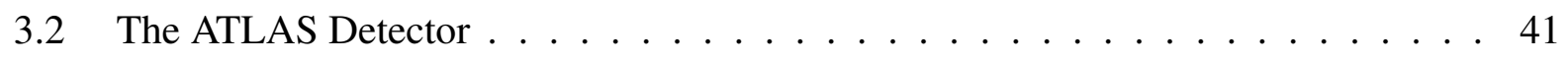

$3.2 .1 \quad$ Inner Detector $\ldots \ldots \ldots \ldots \ldots \ldots \ldots$

$3.2 .2 \quad$ ATLAS Calorimetry $\ldots \ldots \ldots \ldots \ldots \ldots$

3.2.2.1 Electromagnetic Calorimeter $\ldots \ldots \ldots \ldots$

3.2.2.2 Hadronic Calorimeter . . . . . . . . . . . . . . . . 47

3.2 .3 Muon Spectrometer . . . . . . . . . . . . . . . . . . . 49

3.2 .4 ATLAS magnet system . . . . . . . . . . . . . 51

3.2.5 Trigger and Data Acquisition System $\ldots \ldots \ldots \ldots$

3.3 ATLAS Upgrade projects $\ldots \ldots \ldots \ldots \ldots$

4 General physics analysis strategy $\ldots \ldots \ldots \ldots \ldots \ldots$

4.1 Physics object reconstruction . . . . . . . . . . . . . 57

4.1.1 Charged particle tracks and vertices $\ldots \ldots \ldots \ldots \ldots$

4.1 .2 Leptons . . . . . . . . . . . . . . . . . . . 60 60

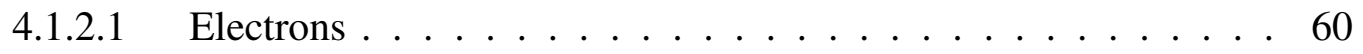

4.1.2.2 Muons ....................61

4.1 .3 Jets ....................... 64

4.1.3.1 Jet reconstruction $\ldots \ldots \ldots \ldots \ldots$

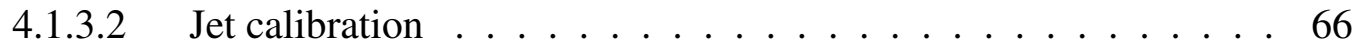

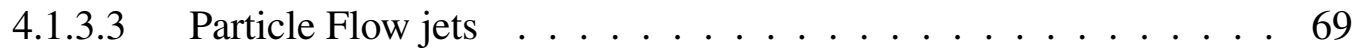

4.1.3.4 Variable radius track jets $\ldots \ldots \ldots \ldots \ldots$

4.1 .4 Jet flavour tagging $\ldots \ldots \ldots \ldots \ldots \ldots \ldots \ldots$

4.1.5 Missing transverse energy $\ldots \ldots \ldots \ldots \ldots$

4.2 Monte Carlo Simulation $\ldots \ldots \ldots \ldots \ldots$

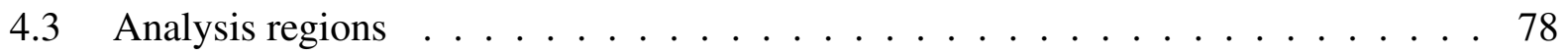


4.4 Statistical analysis and Hypothesis testing . . . . . . . . . . . . . 79

5 Dark Matter and Dark Energy Analyses . . . . . . . . . . . . . . . 86

5.1 mono- $h(b \bar{b})$ dark matter analysis . . . . . . . . . . . . . 86

5.1 .1 Analysis in a nutshell . . . . . . . . . . . . . 87

5.1.2 Characteristics of $2 \mathrm{HDM}+a$ and $\mathrm{Z}^{\prime}-2 \mathrm{HDM}$ models $\ldots \ldots$. . . . . . 91

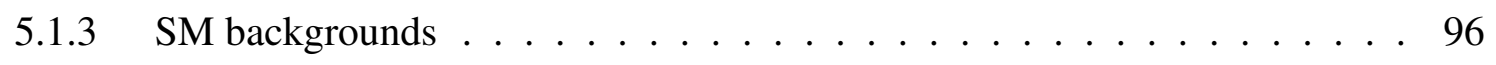

5.1.4 Monte Carlo simulation ................... 100

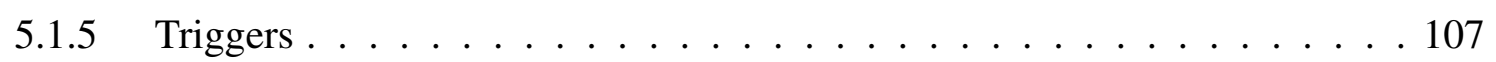

5.1 .6 Physics object definitions $\ldots \ldots \ldots \ldots \ldots$

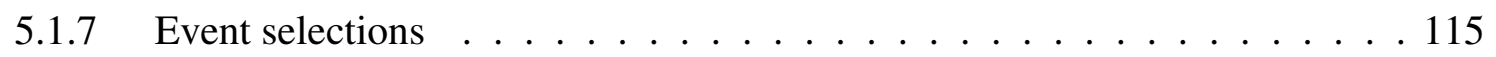

5.1.7.1 0 -lepton signal region . . . . . . . . . . . . 116

5.1.7.2 1-lepton control region ................. 121

5.1.7.3 2-lepton control region .................. 121

5.1.8 Background compositions ...................... 123

5.1 .9 Treatment of $V+$ jets in the analysis $\ldots \ldots \ldots \ldots$

5.1 .10 Systematic uncertainties . . . . . . . . . . . . . . . . . . . . . . . .

5.1.11 Statistical analysis model . . . . . . . . . . . . . 140

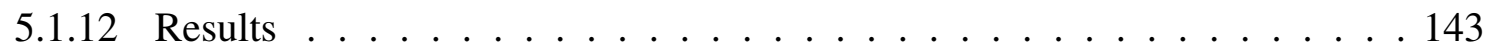

5.2 Dark Energy analysis . . . . . . . . . . . . . . . . . . . 158

5.2 .1 Introduction $\ldots \ldots \ldots \ldots \ldots$

5.2 .2 Dark Energy model . . . . . . . . . . . . . . . 158

5.2.3 Dark Energy model implementation . . . . . . . . . . . . . 159

5.2.4 Signal regions and event selections . . . . . . . . . . . . 161

5.2 .5 Uncertainties .............................. 163

5.2.6 Validity of EFT approximation ................... 164

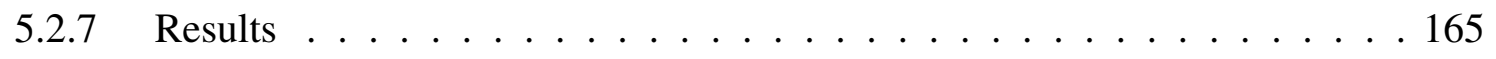


6 Summary, Conclusions and Future prospects . . . . . . . . . . . . . . . . . 168

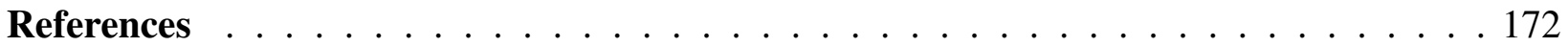

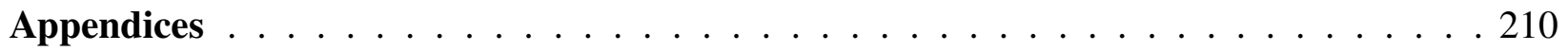

A Combining different background samples . . . . . . . . . . . . . . . . . . 211

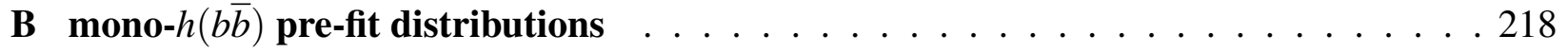

B.1 0-lepton SR . . . . . . . . . . . . . . . . . . . . . . . . . . . . . . . 218

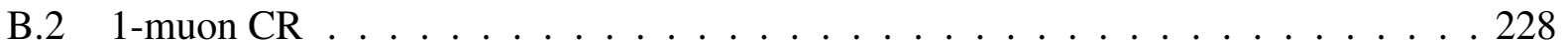

B.3 2-lepton CR . . . . . . . . . . . . . . . . . 235

C Sensitivity improvements with $\geq 3 b$-tag regions . . . . . . . . . . . . . . . . 240

D $m_{h}$ window for mono-higgs model independent limits . . . . . . . . . . . . . . . 241 


\section{LIST OF TABLES}

Table 1. Summary of the fundamental forces in SM interactions, the gauge bosons associated with it and their properties [4]. The QED represents Quantum Electrody-

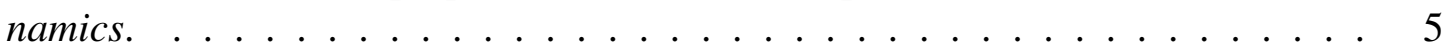

Table 2. Global symmetries and the conserved quantities corresponding to different SM fermionic interaction types. The weak isospin represents a quantum number related to weak interactions and is denoted by $I \ldots \ldots \ldots$. . . . . . . 7

Table 3. Probable DM candidates from different models and their characteristics. . . . . . 23

Table 4. Different types of chambers used in the MS with their locations and functionality. 51

Table 5. Summary of lepton isolation WPs relevant for the thesis, FC: Fixed Cut [132, 133]. . . . . . . . . . . . . . . . . . . . 664

Table 6. Summary of the MC simulations for the SM processes used in the analysis. Abbreviations used: UE: underlying events, LO: Leading-order, NLO: next-toleading order, NNLO: next-to-next-to leading order, NNLL: next-to-next-toleading logarithmic. . . . . . . . . . . . . . . . . . 106

Table 7. Unprescaled $E_{\mathrm{T}}^{\text {miss }}$ triggers used in the analysis corresponding to different data taking periods (sub-periods) and integrated luminosity. . . . . . . . . . 107

Table 8. Unprescaled single-lepton triggers used in the analysis across different datataking periods. The $\mathbf{O R}$ indicates that an event passing any of those specified triggers are recorded. . . . . . . . . . . . . . . . . . . . 109

Table 9. Summary of small-R jet reconstruction criteria used in the analysis. . . . . . . 110

Table 10. Summary of large-R jet reconstruction criteria used in the analysis. . . . . . . . 111

Table 11. Summary of VR track jet reconstruction criteria used in the analysis. . . . . . . 112

Table 12. Summary of $b$-tagging criteria used in the analysis. . . . . . . . . . . . 112

Table 13. Summary of electron reconstruction criteria used in the analysis. The $d_{0}$ and $z_{0}$ are the transverse and longitudinal impact parameters respectively, $d_{0}(\sigma)$ represents the significance of the transverse impact parameter and $\theta$ is the polar angle of the track momentum at reference. . . . . . . . . . . . . . . 113

Table 14. Summary of muon reconstruction criteria used in the analysis. . . . . . . . . 113

Table 15. Summary of tau reconstruction criteria used in the analysis. . . . . . . . . . . 114

Table 16. Summary of $E_{\mathrm{T}}^{\text {miss }}$ reconstruction criteria used in the analysis. . . . . . . . . . 114 
Table 17. Summary of overlap removal criteria for different physics objects used in the analysis. . . . . . . . . . . . . . . . . . 115

Table 18. Summary of muon reconstruction criteria used in the muon-in-jet correction. . . . 119

Table 19. Summary of the selection criteria used in the mono- $h(b \bar{b})$ analysis [234]. DataMC comparisons for these variables are shown in Appendix $B$. . . . . . . 122

Table 20. Table showing the fit results from the comparison of different $Z+$ jets shapes in step 1 of $Z+$ jets shape comparison procedure. . . . . . . . . . . . . 133

Table 21. Table format adapted from [162] summarises different sources of systematic uncertainties considered in the mono- $h(b \bar{b})$ analysis. It also summarises the SM backgrounds affected by different modelling uncertainties. . . . . . . . . . . 137

Table 22. Dominant sources of uncertainties for a few representative $Z^{\prime}-2 H D M$ signal points, the numbers represent fractional uncertainties on the signal strength $(\mu)$

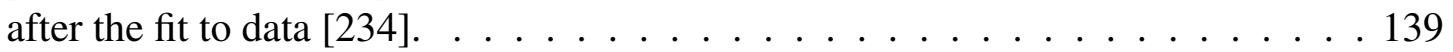

Table 23. Dominant sources of uncertainties for a few representative $2 \mathrm{HDM}+a$ signal points, the numbers represent fractional uncertainties on the signal strength $(\mu)$ after the fit to data $[234] . \ldots \ldots \ldots \ldots \ldots$

Table 24. Table adapted from [234] summarises different analysis regions: lepton regions, $E_{\mathrm{T}}^{\text {miss }}$ bins and $b$-tag regions along with the fit discriminants used in the simulta-

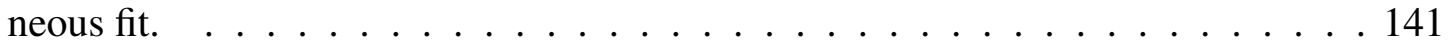

Table 25. Table adapted from [234] summarises the $m_{h}$ binning used in the different fit regions. . . . . . . . . . . . . . . . . . 141

Table 26. Summary of the MC templates used in the analysis for the SM backgrounds. . . . 141

Table 27. Table adapted from [234] summarises the NPs associated to uncertainties affecting the overall normalization of the fit templates. The Gaussian prior uncertainties correspond to the $1 \sigma$ prefit uncertainties. These uncertainties are derived by comparing the $\mathrm{MC}$ samples produced with different $\mathrm{MC}$ generators, while the luminosity prior is calculated by the luminosity group. A flat prior means that there are no prior uncertainties on those NPs. . . . . . . . . . . 142

Table 28. Normalization factors for the three major SM backgrounds obtained from the background-only fit. . . . . . . . . . . . . . . . . . . . 144

Table 29. Pre-fit and Post-fit (background-only) yields for the $1-\mu \mathrm{CR}$ in the $2 b$-tag region across different $E_{\mathrm{T}}^{\text {miss }}$ bins $[234] \ldots \ldots \ldots \ldots \ldots \ldots \ldots \ldots$

Table 30. Pre-fit and Post-fit (background-only) yields for the 1- $\mu$ CR in the $\geq 3 b$-tag region across different $E_{\mathrm{T}}^{\mathrm{miss}}$ bins $[234] . \ldots \ldots \ldots \ldots \ldots$ 
Table 31. Pre-fit and Post-fit (background-only) yields for the combined 2-lepton CR in the $2 b$-tag region across different $E_{\mathrm{T}}^{\text {miss }}$ bins $[234] \ldots \ldots \ldots \ldots \ldots$

Table 32. Pre-fit and Post-fit (background-only) yields for the combined 2-lepton CR in the $\geq 3 b$-tag region across different $E_{\mathrm{T}}^{\text {miss }}$ bins $[234] \ldots \ldots \ldots \ldots \ldots$

Table 33. Pre-fit and Post-fit (background-only) yields for the 0-lepton SR in the $2 b$-tag

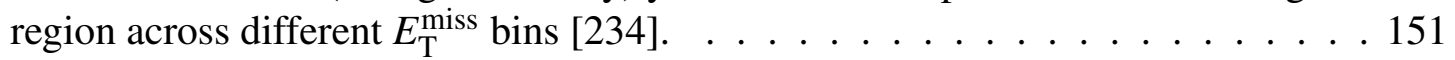

Table 34. Pre-fit and Post-fit (background-only) yields for the 0-lepton SR in the $\geq 3 b$ tag region across different $E_{\mathrm{T}}^{\text {miss }}$ bins $[234] . \ldots \ldots \ldots \ldots \ldots \ldots$

Table 35. Table summarizing the observed (obs) and expected (exp) upper limits at a 95\% $\mathrm{CL}$ on $\sigma_{\mathrm{vis}, h(b \bar{b})+\mathrm{DM}} \equiv \sigma_{h+\mathrm{DM}} \times \mathscr{B}(h \rightarrow b \bar{b}) \times \mathscr{A} \times \varepsilon$ across different analysis

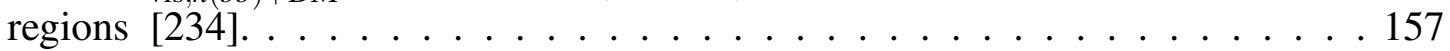

Table 36. Dark energy MC signal samples used for the two operators along with the production cross sections for the two operators [252] . . . . . . . . . . . . 161

Table 37. Selections for 0-lepton channel, details about the variables are described in [254]. 162

Table 38. Selections for 1-lepton channel, details about the variables are described in [255]. 162

Table 39. Selections for 2-lepton channel, details about the variables are described in [256]. 163

Table 40. Acceptance uncertainties for $\mathcal{L}_{1}$ and $\mathcal{L}_{2}$ operators [252]. . . . . . . . . . 163

Table 41. Cross section uncertainties for $\mathcal{L}_{1}$ and $\mathcal{L}_{2}$ operators [252] . . . . . . . . . . 164

Table 42. Expected and observed lower limits on the suppression scale $M$ (in $\mathrm{GeV}$ ) before rescaling (EFT validity criterion not applied). The errors on the expected limits correspond to the uncertainty on the signal production cross section [252]. . . . 165 


\section{LIST OF FIGURES}

Figure 1. Standard Model elementary particles and the force carriers $[5] . \ldots \ldots$

Figure 2. Feynman diagram of a QED vertex for an interaction between a photon and

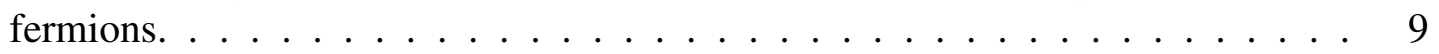

Figure 3. Feynman diagrams of QCD interactions corresponding to, quark and gluon (left) interaction, 3-gluon self-interaction (middle) and 4-gluon self-interaction $($ right $) \ldots \ldots \ldots \ldots \ldots \ldots \ldots \ldots$

Figure 4. Feynman diagram of an EW charged-current interaction vertex. . . . . . . . . 13

Figure 5. Feynman diagrams of an EW neutral-current vertex with photon as the force carrier (left) and $Z$ boson as the force carrier $(r i g h t)$. . . . . . . . . . . . 14

Figure 6. Feynman diagrams of gauge boson self-interaction vertices. . . . . . . . . . . 14

Figure 7. Left plot: Potential with $\mu^{2}>0$ which has a stable minima at $\phi=0$. Right plot: Potential with $\mu^{2}<0$ which does not have a stable equilibrium, instead there is a continuous set of degenerate vacuum states. The Higgs attains a nonzero vacuum expectation value at $\pm v$ which breaks the $S U(2)_{L} \times U(1)_{Y}$ gauge

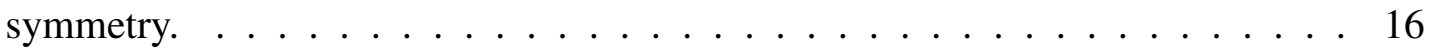

Figure 8. Feynman diagrams for gauge boson-Higgs boson and Higgs boson self-interaction

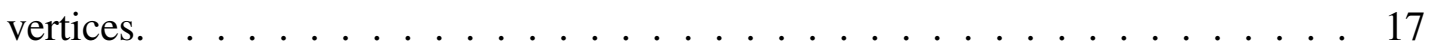

Figure 9. Feynman diagram for fermion-Higgs boson interaction vertex. . . . . . . . . 18

Figure 10. Summary of total cross-section measurements of various SM processes, compared with the theoretical predictions, with Run 1 and Run 2 ATLAS data [17]. . 19

Figure 11. Left plot shows the comparisons between the $m_{W}$ measurements from various experiments and the SM prediction from the global EW fit. Right plot shows the comparisons between the global EW fit and ATLAS measurements with the 2D contour plot of $m_{W}$ and $m_{t}[18] \ldots \ldots \ldots \ldots$. . . . . . . . . . . .

Figure 12. Schematic of different types of DM detection experiments, $\chi$ represents DM

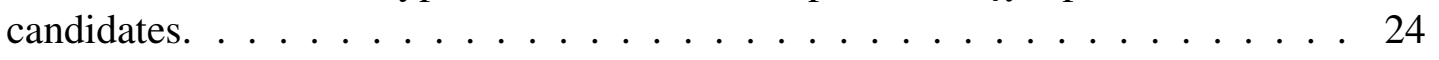

Figure 13. FCNC leads to a Kaon-antikaon oscillation at tree-level. . . . . . . . . . . . . 28

Figure 14. Exclusion limit [56] of the $\cos (\beta-\alpha)-\tan \beta$ plane where the regions are excluded (yellow) at a 95\% CL. The red dashed line is the SM prediction and the cross represents the best fit observed value. . . . . . . . . . . . . 29

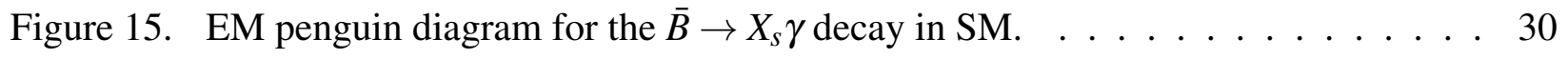


Figure 16. Feynman diagrams of $b b$-initiated (fig. 17b) and ggF (fig. 16b) production mechanisms in $2 \mathrm{HDM}+a$ model with $h+\chi \bar{\chi}$ final state. . . . . . . . . 32

Figure 17. Top panel: Feynman diagrams for $2 \mathrm{HDM}+a$ ggF mechanism; non-resonant (Figure 17a and 17b), Higgs-Strahlung (Figure 17c), single resonant (Figure 17d). Bottom panel: Feynman diagrams for $2 \mathrm{HDM}+a b b$ induced mechanism; non-resonant (Figure 17e and 17f), Higgs-Strahlung (Figure 17g). Figure $17 \mathrm{~h}$ represents the $2 \mathrm{HDM}+a b b$ induced mechanism with additional $b$-jets. . 33

Figure 18. Spin independent one loop $2 \mathrm{HDM}+a$ processes that generate DM-nucleon scattering cross sections in direct detection experiments. . . . . . . . . . 33

Figure 19. Tree level diagrams where DM pairs annihilate to pair of SM fermions (f) or spin-0 states such as $A, a, h, H$. These processes are sensitive to ID dark matter

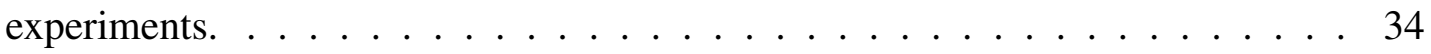

Figure 20. Feynman diagram corresponding to the final state $h+\chi \bar{\chi}$ in the $Z^{\prime}-2$ HDM model. 35

Figure 21. Geographical representation of the LHC: the two big circles represent the LHC accelerator rings and the smaller circles represent the SPS rings. The figure also shows the locations of the four large detectors: ATLAS, CMS, ALICE and LHCb. The whole system is underground with only control rooms at ground level $[68] . \ldots \ldots \ldots \ldots \ldots$

Figure 22. LHC ring schematic: two counter-rotating proton beams marked in blue and red are accelerated in the ring before they collide in four IPs where the four large detectors are located $[67]$. . . . . . . . . . . . . . . . 39

Figure 23. The LHC design layout with the beam injection components [73]. . . . . . . . 40

Figure 24. Cutaway view of the ATLAS detector with different sub-modules [77]. . . . . . 42

Figure 25. Cutaway view of ATLAS inner detector with its sub-modules [79]. . . . . . . 43

Figure 26. Figure shows the three ID modules: the Pixel, SCT and TRT. The red line represents a charged particle of $p_{\mathrm{T}}=10 \mathrm{GeV}$ passing through the modules [79]. . . 44]

Figure 27. Left plot [84]: schematic of a Hadronic shower development which has a Hadronic component and an Electromagnetic component. Right plot [85]: schematic of an Electromagnetic shower development. . . . . . . . . . . . 45

Figure 28. The ATLAS calorimeter system with different sub-systems [87]. . . . . . . . 46

Figure 29. Barrel module of the ATLAS EM calorimeter system with different layers and granularity in $\phi$ and $\eta$ for each layer $[88] . \ldots \ldots \ldots$. . . . . . . . . . . .

Figure 30. Schematic of the Tile calorimeter, the wavelength shifting fibre cables are kept at the edge of the tiles for complete azimuthal coverage and the readout fibres are grouped together into the PMTs $[91] \ldots \ldots \ldots$. . . . . . . . 48 
Figure 31. Schematic of the ATLAS HEC in the R- $\phi$ (left) and R-Z (right) plane, the dashed lines represent the readout cables [92]. . . . . . . . . . . . 48

Figure 32. The ATLAS FCal detector schematic, the black sections represent the cryostats and additional support structures $[93] \ldots \ldots \ldots$

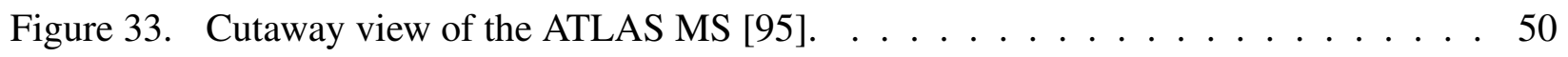

Figure 34. Graphical representation of the ATLAS superconducting magnet system [97]. . . 51]

Figure 35. Schematic adapted from [101] shows the ATLAS TDAQ System with event rates at different stages of the process. In Run 2 the FTK was under commissioning and was not used by the HLT for trigger purposes. . . . . . . . . . 54

Figure 36. The HL-LHC upgrade timeline; the red line shows the collision energy and green line shows the integrated luminosity targets at different upgrade stages [120]. 55

Figure 37. Diagram showing different vertex topologies from a $p p$ collision [127]. . . . . 5 59

Figure 38. A graphical representation of the trajectory of an electron passing through the ATLAS detector. The solid red line represents the electron trajectory and the red dashed line represents the trajectory of a photon, produced through the electron-detector interaction $[128] . \ldots \ldots \ldots$. . . . . . . . . . . . . . .

Figure 39. Jet evolution schematic inside the ATLAS detector: starts from a $p p$ collision followed by partons and hadrons grouped together and eventually depositing energy in the $\mathrm{HCal}[134]$. . . . . . . . . . . . . . . . . . . . 64 64

Figure 40. Figure shows the topo-cluster formation with different $S / N$ criteria across the three HCal layers in the barrel: seed cell (red), neighbour cell (blue) and perimeter cells (green) [136]. . . . . . . . . . . . . . . . . . 65

Figure 41. Jet calibration chain used in ATLAS for small-R and large-R jets [139]. . . . . . 67

Figure 42. ATLAS PFlow jet reconstruction chain $[146] \ldots \ldots \ldots \ldots \ldots$. . . . . . . 71

Figure 43. Figure shows jet structure reconstruction using VR track jets [149]. . . . . . . 72

Figure 44. Schematic of a $b$-jet topology where a $B$-hadron produced at a primary-vertex (red) traverses a microscopic length and forms a displaced secondary-vertex (green) from which additional decay products are produced and forms a $b$-jet. . . 72

Figure 45. Figure 45a shows the $b$-tagging efficiency as a function of jet $p_{\mathrm{T}}$ for different ATLAS $b$-taggers studied with $t \bar{t}$ MC samples. Figure $45 b$ shows the $b$-tagging $\mathrm{SF}$ comparisons between data and $t \bar{t} \mathrm{MC}$ samples for different DL1 $b$-tagger OPs $[150,157] . \ldots \ldots \ldots \ldots \ldots \ldots \ldots$ 
Figure 46. Figure illustrates the different steps of a MC sample generation procedure: hard process (dark red blob), parton shower (red lines), hadronisation (light green ellipses), hadron decays (dark green blob), underlying events (purple blob), unstable particle decay (green lines). The yellow lines represent photon

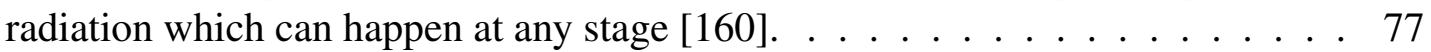

Figure 47. Schematic demonstrating test statistic distributions for the $H_{0}$ and $H_{1}$ hypotheses and the critical region, where the $t_{\text {cut }}$ represents the predefined cut value of the observed test statistic. The parameters $\alpha$ and $\beta$ represent the type-I and type-II errors rates. . . . . . . . . . . . . . . . . . . 80

Figure 48. Schematic [162] demonstrating the exclusion regions associated with a signal hypothesis with a signal strength $\mu$, determined by the $C L_{s}$ values. The blue line represents the $C L_{s}$ values as a function of $\mu$ and the $\mu$ value which crosses the $C L_{s}$ curve at $C L_{s}=0.05$ is the upper limit on $\mu, \mu^{\mathrm{UL}}$. Signal points with $\mu \leq \mu^{\mathrm{UL}}$ are compatible with the observed data and cannot be excluded, while

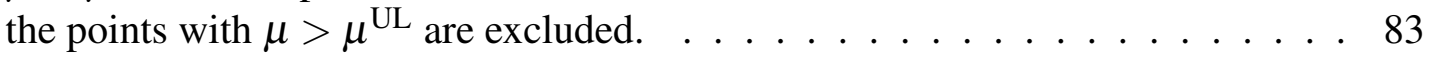

Figure 49. Higgs boson decay BRs and their related uncertainties as a function of the Higgs boson mass [166]. The black vertical line represents the SM Higgs boson mass of $m_{h}=125 \mathrm{GeV}$. . . . . . . . . . . . . . . . 87

Figure 50. Figures show the Resolved (left) and Merged (right) topologies of the mono$h(b \bar{b})$ analysis phase space. The Higgs candidate is reconstructed with $2 b$ tagged central small-R jets in the Resolved topology, while in Merged topology a large-R jet with $2 b$-tagged VR track jets is used. The DM candidates, represented by $\chi$, produce the $E_{\mathrm{T}}^{\text {miss }} \ldots \ldots \ldots \ldots \ldots \ldots$

Figure 51. Classifications of different analysis regions in the mono- $h(b \bar{b})$ phase space. The $2 b$ and $\geq 3 b$ represent the 2 and $\geq 3 b$-tag categories respectively. . . . . 89

Figure 52. The plots show the $m_{h}$ (fig. 52a) and $E_{\mathrm{T}}^{\text {miss }}$ (fig. 52b) distributions in 0-lepton Resolved $2 b$-tag region for different $Z^{\prime}-2$ HDM points and few SM backgrounds. The black dashed lines represent different $E_{\mathrm{T}}^{\text {miss }}$ boundaries for the Resolved regions. . . . . . . . . . . . . . . . . . . 90

Figure 53. Fractional contribution of the $b b$-induced process to the inclusive $2 \mathrm{HDM}+a$ $(b b+\mathrm{ggF})$ cross section as a function of $\tan \beta($ left $)$ and $\sin \theta(r i g h t) . \quad \ldots . . .91$

Figure 54. Cross section as a function of $\tan \beta$ for $b b$-induced (left) and ggF mechanism

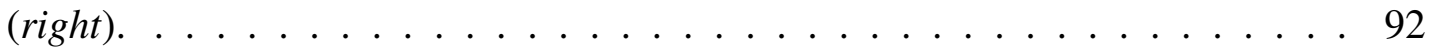

Figure 55. Cross section as a function of $\sin \theta$ for $b b$-induced (left) and $g g F$ mechanism

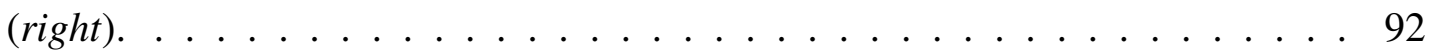

Figure 56. Cross section as a function of $m_{A}$ for $b b$-induced (left) and ggF mechanism

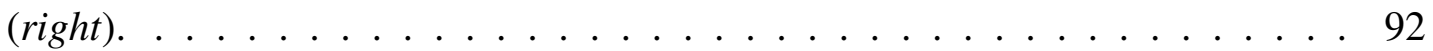


Figure 57. Cross section as a function of $m_{a}$ for $b b$-induced (left) and ggF mechanism

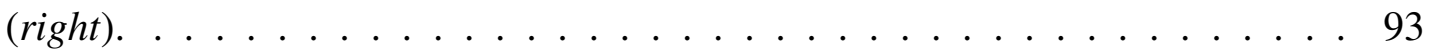

Figure 58. Figures show the different production mechanisms (left plot) and $m_{\chi \bar{\chi}} h$ distributions (right plot) for the $2 \mathrm{HDM}+a \mathrm{ggF}$ process for various $\left(m_{A}, m_{a}\right)$ points. The plots are produced with particle level MC samples. Plot credits to Dr. Andrea Matic.

Figure 59. Figures show the fractional contribution of different production mechanisms for $2 \mathrm{HDM}+a \mathrm{ggF}$ and $b b$-induced processes (left plot) and the associated $m_{\chi \bar{\chi}} h$ distributions (right plot) for $\left(m_{A}, m_{a}\right)=(1300,500) \mathrm{GeV}$ point. The plots are produced with particle level MC samples. Plot credits to Dr. Andrea Matic. . . . 94

Figure 60. $\quad E_{\mathrm{T}}^{\mathrm{miss}}$ distributions for various mass points of $2 \mathrm{HDM}+a \operatorname{ggF}(l e f t)$ and $b b$ induced (right) mechanisms. The Res (Mer) labels indicate that the corresponding point has Resolved (Merged) selections applied. For the Resolved and Merged selections, $E_{\mathrm{T}}^{\text {miss }}<500 \mathrm{GeV}$ and $E_{\mathrm{T}}^{\text {miss }}>500 \mathrm{GeV}$ cuts are removed respectively to obtain the full $E_{\mathrm{T}}^{\text {miss }}$ spectra, and a $\geq 2 b$-jets cut is applied. The black dashed vertical line represents the $E_{\mathrm{T}}^{\text {miss }}=500 \mathrm{GeV}$ point. . . . 95

Figure 61. $E_{\mathrm{T}}^{\text {miss }}$ distribution for the $\mathrm{Z}^{\prime}$-2HDM model for various mass points. The Res (Mer) label indicates that the corresponding point has Resolved (Merged) selections applied. For the Resolved and Merged selections the $E_{\mathrm{T}}^{\text {miss }}<500 \mathrm{GeV}$ and $E_{\mathrm{T}}^{\text {miss }}>500 \mathrm{GeV}$ cuts are removed respectively to obtain the full $E_{\mathrm{T}}^{\text {miss }}$ spectra. A requirement of $\geq 2 b$-jets is applied in both selections. The black dashed vertical line represents the $E_{\mathrm{T}}^{\text {miss }}=500 \mathrm{GeV}$ point. . . . . . . . . 96

Figure 62. Representative Feynman diagrams of the leading SM background processes for mono- $h(b \bar{b})$ analysis. Figure 62a shows the semi-leptonic $t \bar{t}$ decay, while Figures $62 \mathrm{~b}$ and $62 \mathrm{c}$ show the $Z+$ jets and $W+$ jets processes respectively. . . . 98

Figure 63. Representative Feynman diagrams of the sub-leading SM background processes for mono- $h(b \bar{b})$ analysis. Figure 63a shows the single-top $W t$-channel diagram, Figures $63 \mathrm{~b}$ and $63 \mathrm{c}$ show the Diboson and SM $V h(b \bar{b})$ processes respectively. ....................... 99

Figure 64. Representative Feynman diagram of the top-pair production along with a SM Higgs boson decaying to two $b$-quarks. . . . . . . . . . . . . . . . 99

Figure 65. Leading-order QCD Feynman diagrams [191] corresponding to $t \bar{t}$ production at hadron colliders. . . . . . . . . . . . . . . . . . . . . 102

Figure 66. Leading-order QCD Feynman diagrams [191] corresponding to single top production at hadron colliders: $t$-channel as flavor excitation (left) and $W$-gluon fusion (middle), $s$-channel (third) and $W t$-channel (right) production, the $\star$ represents off-shell particles. . . . . . . . . . . . . . . . . . 103 
Figure 67. LO QCD Feynman diagrams corresponding to $V+$ jets production at hadron colliders. . . . . . . . . . . . . . . . . . . . . . . . . . . 104

Figure 68. Leading-order QCD Feynman diagrams [209] corresponding to diboson production at hadron colliders, here $V_{i}=W^{ \pm}, Z$ or $\gamma$. The blue dot represents the Triple Gauge boson Coupling (TGC) vertex. . . . . . . . . . . . . . . . . . 105

Figure 69. Leading-order QCD Feynman diagrams corresponding to SM $V h(b \bar{b})$ production at hadron colliders, here $V=W^{ \pm}$or $Z$ and $V^{\star}=W^{ \pm \star}$ or $Z^{\star}$, the $\star$ represents off-shell particles. . . . . . . . . . . . . . . . 105

Figure 70. LO Feynman diagrams corresponding to $t$ th (left and second), $t \mathrm{t} W$ (third) and $t t Z($ right $)$ production at hadron colliders. . . . . . . . . . . . . . . 106

Figure 71. The $m_{h}$ distributions with (red) and without (blue) the muon-in-jet corrections for a representative $Z^{\prime}-2 \mathrm{HDM}$ signal point in the 0-lepton $E_{\mathrm{T}}^{\text {miss }}$ inclusive Resolved $2 b$-tag region. . . . . . . . . . . . . . . . . . 120

Figure 72. Figures showing a candidate data event in the SR for Resolved (fig. 72a) and Merged (fig. 72b) topologies. The Resolved event contains two $b$-tagged small$R$ jets, represented by the white cone and the cyan lines represent the ID tracks. The Merged event contains a large-R jet (large white cone) with $2 b$-tagged VR track jets inside (cyan lines). In both figures the red line represents the

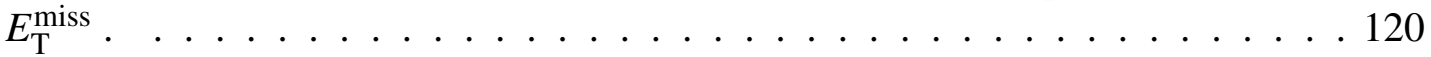

Figure 73. SM Background compositions in different $E_{\mathrm{T}}^{\text {miss }}$ bins in the 0-lepton SR for the $2 b$-tagged regions. . . . . . . . . . . . . . . . . . 124

Figure 74. SM Background compositions in different $E_{\mathrm{T}}^{\text {miss }}$ bins in the 0-lepton SR for the $\geq 3$ b-tagged regions. . . . . . . . . . . . . . . . . 125

Figure 75. SM Background compositions in different $E_{\mathrm{T}}^{\text {miss }}$ bins in the $1-\mu \mathrm{CR}$ for the 2 $b$-tagged regions. . . . . . . . . . . . . . . . 126

Figure 76. SM Background compositions across different $E_{\mathrm{T}}^{\mathrm{miss}}$ bins in the 1- $\mu \mathrm{CR}$ for the $\geq 3$ b-tagged regions. . . . . . . . . . . . . . . . . . 127

Figure 77. SM Background compositions in different $E_{\mathrm{T}}^{\text {miss }}$ bins in the combined 2 lepton CR for the $2 b$-tagged regions. . . . . . . . . . . . . . . . . 128

Figure 78. SM Background compositions in different $E_{\mathrm{T}}^{\text {miss }}$ bins in the combined 2 lepton CR for the $\geq 3 b$-tagged regions. . . . . . . . . . . . . . . . . . 129

Figure 79. SM Background composition of all the backgrounds (left) and only $V+$ jets (right) in the 0-lepton Resolved $2 b$-tagged $150<E_{\mathrm{T}}^{\text {miss }}<200 \mathrm{GeV}$ region. Backgrounds contributing $<3 \%$ to the total are combined in other category. . . 131 
Figure 80. The upper and bottom panel plots represent the $Z_{\text {jets }} / \sum Z_{\text {jets }}$ as a function of $E_{\mathrm{T}}^{\text {miss }}$, and $m_{h}$ respectively in the 0-lepton Resolved region for different $b$ tagged categories. The nature of the flavor decomposition does not change

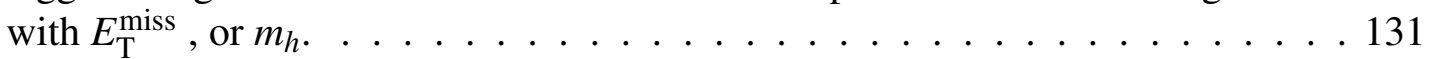

Figure 81. Left plot: compatibility between $Z c c$ and $Z c l$ shapes, the second panel represents the ration between the two flavors, the solid red line represents the linear fit and the third panel (bottom most) represents the normalized residuals from the KS test. Right plot: $Z+$ jets merging template with the most distinct shape: $Z b b+b c+b l, Z c c+c l, Z l l$. The second and third canvas represents the ratio between $Z b b+b c+b l$ and $Z c c+c l$, and the normalized residuals from KS test

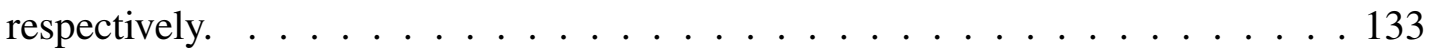

Figure 82. Comparisons between shape driven, previous mono- $h(b \bar{b})$ analysis, and VHbb Resonance merging template. The pink line in Figure $82 b$ represents the linear fit from the shape driven scheme in the Figure 82a. No scheme has a clear preference as shown in Figures $82 \mathrm{a}, 82 \mathrm{~b}$ and $82 \mathrm{c}$. . . . . . . . . . . . 134

Figure 83. The $\mu$-charge distributions in the $1-\mu \mathrm{CR}$ across different $b$-tag categories and $E_{\mathrm{T}}^{\text {miss }}$ bins after the background-only fit. The blue dashed line represents the background prediction from MC before the simultaneous fit. The second canvas shows the ratio between the fitted background prediction and the observed data, and the dashed gray band represents the total MC uncertainty. . . . . . . 145

Figure 84. The total yield in the combined 2-lepton CR across different $b$-tag and $E_{\mathrm{T}}^{\text {miss }}$ regions after the background-only fit. The blue dashed line represents the background prediction from MC before the simultaneous fit. The second canvas shows the ratio between the fitted background prediction and the observed data, and the dashed gray band represents the total MC uncertainty. . . . . . . 147

Figure 85. The Higgs candidate mass distribution in the 0-lepton SR for the $2 b$-tag category across different $E_{\mathrm{T}}^{\text {miss }}$ bins after the background-only fit. The blue dashed line represents the background prediction from MC before the simultaneous fit. The second canvas shows the ratio between the fitted background prediction and the observed data, and the dashed gray band represents the total MC uncertainty. The red dashed line shows an example signal point from the $Z^{\prime}$ 2HDM model, which is scaled by a constant factor to make it visible [234]. . . 149

Figure 86. The Higgs candidate mass distribution in the 0-lepton SR for the $\geq 3 b$-tag category across different $E_{\mathrm{T}}^{\text {miss }}$ bins after the background-only fit. The blue dashed line represents the background prediction from MC before the simultaneous fit. The second canvas shows the ratio between the fitted background prediction and the observed data, and the dashed gray band represents the total MC uncertainty. The red dashed line shows an example signal point from the $2 \mathrm{HDM}+a b b$-induced model, which is scaled by a constant factor to make it visible $[234] . \quad \ldots \ldots \ldots$ 
Figure 87. $\quad E_{\mathrm{T}}^{\mathrm{miss}}$ distributions in the 0-lepton (fig. 87a, 87b), 1- $\mu$ (fig. 87c, 87d), and 2lepton (fig. 87e, 87f) regions for the 2 (left plots) and $\geq 3$ b-tag (right plots) categories across different $E_{\mathrm{T}}^{\text {miss }}$ bins after the background-only fit. The second canvas shows the ratio between the fitted background prediction and the observed data (black dots). The dashed black band represents the total MC uncertainty. The red dashed line in the SR plots shows an example $Z^{\prime}$-2HDM

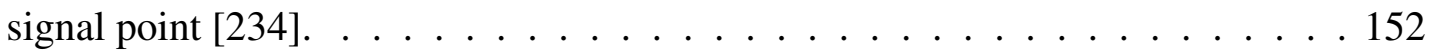

Figure 88. Observed significance against the background-only hypothesis for the signal points of $2 \mathrm{HDM}+a \mathrm{ggF}$ (fig. 88a), 2HDM+a bb-induced (fig. 88b), and $Z^{\prime}$ 2HDM (fig. 88c) [234]. . . . . . . . . . . . . . . . . . . . 153

Figure 89. Exclusion contours for the signal points of $2 \mathrm{HDM}+a$ ggF (Figure 89a), $2 \mathrm{HDM}+a$ $b b$-induced (Figure 89b), and $Z^{\prime}-2 \mathrm{HDM}$ (Figure 89c) models [248]. The solid black line represents the observed limit at a 95\% CL, the dashed black line represents the expected limit, the green and yellow bands represent the $\pm 1 \sigma$ and $\pm 2 \sigma$ uncertainties on the expected limits respectively. The gray area in Figure 89 a represents the exclusion contour obtained from the analysis with $36 \mathrm{fb}^{-1}$ ATLAS dataset [62]. The shaded area in Figure 89a represents the region where the width of at least one of the Higgs bosons $\left(A, H\right.$ or $\left.H^{ \pm}\right)$or of the pseudoscalar $a$ is $>20 \%$ of its mass. In all three plots the parameter space enclosed by the curves are excluded: regions to the left of the curve for $2 \mathrm{HDM}+a$, and regions below the curve for $Z^{\prime}-2 \mathrm{HDM} \ldots \ldots \ldots \ldots$

Figure 90. Figure shows the observed and expected upper limits at a 95\% CL on $\sigma_{\mathrm{vis}, h(b \bar{b})+\mathrm{DM}} \equiv$ $\sigma_{h+\mathrm{DM}} \times \mathscr{B}(h \rightarrow b \bar{b}) \times \mathscr{A} \times \varepsilon$ across different analysis regions [248]. . . . . 157

Figure 91. Feynman diagrams corresponding to the production of a pair of DE particles $(\phi)$ along with $t \bar{t}$ for the $\mathcal{L}_{1}$ (fig. 91a with red vertex) and $\mathcal{L}_{2}$ (fig. 91b with blue vertex) operators. . . . . . . . . . . . . . . 160

Figure 92. Figures show the dependency of $t \bar{t}+\phi \phi$ production cross section (figure 92a) and particle-level $E_{\mathrm{T}}^{\text {miss }}$ (figure $92 \mathrm{~b}$ on $m_{\phi}$ for the $\mathcal{L}_{1}$ operator without any

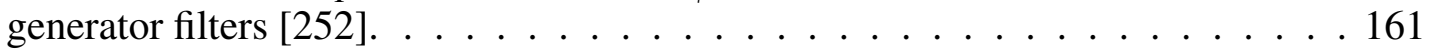

Figure 93. Exclusion plots for $\mathcal{L}_{1}$ (left) and $\mathcal{L}_{2}$ (right) operators in 0-lepton channel. The errors on the predicted cross sections correspond to the scale and PDF uncertainties. Suppression scale $(M)$ values to the left of the crossings of solid black-red $\left(\mathcal{L}_{1}\right)$ and solid black-blue $\left(\mathcal{L}_{2}\right)$ lines are excluded. Figure credits to Dr. Spyridon Argyropoulos. . . . . . . . . . . . . . . . . . . . . 166

Figure 94. Exclusion contour in $\left\{g_{*}, M\right\}$ plane for $\mathcal{L}_{1}($ left $)$ and $\mathcal{L}_{2}$ (right) operators in 0lepton channel, after taking into account the EFT validity criterion. Parameter points enclosed by the curve, shown by red hashed lines, are excluded. Figure credits to Dr. Spyridon Argyropoulos. . . . . . . . . . . . . . . . . . . . . . . 166 


\section{INTRODUCTION}

Understanding our universe, its constituent parts, and the forces that govern the interactions have always been a topic of interest to physicists. From the discovery of electrons in 1897 to the Higgs boson in 2012, a plethora of elementary particles have been discovered. The Standard Model (SM) of particle physics has been immensely successful in describing the newly discovered particles and their interactions. However, SM alone is not sufficient to describe the universe. One of the shortcomings of SM is its failure to explain the dark sector of the universe. It has been observed that about $95 \%$ of the universe consists of Dark Matter (DM) and Dark Energy (DE). This thesis presents searches for DM and DE with the ATLAS detector at the LHC.

The primary focus of the thesis is the DM search. Astrophysical observations suggest that the DM does not interact through strong or electromagnetic force. Hence, DM particles would interact very weakly with the detector and would escape undetected. This will induce a momentum imbalance in the transverse plane of the plane where interactions are taking place, also known as the missing transverse energy ( $E_{\mathrm{T}}^{\text {miss }}$ ). Since at the $\mathrm{LHC}$ the $E_{\mathrm{T}}^{\text {miss }}$ can only be evaluated in the presence of visible SM particles, the DM searches are performed in association with SM particles that can be detected at the LHC. The DM analysis described in this thesis searches for DM produced in association with a SM Higgs boson, that decays to two $b$-jets. The final state consists of $E_{\mathrm{T}}^{\text {miss }}$ coming from the undetected DM particles and two $b$-jets from the SM Higgs decay, known as the mono-Higgs channel. The search uses the full Run 2 dataset of $139 \mathrm{fb}^{-1}$ recorded by the ATLAS detector, and the results are interpreted in terms of the Two-Higgs Doublet Models (2HDM). The 2HDM models are related to the so-called Higgs portal models where the DM particles interact only with the Higgs sector. A statistical analysis based on a binned profile-likelihood method is performed to obtain the final results.

Similar to the DM searches, DE searches at the LHC are also model dependent and a significant amount of $E_{\mathrm{T}}^{\mathrm{miss}}$ in the final state indicates the presence of DE. The second analysis described is a DE-search in a $t \bar{t}+E_{\mathrm{T}}^{\text {miss }}$ final state. The analysis is a reinterpretation of a search for top super- 
partners in the $t \bar{t}+E_{\mathrm{T}}^{\text {miss }}$ final state. The analysis is based on an Effective Field Theory (EFT) based DE model in which the DE-SM interactions and kinematic properties of DE are characterized by operators that preserve a specific symmetry. A profile-likelihood statistical analysis is performed to obtain the final results. This is the first DE analysis [1] from collider experiments, albeit a reinterpretation of a different search.

At the end of Run 2, the total dataset available for physics analysis is $139 \mathrm{fb}^{-1}$, but there are plans to upgrade the detector components and introduce new detectors to increase luminosity. The High-Luminosity LHC (HL-LHC), scheduled to start in 2027, is expected to achieve a peak luminosity of $7.5 \times 10^{34} \mathrm{~cm}^{2} \mathrm{~s}^{-1}$ and an integrated luminosity of $4000 \mathrm{fb}^{-1}$. To cope with the high luminosity a High Granularity Timing Detector (HGTD) with 30-50 ps timing resolution is proposed to be added to the ATLAS detector. Silicon Low Gain Avalanche Detector (LGAD) sensors are to be used at the HGTD. The sensors are tested through beam tests at CERN and other laboratories. A part of my ATLAS authorship qualification task was to develop software to communicate between the sensors and an oscilloscope, used in the beam tests. The second part of my qualification task was to develop a database where the sensor test results are to be stored. 


\section{THEORETICAL FRAMEWORK}

Richard Feynman once said “It doesn't matter how beautiful your theory is, it doesn't matter how smart you are. If it doesn't agree with experiment, it's wrong”. Therefore, theoretical predictions are required to be verified with experimental observations. The foundation of particle physics was laid by the Greek philosopher Democritus in $400 \mathrm{BC}$ when he postulated that all particles have a constituent fundamental unit. He named this basic unit atomo, which means indivisible and is known as atom. According to his theory, the atoms are completely solid with no internal structure and the atomic dimensions vary for different objects. Although the theory was revolutionary at that time it was ultimately proven wrong when J.J Thompson discovered electrons in 1898. In 1917 Ernest Rutherford with the famous gold foil experiment discovered nucleus with positively charged particles protons; James Chadwick discovered the neutral particle neutron in 1932. The atomic model was completed in the quantum mechanics framework by Neils Bohr and then Erwin Schrödinger in the early 20th century and has evolved over the years with experimental observations providing pathways.

When highly energetic cosmic particles such as protons enter the Earth's atmosphere, they interact with the particles on Earth and create rich cosmic showers of particles, such as muons, pions, etc. With the advancement of experimental technologies, a plethora of particles were observed from cosmic rays. To categorize these many newly discovered particles and understand their characteristics a new theoretical model was required. In the 1960s and 1970s physicists came up with a theory that could explain the characteristics and interaction properties of these particles. Different parts of the model were developed by many physicists and the collection is now commonly known as the Standard Model (SM) of particle physics. Although the SM has been very successful, it is not complete. This chapter gives an overview of the SM, its triumphs, shortcomings, an introduction to dark matter and dark energy, the subject matter of the thesis. 


\subsection{The Standard Model of particle physics}

The SM [2, 3] of particle physics is a relativistic quantum field theory based on the gauge symmetry group $S U(3)_{C} \times \mathrm{SU}(2)_{L} \times \mathrm{U}(1)_{Y}$. It provides a field theoretical description of the three fundamental forces in nature: strong, weak and electromagnetic force. The $\mathrm{SU}(3)_{C}$ group represents the strong force which is described by Quantum Chromodynamics (QCD) where the color charge (C) is conserved. The $S U(2)_{L} \times \mathrm{U}(1)_{Y}$ represents the unified weak and electromagnetic (EM) force, the electroweak (EW) sector . Here $L$ indicates that the weak force acts on left-handed particles only, and $Y$ represents the conserved weak hypercharge. In addition to this, there is a SU(2) doublet corresponding to the single scalar Higgs field. The following points encapsulate the critical aspects of the SM:

- The SM encompasses three of the four fundamental forces in nature: strong, weak, and EM force.

- All matter is composed of fundamental particles called fermions which interact with each other through the exchange of force carriers called bosons.

- The interaction force dynamics are described by a gauge theory with $S U(3)_{C} \times \mathrm{SU}(2)_{L} \times \mathrm{U}(1)_{Y}$ symmetry.

- The fermions and gauge particles get their masses by interacting with the Higgs field, known as the Higgs mechanism.

Besides explaining the phenomena observed in elementary particles, the SM transcends to larger distances such as atomic and nuclear scales, forming the basis of chemical, biological and other sciences.

\subsubsection{Elementary particles and their interactions}

All the observed elementary particles are classified into two groups: fermions and bosons. Fermions have half-integral spin $(s \in[1 / 2,3 / 2, \ldots])$ and bosons have integral spin $(s \in[0,1,2, \ldots])$ 
quantum numbers. All the observed stable particles in the universe consist of fermions. The fermions are further divided into two categories: leptons and quarks. Six leptons and six quarks have been observed thus far which are grouped into three generations: first, second and third. Properties of the fermions in each generation are similar but for the masses.

The quarks cannot stay in a free state due to color confinement and form composite particles by combining with two or three quarks. These composite particles are known as hadrons. Depending on the number of quarks the hadrons are grouped into two categories: baryons and mesons. The baryons have half-integer spin and consist of three quarks (or anti-quarks for anti-baryons), such as proton $(u u d)$, neutron $(u d d)$ etc. The mesons have spin 1 or 0 and consist of a quark and anti-quark pair, such as neutral pion $\left(\pi^{0}: u \bar{u}\right)$, neutral kaon $\left(k^{0}: d \bar{s}\right)$, charged pion $\left(\pi^{+}: u \bar{d}\right)$ etc.

Each type of particle interaction involves a gauge boson exchange. The mediators in strong interactions are gluons, which are massless and electrically neutral. The photon $(\gamma)$ is the gauge boson for the EM interactions, while $W^{ \pm}$and neutral $Z$ bosons are the gauge bosons for weak interaction. Table 1 summarizes different forces, their scales, and properties of the gauge bosons associated with the interactions.

\begin{tabular}{|c|c|c|c|}
\hline Characteristics & Strong & EM & Weak \\
\hline \hline Strength & 1 & $1 / 137$ & $10^{-7}$ \\
\hline Range & $10^{-13} \mathrm{~cm}$ & $\infty$ & $10^{-16} \mathrm{~cm}$ \\
\hline Acts on & color & $\begin{array}{c}\text { electric } \\
\text { charge }\end{array}$ & flavour \\
\hline Force carrier & gluon $(g)$ & photon $(\gamma)$ & $W^{ \pm}$and $Z$ \\
\hline Force carrier mass $[\mathrm{GeV}]$ & 0 & 0 & 80.4 and 91.2 \\
\hline Charge & 0 & 0 & \pm 1 and 0 \\
\hline Spin number & 1 & 1 & 1 \\
\hline Theory & QCD & QED & EW \\
\hline
\end{tabular}

Table 1: Summary of the fundamental forces in SM interactions, the gauge bosons associated with it and their properties [4]. The QED represents Quantum Electrodynamics.

The masses of the force carriers and the interaction lengths are correlated. Since the photon is massless, the EM interaction range goes up to infinity, but the interaction strength falls with distance $r$ as $1 / r^{2}$. Electrically charged particles can interact with each other through an exchange 
of a photon and the interaction strength is characterized by the fine structure $\alpha=\frac{e^{2}}{4 \pi \varepsilon_{0} \hbar c}=1 / 137$. The strong interactions, on the other hand, are mediated by gluons, have a much stronger coupling constant $\alpha_{S} \approx 1$, and the interaction range is limited to inside the atomic nucleus. While the EM force acts on an electric charge, the strong force interacts through color charge. Each quark (antiquark) can carry three possible colors (anti-colors): red $(r)$, green $(g)$ and blue $(b)$ and each gluon carries a color and an anti-color which can produce $3 \times 3=9$ gluon states. However, one color combination gives a colorless gluon: $\frac{1}{\sqrt{3}}(|r \bar{r}\rangle+|g \bar{g}\rangle+|b \bar{b}\rangle)$. Hence, there are eight orthogonal gluon states which forms the color octet. For each strong interaction, the color is conserved at each interaction vertex like the electric charge in EM interaction. The weak interaction is mediated by either a neutral $Z$ boson or one of the two charged $W^{ \pm}$bosons. The weak interaction is extremely short range because the $W^{ \pm}$and $Z$ bosons are massive. Figure 1 shows all the elementary particles along with force carriers for the three fundamental forces described above.

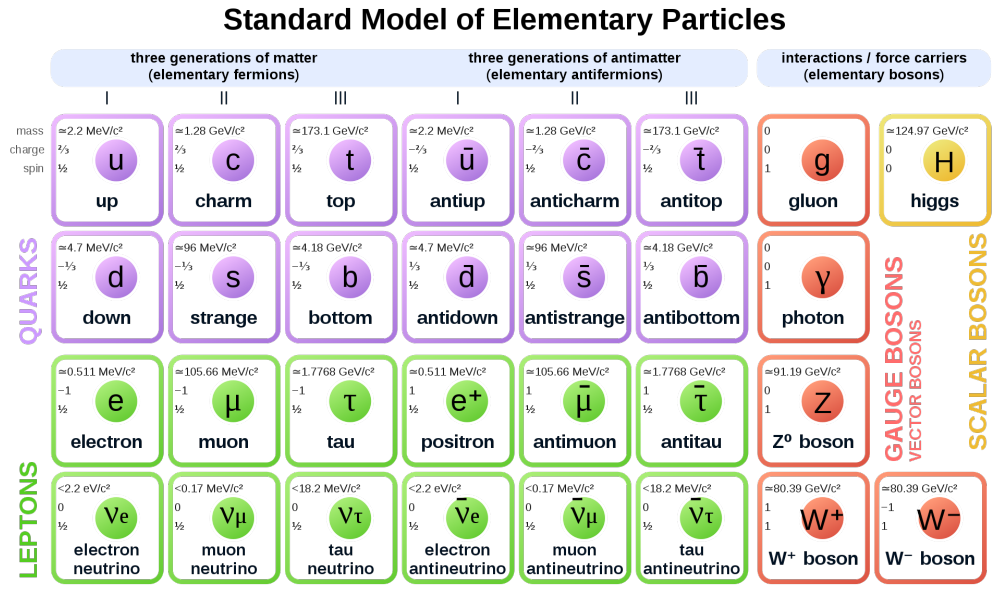

Figure 1: Standard Model elementary particles and the force carriers [5].

\subsubsection{Symmetry and Gauge fields}

The SM is based on a relativistic Quantum Field Theory (QFT). The quantum fields have an underlying symmetry called gauge symmetry. In a field theory framework, the basis is formed by a field, e.g., a complex scalar field $\phi(x)$ and the particles are represented as excited states of the field. The properties of the field are described by a Lorentz invariant Lagrangian, a function of 
the field and its derivative $\mathcal{L}=\mathcal{L}\left(\phi, \partial_{\mu} \phi\right)$. The space-time evolution of the field is described by $\mathcal{S}$, defined as:

$$
\mathcal{S}=\int d^{4} x \mathcal{L}
$$

Using the principle of least action one can write the Euler-Lagrange equation, which describes the equation of motion for the field $\phi$ :

$$
\frac{\delta \mathcal{S}}{\delta \phi}=\frac{\delta \mathcal{L}}{\delta \phi}-\partial_{\mu} \frac{\delta \mathcal{L}}{\partial\left(\partial_{\mu} \phi\right)}=0
$$

where $\delta \mathcal{S}$ represents the variation of $\mathcal{S}$ for an arbitrary perturbation $\delta \phi$ of the field $\phi$. The essence of equation 2 is that for any given field $\phi$, the $\delta \mathcal{S}$ vanishes for an arbitrary perturbation $\delta \phi$ given that $\phi$ satisfies the equation of motion. In a more physical language for a field transformation $\phi \rightarrow \phi+\delta \phi$, for which $\mathcal{L}$ is Lorentz invariant, $\delta \mathcal{L}=0$ implies that the Lagrangian is symmetric under the transformation. According to Noether's theorem, for every differentiable symmetry of the action of any physical system there is a corresponding conservation law. In the SM each type of fermionic interaction is associated with a gauge symmetry, which is invariant under local gauge transformations, corresponding to a conserved quantity as listed in Table 2 .

\begin{tabular}{|c|c|c|}
\hline Interaction & Symmetry & Conserved quantity \\
\hline \hline EM & $U(1)$ & electric charge \\
\hline strong & $S U(3)_{C}$ & color \\
\hline Weak & $S U(2)_{L}$ & weak isospin \\
\hline
\end{tabular}

Table 2: Global symmetries and the conserved quantities corresponding to different SM fermionic interaction types. The weak isospin represents a quantum number related to weak interactions and is denoted by $I$.

\subsubsection{Electromagnetic force and Quantum Electrodynamics}

The theory of Quantum Electrodynamics (QED) describes the EM interaction between fermions and is invariant under a local $U(1)$ symmetry. The Dirac Lagrangian for a fermion of mass $m$ in a complex field $\psi$ can be written as,

$$
\mathcal{L}_{D}=\bar{\psi}\left(i \gamma^{\mu} \partial_{\mu}-m\right) \psi
$$


where $\gamma^{\mu}$ are the Dirac matrices, satisfying the anti-commutation relationship $\left\{\gamma^{\mu}, \gamma^{\nu}\right\}=2 g^{\mu \nu}$, and $\bar{\psi}=\psi^{\dagger} \gamma^{0}$, with $\psi^{\dagger}$ representing the Hermitian adjoint of the field $\psi$. The Lagrangian is invariant under a global $U(1)$ symmetry, where the field transforms as:

$$
\psi(x) \rightarrow e^{-i \alpha Q} \psi(x)
$$

Here $\mathrm{Q}$ is the electric charge and $\alpha$ is an arbitrary real number. However, $\mathcal{L}_{D}$ is not invariant under a local gauge symmetry, i.e., when the transformation of $\alpha$ has a space-time dependency $\alpha \rightarrow \alpha(x)$. To make $\mathcal{L}_{D}$ invariant under local gauge transformations a covariant derivative $D_{\mu}$ is introduced:

$$
D_{\mu}=\partial_{\mu}+i Q A_{\mu}(x)
$$

In an attempt to make the Dirac Lagrangian invariant to local gauge transformations a new vector field $A_{\mu}$ is introduced. This field is known as the gauge field and it transforms as:

$$
A_{\mu}^{\prime}=A_{\mu}+\partial_{\mu} \alpha(x)
$$

Replacing the partial derivative $\partial_{\mu}$ in $\mathcal{L}_{D}$ (equation 3 by the covariant derivative $D_{\mu}$ from equation 5 one can write

$$
\mathcal{L}=\mathcal{L}_{D}-Q \bar{\psi}(x) \gamma^{\mu} A_{\mu} \psi(x)
$$

With the inclusion of the covariant derivative, the Lagrangian becomes invariant but loses its freedom. The extra term $Q \bar{\psi}(x) \gamma^{\mu} A_{\mu} \psi(x)$ represents the interaction between a fermion and a photon. The kinematic $\left(\mathcal{L}_{\text {kin }}\right)$ and mass $\left(\mathcal{L}_{\gamma}\right)$ terms for the photon can be written as:

$$
\mathcal{L}_{k i n}=-\frac{1}{4} F_{\mu v} F^{\mu v}, \quad \mathcal{L}_{\gamma}=\frac{1}{2} m_{\gamma}^{2} A_{\mu} A^{\mu} \quad \text { where } F_{\mu \nu}=\partial_{\mu} A_{v}-\partial_{v} A_{\mu}
$$

Here $F_{\mu \nu}$ is the field tensor, which is gauge invariant under the transformation of $A_{\mu}$. However, the presence of the mass term breaks the local gauge invariance:

$$
\frac{1}{2} m_{\gamma}^{2} A_{\mu}^{\prime} A^{\prime \mu} \rightarrow \frac{1}{2} m_{\gamma}^{2}\left(A_{\mu}+\partial_{\mu} \alpha(x)\right)\left(A^{\mu}+\partial^{\mu} \alpha(x)\right) \neq \frac{1}{2} m_{\gamma}^{2} A_{\mu} A^{\mu}
$$

Hence, the photon field is massless and the full QED Lagrangian can be written as: 


$$
\mathcal{L}_{Q E D}=\mathcal{L}_{D}-Q A_{\mu} \bar{\psi}(x) \gamma^{\mu} \psi(x)-\frac{1}{4} F_{\mu v} F^{\mu \nu}
$$

The term $\bar{\psi}(x) \gamma^{\mu} \psi(x)$ is also known as the Noether current $J_{\mu}$ associated with the $U(1)$ symmetry and the conserved charge is represented by $Q$ :

$$
J^{\mu}=\bar{\psi}(x) \gamma^{\mu} \psi(x), \quad Q=\int d^{3} x J^{0}=\int d^{3} x \bar{\psi}^{\dagger}(x) \psi(x)
$$

The interaction term between a photon and fermion, $Q \bar{\psi}(x) \gamma^{\mu} A_{\mu} \psi(x)$, can be visualized by the Feynman diagram shown in Figure 2

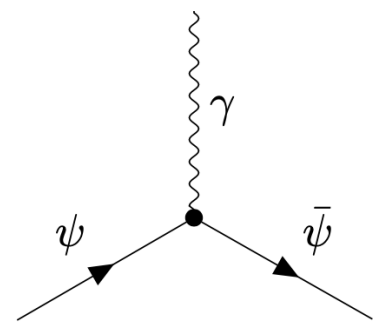

Figure 2: Feynman diagram of a QED vertex for an interaction between a photon and fermions.

\subsubsection{Strong force and Quantum Chromodynamics}

The strong interaction is described by Quantum Chromodynamics (QCD), which is a gauge theory based on $S U(3)_{C}$ symmetry with the color being the conserved quantity. The same technique used to derive the QED Lagrangian can be applied to obtain the QCD Lagrangian:

(i) Find the global symmetry of a free Lagrangian and transform the global symmetry to a local symmetry by replacing the $\partial_{\mu}$ with the covariant derivative $D_{\mu}$ and introducing a vector field.

(ii) Evaluate the kinematic terms of the newly added vector field.

The $S U$ (3) group has 8 Hermitian generators $\left(T^{a}\right)$ which can be expressed in terms of Gell-Mann matrices $\left(\lambda^{a}\right)[6]$ and follow a commutation relationship as shown in the following equations:

$$
T^{a}=\lambda^{a} / 2, \quad \text { where } a \in\{1, \ldots, 8\} \quad \text { and } \quad\left[T^{a}, T^{b}\right]=i f^{a b c} T^{c}
$$

where $f^{a b c}$ is known as the structure constant of the group. The gluons, which are the gauge bosons for the strong interactions, are represented by eight distinct vector fields denoted as $G_{\mu}^{a}(x)$, while 
the QCD covariant derivative is given as:

$$
D_{\mu}=\partial_{\mu}-i g_{s} \frac{\lambda^{a}}{2} G_{\mu}^{a}
$$

Similar to the photon field in QED, using the gluon fields and covariant derivatives one can write the kinetic term of the gluon vector fields as:

$$
G_{\mu \nu}^{a}=\left[\partial_{\mu} G_{v}^{a}-\partial_{\nu} G_{\mu}^{a}+g_{s} f^{a b c} G_{\mu}^{b} G_{v}^{c}\right] .
$$

With all these ingredients one can write the QCD Lagrangian as:

$$
\mathcal{L}_{Q C D}=\sum_{q} \bar{q}\left(i \gamma^{\mu} \partial_{\mu}-m_{q}\right) q-g_{s} \sum_{q}\left(\bar{q} \gamma^{\mu} \frac{\lambda_{a}}{2} q\right) G_{\mu}^{a}-\frac{1}{4} G_{\mu v}^{a} G^{a, \mu v}
$$

where the summation $q$ runs over six quarks. The first term describes the mass and kinetic energy of the quarks, the second term represents the interaction between quark and gluon and contains the quark current $J_{q}^{a, \mu}=-g_{s} \bar{q} \gamma^{\mu} \frac{\lambda_{a}}{2} q$, analogous to Noether's current in $U(1)$ symmetry, and the third term represents the gluon-gluon self-interactions. Unlike photons in EM interactions, the gluons can have self-interactions, resulting in interaction vertices with 3 or 4-gluons, as shown in Figure 3 .
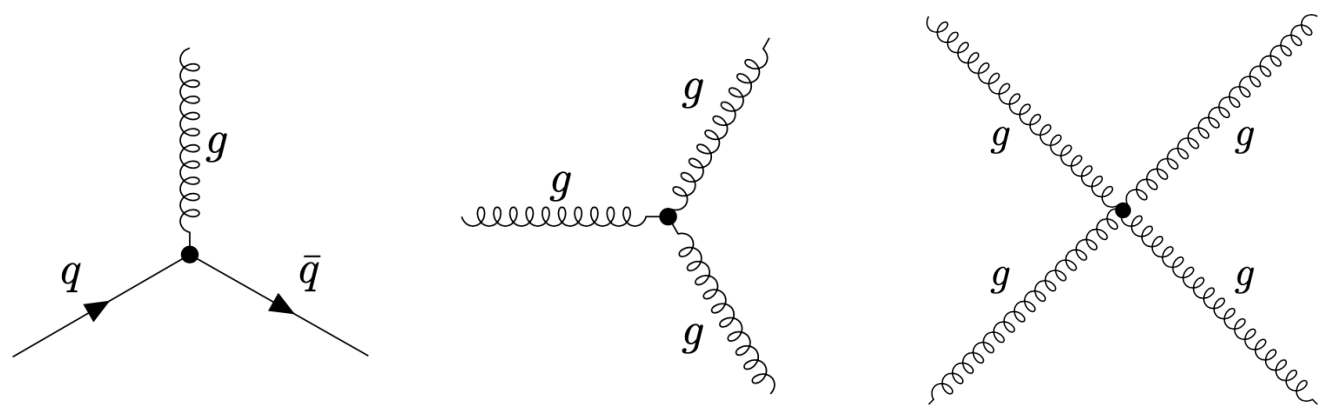

Figure 3: Feynman diagrams of QCD interactions corresponding to, quark and gluon (left) interaction, 3-gluon self-interaction (middle) and 4-gluon self-interaction (right).

The distinct feature of the strong interaction is the asymptotic freedom. This property makes the interaction strength between the quarks and gluons asymptotically weaker with increasing energy scales $\left(Q^{2}\right)$ and decreasing distances, i.e. $\alpha_{s} \rightarrow 0$ as $Q^{2} \rightarrow \infty$. It becomes obvious by expressing the strong interaction coupling constant $\alpha_{s}$ in terms of the QCD scale parameter $\Lambda$ :

$$
\alpha_{s}\left(Q^{2}\right) \propto \frac{1}{\ln \left(Q^{2} / \Lambda^{2}\right)} .
$$


The interpretation of the scale parameter is that it acts as a boundary between quasi-free quarks and gluons and hadrons in bound states. At high energy level, it is easier to have a quark anti-quark pair-production than to pull apart two quarks. As a consequence the initial quarks form hadrons, and this phenomenon is known as quark confinement.

\subsubsection{Weak force and Electroweak unification}

With the success of QED and QCD, it was tempting to come up with similar theories to explain the weak interactions. However, with extensive studies from various physics processes such as the $\beta$-decay $\left(n \rightarrow p+e^{-}+\bar{v}_{e}\right)$, muon decay $\left(\mu^{-} \rightarrow e^{-}+\bar{v}_{e}+v_{\mu}\right)$, it became obvious that the weak interaction has many differences with QED. The weak interaction has two categories: charged current and neutral current interactions. The electrically charged weak interaction has unique features such as change of quark flavour (e.g., $u \rightarrow d$ ), Charge-Parity (CP) violation, and much heavier force carriers compared to EW interaction. Besides, the charged current interactions involve left-handed (LH) fermions only, while neutral current interactions involve both LH and right-handed (RH) fermions. The electroweak field has the $S U(2)_{L} \times S U(1)_{Y}$ symmetry, where $L$ indicates that the $S U(2)_{L}$ gauge fields couple to $\mathrm{LH}$ fermions only and $Y$ refers to weak hypercharge. The RH fermions are $S U(2)$ singlets and do not undergo weak interactions. The weak force and electromagnetic theories were combined into a unified theory, known as the electroweak (EW) theory or GWS (Glashow-Weinberg-Salam) theory [7-9] for which Nobel prize was awarded to Glashow, Weinberg, and Salam in 1979.

The cornerstone of EW theory [10] is the fact that it is a chiral theory, i.e., it distinguishes between LH and RH fermions and the corresponding fields are denoted by:

$$
\psi_{L}=\frac{1}{2}\left(1-\gamma_{5}\right) \psi \quad \text { and } \quad \psi_{R}=\frac{1}{2}\left(1+\gamma_{5}\right) \psi
$$

These fields can also be categorized according to weak isospin number $I, I_{3}$ ( $Z$ component of $I$ ), and weak hypercharge $Y$. The LH fermionic fields have $I=1 / 2$, hence forms doublets with one part having $I=+1 / 2$ and the other with $I=-1 / 2$. On the other hand, the RH fermionic fields have $I=0$ and form singlets. The doublets can have two types of pairs: (i) a charged lepton and its 
associated neutrino (ii) up-down quark pair. There are no RH neutrinos in SM, hence no neutrinos in the singlets. The following are the doublet and singlet structures:

$$
\left(\begin{array}{l}
u \\
d
\end{array}\right)_{L},\left(\begin{array}{l}
c \\
s
\end{array}\right)_{L},\left(\begin{array}{l}
t \\
b
\end{array}\right)_{L},\left(\begin{array}{c}
v_{e} \\
e^{-}
\end{array}\right)_{L},\left(\begin{array}{c}
v_{\mu} \\
\mu^{-}
\end{array}\right)_{L},\left(\begin{array}{c}
v_{\tau} \\
\tau^{-}
\end{array}\right)_{L}, u_{R}, d_{R}, c_{R}, s_{R}, t_{R}, b_{R}, e_{R}^{-}, \mu_{R}^{-}, \tau_{R}^{-} .
$$

The weak hypercharge $Y$ is a conserved quantum number and can be expressed in terms of electric charge $Q$ and isospin $I_{3}$, which is known as the Gell-Mann-Nishijima scheme: $Q=I_{3}+\frac{Y}{2}[11$, 12]. The free Lagrangian for fermionic fields can be written as:

$$
\mathcal{L}_{0}=i \bar{u}(x) \gamma^{\mu} \partial_{\mu} u(x)+i \bar{d}(x) \gamma^{\mu} \partial_{\mu} d(x)=\sum_{j=1}^{3} i \bar{\psi}_{j}(x) \gamma^{\mu} \partial_{\mu} \psi_{j}(x)
$$

To have local gauge invariant $U(1)_{Y}$ and $S U(2)_{L}$ groups, one requires an additional vector field $B_{\mu}$ associated with the $U(1)_{Y}$ group, along with the field tensor $B_{\mu \nu}$ and a triplet auxiliary gauge filed $W_{\mu}^{i}$ associated with the $S U(2)_{L}$ group with the field tensor $W_{\mu \nu}^{i}$.

$$
\begin{aligned}
B_{\mu \nu} & =\partial_{\mu} B_{v}-\partial_{\nu} B_{\mu} \\
W_{\mu \nu}^{i} & =\partial_{\mu} W_{v}^{i}-\partial_{\nu} W_{\mu}^{i}+g_{W} \varepsilon^{a b c} W_{\mu}^{j} W_{v}^{k} \quad \text { where } a=1,2,3
\end{aligned}
$$

where $g_{W}$ is the coupling constant of $S U(2)_{L}$ and $\varepsilon^{a b c}$ is the $S U(2)$ structure constant. The $W_{\mu \nu}^{i}$ has similar structure as the $G_{\mu \nu}$ in QCD (equation 14). The covariant derivatives are given as:

$$
\begin{aligned}
D_{\mu, L} & =\left[\partial_{\mu}-i g_{W} \frac{\sigma_{i}}{2} W_{\mu}^{i}(x)-i g_{Y} \frac{Y}{2} B_{\mu}(x)\right] \\
D_{\mu, R} & =\left[\partial_{\mu}-i g_{Y} \frac{Y}{2} B_{\mu}(x)\right]
\end{aligned}
$$

where $g_{Y}$ is the coupling constant of $U(1)_{Y}$ and $\sigma_{i}$ are the Pauli matrices. The free Lagrangian in equation 18 , becomes invariant under local gauge transformation with the introduction of the covariant derivative $D_{\mu}$. The kinematic terms can be written as:

$$
\mathcal{L}_{k i n}=-\frac{1}{4} B_{\mu v} B^{\mu v}-\frac{1}{4} W_{\mu v}^{i} W_{i}^{\mu v}
$$

It is evident from $W_{\mu \nu}^{i}$ that the $\mathcal{L}_{k i n}$ has cubic and quadratic terms, which would result in selfinteractions among the gauge fields. The self-interactions of the gauge fields are analogous to the 
gluon self-interactions in QCD. The charged-current interaction between the LH fermions $(f)$ and the complex vector boson field $W_{\mu}$ comes from the $S U(2)_{L}$ matrix term and the Lagrangian $\left(\mathcal{L}_{C C}\right)$ is given as:

$$
\mathcal{L}_{C C}=\frac{g}{2 \sqrt{2}}\left\{W_{\mu}^{\dagger}\left[\bar{f} \gamma^{\mu}\left(1-\gamma_{5}\right) f^{\prime}+\text { h.c }\right\} \quad \text { where } W_{\mu}^{ \pm} \equiv \frac{1}{\sqrt{2}}\left(W_{\mu}^{1} \pm i W_{\mu}^{2}\right) .\right.
$$

Figure 4 represents the charged-current interaction vertex. The neutral-current interaction compo-

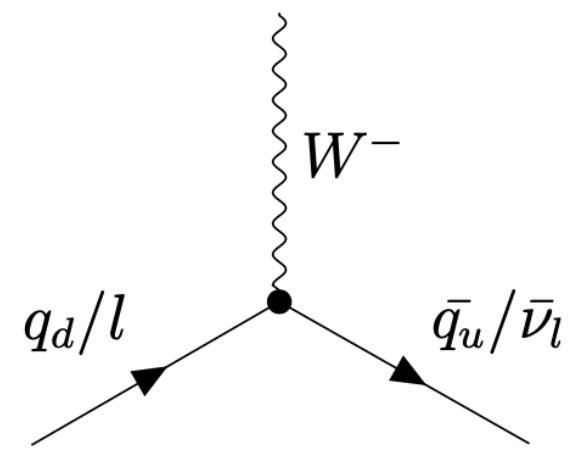

Figure 4: Feynman diagram of an EW charged-current interaction vertex.

nents originate from the neutral gauge fields $W_{\mu}^{3}$ and $B_{\mu}$ and the force carriers associated with it are the $Z$ boson and $\gamma$. The neutral-current interaction Lagrangian can be written as:

$$
\mathcal{L}_{N C}=\sum_{j} \bar{\psi}_{j} \gamma^{\mu}\left\{A_{\mu}\left[g_{W} \frac{\sigma_{3}}{2} \sin \theta_{w}+g_{Y} y_{j} \cos \theta_{w}\right]+Z_{\mu}\left[g_{W} \frac{\sigma_{3}}{2} \cos \theta_{w}-g_{Y} y_{j} \sin \theta_{w}\right]\right\} \psi_{j}
$$

where $Z_{\mu}$ is the $Z$ boson field, $A_{\mu}$ is the QED photon field and $\theta_{w}$ is the weak mixing angle, and $y_{j}$ are hypercharges. Figure 5 shows the Feynman diagrams for neutral-current interaction vertices. Analogous to the 3-gluon and 4-gluon interactions in QCD, the Lagrangian in equation 21 also has self-interaction terms between 3 and 4-gauge bosons and the corresponding interaction vertices are shown in Figure 6 .

A critical aspect of these self-interaction vertices is the presence of charged $W^{ \pm}$boson pairs in all the possible interactions. There are no neutral vertices with only photon and $Z$ bosons. The EW Lagrangian does not have any mass terms, meaning the gauge bosons would be massless. While in reality, this is true for the photons, but the $W^{ \pm}$and $Z$ bosons are heavy. The gauge bosons acquire masses by the Spontaneous Electroweak Symmetry Breaking. 

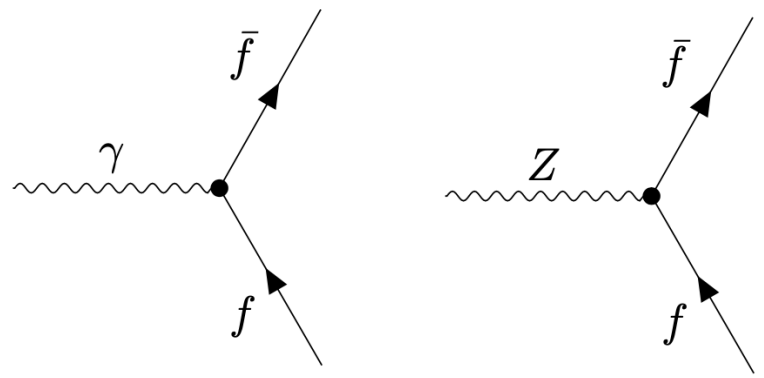

Figure 5: Feynman diagrams of an EW neutral-current vertex with photon as the force carrier (left) and $Z$ boson as the force carrier (right).
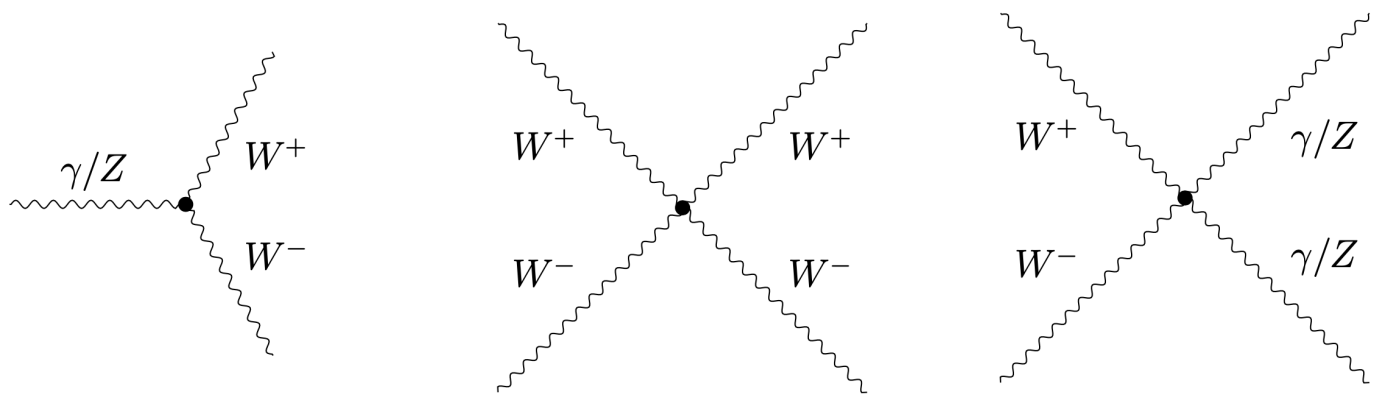

Figure 6: Feynman diagrams of gauge boson self-interaction vertices.

\subsubsection{Electroweak Symmetry Breaking and Higgs Mechanism}

The EW Lagrangian with the $S U(2)_{L} \times U(1)_{Y}$ symmetry successfully describes the EW interaction properties. However, the EW Lagrangian does not have any mass terms, making the gauge bosons massless. If additional mass terms are included, the Lagrangian no longer remains Lorentz-invariant, as seen for photons in equation 9 . To have non-zero $W^{ \pm}$and $Z$ boson masses another ingredient is required, which would break the symmetry but still conserve the Lorentzinvariance of the Lagrangian. This is where the Brout-Englet-Higgs mechanism [13-15] comes into play, which breaks the EW symmetry spontaneously and gives masses to $W^{ \pm}$and $Z$, while the photon remains massless. The SM Higgs sector is composed of a complex $S U(2)$ doublet of scalar fields $\Phi$ :

$$
\Phi=\left(\begin{array}{c}
\phi^{+} \\
\phi^{0}
\end{array}\right)=\frac{1}{\sqrt{2}}\left(\begin{array}{c}
\phi_{1}+i \phi_{2} \\
\phi_{3}+i \phi_{4}
\end{array}\right)
$$


Where the $\phi_{i}$ 's represent four real scalar fields, $\phi^{+}$has an electric charge of +1 and $\phi^{0}$ is neutral. In terms of covariant derivatives, the scalar part of the corresponding Lagrangian is written as:

$$
\mathcal{L}_{\text {Higgs }}=\left(D^{\mu} \Phi\right)^{\dagger}\left(D_{\mu} \Phi\right)-V(\Phi) \quad \text { where } V(\Phi)=\mu^{2} \Phi^{\dagger} \Phi+\lambda\left(\Phi^{\dagger} \Phi\right)^{2}
$$

To have ground states the potential must have lower bounds, hence $\lambda>0$. Depending on the $\mu^{2}$ values there are two distinct cases: (i) $\mu^{2}>0$, and (ii) $\mu^{2}<0$. As shown in the left plot in Figure 7 , for $\mu^{2}>0$ the potential has one minima at $\phi=0$ and describes scalar particles with mass $\mu$ and coupling $\lambda$. For $\mu^{2}<0$ there is not a single vacuum at $\phi=0$, instead there is a continuous set of degenerate vacuum states which satisfy the following condition:

$$
\Phi^{\dagger} \Phi=-\frac{\mu^{2}}{2 \lambda}=\frac{v^{2}}{2} .
$$

Here $v$ is the radius of the circle of minima of the potential $V(\phi)$. There are many possible solutions for the $\phi$ 's which will satisfy the minima, and a convenient choice is $\phi_{1}=\phi_{2}=\phi_{4}=0$, which preserves the charge conservation in $U(1)$ symmetry. Hence, the scalar field $\phi_{3}$ gives a non-zero vacuum expectation value,

$$
\langle\Phi\rangle_{0}=\langle 0|\Phi| 0\rangle=\frac{1}{\sqrt{2}}\left(\begin{array}{l}
0 \\
v
\end{array}\right) \quad \text { where }-\frac{\mu^{2}}{\lambda}=v^{2}
$$

and it is observed that $v \approx 246 \mathrm{GeV}$. The selection of a particular minima breaks the symmetry of $\mathcal{L}_{\text {Higgs }}$, which is known as the spontaneous symmetry breaking and since it breaks the EW symmetry it is commonly known as the Electroweak Symmetry Breaking (EWSB).

To understand the consequences of the EWSB, the Higgs field needs to be studied under a small perturbation around its minima. In terms of unitary gauge the perturbed Higgs field can be written as:

$$
\phi(x)=\frac{1}{\sqrt{2}}\left(\begin{array}{c}
0 \\
v+h(x)
\end{array}\right)
$$

where $h(x)$ represents the excitation of the Higgs field which represents the physical Higgs boson and has a mass of $m_{h}=\sqrt{2}|\mu|=125 \mathrm{GeV}$. The $W^{ \pm}$and $Z$ boson gets their masses through the interaction of $B_{\mu}$ and $W_{\mu \nu}^{i}$ with $h$ and the interaction terms are contained within $\left(D_{\mu} \phi\right)^{\dagger}\left(D^{\mu} \phi\right)$ : 

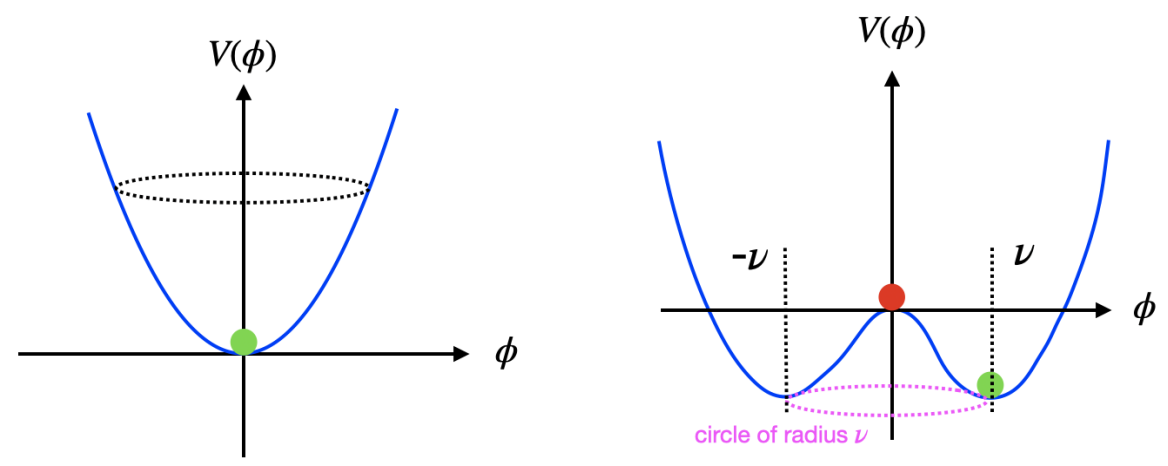

Figure 7: Left plot: Potential with $\mu^{2}>0$ which has a stable minima at $\phi=0$. Right plot: Potential with $\mu^{2}<0$ which does not have a stable equilibrium, instead there is a continuous set of degenerate vacuum states. The Higgs attains a non-zero vacuum expectation value at $\pm \boldsymbol{v}$ which breaks the $S U(2)_{L} \times U(1)_{Y}$ gauge symmetry.

$$
\left(D_{\mu} \phi\right)^{\dagger}\left(D^{\mu} \phi\right)=\frac{1}{2} \partial_{\mu} h \partial^{\mu} h+(v+h)^{2}\left(\frac{g_{W}^{2}}{4} W_{\mu}^{\dagger} W^{\mu}+\frac{g_{W}^{2}}{8 \cos ^{2} \theta_{w}} Z_{\mu} Z^{\mu}\right) .
$$

Equation 29 has no $A_{\mu}$ terms, meaning the photon remains massless, while there are mass terms for the $W^{ \pm}$and $Z$ bosons. The physically observable states of $W^{ \pm}, Z$ bosons, and the photon, masses of the bosons, and the weak mixing angle are given as:

$$
\begin{aligned}
Z_{\mu} & =\cos \theta_{w} W_{\mu}^{3}-\sin \theta_{w} B_{\mu} \quad \text { with } m_{Z}=\frac{1}{2} \nu \sqrt{g_{W}^{2}+g_{Y}^{2}} \\
W_{\mu}^{ \pm} & =\frac{1}{\sqrt{2}}\left(W_{\mu}^{1} \pm i W_{\mu}^{2}\right) \quad \text { with } \quad m_{W^{ \pm}}=\frac{1}{2} v g_{W} \\
A_{\mu} & =\sin \theta_{w} W_{\mu}^{3}+\cos \theta_{w} B_{\mu} \quad \text { with } m_{A}=0 \\
m_{h} & =\sqrt{2} \mu \text { and } \cos \theta_{w}=\frac{g_{W}}{\sqrt{g_{W}^{2}+g_{Y}^{2}}}=\frac{m_{W}}{m_{Z}} .
\end{aligned}
$$

The $\mathcal{L}_{\text {Higgs }}$ contains gauge boson-Higgs boson and Higgs boson self-interaction terms and the corresponding Feynman diagrams for the vertices are shown in Figure 8

The SM Lagrangian needs one more component to be complete. The Higgs mechanism gives masses to the gauge bosons, but the fermions are still massless. The fermionic masses come from the Yukawa interactions between the fermionic fields and the Higgs field $(\Phi)$. The Yukawa Lagrangian is given as:

$$
\mathcal{L}_{Y}=C_{\ell} \bar{L}_{L} \phi \ell_{R}+C_{u} \bar{Q}_{L} \phi^{c} u_{R}+C_{d} \bar{Q}_{L} \phi d_{R}+\text { h.c. }
$$



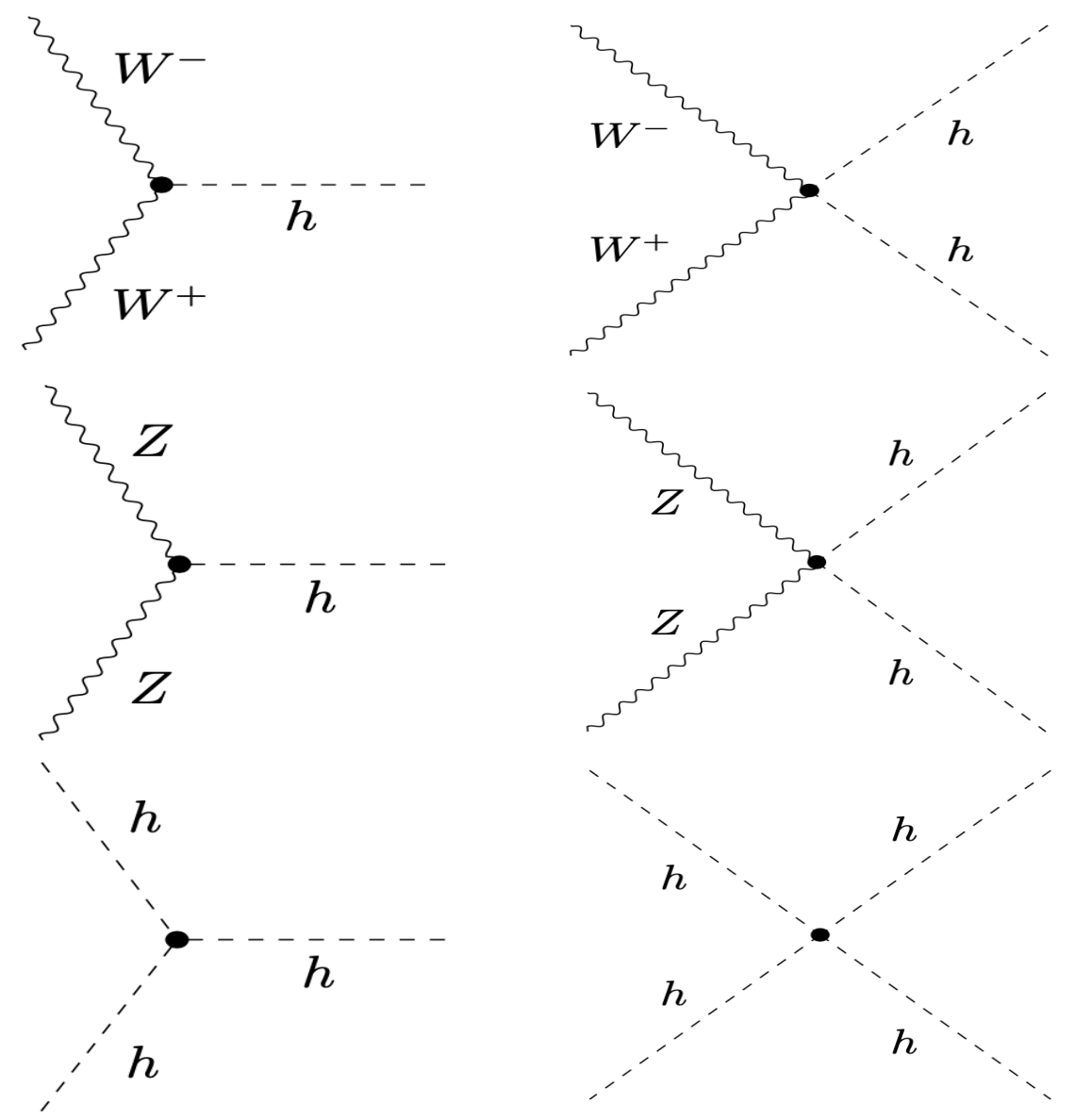

Figure 8: Feynman diagrams for gauge boson-Higgs boson and Higgs boson self-interaction vertices.

where $\phi^{c}=i \sigma_{2} \phi^{\star}$ is the charge-conjugate of the Higgs field; $\ell_{R}, u_{R}$ and $d_{R}$ represent the RH fields for charged leptons, up-type quark and down-type quarks respectively; $L_{L}$ and $Q_{L}$ represent the LH lepton and quark doublets while $C_{\ell}, C_{u}$ and $C_{d}$ represent the coupling constants for leptons, up-type and down-type quarks respectively. In the context of Spontaneous Symmetry Breaking and using the unitary gauge, the fermionic masses are given as:

$$
m_{f}=-C_{f} \frac{v}{\sqrt{2}} \quad \text { where } f=\ell, u, d .
$$

Figure 9 represents the Higgs boson and fermion interaction vertex. The fermionic interaction term 


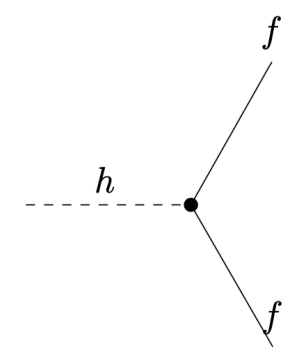

Figure 9: Feynman diagram for fermion-Higgs boson interaction vertex.

completes the SM Lagrangian and has the following form:

$$
\mathcal{L}_{S M}=\mathcal{L}_{Q C D}+\mathcal{L}_{E W}+\mathcal{L}_{\text {Higgs }}+\mathcal{L}_{Y}
$$

\subsection{The success and limitations of Standard Model}

Although the SM has succeeded in describing the elementary particles and their interactions, there are many open questions that the SM does not answer. The following section describes the successes and failures of the SM.

\section{The success of Standard Model}

Figure 10 shows the cross section measurements of different SM processes and the theoretical predictions. The measurements and predictions are in excellent agreement over a range of 12 orders of magnitude, showcasing the immense success of the SM. Another example where the SM prediction is experimentally verified is the $W^{ \pm}$boson mass $\left(m_{W}\right)$ measurement. Left plot in Figure 11 shows the $m_{W}$ measured from different experiments and the SM prediction, derived from a global electroweak fit [16]. Right plot in Figure 11] shows the comparison between contours for the $m_{W}$ and $m_{t}$ prediction from the electroweak fit and ATLAS results. In both cases, the SM prediction and experimental results are in good agreement.

\section{Limitations of Standard Model}

Despite being a massively successful and elegant theory, the SM is incomplete. It does not fully describe the known universe. This section outlines the major open questions in particle physics, which the SM does not answer. 


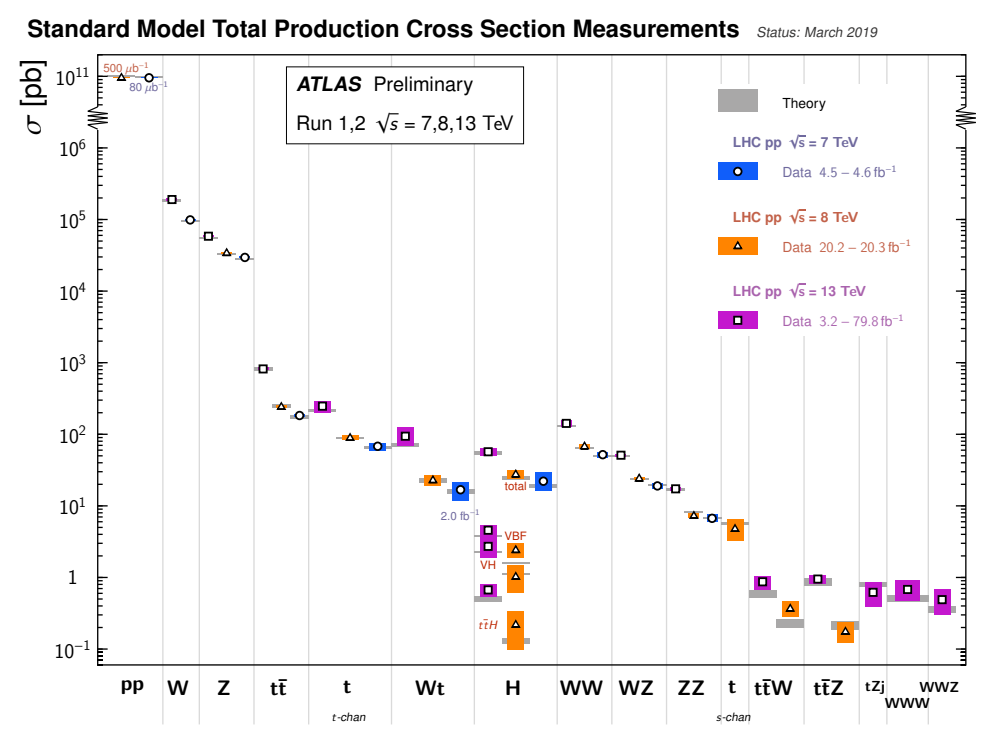

Figure 10: Summary of total cross-section measurements of various SM processes, compared with the theoretical predictions, with Run 1 and Run 2 ATLAS data [17].
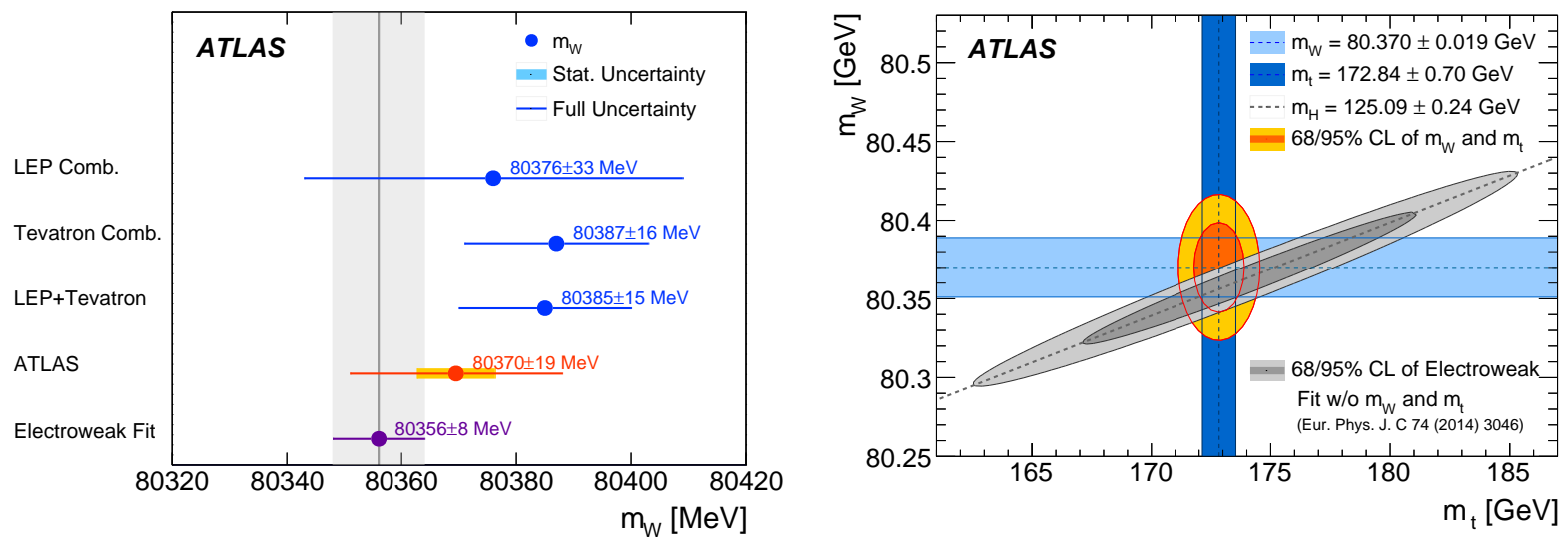

Figure 11: Left plot shows the comparisons between the $m_{W}$ measurements from various experiments and the SM prediction from the global EW fit. Right plot shows the comparisons between the global EW fit and ATLAS measurements with the 2D contour plot of $m_{W}$ and $m_{t}$ [18].

- Dark matter: The ordinary matter that has so far been observed through different experiments constitutes only $\sim 5 \%$ of the energy density of the universe, while $\sim 27 \%$ is in the form of DM. While the nature of the DM is still unknown, one of the leading hypotheses suggests that DM candidates might be Weakly Interacting Massive Particles (WIMPs). The particle nature of the DM candidates has not yet been experimentally verified, and the SM does not predict any likely DM candidates. 
- Dark energy: Cosmological and Astrophysical observations have shown that the expansion of the universe is accelerating. One possible explanation of the accelerating expansion is that there is a flow of energy that counteracts the gravitational pull. This mysterious energy is called DE and the SM has no explanation for the accelerating expansion of the universe.

- Neutrino mass: According to the SM, there are three massless neutrinos. However, neutrino oscillation experiments [19] have observed that the three neutrino flavours oscillate among themselves. The neutrino oscillation is only possible for non-zero neutrino masses. Including additional fields in the SM, to add neutrino mass terms, would introduce theoretical complications because the neutrino masses are tiny. Moreover, some theories propose the existence of sterile neutrinos as a possible explanation for DM, which is not in SM.

- Matter-antimatter asymmetry: The particle component of the universe is mostly matter. According to SM, there should have been no preference between matter and antimatter in the early days of the universe, which is not the case in the universe today. A small part of the discrepancy could be explained by the $\mathrm{CP}$ violation via the $\mathrm{CKM}$ matrix, but there are no SM mechanisms that can describe such a large matter-antimatter asymmetry.

- Gravity: The SM does not explain gravity. Every interaction in SM has bosons as mediators, but the SM predicts no such particle for gravitational interactions. Some theories postulate the existence of a Graviton. Although this has not yet been discovered, the SM also does not postulate an alternative mechanism.

\subsection{Dark matter and dark energy}

In the previous section, the SM of particle physics was introduced along with its shortcomings such as the DM and DE problem. The following sections give an introduction to the observational evidences of DM, experimental methods to detect DM, and introduces DE. 


\subsubsection{Evidence of dark matter}

The first evidence of DM came from Fritz Zwicky's studies of the Coma cluster in 1933. By applying the Virial theorem to the Coma cluster he showed that the luminous matter was not enough to keep the cluster bound. He postulated the existence of DM, responsible for holding the galaxy cluster together [20]. The velocity dispersion curves of galaxies also indicate the existence of DM. Studies by Vera Rubin and Kent Ford in the 1960s with the Andromeda galaxy showed that the visible mass is not enough for the galaxy to be bound by Newtonian gravity. Velocities of stars in spiral galaxies also show that the mass of the galaxy cannot be defined only by known objects identified through telescopes, and a large mass density in the form of DM is necessary. Further evidence came from the temperature anisotropies in the Cosmic Microwave Background (CMB), measured by WMAP [21] and Planck satellite [22]. CMB [23] is the remnant electromagnetic radiation from the hot early stage of the universe. By studying the scale and height of the peaks in the temperature fluctuations in the cosmic microwave background across different angular scales on the sky, the amount of DM in the universe can be estimated. In 2006 observations made by the Hubble Space Telescope and Chandra X-ray observatory on the bullet cluster mass spectra [24] gave another evidence of DM. The bullet cluster is formed by a high-speed collision between

two galaxy clusters. The after collision mass density of the cluster was measured by the X-ray observatory (for the ordinary matter) and gravitational lensing. The discrepancy in identifying the mass density locations by the two methods gave a direct indication of the existence of DM. The mass distribution from the gravitational lensing was outside the luminous matter of the cluster, which indicates that DM interacts very weakly with ordinary matter.

\subsubsection{Dark matter candidates}

Searches for DM are model dependent and the majority of these models are based on QFT where the proposed DM particles can be characterized by their spin and mass. Depending on the model hypothesis masses of the DM candidates can be of a wide range [25]. A few of the probable DM particles predicted by different models, listed in Table 3, are described in this section. 
- Weakly Interacting Massive Particles (WIMPs) : Many leading hypotheses suggest that DM particles are WIMPs [26]. The WIMPs are predicted to be stable, weakly interacting particles and in the mass range of $1-10^{5} \mathrm{GeV}$. The WIMPs are assumed to be produced thermally in early universe and were in equilibrium with the thermal plasma. As the universe expanded and cooled down, the WIMPs started moving with non-relativistic velocities - making them a prime candidate for cold dark matter. Another interesting aspect is that the cross section necessary to observe the current DM density is of weak interaction scale. Hence, a weakly interacting particle such as WIMP is a theoretically well-motivated DM candidate.

- Axion : The Axion [27-29], originally postulated to solve the strong-CP problem, is one of the earliest suggestions for a probable DM candidate. The interaction between strong and weak force results in an effective strong $C P$ violating term $\bar{\Theta}$. While $\bar{\Theta}$ could be between 0 and $2 \pi$, experimental observations suggest that the value of $\bar{\Theta}$ must be extremely small. This constitutes a naturalness problem of SM. In 1977 Roberto Peccei and Helen Quinn proposed a solution, called Peccei-Quinn mechanism [30], to this naturalness problem where $\bar{\Theta}$ is promoted to a dynamical field by adding a new global symmetry, which gets spontaneously broken and results in a new light pseudoscalar boson - Axions.

- Sterile Neutrinos : Sterile Neutrinos [31] are hypothetical DM candidates from the neutrino sector. The neutrinos are a natural fit for the DM problem. However, known neutrinos cannot possibly comprise all the DM due to its interaction scales and mass which is constrained to be less than $2 \mathrm{eV}$ observed from the electron spectrum of $\beta$-decay [32]. The mass scale is much lower compared to DM mass scales. As an alternate, a heavier sterile neutrino with weaker interactions to SM is proposed. It is also theoretically well-motivated by the Seesaw Mechanism [33-36].

- Neutralino : The Neutralino [37] is a hypothetical Supersymmetric (SUSY) particle which in the context of Minimal Supersymmetric Standard Model (MSSM) [38, 39] has four states. The lightest Neutralino is a prime candidate for the cold dark matter (type of DM that moves slowly compared to light) [40] as it is stable and weakly interacting. SUSY predicts that the lightest Neutralino is in the mass range of $10 \mathrm{GeV}-10 \mathrm{TeV}$ and closely related to the supersymmetric partners 
of the EW force carriers: photon, $W^{ \pm}$and $Z$ bosons, and two neutral CP-even Higgs bosons.

\begin{tabular}{|c|c|c|c|c|}
\hline DM particle & Origin & Particle spin & Particle mass scale & Interaction \\
\hline \hline Axion & Strong CP & 0 & $\mu \mathrm{eV}-\mathrm{meV}$ & Weak \\
\hline WIMP & early universe & 0 & $\mathrm{GeV}$ & Weak \\
\hline Sterile Neutrino & neutrino sector & $1 / 2$ & $\mathrm{keV}$ & fermion mixing \\
\hline Neutralino & SUSY & $1 / 2$ & $10 \mathrm{GeV}-10 \mathrm{TeV}$ & Weak \\
\hline
\end{tabular}

Table 3: Probable DM candidates from different models and their characteristics.

\subsubsection{Dark matter detection}

The DM detection experiment types can be categorized into three categories: direct detection [41-43], indirect detection [44], and collider experiments. The collider DM searches are complementary to the other two procedures. Figure 12 shows the schematic representation of possible DM detection procedures.

- Direct detection: Direct detection experiments observe the nuclear recoil energy produced when the particles collide with the detector's target nuclei [45]. This recoil energy can be measured through different channels: ionization (charge), scintillation (light), and heat (phonons). In the direct detection experiments, usually two different types of signal channels are observed to avoid common backgrounds such as gamma rays. A WIMP with a mass between 10 - $1000 \mathrm{GeV}$ can produce nuclear recoil energy between $1-100 \mathrm{keV}$. The advantage of using a nuclear recoil instead of an electron recoil is in background rejection, as the electron recoil transforms all its kinetic energy in ionization.

- Indirect detection: Indirect DM detection experiments search for products originating from self-annihilation or decays of DM particles in outer space. These experiments mostly target detecting SM particles (e.g., gamma rays) produced through annihilation or decay of DM particles that are already present in the cosmos. Since the amount of ambient DM is almost five times higher than ordinary matter, indirect experiments are excellent methods to detect DM. However, since the DM-SM interaction is very weak it is difficult to distinguish DM products from astrophysical backgrounds. 
- Collider experiments: Collider experiments aim to infer the existence of DM particles by producing them in highly energetic particle accelerators. Since the DM interacts weakly with SM particles, the DM particles will escape the detectors undetected. However, the existence of a DM particle can be inferred if a significant $E_{\mathrm{T}}^{\text {miss }}$ is observed. One major advantage of collider experiments over the direct and indirect searches is the energy scale of the collider experiments. Not only the WIMPs but other DM candidates postulated by various theories can be tested at collider experiments. The ATLAS and CMS experiments at the Large Hadron Collider are actively involved in DM searches.

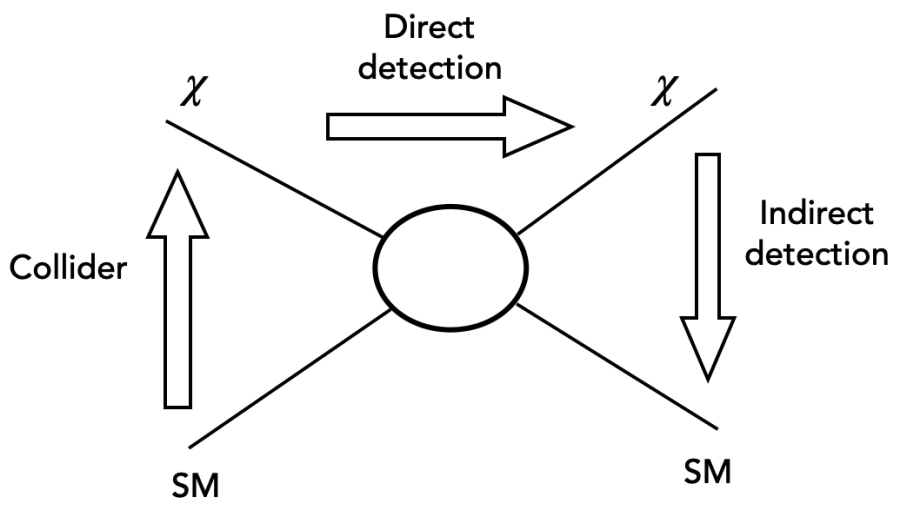

Figure 12: Schematic of different types of DM detection experiments, $\chi$ represents DM candidates.

\subsubsection{Accelerating universe and dark energy}

In 1929 studies from Edwin Hubble showed that our universe is expanding. Although the expansion rate or the evolution of the expansion was unknown scientists agreed that over time the expansion would slow down due to the gravitational pull. In the 1990s observations from Perlmutter [46] and Riess [47] with the Hubble Space Telescope (HST) of distant supernovae showed completely opposite results. Not only the expansion is not slowing down it is accelerating. Although the reason for this accelerating expansion is unknown, it is postulated that some mysterious energy density is counteracting the gravitational pull. This unknown energy density is called dark energy. To explain DE, many theoretical models are proposed, such as the quintessence [48, 49]. 
The quintessence is a possible cosmic energy density that is a time-dependent scalar field in nature. However, no such signature has been found yet. Another set of theoretical models are based on the modified gravity models involving scalar fields with potential energy. One such class of models is the Horndeski [50] theories, where a scalar field couples to gravity. The Horndeski models can be studied experimentally over a wide range of energy scales, and the LHC could pioneer DE searches and complement other cosmological experiments.

\subsection{The Two Higgs Doublet Model (2HDM)}

Since the SM does not explain DM, new theoretical models are developed to provide possible explanations of DM. The DM searches at the LHC are based on specific signal models. Although, there are many models predicting DM candidates, not all are equally viable or experimentaly feasible. The theoretical benchmark models help to optimize the analysis parameter space

and interpret the results. During the initial data taking period, the LHC probed the EFT based DM models. Although since Run 2, the focus has shifted to simplified DM models [51]. Any new DM model to be studied at the LHC, should preferably be theoretically consistent with the simplified DM models used at the LHC, following the recommendations of the ATLAS/CMS DM community [52]. A possible avenue for DM models is the extension of the scalar sector of the SM. To construct a theory based on the extension of the SM scalar sector, one has to abide by the theoretical and experimental constraints:

(i) Constraint on the $\rho$ parameter $(\rho=1)$.

(ii) No Flavor-Changing Neutral Current (FCNC).

The $\rho$ parameter is the neutral to charged coupling constant ratio. In $S U(2) \times U(1)$ gauge theory, with $n$ number of scalar multiplets $\phi_{i}$, at tree level the generic form of $\rho$ can be written as,

$$
\rho=\frac{\sum_{i=1}^{n}\left[I_{i}\left(I_{i}+1\right)-\frac{1}{4} Y_{i}^{2}\right] v_{i}}{\sum_{i=1}^{n} \frac{1}{2} Y_{i}^{2} v_{i}}=\frac{M_{W}^{2}}{M_{Z}^{2} \cos ^{2} \theta_{w}}
$$


where $I_{i}$ and $Y_{i}$ represent the weak isospin and weak hypercharge of the multiplets respectively and $v_{i}$ represents the vacuum expectation value $(\mathrm{VEV})$ of the neutral components. The $S U(2)$ signlets with $Y=1$ and $S U(2)$ doublet with $Y= \pm 1$ satisfies

$$
I(I+1)=\frac{3}{4} Y^{2}
$$

which makes $\rho=1$ and is consistent with the experimental value of $\rho \approx 1[4]$. Condition (i) is satisfied automatically for scalar multiplets, the FCNC criterion is satisfied by constraining the couplings between the fermions and the multiplets. Hence, the simplest extension would be to add an extra Higgs doublet. This extended model is known as the Two-Higgs-Doublet Model (2HDM) [53].

The scalar potential of the $2 \mathrm{HDM}$ model has $S U(2)_{L} \times U(1)_{Y}$ gauge symmetry and 14 parameters built out of the two Higgs doublets: $\phi_{1}$ and $\phi_{2}$. For two Higgs doublets, the most general renormalizable potential can be written as:

$$
\begin{aligned}
V_{H} & =m_{11}^{2} \phi_{1}^{\dagger} \phi_{1}+m_{22}^{2} \phi_{2}^{\dagger} \phi_{2}-m_{12}^{2}\left(\phi_{1}^{\dagger} \phi_{2}+\phi_{2}^{\dagger} \phi_{1}\right)+\frac{\lambda_{1}}{2}\left(\phi_{1}^{\dagger} \phi_{1}\right)^{2}+\frac{\lambda_{2}}{2}\left(\phi_{2}^{\dagger} \phi_{2}\right)^{2}+\lambda_{3}\left(\phi_{1}^{\dagger} \phi_{1}\right)\left(\phi_{2}^{\dagger} \phi_{2}\right) \\
& +\lambda_{4}\left(\phi_{1}^{\dagger} \phi_{2}\right)\left(\phi_{2}^{\dagger} \phi_{1}\right)+\left[\frac{\lambda_{5}}{2}\left(\phi_{1}^{\dagger} \phi_{2}\right)^{2}+\lambda_{6}\left(\phi_{1}^{\dagger} \phi_{1}\right)\left(\phi_{1}^{\dagger} \phi_{2}\right)+\lambda_{7}\left(\phi_{2}^{\dagger} \phi_{2}\right)\left(\phi_{1}^{\dagger} \phi_{2}\right)+\text { h.c. }\right]
\end{aligned}
$$

where $m_{11}^{2}, m_{22}^{2}, \lambda_{1,2,3,4}$ are real and $m_{12}^{2}, \lambda_{5,6,7}$ are complex. The 2 HDM vacuum has a rich phenomenology, e.g., unlike SM with only one possible type of minimum, it has three types of vacua each corresponds to a physical state of the potential: (i) CP-conserving or Normal (N) vacua where the VEVs are real, (ii) CP-violating vacua where the VEVs differ by a complex phase, and (iii) electric charge conservation breaking vacua where one VEV includes an electric charge. The 2HDM model discussed in the thesis has some simplifying assumptions. The model considers only the Higgs sector with the $\mathrm{CP}$ conserving vacua:

$$
\left\langle\phi_{1}\right\rangle_{N}=\left(\begin{array}{c}
0 \\
\frac{v_{1}}{\sqrt{2}}
\end{array}\right), \quad\left\langle\phi_{2}\right\rangle_{N}=\left(\begin{array}{c}
0 \\
\frac{v_{2}}{\sqrt{2}}
\end{array}\right) .
$$

An important parameter of the $2 \mathrm{HDM}$ model is $\tan \beta$ defined as, 


$$
\tan \beta=\frac{v_{1}}{v_{2}}
$$

and to have the $W^{ \pm}$and $Z$ masses as in SM one requires $v=\sqrt{v_{1}^{2}+v_{2}^{2}} \approx 246 \mathrm{GeV}$. The model also assumes that there is no spontaneous $\mathrm{CP}$ violation. With these assumptions, the potential can be written as,

$$
\begin{aligned}
V_{H} & =m_{11}^{2} \phi_{1}^{\dagger} \phi_{1}+m_{22}^{2} \phi_{2}^{\dagger} \phi_{2}-m_{12}^{2}\left(\phi_{1}^{\dagger} \phi_{2}+\phi_{2}^{\dagger} \phi_{1}\right)+\frac{\lambda_{1}}{2}\left(\phi_{1}^{\dagger} \phi_{1}\right)^{2}+\frac{\lambda_{2}}{2}\left(\phi_{2}^{\dagger} \phi_{2}\right)^{2} \\
& \left.+\lambda_{3}\left(\phi_{1}^{\dagger} \phi_{1}\right)\left(\phi_{2}^{\dagger} \phi_{2}\right)+\lambda_{4}\left(\phi_{1}^{\dagger} \phi_{2}\right)\left(\phi_{2}^{\dagger} \phi_{1}\right)+\frac{\lambda_{5}}{2}\left[\left(\phi_{1}^{\dagger} \phi_{2}\right)^{2}+\left(\phi_{2}^{\dagger} \phi_{1}\right)^{2}\right)\right]
\end{aligned}
$$

and the associated eight real mass eigenstates are given as

$$
\phi_{i}=\left(\begin{array}{c}
\phi_{i}^{\dagger} \\
\frac{v_{a}+\rho_{a}+i \eta_{a}}{\sqrt{2}}
\end{array}\right) \quad \text { where } \mathrm{a}=1,2 .
$$

Out of the eight eigenstates, three provide masses for the massive $W^{ \pm}$and $Z$ bosons, and the remaining five corresponds to five different Higgs bosons: two neutral $\mathrm{CP}$-even $H$ and $h$, one neutral CP-odd $A$, and two charged Higgs bosons $H^{ \pm}$. The rotation angle $\beta$ diagonalizes the mass matrices of $H^{ \pm}$and $A$. Another parameter of the 2HDM model is $\alpha$, which diagonalizes the mass matrices of $H$ and $h$. Conventionally $h$ is the lighter CP-even state, and $H$ is the heavy CP-even state, while the SM Higgs is a mixture of these two:

$$
H_{S M}=h \sin (\alpha-\beta)-H \cos (\alpha-\beta)
$$

In the 2HDM model, the FCNC can arise from the Yukawa couplings between the 2HDM scalars and SM fermions, given as:

$$
\mathcal{L}_{Y}=-\sum_{i=1,2}\left(\bar{Q} Y_{u}^{i} \tilde{H}_{i} u_{R}+\bar{Q} Y_{d}^{i} H_{i} d_{R}+\bar{L} Y_{\ell}^{i} H_{i} \ell_{R}+\text { h.c. }\right)
$$

where $Y_{f}^{i}$ are Yukawa matrices acting on fermions; $Q, L$ are LH quarks and lepton doublets respectively, $u_{R}, d_{R}$, and $l_{R}$ are RH up-type, down-type quarks, and RH leptons respectively, and $\tilde{H}_{i}=$ $\varepsilon H_{i}^{\star}$ with $\varepsilon$ being the $2 \mathrm{D}$ asymmetric tensor. The FCNC induces a $H \rightarrow d \bar{s}$ decay which will result in a kaon-antikaon $(K-\bar{K})$ oscillation at tree-level as shown in Figure 13 . As a consequence of the oscillation, the neutral flavour changing mediator would require a mass of $10 \mathrm{TeV}$. According to 


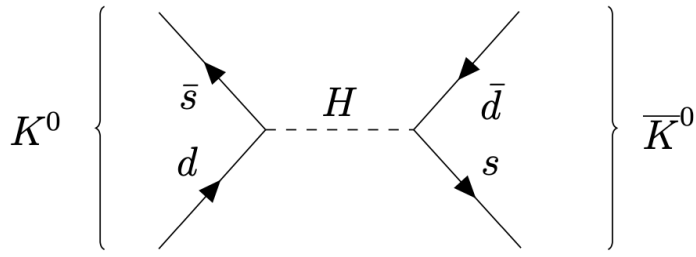

Figure 13: FCNC leads to a Kaon-antikaon oscillation at tree-level.

the Paschos-Glasgow-Weinberg [54, 55] theorem, the FCNCs at tree-level can be avoided if the all $\mathrm{RH}$ fermions of a given electric charge couple only to one of the Higgs doublets. This can be achieved by invoking a $Z_{2}$ symmetry, i.e., $\phi_{1} \rightarrow-\phi_{1}$ and $\phi_{2} \rightarrow \phi_{2}$. Depending on the transformation of fermions under the $Z_{2}$ symmetry, there are four types of 2HDM model where there are no FCNCs:

- Type-I 2HDM: all fermions couple to only one of the Higgs doublets, which is by convention chosen to be $\phi_{2}$. Type-I $2 \mathrm{HDM}$ is enforced with a $\phi_{1} \rightarrow-\phi_{1}$ discrete symmetry.

- Type-II 2HDM: up-type RH quarks couple to $\phi_{2}$ while down-type RH quarks and charged RH leptons couple to $\phi_{1}$. Type-II $2 \mathrm{HDM}$ is enforced with a $\phi_{1} \rightarrow-\phi_{1}, d_{R}^{i} \rightarrow-d_{R}^{i}$ discrete symmetry.

- Lepton specific 2HDM: all RH quarks couple to $\phi_{2}$ and charged RH leptons couple to $\phi_{1}$.

- Flipped 2HDM: up-type RH quarks couple to $\phi_{2}$, down-type RH quarks couple to $\phi_{1}$ as in Type-II, but the charged RH leptons couple to $\phi_{2}$.

The Type-II 2HDM is equivalent to MSSM with some constraints and the DM models discussed in this thesis are based on Type-II 2HDM. A prerequisite of any theoretical model is that perturbative calculations must yield stable minima. In the case of $2 \mathrm{HDM}$, this implies that the potential must not diverge at minus infinity. This stability is achieved by constraining the $\lambda$ parameters [53]:

$$
\lambda_{1}, \lambda_{2} \geq 0, \quad \lambda_{3} \geq-\sqrt{\lambda_{1} \lambda_{2}}, \quad \lambda_{3}+\lambda_{4}-\left|\lambda_{5}\right| \geq-\sqrt{\lambda_{1} \lambda_{2}} .
$$

However, these $\lambda$ parameters are not measurable experimentally and can be replaced by experi- 
mentally measurable parameters:

$$
\left\{\begin{array}{c}
m_{11}, m_{22}, m_{12} \\
\lambda_{1}, \lambda_{2}, \lambda_{3}, \lambda_{4}, \lambda_{5}
\end{array}\right\} \longleftrightarrow\left\{\begin{array}{c}
v, M_{h}, M_{H}, M_{A}, M_{H^{ \pm}} \\
\tan \beta, \cos (\beta-\alpha), \lambda_{3} .
\end{array}\right\}
$$

In addition to the $v \approx 246 \mathrm{GeV}$ constraint, there are constraints on $\alpha$ and $\beta$. As the top-quark Yukawa coupling is required to be perturbative, $\tan \beta$ is constrained to have a lower bound of 0.3. Another constraint is in the $\tan \beta-\cos (\beta-\alpha)$ plane, where $\cos (\beta-\alpha) \approx 0$ for arbitrary values of $\tan \beta$. The $\cos (\beta-\alpha) \approx 0$ condition is also known as the alignment limit, which implies that one of the CP-even mass eigenstates is SM-like if they are aligned with the direction of the vacuum expectation value. Figure 14 shows the region in the $\cos (\beta-\alpha)-\tan \beta$ plane for the Type-II 2HDM model that is excluded at a 95\% confidence level (CL). At the alignment limit the coupling of $h$ is the same as the SM Higgs, i.e., $m_{h}=125 \mathrm{GeV}$. Constraints on the charged Higgs

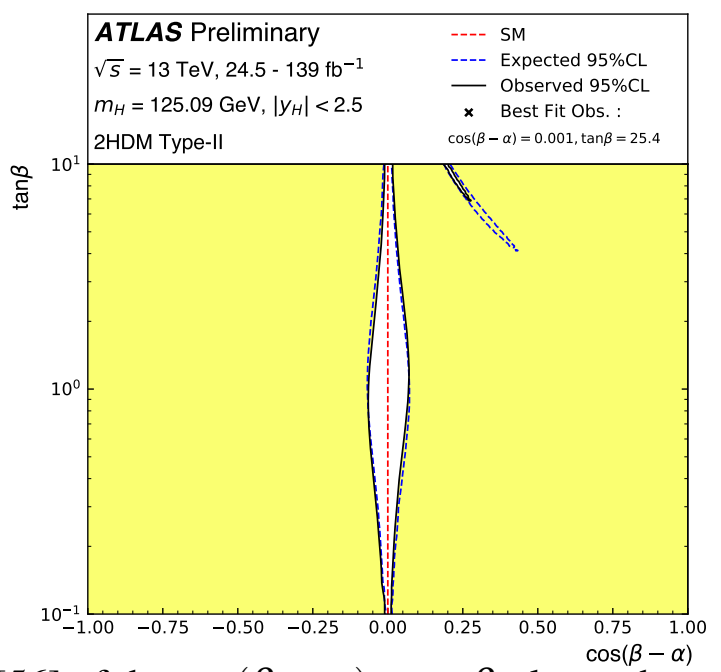

Figure 14: Exclusion limit [56] of the $\cos (\beta-\alpha)-\tan \beta$ plane where the regions are excluded (yellow) at a 95\% CL. The red dashed line is the SM prediction and the cross represents the best fit observed value.

boson masses $\left(M_{H^{ \pm}}\right)$come from $\bar{B} \rightarrow X_{s} \gamma$ decay and $B_{q}-\bar{B}_{q}$ mixing measurements. Figure 15 shows the $\bar{B} \rightarrow X_{s} \gamma$ decay where the $\bar{B}$ meson $(b \bar{u})$ decays to a $X_{s}$ (containing an $s$ quark) and a photon. The underlying decay is $b \rightarrow s \gamma$, which proceeds through a loop diagram with a $W^{-}$ boson. If $H^{ \pm}$exists, the loop diagram would have contribution from $H^{ \pm}$in addition to the $W^{-}$ 
boson, inducing a deviation of the branching ratio $(\mathrm{BR})\left(B \rightarrow X_{s} \gamma\right)$ from SM predictions. From the BR calculations [57] at NNLO QCD the $\tan \beta$ independent lower bound on $M_{H^{ \pm}}$is set at $295 \mathrm{GeV}$ at a $95 \%$ CL.

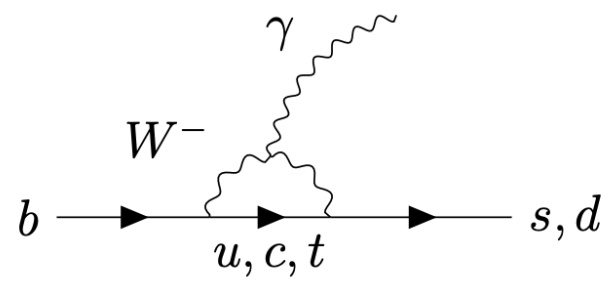

Figure 15: EM penguin diagram for the $\bar{B} \rightarrow X_{s} \gamma$ decay in SM.

\subsubsection{HDM+ $a$ model}

The gauge-invariant and renormalisable $2 \mathrm{HDM}+a$ [58] model is an extension of the TypeII 2HDM. Here an additional pseudoscalar singlet $P$ is incorporated along with the two Higgs doublets $\phi_{1}$ and $\phi_{2}$. The pseudoscalar $a$ is a superposition of the CP-odd components of $\phi_{1}, \phi_{2}$, and $P$ which mediates the interactions between the Higgs sector and the invisible DM candidates $(\chi)$. The DM models with scalar mediators are constrained strongly from DM direct detection experiments. Hence a pseudoscalar based model such as the $2 \mathrm{HDM}+a$ is complementary to the direct detection experiments. A $Z_{2}$ symmetry is imposed under which $\phi_{1} \rightarrow \phi_{1}, \phi_{2} \rightarrow-\phi_{2}$, singlet $P$ is even $P \rightarrow P$, and Dirac fermion DM $\chi$ is odd $\chi \rightarrow-\chi$. The Yukawa interaction between the Higgs doublets and the fermions is represented by the Lagrangian in equation 42 , and the following Lagrangian describes the coupling between $\chi$ and $P$ :

$$
\mathcal{L}_{\chi}=-i g_{\chi} P \bar{\chi} \gamma_{5} \chi
$$

where $g_{\chi}$ is the dark-sector Yukawa coupling. A general scalar potential with two Higgs doublets and a pseudoscalar singlet can be written as:

$$
V\left(\phi_{1}, \phi_{2}, P\right)=\underbrace{V_{2 H D M}\left(\phi_{1}, \phi_{2}\right)}_{\text {2HDM potential }}+\underbrace{V_{P 2 H D M}\left(\phi_{1}, \phi_{2}, P\right)}_{\begin{array}{c}
\text { interaction between P and } \\
\text { Higgs doublets }
\end{array}}+\underbrace{V_{P}(P)}_{\text {potential for } \mathrm{P}} .
$$

The $V_{2 H D M}\left(\phi_{1}, \phi_{2}\right)$ is given in equation 39, while 


$$
V_{P 2 H D M}\left(\phi_{1}, \phi_{2}, P\right)=P\left(i b_{P} \phi_{1}^{\dagger} \phi_{2}+h . c\right)+P^{2}\left(\lambda_{P 1} \phi_{1}^{\dagger} \phi_{1}+\lambda_{P 2} \phi_{2}^{\dagger} \phi_{2}\right)
$$

where $b_{p}$ is the trilinear portal coupling constant, and $\lambda_{P 1}, \lambda_{P 2}$ are quartic interaction terms between $P$ and the two Higgs doublets. The potential for the singlet $P$ is given by:

$$
V_{P}(P)=\frac{1}{2} m_{P}^{2} P^{2}+\frac{1}{4} \lambda_{R} P^{4}
$$

where $m_{p}$ is the mass term and $\lambda_{P}$ is the self-coupling quartic term. However, the $P^{4}$ term does not have any effect on kinematic variables like $E_{\mathrm{T}}^{\text {miss }}$. Hence, the term is excluded from the potential in the context of this thesis. Similar to $2 \mathrm{HDM}$, the parameter space of the $2 \mathrm{HDM}+a$ model can be expressed in mass eigenbasis where the parameters are experimentally measurable:

$$
\left\{\begin{array}{c}
m_{11}, m_{22}, m_{12}, b_{P}, m_{P}, m_{\chi} \\
\lambda_{1}, \lambda_{2}, \lambda_{3}, \lambda_{4}, \lambda_{5}, \\
g_{\chi}, \lambda_{P 1}, \lambda_{P 2}
\end{array}\right\} \longleftrightarrow\left\{\begin{array}{c}
v, M_{h}, M_{H}, M_{A}, M_{H^{ \pm}}, M_{a}, m_{\chi} \\
\tan \beta, \cos (\beta-\alpha), \sin \theta, \\
g_{\chi}, \lambda_{3}, \lambda_{P 1}, \lambda_{P 2}
\end{array}\right\} .
$$

Here $m_{\chi}$ is the mass of the DM candidate, $M_{a}$ is the mass of the pseudoscalar $a, \theta$ is the mixing angle between the two CP-odd mass eigenstates $A$ and $a$.

Constraints on the parameters from the 2HDM model apply to the $2 \mathrm{HDM}+a$ model, where the $\mathrm{VEV} v \approx 246 \mathrm{GeV}, M_{h}=125 \mathrm{GeV}, \cos (\beta-\alpha) \simeq 0$. The $W^{ \pm}$and $Z$ masses are crucial in calculating the $\rho$ parameter, as shown in equation 34 . To satisfy the constraint on $\rho$, one needs to minimize the corrections to the $W^{ \pm}$and $Z$ boson propagators, i.e., maintain custodial symmetry. The custodial symmetry is satisfied if $M_{A}=M_{H^{ \pm}}$or $M_{H}=M_{H^{ \pm}}$. To avoid invisible Higgs decays like $h \rightarrow a a^{\star} \rightarrow \chi \bar{\chi}$, where $a^{\star}$ is the complex conjugate of $a, M_{a}$ is constrained to be $M_{a}>100$ $\mathrm{GeV}$ [59]. The model also assumes that the pseudoscalar $a$ decays only to $\chi$, i.e., $\mathrm{BR}(a \rightarrow \chi \bar{\chi})=$ $100 \%$, hence the DM-pseudoscalar coupling constant $g_{\chi}=1$. For on shell processes like $a \rightarrow \chi \bar{\chi}$, the pseudoscalar mass is constrained to be $M_{a}>2 m_{\chi}$. The benchmark model considered in this thesis has $m_{\chi}=10 \mathrm{GeV}$, to allow probing low $M_{a}$ regions. To avoid divergences at negative infinity, the scalar potential is required to be bounded from below. To satisfy this criterion along with the requirement that $\lambda_{3}$ has to be perturbative, the benchmark model sets $\lambda_{3}=\lambda_{P 1}=\lambda_{P 2}=3$. Since 
$\sin \theta=0.35$ [58] gives the highest sensitivity of the mono-Higgs signal to the 2HDM+a parameter space, it is used as a benchmark value.

The $2 \mathrm{HDM}+a$ model produces a $h+\chi \bar{\chi}$ final state, where $A$ decays to a SM Higgs $h$ and a pseudoscalar $a$, followed by a subsequent decay of the pseudoscalar to DM particle $\chi(a \rightarrow \chi \bar{\chi})$. There are two production mechanisms corresponding to the $h+\chi \bar{\chi}$ final state: (i) $b b$ initiated process, and (ii) gluon-gluon fusion (ggF) process, as shown in Figure 16

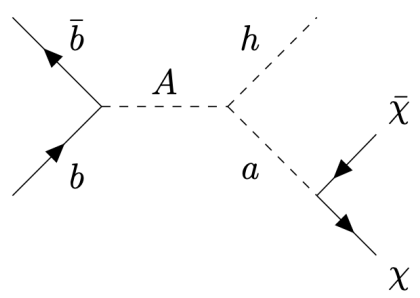

(a)

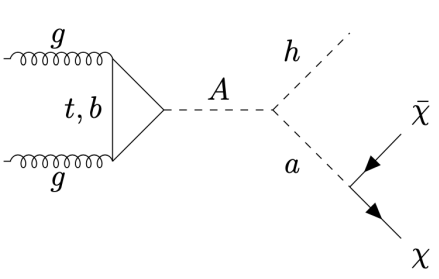

(b)

Figure 16: Feynman diagrams of $b b$-initiated (fig. 17b) and ggF (fig. 16b) production mechanisms in $2 \mathrm{HDM}+a$ model with $h+\chi \bar{\chi}$ final state.

In the Type-II $2 \mathrm{HDM}+a$ model, the $q \bar{q} A$ coupling is proportional to $1 / \tan \beta$ for up-type quarks and $\tan \beta$ for down-type quarks respectively. As a consequence, the cross sections for the $\mathrm{ggF}$ and $b b$-induced models are dependent on the choice of $\tan \beta$. The ggF mechanism is dominant for low $\tan \beta$, while the $b b$-induced mechanism is dominant for high $\tan \beta$ regions. For the benchmark models considered in this thesis, $\tan \beta=1$ (ggF dominant) and $\tan \beta=10$ ( $b b$ dominant) are chosen. Both the processes have similar cross sections for $\tan \beta \sim 5$ [58].

In the $2 \mathrm{HDM}+a$ model, the $E_{\mathrm{T}}^{\text {miss }}$ signatures are produced both resonantly and non resonantly. In the resonant channels such as the $h+E_{\mathrm{T}}^{\mathrm{miss}}$ (c.f. Figure 16), one has to satisfy the kinematic criterion of $M_{A}>M_{h}+M_{a}$. In addition to the diagrams shown in Figure 16, other $2 \mathrm{HDM}+a$ processes can have $h+\chi \bar{\chi}$ final state and are shown in Figure 17. One such example is the non-resonant production $a \rightarrow A h$, where an off-shell $a$ decays to $h$ and $A$, with $A \rightarrow \chi \bar{\chi}$. Other possible processes include Higgs-Strahlung where either $A$ or $a$ is produced and decays to $\chi \bar{\chi}$ after radiating a $\mathrm{SM} h$.

The $2 \mathrm{HDM}+a$ parameter space is also constrained from both direct detection and indirect 


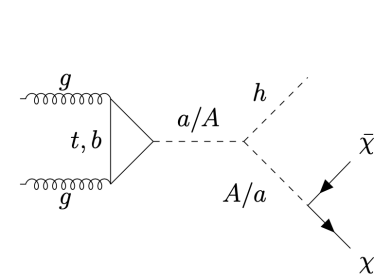

(a)

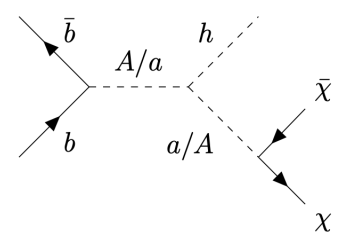

(e)

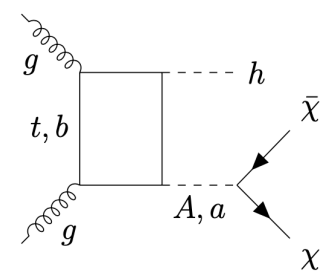

(b)

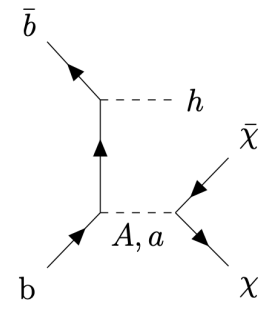

(f)

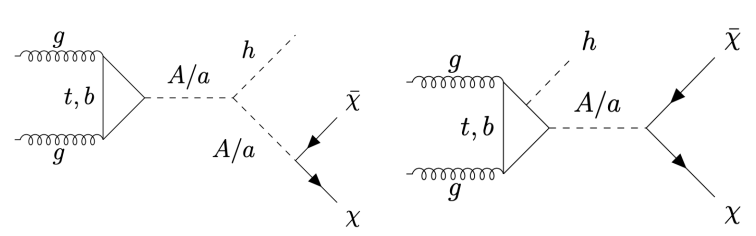

(c)

(d)

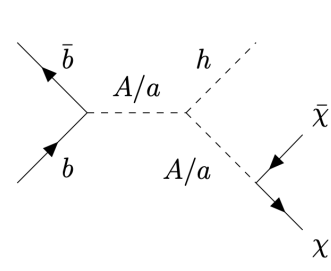

(g)

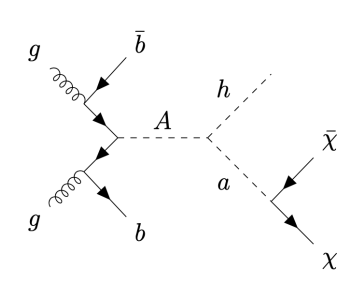

(h)

Figure 17: Top panel: Feynman diagrams for 2HDM+a ggF mechanism; non-resonant (Figure 17a and 17b), Higgs-Strahlung (Figure 17c), single resonant (Figure 17d). Bottom panel: Feynman diagrams for $2 \mathrm{HDM}+a b b$ induced mechanism; non-resonant (Figure 17e and 17f), Higgs-Strahlung (Figure 17g). Figure $17 \mathrm{~h}$ represents the $2 \mathrm{HDM}+a b b$ induced mechanism with additional $b$-jets.

detection dark matter experiments. The 2HDM+a one-loop Feynman diagrams, as shown in Figure 18, are dominant in the direct detection cross sections. Direct detection experiments provide constraints to regions with $M_{a}<2 m_{\chi}$, while the mono- $h$ search conducted in the thesis aims to constrain $M_{a}>2 m_{\chi}$ regions.
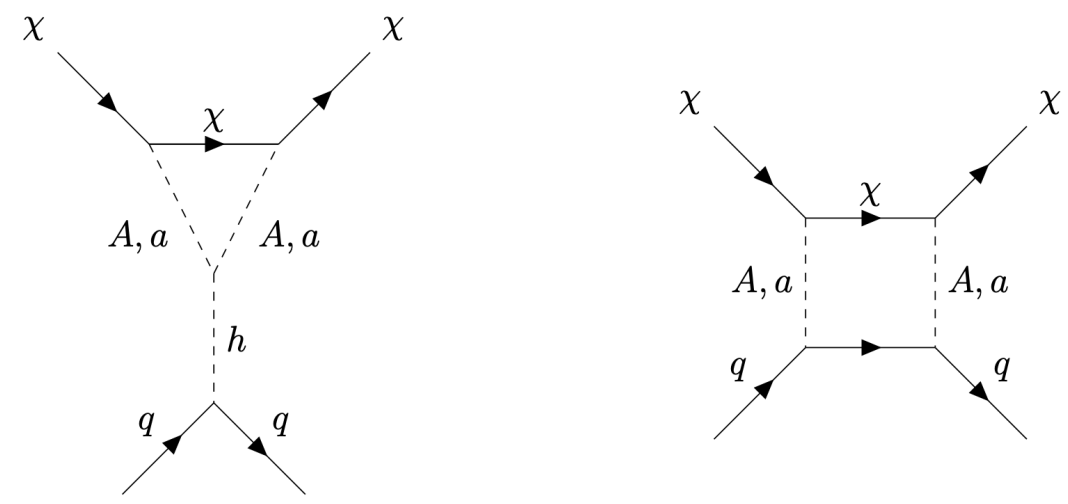

Figure 18: Spin independent one loop $2 \mathrm{HDM}+a$ processes that generate DM-nucleon scattering cross sections in direct detection experiments.

Since the pseudoscalars $A$ and $a$ have strong coupling constants with SM, the $2 \mathrm{HDM}+a$ 
provides many possible signals. Figure 19 shows the diagrams sensitive to indirect detection experiments where the DM particles annihilate and produce final states with SM fermions $(f \bar{f})$ or combinations of $2 \mathrm{HDM}$ parameters such as, $A a, A A, h a, h A, H a, H A$. The cross sections of these different processes are highly dependent on $m_{\chi}$. For example, processes such as $\chi \bar{\chi} \rightarrow f \bar{f}$ are resonantly enhanced for $m_{\chi} \simeq M_{a} / 2$. All other processes such as $\chi \bar{\chi} \rightarrow X Y$, where $X, Y$ are spin-0 bosons are dominant for $m_{\chi}>\left(m_{X}+m_{Y}\right) / 2$. As the benchmark model considers a fixed $m_{\chi}=10$ $\mathrm{GeV}$, it is complementary to indirect detection experiments as well.
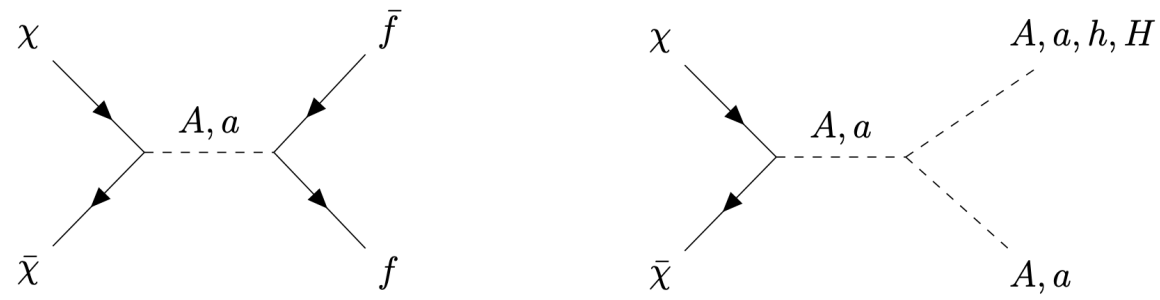

Figure 19: Tree level diagrams where DM pairs annihilate to pair of SM fermions (f) or spin-0 states such as $A, a, h, H$. These processes are sensitive to ID dark matter experiments.

\subsection{2 $Z^{\prime}$-2HDM model}

The $Z^{\prime}-2 \mathrm{HDM}$ model is an extension of the Type-II 2 HDM model with a $U(1)_{Z^{\prime}}$ symmetry, where $U(1)_{Z^{\prime}}$ gauge symmetry gets broken spontaneously above the EW scale producing the massive spin-1 mediator - the $Z^{\prime}$ boson. An important specification of the model is that only the RH up-type quarks $\left(u_{R}\right)$ are charged under $U(1)_{Z^{\prime}}$ symmetry. As a consequence, although $Z^{\prime}$ might be produced at the LHC, since the leptons are neutral the model would avoid stringent constraints from dilepton resonances $\left(Z^{\prime} \rightarrow \ell \ell\right)$. In addition to the $2 \mathrm{HDM}$ model parameters, the parameters of the $Z^{\prime}-2 H D M$ model are:

$$
\left(M_{Z^{\prime}}, M_{A}, g_{Z^{\prime}}, g_{\chi}\right)
$$

where $M_{Z^{\prime}}$ is the mass of $Z^{\prime}, M_{A}$ is mass of the neutral CP-odd $A, g_{Z^{\prime}}$ is the the universal coupling strength of $Z^{\prime}$ and $g_{\chi}$ is the $A-\chi$ coupling constant. The $h+\chi \bar{\chi}$ signature is produced via $p p \rightarrow Z^{\prime} \rightarrow A h$, and a subsequent decay of $A \rightarrow \chi \bar{\chi}$ as shown in Figure 20, Parameters listed 


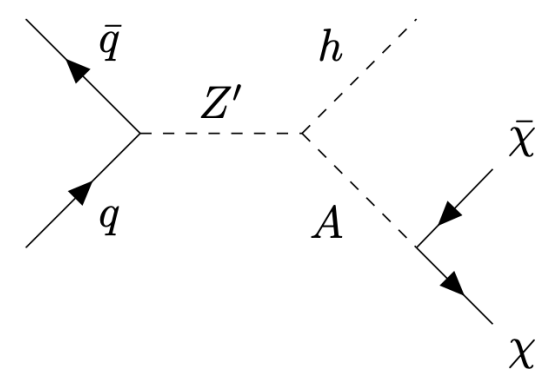

Figure 20: Feynman diagram corresponding to the final state $h+\chi \bar{\chi}$ in the $Z^{\prime}-2 \mathrm{HDM}$ model.

in equation 50 are free parameters of the model and can take any values within kinematic limits. However, the thesis only considers $m_{Z^{\prime}}$ and $M_{A}$ as free parameters, while the rest of the variables constrained. Variations of $\tan \beta$ and $g_{Z^{\prime}}$ do not alter the kinematics of the signal process. To maximize the production cross section of $p p \rightarrow Z^{\prime}$, the $\mathrm{BR}\left(Z^{\prime} \rightarrow h A\right)$, and to be consistent with the dijet searches, the chosen benchmark values for these two parameters $\operatorname{are} \tan \beta=1$ and $g_{Z^{\prime}}=0.8$. The model assumes that BR $(A \rightarrow \chi \bar{\chi})=100 \%$, i.e., $A$ decays exclusively to $\chi \bar{\chi}$, hence, $g_{\chi}=1$. The masses of the heavy Higgs bosons are chosen in accordance with the ATLAS and CMS agreement, $M_{A}=M_{H^{ \pm}}$or $M_{H}=M_{H^{ \pm}}$. The choice of the DM mass $m_{\chi}$ is dependent on two phenomena: (i) on-shell production of $A$, and (ii) invisible Higgs decays $(h \rightarrow \chi \bar{\chi})$. For on-shell production of $A$, $M_{A}>2 m_{\chi}$ condition has to be satisfied. Besides, to avoid invisible Higgs decays, one needs to satisfy $m_{\chi}>M_{h} / 2$. To comply with the above-mentioned criterion and to be able to probe low $M_{A}$ regions, the DM mass is set at $m_{\chi}=100 \mathrm{GeV}$. Similar to the 2HDM scenario where the $H$ and $h$ are connected to the SM Higgs, in $Z^{\prime}-2 \mathrm{HDM}$ the heavy $Z^{\prime}$ is also connected to the $\mathrm{SM} Z$ boson. The tree-level masses of $Z$ and $Z^{\prime}$ are given as:

$$
\begin{gathered}
M_{Z}^{2} \approx\left(M_{Z}^{0}\right)^{2}-\varepsilon^{2}\left[\left(M_{Z^{\prime}}^{0}\right)^{2}-\left(M_{Z}^{0}\right)^{2}\right] \\
M_{Z^{\prime}}^{2} \approx\left(M_{Z^{\prime}}^{0}\right)^{2}+\varepsilon^{2}\left[\left(M_{Z^{\prime}}^{0}\right)^{2}-\left(M_{Z}^{0}\right)^{2}\right] .
\end{gathered}
$$

where $\varepsilon$ is the mixing parameter and $M_{Z}^{0}, M_{Z^{\prime}}^{0}$ are the masses of $Z$ and $Z^{\prime}$ without mixing.

The $Z^{\prime}$ can also decay as $Z^{\prime} \rightarrow Z h$, where the $Z$ subsequently decays to neutrinos $Z \rightarrow v \bar{v}$. Similar to $\chi$, the neutrinos $(v)$ will leave $E_{\mathrm{T}}^{\text {miss }}$ signature in the detector. Hence, $h+v \bar{v}$ and $h+\chi \bar{\chi}$ 
signatures become indistinguishable from each other. Besides, the $Z^{\prime} \rightarrow Z h$ and $Z^{\prime} \rightarrow A h$ signatures are experimentally identical, having comparable production cross sections. For low $\tan \beta$, the cross section of $Z^{\prime} \rightarrow A h$ scales as $1 / \tan \beta^{2}$ and since the benchmark model sets $\tan \beta=1$, both these processes will contribute equally to the total cross section. Besides, the $M_{Z^{\prime}}-M_{A}$ phase space is highly constrained from dijet $\left(Z^{\prime} \rightarrow q \bar{q}\right)$ searches $[60-62]$. The dijet constraints can be avoided by choosing a small $g_{Z^{\prime}}$. However, it will reduce the mono-Higgs signal strength by a factor of $\left(0.8 / g_{Z^{\prime}}\right)^{2}$.

Despite these shortcomings, the $Z^{\prime}-2 \mathrm{HDM}$ model is still an important model to consider for DM searches at the LHC. The model has a high sensitivity to boosted Higgs decays which are not common in other existing models. Studying the $Z^{\prime}$-2HDM model can give insights into new theories targeting highly boosted Higgs phase space and serve as a benchmark model. 


\section{LHC AND THE ATLAS DETECTOR}

The number of particles reaching earth from astrophysical and cosmic sources are statistically limited to verify SM predictions and discover new particles. Thus increasingly high energy colliders have been constructed to find massive and rare new particles. In a controlled collider environment, the interesting events can be studied carefully. The Large Hadron Collider (LHC) at CERN is the highest energy collider to date, colliding $6.5 \mathrm{TeV}$ proton beams circulating in opposite directions. As a result of these very high energetic collisions, many new particles are generated in a very short time and the outcome can be studied under controlled environment. The particles generated in the $p p$ collisions are detected and specially reconstructed in detectors surrounding the collision points. There are four such large particle detectors in four $p p$ intersection points: ATLAS, CMS, ALICE, and LHCb [63-66]. This chapter describes the design and operation of the LHC in section 3.1 and gives an overview of the ATLAS detector in section 3.2 .

\subsection{The Large Hadron Collider}

The LHC [67] is a two-ring superconducting hadron accelerator installed in a tunnel with a radius of $\sim 26.7 \mathrm{~km}$ and a depth in the range of $45-170 \mathrm{~m}$. The geographical overview of the LHC is shown in Figure 21. The LHC [69-71] was built to search for the origin of EWSB, Higgs

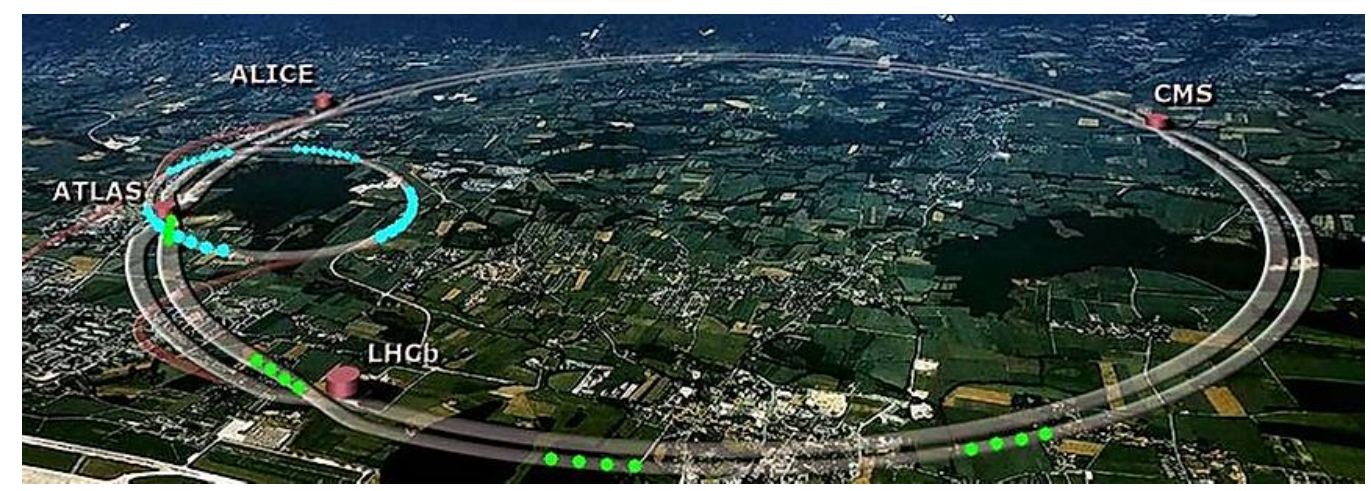

Figure 21: Geographical representation of the LHC: the two big circles represent the LHC accelerator rings and the smaller circles represent the SPS rings. The figure also shows the locations of the four large detectors: ATLAS, CMS, ALICE and LHCb. The whole system is underground with only control rooms at ground level [68]. 
boson, and Beyond Standard Model (BSM) physics. It accelerates proton beams using the $\sim 26.7$ $\mathrm{km}$ ring and the proton-proton collisions are recorded by the four large detectors located across the ring. In a collider experiment new particles with high masses can be generated as the colliding particles can have high energy levels. For the discovery of new particles a high level of statistical power is necessary to minimise experimental fluctuations. The total number of events $\left(N_{\text {event }}\right)$ can be written as:

$$
N_{\text {event }}=\sigma_{\text {event }} \times L
$$

where $\sigma_{\text {event }}$ is the event cross-section and $L$ is the luminosity. Luminosity [72] quantifies how many collisions a collider can produce per $\mathrm{cm}^{2}$ per second. For a Gaussian like beam profile the luminosity can be written as:

$$
L=\frac{N^{2} n_{b} f_{r e v}}{4 \pi \sigma_{x} \sigma_{y}} F .
$$

Here $\mathrm{N}$ is the number of particles (protons for LHC) per bunch, $n_{b}$ is the number of bunches in one

beam, $f_{r e v}$ is the revolution frequency, $\sigma_{x, y}$ are transverse beam widths in the Gaussian approximation, $\mathrm{F}$ is the geometric luminosity reduction factor due to the crossing angle at the Interaction Points (IPs). Luminosity has a unit of $\mathrm{cm}^{-2} \mathrm{~s}^{-1}$ and the LHC delivered a peak luminosity of $2.1 \times 10^{34} \mathrm{~cm}^{-2} \mathrm{~s}^{-1}[69]$.

The circulating protons are in bunches and each bunch contains $\approx 10^{11}$ protons. When these bunches collide, several proton interactions can take place in addition to hard collisions which are of interest to us. These soft extra collisions are called pile-up events, denoted by $<\mu>$ (number of interactions per bunch crossing), and makes analyses challenging at the LHC.

\subsubsection{Design of the $\mathrm{LHC}$}

The LHC accelerator ring has eight arcs and eight straight sections and as shown in Figure 22, the ATLAS and CMS detectors are located at diametrically opposite straight sections, commonly known as Point 1 and Point 5 respectively, while LHCb and ALICE are located at Point 2 and Point 8, respectively. Two counter-rotating proton beams are accelerated through the rings and the injection systems for Beam 1 and Beam 2 are located at Point 1 and Point 8 respectively. When needed, the beams are dumped out from the ring at the straight section at Point 6 . The 
acceleration of the charged particles at the LHC is done through Radio-Frequency (RF) cavities, with EM fields. The LHC has 16 superconducting RF cavities with an oscillating frequency of $400 \mathrm{MHz}$ across the acceleration ring, through which particles travel in a vacuum. Depending on the time of arrival, the RFs accelerate or decelerate the protons, thus creating discrete bunches of protons. A maximum of 2808 such bunches with a bunch spacing of $25 \mathrm{~ns}$ can be accommodated at the LHC.

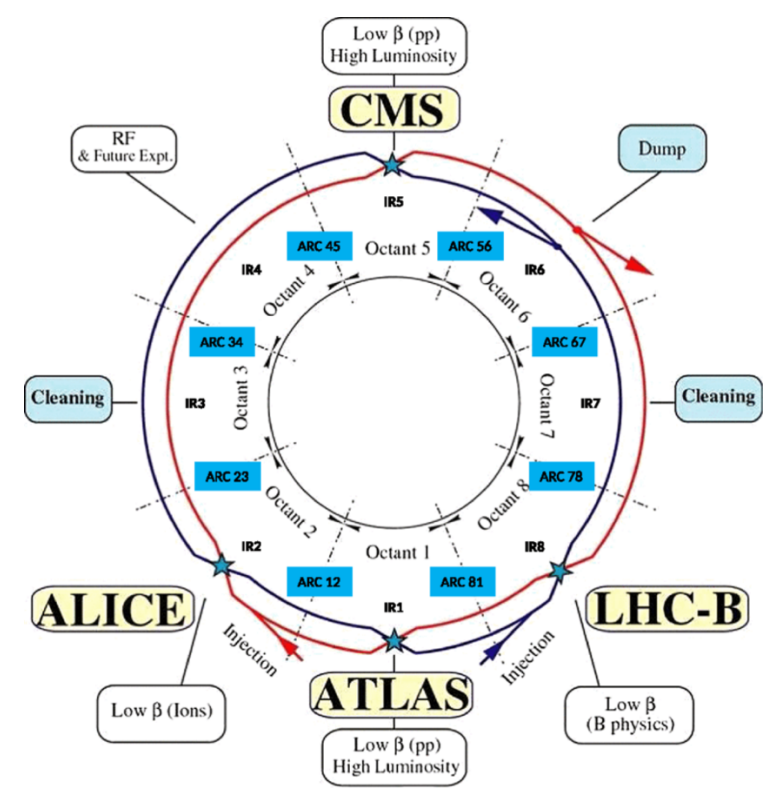

Figure 22: LHC ring schematic: two counter-rotating proton beams marked in blue and red are accelerated in the ring before they collide in four IPs where the four large detectors are located [67].

\subsubsection{Beam acceleration and injection}

As shown in Figure 23, the protons are created and sent through a beam injection and preacceleration chain before reaching the LHC ring and the RF cavities as: Linac $2 \rightarrow$ Proton Synchrotron Booster (PSB) $\rightarrow$ Proton Synchrotron (PS) $\rightarrow$ Super Proton Synchrotron (SPS). Starting with a Hydrogen gas source in a magnetic field, the Hydrogen atoms are broken into their constituent electrons and protons. The protons are accelerated to $50 \mathrm{MeV}$ in the linear accelerator Linac 2, which injects the protons to the PSB, consisting of four rings. Each PSB ring is filled with one proton bunch, which is accelerated up to an energy of $1.4 \mathrm{GeV}$. The proton bunches are next 
injected to the PS, which has many RF systems with different frequencies and create a bunch spacing of $25 \mathrm{~ns}$ appropriate to the LHC bunch crossing time. The PS accelerates the proton beams to an energy level of $26 \mathrm{GeV}$ and splits them into 81 bunches. These proton bunches are next injected to the SPS which accelerates the bunches to $450 \mathrm{GeV}$, the injection energy of the LHC. Finally they are injected into the LHC ring, where while passing the RF cavities the bunches gain energy. It reaches an energy level of $6.5 \mathrm{TeV}$ from $450 \mathrm{GeV}$ in $\sim 20$ minutes after passing the RF cavities more than 10 million times.

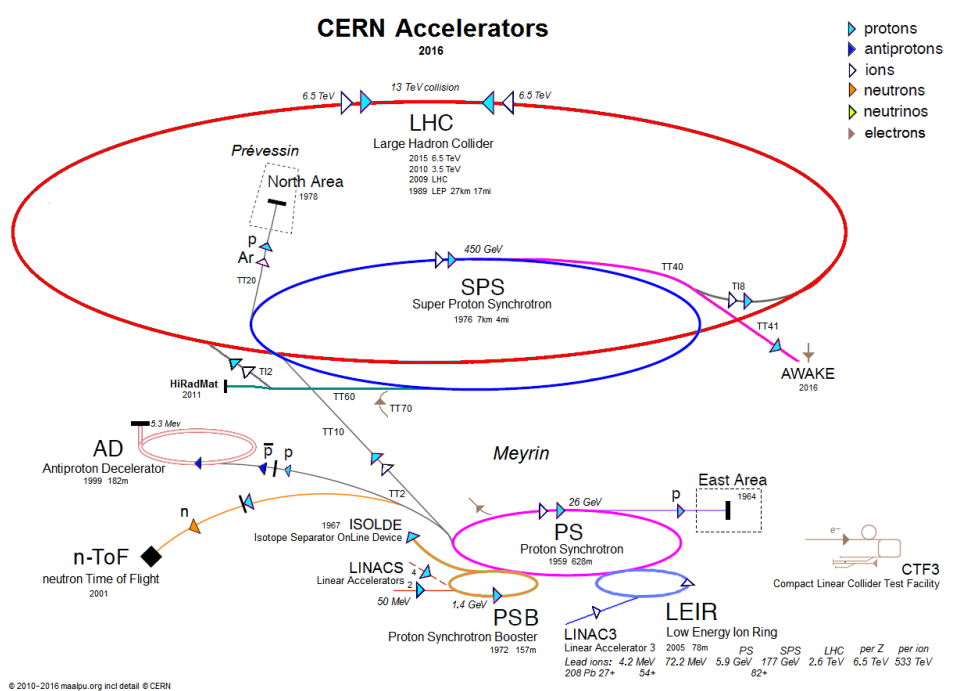

Figure 23: The LHC design layout with the beam injection components [73].

\subsubsection{LHC magnet system}

The LHC aims to operate at TeV energy scale. The relativistic total kinetic energy $E$ of a charged particle of mass $m$ and charge $q$ rotating in a magnetic field $B$ with relativistic velocity $v$ can be written as:

$$
E=m c^{2} \gamma=\frac{q B c^{2}}{\omega} \quad \text { where } \omega=\frac{q B}{\gamma m}
$$

where the relativistic Lorentz factor $\gamma=\frac{1}{\sqrt{1-\frac{v^{2}}{c^{2}}}}$ and $\omega$ is the synchrotron frequency. The LHC requires a high magnetic field to bend the protons across the arcs in the ring and keep them collimated. The LHC magnetic strength is $8.33 \mathrm{~T}$ and to generate such high magnetic fields, the LHC uses $\mathrm{NbTi}$ superconducting magnets which are cooled down to $1.9 \mathrm{~K}$ using superfluid helium [74]. 
To bend the particles across the arcs of the LHC tunnel, 1232 dipole magnets (Main Bends, MBs) [69, 75] are used with $8.33 \mathrm{~T}$ magnetic field ( 11600 A). To achieve maximum number of proton-proton collisions, the proton bunches are focused using 392 Main Quadrupoles (MQ) which can produce a maximum magnetic field of 7.5 T. The MQs are also known as the insertion magnets as they are used in the insertion regions of the LHC. Since the magnets are very close to each other, they are coupled both magnetically and mechanically, corrector magnets are used to correct for the magnetic interference effects. The magnets are used to clean up beams after collisions to protect the detector from stray particles near the IP.

\subsection{The ATLAS Detector}

The ATLAS (A Toroidal LHC ApparatuS) [76] detector is one of the two general-purpose detectors hosted at the LHC with a design luminosity of $10^{34} \mathrm{~cm}^{-2} \mathrm{~s}^{-1}$ to probe $p p$ and heavy-ion collisions. The ATLAS detector is located at Point 1 of the LHC and has a height of $25 \mathrm{~m}$, length of $44 \mathrm{~m}$, and a weight of 7000 tonnes. The ATLAS detector uses a right-handed coordinate system with its origin at the particle IP. The beam direction defines $z$-axis, the $x$-axis points towards the center of the LHC ring, and the $y$-axis points upwards. It has a cylindrical coordinate $(r, \theta, \phi)$ system: the azimuthal angle $\phi$ is about the $z$-axis and $\phi=0$ along positive $x$-axis; the spherical polar angle $\theta$ is defined with respect to the $z$-axis and $\theta=0$ along the $z$-axis. A commonly used variable to describe a particle's location inside the detector is pseudorapidity, $\eta$, defined as $\eta=-\ln [\tan (\theta / 2)]$, where $\theta$ represents the angle between the particle and $z$-axis. Figure 24 is a cutaway view of the ATLAS detector which shows the four main sub-detectors: Inner Detector, Electromagnetic Calorimeter, Hadronic Calorimeter and Muon Spectrometer. The ATLAS data taking started in November 2009 with a pp collision energy of $900 \mathrm{GeV}$. In 2010 high energy collisions started with an energy of $7 \mathrm{TeV}$ and was increased to $8 \mathrm{TeV}$ in 2012. The data taking period between 2009-2013 is known as LHC Run 1. The LHC had a long shutdown for two years, for operations at higher energies. It restarted in 2015 with a pp collision Center of Mass (COM) energy of $\sqrt{s}=13 \mathrm{TeV}$ and ATLAS recorded a total of $139 \mathrm{fb}^{-1}$ of $p p$ collision data between 2015- 
2018 (Run 2), which are used for this thesis. The following sections give an introduction to the ATLAS detector and its different sub-detectors: Inner Detector in Section 3.2.1, Electromagnetic and Hadronic calorimeters in sections 3.2.2 and 3.2.2.2 respectively, and Muon Spectrometer in section 3.2.3. The ATLAS magnet system is described in section 3.2.4, and the Trigger and Data Acquisition System (DAQ) are described in section 3.2.5.

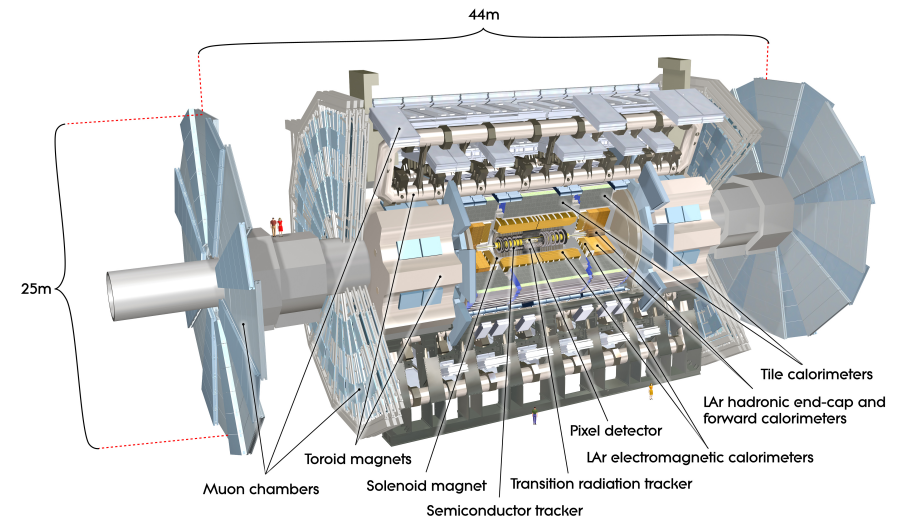

Figure 24: Cutaway view of the ATLAS detector with different sub-modules [77].

\subsubsection{Inner Detector}

The Inner Detector (ID) [78] is the inner most first part of the ATLAS $(|\eta|<2.5)$ detector which measures charge, momentum, direction of charged particles, and identifies primary and secondary vertices. The ID components record energy deposits (hits) from charged particles, which with appropriate software reconstructs particle tracks. Due to close proximity to the IP, the ID endures a very high particle density, thus necessitating high granularity to achieve good resolution in position, momentum, and vertex measurements. Semiconductor tracking devices, using pixel technology and silicon strips, provide the desired performance goals. The ATLAS ID consists of three modules: (i) Pixel Detector, (ii) Semiconductor Tracker (SCT), and (iii) Transition Radiation Tracker (TRT). The whole setup is immersed in a magnetic field of $2 \mathrm{~T}$ generated by the superconducting solenoid magnet.

The silicon Pixel Detector, the innermost part of the ID, consists of three sections: a barrel, 


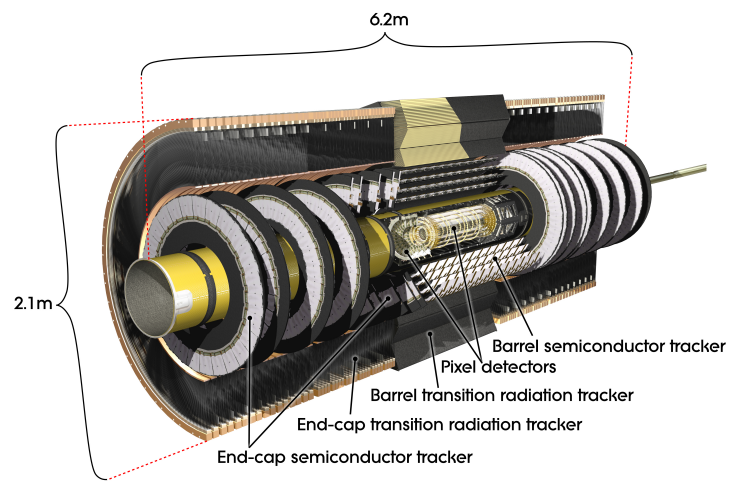

Figure 25: Cutaway view of ATLAS inner detector with its sub-modules [79].

and two end-caps. Its sensors are $250 \mu \mathrm{m}$ thick detectors of pixels of reverse-biased n-type silicon semiconductor of size $50 \times 400 \mu \mathrm{m}^{2}$. The barrel section has four layers and each end-cap consists of three pixel disks. For Run 2 an additional layer, the Insertable $B$-Layer (IBL), was installed between the beam pipe and the $B$-layer of the Pixel detector to enhance vertexing, improve $b$-jet identification performance and robustness against high luminosity, radiation dose, and hardware lifetime. The pixels are individually connected to 16 Front-End (FE) [80] chips and the chips are read out by a Module Control Chip (MCC) [81]. Each module is connected to the off-detector Read-out Drivers (RODs) through optical-fibre links to read out events.

The Semiconductor Tracker (SCT) also uses silicon semiconductor and helps provide momentum measurement, vertex position, and impact parameter information in the radii region of $30-56 \mathrm{~cm}$. It has a strip-like geometry and the modules are arranged into four coaxial cylindrical tile layers in the barrel region, and nine disk layers in each of the two end-caps. The barrels cover $|\eta|<1.4$, while the disks cover $|\eta|<2.5$. Figure 26 shows a charged particle (red) passing four layers of the SCT. The SCT readout system is based on 12 ABCD3TA [82] chips, an Application Specific Integrated Circuit (ASIC), that amplifies, shapes, and converts the strip signals into an output signal. The ABCD3TAs are connected to optical links [83] which sends data to the offdetector electronics.

The Transition Radiation Tracker (TRT) detector is the outermost part of the ID, covering $|\eta|<2.0$, and is composed of drift tubes (straws) of $4 \mathrm{~mm}$ diameter. The straws, filled with a gas 


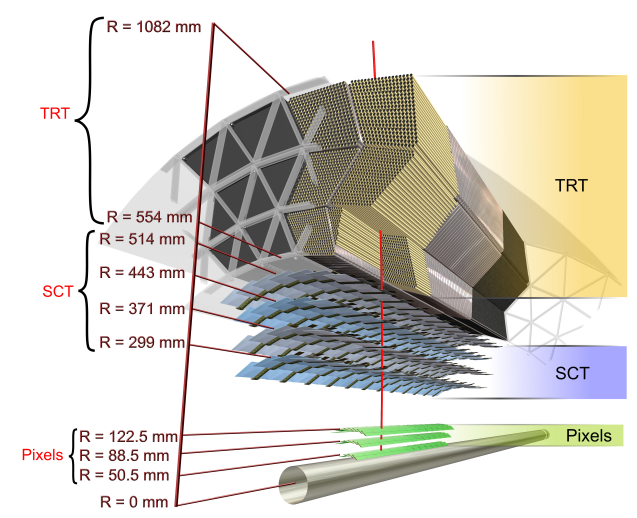

Figure 26: Figure shows the three ID modules: the Pixel, SCT and TRT. The red line represents a charged particle of $p_{\mathrm{T}}=10 \mathrm{GeV}$ passing through the modules [79].

mixture of $70 \% \mathrm{Xe}, 20 \% \mathrm{CO}_{2}$ and $3 \% \mathrm{O}_{2}$, act as proportional counters. While interacting with charged particles, the gas medium gets ionised and the resulting ions drift to the inner walls of the straws, producing the track hits. The TRT consists of a barrel part $(|\eta|<1)$ and two end-caps $(1<|\eta|<2)$. A charged particle with $p_{\mathrm{T}}>0.5 \mathrm{GeV}$ and $|\eta|<2.0$ will pass through at least 36 straws through most of the TRT regions. Instead of being stacked directly on top of each other, the intermediate space between two straws is filled with polymer fibre and coils in the barrel and end-cap regions respectively, creating a Transition Radiation (TR) (when charged particles interact with the transition region between the straws), which provides additional particle identification in addition to track position information. The TRT FE readout system consists of analog and digital components. The analog readout is based on Amplifier Shaper Discriminator Baseline Restorer (ASDBLR), which amplifies and shapes outputs from the straws and generates low and high threshold signals. These signals are then read by a Digital Time Measurement ReadOut Chip (DTMROC). The DTMROC performs the signal timing measurements and sends digitised signals to the off-detector electronics. 


\subsubsection{ATLAS Calorimetry}

In nuclear and particle physics, calorimeters typically measure the energies and some properties of the traversing particles via the type of their interactions in the calorimeter materials. A calorimeter typically contains two types of materials, active and passive/absorber. When particles interact with the absorber, they create showers of secondary particles which later on are detected by the active medium. As shown in Figure 27, the particle showers are classified into two categories: (i) Electromagnetic shower developed through the EM interaction, and (ii) Hadronic shower developed via the strong and EM interaction. The EM shower is developed by successive
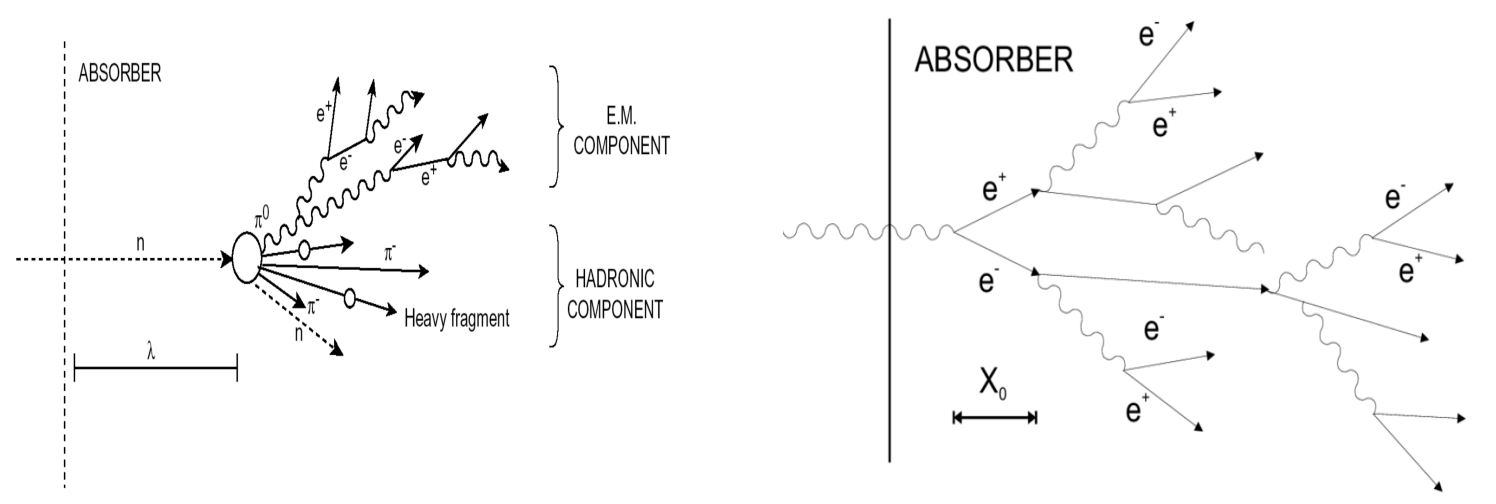

Figure 27: Left plot [84]: schematic of a Hadronic shower development which has a Hadronic component and an Electromagnetic component. Right plot [85]: schematic of an Electromagnetic shower development.

pair-production and Bremsstrahlung and it continues until the photon energy is insufficient for pair production or the lepton energy goes below a critical energy threshold $\left(\mathrm{E}_{c}\right.$ : energy below which ionization dominates) necessary for Bremsstrahlung. An important parameter for EM shower development is the radiation length $\left(X_{0}\right)$, which depends on the characteristics of the calorimeter material [86]:

$$
X_{0} \approx \frac{716 A}{Z(Z+1) \ln (287 / \sqrt{Z})} \mathrm{g} \mathrm{cm}^{-2},
$$

where $A$ and $Z$ are atomic weight and numbers of the material respectively. For liquid $\operatorname{Ar} X_{0} \approx 14$ $\mathrm{cm}$ and it represents the average distance an electron needs to travel to reduce its energy $(E)$ to $1 / e^{\text {th }}$ of its initial energy $\left(E_{0}\right): E=E_{0} e^{-x / X_{0}}$. Similarly a photon looses $1 / e^{\text {th }}$ of its intensity $(I)$

after traveling a distance of $\frac{9 X_{0}}{7}: I=I_{0} \exp \left(-\frac{7 \mathrm{x}}{9 \mathrm{X}_{0}}\right)$. The hadronic shower is more complex than 
the EM shower and the shower development is determined by the interaction length $\lambda$, which is characterized as: $\lambda \approx 35 \mathrm{~A}^{1 / 3} \mathrm{~g} \mathrm{~cm}^{-2}$, where $A$ is the atomic weight of the material; for liquid Ar $\lambda \approx 83.7 \mathrm{~cm}$. The hadronic shower formation takes place when the incoming hadron interacts with a nucleus and produces secondaries in the nuclear interaction. This includes unobserved energies, such as neutrinos produced in the interaction, neutrons which may or may not produce further interactions, and very importantly, the loss in binding energy to break up the nucleus, which varies depending on the specific nuclear interactions taking place.

This section describes the two dedicated ATLAS calorimeter systems (i) Electromagnetic, which detects $\mathrm{e}^{ \pm}$and neutral particles such as photon $\left(\gamma\right.$ and $\left.\pi^{0}\right)$, and (ii) Hadronic calorimeter which detects $\pi^{ \pm}, \mathrm{K}^{ \pm}, \mathrm{p}^{ \pm}$and neutral particles such as $\mathrm{n}, \mathrm{K}_{L}^{0}$, while muons $\left(\mu^{ \pm}\right)$escape the calorimeters undetected.

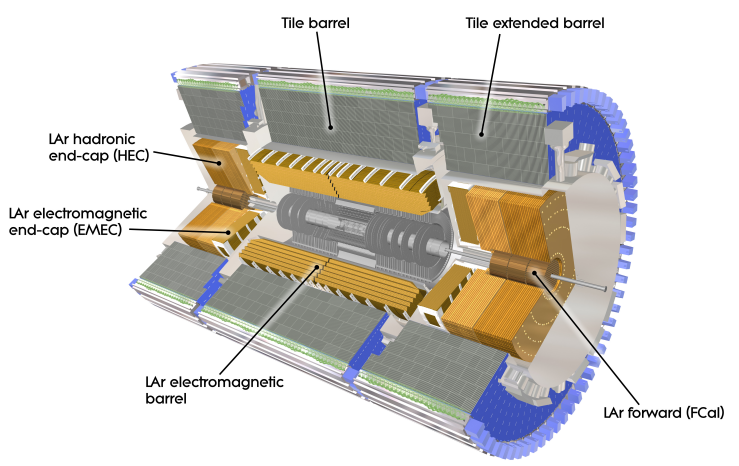

Figure 28: The ATLAS calorimeter system with different sub-systems [87].

\subsubsection{Electromagnetic Calorimeter}

As shown in Figure 29, ATLAS electromagnetic calorimeter (EM calorimeter) has an accordion geometry and provides complete coverage in $\phi$ without azimuthal cracks, fast signal extraction and uniform resolution. It uses lead ( $2 \mathrm{~mm}$ thick plates) as the absorber material and liquid Argon (LAr) $\left(2.1 \mathrm{~mm}\right.$ thick and an operating temperature of $\left.-183^{\circ} \mathrm{C}\right)$ [89] as the active medium. The EM calorimeter is divided into three components: a barrel part with $\eta \mid<1.475$, and two end-caps with $1.375<|\eta|<3.2$. The end-caps are further divided into two coaxial wheels: outer 


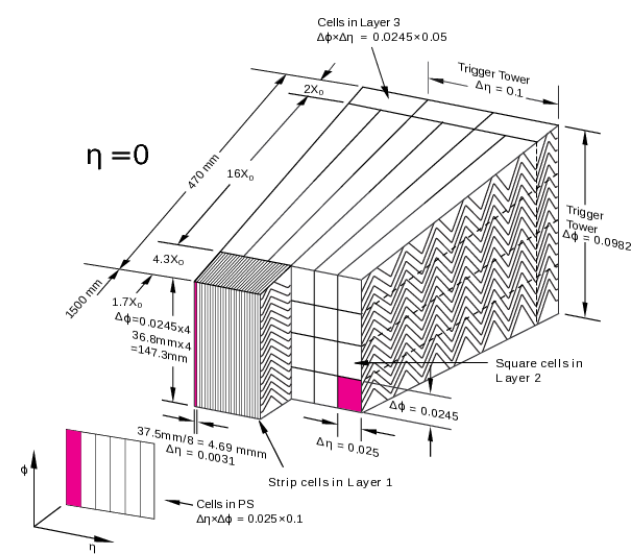

Figure 29: Barrel module of the ATLAS EM calorimeter system with different layers and granularity in $\phi$ and $\eta$ for each layer [88].

wheel spanning $1.375<|\eta|<2.5$ and inner wheel covering $2.5<|\eta|<3.2$. There are three LAr layers in the region of $0<|\eta|<2.5$ which is also known as precision measurement region, and two LAr layers in the high- $\eta$ region $(2.5<|\eta|<3.2)$ and in the transition region between the barrel and EM end-caps. To rectify the energy loss from electrons and photons upstream of the EM calorimeter, a presampler detector is used in the region of $|\eta|<1$. The presampler consists of a LAr layer of thickness $1.1 \mathrm{~cm}$ in the barrel and $0.5 \mathrm{~cm}$ in end-cap regions respectively.

\subsubsection{Hadronic Calorimeter}

The ATLAS Hadronic Calorimeter (HCal) has three main components: (i) Tile calorimeter, (ii) LAr Hadronic end-cap Calorimeter, and (iii) LAr forward Calorimeter, as shown in Figure 28.

The Tile calorimeter [90] is a sampling calorimeter which uses steel as the absorber and scintillating plastic tiles as the active material. It covers the region of $|\eta|<1.7$ and is installed directly outside of the LAr EM calorimeter. The Tile calorimeter is divided intro three components: a central barrel covering $|\eta|<1.0$ region and two extended barrels in the region of $0.8<|\eta|<1.7$. Both the central and extended barrels, azimuthally divided into 64 modules, have layers of active region and absorbers. For complete coverage in $\phi$ plane, the scintillator tiles are arranged radially and normal to the beam line. A key element of the Tile calorimeter are the Photomultiplier tubes (PMTs), which converts the ultraviolet light produced in scintillating tiles into electric currents. 
Figure 30 shows the ATLAS Tile calorimeter design layout.

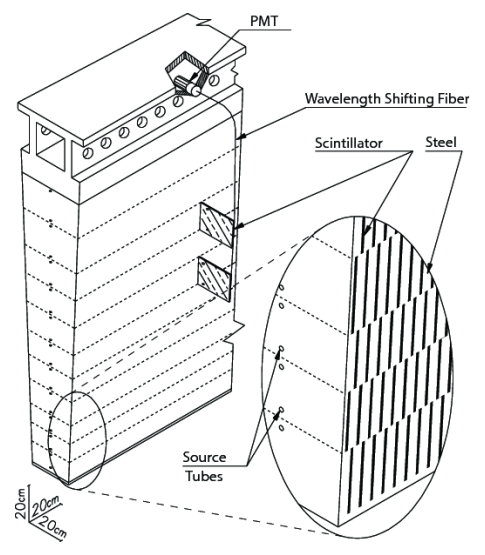

Figure 30: Schematic of the Tile calorimeter, the wavelength shifting fibre cables are kept at the edge of the tiles for complete azimuthal coverage and the readout fibres are grouped together into the PMTs [91].

The Hadronic End-cap Calorimeter (HEC) is also a sampling calorimeter like the Tile calorimeter and it uses LAr and copper as the active medium and the absorber respectively. The HEC consists of two independent wheels in each end-cap, a front-wheel (HEC1) and a rear wheel (HEC2), and is installed behind the end-cap EM calorimeter in the region of $1.5<|\eta|<3.2$. To avoid a drop in material density, the $\eta$ range of the HEC is chosen such that it overlaps with the region between end-cap and forward calorimeter at $|\eta|=3.1$ and with the Tile calorimeter in the region of $|\eta|=1.7$. The HEC uses the same cryostats as the electromagnetic end-cap (EMEC) and forward calorimeters (FCal). Figure 31 shows the ATLAS HEC design layout.

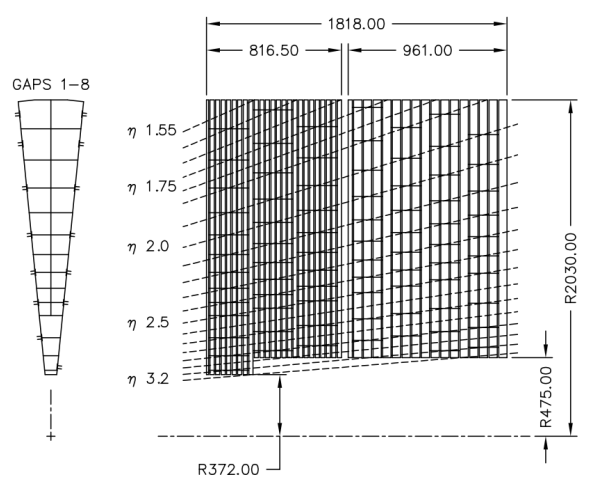

Figure 31: Schematic of the ATLAS HEC in the R- $\phi$ (left) and R-Z (right) plane, the dashed lines represent the readout cables [92]. 
The third component of the HCal is the Forward calorimeters (FCal) which covers the region of $3.1<|\eta|<4.9$ and shares the cryostats with the end-cap calorimeters. It uses LAr as the active medium and copper and tungsten as the absorber. The positioning of the FCal detector makes the coverage uniform and minimizes losses due to calorimeter cracks and works as a shield for the Muon Spectrometer from backgrounds. The FCal design has smaller LAr gaps $(<2 \mathrm{~mm})$ compared to Tile and HEC. Each FCal is composed of three components: one EM module FCal 1, and two hadronic modules FCal 2 and FCal 3. Figure 32 shows the ATLAS FCal design layout.

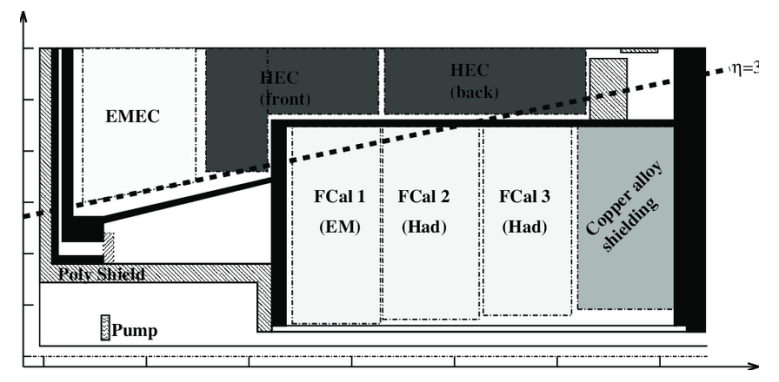

Figure 32: The ATLAS FCal detector schematic, the black sections represent the cryostats and additional support structures [93].

The readout system for the LAr calorimeters (both EM and hadronic) is based on Front End Boards (FEB) [94] installed on the detector. When charged particles ionize the LAr, the ionization current signal gets pre-amplified, shaped, buffered, and digitized by the FEBs. The digitized signals are sent to the RODs via optical links and after calculating the energy deposits, the RODs send the data to off-detector electronics via S-links. The Tile calorimeter readout is segmented into multiple cells (longitudinally and transversely) are organized into 4 partitions: two long barrels called LBA and LBC, an two extended barrels called EBA and EBC. Signal from each cell is read out by two PMTs via wavelength shifting fibers. The PMT signals are shaped, amplified and are sent to the off-detector electronics.

\subsubsection{Muon Spectrometer}

High energy prompt leptons can be signatures of many interesting physics processes. Hence, it is critical to detect muons and measure muons from the IP (for e.g. to study the Higgs decay). 
The Muon Spectrometer (MS) is the outermost part of the ATLAS detector, designed to measure high- $p_{\mathrm{T}}$ muons independently of the ID and to provide an independent muon trigger (c.f. section 3.2.5). The MS is surrounded by superconducting air-core toroid magnets (c.f. section 3.2.4), which deflects the muon tracks and allows the momentum measurements. The ATLAS MS consists of four types of chambers and has the largest surface area among all other modules of the ATLAS detector.

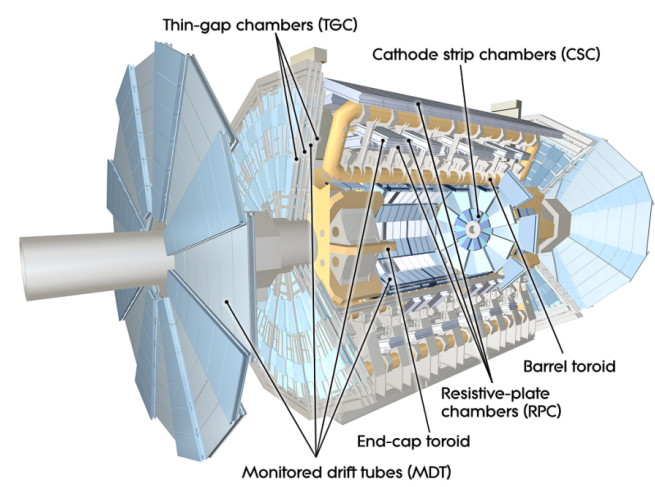

Figure 33: Cutaway view of the ATLAS MS [95].

Depending on $\eta$, different types of chambers are used for tracking and triggering system. For majority of the $\eta$ coverage the Monitored Drift Tubes (MDT's) provide precise measurement of the track coordinates in the principal bending direction of the magnetic field. In the regions of $2.0<|\eta|<2.7$ (close to the beam line), Cathode Strip Chambers (CSC's) are used instead of the MDT's since the CSC's have much higher granularity to withstand the high particle flux. The trigger system is installed in $|\eta|<2.4$ region and it consists of two types of chambers: Resistive Plate Chambers (RPC's) in the barrel region and Thin Gap Chambers (TGC's) in the end-caps. In addition to providing the momentum dependent muon trigger, these trigger chambers also identify bunch crossing and measure the second coordinate along the tube in MDT chambers. Table 4 summarises the different MS chambers and their functionality. 


\begin{tabular}{|c|c|c|c|}
\hline $\begin{array}{c}\text { Chamber } \\
\text { type }\end{array}$ & Functionality & Location & Coverage \\
\hline \hline MDT & Tracking & barrel+end-cap & $|\eta|<2.7$ \\
\hline CSC & Tracking & end-cap layer 1 & $2.0<|\eta|<2.7$ \\
\hline RPC & Trigger & barrel & $|\eta|<1.05$ \\
\hline TGC & Trigger & end-cap & $1.05<|\eta|<2.7$ \\
\hline
\end{tabular}

Table 4: Different types of chambers used in the MS with their locations and functionality.

\subsubsection{ATLAS magnet system}

As shown in Figure 34, the ATLAS magnet system [96] consists of four superconducting magnets: one Barrel Toroid (BT), two End-Cap Toroids (ECT), and one Central Solenoid (CS). The primary reason for using superconducting magnets is that the center of the superconducting magnet is empty, while regular resistive non-superconducting magnets have iron core. Hence, the particles generated from the $p p$ collisions have minimum interactions with the magnet system, resulting in improved particle identification and measurements. The central solenoid [98] is aligned along the

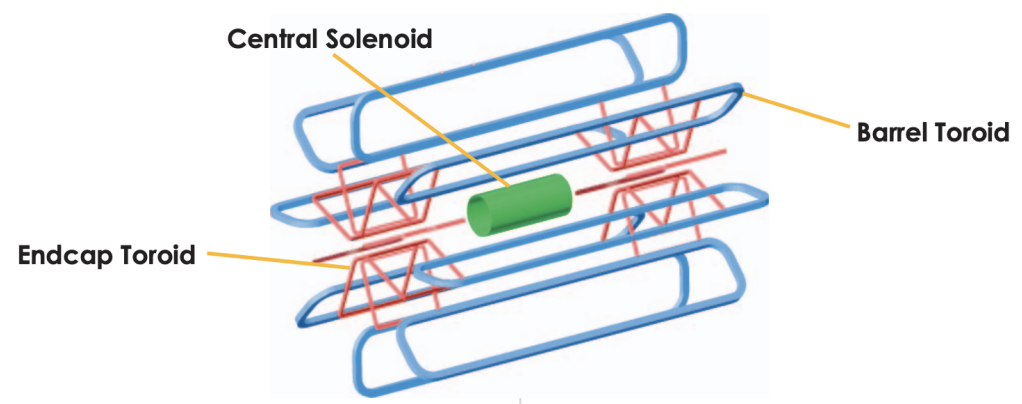

Figure 34: Graphical representation of the ATLAS superconducting magnet system [97].

beam axis and is designed to provide a magnetic field of $2 \mathrm{~T}$ to the ID. Each of the Barrel and End-Cap Toroids [99, 100] has an air-core design and consist of 8 coils assembled symmetrically and radially around the beam axis. To cover the transition gap between the barrel and the end-caps, the End-Cap toroids are placed at an angle of $22.5^{\circ}$ with respect to the Barrel toroid. The Barrel and End-Cap Toroids provide a magnetic field of strength $\sim 4 \mathrm{~T}$ each to the MS. 


\subsubsection{Trigger and Data Acquisition System}

At the peak luminosity of $2.1 \times 10^{34} \mathrm{~cm}^{-2} \mathrm{~s}^{-1}$, there are $\sim 1$ billion $(1 \mathrm{GHz})$ collisions taking place per second within the ATLAS detector. As all SM processes take place in each interaction, to store all the event data, $\sim 1 \mathrm{~PB}\left(10^{15}\right)$ of space is required per second, which is not technically feasible nor sensible. However, events of current physics interest are rare, e.g., the Higgs boson production rate is $\sim 33 /$ minute. Hence, a trigger system is used, which is a mechanism designed to target events with specific objects that are of current physics interest, to decide in real time if an event is not of interest or background of many possible types, and then decide whether to record an event from a given collision. The trigger and data collection process follows the following steps: Event identification (Trigger) $\rightarrow$ Event recording (data acquisition) $\rightarrow$ Data-processing (computing). The ATLAS trigger system is essentially a serial device with multiple parallel paths, divided into different levels, each level selecting the data as input to the next level. The rest of the events get rejected. This section describes the ATLAS Trigger and Data Acquisition System (DAQ), together known as the TDAQ.

The ATLAS trigger system [101] consists of two distinct levels: a hardware-based first-level trigger (L1), and a software-based high-level trigger (HLT). Figure 35 shows the trigger system diagram, which operates in a sequence where the successful output of L1 is the input to the HLT.

The L1 trigger receives inputs from the L1 calorimeter (L1Calo) and L1 muon (L1Muon) trigger systems. The L1Calo trigger receives inputs from the calorimeter cells and the analog signals are digitized and calibrated before sending to the Cluster Processor (CP) and Jet/Energy-sum Processor (JEP). The CP identifies electrons, $\tau$-leptons, and photons, while the JEP identifies jet candidates and calculates global sums of total and missing transverse energy. L1Muon trigger uses the hits from the RPCs (in barrels) and TGCs (in end-caps) as inputs. The final L1 trigger decision is processed by the Central Trigger Processor (CTP), which takes inputs from the L1Calo, L1Muon (through Muon Central Trigger Processor Interface (MUCTPI)), L1 topological trigger (L1Topo) [102] and other detector subsystems such as Minimum Bias Trigger Scintillators (MBTS) [103], LUCID Cherenkov counter [104], and Zero Degree Calorimeter (ZDC) [105]. The 
L1 accept decisions are distributed to the Front-End (FE) detector electronics by the TTC (Timing, Trigger and Control) [106-108] system. The data are sent to the ReadOut Drivers (RODs) first for processing and formatting and then are sent to the ReadOut System (ROS) to buffer the data to the HLT. The CTP also applies the dead times, which restricts the L1 acceptance rate to be within the detector read-out latency. There are two types of preventive dead-times: (i) simple dead-time, applied to constrain the minimum time between the two L1 accepts to avoid overlapping read-out windows, and (ii) complex dead-time, applied to restrict the number of L1 accepts in a given number of bunch-crossings to avoid overflowing of FE buffers. At the end of Run 2 the simple dead-time was $100 \mathrm{ns,}$, while the complex dead-time was configured with different algorithms depending on the read-out limitations of various sub-detectors. The L1 trigger reduces the event rate from $40 \mathrm{MHz}$ to the maximum detector read-out rate of $100 \mathrm{kHz}$ within a latency of 2.5 $\mu$ s. The L1 trigger also identifies Regions-of-Interest (ROI) in $\eta$ and $\phi$ within the detector that are investigated in HLT.

The software based HLT uses dedicated algorithms to process the ROIs. It starts by using dedicated fast trigger algorithms for early rejection of events and then uses more precise and CPU consuming algorithms to take a final decision. The HLT algorithms run on a dedicated computing facility, called the Processing Units (PU) and the PUs take trigger decisions within few hundred milliseconds. Once the events are accepted by the HLT, they are transferred to the local storage before moving them to permanent storage centers at CERN. The HLT physics output rate is on average $1.2 \mathrm{kHz}$ which on takes $\sim 1.2 \mathrm{~GB} / \mathrm{s}$ disk space.

The total RAW data size of ATLAS Run 2 dataset is $\sim 34 \mathrm{~PB}$. To make this massive amount of data accessible to users, a pyramid-structured global computing model is designed, called Worldwide LHC Computing Grid (WLCG) [109]. The WLCG consists of four layers: Tier-0, Tier-1, Tier-2, and Tier-3. The Tier-0 is located at CERN and processes the raw data emerging from the DAQ systems. The processed data from Tier-0 is sent to Tier-1, which consists of 13 national computer centers located around the world with the capacity to store large fraction of data. The Tier- 1 centres make the data available to the Tier- 2 centers, which consist of several collab- 


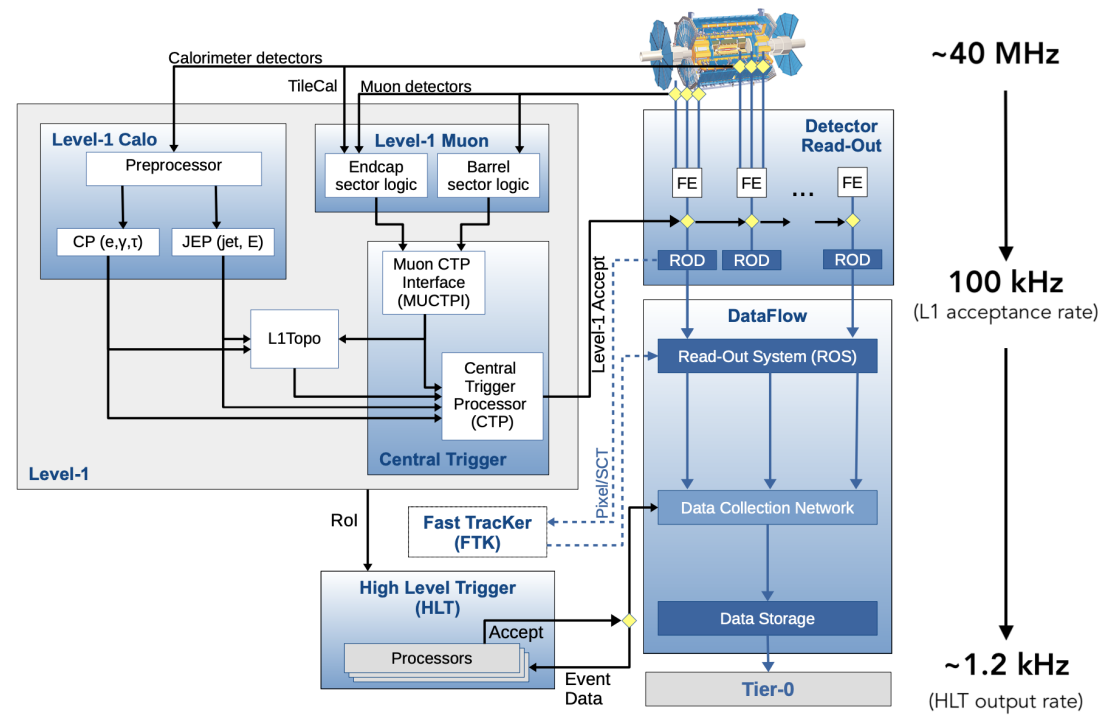

Figure 35: Schematic adapted from [101] shows the ATLAS TDAQ System with event rates at different stages of the process. In Run 2 the FTK was under commissioning and was not used by the HLT for trigger purposes.

orating computing facilities. The Tier-2 centers have sufficient storage and computing power to perform tasks like MC simulation. The final part of the WLCG is Tier-3 which consists of local clusters in universities. The analysers mostly use Tier-3 facilities to perform physics analyses.

\subsection{ATLAS Upgrade projects}

The LHC was designed to collide protons at a center-of-mass energy of $\sqrt{s}=14 \mathrm{TeV}$ and deliver an instantaneous luminosity of $1 \times 10^{34} \mathrm{~cm}^{2} \mathrm{~s}^{-1}$. The LHC has already exceeded the peak luminosity target by delivering $2.1 \times 10^{34} \mathrm{~cm}^{2} \mathrm{~s}^{-1}$ in 2016 with $\sqrt{s}=13 \mathrm{TeV}$. However, for precision measurements of EWSB, BSM Higgs etc. higher luminosity is needed to increase the statistical prowess. As higher energy requires much more drastic changes, upgrades in the detector and the readouts, DAQ etc. are required and this section gives an overview. During 2013-2014 (Long Shutdown 1: LS1), the first upgrade project, phase-0, was performed and it included the addition of the new IBL inside in the Pixel detector, trigger updates including the new CTP, installation of Forward Proton Taggers Detectors (AFP) [110]. The second ATLAS upgrade project is called phase-I and was originally scheduled to be completed during LS2 (2019-2020). The phase-I up- 
grade project includes the addition of New Small Wheel (NSW) detector [111], new LAr calorimeter FE electronics [112], and the TDAQ system [113]. The LHC will increase its luminosity to a maximum instantaneous luminosity of $7.5 \times 10^{34} \mathrm{~cm}^{2} \mathrm{~s}^{-1}$ for the high luminosity (Hi-Lumi) upgrade, and thus detector upgrades were also needed to commensurate with the luminosity upgrade. The next ATLAS upgrade project, phase-II, is scheduled to be performed during LS3 (2024-2026). The phase-II upgrades are designed to cope with the requirements of the High-Luminosity LHC (HL-LHC) [114]. The HL-LHC is scheduled to start data taking in 2027 and is expected to obtain a total integrated luminosity of $4000 \mathrm{fb}^{-1}$. From a detector's perspective, the main challenges in the HL-LHC would be to maintain or improve the performance in an environment with increased pile-up events, high radiation doses and high trigger rates. The upgrades include: a new fully silicon Inner Tracking (ITK) [115, 116] detector with better $\eta$ coverage, upgraded TDAQ [117], a new High Granularity Timing Detector [118], and an upgraded forward muon system [119].

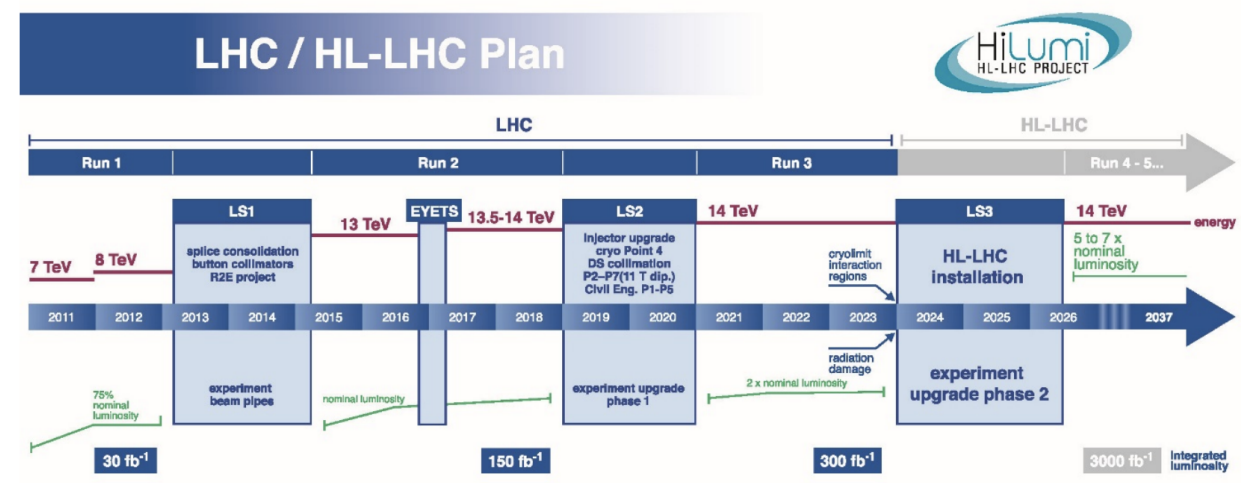

Figure 36: The HL-LHC upgrade timeline; the red line shows the collision energy and green line shows the integrated luminosity targets at different upgrade stages [120].

A major challenge of the HL-LHC would be the handling of the high pile-up interactions which could go up to $\langle\mu>=200$. A possible solution is to use the time spread of the $p p$ collisions in each bunch crossing to distinguish the tracks originating from collisions in close spatial space but well-separated in time. To obtain the timing information, a new component to the ATLAS detector is proposed, the High-Granularity Timing Detector (HGTD). The HGTD has an expected time resolution of 30-50 ps for minimum ionizing particles and will reduce the pile-up by a factor 
of $\sim 6$. Apart from pile-up reduction, the ATLAS HGTD would also improve the performance of other important items such as $b$-tagging, lepton isolation efficiency, identification of long-lived particles, luminosity measurement etc. The HGTD is proposed to be built in front of the two endcap calorimeter cryostats with the active layers between $|z|=3.5 \mathrm{~m}$ and will cover the forward region of $2.4<|\eta|<4$. The HGTD detector will be based on a Low Gain Avalanche Detector (LGAD) [121] which is a silicon sensor that provides a timing resolution between 30-50 ps/hit.

At CERN beam tests are conducted to test different LGAD sensors and study their characteristics. The signal shapes from different sensors are monitored by the oscilloscope. One of my ATLAS authorship qualification tasks was to develop a software to communicate between the oscilloscope and the sensors being tested. Apart from beam tests at CERN many institutes across the ATLAS HGTD community perform sensor bench tests such as I-V, C-V characteristics. I also developed a fully functional $M y S Q L$ database with a web interface using $h t m l$ and $p h p$, hosted at CERN. Using the web interface an user can insert sensor test results to the database and retrieve the results using different search criterion such as sensor names, institutions etc. 


\section{GENERAL PHYSICS ANALYSIS STRATEGY}

All the physics analyses at the LHC, whether SM measurements or BSM studies have few essential items in common. One such item is the identification of physics objects in an event produced by the $p p$ collision which are mostly important characteristics to define an event, like measuring critical information needed to perform a complete analysis such as transverse momentum $\left(p_{\mathrm{T}}\right)$, energy, charge etc. Section 4.1 describes the physics objects relevant to the thesis such as vertices, jets, leptons, $E_{\mathrm{T}}^{\text {miss }}$ etc. The observed data recorded by the detector is compared with the predictions from simulated events generated from scratch using SM cross sections, and in cases of searches, from following the specific BSM models. These generated event four-vectors are then further developed according to specific hadronisation and fragmentation. Monte Carlo (MC) descriptions are commonly accepted and used in the field and passed through the simulated detector and readout chains as in real events. Section 4.2 gives an overview of the MC event generation. An ATLAS physics analysis targets a specific final state, e.g., the mono- $h(b \bar{b})$ analysis described in the thesis targets final state containing $E_{\mathrm{T}}^{\text {miss }}+h(\rightarrow b \bar{b})$ events. However, other physics processes can produce the same final state and are known as background processes to the analysis. To observe the targeted final state as efficiently as possible, the analysis phase space is divided into multiple regions. The philosophy of analysis region classification is similar in all ATLAS analyses and is described in section 4.3 . Once the analysis regions are defined, a statistical analysis is performed where a hypothesis is tested to check the compatibility between the observed data and MC simulated events. Section 4.4 describes the relevant statistical concepts and techniques.

\subsection{Physics object reconstruction}

The quality of a physics analysis depends on the precision of physics object reconstruction. Physics objects such as leptons, jets, jet flavour tagging, $E_{\mathrm{T}}^{\text {miss }}$, etc. and their qualities are explained here. Section 4.1.1 describes the reconstruction of charged particle tracks and identification of the $p p$ interaction vertices, section 4.1 .2 describes identification of leptons: electrons and muons. 
The most dominant physics process at the LHC is QCD and the fundamental characteristic of a QCD process is color confinement. Quarks and gluons produced in a QCD process carry a color charge and due to color confinement, which allows only colorless states, the quarks and gluons cannot stay isolated for a physically observable time. Instead, they follow a process called hadronization, where they radiate additional quarks and gluons and eventually produce collimated color-neutral hadrons. This collimated spray of hadrons is known as jet and section 4.1 .3 describes jet reconstruction procedure. The jets are classified into different flavour categories depending on the parent hadrons: e.g. $b$-jets containing $b$-hadrons, $c$-jets containing $c$-hadrons, and lightjets containing neither $b$-hadrons nor $c$-hadrons. Distinguishing different jet flavours is critical for analyses which involve specific decay channels, such as $h \rightarrow b \bar{b}$. Section 4.1 .4 describes the $b$-jet identification procedure used to identify jets originating from $b$-quark (hadronized as $B$ ) decays. Another important variable in the context of BSM searches is $E_{\mathrm{T}}^{\text {miss }}$ which is defined as the negative $p_{\mathrm{T}}$ sum of all the physics objects in an event. The $E_{\mathrm{T}}^{\text {miss }}$ can originate from misidentification of leptons, jets, and from particles which escape the detector undetected such as neutrinos and exotic particles such as DM and DE. By the momentum conservation law, in the transverse plane of the beam axis, the sum of the $\overrightarrow{p_{\mathrm{T}}}$ of all the particles produced in a $p p$ collision should be zero. Any significant deviation from zero would indicate the existence of neutrinos or exotic particles. Hence reconstruction of $E_{\mathrm{T}}^{\text {miss }}$ is crucial in the context of this thesis and is described in section 4.1.5.

\subsubsection{Charged particle tracks and vertices}

The primary vertex is defined as the point at which a $p p$ interaction occurred. The particles produced in the collision traverse through the detector medium and depending on the particle properties go through further decay processes. Decays of long-lived particles produce secondary and tertiary vertices and charged particles produce tracks inside the detector. To identify charged particles and fully reconstruct their decay chain, the tracks and vertices must be identified. This task is performed by the ATLAS ID.

The process of track reconstruction [122, 123] starts with a conversion of the Pixel and SCT 
hits to 3D measurements, known as space-point, representing the points in the active material of the ID through which charged particles passed. After space-point formation, a seed is constructed from it, combining different ID sub-detectors. The seeds are then used to build track candidates using a combinatorial Kalman filter [124]. Finally, a track refit is performed to improve the track momentum resolution. This track reconstruction procedure is known as the inside-out pattern recognition procedure. Although majority of the tracks in ATLAS are reconstructed with the inside-out approach, a complementary outside-in approach is also used, where the process starts from the TRT hits and moves inward. This is used for reconstructing tracks, for which enough space-points were not available from the Pixel detector.

The current ATLAS primary vertex reconstruction method follows the Adaptive Multi Vertex Finder (AMVF) [125, 126] procedure. The primary vertex reconstruction algorithm uses the ID tracks as inputs and have two components: (i) primary vertex finding algorithm which associates the reconstructed tracks to vertex candidates, and (ii) the vertex fitting algorithm, which reconstructs the vertex position and its corresponding error matrix. The vertex fitting stage is an iterative process and in each iteration less compatible tracks are down-weighted. Once a vertex position is determined, the tracks which are incompatible with that vertex are removed and are used to reconstruct other vertices.

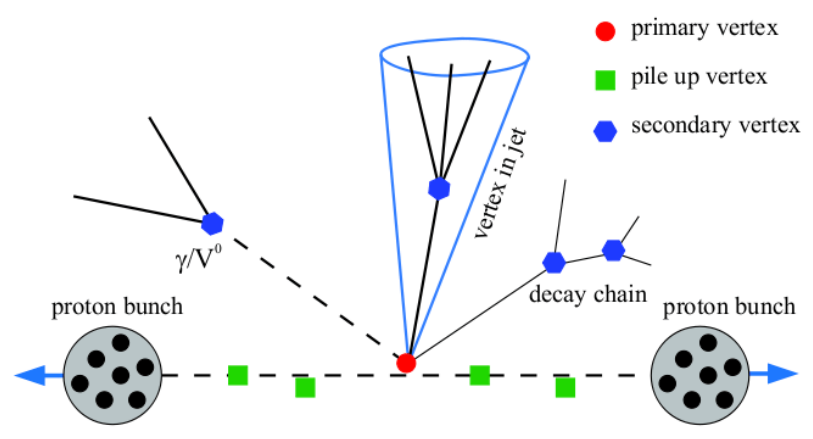

Figure 37: Diagram showing different vertex topologies from a $p p$ collision [127]. 


\subsubsection{Leptons}

Leptons, being charged particles, leave tracks when traversing through the ID. The electrons deposit their energy in the EM calorimeter and the muons travel up to the MS. The ATLAS lepton identification and reconstruction procedure use ID tracking information, energy deposits in the EM calorimeter, and MS. Once the leptons are identified, they go through an isolation procedure, where prompt leptons (originating from hard-scattering vertex, decay of heavy resonances such as $W^{ \pm} / Z$ and Higgs bosons) are separated from non-prompt leptons (originating from misidentification of particles, photon conversions, semi-leptonic decays of heavy quarks etc.). The isolation process uses both the tracking and calorimeter information.

\subsubsection{Electrons}

The ATLAS electron identification is a two step process:(i) electron reconstruction, and (ii) electron identification.

\section{(i) Electron reconstruction}

The electron reconstruction [128] is based on three fundamental characteristics of an electron signature: (i) localised clusters of energy deposit conforming to EM shower shape found within the EM calorimeter, (ii) charged-particle tracks identified in the ID, and (iii) close matching in $\eta \times \phi$ space of the tracks that form final electron candidate.

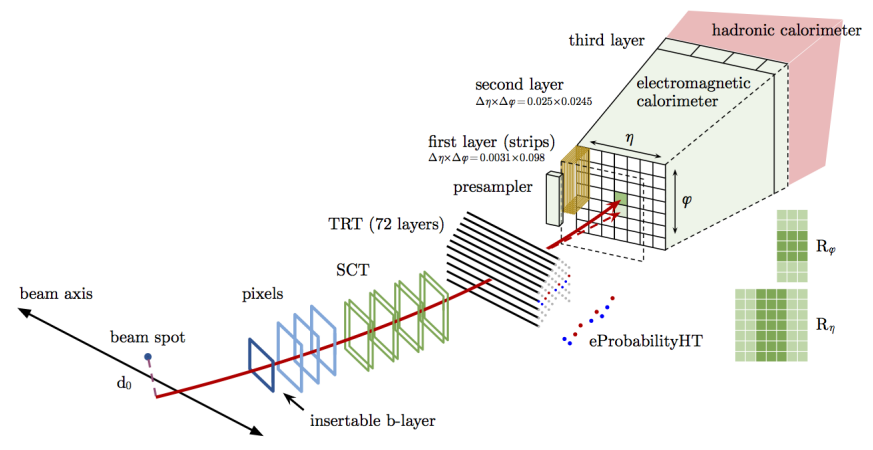

Figure 38: A graphical representation of the trajectory of an electron passing through the ATLAS detector. The solid red line represents the electron trajectory and the red dashed line represents the trajectory of a photon, produced through the electron-detector interaction [128]. 
The reconstruction of the energy deposits in the EM calorimeter starts with dividing the $\eta \times \phi$ space of the EM calorimeter into a grid or towers. Energy deposits in each of the EM calorimeter layers are summed over to compute the total energy in each tower. A sliding window algorithm [129] is used to find the EM energy cluster candidates and form a set of towers $(3 \times 5)$ where the localised energy deposits are greater than $2.5 \mathrm{GeV}$. Once a cluster is found, it is used as a seed to match the track reconstructed in the ID. A Gaussian-Sum-Fitter (GSF) is used to refit the reconstructed tracks which accounts for the energy loss of the electrons due to Bremsstrahlung. The GSF track candidates are then matched to the calorimeter seed cluster candidates. If there is a good matching the cluster is reconstructed as an electron, otherwise it is reconstructed as a photon.

\section{(ii) Electron identification}

The electron identification procedure separates prompt electrons from non-prompt electrons. Identification of prompt electrons in the central region of the ATLAS detector $(|\eta|<2.47)$ is based on a likelihood (LH) identification, which is constructed with inputs from the ID tracks and EM calorimeter. The recommended ATLAS electron identification LH discriminant has three values, which correspond to three working points (WP): Loose, Medium and Tight. These WPs correspond to identifying a prompt electron with $E_{\mathrm{T}}=40 \mathrm{GeV}$ at $93 \%, 88 \%$, and $80 \%$ efficiency for Loose, Medium and Tight WPs respectively [128].

\subsubsection{Muons}

The ATLAS muon reconstruction [130] procedure is a two steps process: (i) muon reconstruction, and (ii) muon identification.

\section{(i) Muon reconstruction}

Muons are reconstructed independently in the ID and MS and the final muon candidate is reconstructed by combining information from both. Muon reconstruction in the ID follows the same procedure as any other charged particle (c.f. section 4.1.1). The reconstruction procedure in the MS starts by forming segments in each muon chamber utilizing the hit patters. The MDT hits correspond to the bending plane alignment, while the RPC and TGC hits provide information 
about the coordinate orthogonal to the bending plane. These segments are used as seeds in a fitting procedure which starts from the middle layers of the MS and successively moves to the inner and outer layer segments. A segment is selected if it satisfies the benchmark selection criterion such as fit quality, hit multiplicity etc. To build a track, at least two matching segments are necessary in each $\eta$ region of the MS, except for the transition region between the barrel and end-cap where only one high-quality segment is sufficient. Due to the proximity of many tracks multiple track candidates can share the same segments. An overlap removal procedure is applied to assign the best segment to a single track or share a segment between two tracks. A global $\chi^{2}$ fit is performed on the hits associated with each track candidate and a candidate is selected if the $\chi^{2}$ value satisfies the selection criterion. The reconstructed muons are classified into four types:

- Combined (CB) muon: Initially tracks are reconstructed independently in the ID and MS and later on a global fit is performed using the ID and MS information to reconstruct a combined track. For most muons, the fitting procedure uses an outside-in pattern recognition approach, where the muons reconstructed in the MS are extrapolated inward and are matched with the ID track. An inside-out muon reconstruction is also performed as a complementary approach.

- Segment-tagged (ST) muon: The ST muons are reconstructed using the ID tracks and parts of the MS. An ID track is extrapolated to the MS and if the track is associated with at least one local track segment in the MDT or CSC chambers, the ID track is classified as a muon. This procedure is useful for muons which cross only one MS layer either due to low $p_{\mathrm{T}}$ or falling in the low MS acceptance regions.

- Calorimeter-tagged (CT) muon: The CT muons have the lowest purity, but particularly important as it provides coverage where the MS is not fully functional. The CT muon reconstruction procedure utilises the Minimum Ionizing Particle (MIP) characteristic of muons. An ID track is accepted as a muon if the track is matched to an energy deposit in the calorimeter which is compatible with a MIP. The CT muons are optimised for the region of $|\eta|<0.1$ 
and $15<p_{\mathrm{T}}<100 \mathrm{GeV}$.

- Extrapolated (ME) muon: The ME muons are reconstructed using the MS track only, with an additional loose requirement on the compatibility between the track candidate and impact parameter. The ME muons are required to traverse two layers of MS chamber and three layers in the forward region. These muons provide coverage in the region of $2.5<|\eta|<2.7$ where the ID has no acceptance.

\section{(ii) Muon identification}

The reconstructed muon candidates go through an identification process where additional quality criteria are applied to suppress background sources that mimic muon signatures (e.g. pion and kaon decays) and ensure high efficiency of prompt-muon acceptance. In ATLAS experiment, four muon identification selections are available: Medium, Loose, Tight, and High- $p_{\mathrm{T}}$.

The Medium type is the default muon selection in ATLAS. It only uses the CB and ME tracks and has the lowest systematic uncertainty associated with muon reconstruction and momentum calibration. The Loose identification criterion uses all muon types and provides good-quality tracks while maximising the reconstruction efficiency. The Tight identification criterion identifies muons by maximising the purity of muons at the expense of reconstruction efficiency. It uses only $\mathrm{CB}$ muons with hits in at least two MS stations and satisfying the Medium selection criterion. The High- $p_{\mathrm{T}}$ muons, which are used in new high-mass resonance searches, are optimised to maximize the momentum resolution for tracks with $p_{\mathrm{T}}>100 \mathrm{GeV}$. It uses $\mathrm{CB}$ muons with at least three hits in the MS and satisfying the Medium selection criterion.

The lepton reconstruction and identification procedure described above is efficient in discriminating prompt leptons from other particles. However, the non-prompt leptons are surrounded by additional tracks and are generally embedded in jets. Hence, to distinguish between the prompt and non-prompt leptons, a lepton isolation [130, 131] procedure is applied. In this process isolation variables are calculated to quantify the amount of detector activity around a lepton candidate. There are two types of isolations: (i) calorimeter based where the variables are calculated by using the transverse energies of the topological clusters (both EM and hadronic) $E_{T, \Delta R=0.2}^{\text {isol }}$ in a fixed 
cone of $\Delta R=0.2$, (ii) track based where the variables $\left(p_{T, v a r \Delta \mathrm{R}}^{\text {isol }}\right)$ are defined as the scalar sum of the $p_{\mathrm{T}}$ of tracks with $p_{\mathrm{T}}>1 \mathrm{GeV}$ in a variable cone ( $p_{\mathrm{T}}$ dependent) around the lepton, excluding the lepton track itself. The track-isolation variable values are: $p_{T, \Delta \mathrm{R}_{\max }=0.2}^{\text {isol }}$ and $p_{T, \Delta \mathrm{R}_{\max }=0.3}^{\text {isol }}$ for maximum cone sizes of $\Delta R_{\max }=0.2$ and 0.3 respectively. Table 5 summarizes the different lepton isolation WPs relevant to the thesis.

\begin{tabular}{|c|c|c|c|}
\hline Object & WP & Calorimeter isolation & Track isolation \\
\hline Electron & $\begin{array}{c}\text { FCLoose } \\
\text { FCHighPtCaloOnly }\end{array}$ & $\begin{array}{c}E_{\mathrm{T}, \Delta \mathrm{R}=0.2}^{\mathrm{isol}} / p_{\mathrm{T}}<0.2 \\
E_{\mathrm{T}, \Delta \mathrm{R}=0.2}^{\text {isol }} / p_{\mathrm{T}}<3.5 \mathrm{GeV}\end{array}$ & $\begin{array}{c}p_{\mathrm{T}, \mathrm{R}_{\max }=0.2}^{\mathrm{isol}} / p_{\mathrm{T}}<0.15 \\
-\end{array}$ \\
\hline Muon & $\begin{array}{c}\text { FCLoose } \\
\text { FCTightTrackOnly }\end{array}$ & $\begin{array}{c}E_{\mathrm{T}, \Delta \mathrm{R}=0.2}^{\mathrm{isol}} / p_{\mathrm{T}}<0.3 \\
-\end{array}$ & $\begin{array}{l}p_{\mathrm{T}, \mathrm{T}, \mathrm{R}_{\max }=0.3}^{\text {isol }} / p_{\mathrm{T}}<0.15 \\
p_{\mathrm{T}, \mathrm{R}}^{\text {isol }}=0.3 / p_{\mathrm{T}}<0.06 \\
\end{array}$ \\
\hline
\end{tabular}

Table 5: Summary of lepton isolation WPs relevant for the thesis, FC: Fixed Cut [132, 133].

\subsubsection{Jets}

Jets are physically observable in the detectors and carry the characteristics of the initial quarks and gluons. This section describes the ATLAS jet reconstruction, calibration procedure, and specific types of jets used in the thesis.

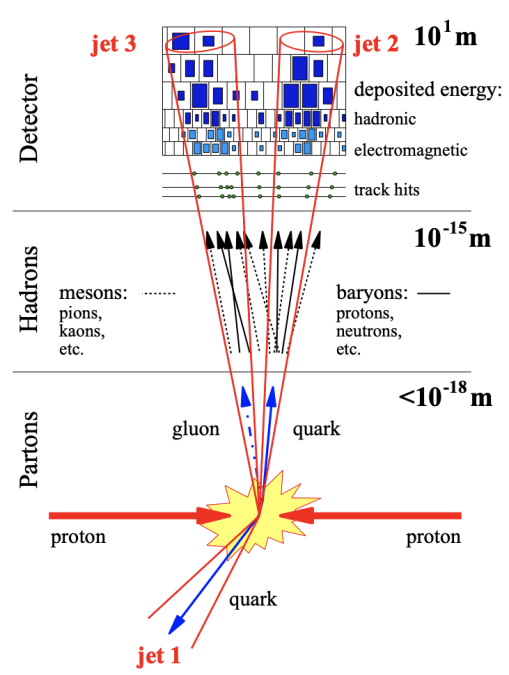

Figure 39: Jet evolution schematic inside the ATLAS detector: starts from a $p p$ collision followed by partons and hadrons grouped together and eventually depositing energy in the HCal [134] . 


\subsubsection{Jet reconstruction}

\section{$\underline{\text { Topo-cluster formation }}$}

The ATLAS jet reconstruction procedure starts with the topological cluster (topo-cluster) formation [129, 135], where 3D clusters of the hadronic calorimeter cells are reconstructed. The cluster formation begins by identifying seed cells with a certain cell signal $(S)$ to expected noise $(N)$ ratio. A cell is considered as a seed if it satisfies $|S / N| \geq 4$. Once a seed cell is selected, neighbouring cells with $|S / N| \geq 2$ are added to the 3D cluster. Finally, the topo-cluster formation is completed by adding cells with $|S / N| \geq 0$ to account for the shower tails. Figure 40 represents a schematic of the cluster formation. The next step is the calibration of the topo-clusters, which has two choices: (i) calibrate to EM scale (EM topo-cluster) or (ii) hadronic scale with local calibration weighting (LCW [135] topo-cluster). The LCW calibration starts by dividing the LCW topo-clusters into EM or hadronic types, followed by a reweighting procedure to correct for the different electron-pion responses in calorimeter cells.

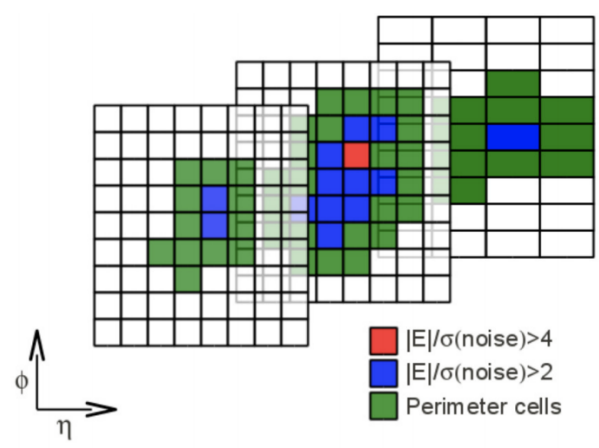

Figure 40: Figure shows the topo-cluster formation with different $S / N$ criteria across the three HCal layers in the barrel: seed cell (red), neighbour cell (blue) and perimeter cells (green) [136].

\section{Jet-finding}

Once the topo-clusters are formed a jet-finding algorithm associates the appropriate calorimeter topo-clusters with each other to form the final jet object. In ATLAS, the anti- $k_{T}$ algorithm [137] is the default jet-finding algorithm, which is a sequential combination algorithm. A $k_{T}$ algorithm computes the following variables: 


$$
d_{i j}=\min \left(k_{T i}^{2 p}, k_{T j}^{2 p}\right) \frac{\Delta R_{i j}^{2}}{R^{2}}, d_{i B}=k_{T i}^{2 p} .
$$

The name 'anti' is an artifact of setting the power $p=-1$ in the algorithm. Here $R$ is the parameter that controls the radius of the jet, $\Delta R_{i j}^{2}=\left(\eta_{i}-\eta_{j}\right)^{2}+\left(\phi_{i}-\phi_{j}\right)^{2}, k_{T i}$ is the transverse momentum of the $i^{\text {th }}$ constituent of the jet, the distance parameters $d_{i j}$ and $d_{i B}$ represent the distance between the jet constituents $i$ and $j$, and between the jet constituent $i$ and the beam respectively. The $d_{i B}$ parameter separates the jet constituents originating from hard-scatter interactions from the proton residuum. The anti-k $k_{T}$ algorithm begins by identifying the smallest distances among $d_{i j}$ and $d_{i B}$. If

the smallest distance turns out to be $d_{i j}$, the algorithm combines the $i^{\text {th }}$ and $j^{\text {th }}$ jet constituents and treats them as one constituent for the next iteration. If the smallest distance is $d_{i B}$, the algorithm identifies the $i^{\text {th }}$ constituent as a jet and removes it from the list of jet constituents. The distance calculation and constituent combination process continues until no constituents are available. The anti- $k_{T}$ algorithm uses the EM and LCW topo-clusters as inputs and provides jets with different cone radius such as small-R jets $(R=0.4)$, large- $\mathrm{R}$ jets $(R=1.0)$.

\subsubsection{Jet calibration}

After reconstruction, a jet candidate passes through a calibration process to correctly assign the energy and momentum scales, known as Jet Energy Scale (JES), of the underlying particles from which jets are produced after QCD fragmentation and hadronization. Although the jet calibration procedure is different between large- $\mathrm{R}$ and small- $\mathrm{R}$ jets, as shown in Figure 41 the overall workflow is similar. The ATLAS jet calibration process [138] has three principal steps: pile-up corrections, followed by calibration to MC truth scale and finally account for the differences with the data.

\section{Jet origin correction}

The first step in a jet calibration chain for small-R jets is the jet origin correction. The EMscale jet reconstruction process assumes that the origin of the small-R jets is the geometric center of the detector. Although in reality the center is the primary hard-scatter interaction vertex. This 


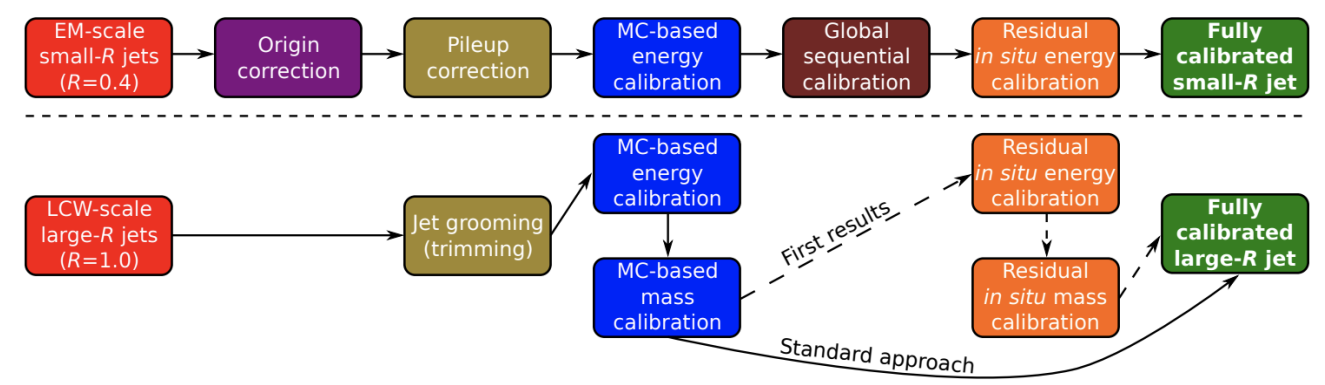

Figure 41: Jet calibration chain used in ATLAS for small-R and large-R jets [139].

correction rectifies the four-momentum vector of the jets and points the jet's origin to the primary vertex. It improves the angular resolution of the jets without changing jet energy.

\section{$\underline{\text { Pile-up correction }}$}

After the jet origin correction, the jets go through a pile-up correction procedure. Due to the large catchment area [140] of the jets, they are susceptible to pile-up effects, which reduces the accuracy of jet energy estimation. The pile-up correction for small-R jets has two components: (i) area-based correction, and (ii) residual dependence on the number of primary vertices and interactions. The final jet $p_{\mathrm{T}}$ is obtained by subtracting the resulting $p_{\mathrm{T}}$ of the two above mentioned corrections from the total reconstructed jet $p_{\mathrm{T}}$. The area based pile-up correction is effective in $|\eta|<2.0$ regions, while in $|\eta|>2.0$ regions residual corrections are required. In these corrections the reconstructed jet $p_{\mathrm{T}}$ is corrected by subtracting the $p_{\mathrm{T}}$ corrections from the above mentioned two pile-up correction components.

\section{$\underline{\text { Jet grooming }}$}

The large-R jets are vulnerable to pile-up effects since they have large jet area. It obscures the angular structure of the jet and as a consequence the hadronic particle decays contained inside the jet cone are not fully reconstructed. The pile-up correction procedure for large-R jets is performed through jet grooming, where the grooming algorithm selects a jet following some specific criteria. The ATLAS jet grooming algorithm uses a trimming [141] procedure for anti- $k_{T}$ jets. The algorithm re-clusters the jet constituents with a distance parameter $R_{\text {sub }}=0.2$, and trims any constituents in a $k_{T}$ sub-jet which has $p_{\mathrm{T}}<5 \%\left(f_{\text {cut }}\right)$ of the large-R jet $p_{\mathrm{T}}$. The trimming process 
vastly improves the mass resolution of large-R jets which is important in reconstruction of massive objects such as Higgs bosons. The jet grooming process is used for the large-R jets used in the mono- $h(b \bar{b})$ analysis (c.f. section 5.1).

\section{Jet energy calibration}

The jet energy calibration is purely MC-based correction which corrects the reconstructed jet energy to the MC particle-level jets. It accounts for detector effects such as the hadronic decays

not being fully reconstructed as showers might end up in inactive detector regions as well as energy deposits of particles which did not go in the reconstructed jet cluster. These energies are a part of the original jet particles, therefore must be recovered to measure the JES accurately. The reconstructed jet energy $\left(E^{r e c o}\right)$ is matched to the particle-level jet energy $\left(E^{\text {truth }}\right)$ and the correction factor $E^{\text {truth }} / E^{\text {reco }}$ is applied to the reconstructed jets to account for the lost energy.

The MC based jet correction is applied for both small-R and large- $\mathrm{R}$ jets, although each has some differences. Typically the large-R jets are used to reconstruct massive particles which decay hadronically. Hence, to identify the parent particle, the large-R jet needs to have a well-defined mass corresponding to the parent particle. This is achieved by matching the reconstructed jets to the particle-level jets with the mass response rather than energy. This is known as Jet Mass Scale (JMS) calibration.

\section{Global sequential calibration}

After the jet energy calibration, the JES is further refined for small-R jets through a Global Sequential Calibration (GSC) [142]. Depending on whether the jet-initiating particles are quarks or gluons, the jet composition and shower shapes vary. The quark initiated jets, having higher $p_{\mathrm{T}}$ than gluon initiated jets, provoke a higher calorimeter response compared to gluon initiated jets. In general the particle showers generated inside the calorimeters deposit their energy in the calorimeter cells. However, shower shapes (for incident particles with high energy) can also traverse through the calorimeter to the MS; these types of jets are called punch-through jets. The GSC improves the calorimeter response, energy distribution inside the jet cone, and punch-through effects. The punch-through correction changes the central value of JES, however other GSC cor- 
rections are designed to improve the Jet Energy Resolution (JER) only.

\section{$\underline{\text { In-situ calibration }}$}

The final step in jet calibration is the in-situ calibration where the JES and JMS calibrated jets are compared with the data. A deviation from data indicates possible biases in the jet energy or mass calibration. For small-R jets, the in-situ calibration is done in two steps. The first one is the dijet $\eta$ calibration [143], where well-understood central jets $(|\eta|<0.8)$ are used as references

to calibrate the forward jets $(0.8<|\eta|<4.5)$. The second in-situ correction step is a series of $p_{\mathrm{T}}$ balance method where well-understood reference objects are used to correct for the JES between data and MC for central jets.

For the large-R jets the in-situ calibration [144] uses the $R_{t r k}[144]$ parameter, defined as:

$$
R_{\text {trk }}=\frac{\left.\left(X_{\text {calo }}\right) / X_{\text {track }}\right)_{\text {data }}}{\left.\left(X_{\text {calo }}\right) / X_{\text {track }}\right)_{M C}}
$$

where $X$ represents the calorimeter observable. The $R_{t r k}$ parameter estimates the differences between the calorimeter and tracker jet representations, hence one can assign uncertainties on $X$. To constrain the JMS for both data and MC, the forward-folding [145] procedure is followed. The complete large-R jet in-situ calibration uses a combination of the forward-folding and $R_{t r k}$ method.

\subsubsection{Particle Flow jets}

During Run 1, the reconstruction process of hadronic jets in ATLAS used either the calorimeter or ID information. The jet reconstruction procedure started from the calorimeter topo-cluster formation, followed by calibrations to match the reconstructed jet energy scale to that of particlelevel jets. Towards the end of Run 1, the Particle Flow (PFlow) [146] algorithm was introduced which uses the combination of tracking and calorimeter information to reconstruct a jet. This method mimics the individual particle reconstruction procedure, such as leptons and vertices. The usage of PFlow jets improves the JER and has the following advantages:

- Resolution: The calorimeter energy resolution for a single charged pion at the center of the ATLAS detector is [147]: 


$$
\frac{\sigma(E)}{E}=\frac{50 \%}{\sqrt{E}} \oplus \frac{1 \%}{E} \oplus 3.4 \%
$$

while the inverse $p_{\mathrm{T}}$ resolution for the tracker is:

$$
\sigma\left(\frac{1}{p_{\mathrm{T}}}\right) \cdot p_{\mathrm{T}}=0.036 \% \cdot p_{\mathrm{T}} \oplus 1.3 \%
$$

Hence, for charged particles with low-energy the $p_{\mathrm{T}}$ resolution of the tracker is better than the energy resolution of the calorimeter. On the other hand, the calorimeter performs better for high-energy charged particles.

- Increased acceptance: Particles with low energies cannot pass the $|S / N|$ criterion to be included in a topo-cluster. The use of tracker extends the acceptance for low-energy particles, as charged particle tracks can be reconstructed for $p_{\mathrm{T}}>400 \mathrm{MeV}$.

- Pile-up reduction: Using the tracks one can identify the vertices associated to the tracks, hence multiple in-time pile-up interactions can be eliminated by rejecting jets originating from pile-up vertices.

- Low- $\boldsymbol{p}_{\mathrm{T}}$ sensitivity: Charged particles with low- $p_{\mathrm{T}}$ inside a hadronic jet are swept out of the jet cone by the high magnetic field before they reach the calorimeters. By using the tracker these low- $p_{\mathrm{T}}$ particles can be reintegrated as jet constituents.

In the PFlow method, the tracker and calorimeter information complement each other. Charged particles with low- $p_{\mathrm{T}}$ have a higher resolution with the tracker, while for neutral, high- $p_{\mathrm{T}}$ particles and for regions outside the acceptance of the ID $(2.5<|\eta|<4.5)$ only the calorimeter information is used. An overlap removal procedure is applied to remove the overlaps between the momentum and energy measurements performed in the ID and calorimeters to avoid double counting. The ATLAS PFlow algorithm chain, shown in Figure 42, starts with a set of tracks and topo-clusters. Each well-measured track is matched with a single topo-cluster and the expected energy deposit of the particle corresponding to the track is calculated. A particle can deposit energy in multiple topoclusters. The algorithm computes such probabilities and adds additional topo-clusters if necessary 
to measure the full shower energy. Once the topo-cluster(s) corresponding to a track is identified, the energy deposits are removed cell by cell. Once the energy of the remaining topo-cluster is consistent with the expected shower energy the topo-cluster remnants are removed. The PFlow algorithm starts with tracks which are sorted in descending $p_{\mathrm{T}}$ order and are matched to only one topo-cluster, followed by tracks associated with multiple topo-clusters.

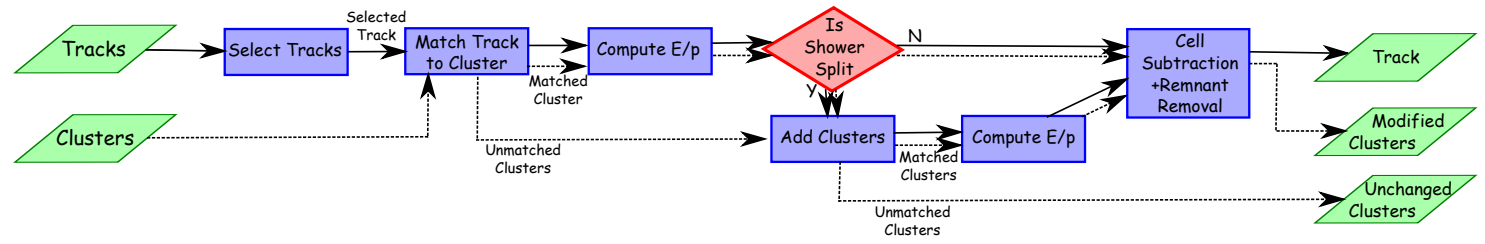

Figure 42: ATLAS PFlow jet reconstruction chain [146].

\subsubsection{Variable radius track jets}

The jet reconstruction procedure discussed in the previous sections reconstructs hadronic jets with a fixed radii $\Delta R=\sqrt{(\Delta \eta)^{2}+(\Delta \phi)^{2}}$ on the $(\eta-\phi)$ plane. An alternative approach to the fixed radius track-based jets is the variable radius (VR) [148] jets, where instead of a fixed radii the jet algorithm reconstructs jets with a variable radii $\Delta R$ using ID tracks and anti- $k_{T}$ algorithm. The VR jet algorithm captures the jet structure more accurately, which can be explained by a resonance decaying to two partons in the lab rest frame. Regardless of the decay orientation relative to the beam axis, the hadrons from the parton decay will be inside a circular cone of fixed angular size. But a fixed cone size $(\Delta R)$ corresponds to a variable angular size in the decay frame, hence a fixed $\Delta R$ jet does not depict the decay properties properly. The VR jet algorithm introduces a $p_{\mathrm{T}}$ dependency for the radius parameter:

$$
\Delta R \rightarrow \Delta R_{V R}\left(p_{\mathrm{T}}\right)=\frac{\rho}{p_{\mathrm{T}}},
$$

where the $\Delta R_{V R}$ is the variable radii and $\rho$ is the parameter that determines the shrinking rate of the radii. From equation 60, it is evident that the jet can become too large for low- $p_{\mathrm{T}}$ and shrink beyond the detector resolution for high- $p_{\mathrm{T}}$. To avoid such scenarios the VR jet algorithm has two more additional parameters, $R_{\min }$ and $R_{\max }$ which represent the lower and upper cut-offs on the track-jet radius respectively [149]. The VR jet algorithm is used to reconstruct track jets from 


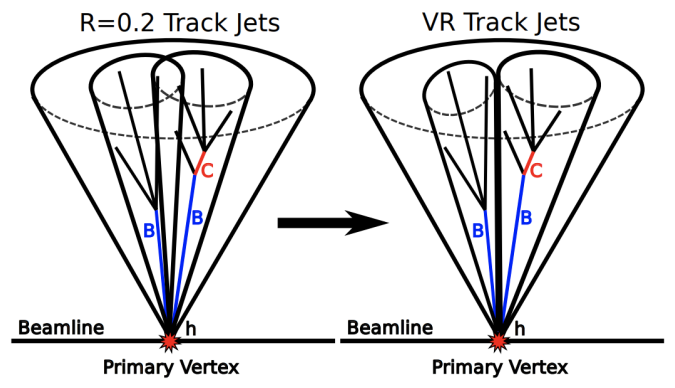

Figure 43: Figure shows jet structure reconstruction using VR track jets [149].

boosted decays of heavy bosons such as $h \rightarrow b \bar{b}$ where the $b$-quarks form jets which are very close to each other are reconstructed as large-R jets.

\subsubsection{Jet flavour tagging}

The jet flavour tagging is a critical aspect of the mono- $h(b \bar{b})$ analysis described in this thesis, where the SM Higgs boson is reconstructed using $b$-jets. Besides, due to high $\mathrm{BR}(t \rightarrow W b)$ $\approx 100 \%, b$-jet identification, known as $b$-tagging, is important for physics analyses involving topquark. It utilises the relatively long lifetime of $B$-hadrons. A $B$-hadron has a lifetime $\approx 1.6 \mathrm{ps}$ $(c \tau \approx 450 \mu \mathrm{m})$ and can travel macroscopic distances inside the detector before decaying. As a consequence, there will be at least one displaced secondary vertex where the heavy-flavour hadron decays, with respect to the primary hard-scatter vertex. Figure 44 shows a $B$-hadron traversing a decay length and producing a secondary vertex before forming a jet. The ATLAS $b$-tagging [150]

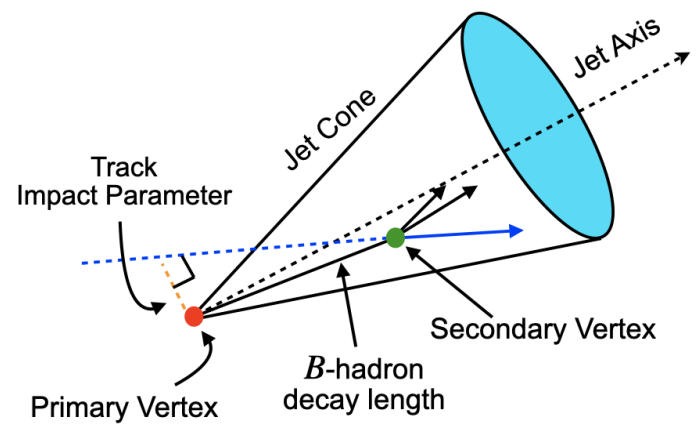

Figure 44: Schematic of a $b$-jet topology where a $B$-hadron produced at a primary-vertex (red) traverses a microscopic length and forms a displaced secondary-vertex (green) from which additional decay products are produced and forms a $b$-jet. 
process has two steps: (i) low-level algorithms to reconstruct the $b$-jets using the $B$-hadron decay properties, and (ii) combining the output of low-level algorithms with a high-level algorithm to maximize the $b$-tagging performance.

\section{Low level taggers}

The ATLAS $b$-tagging process starts with the low-level taggers which reconstruct the characteristics of a $b$-jet in two complementary approaches based on: (i) the impact parameter information of the tracks associated to the jets, and (ii) the secondary vertex information which are reconstructed using the tracks.

- Impact parameter based algorithms (IP2D and IP3D): The IP2D and IP3D [150, 151] are impact parameter (IP) based algorithms which are complementary to each other. To construct a discriminating variable, the IP2D algorithm uses the track signed transverse IP significance and IP3D uses track signed transverse and longitudinal IP significance. The Probability Density Functions (PDFs) are formed from MC simulations corresponding to the jet flavours ( $b, c$ and light-jets). The PDFs are used to form the Log-likelihood ratio (LLR) discriminant for each jet-flavour hypothesis and all three LLRs are used as inputs to high-level taggers.

- Secondary vertex finding algorithm (SV1): The SV1 [152] algorithm reconstructs a single displaced secondary vertex inside a jet cone. The algorithm takes all the tracks associated with a jet as inputs and identifies all the two-track vertices. It rejects vertices which are compatible with the decay of long-lived particles such as $K_{s}, \Lambda$, photon conversion and vertices due to hadronic interactions with detector material. The SV1 is an iterative algorithm where in each iteration the track with the largest $\chi^{2}$ value is removed until a secondary vertex is found with an acceptable $\chi^{2}$. The secondary vertex reconstructed from a SV1 algorithm is common to both $B$ and $C$-hadron decay products.

- Multi-vertex finding algorithm (JetFitter): The JetFitter [153, 154] algorithm is designed to reconstruct the full $B$-hadron decay chain inside a jet cone. It uses a modified Kalman 
filter [124] to find a single line on which the primary, $b$, and $c$ vertices lie by approximating the $B$-hadron flight path and the position of the vertices. Unlike SV1 the JetFitter algorithm is able to distinguish between $B$ and $C$-hadron vertices.

\section{High level tagger}

The low-level tagger outputs are the inputs to the high-level tagger, such as the DL1 tagger.

- DL1: The DL1 [151] $b$-tagging algorithm is based on a deep feed-forward neural network (NN) and is trained using KERAS with THEANO [155] backend and the ADAM [156] optimizer. The inputs to the DL1 tagger are the outputs of IP2D/IP3D, SV1, JetFitter, and JetFitter $c$-tagger. The outputs of the DL1 NN are multidimensional corresponding to the probabilities of a jet being $b, c$, or light-flavoured. Since all jet flavours are treated equally during the training, the NN can be used for both $b$ and $c$-jets.

\section{b-tagging calibration}

Depending on the selection criterion on the tagger discriminant distribution, there are multiple single-cut operating points (OP) of the $b$-tagging algorithm. The DL1 tagger has four OPs, each corresponding to different $b$-tagging efficiencies such as $60 \%, 70 \%, 77 \%$, or $85 \%$. The $b$ tagging algorithm performance is characterized by the probability of identifying a $b$-jet ( $b$-tagging efficiency $\varepsilon_{b}$ ) and the probability of mis-tagging a $c$ or light-flavoured jet as a $b$-jet, denoted as $\varepsilon_{c}$ and $\varepsilon_{l}$ respectively. The mis-tag rates are more commonly expressed in terms of $c$-jet or light-jet rejection rates, denoted as $1 / \varepsilon_{c}$ and $1 / \varepsilon_{l}$ respectively. Figure $45 \mathrm{a}$ shows $\varepsilon_{b}$ as a function of jet $p_{\mathrm{T}}$ across different $b$-taggers at $77 \%$ OP. The $b$-tagging efficiencies at each OP are MC based and are calibrated to match the efficiencies observed in data. The calibration rectifies the mis-modelings in the input variables of the tagger. The efficiencies are measured in data $\left(\varepsilon_{d a t a}\right)$ using samples enriched with $b, c$, and light-jets and are compared to the efficiencies obtained from $\mathrm{MC}\left(\varepsilon_{M C}\right)$. The efficiencies are used to calculate the $b$-tagging scale factors $\mathrm{SF}=\varepsilon_{d a t a} / \varepsilon_{M C}$ and are applied as event weight corrections to the MC events. Figure $45 \mathrm{~b}$ shows the SF comparisons between data and MC for DL1 $b$-tagger as a function of the OPs. 


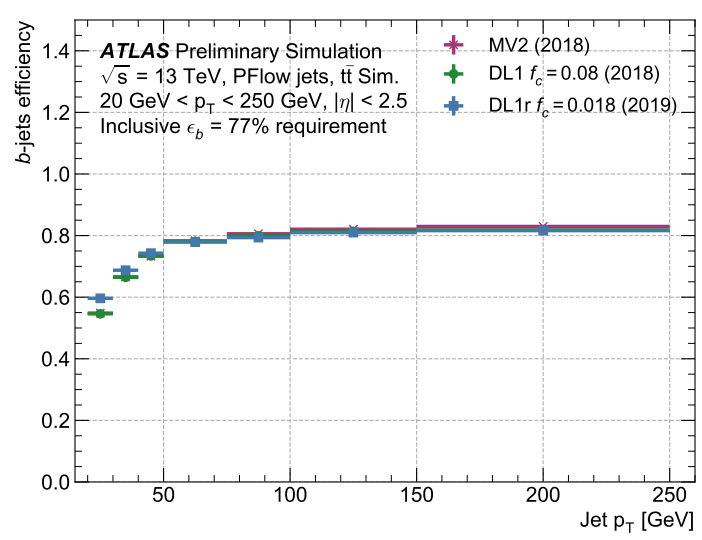

(a) $b$-tagging efficiency

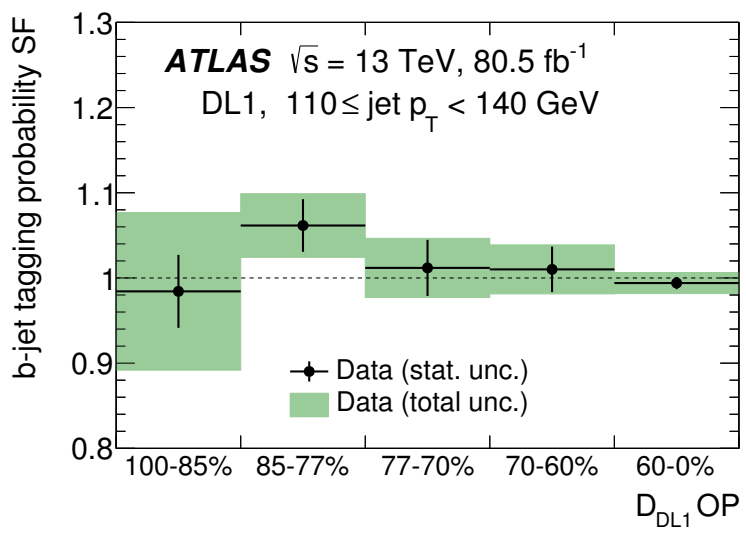

(b) $b$-tagging SF

Figure 45: Figure 45a shows the $b$-tagging efficiency as a function of jet $p_{\mathrm{T}}$ for different ATLAS $b$-taggers studied with $t \bar{t}$ MC samples. Figure $45 b$ shows the $b$-tagging SF comparisons between data and $t \bar{t}$ MC samples for different DL1 $b$-tagger OPs [150, 157].

\subsubsection{Missing transverse energy}

The challenge in $E_{\mathrm{T}}^{\mathrm{miss}}$ reconstruction $[158,159]$ is that it involves all the detector subsystems and requires precise information about the hard and soft interactions from the calorimeters and ID. The objects used in $E_{\mathrm{T}}^{\mathrm{miss}}$ reconstruction are categorized as: (i) hard-term, and (ii) softterm. Hard objects such as electrons, muons, photons, $\tau$-leptons, and jets. The soft-term components are reconstructed from charged-particle tracks and momentum deposits in the calorimeters which are associated with the hard-scatter but not associated with the hard objects. In terms of the components $p_{x(y)}$ of the $\overrightarrow{p_{T}}$ of the hard and soft term contributions, the $E_{\mathrm{T}}^{\text {miss }}$ components are expressed as:

$$
\begin{aligned}
& E_{x(y)}^{\text {miss }}=-\sum_{h \in\{\text { hard objects }\}} p_{x(y), h}-\sum_{s \in\{\text { soft term }\}} p_{x(y), s} \\
& =\underbrace{-\sum_{\text {electrons }} p_{x(y)}^{e}}_{E_{x(y)}^{\text {miss,e }}}-\underbrace{\sum_{\text {muons }} p_{x(y)}^{\mu}}_{E_{x(y)}^{\text {miss }, \mu}}-\underbrace{\sum_{\text {hhotons }} p_{x(y)}^{\gamma}}_{E_{x(y)}^{\text {miss }, \gamma}}-\underbrace{\sum_{\tau-l e p t o n s} p_{x(y)}^{\tau_{\text {had }}}}_{E_{x(y)}^{\text {miss }, \tau_{\text {had }}}} \underbrace{-\sum_{j e t s} p_{x(y)}^{\text {jet }}}_{E_{x(y)}^{\text {miss jet }}} \underbrace{-\underbrace{\sum_{\text {unused tracks }} p_{x(y)}^{\text {track }}}_{E_{x(y)}^{\text {miss soft }}} .}_{\text {soft terms }}
\end{aligned}
$$

The final $E_{\mathrm{T}}^{\text {miss }}$ is calculated using the following equation: 


$$
E_{\mathrm{T}}^{\mathrm{miss}}=\sqrt{\left(E_{x}^{\mathrm{miss}}\right)^{2}+\left(E_{y}^{\mathrm{miss}}\right)^{2}}
$$

In the ATLAS experiment there are three $E_{\mathrm{T}}^{\text {miss }}$ reconstruction algorithms: (i) Calorimeter Soft Term (CST) $E_{\mathrm{T}}^{\mathrm{miss}}$, (ii) Track $E_{\mathrm{T}}^{\mathrm{miss}}$, and (iii) Track Soft Term (TST) $E_{\mathrm{T}}^{\mathrm{miss}}$.

- CST $E_{\mathrm{T}}^{\text {miss }}$ : The CST $E_{\mathrm{T}}^{\text {miss }}$ algorithm reconstructs $E_{\mathrm{T}}^{\text {miss }}$ based on the energy deposits in the ATLAS calorimeter cells. This includes the calorimeter soft terms, originating from energy deposits which are not associated with hard objects. This method does not include any track information, hence it is vulnerable to pile-up interactions and the total $E_{\mathrm{T}}^{\text {miss }}$ includes additional contribution from these pile-up interactions. Although this method was used in Run 1 analyses, it is not suitable for Run 2.

- Track $E_{\mathrm{T}}^{\text {miss }}$ : The track based method uses the ID tracks and vertices, hence it is a more robust approach for Run 2 conditions which has high pile-up density. This method however is insensitive to neutral particles, as they do not leave any tracks in the ID. Besides, the ID can only provide tracking for a limited region of $|\eta|<2.5$.

- TST $E_{\mathrm{T}}^{\mathrm{miss}}$ : This method combines the track based soft term and calorimeter based measurements for hard objects. Only the tracks associated with hard scatter vertex are considered in this algorithm. The calorimeter information provides a much larger $\eta$ coverage $(|\eta|<4.9)$. The TST is the primary ATLAS $E_{\mathrm{T}}^{\text {miss }}$ reconstruction algorithm for Run 2 analyses.

\subsection{Monte Carlo Simulation}

Understanding the physics processes generated in the $p p$ collisions is a cornerstone of any physics analysis at the LHC. The LHC produces particles with momenta ranging over multiple orders of magnitude. To make theoretical predictions about these collisions, one needs to calculate the relevant matrix elements with higher order accuracy in QCD and take into account the confinement problem for QCD processes. For this purpose, computer simulated events are generated using MC methods. The MC simulation helps predict these collisions correctly, calibrate the detector components, and optimize physics analyses. In a physics analysis MC events are used to model 
the physics process of interest as well as the expected SM processes, in this case, as backgrounds. Figure 46 shows the different steps in a MC event generation process which are described below:

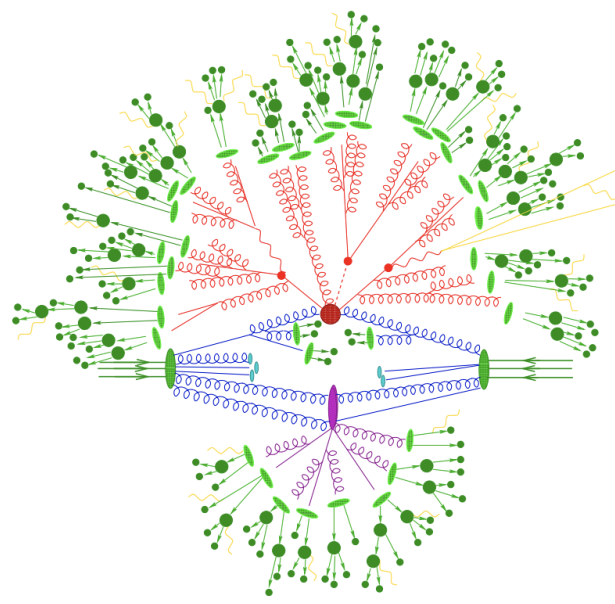

Figure 46: Figure illustrates the different steps of a MC sample generation procedure: hard process (dark red blob), parton shower (red lines), hadronisation (light green ellipses), hadron decays (dark green blob), underlying events (purple blob), unstable particle decay (green lines). The yellow lines represent photon radiation which can happen at any stage [160].

- Hard process: The MC event generation starts with the hard process (dark red blobs) which is the process with the highest momentum transfer in an event. The simulation and matrix element calculations of hard processes are performed at a fixed order perturbation theory.

- Parton shower: The parton shower (red lines) step models the QCD evolution of incoming and outgoing partons (quarks and gluons) involved in the hard scatter process. The parton shower models the QCD Bremsstrahlung at perturbative scales starting from the hard process to low momentum scales up to a point where perturbation theory becomes invalid.

- Hadronization: Once the parton shower is complete, the colored partons go through hadronisation (light green ellipses) and are transformed into primary colorless hadrons. The primary hadrons further decay to stable particles which are visible in the detector. The dark green blobs represents the hadrons.

- Underlying event: The underlying event (UE) (purple blob) is the particle production process which is not associated with the leading hard process. It arises mostly from soft QCD 
interactions between proton remnants. They follow the steps described for hard interaction (e.g., parton shower shown in purple lines) and produce particles in addition to the particles produced in hard interactions. The UE are beyond QCD factorization theorem and are modelled by phenomenological models and are calibrated with data.

- Unstable particle decays: The final component of an event generation is the unstable particle decays (green lines) where unstable hadrons decay and produce other particles.

\subsection{Analysis regions}

In a physics analysis, the events which are analysed are classified into different analysis regions according to their kinematics. This section gives an overview of different types of analysis regions which will be relevant later. In BSM searches such as dark matter, the events can be categorized into two categories: (i) events predicted by the SM, and (ii) events with the potential for new physics that are not explained by the SM. Events in the first category are referred to as $S M$ backgrounds, and the ones in the second category are referred to as the possible signal. The BSM analyses in LHC are conducted to separate the signal like events ( $s$ ) from the SM background events (b) with highest possible purity (i.e., maximizing $s / \sqrt{b}$ ) with full consideration of the uncertainties involved in categorization of the two types of events. The event classification is performed using different physics objects or variables (c.f. section 5.1.6, such as $E_{\mathrm{T}}^{\mathrm{miss}}$ that is supposed to be higher in BSM events compared to SM background events. These variables are called discriminating variables, and an event is selected by applying cuts on a set of chosen discriminating variables

- this process is commonly known as event selection. A Signal Region (SR) is constructed to maximize the presence of signal events while suppressing the backgrounds. For each physics analysis, the event selections are optimized by extensive studies with different MC samples for each background process. Multiple physics analyses might have a common final state, i.e., the SR targets similar types of events. In that case, the SR gets contaminated and does not truly reflect the actual phase space of the search. For example, the signature of mono- $h(b \bar{b}) \mathrm{DM}$ analysis is $b \bar{b}(\mathrm{~h} \rightarrow b \bar{b})+E_{\mathrm{T}}^{\text {miss }}$ and this signature can be also produced by other processes such as $Z(\rightarrow$ 
$v v)+$ jets, semileptonically decaying $t \bar{t}$ events where the leptons are misidentified etc. Estimating backgrounds in the SR is a critical aspect of any physics analysis. For this purpose, Control Regions (CR) are defined, which are enriched with backgrounds and contain negligible signal events. The main criterion in designing a CR is to make the phase space as close as possible to SR, yet disjoint from it. Since the MC generators are based on theoretical assumptions, the background predictions have limited accuracy. Hence, these predictions are required to be validated with the $p p$ collision data. In an analysis all the SR and CRs are a part of the statistical model.

\subsection{Statistical analysis and Hypothesis testing}

In experimental particle physics, searches are often performed to discover new physics which has been predicted by theories but not yet proven, like for BSM searches. The analysis results, i.e. compatibility between the observed data and the proposed theory, are interpreted in the context of a frequentist statistical analysis [161]. This section describes the statistical concepts and methods used for the interpretation of the analysis results described in chapter 5 .

\section{- Hypothesis formation}

The statistical analysis starts with the formulation of the hypothesis under investigation. There are two types of hypotheses in statistical analysis. First is the null hypothesis, denoted by $H_{0}$, which represents the known physics. It is also known as the background-only $(B)$ hypothesis. The second one is the alternate hypothesis, denoted by $H_{1}$, which represents the combination of the known physics and the new signal hypothesis. The alternate hypothesis is also known as the signalplus-background $(S+B)$ hypothesis. For the DM analysis described here, the null hypothesis is the $\mathrm{SM}$, while the alternate hypothesis is the SM along with the DM signals. In a hypothesis testing one evaluates whether the observed data is compatible with $H_{0}$ or $H_{1}$.

\section{- Test statistic and $p$-values}

To quantify the level of compatibility between the observed data and the hypothesis a test statistic $t(x)$ is defined, which is a function of a measured quantity $(x)$ in an analysis. The conclusion of a hypothesis testing depends on which side of a predefined critical region the observed 
test statistic value falls. A critical region is defined by a predefined cut value on the test statistic distribution, as shown in Figure 47. In a simple hypothesis test, i.e. $H_{0}: x=x_{0}$ and $H_{1}: x=x_{1}$, the outcome could be either reject $H_{0}$ and accept $H_{1}$ or accept $H_{0}$ and reject $H_{1}$. However, in composite hypothesis testing, i.e. $H_{0}: x=x_{0}$ and $H_{1}: x \neq x_{1}$, there are other possible outcomes, such as:

1. Reject $H_{0}$ and accept $H_{1}$ when $H_{0}$ is true, known as type-I error.

2. Accept $H_{0}$ and reject $H_{1}$ when $H_{1}$ is true, known as type-II error.

The rates of the type-I and type-II errors are given by $\alpha$ and $\beta$ respectively, which are defined as:

$$
\alpha=\int_{t_{\mathrm{cut}}}^{\infty} f\left(t \mid H_{0}\right) d t \quad \text { and } \quad \beta=\int_{\infty}^{t_{\mathrm{cut}}} f\left(t \mid H_{1}\right) d t
$$

The quantity $f\left(t \mid H_{0}\right)$ and $f\left(t \mid H_{1}\right)$ represent the probability density function for the test statistic under the null and alternate hypothesis respectively. The quantity $1-\beta$ is known as the power of the test and the goal is to maximize the power for a fixed $\alpha$ to increase the accuracy of the hypothesis test.

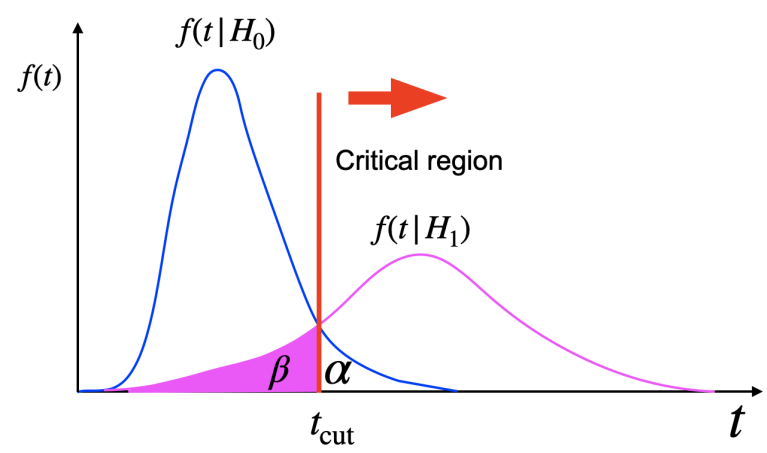

Figure 47: Schematic demonstrating test statistic distributions for the $H_{0}$ and $H_{1}$ hypotheses and the critical region, where the $t_{\text {cut }}$ represents the predefined cut value of the observed test statistic. The parameters $\alpha$ and $\beta$ represent the type-I and type-II errors rates.

Once the hypothesis is formed and a test statistic is defined, the next step in a hypothesis test is to define a parameter that will quantify the level of agreement between the observed data and the given hypothesis. This parameter is known as the $p$-value representing the probability, under the assumption of a hypothesis, of finding data of equal or greater incompatibility with the predictions of the hypothesis. A general mathematical form of the $p$-value, applicable to both $H_{0}$ 
and $H_{1}$ is given as:

$$
p_{\mu}=\int_{t_{\mu, \mathrm{obs}}}^{\infty} f\left(t_{\mu} \mid \mu\right) d t_{\mu} .
$$

Here the parameter $\mu$ is known as the signal strength and it can have a value of 0 or 1 . The $\mu=0$ represents $H_{0}$ and $\mu=1$ represents $H_{1}, t_{\mu \text {,obs }}$ represents the observed test statistic, and $f\left(t_{\mu} \mid \mu\right)$ is the probability density function of $t_{\mu}$ under the assumed $H_{\mu}$. To give an example, $p_{0}$ represents the probability of finding the observed test statistic ( $\left.t_{0, \text { obs }}\right)$ under the assumption of $H_{0}$ if $H_{0}$ is true. As the $p$-value gets smaller, the greater the evidence against $H_{0}$ and a hypothesis is rejected if the observed $p$-value is below a certain predefined threshold.

However, in particle physics an alternate variable, known as the significance $(Z)$, is used to interpret the outcome of an analysis. The significance is defined as:

$$
Z=\Phi^{-1}(1-p)
$$

where $\Phi^{-1}$ is the inverse of the cumulative distribution of the standard Gaussian, known as the quantile function. In a more physical language, if a Gaussian distributed variable is found $Z$ standard deviations above its mean, it has an upper-tail probability of $p$. For low $p_{\mu}$ values, it becomes more likely to reject the assumed hypothesis $(\mu)$. In particle physics a null hypothesis $(\mu=0)$ is rejected, i.e. a discovery is made, if the significance level is at least $Z=5 \sigma$ which corresponds to $p_{0}=2.87 \times 10^{-7}$. On the other hand, the exclusion of a signal hypothesis has much less strict criterion. It requires a significance level of at least $Z=1.64 \sigma$ which corresponds to $p_{0}=0.05$ or 95\% confidence level (known as $C L_{b}$ ).

\section{- $\mathrm{CL}_{s}$ construction}

When a signal hypothesis or a part of its parameter space gets excluded at the LHC, it is most likely that the signal model will not be studied further. Hence such conclusions are of extreme importance for new physics searches. The above mentioned $p$-value and $C L_{b}$ criterion is not robust enough to exclude a signal hypothesis. For e.g., in an analysis where the expected signal is very low, the probability distribution functions of $H_{0}$ and $H_{1}$ would be almost indistinguishable. When the number of observed data events fluctuates far below the expected backgrounds, neither of $H_{0}$ and $H_{1}$ are favoured. Yet due to low $p_{1}$-values, the $H_{1}$ hypothesis could be excluded. In simple 
terms, an analysis which is insensitive to the signal model, exclusion of the $H_{1}$ based on $p$-value might not be accurate. To avoid this, the $C L_{s}[161]$ value is used as the metric to exclude a signal hypothesis. The $C L_{S}$ is defined as:

$$
C L_{s}=\frac{p_{1}}{1-p_{0}}
$$

where $p_{0}$ and $p_{1}$ represent the usual background only and signal + background hypothesis respectively. With this metric a downward fluctuation of data will lead to higher values of $p_{0}$, hence larger $C L_{s}$. On the other hand for larger values of $p_{1}, p_{0}$ would be small, hence $C L_{s} \approx p_{1}$ and $C L_{s} \approx C L_{b}$. As a result a signal hypothesis would not be excluded due to data fluctuation or the analysis being insensitive to the model. A signal hypothesis $(\mu=1)$ is excluded if $C L_{s} \leq \alpha$, where $\alpha$ represents a predefined significance level and at the $\mathrm{LHC}$, it is set at $C L_{S} \leq 0.05$, known as the $95 \% C L_{S}$.

\section{- Upper limits}

Often a signal hypothesis contains one or more parameters of interest (POI) whose values are not specified, e.g., production cross-section of a BSM process. For those cases, upper limits are put on the values of the POIs with a certain predefined CL of $\alpha$. An upper limit at a 95\% CL on a POI of a given signal hypothesis, with a certain $\mu$, represents the maximum value of $\mu$ satisfying $C L_{s} \geq 0.05$. In case of a BSM signal, the upper limit represents the largest value of the production cross-section, satisfying $C L_{s} \geq 0.05$, which cannot be excluded. This also means that the smaller production cross sections, corresponding to smaller $\mu$ values, are compatible with the observed data and cannot be excluded.

\section{- Likelihood function}

The BSM signal shapes are likely to be different than the SM background shapes. Hence to accurately distinguish between $H_{0}$ and $H_{1}$, the hypothesis test is based on a binned likelihood function. For a counting experiment, measuring a variable $x$, a histogram can be formed with the measured values $n=\left(n_{1}, n_{2}, n_{3}, . ., n_{N}\right)$, where $N$ is the total number of histogram bins. The expected number of events $\left(E^{\exp }\right)$ in a particular bin $i$ is given as:

$$
E_{i}^{\exp }(\mu, \theta)=\mu s_{i}^{\exp }(\theta)+b_{i}^{\exp }(\theta)
$$




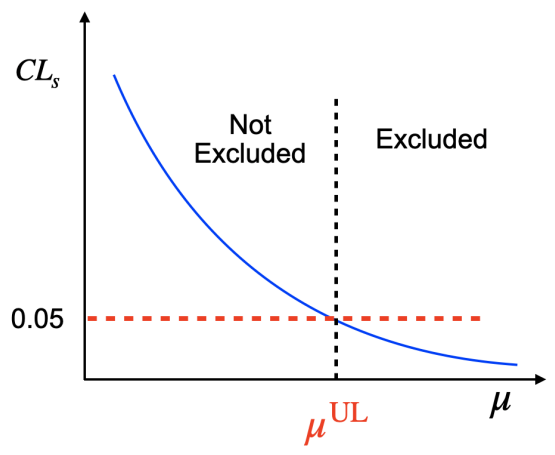

Figure 48: Schematic [162] demonstrating the exclusion regions associated with a signal hypothesis with a signal strength $\mu$, determined by the $C L_{s}$ values. The blue line represents the $C L_{s}$ values as a function of $\mu$ and the $\mu$ value which crosses the $C L_{s}$ curve at $C L_{s}=0.05$ is the upper limit on $\mu, \mu^{\mathrm{UL}}$. Signal points with $\mu \leq \mu^{\mathrm{UL}}$ are compatible with the observed data and cannot be excluded, while the points with $\mu>\mu^{\mathrm{UL}}$ are excluded.

where $\mu$ is the signal strength, $\theta$ represents the set of nuisance parameters (NP) associated with the signal and background systematics (c.f. section 5.1.10), and $s_{i}^{\exp }, b_{i}^{\text {exp }}$ represent the mean number of expected signal and background events in the $i^{\text {th }}$ bin respectively. Similarly in a control region a histogram can be constructed with $M$ bins and a set of values represented by $m=\left(m_{1}, m_{2}, m_{3}, . ., m_{M}\right)$. The expected number of events in a particular bin $i$ can be written as:

$$
E_{i}^{\exp }(\theta)=u_{i}^{\exp }(\theta)
$$

Since a counting experiment follows a Poisson distribution, the likelihood function for an analysis with one signal region and one control region can be written as:

$$
L(\mu, \theta)=\prod_{i=1}^{N} \frac{\left(\mu s_{i}+b_{i}\right)^{n_{i}}}{n_{i} !} e^{-\left(\mu s_{i}+b_{i}\right)} \prod_{j=1}^{M} \frac{u_{k}^{m_{k}}}{m_{k} !} e^{-u_{k}}
$$

Here $n_{i}$ and $m_{k}$ represent the number of observed events in the $i^{t h}$ and $k^{\text {th }}$ bin respectively. The NPs represent the systematic uncertainties for both signal and background processes and these NPs can alter the overall normalisation or shape or both of an observable. These NPs are initially determined by specific object reconstruction groups in ATLAS and are included in the fit, but the final values of these NPs are determined by a global fit to the observed data which includes all the regions of a given analysis. The NPs are typically parameterized in such as way that the nominal value is at $\theta_{i}=0$ and $\theta_{i}= \pm 1$ corresponds to the $\pm 1 \sigma$ variation. These are implemented in the fit 
as a Gaussian distribution centered at zero and with a width of $\sigma=1$ :

$$
G\left(\theta_{i}\right)=\frac{1}{\sqrt{2 \pi}} \exp ^{-\frac{\theta_{i}^{2}}{2}}
$$

In a fit, the $\theta$ parameters vary between $\pm 1 \sigma$ and the probability distribution functions of any combination of $\theta$ 's are constructed by the HISTFACTORY toolkit [163]. With the $\theta$ parameters the full likelihood function is written as:

$$
L(\mu, \theta)=\prod_{i=1}^{N} \frac{\left(\mu s_{i}+b_{i}\right)^{n_{i}}}{n_{i} !} e^{-\left(\mu s_{i}+b_{i}\right)} \prod_{k=1}^{M} \frac{u_{k}^{m_{k}}}{m_{k} !} e^{-u_{k}} \prod_{l=1}^{P} G\left(\theta_{p}\right) .
$$

According to the Neyman-Pearson lemma [164], the accuracy of a hypothesis is maximized if the test-statistic is based on a likelihood ratio:

$$
\lambda(\mu)=\frac{L(\mu, \hat{\hat{\theta}})}{L(\hat{\mu}, \hat{\theta})}
$$

The $\hat{\hat{\theta}}$ is the value of $\theta$ that maximizes $L$ for a specific $\mu$, i.e., the conditional maximum-likelihood (ML) estimator of $\theta ; L(\hat{\mu}, \hat{\theta})$ represents the likelihood which is maximized unconditionally, and $\hat{\mu}, \hat{\theta}$ are the parameters that maximize the likelihood. By definition $0 \leq \lambda(\mu) \leq 1$ and a value close to 1 represents a good agreement between the signal hypothesis and the observed data. The associated test statistic is given as:

$$
t_{\mu}=-2 \ln \lambda(\mu)
$$

Hence, higher values of the test statistic correspond to greater incompatibility between the observed data and the signal hypothesis.

The definition of the test statistic changes depending on whether there is any excess observed in data compared to the predicted backgrounds. If an excess is observed, $H_{0}$ is compared with $H_{1}$ and the goal is to reject $H_{0}$ in favor of $H_{1}$ at a certain significance level. If there is a signal, the total number of events (yields) in the signal region would be higher compared to the predicted backgrounds. Hence, the test statistic is chosen as:

$$
t_{0}= \begin{cases}-2 \ln \lambda(0), & \text { if } \hat{\mu} \geq 0 \\ 0, & \text { if } \hat{\mu}<0\end{cases}
$$


With this choice of the test statistic $H_{0}$ would not be rejected for a downward fluctuation of data. On the other hand if there is a good agreement between the observed data and the background prediction, the signal hypothesis can be excluded and upper limits can be put on the POI (e.g., signal strength $\mu$ ). To set upper limits, the roles of $H_{0}$ and $H_{1}$ are reversed, i.e., the goal is to exclude the signal + background hypothesis in favor of the background only hypothesis. For upper limits the test statistic is chosen as:

$$
t_{\mu}= \begin{cases}-2 \ln \lambda(\mu), & \text { if } \hat{\mu} \leq \mu \\ 0, & \text { if } \hat{\mu}>\mu\end{cases}
$$

Here as well the choice of $q_{\mu}=0$ for $\hat{\mu}>\mu$ ensures that an excess in observed data compared to the signal + background prediction would not indicate a greater incompatibility and reject the signal + background hypothesis.

\section{- Smoothing and pruning}

The NPs corresponding to the shape uncertainties are histograms which serve as alternate templates of the discriminating variables compared to the nominal one. Often these shape systematic histograms have statistical fluctuations and can make the fit unstable. To avoid such scenarios a smoothing mechanism is implemented. There are many smoothing algorithms, and the one used in the analysis described in section 5.1 uses an iterative rebinning of the systematic histogram until two local extremas are attained. The smoothing procedure is applied to the shape systematics only and in the mono- $h(b \bar{b})$ analysis its applied to the Higgs candidate mass and muon-charge distribution shapes, which are the discriminating variables used in the statistical analysis.

Among the various systematic uncertainties some might have a negligible impact on the discriminating variables of the fit. Also if a systematic has limited statistics, it can induce instabilities in the fit. To reduce these types of systematics and to speed up the fit procedure a pruning mechanism is implemented which removes such systematics depending on analysis specific criteria. Unlike the smoothing, pruning is applied to both the shape and normalisation systematics separately. 


\section{DARK MATTER AND DARK ENERGY ANALYSES}

\section{1 mono- $h(b \bar{b})$ dark matter analysis}

DM search is conducted in association with a visible SM particle. These types of final states with exactly one visible SM particle along with DM candidates are known as mono- $X$ signatures, where $X$ is the visible SM particle. There are many possible mono- $X$ signatures, where the $X$ could be a jet, photon, $W^{ \pm} / Z$-bosons, a SM Higgs boson $(h)$ etc. The phenomenology of the mono- $h$ signature is different than the other mono- $X$ signatures. Generally DM- $X$ coupling is much weaker than the couplings between $X$ and SM fermions. Hence in general, for the majority of the mono- $X$ signatures, the visible particle $X$ is generated through Initial State Radiation (ISR). On the contrary, since couplings between the SM Higgs boson and SM fermions are Yukawa suppressed, the Higgs boson gets produced either through Final State Radiation (FSR) or DM production mechanisms. For this characteristic of Higgs boson production, a mono- $h$ signature can probe the SM-DM coupling directly [165]. A DM search with a mono- $h$ signature is performed and the following sections describing the analysis are organized as:

Section 5.1.1: Brief description of the analysis.

Section 5.1.2. Description of the characteristics of the theoretical models.

Section 5.1.3. Description of the relevant SM backgrounds.

Section 5.1.4. MC simulation of signal and SM background events.

Section 5.1.5: Triggers used to select the events used in the analysis.

Section 5.1.6. Physics object definitions such as jets, leptons, $E_{\mathrm{T}}^{\mathrm{miss}}$ etc.

Section 5.1.7, Event selections.

Section 5.1.8. SM background compositions across different analysis regions.

Section 5.1.9. Treatment of $V+$ jets in the analysis.

Section 5.1.10, Analysis uncertainties.

Section 5.1.11. Statistical model used to interpret the observations.

Section 5.1.12, Results. 


\subsubsection{Analysis in a nutshell}

The DM search described in this thesis analyzes two benchmark models, $2 \mathrm{HDM}+a$, and $Z^{\prime}$ 2HDM (c.f., section 2.4), which predict the production of DM particles in association with a SM Higgs boson. Since the DM particles escape the detector undetected, it leaves a $E_{\mathrm{T}}^{\text {miss }}$ signature. The SM Higgs boson decays to two $b$-quarks and the final state contains $h(\rightarrow b \bar{b})+E_{\mathrm{T}}^{\text {miss }}$ signature. Depending on the decay mode of the SM Higgs boson, there can be other possible final states. However, as shown in Figure 49 the BR $(h \rightarrow b \bar{b}) \approx 58 \%$ and is much higher than other decay modes. Hence, the mono- $h(b \bar{b})$ final state has much greater statistical power for potential DM events than other mono- $h$ final states.

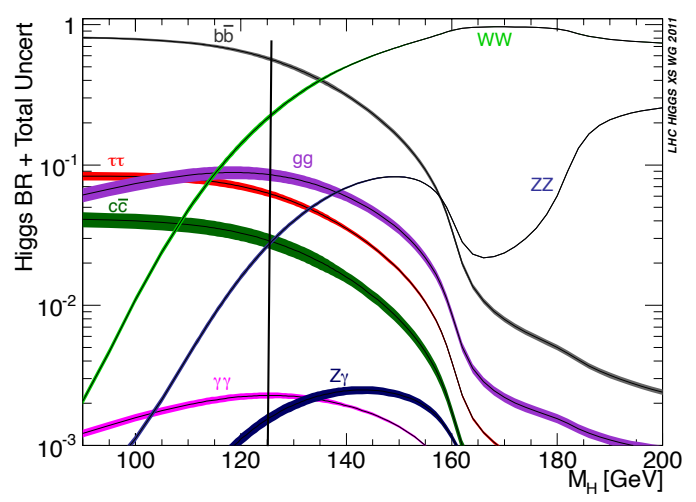

Figure 49: Higgs boson decay BRs and their related uncertainties as a function of the Higgs boson mass [166]. The black vertical line represents the SM Higgs boson mass of $m_{h}=125 \mathrm{GeV}$.

The SM Higgs boson is reconstructed from the $b$-tagged jets, and the reconstruction procedure depends on the SM Higgs boson $p_{\mathrm{T}}$. From momentum conservation, the angle between the two $b$-jets from the Higgs decay is inversely proportional to the Higgs $p_{\mathrm{T}}$. For low $p_{\mathrm{T}}$ Higgs, the two $b$-jets can be identified separately, and the Higgs candidate is reconstructed with two central $(|\eta|<2.5)$ small-R $b$-tagged jets. However, for highly boosted Higgs bosons, the $b$-jets get collimated and it becomes difficult to distinguish the two $b$-jets separately. In these scenarios, the Higgs candidate is reconstructed with a large- $\mathrm{R}$ jet with two associated $b$-tagged variable radius track jets. For this reason, the analysis phase space is divided into two topologies: Resolved and Merged. Since the Higgs $p_{\mathrm{T}}$ is correlated to the $E_{\mathrm{T}}^{\mathrm{miss}}$, the $E_{\mathrm{T}}^{\mathrm{miss}}$ is used as the discriminating 
variable to define the Resolved and Merged topologies, Resolved: $150<E_{\mathrm{T}}^{\text {miss }}<500 \mathrm{GeV}$ and Merged: $E_{\mathrm{T}}^{\text {miss }}>500 \mathrm{GeV}$. Figure 50 shows the schematic of the Resolved and Merged topologies of the mono- $h(b \bar{b})$ analysis phase space.

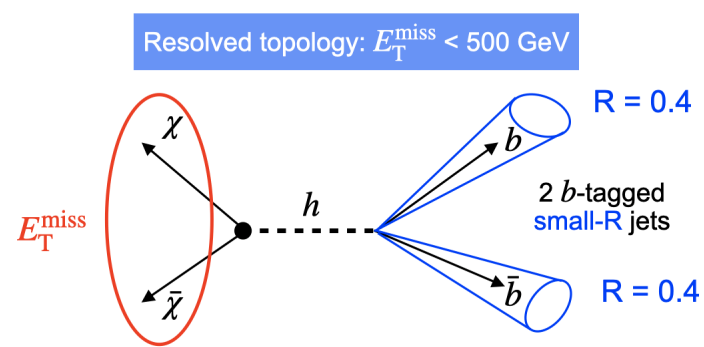

(a)

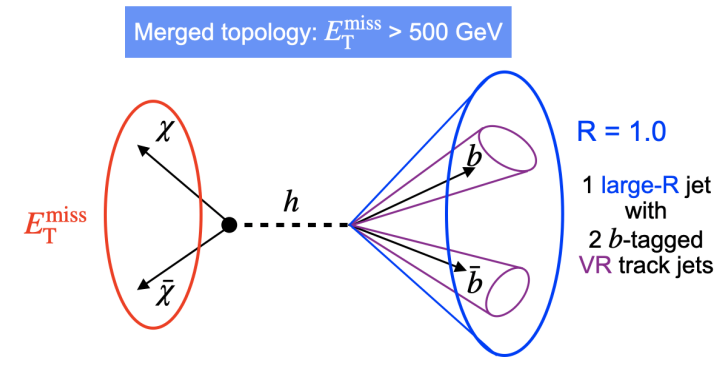

(b)

Figure 50: Figures show the Resolved (left) and Merged (right) topologies of the mono$h(b \bar{b})$ analysis phase space. The Higgs candidate is reconstructed with 2 -tagged central small-R jets in the Resolved topology, while in Merged topology a large-R jet with $2 b$-tagged VR track jets is used. The DM candidates, represented by $\chi$, produce the $E_{\mathrm{T}}^{\mathrm{miss}}$.

The signal region of the mono- $h(b \bar{b})$ DM analysis phase space contains a Higgs boson which decays to two $b$-quarks $(h \rightarrow b \bar{b})$ and DM candidates that produce a significant $E_{\mathrm{T}}^{\text {miss }}$. The SR does not contain any leptons and is also referred to as the 0-lepton region. BSM signal events would be rare, the backgrounds in these types of searches are mainly from SM. In the mono$h(b \bar{b})$ analysis the major backgrounds are: top-pair production $(t \bar{t})$, and $W^{ \pm} / Z$ bosons produced in association with jets. The $t \bar{t}$ events can enter the SR where the $t \bar{t}$ decays semi-leptonically $\left(t \bar{t} \rightarrow b W^{+} \bar{b} W^{-} \rightarrow b \ell^{+} v \bar{b} q \bar{q}\right)$ and the lepton is misidentified due to detector limitations. Similarly, the $W^{ \pm}+$jets events can end up in the SR where the leptons coming from the $W^{ \pm}$boson decay get misidentified. A 1-lepton CR containing exactly one muon $\left(\mu^{+} / \mu^{-}\right)$is defined to estimate the $t \bar{t}$ and $W^{ \pm}+$jets backgrounds. On the other hand, the $Z(\rightarrow v v)+$ jets events can have exactly the same signature as $E_{\mathrm{T}}^{\text {miss }}+b \bar{b}$; these types of backgrounds are called irreducible backgrounds. The kinematics of $Z$-boson does not depend on its decay products, hence the $Z(\rightarrow v \bar{v})+$ jets backgrounds in the SR are estimated with $Z(\rightarrow \ell \bar{\ell})+$ jets events. For this purpose, two 2-lepton CRs with exactly two leptons that can be either two opposite flavor electrons $\left(e^{+} e^{-}\right)$or muons $\left(\mu^{+} \mu^{-}\right)$are constructed. Hence, depending on the number of leptons the mono- $h(b \bar{b})$ phase space 
is classified as : (i) 0-lepton SR, (ii) 1-lepton CR, and (iii) 2-lepton CR.

The analysis phase space is also categorized according to the number of $b$-jets. As shown in Figure $17 \mathrm{~h}$, the $2 \mathrm{HDM}+a b b$-induced mechanism contains extra $b$-quarks (apart from $h \rightarrow b \bar{b}$ ) in the final state from gluon splitting. On the other hand, the sensitivity for $Z^{\prime}-2 \mathrm{HDM}$ and $2 \mathrm{HDM}+a$ ggF models come from the $2 b$-tag regions. Hence, to gain sensitivity to all the benchmark models, the analysis phase space is divided into different $b$-tag categories such as: (i) $2 b$-tag category with exactly two $b$-jets, and (ii) $\geq 3 b$-tag category with at least three $b$-jets. For the Resolved topology, the 2 and $\geq 3 b$-tag categories are defined to have exactly 2 and $\geq 3 b$-tagged small-R jets. For the Merged topology the $2 b$-tag category is defined to have exactly two $b$-tagged VR-track jets associated to the large-R jet and no $b$-tagged VR-track jets outside the large-R jet, while the $\geq 3 b$ jets category is defined to have exactly two $b$-tagged VR-track jets associated to the large-R jet and $\geq 1 b$-tagged VR-track jets outside the large-R jet. Figure 51 summarises the mono- $h(b \bar{b})$ analysis phase space classifications.

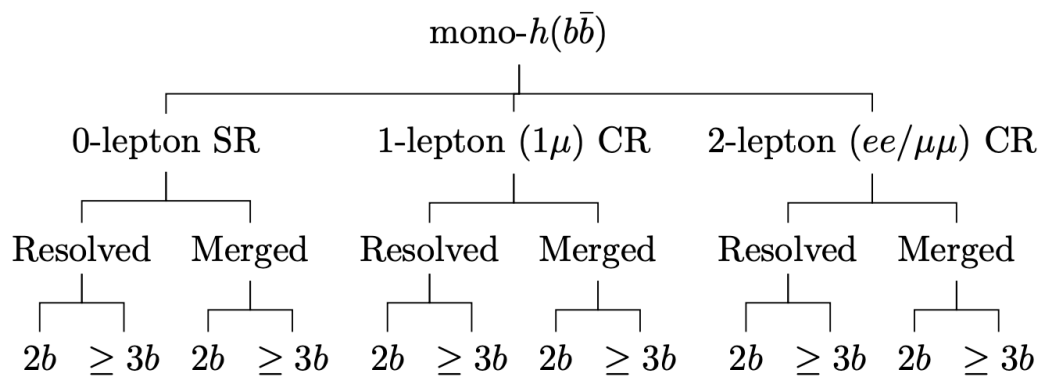

Figure 51: Classifications of different analysis regions in the mono- $h(b \bar{b})$ phase space. The $2 b$ and $\geq 3 b$ represent the 2 and $\geq 3 b$-tag categories respectively.

The statistical interpretation of the observed data, in the context of a possible mono- $h(b \bar{b}) \mathrm{DM}$ signal, is performed with a profile likelihood (PL) fit where the SR and CRs are fitted simultaneously. The fit discriminant used in the SR is the Higgs candidate mass distribution. It is labeled as $m_{j j}$ in Resolved topology where " $j j$ " represents two $b$-tagged small-R jets, and $m_{J}$ in Merged topology where " $J$ " represents a large-R jet with two associated $b$-tagged VR track jets; the Higgs candidate mass is generically labeled as $m_{h}$. The results are interpreted in the context of the two benchmark models. 
The precision of a physics analysis searching for a specific signal depends on accurate background separation from the potential signals. Hence, the choice of binning for the discriminant variable histogram is important. For the signal process, the $m_{h}$ distribution will have a peak around $m_{h} \approx 125 \mathrm{GeV}$, while for SM backgrounds, the peak and shape would be different from the signal. Figure 52a shows the $m_{h}$ distribution for a few $Z^{\prime}$-2HDM signal points and SM backgrounds $(t \bar{t}$ and $Z+$ jets) in the SR for the Resolved $2 b$-tagged category. As seen from the plot, by dividing the $m_{h}$ distribution into multiple bins and using the shape differences, the signals can be distinguished from the backgrounds. The binning choice is such that the shape differences are distinguishable, and each bin has enough statistics for accurate background estimation.

As shown in Figure $52 \mathrm{~b}$, the shape of the $E_{\mathrm{T}}^{\text {miss }}$ spectra is correlated with the mass parameters, such as $\left(m_{Z^{\prime}}, m_{A}\right)$ in the $Z^{\prime}$-2HDM model (c.f. Figure 20p. For high $m_{Z^{\prime}}, E_{\mathrm{T}}^{\text {miss }}$ goes higher and the peak position of the $E_{\mathrm{T}}^{\text {miss }}$ spectra changes with $m_{Z^{\prime}}$. To increase sensitivity for different signal points with different $E_{\mathrm{T}}^{\text {miss }}$ peaks, the phase space is also binned in $E_{\mathrm{T}}^{\text {miss }}$. For both 2 and $\geq 3$ $b$-tag categories the Resolved topology is divided into three $E_{\mathrm{T}}^{\text {miss }}$ bins: $E_{\mathrm{T}}^{\text {miss }}=[150-200,200-350$, 350-500] GeV. Due to lower data statistics, the Merged topology has wider $E_{\mathrm{T}}^{\text {miss }}$ bins: $E_{\mathrm{T}}^{\text {miss }}=$ [500-750, $>750] \mathrm{GeV}$ and $E_{\mathrm{T}}^{\text {miss }}>500 \mathrm{GeV}$ for $2 b$ and $\geq 3 b$-tagged regions respectively.

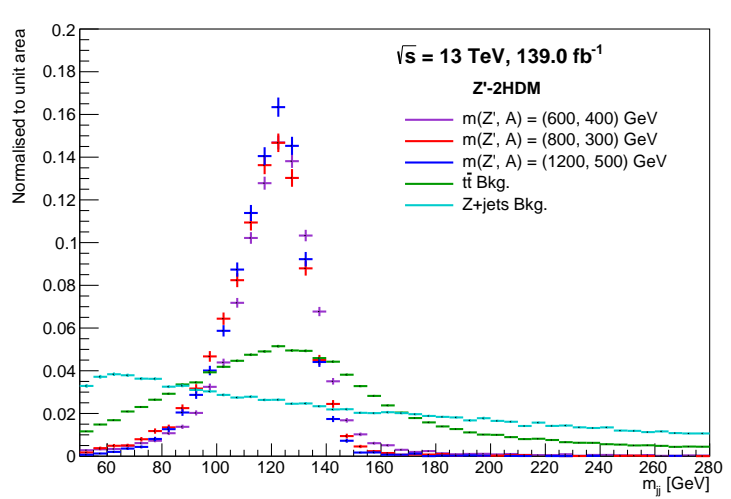

(a)

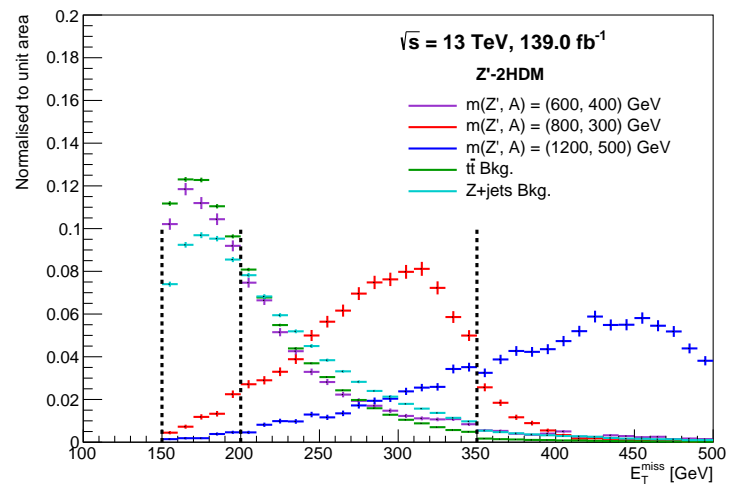

(b)

Figure 52: The plots show the $m_{h}$ (fig. 52a) and $E_{\mathrm{T}}^{\text {miss }}$ (fig. 52b) distributions in 0-lepton Resolved $2 b$-tag region for different $Z^{\prime}-2 H D M$ points and few SM backgrounds. The black dashed lines represent different $E_{\mathrm{T}}^{\text {miss }}$ boundaries for the Resolved regions. 


\subsubsection{Characteristics of 2HDM+ $a$ and $Z^{\prime}-2 H D M$ models}

\section{- $2 \mathrm{HDM}+a$}

The cross sections for the two $2 \mathrm{HDM}+a$ processes (c.f. Figure 17) depend on the choices of the model parameters. Figure 53a shows the fractional contribution of the $b b$-induced process to the inclusive $2 \mathrm{HDM}+a(b b+\mathrm{ggF})$ cross section as a function of $\tan \beta$ for a fixed $\sin \theta=0.35, m_{A}=600$ $\mathrm{GeV}$, and $m_{a}=200 \mathrm{GeV}$. The $b b$-induced process has negligible contribution for $\tan \beta=1$ and the ggF process contributes $\sim 10 \%$ of the total cross section for $\tan \beta=10$. Figure $53 \mathrm{~b}$ shows the cross section comparisons between the two processes as a function of $\sin \theta$ for a fixed $\tan \beta=1$, $m_{A}=600 \mathrm{GeV}$, and $m_{a}=200 \mathrm{GeV}$. Figures $54 \mathrm{a}$ and $54 \mathrm{~b}$ show the cross section as a function of $\tan \beta$ for the $b b$-induced and ggF mechanism respectively. The $b b$-induced and ggF processes are dominant in high $\tan \beta$ and low $\tan \beta$ regions respectively due to the coupling characteristics of the Type-II 2HDM+a model (c.f. section 2.4.1). Figures 55, 56, and 57 show the cross section comparisons as a function of $\sin \theta, m_{A}$, and $m_{a}$ respectively. For both the processes the cross section scales as $1 / m_{A}$ and $1 / m_{a}$, hence the cross section drops with increasing $m_{A}$ or $m_{a}$.

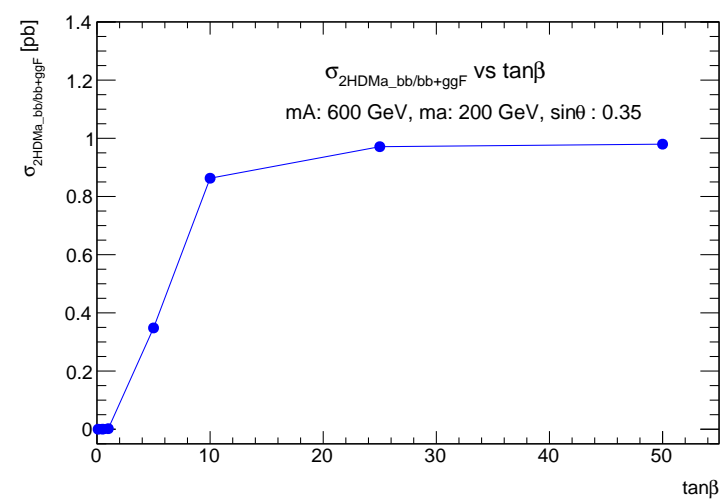

(a)

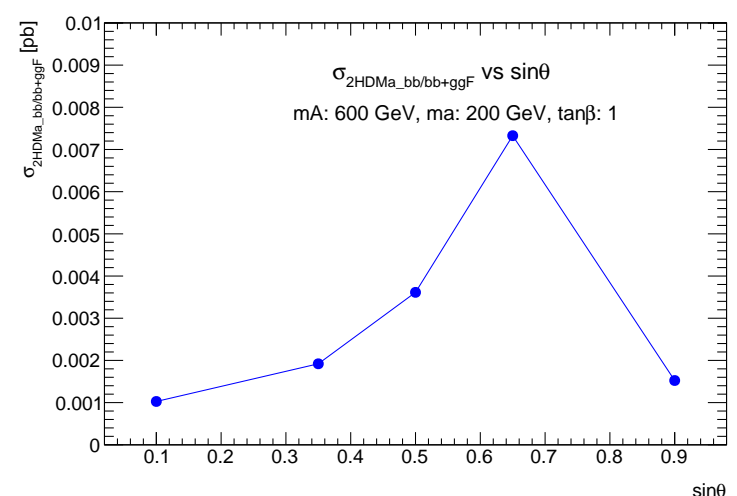

(b)

Figure 53: Fractional contribution of the $b b$-induced process to the inclusive $2 \mathrm{HDM}+a(b b+$ $\mathrm{ggF}$ ) cross section as a function of $\tan \beta$ (left) and $\sin \theta$ (right). 


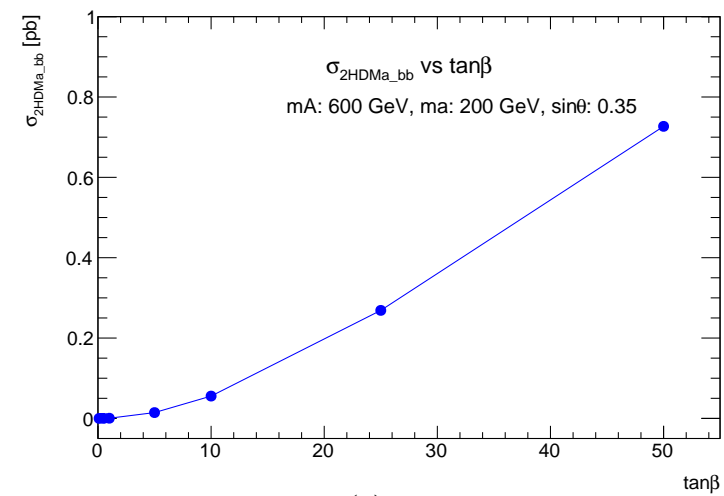

(a)

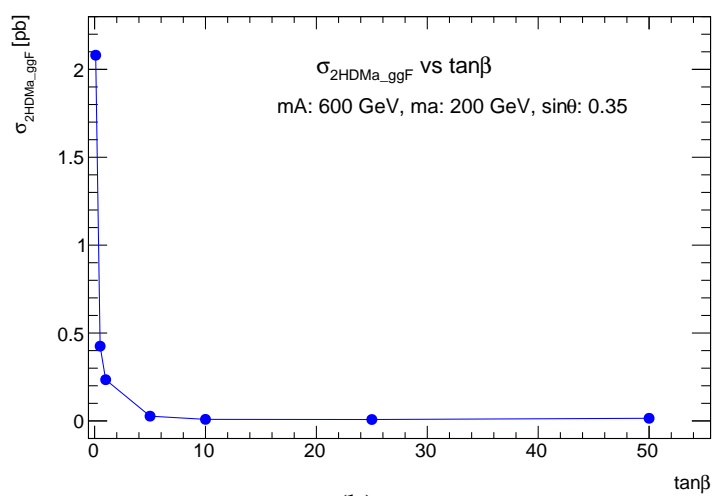

(b)

Figure 54: Cross section as a function of $\tan \beta$ for $b b$-induced (left) and ggF mechanism (right).

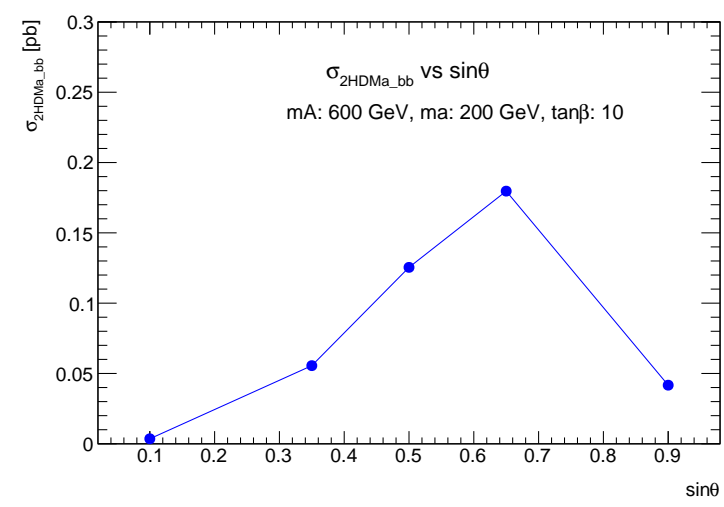

(a)

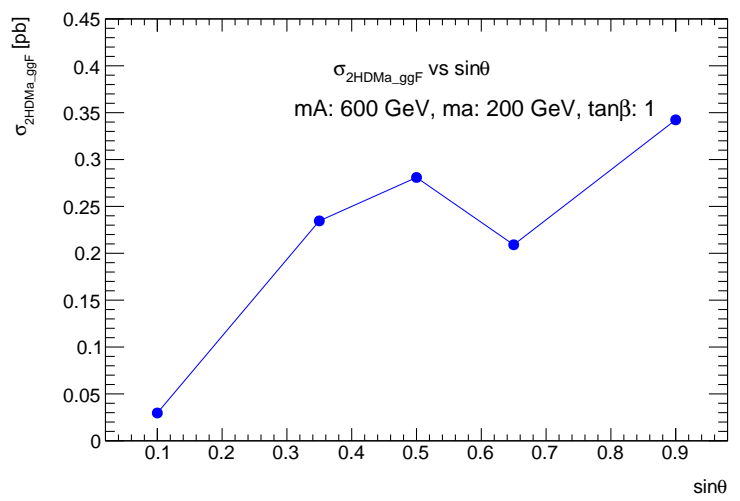

(b)

Figure 55: Cross section as a function of $\sin \theta$ for $b b$-induced (left) and ggF mechanism (right).

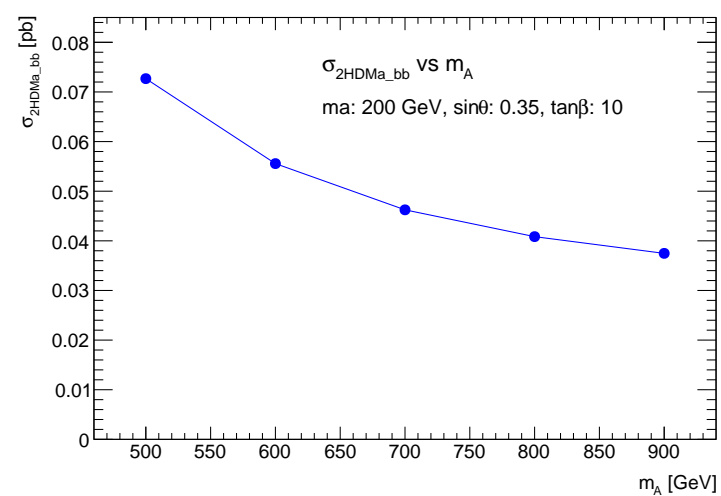

(a)

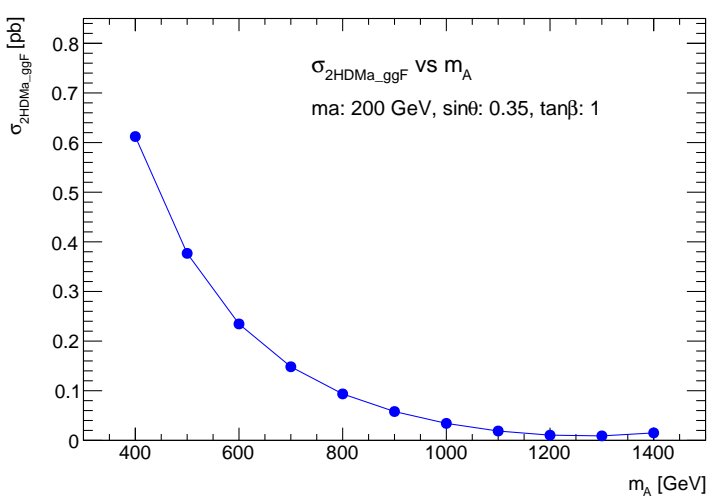

(b)

Figure 56: Cross section as a function of $m_{A}$ for $b b$-induced (left) and ggF mechanism (right). 


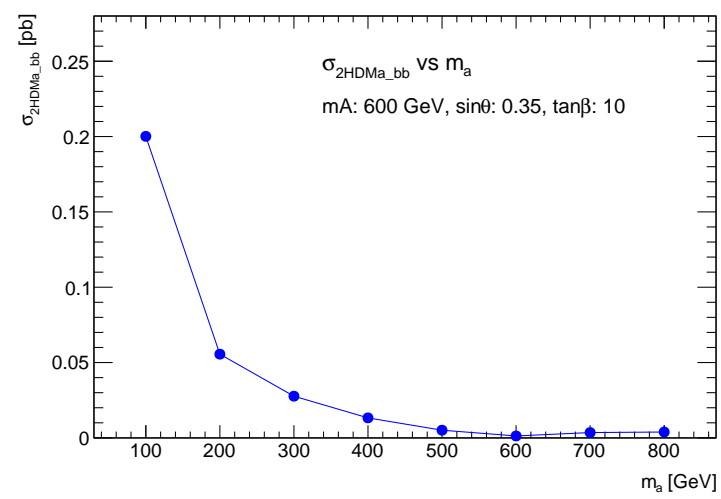

(a)

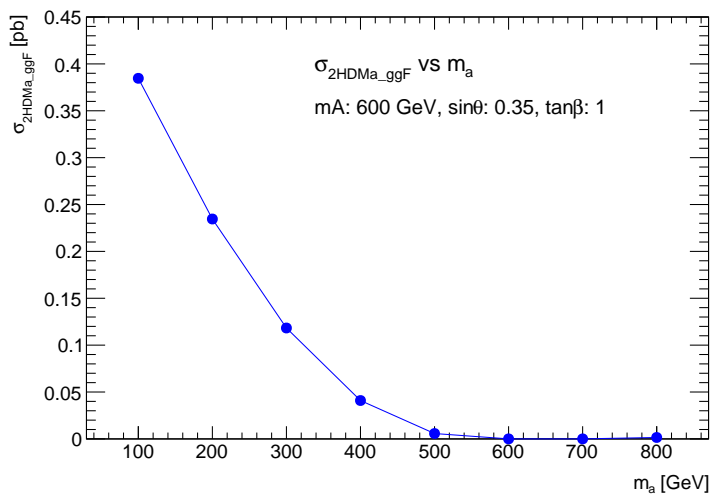

(b)

Figure 57: Cross section as a function of $m_{a}$ for $b b$-induced (left) and ggF mechanism (right).

Depending on the choices of $m_{A}$ and $m_{a}$, different $2 \mathrm{HDM}+a$ processes (c.f. Figure 17) become dominant and is demonstrated by the invariant mass distribution of the Higgs boson and the DM pair denoted as $m_{\chi \bar{\chi} h}$. The variable $m_{\chi \bar{\chi} h}$ is computed at particle level without any event selections. Figures $58 \mathrm{a}$ and $58 \mathrm{~b}$ show the contributions from different production mechanisms for the ggF process and the $m_{\chi \bar{\chi} h}$ distribution respectively, for various mass points. The light

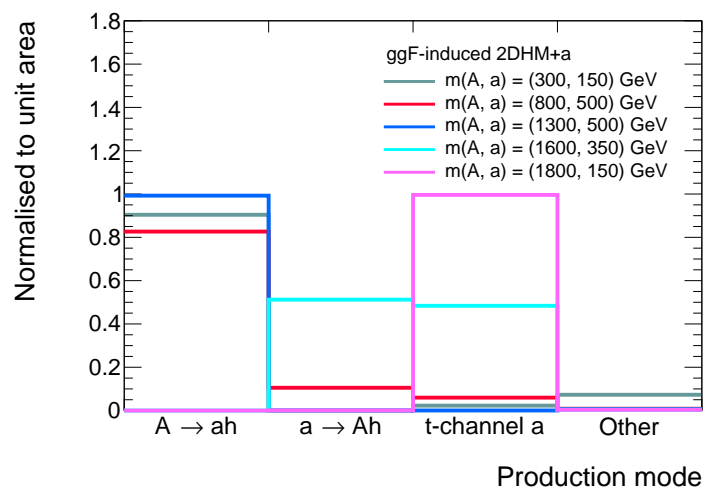

(a)

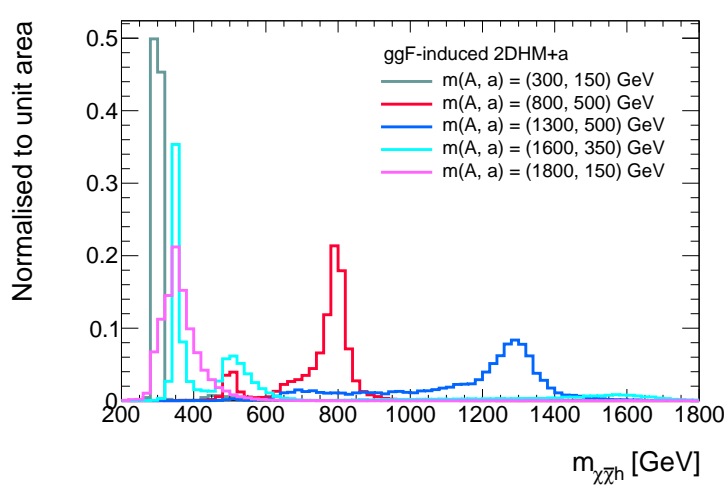

(b)

Figure 58: Figures show the different production mechanisms (left plot) and $m_{\chi \bar{\chi}} h$ distributions (right plot) for the $2 \mathrm{HDM}+a \mathrm{ggF}$ process for various $\left(m_{A}, m_{a}\right)$ points. The plots are produced with particle level MC samples. Plot credits to Dr. Andrea Matic.

green plot in Figure 58a shows that $A \rightarrow a h$ is the dominant process (c.f. Figure 16b) for the $\left(m_{A}\right.$, $\left.m_{a}\right)=(300,150) \mathrm{GeV}$ point. This characteristic translates to the $m_{\chi \bar{\chi} h}$ distribution that peaks at $m_{\chi \bar{\chi} h}=300 \mathrm{GeV}$. For the $\left(m_{A}, m_{a}\right)=(800,500) \mathrm{GeV}$ point (red plot in Figure 58a), $A \rightarrow a h$ is the dominant process, while there are contributions from the off-shell $a \rightarrow A h$ (Figure 17a) and 
$t$-channel (Figure 17b) processes. Although the off-shell process has no characteristic feature in $m_{\chi \bar{\chi} h}$, there are two peaks at $m_{\chi \bar{\chi} h}=800 \mathrm{GeV}$ and $m_{\chi \bar{\chi} h}=500 \mathrm{GeV}$ representing the $A \rightarrow a h$ and $t$-channel processes respectively. The dominant production mode for the $\left(m_{A}, m_{a}\right)=(1300,500)$ $\mathrm{GeV}$ point (blue plot) is $A \rightarrow a h$, hence the $m_{\chi \bar{\chi} h}$ peaks at $1300 \mathrm{GeV}$. The $\left(m_{A}, m_{a}\right)=(1600,350)$ $\mathrm{GeV}$ point (cyan plot) has similar contributions from the off-shell $a \rightarrow A h$ and $t$-channel processes. The off-shell $a \rightarrow A h$ process is manifested by the peak at $m_{\chi \bar{\chi} h}=350 \mathrm{GeV}$, and the $t$-channel process produces the shape at $m_{\chi \bar{\chi} h}=500 \mathrm{GeV}$. The $\left(m_{A}, m_{a}\right)=(1800,500) \mathrm{GeV}$ point (pink plot) has most of the events coming from the $t$-channel process and results in soft $m_{\chi \bar{\chi} h}$ spectra.

As shown by the blue plots in Figure 59, the $b b$-induced process has similar characteristics to the ggF process. The $\left(m_{A}, m_{a}\right)=(1300,500) \mathrm{GeV}$ point has three dominant production modes for the $b b$-induced process: the $A \rightarrow a h$, off-shell $a \rightarrow A h$, and $t$-channel. The $A \rightarrow a h$ and $t$-channel processes are represented by the peaks at $m_{\chi \bar{\chi} h}=1300 \mathrm{GeV}$ and $m_{\chi \bar{\chi} h}=500 \mathrm{GeV}$ respectively, while the broad $m_{\chi \bar{\chi} h}=$ distribution between $600-1000 \mathrm{GeV}$ represents the off-shell $a \rightarrow A h$ process.

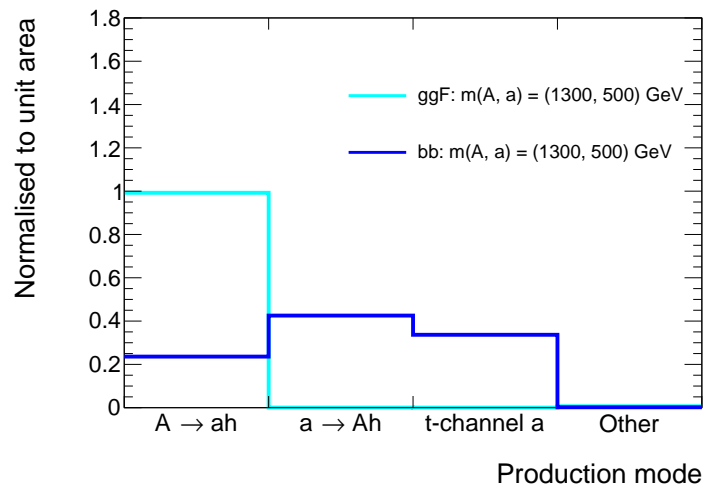

(a)

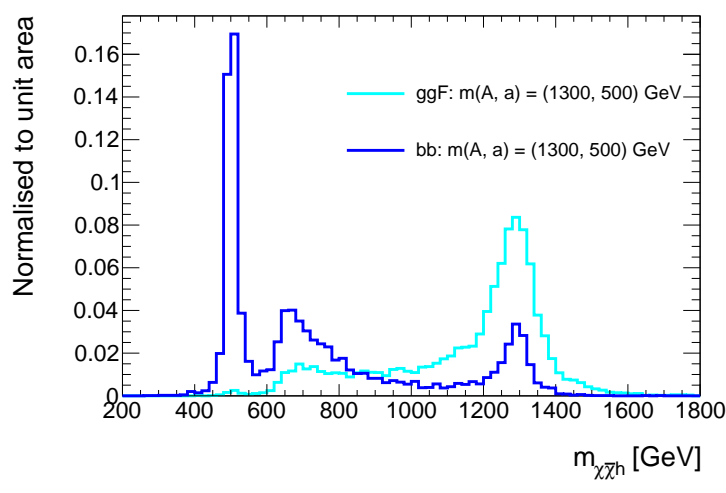

(b)

Figure 59: Figures show the fractional contribution of different production mechanisms for $2 \mathrm{HDM}+a \mathrm{ggF}$ and $b b$-induced processes (left plot) and the associated $m_{\chi \bar{\chi} h}$ distributions (right plot $)$ for $\left(m_{A}, m_{a}\right)=(1300,500) \mathrm{GeV}$ point. The plots are produced with particle level MC samples. Plot credits to Dr. Andrea Matic.

Figure 60 represents the above-mentioned characteristics at reconstruction level (i.e., with full ATLAS detector simulation) using the $E_{\mathrm{T}}^{\text {miss }}$ spectrum for the two $2 \mathrm{HDM}+a$ production modes. 


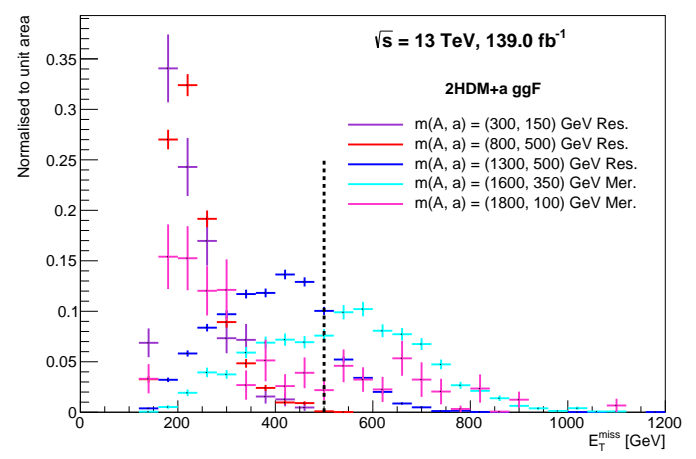

(a)

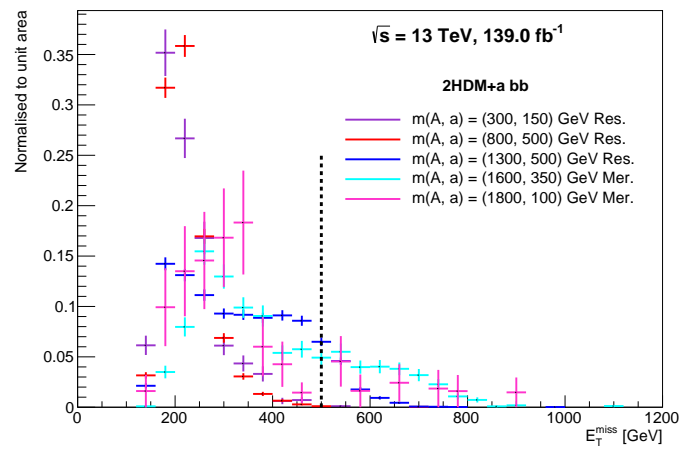

(b)

Figure 60: $E_{\mathrm{T}}^{\mathrm{miss}}$ distributions for various mass points of $2 \mathrm{HDM}+a \mathrm{ggF}($ left $)$ and $b b$-induced (right) mechanisms. The Res (Mer) labels indicate that the corresponding point has Resolved (Merged) selections applied. For the Resolved and Merged selections, $E_{\mathrm{T}}^{\text {miss }}<500 \mathrm{GeV}$ and $E_{\mathrm{T}}^{\text {miss }}>500 \mathrm{GeV}$ cuts are removed respectively to obtain the full $E_{\mathrm{T}}^{\text {miss }}$ spectra, and a $\geq 2 b$-jets cut is applied. The black dashed vertical line represents the $E_{\mathrm{T}}^{\mathrm{miss}}=500 \mathrm{GeV}$ point.

The $E_{\mathrm{T}}^{\text {miss }}$ spectra moves to higher values with increasing $m_{A}$ and with greater $\Delta\left(m_{A}-m_{a}\right)$. However, at $\left(m_{A}, m_{a}\right)=(1800,100) \mathrm{GeV}$ the $E_{\mathrm{T}}^{\text {miss }}$ spectrum moves to the left again due to reasons explained above. For most of the $2 \mathrm{HDM}+a$ signal mass points, the majority of the events are in the Resolved topology, represented by the black dotted line.

\section{- $\mathrm{Z}^{\prime}$-2HDM}

The $Z^{\prime}$-2HDM model has much simpler characteristics compared to the $2 \mathrm{HDM}+a$ model. Figure 61 shows the $E_{\mathrm{T}}^{\text {miss }}$ distribution at reconstruction level for different $\left(m_{Z^{\prime}}, m_{A}\right)$ points of the $Z^{\prime}$-2HDM model. As $m_{Z^{\prime}}$ increases the energies of its decay products, i.e., the SM Higgs boson and the neutral $\mathrm{CP}$-odd Higgs $A$, also increase. Hence, the $E_{\mathrm{T}}^{\text {miss }}$ spectra has higher values at high $m_{Z^{\prime}}$ points. The $E_{\mathrm{T}}^{\text {miss }}$ is also correlated to $\Delta\left(m_{Z^{\prime}}-m_{A}\right)$, the $E_{\mathrm{T}}^{\text {miss }}$ spectra has higher values for higher $\Delta\left(m_{Z^{\prime}}-m_{A}\right)$. For e.g., the $E_{\mathrm{T}}^{\text {miss }}$ peak for $\left(m_{Z^{\prime}}, m_{A}\right)=(3200,1100) \mathrm{GeV}$ (pink plot) is at $E_{\mathrm{T}}^{\text {miss }}=1200 \mathrm{GeV}$, while for $\left(m_{Z^{\prime}}, m_{A}\right)=(3200,300) \mathrm{GeV}$ (green plot) the $E_{\mathrm{T}}^{\text {miss }}$ peaks at $E_{\mathrm{T}}^{\text {miss }}=$ $1500 \mathrm{GeV}$. A distinct feature of the $Z^{\prime}-2 \mathrm{HDM}$ model is the events from low $m_{Z^{\prime}}$ points mostly fall in the Resolved topology, while the high $m_{Z^{\prime}}$ points dominate the Merged topology. 


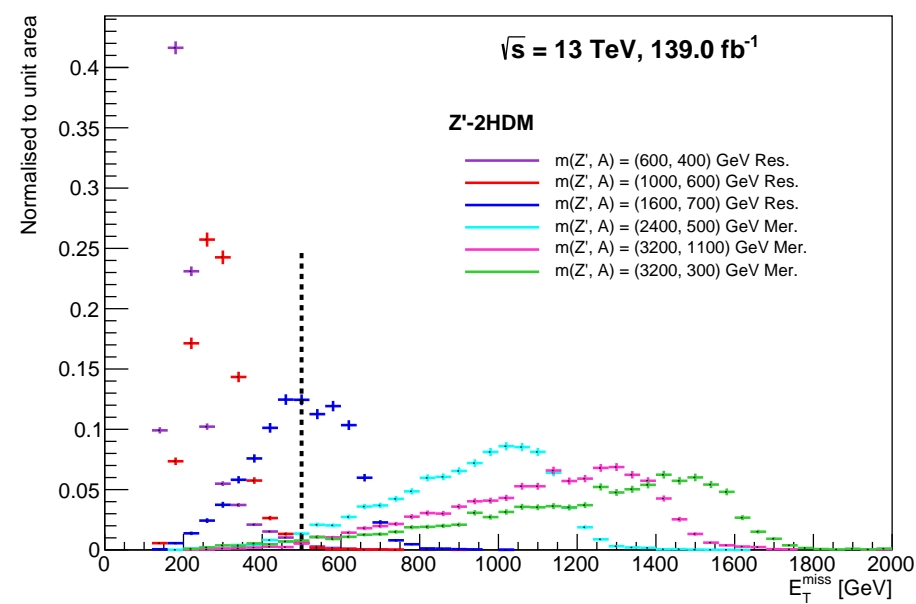

Figure 61: $E_{\mathrm{T}}^{\text {miss }}$ distribution for the $\mathrm{Z}^{\prime}-2 \mathrm{HDM}$ model for various mass points. The Res (Mer) label indicates that the corresponding point has Resolved (Merged) selections applied. For the Resolved and Merged selections the $E_{\mathrm{T}}^{\text {miss }}<500 \mathrm{GeV}$ and $E_{\mathrm{T}}^{\text {miss }}>500 \mathrm{GeV}$ cuts are removed respectively to obtain the full $E_{\mathrm{T}}^{\text {miss }}$ spectra. A requirement of $\geq 2 b$-jets is applied in both selections. The black dashed vertical line represents the $E_{\mathrm{T}}^{\mathrm{miss}}=500 \mathrm{GeV}$ point.

\subsubsection{SM backgrounds}

This section describes the $\mathrm{SM}$ processes that can have $E_{\mathrm{T}}^{\mathrm{miss}}+b \bar{b}$ final state and are backgrounds to the mono- $h(b \bar{b})$ analysis. The leading SM backgrounds in the analysis are $t \bar{t}, Z+$ jets, and $W^{ \pm}+$jets. To estimate these backgrounds CRs are formed: (i) 1-lepton CR for $t \bar{t}$ and $W^{ \pm}+$ jets, (ii) 2-lepton $\mathrm{CR}$ for $Z+$ jets. Other important background processes are Diboson, Single-top, SM $V h(b \bar{b})$ productions. Another source of SM background is the $t \bar{t}$ production in association with a SM Higgs boson $(t \bar{t}+h)$, or $W^{ \pm} / Z$ bosons $(t \bar{t}+V)$. Due to small cross sections, these processes have very little contributions to the total backgrounds.

\section{- Top-pair production $(t \bar{t})$}

The top-pair production $(t \bar{t})$ is one of the major SM backgrounds in the mono- $h(b \bar{b})$ analysis in both Resolved and Merged topologies. The $t \bar{t}$ process (c.f. Figure 62a) has three possible decay modes: (i) all-hadronic where $t \bar{t}$ decays fully hadronically $\left(t \bar{t} \rightarrow b W^{+} \bar{b} W^{-} \rightarrow b q \overline{q^{\prime}} \bar{b} q \overline{q^{\prime}}\right)$, (ii) non-all hadronic where $t \bar{t}$ decays semi-leptonically $\left(t \bar{t} \rightarrow b W^{+} \bar{b} W^{-} \rightarrow b \ell^{+} v_{\ell} \bar{b} q \bar{q}\right)$, and (iii) non-all hadronic where $t \bar{t}$ decays leptonically $\left(t \bar{t} \rightarrow b W^{+} \bar{b} W^{-} \rightarrow b \ell^{+} v_{\ell} \bar{b} \ell^{-} \bar{v}_{\ell}\right)$. Since the all- 
hadronic $t \bar{t}$ processes have low $E_{\mathrm{T}}^{\text {miss }}$, these events do not pass the mono- $h(b \bar{b})$ selection criterion of $E_{\mathrm{T}}^{\text {miss }}>150 \mathrm{GeV}$ and is not a part of the analysis. Events from non-all hadronic processes can produce $E_{\mathrm{T}}^{\text {miss }}+b \bar{b}$ final state, where the top-quark decays to a $b$-quark and a $W^{ \pm}$boson, and $E_{\mathrm{T}}^{\text {miss }}$ comes from the misidentification of the lepton produced from the $W^{+} \rightarrow \ell^{+} v_{\ell}$ decay. In principle with fully efficient object reconstruction and analysis techniques the $t \bar{t}$ events can be completely removed from the analysis, hence these types of backgrounds are known as reducible backgrounds. However, this is not the case in an actual analysis and a dedicated 1-lepton CR is designed to estimate the $t \bar{t}$ background. Figure 62a shows the semi-leptonic $t \bar{t}$ decay process.

\section{- $\underline{Z+\text { jets }}$}

The $Z+$ jets are also one of the dominant SM backgrounds, and as shown in Figure 62b, it can produce $E_{\mathrm{T}}^{\text {miss }}+b \bar{b}$ final state where $b$-quarks are produced from gluon splittings, and the $E_{\mathrm{T}}^{\text {miss }}$ comes from the $Z$-boson decaying to neutrinos $Z \rightarrow v \bar{v}$. This type of background is also known as the irreducible background. There are other $Z+$ jets events where the jets could be $c$ or lightflavored. The jet flavors can also be misidentified, i.e., a $c$ or light-flavored jet can get misidentified as $b$-jet. Since the Higgs boson in this analysis is reconstructed from the leading two $b$-jets, the $c$ or light-flavored jets can also be a part of the Higgs reconstruction due to misidentification. Depending on the jet flavors, the $Z+$ jets events are categorized as: $Z+\mathrm{HF}^{1}$ where the flavors of the leading two jets are: $b b, b c, b l$, or $c c$, and $Z+\mathrm{LF}^{1}$ where the flavors of the leading two jets are: $c l$ or $l l$. Since the $Z$-boson kinematics does not depend on its decay products, the $Z+$ jets backgrounds are estimated with $Z \rightarrow \ell \bar{\ell}+$ jets events. For this reason, two 2-lepton CRs are formed where the leptons are either two electrons or two muons of opposite flavors $\left(e^{+} e^{-}\right.$or $\left.\mu^{+} \mu^{-}\right)$.

\section{- $W+$ jets}

As shown in Figure 62c, the $W^{ \pm}+$jets have similar production mechanisms as the $Z+$ jets. It can produce the $E_{\mathrm{T}}^{\text {miss }}+b \bar{b}$ final state where the $b$-quarks are produced via gluon splitting, and the $E_{\mathrm{T}}^{\text {miss }}$ comes from the misidentified leptons from the $W^{ \pm}$-boson decay. Similar to the $Z+$ jets, the $W^{ \pm}+$jets also contribute to the Higgs reconstruction due to the jet flavor misidentification.

\footnotetext{
${ }^{1} \mathrm{HF}$ : heavy flavor, LF: light flavor
} 
The $W^{ \pm}+$jets are also split into $W+\mathrm{HF}$ and $W+$ LF components. However, due to the $E_{\mathrm{T}}^{\text {miss }}$ $>150 \mathrm{GeV}$ cut, the contributions from $W^{ \pm}+$jets backgrounds are much less than the $t \bar{t}$ or $Z+$ jets backgrounds. The $W^{ \pm}+$jets backgrounds are estimated using the 1-lepton CR similar to $t \bar{t}$.

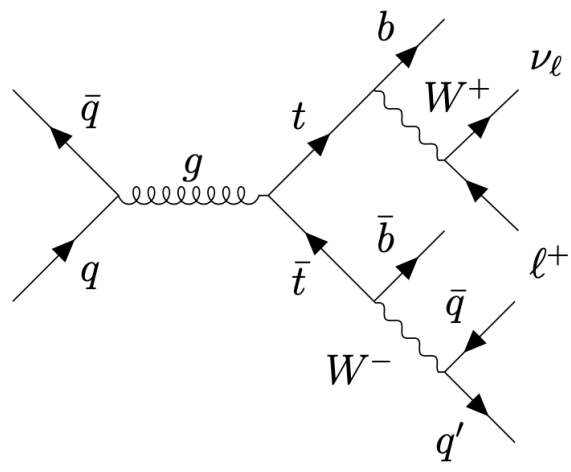

(a) Semi-leptonic $t \bar{t}$ decay

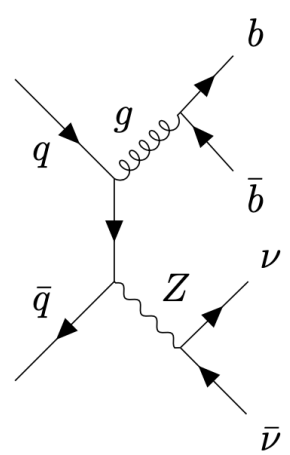

(b) $Z+$ jets

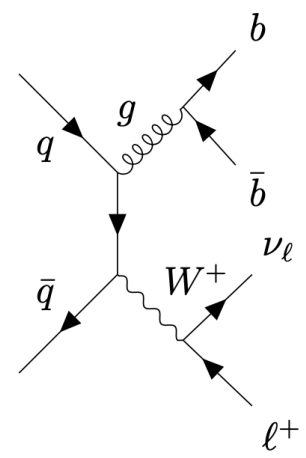

(c) $W+$ jets

Figure 62: Representative Feynman diagrams of the leading SM background processes for mono$h(b \bar{b})$ analysis. Figure 62a shows the semi-leptonic $t \bar{t}$ decay, while Figures $62 \mathrm{~b}$ and $62 \mathrm{c}$ show the $Z+$ jets and $W+$ jets processes respectively.

\section{- Single top}

The single-top process is a part of the sub-leading SM backgrounds in the analysis with three possible production modes: (i) $t$-channel, (ii) $s$-channel, and (iii) $W t$-channel. Among the three modes, the $W t$-channel has the highest contribution to the total backgrounds. The singletop events can produce $E_{\mathrm{T}}^{\text {miss }}+b \bar{b}$ signature where the leptons from $t \rightarrow W b \rightarrow v \ell b$ decay get misidentified . Figure 63a shows the production and decay of single-top $W t$-channel.

\section{- Diboson}

The diboson process has three production modes: (i) $W W$, (ii) $W Z$, and (iii) $Z Z$ and is one of the sub-leading SM backgrounds in the analysis. Figure $63 \mathrm{~b}$ shows the production and decay of the $Z Z$ process. One of the $Z$-bosons can decay to two neutrinos $(Z \rightarrow v \bar{v})$, while the other can decay hadronically to two $b$-quarks $(Z \rightarrow b \bar{b})$ and produce the $E_{\mathrm{T}}^{\text {miss }}+b \bar{b}$ final state.

- $\mathbf{S M} V h(b \bar{b})$

Figure 63c represents the Feynman diagram of a SM Higgs boson production along with a $Z$-boson. The SM Higgs boson decays to two $b$-quarks and the $Z$-boson decays to two neutrinos, producing a final state of $E_{\mathrm{T}}^{\text {miss }}+b \bar{b}$. This process is known as the SM $V h(b \bar{b})$ process and is one 
of the SM backgrounds in the analysis.

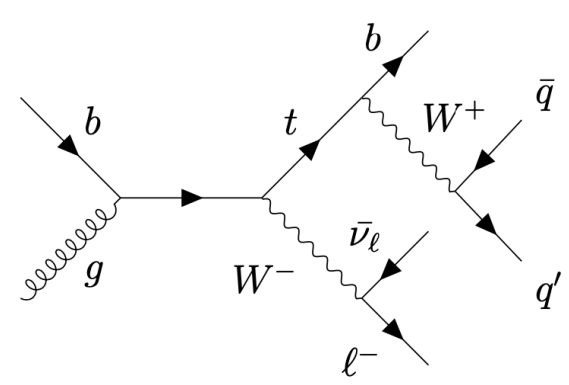

(a) Single-top $W t$-channel

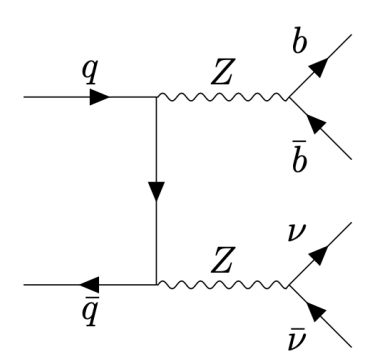

(b) Diboson

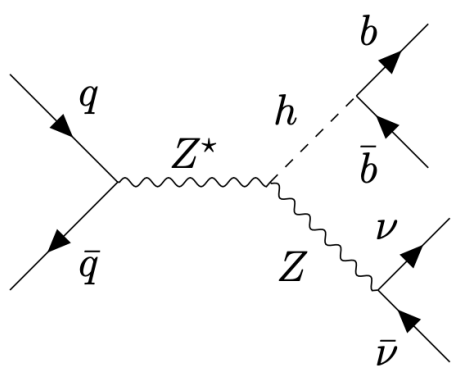

(c) $\operatorname{SM} V h(b \bar{b})$

Figure 63: Representative Feynman diagrams of the sub-leading SM background processes for mono- $h(b \bar{b})$ analysis. Figure $63 \mathrm{a}$ shows the single-top $W t$-channel diagram, Figures $63 \mathrm{~b}$ and $63 \mathrm{c}$ show the Diboson and SM $V h(b b)$ processes respectively.

- $\underline{t \bar{t}+W / Z / h}$

Another set of SM background to the analysis is the top-pair production along with a SM Higgs boson or with $W^{ \pm} / Z$-boson. Figure 64 shows the Feynman diagram of the $t \bar{t}+h$ process. The SM Higgs boson can decay to two $b$-quarks and from the semi-leptonic top decay one can get additional $b$-jets and leptons. Similar to $t \bar{t}$ process, these leptons can be misidentified and produce a final state of $E_{\mathrm{T}}^{\mathrm{miss}}+b \bar{b}$. Since these processes have at least four $b$-quarks, they are more dominant in the $\geq 3 b$-tag regions.

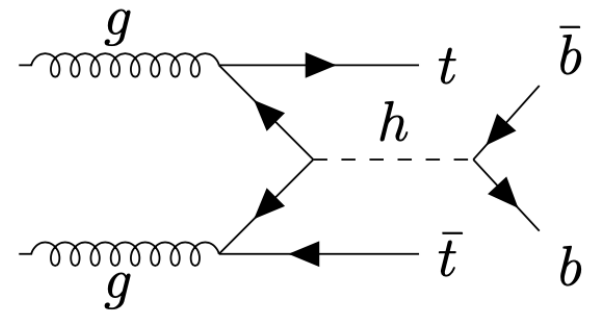

Figure 64: Representative Feynman diagram of the top-pair production along with a SM Higgs boson decaying to two $b$-quarks.

\section{- Multijet}

At the LHC, when proton bunches collide, quarks and gluons produce multiple QCD jets. This is known as multijet process and can be a source of background to the analysis, e.g., $t \rightarrow$ $b W^{+} \rightarrow b v \ell^{+}$. Typically the multijet backgrounds have lower $E_{\mathrm{T}}^{\text {miss }}$ and are mostly eliminated by 
the high $E_{\mathrm{T}}^{\text {miss }}>150 \mathrm{GeV}$ selection applied in the analysis. To further suppress the multijet backgrounds a selection on the object-based $E_{\mathrm{T}}^{\text {miss }}$ significance $S>12$, and angular separation between jet and $E_{\mathrm{T}}^{\mathrm{miss}} \min _{j \in\{1,2,3\}} \Delta \phi\left(\right.$ jet,$\left.E_{\mathrm{T}}^{\mathrm{miss}}\right)>20^{\circ}$ is applied (c.f. section 5.1.7). After these selections the multijet backgrounds in the SR were found to be significantly smaller than the expected signal uncertainty of the data. Hence, the impact of the multijet backgrounds is not a part of the final background estimation.

\subsubsection{Monte Carlo simulation}

\section{- 2HDM+a signal}

The MC simulation events for the $2 \mathrm{HDM}+a$ signal model corresponding to the physics process $p p \rightarrow h A / a(\chi \bar{\chi})$ (c.f. Figure 17) are produced with MADGRAPH5_AMC@ NLO v2.6.7 [167], based on the UFO model [58] at leading-order (LO) accuracy using NNPDF3.0NLO PDF set with $\alpha_{s}=0.118$ [168]. The parton shower and hadronization are performed with PYTHIA8.244 [169] with NNPDF2.3LO PDF [170] set, and the tuned parameters are chosen from the A14 (ATLAS 2014) set [171]. The decay of Higgs boson to $b \bar{b}$ is modelled with PyTHIA8.230, where the BR $(h \rightarrow b \bar{b})$ is fixed at SM prediction. The $b b$-induced samples are generated using a five-flavor scheme, while the ggF samples are generated with a four-flavor scheme (the bottom quarks are included in the loop diagrams). In the event generation a filter of $E_{\mathrm{T}}^{\mathrm{miss}}>100 \mathrm{GeV}$ is applied at particle level to increase the acceptance of the samples. The $2 \mathrm{HDM}+a$ samples are generated for different values of $m_{A}$ and $m_{a}$ with the following parameter set [58](details in section 2.4.1):

$$
\begin{gathered}
M_{A}=M_{H^{ \pm}} \text {or } M_{H}=M_{H^{ \pm}}, \quad M_{h}=125 \mathrm{GeV}, \quad m_{\chi}=10 \mathrm{GeV}, \quad g_{\chi}=1, \quad \cos (\beta-\alpha)=0 \\
\sin \theta=0.35, \quad \tan \beta=1(\mathrm{ggF}) \text { and } 10(\mathrm{bb}), \quad \lambda_{3}=\lambda_{P 1}=\lambda_{P 2}=3 .
\end{gathered}
$$

\section{- $Z^{\prime}$-2HDM signal}

The MC simulation events for the $Z^{\prime}-2 \mathrm{HDM}$ model corresponding to the physics process $p p \rightarrow Z^{\prime} \rightarrow h A(\chi \bar{\chi})$ (c.f. Figure 20) are produced in a five-flavor scheme at LO accuracy with MADGRAPH5_AMC@NLO v2.6.5 [167] using NNPDF3.0NLO PDF set with $\alpha_{s}=0.118$. The 
parton shower and hadronization is performed with PYTHIA8.240 [172] and NNPDF2.3LO PDF set, using the A14 tuned parameter set. The $h \rightarrow b \bar{b}$ decay is modelled with PYTHIA8.230, where the $\mathrm{BR}(h \rightarrow b \bar{b})$ is fixed at $\mathrm{SM}$ prediction. In the event generation a filter of $E_{\mathrm{T}}^{\text {miss }}>100 \mathrm{GeV}$ is applied at particle level to increase the acceptance of the samples. Samples with different values of $m_{Z^{\prime}}$ and $m_{A}$ are produced with the following parameter set (details in section 2.4.2):

$$
\begin{gathered}
M_{A}=M_{H^{ \pm}} \text {or } M_{H}=M_{H^{ \pm}}, \quad M_{h}=125 \mathrm{GeV}, \quad m_{\chi}=100 \mathrm{GeV} \\
\cos (\beta-\alpha)=0, \quad \tan \beta=1, \quad g_{Z^{\prime}}=0.8, \quad g_{\chi}=1 .
\end{gathered}
$$

\section{- Top-pair production $(t \bar{t})$}

The $t \bar{t}$ events are generated with PowhegBox v2 generator [173-176], which calculates the matrix elements at next-to-leading order (NLO) accuracy, using the NNPDF3.0NLO PDF set. The parton showering and hadronization are performed with PYTHIA8.230 [169], using the NNPDF2.3LO [168] PDF set, and the tuned parameters are from the A14 set. The bottom and charm hadron decays are modelled by the EVTGEN v1.6.0 generator [177]. The theoretical corrections of the inclusive NLO $t \bar{t}$ production cross section are performed at QCD next-to-next-leading order (NNLO) accuracy and include the resummation of next-to-next-leading logarithmic (NNLL) soft-gluon terms calculated by Top $++2.0[178-184]$. The cross section uncertainties due to choices of PDF set and $\alpha_{s}$ are calculated using the PDF4LHC [185] prescription with these PDF sets: MSTW2008 68\% CL NNLO [186, 187], CT10 NNLO [188, 189], and NNPDF2.3 5f FFN [190]. Uncertainties from different PDF sets are added in quadrature to the $\alpha_{s}$ uncertainty to obtain the

final cross section uncertainty. Figure 65 shows the LO QCD Feynman diagrams corresponding to the $t \bar{t}$ event production in hadron colliders. The top quark mass $\left(m_{t o p}\right)$ is set as $m_{t o p}=172.5 \mathrm{GeV}$ in all the simulation processes. In the analysis only the non-all hadronic $t \bar{t}$ samples are used which has two types:

1. $E_{\mathrm{T}}^{\text {miss }}$ inclusive $t \bar{t}$ samples.

2. $E_{\mathrm{T}}^{\mathrm{miss}}$ filtered $t \bar{t}$ samples. 
In the previous iteration of the analysis, only the $E_{\mathrm{T}}^{\text {miss }}$ inclusive $t \bar{t}$ samples were used, while in this iteration the $E_{\mathrm{T}}^{\text {miss }}$ filtered $t \bar{t}$ samples are also used. The inclusion of the $E_{\mathrm{T}}^{\text {miss }}$ filtered $t \bar{t}$ samples reduces the $\mathrm{MC}$ statistical uncertainty in high- $E_{\mathrm{T}}^{\mathrm{miss}}$ regions. Appendix $\mathrm{A}$ describes the procedure used to combine these two types of samples.
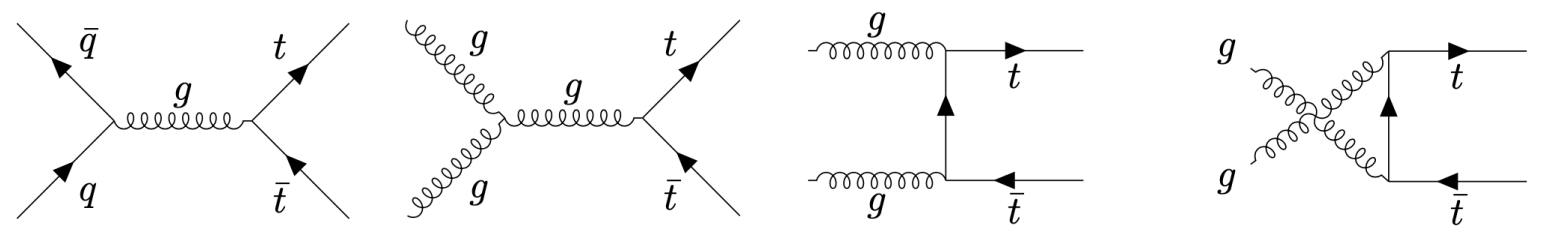

Figure 65: Leading-order QCD Feynman diagrams [191] corresponding to $t \bar{t}$ production at hadron colliders.

\section{- Single top}

The single-top $t$-channel events are produced with POWHEGBOX v2 generator [192], and the matrix elements are calculated using the NNPDF3.0NLOnf4 PDF set, at NLO accuracy in the four-flavor scheme. The top-quark decays are modelled at LO using MADSPIN [193, 194]. The inclusive production cross sections are corrected at QCD NLO accuracy using HATHOR v2.1 [195, 196].

The single-top s-channel events are produced with PowHEGBox v2 generator [197], and the matrix elements are calculated using the NNPDF3.0NLO PDF set at NLO accuracy in the fiveflavor scheme. The inclusive production cross sections are corrected at QCD NLO accuracy using HATHOR v2.1.

The single-top $W t$-channel events are produced with POWHEGBox v2 generator, and the matrix elements are calculated using the NNPDF3.0NLO PDF set at NLO accuracy in the fiveflavor scheme. The diagram removal scheme [198] $]^{2}$ is used in the $W t$ events to remove the interference with the $t \bar{t}$ events [199]. The theoretical corrections of the inclusive NLO $W t$-channel production cross sections are performed at QCD NNLO accuracy and include the resummation of NNLL soft-gluon terms.

\footnotetext{
${ }^{2}$ The NLO $W t$ diagrams are removed that are doubly-resonant $(t \bar{t})$ to handle the interference between $t \bar{t}$ and single-top $W t$ production.
} 
For all three processes the parton showering and hadronization are performed with PYTHIA 8.230, using the NNPDF2.3LO PDF set, and the tuned parameters are from the A14 series. The bottom and charm hadron decays are modelled by the EVTGEN v1.6.0 generator for all three processes. The cross section uncertainties due to choices of PDF set and $\alpha_{s}$ are calculated using the PDF4LHC prescription. The PDF sets used for the PDF uncertainty calculations are: MSTW2008 68\% CL NNLO, CT10 NNLO, and NNPDF2.3 NLO [190] PDF set for $s$ and $t$-channel, and MSTW2008 68\% CL NNLO PDF set for $W t$-channel. The PDF uncertainties are added in quadrature to the $\alpha_{s}$ uncertainty to obtain the final cross section uncertainty. The top quark mass is set at $m_{\text {top }}=172.5 \mathrm{GeV}$ in all the simulation processes. Figure 66 shows the single-top process.
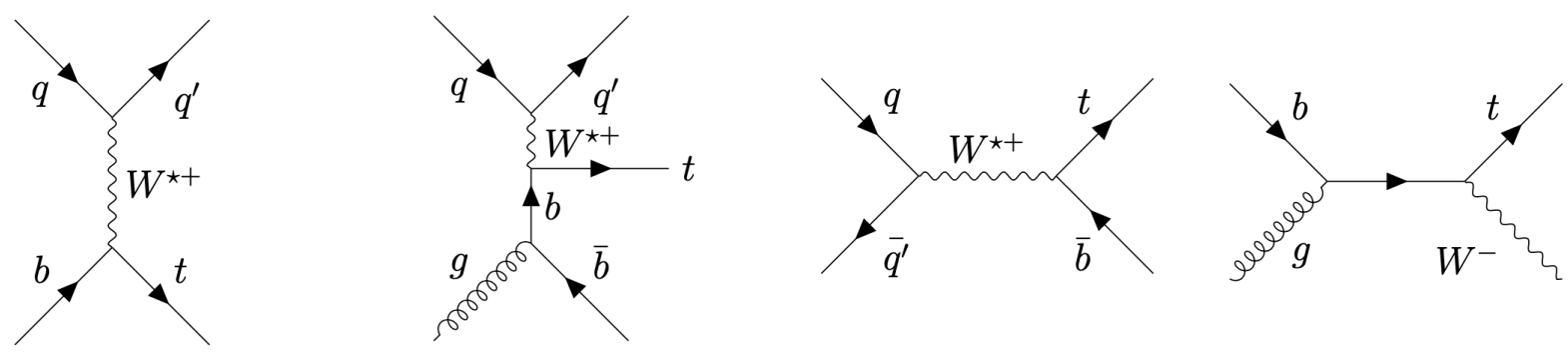

Figure 66: Leading-order QCD Feynman diagrams [191] corresponding to single top production at hadron colliders: $t$-channel as flavor excitation (left) and $W$-gluon fusion (middle), $s$-channel (third) and $W t$-channel (right) production, the $\star$ represents off-shell particles.

\section{- $V+$ jets}

The QCD $V+$ jets $\left(V: W^{ \pm} / Z\right)$ samples are produced with SHERPA v2.2.1 [200] and the matrix elements are calculated for up to two jets at NLO accuracy and up to four jets at LO accuracy with the COMIX [201] and OPENLoop [202, 203] libraries. The samples are matched with the SHERPA parton shower [204] using the MEPS@NLO prescription [205-208]. The NNPDF3.0NNLO set and the tuned parameters from SHERPA are used to generate the samples. Figure 67 shows the LO QCD Feynman diagrams corresponding to the $V+$ jets production in hadron colliders. The $V+$ jets samples are classified according to the jet flavors requirements in the MC production stage:

1. BFilter: sample contains a $B$ hadron with $p_{\mathrm{T}}>5 \mathrm{GeV}$ and $|\eta|<2.9$.

2. CFilterBVeto: sample contains a $C$ hadron with $p_{\mathrm{T}}>4 \mathrm{GeV}$ and $|\eta|<3$. 
3. CVetoBVeto: sample contains events orthogonal to the above two.

Depending on the types of filters used in the MC production, the samples are further classified as:

1. PTV: uses the $V$ boson $p_{\text {T }}$ produced by SHERPA $\left(p_{\mathrm{T}}(V)\right)$.

2. $M A X(H T, P T V)$ : uses the $\max p_{\mathrm{T}}(V), H_{T}$ where $H_{T}=\sum_{j e t s} p_{\mathrm{T}}$ for jets with $p_{\mathrm{T}}>20 \mathrm{GeV}$.

In the previous iteration of the analysis only MAX (HT, PTV) samples were used, while in this iteration both MAX (HT, PTV) and PTV filtered $Z(\rightarrow v v)+$ jets samples are used. These two types of samples are merged together and the procedure is described in appendix A.
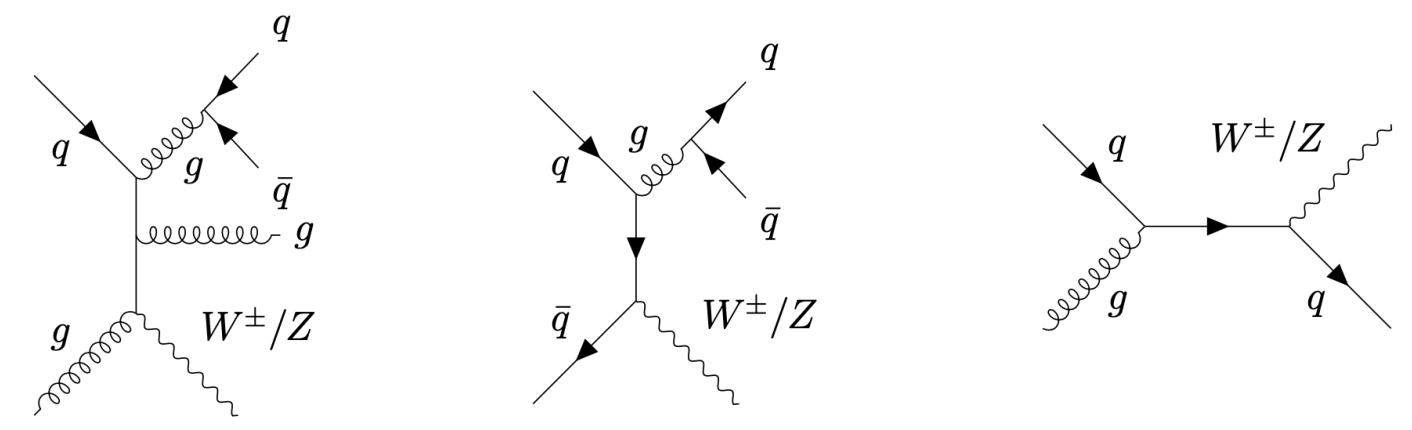

Figure 67: LO QCD Feynman diagrams corresponding to $V+$ jets production at hadron colliders.

\section{- Diboson}

There are two types of diboson samples used in the analysis: (i) $q \bar{q}$-initiated that are simulated with SHERPA v2.2.1, and (ii) $g g$-initiated that are simulated with SHERPA v2.2.2 [200]. The matrix element calculations also vary for the two types: (i) for $q \bar{q}$-initiated process, matrix elements for up to 3 additional jets at LO and up to 1 additional jet at NLO in QCD are used, and for (ii) $g g$-initiated processes, matrix elements for up to 1 additional jet at LO QCD are used. The merging and matching of matrix elements with the SHERPA parton shower [204] are performed using the MEPS@NLO prescription [205-208]. The virtual NLO QCD corrections for matrix elements are obtained from the OPENLoOPs library [202, 203]. The NNPDF3.0NNLO set [168] and tuned parameters from SHERPA are used to generate the samples. Figure 68 shows the LO QCD Feynman diagrams for the production of diboson events in hadron colliders. 
Diboson samples generated with $B$-filters $(Z \rightarrow b \bar{b})$ are also used in the analysis and are combined with the inclusive diboson samples $(Z \rightarrow q \bar{q})$, as described in appendix A.
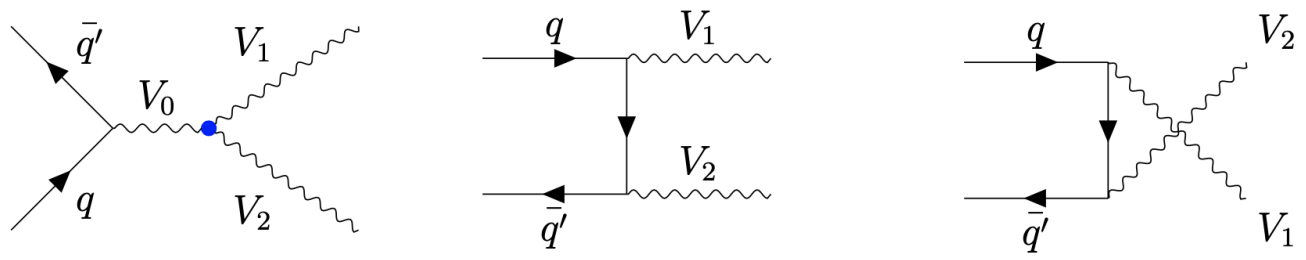

Figure 68: Leading-order QCD Feynman diagrams [209] corresponding to diboson production at hadron colliders, here $V_{i}=W^{ \pm}, Z$ or $\gamma$. The blue dot represents the Triple Gauge boson Coupling (TGC) vertex.

\section{- $\mathbf{S M} V h(b \bar{b})$}

As shown in Figure 69, the SM $V h(b \bar{b})$ process has two production mechanisms: (i) $q \bar{q}-$ induced, and (ii) $g g$ loop-induced. The $q \bar{q}$ process is modelled using PowHEGBox v2 generator, using the MINLO [210, 211] procedure with NNPDF3.0NLO PDF set, and the $g g$ process is modelled with PowHEGBox v2 generator with NNPDF3.0NLO PDF set. For both the processes, the hadronization and parton showering are performed with PYTHIA8.212 [169], using AZNLO [212] tune, and CTEQ6L1 [213] PDF set. The cross sections for $q \bar{q}$ and $g g$ are calculated at NNLO $(\mathrm{QCD})+\mathrm{NLO}(\mathrm{EW})[214-220]$ and NLO + NLL (QCD) [221-223] accuracy respectively.
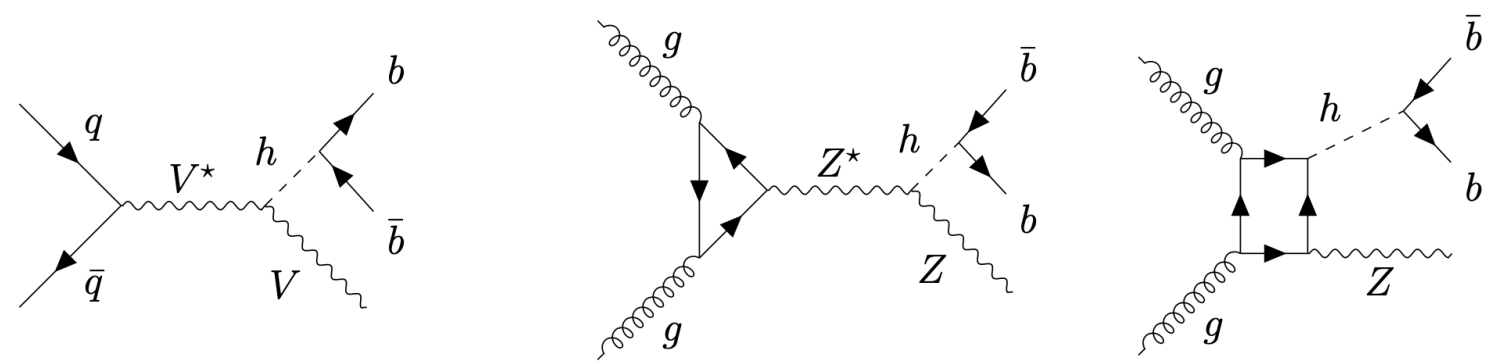

Figure 69: Leading-order QCD Feynman diagrams corresponding to SM $V h(b \bar{b})$ production at hadron colliders, here $V=W^{ \pm}$or $Z$ and $V^{\star}=W^{ \pm \star}$ or $Z^{\star}$, the $\star$ represents off-shell particles.

$$
\text { - } t \bar{t}+W / Z / h
$$

The $t \bar{t} V$ events are generated with MADGRAPH_AMC@NLO v2.3.3 [167] generator, and the matrix elements are calculated at NLO accuracy using NNPDF3.0NLO PDF set. The parton showering and hadronization are performed with PYTHIA8.210 [169] using the NNPDF2.3LO 
PDF set and the A14 tune parameter set. The top quark decays are modelled by MADSPIN at LO, and the bottom and charm hadron decays are modelled by the EVTGEN v1.2.0 generator [177].

The $t \bar{t} h$ events are generated with PowhEGBox generator, and the matrix elements are calculated at NLO accuracy with NNPDF3.0NLO PDF set. The parton showering and hadronization are performed with PYTHIA8.230, using the NNPDF2.3LO PDF set, and A14 tuned parameters.

For all three samples, the cross sections are calculated with MADGRAPH5_AMC@NLO at $\mathrm{NLO}(\mathrm{QCD})+\mathrm{NLO}(\mathrm{EW})$ accuracy [224, 225], and the top quark mass is set as $m_{\text {top }}=172.5 \mathrm{GeV}$. Figure 70 shows the LO Feynman diagrams corresponding to the $t \bar{t} V$ and $t \bar{t} h$ production.
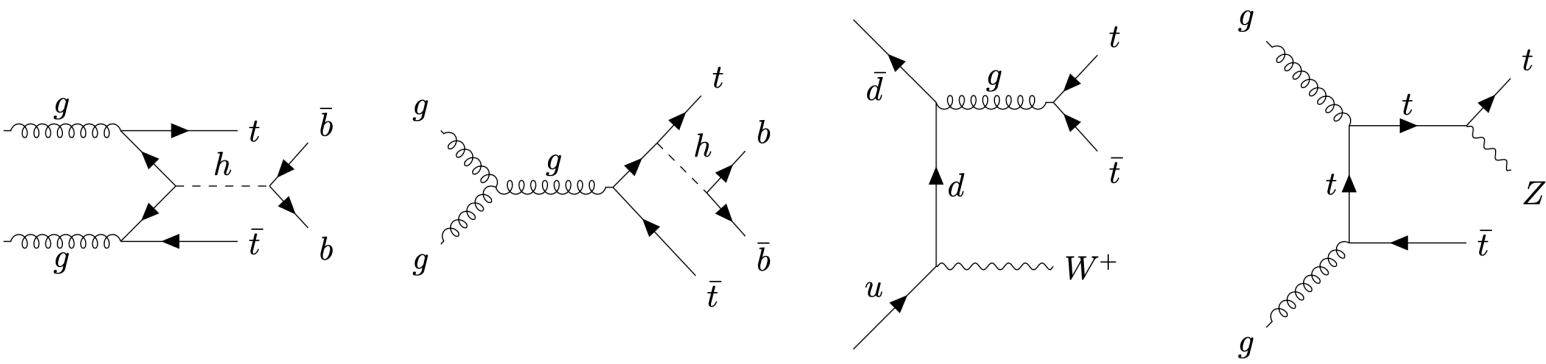

Figure 70: LO Feynman diagrams corresponding to $t$ th (left and second), $t t W$ (third) and $t t Z$ (right) production at hadron colliders.

Table 6 summarises the MC simulations of SM processes used in the analysis.

\begin{tabular}{|c|c|c|c|c|c|c|}
\hline MC sample & Generator & Matrix element order & Parton Showering & UE tune & Cross section order & PDF sets used \\
\hline$t \bar{t}$ & PowhegBox v2 & NLO & PYThia8.230 & A14 & NNLO+NNLL & $\begin{array}{c}\text { NNPDF2.3LO } \\
\text { MSTW2008 NNLO } \\
\text { CT10 NNLO } \\
\text { NNPDF2.3 5f FFN }\end{array}$ \\
\hline Single-top $t$ & PowhegBox v2 & NLO & PYTHIA 8.230 & A14 & NLO & $\begin{array}{c}\text { NNPDF2.3LO } \\
\text { MSTW2008 NNLO } \\
\text { CT10 NNLO } \\
\text { NNPDF2.3 NLO }\end{array}$ \\
\hline Single-top $s$ & PowhegBox v2 & NLO & PYTHIA8.230 & A14 & NLO & $\begin{array}{c}\text { NNPDF2.3LO } \\
\text { MSTW2008 NNLO } \\
\text { CT10 NNLO } \\
\text { NNPDF2.3 NLO }\end{array}$ \\
\hline Single-top $W t$ & PowhegBox v2 & NLO & PYTHia 8.230 & A14 & NNLO + NNLL & $\begin{array}{c}\text { NNPDF2.3LO } \\
\text { MSTW2008 NNLO }\end{array}$ \\
\hline$V+$ jets & SHERPA v2.2.1 & NLO ( $\leq 2$ jets), LO ( $\leq 4$ jets $)$ & SHERPA & SHERPA default & NLO & NNPDF3.0NNLO \\
\hline Diboson & $\begin{array}{l}q \bar{q}: \text { SHERPA v2.2.1 } \\
g g: \text { SHERPA v2 } 2.2 .2\end{array}$ & $\begin{array}{l}q \bar{q}: \mathrm{LO}(\leq 3 \text { jets }), \mathrm{NLO}(\leq 1 \text { jets }) \\
g g: \mathrm{LO}(\leq 1 \text { jets }), \mathrm{NLO}(\leq 1 \text { jets })\end{array}$ & $\begin{array}{l}\text { SHERPA } \\
\text { SHERPA }\end{array}$ & $\begin{array}{l}\text { SHERPA default } \\
\text { SHERPA default }\end{array}$ & $\begin{array}{l}\text { NLO } \\
\text { NLO }\end{array}$ & $\begin{array}{l}\text { NNPDF3.0NNLO } \\
\text { NNPDF3.0NNLO }\end{array}$ \\
\hline SM $V h(b \bar{b})$ & $\begin{array}{l}q \bar{q}: \text { PowhegBox v2 } \\
g g: \text { PowhegBox v2 }\end{array}$ & $\begin{array}{l}q \bar{q}: \text { NLO } \\
g g: \text { NLO }\end{array}$ & $\begin{array}{l}\text { PYTHIA 8.212 } \\
\text { PYTHIA 8.212 }\end{array}$ & $\begin{array}{l}\text { AZNLO } \\
\text { AZNLO }\end{array}$ & $\begin{array}{l}\text { NNLO (QCD) + NLO (EW) } \\
\text { NLO + NLL (QCD) }\end{array}$ & $\begin{array}{l}\text { CTEQ6LI } \\
\text { CTEQ6LI }\end{array}$ \\
\hline$t \bar{t}+Z$ & MADGRAPH_AMC@NLO v2.3.3 & NLO & PYTHIA 8.210 & A14 & $\mathrm{NLO}(\mathrm{QCD})+\mathrm{NLO}(\mathrm{EW})$ & NNPDF2.3LO \\
\hline$t \bar{t}+W$ & MADGRAPH_AMC@NLO v2.3.3 & NLO & PYThia8.210 & A14 & $\mathrm{NLO}(\mathrm{QCD})+\mathrm{NLO}(\mathrm{EW})$ & NNPDF2.3LO \\
\hline$t \bar{t}+h$ & PowehegBox v2.3.3 & NLO & PYTHiA8.230 & A14 & $\mathrm{NLO}(\mathrm{QCD})+\mathrm{NLO}(\mathrm{EW})$ & NNPDF2.3LO \\
\hline
\end{tabular}

Table 6: Summary of the MC simulations for the SM processes used in the analysis. Abbreviations used: UE: underlying events, LO: Leading-order, NLO: next-to-leading order, NNLO: nextto-next-to leading order, NNLL: next-to-next-to-leading logarithmic. 


\subsubsection{Triggers}

In the mono- $h(b \bar{b})$ analysis there are two types of triggers used to select the events: (i) unprescaled $E_{\mathrm{T}}^{\text {miss }}$ trigger [226] in the 0 and 1-lepton channels, and (ii) unprescaled single-lepton triggers, i.e., at least one electron [132] or muon [227], in the 2-lepton channels.

\section{- Unprescaled $E_{\mathrm{T}}^{\text {miss }}$ triggers}

In the 0-lepton $\mathrm{SR}$, unprescaled $E_{\mathrm{T}}^{\text {miss }}$ trigger is used to select events with a considerable amount of $E_{\mathrm{T}}^{\mathrm{miss}}$. Unprescaled triggers record all the events passing a specific $p_{\mathrm{T}}$ or $E_{\mathrm{T}}^{\text {miss }}$ threshold, as opposed to prescaled triggers which record a certain fraction of events to reduce the event recording rate. Since BSM events are likely to be rare, unprescaled triggers are used in this search. The ATLAS $E_{\mathrm{T}}^{\text {miss }}$ triggers are based on the calorimeter information only, and since muons are MIPs, they act as invisible particles in $E_{\mathrm{T}}^{\text {miss }}$ trigger calculations. Hence, these $E_{\mathrm{T}}^{\text {miss }}$ triggers can also select events containing muons, such as muons in the final states of $t \bar{t}$ and $W^{ \pm}+$jets decays. Hence, these are also used to select the events in the 1- $\mu$ CR. Table 7 summarises the unprescaled $E_{\mathrm{T}}^{\text {miss }}$ triggers used in the 0-lepton SR and 1- $\mu \mathrm{CR}$ for different data-taking periods.

\begin{tabular}{ccc}
\hline & Unprescaled $E_{\mathrm{T}}^{\text {miss }}$ triggers in 0 and 1-lepton \\
\hline Period & Integrated luminosity & Trigger \\
\hline 2015 & $3.2 \mathrm{fb}^{-1}$ & HLT_XE70_MHT_L1XE50 \\
$2016($ A-D3) & $6.1 \mathrm{fb}^{-1}$ & HLT_XE90_MHT_L1XE50 \\
$2016(\mathrm{D} 4-)$ & $26.9 \mathrm{fb}^{-1}$ & HLT_XE110_MHT_L1XE50 \\
$2017($ B-D5) & $12.8 \mathrm{fb}^{-1}$ & HLT_XE110_PUFIT_L1XE55 \\
$2017($ D6-) & $31.6 \mathrm{fb}^{-1}$ & HLT_XE110_PUFIT_L1XE50 \\
2018 & $58.5 \mathrm{fb}^{-1}$ & HLT_XE110_PUFIT_70_L1XE55 \\
\hline
\end{tabular}

Table 7: Unprescaled $E_{\mathrm{T}}^{\text {miss }}$ triggers used in the analysis corresponding to different data taking periods (sub-periods) and integrated luminosity.

The ATLAS $E_{\mathrm{T}}^{\text {miss }}$ trigger menu evolves with different data-taking periods to cope with the increasing luminosity and pile-up effects. The different $E_{\mathrm{T}}^{\text {miss }}$ trigger algorithms, and L1, HLT thresholds are included in the trigger names. For e.g., HLT_XE70_MHT_L1XE50, here 
HLT_XE70 represents a HLT $E_{\mathrm{T}}^{\text {miss }}$ (XE) threshold of $70 \mathrm{GeV}$, MHT represents the HLT trigger algorithm, and L1XE50 represents the $\mathrm{L} 1 E_{\mathrm{T}}^{\text {miss }}$ threshold of $50 \mathrm{GeV}$. The triggers used in 2017 also had an additional requirement of cell $E_{\mathrm{T}}^{\text {miss }}>50 \mathrm{GeV}$, which is not specified in the name. The $E_{\mathrm{T}}^{\text {miss }}$ triggers used in the analysis are based on three different algorithms briefly described below, more detailed descriptions can be found in [228].

- Calorimeter cell algorithm (cell): This algorithm uses the measured energies in each LAr and Tile calorimeter cell which passes the required quality criterion. The final $E_{\mathrm{T}}^{\text {miss }}$ is calculated from the vector sum of the $p_{\mathrm{T}}$ deposited in the cells, $E_{\mathrm{T}}^{\text {miss }}=\left|\vec{p}_{\mathrm{T}}^{\text {miss }}\right|$.

- Jet based algorithm (mht): This algorithm calculates the $E_{\mathrm{T}}^{\text {miss }}$ from the negative vector sum of the $p_{\mathrm{T}}$ of all the jets in an event. The jets are reconstructed using the calorimeter topoclusters, and using the anti- $K_{T}$ algorithm with a radius parameter of $R=0.4$ and $R=1.0$. These jet energies are also corrected for the pile-up effects. The name $m h t$ comes from Missing $H_{T}$, where the $H_{T}$ represents the scalar sum of all the jet $p_{\mathrm{T}}$ in an event.

- Pile-up fit algorithm (pufit): In the pufit algorithm, the $E_{\mathrm{T}}^{\text {miss }}$ is calculated by taking the negative vector sum of the $p_{\mathrm{T}}$ of all the calorimeter topological clusters corrected for pile-up. The clusters are grouped into towers and a tower is marked as pile-up if the $E_{\mathrm{T}}$, calculated from that tower, falls below a certain pile-up dependent threshold. A fit to the below-threshold towers is performed to estimate the $E_{\mathrm{T}}$ contribution from the pile-up. The pile-up contribution is subtracted from the $E_{\mathrm{T}}$ to obtain the corrected $E_{\mathrm{T}}$, which is used to calculate the final $E_{\mathrm{T}}^{\text {miss }}$ values.

\section{- Unprescaled single-electron triggers}

The unprescaled single-electron triggers, listed in Table 8 , are used to select $Z \rightarrow e^{+} e^{-}$ events in the 2-lepton $\mathrm{CR}\left(e^{+} e^{-}\right)$. The single-electron triggers select at least one electron with a certain $p_{\mathrm{T}}$ threshold, hence, these triggers can be used to select di-electron events. The singleelectron triggers have a nominal $E_{\mathrm{T}}$ threshold which differs between different data-taking periods, e.g., $E_{\mathrm{T}}>20 \mathrm{GeV}$ in 2015 and $E_{\mathrm{T}}>22 \mathrm{GeV}$ for 2016-2018. These $E_{\mathrm{T}}$ thresholds are $\eta$-dependent and can have variations between -2 to $+3 \mathrm{GeV}$ relative to the nominal value. The naming convention of the single-electron triggers is: [Trigger level]_[object multiplicity]_[object type] [minimum 
$E_{\mathrm{T}}$ value in $\mathrm{GeV}$ ] followed by other strings to address specific characteristics. As an example, the trigger HLT_E26_LHTIGHT_NOD0_IVARLOOSE requires the electron to pass the HLT threshold of $E_{\mathrm{T}}>26 \mathrm{GeV}$ and the tight $\mathrm{LH}$ (likelihood) identification criterion. Further, nod0 represents that there are no requirements on the transverse impact parameter $(\mathrm{d} 0)$, and ivarloose represents that the electron has to pass a loose isolation criterion. There are some triggers with high $E_{\mathrm{T}}$ thresholds such as HLT_E140_LHLOOSE_NOD0 where $E_{\mathrm{T}}>140 \mathrm{GeV}$. These triggers are used to select highly energetic electrons. More details about these triggers can be found in [132].

\begin{tabular}{|c|c|c|}
\hline Period & Single-electron triggers & Single-muon triggers \\
\hline 2015 & $\begin{array}{c}\text { HLT_E24_LHMEDIUM_L1EM20VH } \\
\text { OR HLT_E60_LHMEDIUM } \\
\text { OR HLT_E120_LHLOOSE }\end{array}$ & $\begin{array}{c}\text { HLT_MU20_ILOOSE_L1MU15 } \\
\text { OR HLT_MU50 }\end{array}$ \\
\hline $2016(\mathrm{~A})$ & $\begin{array}{l}\text { HLT_E60_LHMEDIUM_NOD0 } \\
\text { OR HLT_E140_LHLOOSE_NOD0 }\end{array}$ & $\begin{array}{c}\text { HLT_MU40 } \\
\text { OR HLT_MU50 }\end{array}$ \\
\hline $2016(\mathrm{~B}-\mathrm{D} 3)$ & $\begin{array}{l}\text { HLT_E60_LHMEDIUM_NOD0 } \\
\text { OR HLT_E140_LHLOOSE_NOD0 }\end{array}$ & $\begin{array}{c}\text { HLT_MU24_IVARMEDIUM } \\
\text { OR HLT_MU50 }\end{array}$ \\
\hline 2016 (D4-) & $\begin{array}{c}\text { HLT_E26_LHTIGHT_NOD0_IVARLOOSE } \\
\text { OR HLT_E60_LHMEDIUM_NOD0 } \\
\text { HLT_E140_LHLOOSE_NOD0 }\end{array}$ & $\begin{array}{c}\text { HLT_MU26_IVARMEDIUM } \\
\text { OR HLT_MU50 }\end{array}$ \\
\hline 2017 (B-D5) & $\begin{array}{c}\text { HLT_E26_LHTIGHT_NOD0_IVARLOOSE } \\
\text { OR HLT_E60_LHMEDIUM_NOD0 } \\
\text { OR HLT_E140_LHLOOSE_NOD0 }\end{array}$ & $\begin{array}{c}\text { HLT_MU26_IVARMEDIUM } \\
\text { OR HLT_MU50 }\end{array}$ \\
\hline 2017 (D6-) & $\begin{array}{c}\text { HLT_E26_LHTIGHT_NOD0_IVARLOOSE } \\
\text { OR HLT_E60_LHMEDIUM_NOD0 } \\
\text { OR HLT_E140_LHLOOSE_NOD0 }\end{array}$ & $\begin{array}{c}\text { HLT_MU26_IVARMEDIUM } \\
\text { OR HLT_MU50 }\end{array}$ \\
\hline 2018 & $\begin{array}{c}\text { HLT_E26_LHTIGHT_NOD0_IVARLOOSE } \\
\text { OR HLT_E60_LHMEDIUM_NOD0 } \\
\text { OR HLT_E140_LHLOOSE_NOD0 }\end{array}$ & $\begin{array}{c}\text { HLT_MU26_IVARMEDIUM } \\
\text { OR HLT_MU50 }\end{array}$ \\
\hline
\end{tabular}

Table 8: Unprescaled single-lepton triggers used in the analysis across different data-taking periods. The OR indicates that an event passing any of those specified triggers are recorded.

\section{- Unprescaled single-muon triggers}

The unprescaled single-muon triggers, listed in Table 8 , are used to select $Z \rightarrow \mu^{+} \mu^{-}$events 
in the 2-lepton $\mathrm{CR}\left(\mu^{+} \mu^{-}\right)$. The single-muon triggers select at least one muon with a certain $p_{\mathrm{T}}$ threshold, hence, these triggers are able to select di-muon events. The muon triggers used in the analysis have a L1 $p_{\mathrm{T}}$ threshold of $>20 \mathrm{GeV}$ across different data-taking periods, which are not specified in the trigger names. The exception to this is the 2015 data taking period where the L1 $p_{\mathrm{T}}$ threshold is $>15 \mathrm{GeV}$ and is represented by L1MU15. The naming convention is similar to the single-electron triggers, e.g., the trigger HLT_MU26_IVARMEDIUM requires the muon to pass a HLT threshold of $E_{\mathrm{T}}>26 \mathrm{GeV}$, and a medium isolation criterion. More details about the single-muon triggers can be found in [227].

\subsubsection{Physics object definitions}

An important part of any physics analysis at the LHC is physics object reconstruction (c.f. section 4.1) and those relevant to the analysis are described in this section.

\section{- Small-R jets}

The small-R jets have a radius parameter of $R=0.4$ and are reconstructed using the anti$k_{T}$ algorithm [137]. The algorithm uses the Particle-Flow (PFlow) jets as input which are reconstructed using the ID and calorimeter information (c.f. section 4.1.3.3). Depending on $\eta$, the smallR jets are categorized as: (i) central jets with $|\eta|<2.5$, and (ii) forward jets with $2.5<|\eta|<4.5$. To remove the impacts of pile-up jets, the central jets have to pass the jet-vertex-tagger (JVT) algorithm at a Medium working point. In the Resolved region only the central small-R jets are used to reconstruct the two jet candidates originating from the $h \rightarrow b \bar{b}$ decay, and its reconstruction criterion are summarized in Table 9.

\begin{tabular}{c|c}
\hline Element & Criterion \\
\hline Algorithm & Anti- $k_{T}$ \\
Radius parameter & 0.4 \\
Jet input constituent & PFlow \\
$p_{\mathrm{T}}$ & $>20 \mathrm{GeV}$ (central jets), $>30 \mathrm{GeV}$ (forward jets) \\
$|\eta|$ & $|\eta|<2.5$ (central jets), $2.5<|\eta|<3.5$ (forward jets) \\
JVT & Applied to central jets with $>20 \mathrm{GeV}$ at Medium working point \\
\hline
\end{tabular}

Table 9: Summary of small-R jet reconstruction criteria used in the analysis. 


\section{- Large-R jets}

The large- $\mathrm{R}$ jets are reconstructed using the anti- $k_{T}$ algorithm with a radius parameter $R=1.0$. The inputs to the algorithm are the calorimeter topo-clusters, that are calibrated to the hadronic scale with the LCW scheme [135]. To remove the pile-up jets, a jet grooming procedure (c.f. section 4.1.3.2) is applied using the trimming [141] algorithm. It removes the large-R jet constituents with $p_{\mathrm{T}}<5 \%\left(f_{\text {cut }}\right)$ of the total large- $\mathrm{R}$ jet $p_{\mathrm{T}}$, and within the distance parameter $R_{\text {trim }}=0.2$. The large- $\mathrm{R}$ jets are used to reconstruct the $h \rightarrow b \bar{b}$ decay in the Merged region and Table 10 summarises its reconstruction criterion used in the analysis. The large- $\mathrm{R}$ jet flavor contents are identified by the VR track jets and are described below.

\begin{tabular}{c|c}
\hline Element & Criterion \\
\hline Algorithm & Anti- $k_{T}$ \\
Radius parameter & 1.0 \\
Jet input constituent & LCTopo \\
Jet grooming algorithm & Trimming with subjet $p_{\mathrm{T}}$ fraction $f_{\text {cut }}=5 \%$ and $R_{\text {trim }}=0.2$ \\
$p_{\mathrm{T}}$ & $>200 \mathrm{GeV}$ \\
$|\eta|$ & $|\eta|<2$ \\
\hline
\end{tabular}

Table 10: Summary of large- $\mathrm{R}$ jet reconstruction criteria used in the analysis.

\section{- Variable-radius track jets}

The VR track jets (c.f. section 4.1.3.4) are reconstructed with the anti- $k_{T}$ algorithm, using the ID tracks as inputs. To reduce the pile-up effects, the track inputs have to pass $p_{\mathrm{T}}>0.5 \mathrm{GeV}$, and $z_{0} \sin \theta<3 \mathrm{~mm}$ selections [149]. Unlike the small-R and large-R jets, the VR track jets do not have a fixed radius, instead the jet radius has a $p_{\mathrm{T}}$ dependency,

$$
R_{V R}\left(p_{\mathrm{T}}\right)=\frac{30 \mathrm{GeV}}{p_{\mathrm{T}}}
$$

The two additional radius parameters, $R_{\min }=0.02$ and $R_{\max }=0.4$, represent a lower and upper cut on the effective track jet radius respectively [149]. The usage of VR track jets increases the efficiency of reconstructing $h \rightarrow b \bar{b}$ decays compared to the fixed radius jets. Hence, the analysis becomes more sensitive to $h \rightarrow b \bar{b}$ signal models as shown in reference [229]. To identify the large-R jet flavors, the VR track jets are matched to the initial un-groomed large-R jets via ghost- 
association $[140,230]^{3}$. Table 11 summarises the VR track jet reconstruction criteria used in the analysis.

\begin{tabular}{c|c}
\hline Element & Criterion \\
\hline Algorithm & anti- $k_{T}$ \\
Radius parameter & $p_{\mathrm{T}}$ dependent radius with $R_{\min }=0.02$ and $R_{\max }=0.4$ \\
Track quality & $p_{\mathrm{T}}>0.5 \mathrm{GeV}$ and $z_{0} \sin \theta<3 \mathrm{~mm}$ \\
\hline
\end{tabular}

Table 11: Summary of VR track jet reconstruction criteria used in the analysis.

\section{- $b$-tagging}

In the mono- $h(b \bar{b})$ analysis a neural-network based algorithm, DL1, is used to identify $b$ jets for both central small-R jets and VR track jets. The inputs to the algorithm are the PFlow small-R jets for the Resolved region, and VR track jets for the Merged region. The working point of the DL1 algorithm is set at a point that tags jets containing $B$-hadrons at $77 \%$ efficiency (c.f. Figure 45a) [151]. Depending on the number of $B$-hadrons associated with a jet, a jet is labeled as 0,1 , or $2 b$-tagged jet. Table 12 summarises the $b$-tagging criterion used in the analysis.

\begin{tabular}{c|c}
\hline Element & Criterion \\
\hline Algorithm & DL1 \\
Jet collection & AnitiKt4EMPFlow (small-R jets), AntiKtVR30Rmax4Rmin02 (VR-track jets) \\
Operating point & $b$-tagging efficiency 77\%,$c$-rejection 4.9, light-rejection 130 \\
\hline
\end{tabular}

Table 12: Summary of $b$-tagging criteria used in the analysis.

\section{- Electrons}

The mono- $h(b \bar{b})$ analysis uses the electrons reconstructed in the central region, i.e., $|\eta|<$ 2.47, with a FCLooseAndBLayer [128] working point (WP) The electron isolation WPs used in the analysis are FCLoose and FCHighPtCaloOnly [128]. To ensure the compatibility between the electrons and the primary vertices, the electron track candidates are required to pass the track to vertex association criteria listed in Table 13 along with the electron reconstruction criteria.

\footnotetext{
${ }^{3}$ For large-R jets, the jet reconstruction process treats the track-jets as 'ghosts': 4-vectors with infinitesimal $p_{\mathrm{T}}$ to keep the properties of the calorimeter-based jets unchanged. A track is associated with a jet if the ghost is contained within the jet after re-clustering.

${ }^{4}$ The FCLooseAndBLayer WP has an identification efficiency of $93 \%$ for a prompt electron with $E_{\mathrm{T}}=40 \mathrm{GeV}$. It also requires at least two hits in the pixel detector and a total of seven hits in the pixel and silicon strip detectors (SCT) combined, and at least one of these seven hits must be in the innermost pixel later.
} 


\begin{tabular}{c|cc}
\hline Element & baseline electron & signal electron \\
\hline $\begin{array}{c}\text { Identification } \\
\text { Isolation }\end{array}$ & $\begin{array}{c}\text { LooseAndBLayer } \\
\text { FCLoose }\end{array}$ & $\begin{array}{c}\text { LooseAndBLayer } \\
\text { FCLoose for } p_{\mathrm{T}}<200 \mathrm{GeV}\end{array}$ \\
& & FCHighPtCaloOnly for $p_{\mathrm{T}}>200 \mathrm{GeV}$ \\
\hline$\eta \mid$ & $|\eta|<2.47$ & $|\eta|<2.47$ \\
$p_{\mathrm{T}}$ & $p_{\mathrm{T}}>7 \mathrm{GeV}$ & $p_{\mathrm{T}}>27 \mathrm{GeV}$ \\
\hline \multirow{2}{*}{ Track to vertex association } & $\left|\Delta z_{0}^{B L} \sin \theta\right|<0.5 \mathrm{~mm}$ & $\left|\Delta z_{0}^{B L} \sin \theta\right|<0.5 \mathrm{~mm}$ \\
\hline
\end{tabular}

Table 13: Summary of electron reconstruction criteria used in the analysis. The $d_{0}$ and $z_{0}$ are the transverse and longitudinal impact parameters respectively, $d_{0}(\sigma)$ represents the significance of the transverse impact parameter and $\theta$ is the polar angle of the track momentum at reference.

\section{- Muons}

In the mono- $h(b \bar{b})$ analysis two muon identification WPs are used: Loose for baseline muons and Medium for signal muons. The muon isolation WPs used in the analysis are FCLoose, and FCTightTrackOnly for baseline and signal muons respectively. Similar to the electrons, the muons also have to pass some track to vertex association criteria to check the track-vertex compatibility. Table 14 summarises the muon reconstruction criteria used in the analysis.

\begin{tabular}{c|ccc}
\hline Element & baseline & sub-leading signal muon & leading signal muon \\
\hline $\begin{array}{c}\text { Selection working point } \\
\text { Isolation working point }\end{array}$ & $\begin{array}{c}\text { Loose } \\
\text { FCLoose }\end{array}$ & $\begin{array}{c}\text { Medium } \\
\text { FCTightTrackOnly }\end{array}$ & $\begin{array}{c}\text { Medium } \\
\text { FCTightTrackOnly }\end{array}$ \\
\hline $\begin{array}{c}p_{\mathrm{T}} \\
\eta \mid\end{array}$ & $\begin{array}{c}>\mathrm{GeV} \\
|\eta|<2.5\end{array}$ & $\begin{array}{c}\mid \eta \mathrm{GeV} \\
|\eta|<2.5\end{array}$ & $>25 \mathrm{GeV}$ \\
\hline$d_{0}$ significance cut & $\begin{array}{c}\left.d_{0} / \sigma(d) 0\right)<3 \\
z_{0} \text { cut }\end{array}$ & $\left|z_{0} \sin \theta\right|<0.5 \mathrm{~mm}$ & $\begin{array}{c}\left.d_{0} / \sigma(d) 0\right)<3 \\
\left|z_{0} \sin \theta\right|<0.5 \mathrm{~mm}\end{array}$ \\
\hline
\end{tabular}

Table 14: Summary of muon reconstruction criteria used in the analysis.

\section{- $\tau$-leptons}

The $\tau$-leptons decay hadronically with a BR of $\sim 65 \%$ and form jets. These jets are reconstructed using the ID and calorimeter information [231] with the anti- $k_{T}$ algorithm and a radius parameter $R=0.4$. The hadronic $\tau$ decays can produce either one $\left(\tau \rightarrow \pi^{ \pm}+\pi^{0}+v\right)$ or three charged pions ( $\tau \rightarrow 3 \pi^{ \pm}+\pi^{0}+v$ ). Hence, the $\tau$-jet identification criteria require either one or three tracks with $\Delta R<0.2$. In this analysis, the tau identification procedure uses a Recurrent 
Neural Network (RNN) [232] classifier that uses the clusters and tracks associated with the taus as inputs. The RNN classifier has a VeryLoose working point and the taus are required to have $|\eta|<2.5, p_{\mathrm{T}}<20 \mathrm{GeV}$. A tau veto is applied to all the signal and control regions of the analysis, and Table 15 summarises the tau reconstruction criteria used in the analysis.

\begin{tabular}{c|c}
\hline Element & Criterion \\
\hline$p_{\mathrm{T}}$ & $>20 \mathrm{GeV}$ \\
$|\eta|$ & $|\eta|<2.5$ \\
Number of tracks & 1 or 3 \\
Charge & $|Q|=1$ \\
Tau energy scale & MVA TES \\
Jet rejection & RNN-based (VeryLoose) \\
Electron rejection & BDT-based \\
Muon rejection & through overlap removal in $\Delta R<0.2$ and $p_{\mathrm{T}}>2 \mathrm{GeV}$ for non Calo-tagged muons \\
\hline
\end{tabular}

Table 15: Summary of tau reconstruction criteria used in the analysis.

\section{- Missing transverse momentum $E_{\mathrm{T}}^{\text {miss }}$}

The $E_{\mathrm{T}}^{\text {miss }}$ reconstruction in this analysis includes baseline muons, electrons while photons and $\tau$-leptons are excluded. All jets passing JVT selection are also included, while jets outside the tracking acceptance, i.e., in $|\eta|>2.5$ region, are required to have $p_{\mathrm{T}}>30 \mathrm{GeV}$. This jet $\eta$ and $p_{\mathrm{T}}$ criteria are known as the Tight $E_{\mathrm{T}}^{\text {miss }}$ working point.

\begin{tabular}{|c|c|}
\hline Element & Criterion \\
\hline Algorithm & Calorimeter-based \\
\hline Soft term & Track-based (TST) \\
\hline$E_{\mathrm{T}}^{\text {miss }}$ operating point & Tight \\
\hline
\end{tabular}

Table 16: Summary of $E_{\mathrm{T}}^{\text {miss }}$ reconstruction criteria used in the analysis.

\section{- Object-based $E_{\mathrm{T}}^{\text {miss }}$ significance $\mathcal{S}$}

Theoretically a genuine source of $E_{\mathrm{T}}^{\text {miss }}$ in a $p p$ collision event could be exotic particles such as DM or neutrinos. However, an event can have $E_{\mathrm{T}}^{\mathrm{miss}}$, from inefficiencies and resolution effects in different object reconstructions and measurements. The object-based $E_{\mathrm{T}}^{\mathrm{miss}}$ significance $\mathcal{S}$ parameter assess whether the measured $E_{\mathrm{T}}^{\text {miss }}$ is genuine and is defined as: 


$$
\mathcal{S}=\frac{\left|\overrightarrow{E_{\mathrm{T}}^{\mathrm{miss}}}\right|^{2}}{\sigma_{L}^{2}\left(1-\rho_{L T}^{2}\right)},
$$

where $\rho_{L T}$ is the correlation factor of the longitudinal $(L)$ and transverse measurements $(T)$ [233], and $\sigma_{L}^{2}$ is the longitudinal variance of $\overrightarrow{E_{\mathrm{T}}^{\text {miss }}}$ which is calculated using the resolutions of the individual objects used in $E_{\mathrm{T}}^{\text {miss }}$ calculation.

\section{- Overlap removal}

As discussed previously in section 4.1, the reconstruction of many physics objects involves common parts of the ATLAS detector. An overlap removal procedure is implemented for all physics objects used in the analysis and is summarized in Table 17 .

\begin{tabular}{c|cc}
\hline Exclude & Against & Criterion \\
\hline Electron & Electron & shared track with $p_{\mathrm{T}, 1}<p_{\mathrm{T}, 2}$ \\
Electron & Muon & shared ID track \\
Electron & Jet & $\Delta R<\min \left(0.4,0.04+10 \mathrm{GeV} / p_{\mathrm{T}}(e)\right)$ \\
\hline Muon & Electron & if its Calo-muon and shared ID track \\
Muon & Jet & $\Delta R<$ min $\left(0.4,0.04+10 \mathrm{GeV} / p_{\mathrm{T}}(\mu)\right)$ \\
\hline Tau & Electron & $\Delta R<0.2$ \\
Tau & Muon & $\Delta R<0.2$ \\
\hline Jet & Electron & $\Delta R<0.2$ \\
Jet & Muon & NumTrack $<3$ and $($ ghost-associated or $\Delta R<0.2)$ \\
Jet & Tau & $\Delta R<0.2$ \\
Fat-jet & Electron & $\Delta R<1.0$ \\
\hline
\end{tabular}

Table 17: Summary of overlap removal criteria for different physics objects used in the analysis.

\subsubsection{Event selections}

Multiple discriminating variables are used to select events with $E_{\mathrm{T}}^{\mathrm{miss}}+b \bar{b}$ final state for the SR and to construct CRs to constrain SM backgrounds. While some discriminating variables are common to both Resolved and Merged topology, some variables differ between the two topologies. This section describes the discriminating variables and event selections used in the analysis and are summarized in Table 19 , 


\subsubsection{0-lepton signal region}

\section{- Common selections:}

(i) $E_{\mathrm{T}}^{\text {miss }}$ trigger: Events in the $\mathrm{SR}$ are selected with a lowest unprescaled $E_{\mathrm{T}}^{\text {miss }}$ trigger.

(ii) $E_{\mathrm{T}}^{\text {miss }}>\mathbf{1 5 0}$ GeV: Since events with DM particles will have high $E_{\mathrm{T}}^{\text {miss }}$ values, the events in the $\mathrm{SR}$ are required to pass $E_{\mathrm{T}}^{\mathrm{miss}}>150 \mathrm{GeV}$ selection.

(iii) Lepton and tau veto: The mono- $h(b \bar{b})$ analysis targets events with $E_{\mathrm{T}}^{\mathrm{miss}}$ and $b$-jets, hence all the baseline electrons, muons, and taus are vetoed from the SR.

(iv) Extended tau veto: Since $\tau$ decays hadronically and produces jets, the $\tau$-veto is not sufficient to veto all the $\tau$-leptons. For this an additional variable, called the extended $\tau$ veto, is calculated using the small-R jets and large- $\mathrm{R}$ jets in Resolved and Merged topologies respectively. This variable reduces the $t \bar{t}$, single-top, and $W^{ \pm}+$jets backgrounds by eliminating $\tau$-leptons from decays such as $t \rightarrow W b \rightarrow \tau \nu b$. To be compatible with the $\tau$ lepton decay properties (one prong and three prong), the extended $\tau$-veto includes a selection criterion of $1 \leq N_{\text {Tracks }} \leq 4$, where $N_{\text {Tracks }}$ represents the track multiplicity in the jet cone. A further selection is applied on the angular separation between the jet and $E_{\mathrm{T}}^{\text {miss }}$ , $\Delta \phi\left(\right.$ jet,$\left.E_{\mathrm{T}}^{\mathrm{miss}}\right) \leq 22.5^{\circ}$, to ensure alignment between the $\tau$-lepton and the neutrino from $W^{+} \rightarrow \tau^{+} v$ decay.

(v) Angular separation between jet and $E_{\mathrm{T}}^{\mathrm{miss}}$ : Even with the $E_{\mathrm{T}}^{\mathrm{miss}}>150 \mathrm{GeV}$ selection, a significant number of multijet backgrounds are still present. The $E_{\mathrm{T}}^{\text {miss }}$ sources for the multijet events are the incorrect jet energy measurements and semi-leptonic hadron decays. Hence, the $E_{\mathrm{T}}^{\text {miss }}$ from a multijet event is aligned to the direction of the jet. To reduce the multijet backgrounds, the variable $\min _{j \in\{1,2,3\}} \Delta \phi\left(j e t, E_{\mathrm{T}}^{\mathrm{miss}}\right)$ is computed. The variable is calculated for three leading jets (both central and forward), and a cut on the minimum angle is applied, $\min _{j \in\{1,2,3\}} \Delta \phi\left(\right.$ jet,$\left.E_{\mathrm{T}}^{\mathrm{miss}}\right)>20^{\circ}$. 
After applying the above-mentioned common selections in both Resolved and Merged topologies, the following region specific selections are applied as well.

\section{- Resolved selections:}

(i) $E_{\mathrm{T}}^{\text {miss }}$ : The events in the Resolved topology have $150<E_{\mathrm{T}}^{\text {miss }} \leq 500 \mathrm{GeV}$ and to increase sensitivity to different signal points the Resolved topology is binned in $E_{\mathrm{T}}^{\text {miss }}: 150<E_{\mathrm{T}}^{\text {miss }}$ $\leq 200 \mathrm{GeV}, 200<E_{\mathrm{T}}^{\text {miss }} \leq 350 \mathrm{GeV}$, and $350<E_{\mathrm{T}}^{\text {miss }} \leq 500 \mathrm{GeV}$.

(ii) Jet multiplicity: The benchmark DM signal models analyzed in this thesis contain different number of $b$-jets in the final state, e.g., $2 b$-jets for the $Z^{\prime}-2 \mathrm{HDM}$ model and $\geq 3 b$-jets for $2 \mathrm{HDM}+a b b$-induced process. On the other hand, from the semi-leptonic $t \bar{t}$ decays there are $\geq 4$ jets ( 2 from top decay and 2 from hadronic $W^{ \pm}$decay). Hence, an upper limit on the number of central small-R jets reduces the $t \bar{t}$ backgrounds and increases the analysis sensitivity. For the $2 b$-tag regions a cut of $2 \leq N_{\text {small-R jets }}^{\text {central }} \leq 4$ is applied, however to ensure enough statistics a loose cut of $2 \leq N_{\text {small-R jets }}^{\text {central }} \leq 5$ is used for the $\geq 3 b$-tag regions.

(iii) $b$-jet multiplicity: In the $2 b$-tag category the number of $b$-jets are set at $N_{b-j e t s}=2$ and for $\geq 3 b$-tag category $N_{b-\text { jets }} \geq 3$. This reduces the $t \bar{t}$ backgrounds.

(iv) Higgs candidate $\boldsymbol{p}_{\mathrm{T}}$ : Since the $E_{\mathrm{T}}^{\text {miss }}$ is correlated with the Higgs- $p_{\mathrm{T}}$, the signal events would have higher Higgs- $p_{\mathrm{T}}$ than SM backgrounds. The Higgs candidate $p_{\mathrm{T}}\left(j_{1}, j_{2}\right)$, where $j_{1}$ and $j_{2}$ are the two leading central $b$-tagged small-R jets, is calculated by the sum of the $\overrightarrow{p_{T}}$ vectors of $j_{1}$ and $j_{2}$. For $150<E_{\mathrm{T}}^{\text {miss }} \leq 350 \mathrm{GeV}$, a cut of $p_{\mathrm{T}}\left(j_{1}, j_{2}\right)>100 \mathrm{GeV}$ is applied, and for $E_{\mathrm{T}}^{\mathrm{miss}} \geq 350 \mathrm{GeV}$ a more strict cut of $p_{\mathrm{T}}\left(j_{1}, j_{2}\right)>200 \mathrm{GeV}$ is applied.

(v) Top-mass proxy: Events from the semi-leptonic $t \bar{t}$ decay are the most dominant SM backgrounds in the Resolved topology. To reduce this background a selection is applied to the top mass proxy variable, defined depending on the angular separation between the $\vec{E}_{\mathrm{T}}$ miss and the $b$-jet from the top decay as:

$$
m_{\mathrm{T}}^{b, \min / \max }=\sqrt{2 p_{\mathrm{T}}^{b, \min / \max } E_{\mathrm{T}}^{\operatorname{miss}}\left(1-\cos \Delta \phi\left({\overrightarrow{p_{\mathrm{T}}}}^{b, \min / \max }, \vec{E}_{\mathrm{T}}^{\text {miss }}\right)\right)},
$$


here $p_{\mathrm{T}}{ }^{b, \min }$ and $p_{\mathrm{T}}{ }^{b, \max }$ are the $p_{\mathrm{T}}$ of the $b$-jet which are closest to (min) or furthest from (max) the $\vec{E}_{\mathrm{T}}{ }^{\text {miss }}$ respectively. For $t \bar{t}$ events the $m_{\mathrm{T}}^{b, \min / \max }$ variable would have a sharp drop near the mass of $t \bar{t}$ system, while for DM signals the values would be much higher. Hence, a cut of $m_{\mathrm{T}}^{b, \min }>170 \mathrm{GeV}$ and $m_{\mathrm{T}}^{b \text {,max }}>200 \mathrm{GeV}$ is applied across all Resolved $E_{\mathrm{T}}^{\text {miss }}$ regions.

(vi) $E_{\mathrm{T}}^{\text {miss }}$ significance $\mathcal{S}$ : To distinguish the genuine $E_{\mathrm{T}}^{\text {miss }}$ (from exotic particles and neutrinos) from the $E_{\mathrm{T}}^{\text {miss }}$ from resolution effects of different object reconstructions, and to suppress the multijet backgrounds, the events in the SR have to pass a selection of $\mathcal{S}>12$.

(vii) Higgs candidate mass: The Higgs candidate mass distribution is the discriminating variable used in the SR for the statistical interpretation. Although the signal points have peaks around $m_{j j}=125 \mathrm{GeV}$, a broader range of $50<m_{j j}<280 \mathrm{GeV}$ is used. The $50 \mathrm{GeV}$ lower bound is chosen since it is the lowest calibrated large- $R$ jet mass. The higher bound of $280 \mathrm{GeV}$ is chosen to be significantly higher than $m_{h}$ and the precise value is motivated by the $m_{h}$ binning choices in the fit. The $m_{j j}$ sidebands are used in the fit to estimate the SM backgrounds.

\section{- Merged selections:}

(i) $E_{\mathrm{T}}^{\text {miss }}$ : Events in the Merged topology have to pass a selection of $E_{\mathrm{T}}^{\text {miss }}>500 \mathrm{GeV}$.

(ii) Jet multiplicity: The Higgs candidate is reconstructed with the central leading large-R jet and a cut of $N_{\text {large-Rjets }}^{\text {central }} \geq 1$ is applied.

(iii) $b$-jet multiplicity: In $2 b$-tag regions, two associated $b$-tagged VR track jets, and for the $\geq 3$ $b$-tag regions, along with the associated $b$-tagged VR track-jets, additional non-associated $b$-tagged VR track jets are necessary to reconstruct the Higgs boson. The complete $b$-jet multiplicity requirement is, $N_{\text {associated track-jets }}^{b-\text { tagged }}=2$ and $N_{\text {non-associated track-jets }}^{b-\text { tagged }}=0$ for the $2 b$ tag region and for the $\geq 3 b$-tag region $N_{\text {associated track-jets }}^{b-\text { tagged }}=2$ and $N_{\text {non-associated track-jets }}^{b-\text { tagged }} \geq 1$.

(iv) VR track jet overlap: Since the two leading $b$-tagged VR track jets $\left(\mathrm{VR}_{1}\right.$ and $\left.\mathrm{VR}_{2}\right)$ are used to reconstruct the Higgs candidate it is important to have transparency in the $b$-tagging. 
For this reason an overlap removal is used where the VR track jets are required to have $\Delta R\left(\mathrm{VR}_{1 / 2}, \mathrm{VR}_{\mathrm{i}}\right)>\mathrm{R}_{\min , \mathrm{i}}$, which ensures that $\mathrm{VR}_{1}$ and $\mathrm{VR}_{2}$ are not concentric to other track jets. Here, $\mathrm{VR}_{i}$ represents any track jet, other than $\mathrm{VR}_{1}$ and $\mathrm{VR}_{2}$, which is associated to the large- $\mathrm{R}$ jet, and $\mathrm{R}_{\text {min }, i}$ represents the minimum radius between $\mathrm{VR}_{1}$ or $\mathrm{VR}_{2}$ and $\mathrm{VR}_{i}$.

(v) Higgs candidate mass: Similar to the Resolved topology, a broader range of $50<m_{J}<270$ $\mathrm{GeV}$ is chosen to use the $m_{J}$ sidebands in the fit for SM background estimation. A $50 \mathrm{GeV}$ lower bound is selected as it is the lowest calibrated large- $R$ jet mass. The higher bound of $270 \mathrm{GeV}$ is chosen to be significantly higher than $m_{h}$ and the precise value is motivated by the $m_{h}$ binning choices in the fit. The difference in the upper bound of $m_{h}$ between Resolved and Merged topologies is purely due to the choice of $m_{h}$ binning in the fit model.

\section{- Muon-in-jet correction:}

The $B$ hadrons inside a jet cone can decay as $b \rightarrow c W^{\star-} \rightarrow c \mu \bar{v}$ and the ATLAS jet reconstruction procedure does not consider the contributions of the muons and neutrinos to the jet $p_{\mathrm{T}}$ and energy. Consequently, $m_{h}$ includes these additional muon contributions, which reduces the $m_{h}$ resolution. Therefore, the jet four-momentum has to be corrected for the additional muon energies, and the correction is commonly known as the muon-in-jet correction. The muons chosen for this correction have to satisfy the quality criteria listed in Table 18 . The correction procedure differs between the Resolved and Merged topologies:

\begin{tabular}{c|c}
\hline Criterion & Selection \\
\hline Selection working point & Loose \\
Isolation working point & non-isolated \\
$p_{\mathrm{T}}$ & $>5 \mathrm{GeV}$ \\
$|\eta|$ & $|\eta|<2.7$ \\
$\Delta R$ in resolved (merged) & $\Delta R<0.4(0.1)$ \\
\hline
\end{tabular}

Table 18: Summary of muon reconstruction criteria used in the muon-in-jet correction.

- Resolved: The correction is applied to the two central small-R $b$-tagged jets. If a muon is within $\Delta R<0.4$ of the jet axis, the jet is corrected by adding the muon four-momentum to 
the jet four-momentum. If multiple muons are present, the correction uses the closest muon. Although, correcting with all the muons, or muons with highest $p_{\mathrm{T}}$ produces similar results.

- Merged: Correction is applied to the leading central large-R jet with the two closest muons. Figure 71 shows the comparison between $m_{h}$ with (red) and without (blue) the muon-in-jet corrections for a representative $Z^{\prime}$-2HDM signal point. The correction improves the expected exclusion limits by up to $7 \%$.

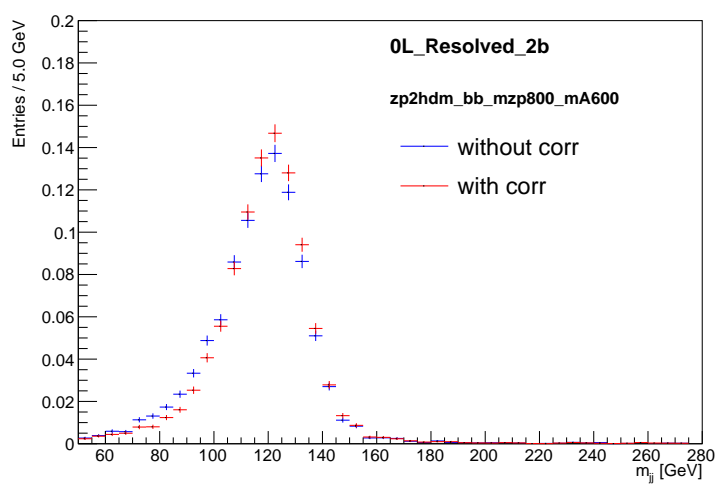

Figure 71: The $m_{h}$ distributions with (red) and without (blue) the muon-in-jet corrections for a representative $Z^{\prime}$-2HDM signal point in the 0-lepton $E_{\mathrm{T}}^{\text {miss }}$ inclusive Resolved $2 b$-tag region.

Figures $72 \mathrm{a}$ and $72 \mathrm{~b}$ show a candidate data event in the Resolved and Merged regions respectively.

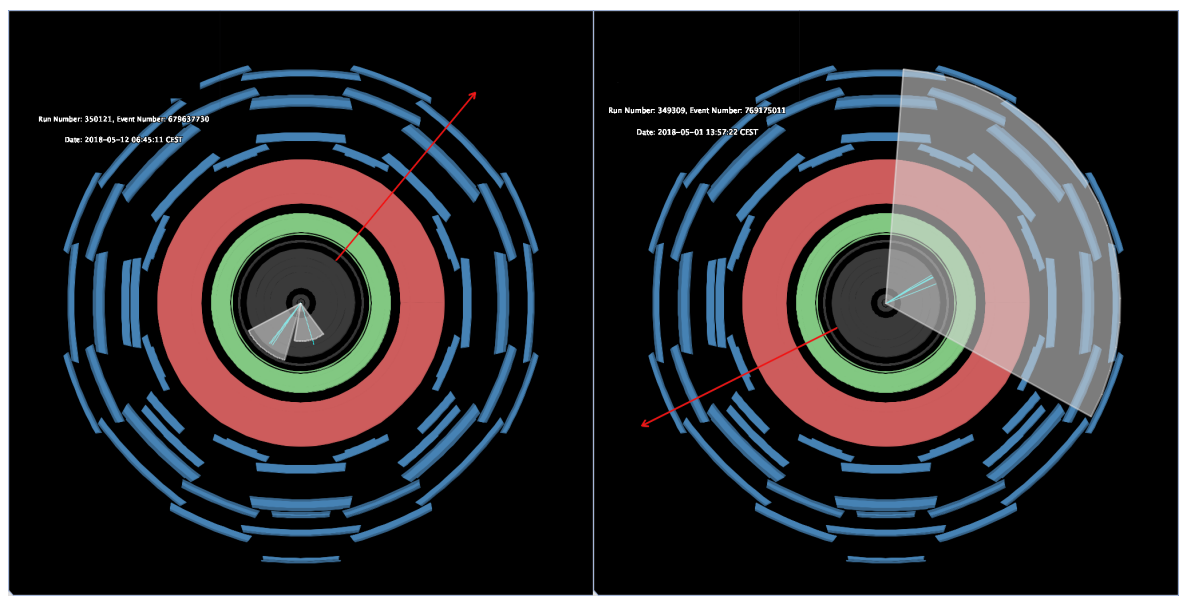

(a) Resolved

(b) Merged

Figure 72: Figures showing a candidate data event in the SR for Resolved (fig. 72a) and Merged (fig. 72b) topologies. The Resolved event contains two $b$-tagged small-R jets, represented by the white cone and the cyan lines represent the ID tracks. The Merged event contains a large-R jet (large white cone) with $2 b$-tagged VR track jets inside (cyan lines). In both figures the red line represents the $E_{\mathrm{T}}^{\text {miss }}$. 


\subsubsection{1-lepton control region}

To estimate the $t \bar{t}$ and $W^{ \pm}+$jets backgrounds in the SR, a 1-lepton CR with exactly one muon $\left(\mu^{+} / \mu^{-}\right)$is defined. In principle, the lepton could also be an electron, but using muons has some advantages. To begin with the $E_{\mathrm{T}}^{\text {miss }}$ triggers used in the SR can be used to target $1-\mu$ events. Besides, the CR phase-space must be as close as possible to the SR, yet disjoint from it. By using the $E_{\mathrm{T}}^{\text {miss }}$ trigger for both $\mathrm{SR}$ and 1-lepton $\mathrm{CR}$, the $E_{\mathrm{T}}^{\text {miss }}$ spectra from $t \bar{t}$ and $W^{ \pm}+$jets events become similar in both regions. The discriminating variables and selections used in the $1-\mu$ CR are mostly similar to the SR, but for some selections which make the two regions disjoint. Events in the $1-\mu \mathrm{CR}$ are required to have exactly one signal muon with $p_{\mathrm{T}}>25 \mathrm{GeV}$, no other baseline leptons, and the muons have to pass the quality criteria listed in Table 14 . To mimic the $E_{\mathrm{T}}^{\mathrm{miss}}$ in the $\mathrm{SR}$, the muons are considered invisible when calculating the $E_{\mathrm{T}}^{\mathrm{miss}}$ and is identified as $E_{\mathrm{T} \text {,lep.invis. }}^{\text {miss }}$ Similarly, when calculating the object-based $E_{\mathrm{T}}^{\text {miss }}$ significance $\mathcal{S}$, the muons are considered invisible, and the variable is identified as $\mathcal{S}_{\text {lep.invis. }}$ The $E_{\mathrm{T} \text {,lep.invis. }}^{\text {miss }}$ also used as inputs in the top-mass proxy $\left(m_{\mathrm{T}}^{b, \min / \max }\right)$ and object based $E_{\mathrm{T}}^{\text {miss }}$ significance $\mathcal{S}$ calculations.

\subsubsection{2-lepton control region}

To constrain the $Z(\rightarrow v \bar{v})+$ jets backgrounds in the SR, two 2-lepton CRs with exactly two opposite sign electrons $\left(e^{ \pm}\right)$or muons $\left(\mu^{ \pm}\right)$are constructed. Similar to the $1-\mu \mathrm{CR}$, most of the selections in the 2-lepton CRs are similar to the SR with some exceptions. The events are selected with unprescaled single-electron or single-muon triggers, as listed in Table 8, The events are also required to have exactly two opposite sign electrons or muons, satisfying the quality criterion listed in Tables 13 and 14 respectively. One of the two electrons (muons) must be a signal electron (muon), and the signal electron (muon) must have $p_{\mathrm{T}}>27 \mathrm{GeV}\left(p_{\mathrm{T}}>25 \mathrm{GeV}\right)$. A veto is applied on any further baseline leptons and $\tau$-leptons. The 2-lepton CRs can also have events from leptonically decaying $t \bar{t}$ events $\left(t \bar{t} \rightarrow b W^{+} \bar{b} W^{-} \rightarrow\left(b \ell^{+} v\right)\left(\bar{b} \ell^{-} \bar{v}\right)\right)$. To reduce these $t \bar{t}$ events a selection of $\left|m_{Z}-m_{\ell \ell}\right|<10 \mathrm{GeV}$ is applied, where the $m_{Z}$ and $m_{\ell \ell}$ are the masses of the $Z$-boson 
and the dilepton system respectively. Similar to the $1-\mu \mathrm{CR}$, to make the $E_{\mathrm{T}}^{\text {miss }}$ distribution similar to $\mathrm{SR}$, the leptons are considered invisible when calculating the $E_{\mathrm{T}}^{\mathrm{miss}}$ and it is labeled as $E_{\mathrm{T}, \text { lep.invis. }}^{\text {miss }}$. The $E_{\mathrm{T} \text {,lep.invis. }}^{\text {miss }}$ is also used as inputs to the top-mass proxy, and the object based $E_{\mathrm{T}}^{\text {miss }}$ significance $\mathcal{S}$ calculations. Apart from the $\mathcal{S}_{\text {lep.invis. }}>12$ cut, which reduces the multijet backgrounds, another $E_{\mathrm{T}}^{\text {miss }}$ significance selection is applied to the 2-lepton CRs to reduce the $t \bar{t}$ backgrounds. For $t \bar{t}$ events $E_{\mathrm{T}}^{\text {miss }}$ arises from the neutrinos $\left.\left(t \rightarrow b W^{+} \rightarrow\left(b \ell^{+} v\right) \ell^{-}\right)\right)$, while for $Z(\rightarrow \ell \bar{\ell})+$ jets events the $E_{\mathrm{T}}^{\text {miss }}$ mostly comes from lepton misidentification and resolution effects. Hence, a cut of $\mathcal{S}<5$ is applied, where $\mathcal{S}$ is calculated with leptons treated as visible objects, similar to the SR.

\begin{tabular}{|c|c|c|}
\hline Selection & Resolved & Merged \\
\hline Trigger & \multicolumn{2}{|c|}{$\begin{array}{l}0 \text { and 1-lepton: lowest unprescaled } E_{\mathrm{T}}^{\text {miss }} \text { triggers } \\
\text { 2-lepton: lowest unprescaled single-lepton triggers }\end{array}$} \\
\hline Lepton multiplicity & \multicolumn{2}{|c|}{$\begin{array}{l}\text { 1-lepton: exactly } 1 \text { signal muon with } p_{\mathrm{T}}>25 \mathrm{GeV} \text {, veto on other baseline leptons } \\
\text { 2-lepton: exactly } 2 \text { signal } \mu\left(p_{\mathrm{T}}>25 \mathrm{GeV}\right) \text { or } e\left(p_{\mathrm{T}}>27 \mathrm{GeV}\right) \text {, veto on other baseline leptons }\end{array}$} \\
\hline$\tau$-lepton veto & \multicolumn{2}{|c|}{ Veto on $\tau$-leptons and extended $\tau$-lepton veto } \\
\hline $\min _{j \in\{1,2,3\}} \Delta \phi\left(j e t, E_{\mathrm{T}}^{\text {miss }}\right)$ & \multicolumn{2}{|l|}{$>20^{\circ}$} \\
\hline$E_{\mathrm{T}}^{\mathrm{miss}}$ & $150<E_{\mathrm{T}}^{\text {miss }}<500 \mathrm{GeV}$ & $E_{\mathrm{T}}^{\mathrm{miss}}>500 \mathrm{GeV}$ \\
\hline Jet multiplicity & $\begin{array}{l}2 \leq N_{\text {small-R jets }}^{\text {central }} \leq 4 \text { for } N_{\text {small-R jets }}^{b-\text { tagged }}=2 \\
2 \leq N_{\text {small-R jets }}^{\text {central }} \leq 5 \text { for } N_{\text {small-R jets }}^{b-\text { tagged }} \geq 3\end{array}$ & $N_{\text {large-R jets }}^{\text {central }} \geq 1$ \\
\hline$b$-tag multiplicity & $N_{\text {small-R jets }}^{b-\text { tagged }} \geq 2$ & $\begin{array}{c}N_{\text {associated track-jets }}^{b-\text { tagged }}=2 \\
N_{\text {non- associated track-jets }}^{b-\text {-agged }} \geq 0\end{array}$ \\
\hline Higgs candidate $p_{\mathrm{T}}$ & $\begin{array}{l}p_{\mathrm{T}}\left(j_{1}, j_{2}\right)>100 \mathrm{GeV} \text { for } 150<E_{\mathrm{T}}^{\text {miss }}<350 \mathrm{GeV} \\
\quad p_{\mathrm{T}}\left(j_{1}, j_{2}\right)>300 \mathrm{GeV} \text { for } E_{\mathrm{T}}^{\text {miss }} \geq 350 \mathrm{GeV}\end{array}$ & - \\
\hline Top-mass proxy & $m_{\mathrm{T}}^{b, \min }>170 \mathrm{GeV}$ and $m_{\mathrm{T}}^{b, \max }>200 \mathrm{GeV}$ & - \\
\hline$E_{\mathrm{T}}^{\mathrm{miss}}$ significance $\mathcal{S}$ & $\mathcal{S}>12$ & - \\
\hline$E_{\mathrm{T}}^{\mathrm{miss}}$ significance $\mathcal{S}$ & $\begin{array}{c}\text { 0-lepton: } \mathcal{S}>12 \\
\text { 1-lepton: } \mathcal{S}_{\text {lep.invis. }}>12 \\
\text { 2-lepton: } \mathcal{S}_{\text {lep.invis. }}>12 \text { and } \mathcal{S}<5\end{array}$ & $\begin{array}{l}- \\
-\end{array}$ \\
\hline Higgs candidate mass & $50<m_{j j}<280 \mathrm{GeV}$ & $50<m_{J}<270 \mathrm{GeV}$ \\
\hline
\end{tabular}

Table 19: Summary of the selection criteria used in the mono- $h(b \bar{b})$ analysis [234]. Data-MC comparisons for these variables are shown in Appendix B. 


\subsubsection{Background compositions}

This section shows the compositions of various SM backgrounds in different regions of the mono- $h(b \bar{b})$ analysis phase space. The numbers represent the fractional contribution of a particular process to the total SM background. Processes with a contribution of $<3 \%$ are merged together and are shown in the "other" category. In the following pie charts the Resolved $2 b$ and $\geq 3 b$-tag categories are represented as " $2 \mathrm{~b}$ " and " $3 \mathrm{pb}$ " respectively, while the Merged $2 b$ and $\geq 3 b$-tag categories are represented as " $2 \mathrm{w} 0 \mathrm{~b}$ " and " $2 \mathrm{w} 1 \mathrm{pb}$ " respectively.

\section{- 0-lepton SR:}

Figures 73 and 74 show the SM background compositions in the 0-lepton SR for the 2 $b$-tagged and $\geq 3 b$-tagged regions respectively, across different $E_{\mathrm{T}}^{\text {miss }}$ regions. In both $2 b$-tagged and $\geq 3 b$-tagged regions, the major backgrounds are the $t \bar{t}$ and $Z / W^{ \pm}+$jets events. In the $2 b$ tagged region the $Z+\mathrm{HF}$ events are the most dominant backgrounds, while in $\geq 3 b$-tagged region the most dominant background is the $t \bar{t}$. Since the $Z+$ HF events are a part of the irreducible $Z+$ jets backgrounds, $Z+\mathrm{HF}$ has a significant contribution to the total background in the SR. 

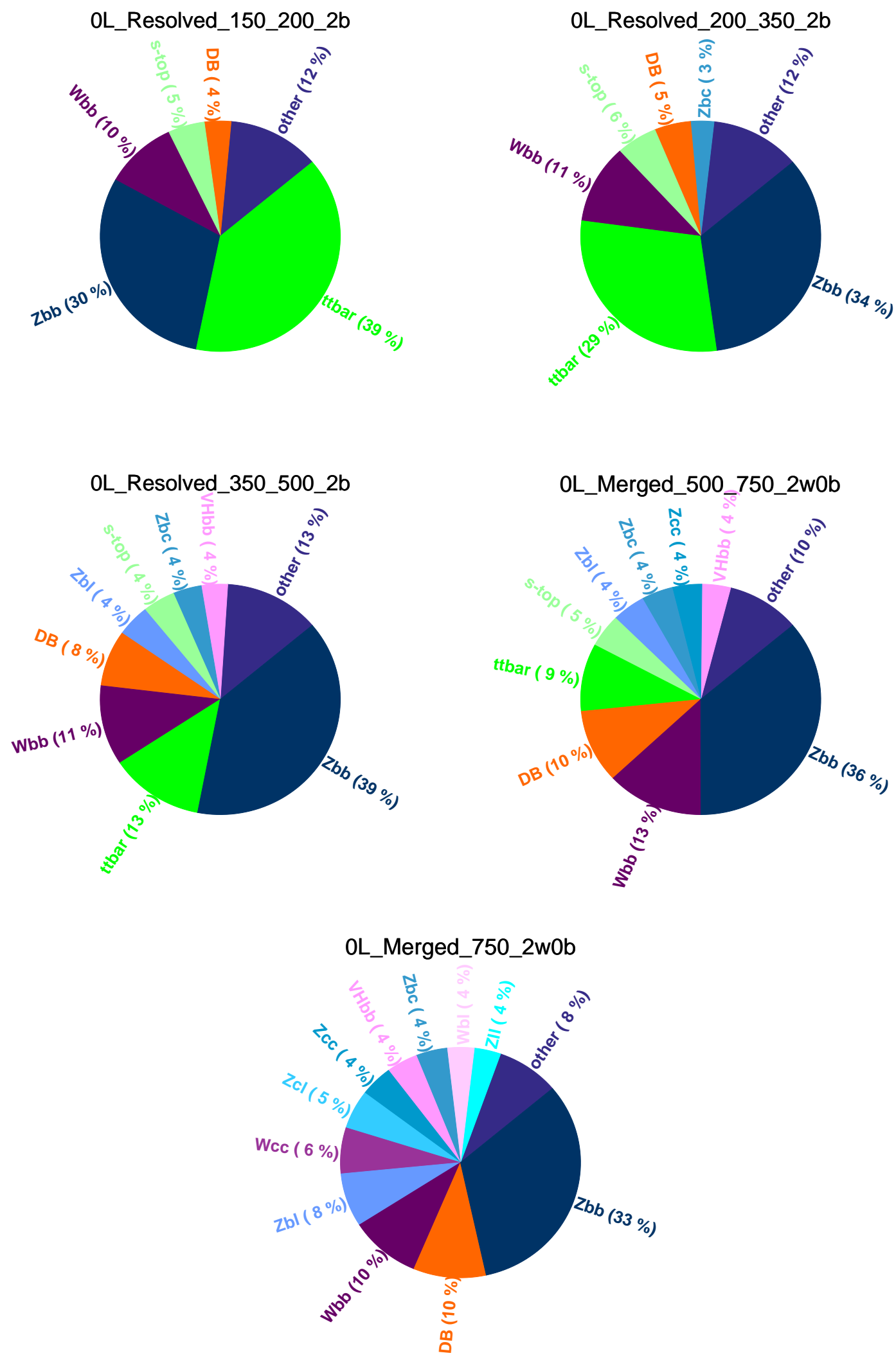

Figure 73: SM Background compositions in different $E_{\mathrm{T}}^{\text {miss }}$ bins in the 0-lepton SR for the $2 b$ tagged regions. 

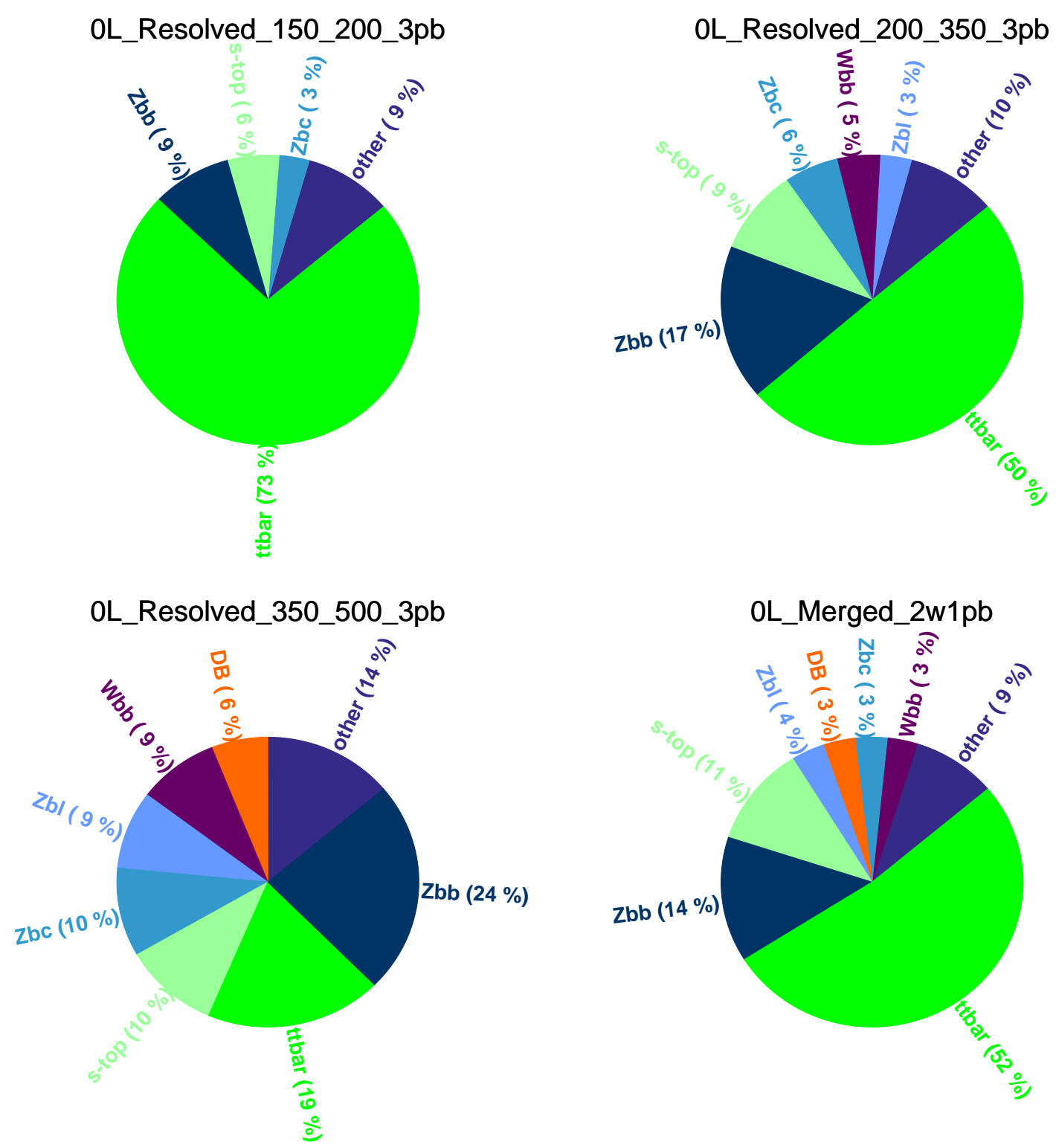

Figure 74: SM Background compositions in different $E_{\mathrm{T}}^{\mathrm{miss}}$ bins in the 0-lepton $\mathrm{SR}$ for the $\geq 3$ $b$-tagged regions. 


\section{- 1- $\mu$ CR:}

Figures 75 and 76 show the SM background compositions in the $1-\mu$ CR for the $2 b$-tagged and $\geq 3 b$-tagged regions respectively across different $E_{\mathrm{T}}^{\mathrm{miss}}$ regions. All the regions in the $1-\mu \mathrm{CR}$ are mostly pure in $t \bar{t}$ and $W^{ \pm}+$jets events, hence these backgrounds are estimated with the $1-\mu$ $\mathrm{CR}$. In the $\geq 3 b$-tagged regions the contribution from $W^{ \pm}+$jets events are negligible compared to $t \bar{t}$.
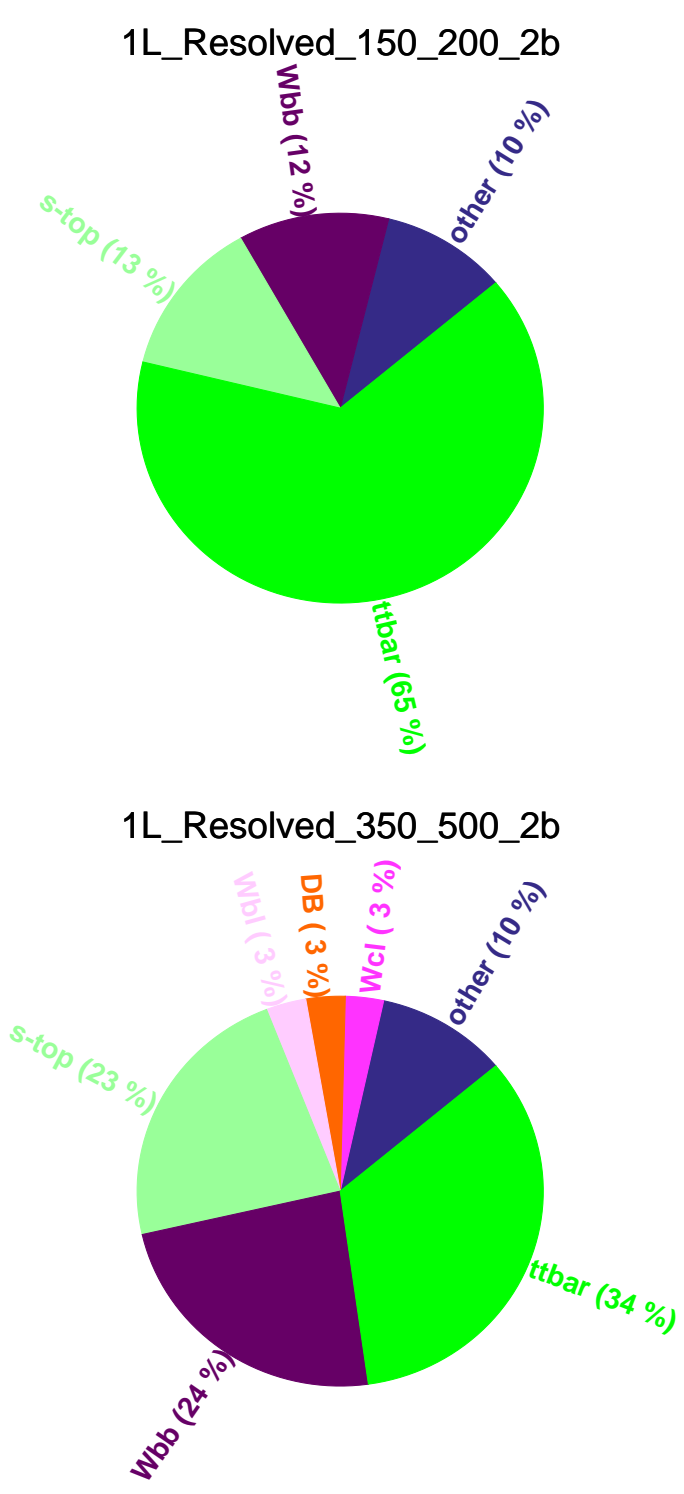
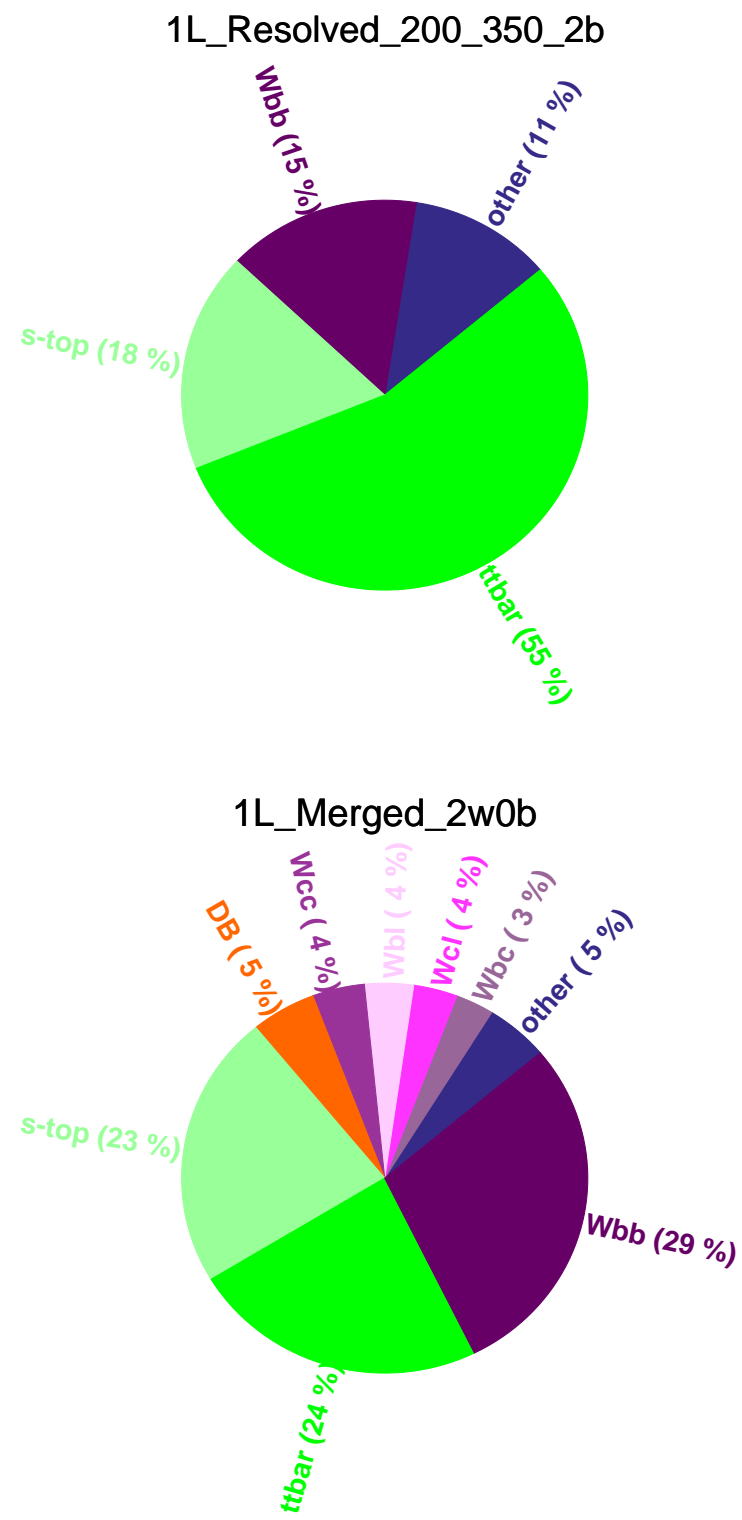

Figure 75: SM Background compositions in different $E_{\mathrm{T}}^{\mathrm{miss}}$ bins in the $1-\mu \mathrm{CR}$ for the $2 b$-tagged regions. 

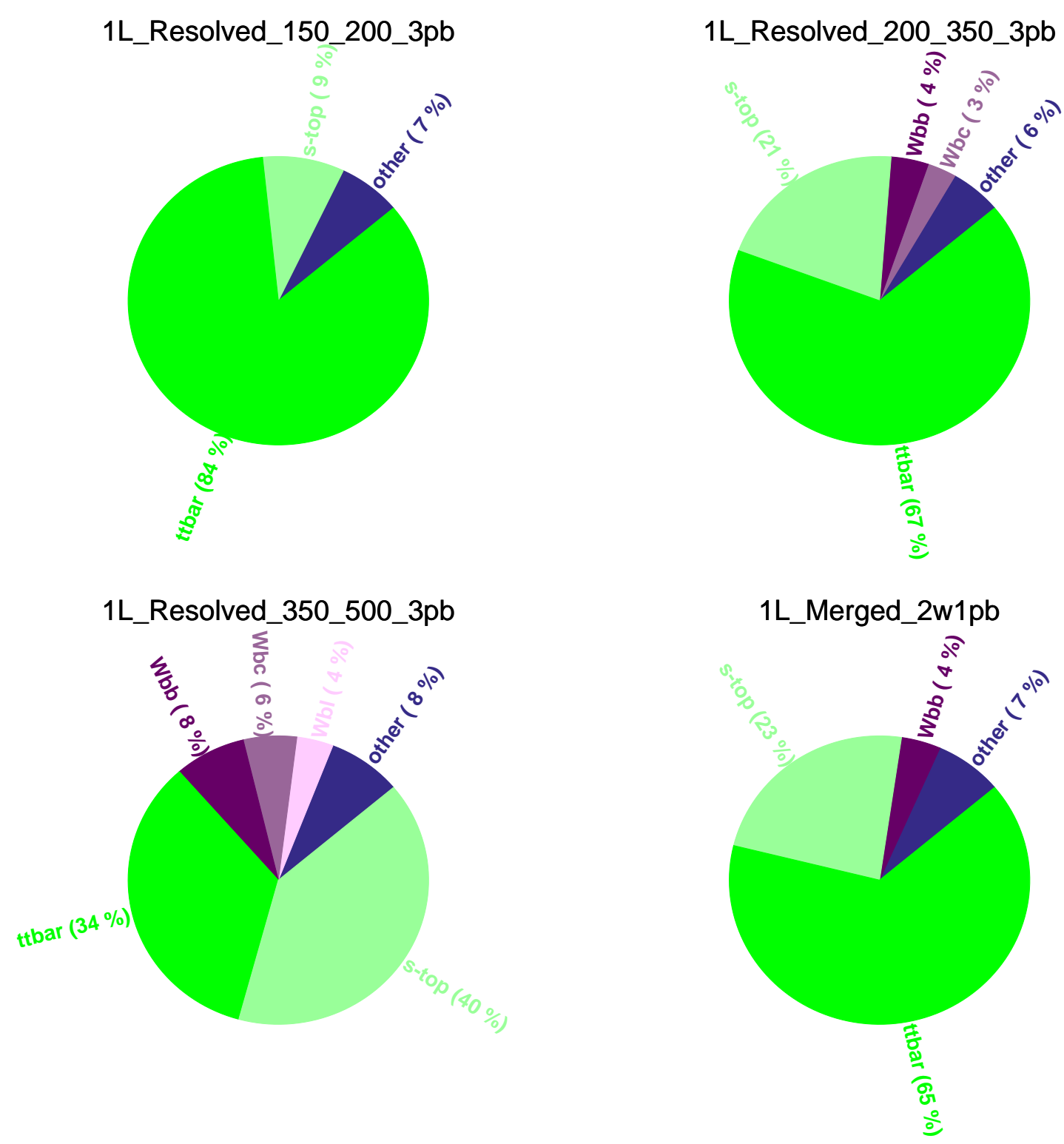

Figure 76: SM Background compositions across different $E_{\mathrm{T}}^{\mathrm{miss}}$ bins in the $1-\mu \mathrm{CR}$ for the $\geq 3$ $b$-tagged regions. 


\section{- 2-lepton CR:}

Figures 77 and 78 show the SM background compositions in the combined 2-lepton CR $\left(e^{+} e^{-}+\mu^{+} \mu^{-}\right)$for the $2 b$-tagged and $\geq 3 b$-tagged regions respectively, across different $E_{\mathrm{T}}^{\text {miss }}$ regions. The $Z+$ jets events are most dominant as expected in the 2-lepton CR.
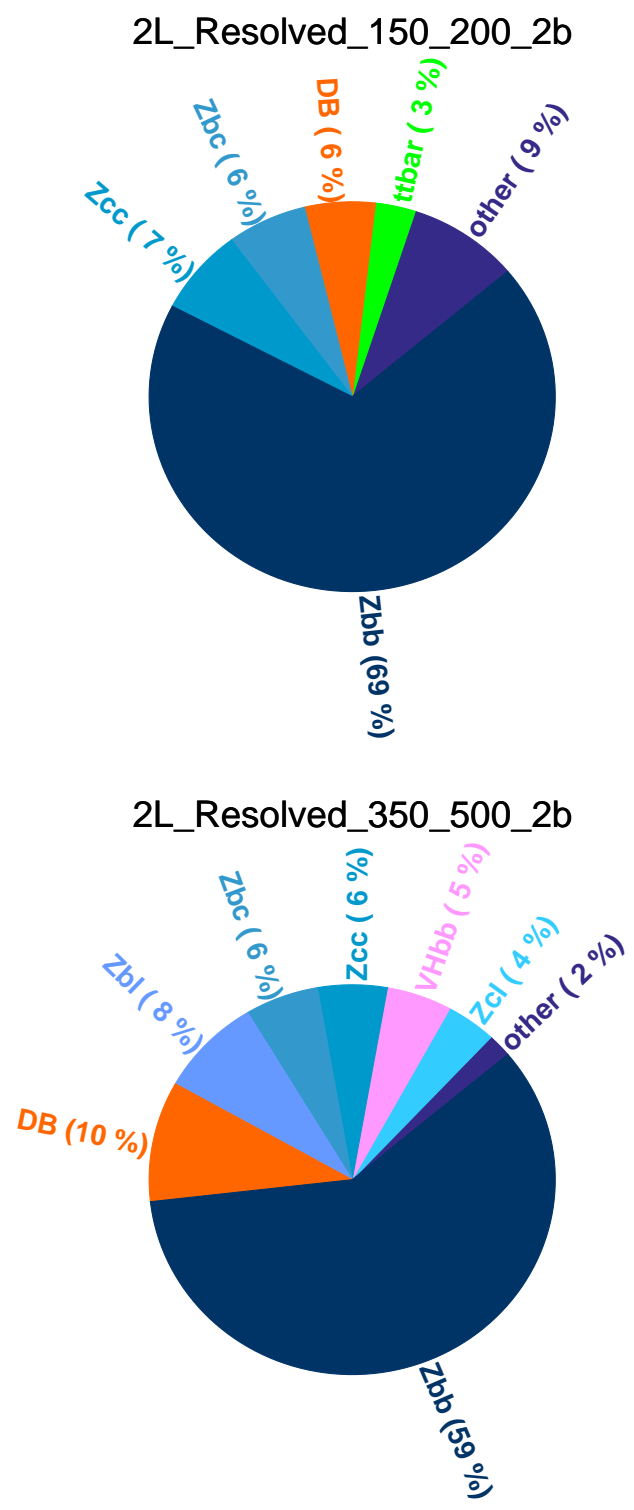

\section{L_Resolved_200_350_2b}
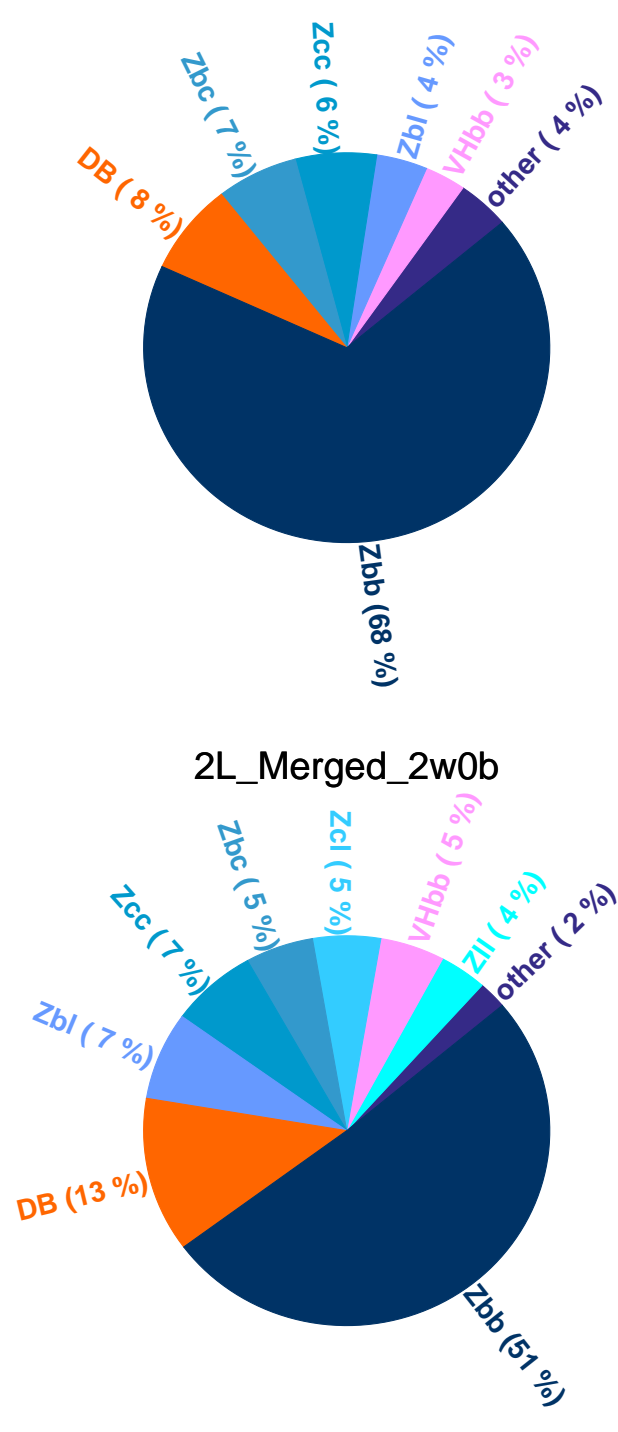

Figure 77: SM Background compositions in different $E_{\mathrm{T}}^{\text {miss }}$ bins in the combined 2 lepton CR for the $2 b$-tagged regions. 

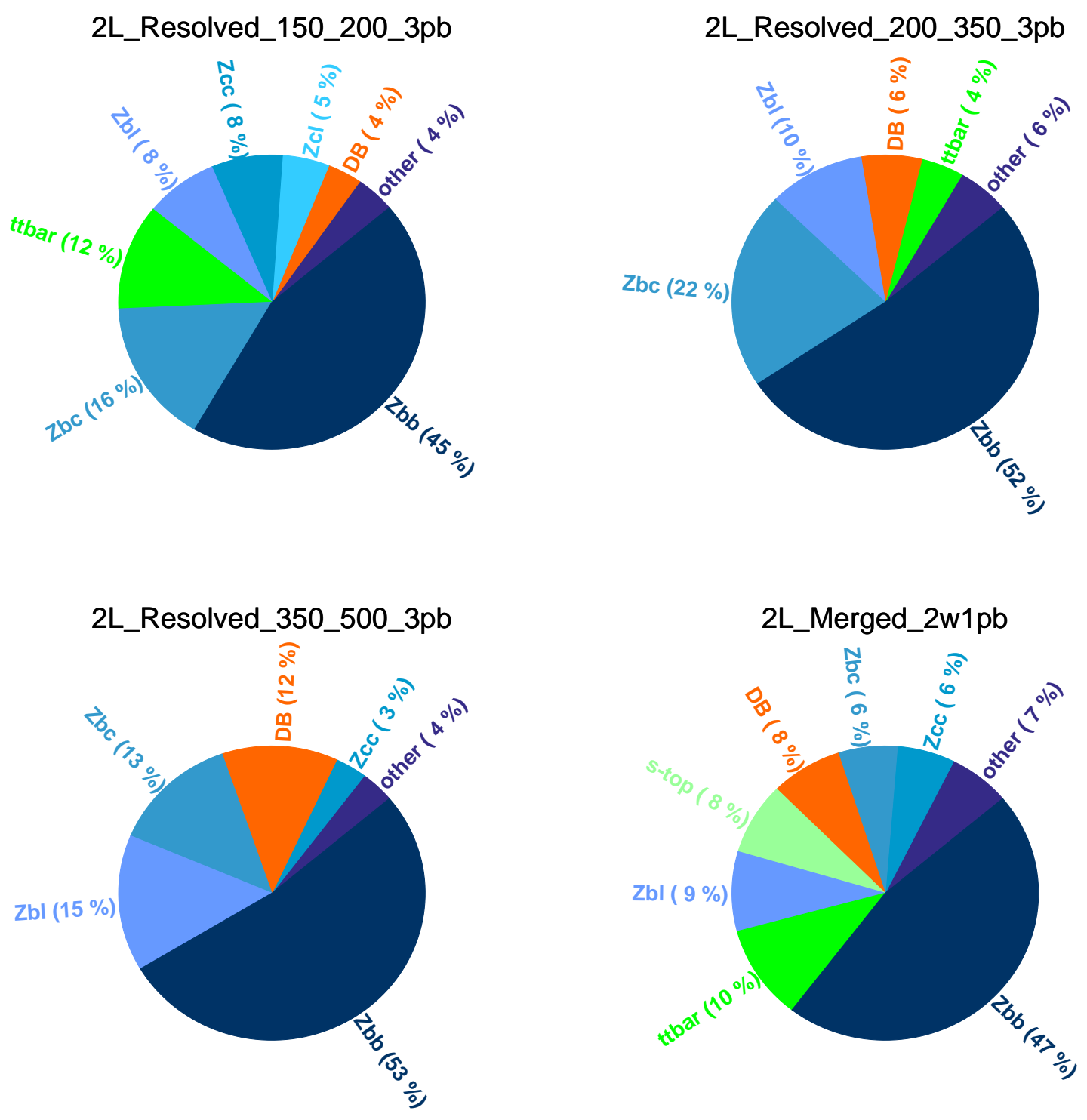

Figure 78: SM Background compositions in different $E_{\mathrm{T}}^{\text {miss }}$ bins in the combined 2 lepton CR for the $\geq 3$-tagged regions. 


\subsubsection{Treatment of $V+$ jets in the analysis}

The $V+$ jets backgrounds and their shapes play a significant role in the fit and the way they are treated in the analysis could impact the fit results. In the mono- $h(b \bar{b})$ analysis, the $V+$ jets events are classified on the basis of the flavors of the two leading small-R jets in the Resolved regions and two leading VR track jets associated with the leading large-R jet in the Merged regions. Hence, the $V+$ jets events are decomposed into the following components: $V b b, V b c, V b l, V c c, V c l, V l l$. In the previous iteration of the mono- $h(b \bar{b})$ analysis [235], no dedicated studies were performed to optimize the handling of the $V+$ jets flavors, a strategy was adopted from the VHbb analysis [236]: (i) HF: $V b b+V b c+V b l+V c c$, (ii) $V c l$, and (iii) $V l l$. The $H F$ (heavy flavor) refers to the $V+$ jets flavors with at least one $b$ or two $c$-jets.

Due to limited MC statistics and systematic fluctuations, the $V+$ jets flavors can affect the fit. For example, systematics with statistical fluctuations can have multiple minima and maxima, which can lead to unphysical NP pulls and can cause convergence issues in the fit: affecting smoothing. On the other hand, if a $V+$ jets flavor has a small systematic variation compared to the nominal, it can be removed from the fit: pruning. The following study describes if the $V+$ jets flavors handling strategy is optimal or needs to be optimized for the analysis. The study has been performed using the $Z(\rightarrow v v)+$ jets samples.

A motivation for merging different $V+$ jets flavor comes from the fact that the compositions of different $V+$ jets backgrounds vary across the regions. For example in the 0-lepton Resolved 2 $b$-tagged category for $150<E_{\mathrm{T}}^{\text {miss }} \leq 200 \mathrm{GeV}, V b b$ is the most dominant background ( $\left.40 \%\right)$, and other $V+$ jets has much less statistics (c.f. Figure 79). As a consequence, the systematic estimation of $V b b$ background has higher precision than other $V+$ jet flavors. Hence, merging different $V+$ jets flavors is necessary since it increases the precision of the systematic estimation.

As shown in Figure 80, the $V+$ jets flavor fraction does not change with the $m_{h}$, or with

the $E_{\mathrm{T}}^{\text {miss }}$. Hence, the optimization studies are performed using the $m_{h}$ distribution in the $E_{\mathrm{T}}^{\text {miss }}$ inclusive $\left(150<E_{\mathrm{T}}^{\text {miss }} \leq 500 \mathrm{GeV}\right) 0$-lepton Resolved $2 b$-tagged region. The merging of different $V+$ jets flavors are well motivated: 

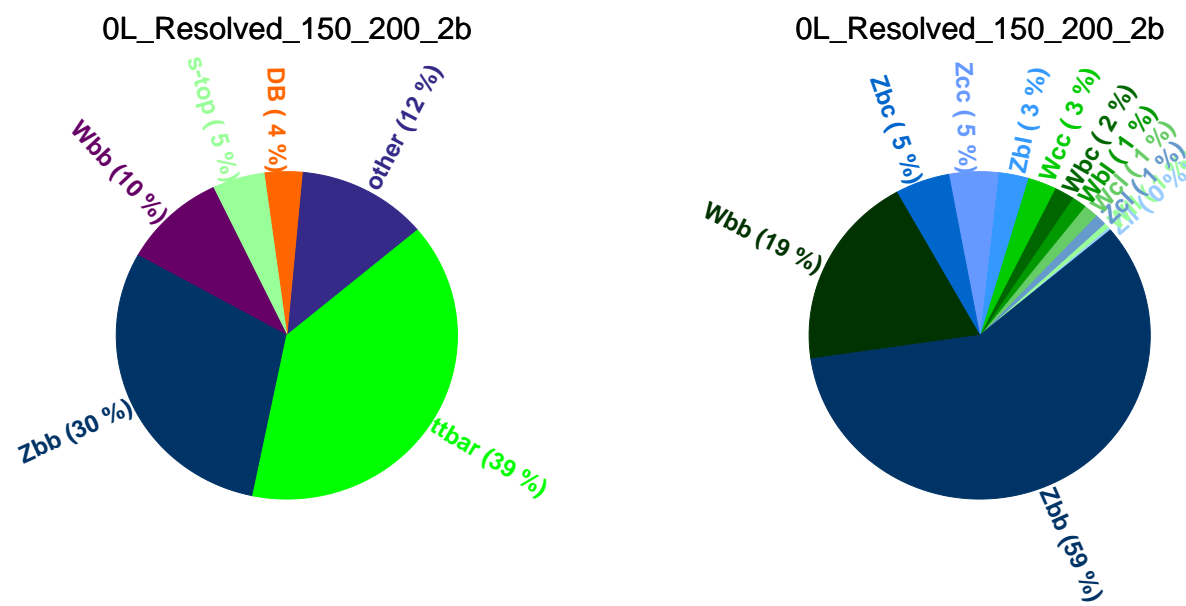

Figure 79: SM Background composition of all the backgrounds (left) and only $V+$ jets (right) in the 0-lepton Resolved $2 b$-tagged $150<E_{\mathrm{T}}^{\text {miss }}<200 \mathrm{GeV}$ region. Backgrounds contributing $<$ $3 \%$ to the total are combined in other category.
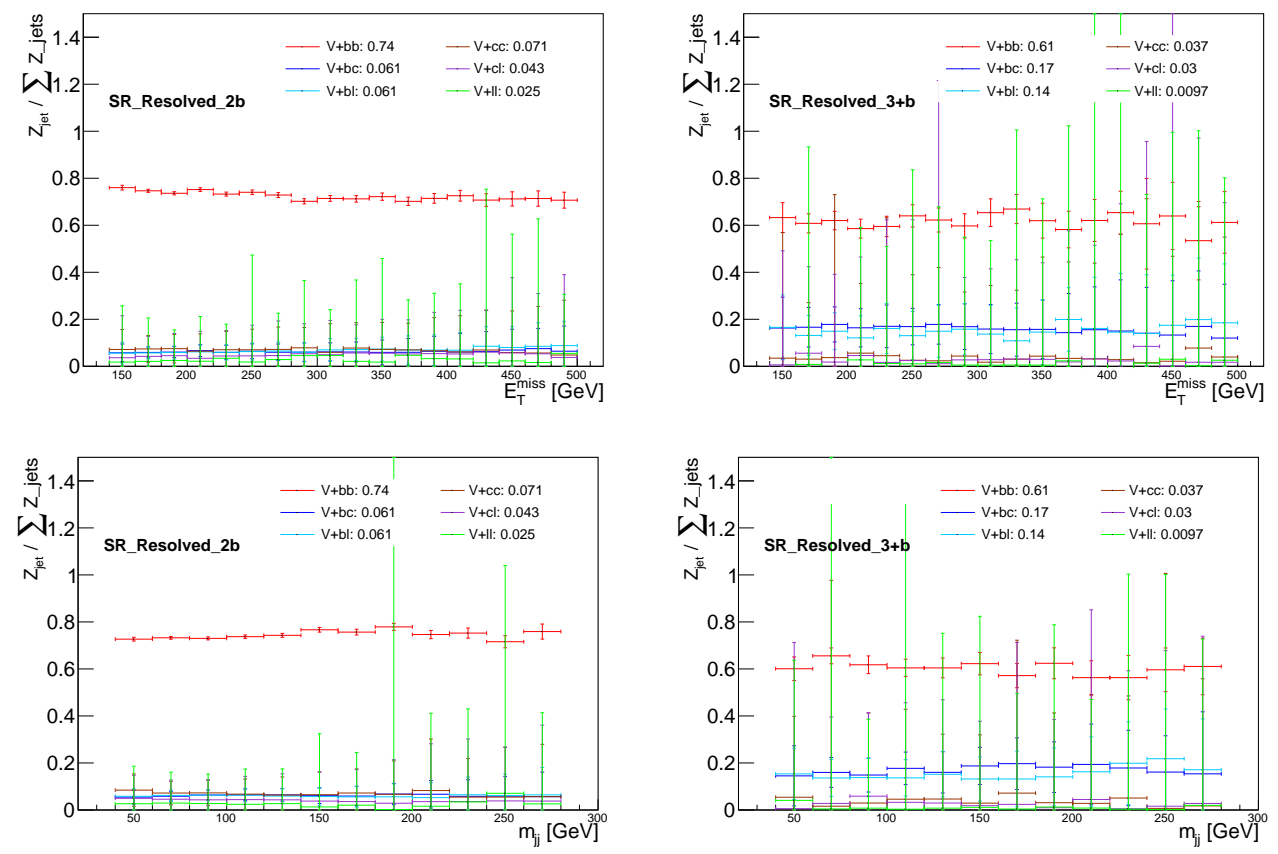

Figure 80: The upper and bottom panel plots represent the $Z_{\text {jets }} / \sum Z_{\text {jets }}$ as a function of $E_{\mathrm{T}}^{\mathrm{miss}}$, and $m_{h}$ respectively in the 0 -lepton Resolved region for different $b$-tagged categories. The nature of the flavor decomposition does not change with $E_{\mathrm{T}}^{\mathrm{miss}}$, or $m_{h}$.

\section{Theory driven:}

- Merge $V+$ jets flavors originating from similar processes (e.g., $b b, c c$ from gluon splitting). 
- Keep $H F$ template separate from the light-flavors, since light-flavor is well modelled, and the $H F$ is not.

\section{Shape driven:}

- Maximize shape differences between the merged $V+$ jets flavors .

The aim of optimising $V+$ jets flavor merging is to:

- Check if any prior knowledge can be injected on what flavors to merge, based on the $V+$ jets flavors shape.

- Find a well motivated and theoretically interpretable $V+$ jets flavors merging strategy.

The $V+$ jets flavor merging optimization is performed following the strategy described below:

1. Perform a liner (polynomial order 1) fit on the ratio of flavor $1 /$ flavor 2 as a function of $m_{h}$.

2. Calculate the slope and error on the slope from the fit and merge the two flavors with the smallest slope and slope/error. E.g., in the 0-lepton Resolved $2 b$-tag category, Zcl and Zcc are merged together since their ratio has the smallest slope and slope/error (Table 20).

3. Compare the merged flavor in step 2 with other flavors (e.g., $Z c l+Z c c$ vs $Z b b$ ) and depending on the slope and slope/error decide whether to merge them or not.

4. The iteration continues until a template is found where the $V+$ jets flavor shapes are distinct.

5. The deciding factor in whether to merge flavors or not is the slope and slope/error, while $\chi^{2}$ and Kolmogorov-Smirnov (KS) tests have been performed to cross-check the results.

Following the procedure described in step 1 and 2, the $Z c l$ and $Z c c$ are found to be the most compatible flavors in the 0-lepton Resolved 2 b-tagged region (lowest slope/error in Table 20) and are merged together as shown in Figure 81a. As described in the strategy, the combined $Z c l+Z c c$ shape is compared with other $Z+$ jets flavors and combinations of other merged flavors. 


\begin{tabular}{|l||l|l|l|}
\hline Flavor & Slope & Error & Slope/Error \\
\hline \hline Zbb_Zbc & 0.000298 & 0.000057 & 5.23 \\
\hline Zbb_Zbl & 0.000702 & 0.000057 & 12.32 \\
\hline Zbb_Zcc & 0.001602 & 0.000057 & 28.11 \\
\hline Zbb_Zcl & 0.002581 & 0.000057 & 45.28 \\
\hline Zbb_Zll & 0.003228 & 0.000057 & 55.26 \\
\hline Zbl_Zbc & 0.000858 & 0.000212 & 4.05 \\
\hline Zbl_Zcl & 0.000858 & 0.000212 & 8.20 \\
\hline Zbl_Zcc & 0.000858 & 0.000212 & 4.34 \\
\hline Zbl_Zll & 0.000858 & 0.000212 & 9.60 \\
\hline ZclZZbc & 0.002309 & 0.00068 & 3.40 \\
\hline Zcl_Zcc & 0.000446 & 0.00068 & 0.66 \\
\hline ZclZZll & 0.00185 & 0.000669 & 2.77 \\
\hline Zbc_Zcc & 0.00118 & 0.000214 & 5.22 \\
\hline Zcc_Zll & 0.003846 & 0.000506 & 7.60 \\
\hline
\end{tabular}

Table 20: Table showing the fit results from the comparison of different $Z+$ jets shapes in step 1 of $Z+$ jets shape comparison procedure.

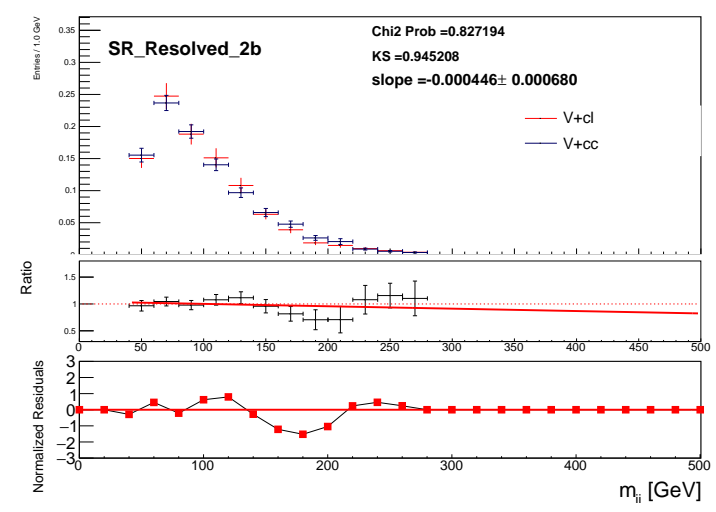

(a) Zcl vs Zcc

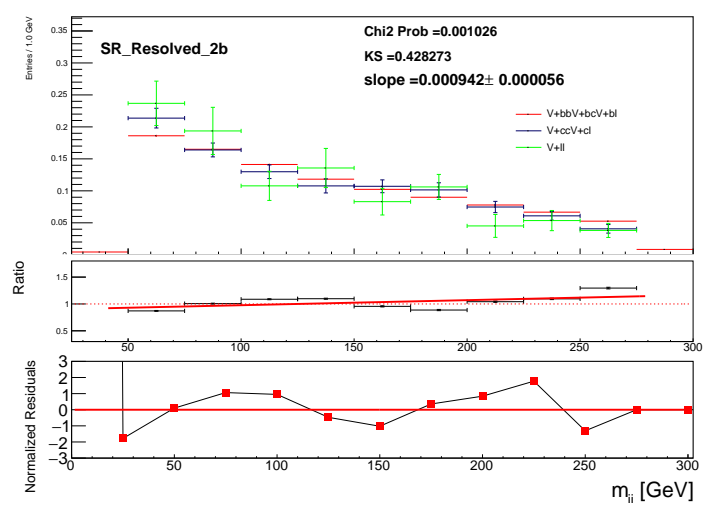

(b) Shape-driven template

Figure 81: Left plot: compatibility between $\mathrm{Zcc}$ and $\mathrm{Zcl}$ shapes, the second panel represents the ration between the two flavors, the solid red line represents the linear fit and the third panel (bottom most) represents the normalized residuals from the KS test. Right plot: $Z+$ jets merging template with the most distinct shape: $Z b b+b c+b l, Z c c+c l, Z l l$. The second and third canvas represents the ratio between $Z b b+b c+b l$ and $Z c c+c l$, and the normalized residuals from KS test respectively.

The merging template with maximum shape difference is found to be: $Z b b+Z b c+Z b l, Z c l+Z c c$, Zll and is shown in Figure $81 \mathrm{~b}$

As shown in Figure 82, the results are compared with the templates used in the previous mono- $h(b \bar{b})$ iteration, and the VHbb Resonance [236]. There are no significant differences ob- 
served, hence no strong preference for any of the templates. For the mono- $h(b \bar{b})$ analysis described in this thesis, the $V+$ jets template used is: HF: $V b b+V b c+V b l+V c c$, (ii) $V c l$, and (iii) $V l l$.

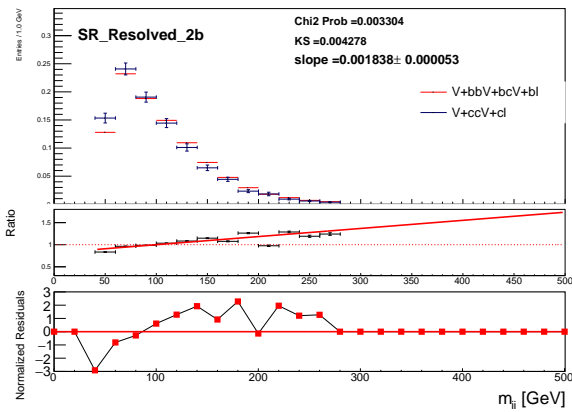

(a) Shape driven template

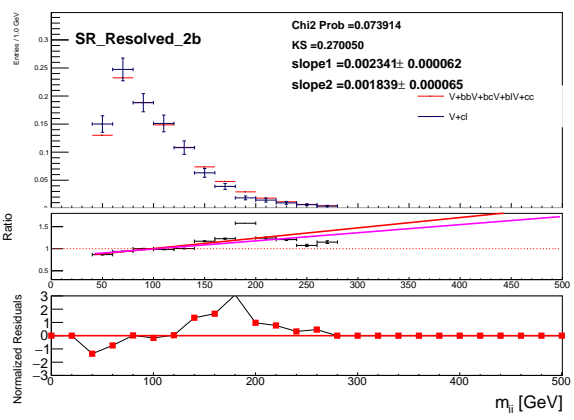

(b) Template in previous mono- $h(b \bar{b})$

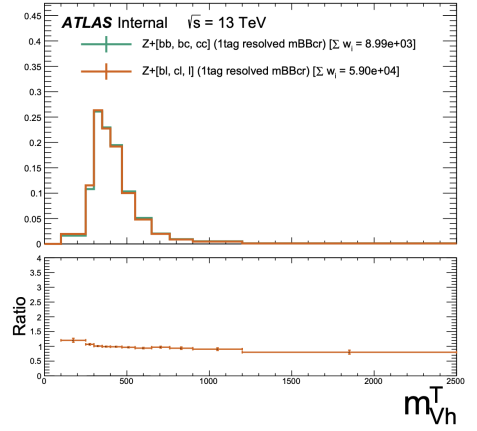

(c) VHbb resonance template 236

Figure 82: Comparisons between shape driven, previous mono- $h(b \bar{b})$ analysis, and VHbb Resonance merging template. The pink line in Figure $82 \mathrm{~b}$ represents the linear fit from the shape driven scheme in the Figure $82 \mathrm{a}$. No scheme has a clear preference as shown in Figures 82a, 82b and $82 \mathrm{c}$

\subsubsection{Systematic uncertainties}

The sources of uncertainties in a physics analysis at the LHC are categorized as: statistical and systematic. The outcome of any measurement is based on a finite set of measurements, and each measurement has some random fluctuations. The source of statistical uncertainties is the random fluctuations in a finite set of measurements. The systematic uncertainties are all other uncertainties which are not directly due to the data statistics. The sources of systematic uncertainties are physics object reconstruction and identification uncertainties (detector systematics), and the MC simulation uncertainties of both the SM backgrounds, signals, and other theoretical ones (theoretical uncertainties). These uncertainties can affect the total yield of a background in specific analysis regions, and the shape of the variables that are relevant for the simultaneous fit, such as $m_{h}$. The detector and theoretical uncertainties together are known as the systematic uncertainties, and this section describes the systematic uncertainties associated with the mono- $h(b \bar{b})$ analysis.

- Detector systematics

Luminosity uncertainty: In the analysis, the MC simulated events are normalized to the 
integrated luminosity. The integrated luminosity uncertainty for the full Run 2 ATLAS dataset is measured to be $1.7 \%$ [237].

Pile-up reweighting uncertainty: A pile-up reweighting factor is applied to rectify the differences between the observed pile-up distribution in data and the predicted pile-up distribution during MC simulation. The uncertainty corresponding to the pile-up reweighting factor is considered to be $4 \%$ in this analysis. The integrated luminosity and pile-up reweighting uncertainties are common to all events and affect the overall normalization of the physics processes.

Jet uncertainties: The dominant source of detector systematics in the analysis is the jet reconstruction and calibration uncertainties. The uncertainties that affect the small-R jet uncertainties (both JES and JER) [238], arise from detector acceptance, pile-up corrections, modelling effects, $\eta$ and $p_{\mathrm{T}}$ dependent effects, jet-flavor compositions, data-MC resolution effects, etc. The JER and JES uncertainties are divided into multiple components, and each acts as a NP in the simultaneous fit. The set of JES and JER systematic uncertainties used in the analysis has 29 and 8 NPs respectively. For large-R jets, the uncertainties on the jet mass scale and resolution [144, 239] are considered in the analysis in addition to JES.

Flavor tagging uncertainties: The jet flavor tagging uncertainties include uncertainties from the $b$-tagging efficiency and the mistagging efficiency of the $c$ and light-flavored jets [150, 240, 241]. These uncertainties arise from detector efficiencies, modelling uncertainties that affect the flavor tagging calibrations, etc. The flavor tagging systematics are divided into many eigenvector components, and each component enters the simultaneous fit as a NP. There are 3, 4, and 5 NPs corresponding to $b, c$, and light- flavor jet tagging efficiencies respectively.

Lepton uncertainties: Uncertainties corresponding to the electron and muon reconstruction [130, 131], identification, trigger, and isolation efficiencies are considered in this analysis. Besides, there are uncertainties related to the lepton kinematics due to the resolution and scale of electron energy measurements and resolution and scale of muon momentum measurements. Since the analysis vetoes $\tau$-leptons, only the energy scale uncertainties related to the $\tau$-lepton reconstruction are considered. 
$E_{\mathrm{T}}^{\text {miss }}$ uncertainties: Since the $E_{\mathrm{T}}^{\text {miss }}$ reconstruction procedure uses various physics objects, the systematic uncertainties associated with it are from those physics objects such as electrons, muons, jets. The $E_{\mathrm{T}}^{\text {miss }}$ systematic uncertainties also include the uncertainties related to the scale, reconstruction, and resolution of the $E_{\mathrm{T}}^{\text {miss }}$ soft terms [158] used in the $E_{\mathrm{T}}^{\text {miss }}$ calculations. Besides, uncertainties on the $E_{\mathrm{T}}^{\text {miss }}$ trigger scale factors $(\mathrm{SF})$ are also derived, which are $\sim 1-2 \%$ for low $E_{\mathrm{T}}^{\mathrm{miss}}$ values.

\section{- Theoretical systematics}

The analysis also considers theoretical uncertainties from the MC modelling of both background and signal processes. The theoretical uncertainty sources are:

Choice of PDF and $\alpha_{s}$ : In the MC sample production, a specific PDF and $\alpha_{s}$ is used. The evaluation of a PDF set and $\alpha_{s}$ involve inputs from experimental measurements with uncertainties. Besides, there are uncertainties in the fit functions used to derive the PDF sets. These uncertainties are considered in this analysis and are estimated using the PDF4LHC prescription [242].

Missing higher orders in matrix element calculations: The MC simulated samples are generated with a finite order of QCD precision. Hence, the cross section of a certain process has uncertainties due to the missing higher-order QCD terms. These uncertainties are estimated by using different pairs of factorization $\left(\mu_{F}\right)$ and renormalization $\left(\mu_{R}\right)$ scales by varying them by a factor of 2 up or down, excluding $\left(\mu_{R}, \mu_{F}\right)=\left(\frac{1}{2}, 2\right),\left(2, \frac{1}{2}\right) \times \mu_{\text {central }}$, which can lead to large logarithm values.

Merging-scale and resummation uncertainties: For the $W^{ \pm} / Z+$ jets samples there are uncertainties associated with the merging of matrix elements (ME). This is because there are different jet multiplicities in these samples, i.e., $W^{ \pm} / Z+1,2, .$. jets, and the merging scale that separates the soft jets from hard jets has an uncertainty. These uncertainties are evaluated by varying the merging scale by a factor of two up and down. For the SHERPA samples, i.e., $W^{ \pm} / Z+$ jets, and DB samples, an additional uncertainty comes from the resummation procedure of the parton shower [243].

Matching uncertainties: For samples which are generated using a NLO matrix element, 
such as $t \bar{t}$, single-top, $W^{ \pm} / Z+$ jets, there in an uncertainty associated to the ME and PS matching. These uncertainties are derived by comparing $t \bar{t}$, single-top, $W^{ \pm} / Z+$ jets samples generated with POWHEG (nominal samples) with MADGRAPH5_AMC@NLO (alternative samples) [167].

Parton shower and hadronization uncertainties: Uncertainties associated with the modelling of the PS and hadronization are also considered in this analysis. These uncertainties are evaluated by comparing samples produced with different showering and hadronization generators such as PYTHIA 8 and HeRWIG 7 [244, 245].

Eigentune uncertainties: These are uncertainties associated with the choice of tune parameters used in the hadronization and PS generators, derived using the procedure described in [171].

\begin{tabular}{|c|c|c|}
\hline \multicolumn{3}{|c|}{ Event } \\
\hline \multicolumn{3}{|c|}{$\begin{array}{c}\text { Luminosity } \\
\text { Pile-up reweighting }\end{array}$} \\
\hline \multicolumn{3}{|c|}{ Object reconstruction } \\
\hline $\begin{array}{c}\text { Jets } \\
\text { Jet Energy Scale (JES) } \\
\text { Jet Energy Resolution (JER) } \\
\text { Jet Mass Scale (JMS) } \\
\text { Jet Mass Resolution (JMR) }\end{array}$ & $\begin{array}{l}\text { Flavor-tagging } \\
b \text {-tagging efficiency } \\
\text { mis-tag efficiency }\end{array}$ & $\begin{array}{cc}\text { Leptons } & E_{\mathrm{T}}^{\text {miss }} \\
\text { Reconstruction efficiency } & E_{\mathrm{T}}^{\text {miss }} \text { soft-term scale } \\
\text { Identification efficiency } & E_{\mathrm{T}}^{\text {miss }} \text { soft-term resolution } \\
\text { Isolation efficiency } & E_{\mathrm{T}}^{\text {miss }} \text { trigger SF } \\
\text { Trigger efficiency } & \end{array}$ \\
\hline \multicolumn{3}{|c|}{ Background modelling } \\
\hline $\begin{array}{c}\text { Source } \\
\text { PDF and } \alpha_{s} \\
\text { Scale variation }\left(\mu_{R}, \mu_{F}\right) \\
\text { PS and hadronization } \\
\text { ME and PS matching } \\
\text { Merging scale and resummation } \\
\text { Tune parameters }\end{array}$ & $t \bar{t}, \mathrm{~s}$ & $\begin{array}{c}\text { Affected backgrounds } \\
\text { ngle-top, } W / Z+\text { jets, DB, SM Vh }(b \bar{b}) \\
\text { ngle-top, } W / Z+\text { jets, DB, SM Vh }(b \bar{b}) \\
\text { ngle-top, } W / Z+\text { jets, DB, SM Vh }(b \bar{b}) \\
t \bar{t}, \text { single-top, } W / Z+\text { jets } \\
\quad W / Z+\text { jets, DB } \\
t \bar{t} \text {, single-top, } W / Z+\text { jets, DB }\end{array}$ \\
\hline \multicolumn{3}{|c|}{ Signal modelling } \\
\hline \multicolumn{3}{|c|}{$\begin{array}{c}\text { PDF and } \alpha_{s} \\
\text { Scale variation }\left(\mu_{R}, \mu_{F}\right)\end{array}$} \\
\hline
\end{tabular}

Table 21: Table format adapted from [162] summarises different sources of systematic uncertainties considered in the mono- $h(b \bar{b})$ analysis. It also summarises the SM backgrounds affected by different modelling uncertainties.

Table 21 summarises the different sources of detector, background modelling, and signal 
uncertainties considered in the analysis. Since backgrounds like $t \bar{t}+W^{ \pm} / Z / h$ have a contribution of $<1 \%$ in different regions, the modelling uncertainties for these samples are not considered in the analysis. The systematic uncertainties can affect the simultaneous fit in different ways:

- Normalization of the MC templates: The detector systematics and the theory systematics originating from cross section uncertainties can affect the normalization of a MC template.

- Shape of fit discriminants: The systematics can distort the shapes of the fit discriminants such as $m_{h}$ and muon-charge distribution in the 0 and 1-lepton regions respectively.

- Relative acceptance: The systematics can also affect the shape of the variables in which the fit discriminant is binned such as $E_{\mathrm{T}}^{\text {miss }}, N_{b-\text { tag }}, N_{\text {leptons }}$.

A detailed description of the how the NPs are handled in the analysis are described in the following section.

Tables 22 and 23 show the impacts of different sources of uncertainties on the signal strength $(\mu)$, i.e., the parameter of interest in the simultaneous fit, for a few representative $Z^{\prime}-2 \mathrm{HDM}$ and $2 \mathrm{HDM}+a$ signal points. The numbers are obtained from a signal + background fit to the observed data where the cross section of each signal point is fixed to the observed cross section upper limit. The fractional impact from each systematics source is calculated as:

$$
\text { Fractional impact } \mathrm{i}_{\mathrm{i}}=\frac{\mu_{\mathrm{i}}^{2}}{\mu^{2}},
$$

where $\mu_{i}$ and $\mu$ represent the uncertainty from a particular systematic and the total uncertainty respectively. Since there are correlations between different sources of systematics, the sum of individual fractional uncertainties might not add up to the total of all the systematic uncertainties. The chosen signal points for the $Z^{\prime}-2 \mathrm{HDM}$ model are: $\left(m_{Z^{\prime}}, m_{A}\right)=(800,500) \mathrm{GeV},\left(m_{Z^{\prime}}, m_{A}\right)=(1400$, 1000) $\mathrm{GeV},\left(m_{Z^{\prime}}, m_{A}\right)=(280,300) \mathrm{GeV}$, which represent a low, medium, and high $Z^{\prime}$ mass points respectively. The chosen signal points for the $2 \mathrm{HDM}+a$ signal model are: $\left(m_{A}, m_{a}, \tan \beta\right)=(500$, $200,1),(1000,400,1),(1700,200,1),(1000,200,10)$, the masses are in $\mathrm{GeV}$. 


\begin{tabular}{c|ccc}
\hline Source of uncertainty & \multicolumn{3}{|c}{$\left(m_{Z^{\prime}}, m_{A}\right) \mathrm{GeV}$} \\
& $(800,500)$ & $(1400,1000)$ & $(2800,300)$ \\
\hline Statistical uncertainty & 27 & 61 & 79 \\
All systematic uncertainties & 73 & 39 & 21 \\
\hline$Z+\mathrm{HF}$ normalization & 11 & 3 & $<1$ \\
$W+$ HF normalization & 2 & 1 & $<1$ \\
$t \bar{t}$ normalization & 16 & 4 & $<1$ \\
$Z$ theoretical uncertainties & 2 & 7 & 3 \\
$W$ theoretical uncertainties & $<1$ & 1 & $<1$ \\
$t \bar{t}$ theoretical uncertainties & 13 & 5 & $<1$ \\
Single $t$ theoretical uncertainties & 18 & 2 & $<1$ \\
Other theoretical uncertainties & 5 & 1 & $<1$ \\
JER/JES & 20 & 6 & 1 \\
$b$-tagging & 1 & 1 & 4 \\
Soft term and pile-up & $<1$ & $<1$ & $<1$ \\
$E_{\mathrm{T}}^{\text {miss }}$ soft & $<1$ & $<1$ \\
Other detector related uncertainties & 1 & $<1$ & 11 \\
Signal uncertainties & $<1$ & $<1$ &
\end{tabular}

Table 22: Dominant sources of uncertainties for a few representative $Z^{\prime}$-2HDM signal points, the numbers represent fractional uncertainties on the signal strength $(\mu)$ after the fit to data [234].

\begin{tabular}{|c|c|c|c|c|}
\hline \multirow{2}{*}{$\begin{array}{l}\text { Source of uncertainty } \\
\qquad\left(m_{A}, m_{a}, \tan \beta\right)\end{array}$} & \multicolumn{4}{|c|}{ Fractional impact on signal strength $(\mu)$ in $\%$} \\
\hline & $(500,200,1)$ & $(1000,400,1)$ & $(1700,200,1)$ & $(1000,200,10)$ \\
\hline Statistical uncertainty & 31 & 65 & 69 & 60 \\
\hline All systematic uncertainties & 69 & 35 & 31 & 40 \\
\hline$Z+$ HF normalization & 6 & 3 & $<1$ & 1 \\
\hline$W+$ HF normalization & 2 & 1 & $<1$ & 1 \\
\hline$t \bar{t}$ normalization & 9 & 3 & 1 & $<1$ \\
\hline$Z$ theoretical uncertainties & 1 & 5 & 8 & 3 \\
\hline$W$ theoretical uncertainties & 1 & 1 & 1 & $<1$ \\
\hline$t \bar{t}$ theoretical uncertainties & 7 & 4 & 1 & 3 \\
\hline Single $t$ theoretical uncertainties & 9 & 2 & 1 & 2 \\
\hline Other theoretical uncertainties & 5 & 1 & 1 & 1 \\
\hline JER/JES & 20 & 6 & 1 & 7 \\
\hline$b$-tagging & $<1$ & 1 & 8 & 3 \\
\hline$E_{\mathrm{T}}^{\text {miss }}$ soft-term and pile-up & 2 & $<1$ & $<1$ & $<1$ \\
\hline Other detector related uncertainties & $<1$ & $<1$ & $<1$ & $<1$ \\
\hline Signal uncertainties & $<1$ & $<1$ & $<1$ & $<1$ \\
\hline MC sample statistical uncertainties & 9 & 7 & 7 & 11 \\
\hline
\end{tabular}

Table 23: Dominant sources of uncertainties for a few representative $2 \mathrm{HDM}+a$ signal points, the numbers represent fractional uncertainties on the signal strength $(\mu)$ after the fit to data [234]. 


\subsubsection{Statistical analysis model}

The interpretation of the final results is performed by a binned profile likelihood fit (c.f section 4.4). The model, consisting of all the SM backgrounds and the DM signals is fitted to the

observed data. The statistical fit, performed simultaneously, includes all the lepton channels, $E_{\mathrm{T}}^{\text {miss }}$ bins, and $b$-tag categories. The likelihood fit is performed to extract upper limits at a 95\% CL on the signal cross section, later used to obtain exclusion limits on $\left(m_{Z^{\prime}}, m_{A}\right)$ and $\left(m_{A}, m_{a}\right)$ plane for the $Z^{\prime}-2 \mathrm{HDM}$ and $2 \mathrm{HDM}+a$ model respectively. The POI in the fit is the signal strength $(\mu)$ :

$$
\mu=\frac{\sigma_{\text {observed }}}{\sigma_{\text {predicted }}}
$$

where $\sigma_{\text {observed }}$, and $\sigma_{\text {predicted }}$ represent the measured and theoretical cross sections of the DM processes. This section describes the fit model implemented in the analysis.

- Fit regions and discriminating variables:

The choice of fit discriminants varies across the different lepton regions. For the 0-lepton $\mathrm{SR}$, the Higgs candidate mass distribution is used as the fit discriminant across all $E_{\mathrm{T}}^{\text {miss }}$ bins and $b$-tag categories.

In the $1-\mu \mathrm{CR}$, the $\mu$-charge distribution is used as the fit discriminant across all $E_{\mathrm{T}}^{\text {miss }}$ bins for the $2 b$-tagged category, but due to limited data statistics, the total yield is used as fit discriminant across all $E_{\mathrm{T}}^{\text {miss }}$ bins for the $\geq 3 b$-tagged category. The $\mu$-charge distribution is a two-bin histogram corresponding to the two $\mu$ charged $\left(\mu^{+} / \mu^{-}\right)$. The shape of the $\mu$-charge distribution helps to distinguish between the $t \bar{t}$ and $W^{ \pm}+$jets events. Since the LHC is a $p p$ collider, the production cross section of $W^{+}$is much higher than that of $W^{-}, \sigma\left(W^{+}\right) \approx 1.3 \sigma\left(W^{-}\right)[246]$ (also known as the $W$-charge asymmetry). Consequently, there are more $\mu^{+}$than $\mu^{-}$events in the $W^{ \pm}+$ jets process. On the other hand, there is no such asymmetry for the $t \bar{t}$ process. Hence, the $\mu$-charge shape can distinguish between the two above mentioned backgrounds. This is prominent for the high $E_{\mathrm{T}}^{\text {miss }}$ regions as shown in Figures 83 and 83 .

In the 2-lepton CR a single bin total yield is used as the fit discriminant across all $E_{\mathrm{T}}^{\mathrm{miss}}$ bins and $b$-tag categories. The binning choice increases the robustness of the fit and serves the purpose 
of constraining the $Z+$ jets background normalization without compromising the sensitivity. Table 24 summarises different $E_{\mathrm{T}}^{\text {miss }}$ bins, $b$-tag categories and discriminating variables entering the fit model, while Table 25 summarises the different $m_{h}$ binnings used in the fit.

\begin{tabular}{|c|c|c|c|}
\hline & 0-lepton SR & 1- $\mu$ CR & 2-lepton CR \\
\hline Fit discriminant & $m_{h}$ distribution & $\begin{array}{l}\mu \text {-charge distribution }(2,2 b \text {-tag/ } 0 \text { add. } b \text {-tags }) \\
\text { Yield }(\geq 3 b \text {-tags, } 2 b \text {-tags } / \geq 1 \text { add. } b \text {-tags })\end{array}$ & Yield \\
\hline Targeted region & signal region & $t \bar{t}$ and $W+$ jets CR & $Z+$ jets $\mathrm{CR}$ \\
\hline$b$-tag multiplicity & Merged: $2 b$ & $\begin{array}{l}\text { Resolved: } 2, \geq 3 b \text {-tags } \\
\text { tags } / 0 \text { add. } b \text {-tags, } 2 b \text {-tags } / \geq 1 \text { add. } b \text {-tags }\end{array}$ & \\
\hline$E_{\mathrm{T}}^{\text {miss }}$ proxy & $E_{\mathrm{T}}^{\mathrm{miss}}$ & $E_{\mathrm{T}, \text { lep.invis. }}^{\text {miss }}$ & $E_{\mathrm{T}, \text { lep.invis. }}^{\text {miss }}$ \\
\hline$E_{\mathrm{T}}^{\text {miss }}$ bins & $\begin{array}{l}\text { Resolved: } \\
2 b \text {-tag 0- }\end{array}$ & $\begin{array}{l}150,200],[200,350] \text { and }[350,500] \mathrm{GeV} \\
\text { epton Merged: }[500,750] \text { and }>750 \mathrm{GeV} \\
\text { other Merged: }>500 \mathrm{GeV}\end{array}$ & \\
\hline
\end{tabular}

Table 24: Table adapted from [234] summarises different analysis regions: lepton regions, $E_{\mathrm{T}}^{\text {miss }}$ bins and $b$-tag regions along with the fit discriminants used in the simultaneous fit.

\begin{tabular}{|c|c|c|c|c|c|}
\hline$E_{\mathrm{N} \text { tags }} E_{\mathrm{T}}^{\text {miss }}$ bin & $150-200 \mathrm{GeV}$ & $200-350 \mathrm{GeV}$ & $350-500 \mathrm{GeV}$ & $500-750 \mathrm{GeV}$ & $>750 \mathrm{GeV}$ \\
\hline $\begin{array}{l}2,2 b \text {-tags } / \geq 0 \text { add } \\
b \text {-tags }\end{array}$ & $5 \mathrm{GeV}$ & $5 \mathrm{GeV}$ & $10 \mathrm{GeV}$ & $20 \mathrm{Gev}$ & $\begin{array}{l}3 \text { bins: } \\
{[50,90,150,270]} \\
\mathrm{GeV}\end{array}$ \\
\hline $\begin{array}{l}\geq 3,2 b \text {-tags } / \geq 1 \text { add. } \\
b \text {-tags }\end{array}$ & $10 \mathrm{GeV}$ & $10 \mathrm{GeV}$ & $\begin{array}{l}3 \text { bins: } \\
{[50,110,150,280]} \\
\mathrm{GeV}\end{array}$ & \multicolumn{2}{|c|}{3 bins: $[50,90,150,270] \mathrm{GeV}$} \\
\hline
\end{tabular}

Table 25: Table adapted from [234] summarises the $m_{h}$ binning used in the different fit regions.

\section{- MC templates and nuisance parameters:}

Some of the SM backgrounds suffer from low statistics, due to fluctuations the fit is unable to constrain these backgrounds accurately. To avoid this, similar processes are combined together, as shown in Table 26. Each source of systematic uncertainty appears in the fit as a NP in the

\begin{tabular}{|c|c|c|c|c|c|}
\hline$Z+$ jets & $W+$ jets & $t \bar{t}$ & Single-top & Diboson & Others \\
\hline $\begin{array}{c}Z \mathrm{HF}=Z b b+Z b c+Z b l+Z c c \\
Z c l \\
Z l\end{array}$ & $\begin{array}{c}W \mathrm{HF}=W b b+W b c+W b l+W c c \\
W c l \\
W l\end{array}$ & $t \bar{t}$ & single-top $W t+t$-channel $+s$-channel & $W W+W Z+Z Z$ & $\begin{array}{l}\text { SM } V h(b \bar{b}), \\
t \bar{t} h, t \bar{t} Z, t \bar{t} W\end{array}$ \\
\hline
\end{tabular}

Table 26: Summary of the MC templates used in the analysis for the SM backgrounds.

likelihood function. The normalization uncertainties appear in the fit either as floating NPs ("flat", 
the final values are obtained by comparing with the observed data) or NPs with Gaussian priors (values obtained from MC only). The NP controlling the signal normalization is a floating NP and is known as the signal strength $(\mu)$. There are four floating NPs controlling the normalization factors of the major SM backgrounds: one each for $t \bar{t}$ and $W+\mathrm{HF}$, and two for the $Z+\mathrm{HF}$ (one each for $2 b$-tag and $\geq 3 b$-tag categories). Since the $t \bar{t}$ production mechanism is same for both $2 b$ and $\geq 3 b$-tag categories, and the $W+\mathrm{HF}$ has negligible contribution in the $\geq 3 b$-tag region, a single NP for the normalization factors is sufficient for both the MC templates. However, a significant amount of $Z+\mathrm{HF}$ events enter the $\geq 3 b$-tag regions because $c$-jets in the $Z+\mathrm{HF}$ events get mistagged as $b$-jets, while in the $2 b$-tag region this is not the case. Hence, there are two NPs used for the $Z+\mathrm{HF}$ normalization factor. There are no NPs corresponding to the normalization factors of the $Z c l, Z l$, and $W l$ templates since their contribution to the total background is $<1 \%$. Table 27 summarises the NPs associated with the uncertainties that affect the overall normalization of the fit templates.

\begin{tabular}{|c|c|c|}
\hline NP Description & Prior & Applied to sample \\
\hline Normalization of $Z+\mathrm{HF}$ template & flat & $Z+\mathrm{HF}$ \\
Normalization of $W+\mathrm{HF}$ template & flat & $W+\mathrm{HF}$ \\
Normalization of $t \bar{t}$ template & flat & $t \bar{t}$ \\
\hline Single-top inclusive normalization & $42 \%$ & single-top $W t, s$ and $t$-channel \\
Inclusive normalization of DB processes & $16 \%$ & $W W, W Z, Z Z$ \\
Inclusive normalization of SM $V h(b \bar{b})$ & $5 \%$ & VHbb \\
Inclusive normalization uncertainty for $W c l$ & $42 \%$ & $\mathrm{Wcl}$ \\
\hline \hline Luminosity uncertainty & $1.7 \%$ & all \\
\hline
\end{tabular}

Table 27: Table adapted from [234] summarises the NPs associated to uncertainties affecting the overall normalization of the fit templates. The Gaussian prior uncertainties correspond to the $1 \sigma$ prefit uncertainties. These uncertainties are derived by comparing the MC samples produced with different MC generators, while the luminosity prior is calculated by the luminosity group. A flat prior means that there are no prior uncertainties on those NPs.

When the NPs are propagated into the fit, one must ensure the stability of the fit. For example, a histogram of a given NP may have statistical fluctuations that can cause instability in the likelihood maximization. To avoid such circumstances, the NP histograms are subjected to some treatments before being propagated into the fit, such as smoothing, pruning (c.f. section 4.4), and 
symmetrization. The smoothing procedure removes statistical fluctuations from a NP histogram and is applied only to those systematics that affect the shapes of the $m_{h}$ and $\mu$-charge distribution. The pruning procedure removes those systematics that has negligible effects on the sensitivity of the analysis and also helps to increase the stability of the likelihood fit. The application of the pruning procedure is different between the systematics affecting normalization and shape. A systematic affecting the normalization is pruned away if the difference between the systematic yield and the nominal value is $<0.5 \%$ or if the upward and downward systematic variations are in the same direction. On the other hand, a systematic variation that affects the shape is pruned away if it satisfies any of the following criteria: (i) the difference between the systematic variation and the nominal one is $<0.5 \%$ in $N_{\text {bins }}-1$, (ii) only the upward or downward systematic variation is present while both systematic variations are expected, and (iii) the difference between the upward and downward variations is smaller than the difference between upward/nominal or downward/nominal, determined by a $\chi^{2}$ test. The symmetrization procedure is used to remove asymmetric uncertainties that may lead to over- or under-constrained systematics. Symmetrization is mainly applied to the one-sided detector systematics, while the two-sided systematics can also be symmetrized by taking an average of the up/down variations.

\subsubsection{Results}

This section describes the results obtained from the statistical interpretations of the observed data for the mono- $h(b \bar{b})$ analysis.

\section{- Background-only fit:}

To assess the impact of the CRs on the estimation of backgrounds in the SR, a backgroundonly fit (i.e., assuming there is no signal) with the 0,1 , and 2-lepton regions is performed.

The post-fit $\mu$-charge distributions in the $1-\mu \mathrm{CR}$ across different $b$-tag and $E_{\mathrm{T}}^{\text {miss }}$ regions are shown in Figure 83 . The asymmetry in the $\mu$-charge distribution for the $W^{ \pm}+$jets is due to the $W$ charge asymmetry at the LHC. The blue dashed line represents the pre-fit background predictions. In the $1-\mu \mathrm{CR}$ there is a normalization disagreement between the $\mathrm{MC}$ prediction and observed data before the fit. This is because the MC generators underestimate the $t \bar{t}$ event rates by $\sim 10 \%$. The 
normalization disagreement is resolved by the floating NPs controlling the normalization factors. Table 28 summaries the values of the normalization factors of $t \bar{t}$ and $W+$ HF backgrounds which are obtained from the background-only fit. Overall there is a good agreement between the MC background predictions and the observed data after the fit. The pre-fit and post-fit yields for the observed data and SM background processes for $2 b$ and $\geq 3 b$-tag regions are summarized in Table 29 and 30 respectively.

The post-fit yields for the combined 2-lepton CR across different $E_{\mathrm{T}}^{\text {miss }}$ and $b$-tag regions are shown in Figure 84. The blue dashed line represents the pre-fit background prediction, and since the MC generators underestimate the $Z+\mathrm{HF}$ event rate by $\sim 20-40 \%$, the pre-fit prediction is underestimated compared to the observed data. This is resolved by the NPs controlling the $Z+\mathrm{HF}$ normalization factors. The values of these normalization factors are obtained from the backgroundonly fit and are summarized in Table 28. Overall, there is good agreement between the post-fit MC background yield and the observed data yield across all the $E_{\mathrm{T}}^{\mathrm{miss}}$ bins and $b$-tag regions for the 2-lepton CR. Tables 31 and 32 show the pre-fit and post-fit background yields, and the observed data yields for the 2-lepton CR across different $E_{\mathrm{T}}^{\text {miss }}$ bins in the $2 b, \geq 3 b$-tag regions respectively.

Figures 85 and 86 show the $m_{h}$ distributions in the SR across different $E_{\mathrm{T}}^{\text {miss }}$ bins for the $2 b$ and $\geq 3 b$-tag regions respectively. Overall, there is good post-fit agreement between the observed data and the MC background prediction across all the $E_{\mathrm{T}}^{\text {miss }}$ bins and $b$-tag categories. However, in the $\geq 3 b$-tag category a data deficit is observed in $350<E_{\mathrm{T}}^{\text {miss }} \leq 500 \mathrm{GeV}$, and $E_{\mathrm{T}}^{\text {miss }}>500 \mathrm{GeV}$ regions. Tables 33 and 34 show the pre-fit and post-fit background yields and the observed data yields for the 0 -lepton SR across different $E_{\mathrm{T}}^{\text {miss }}$ bins in the $2 b, \geq 3 b$-tag regions respectively.

\begin{tabular}{|c|c|}
\hline Normalization factors of & Normalization factor value \\
\hline$t \bar{t}$ & $0.89 \pm 0.8$ \\
$W+\mathrm{HF}$ & $0.98 \pm 0.13$ \\
$Z+\mathrm{HF} 2 b$-tag & $1.42 \pm 0.08$ \\
$Z+\mathrm{HF} \geq 3 b$-tag & $1.88 \pm 0.25$ \\
\hline
\end{tabular}

Table 28: Normalization factors for the three major SM backgrounds obtained from the background-only fit. 

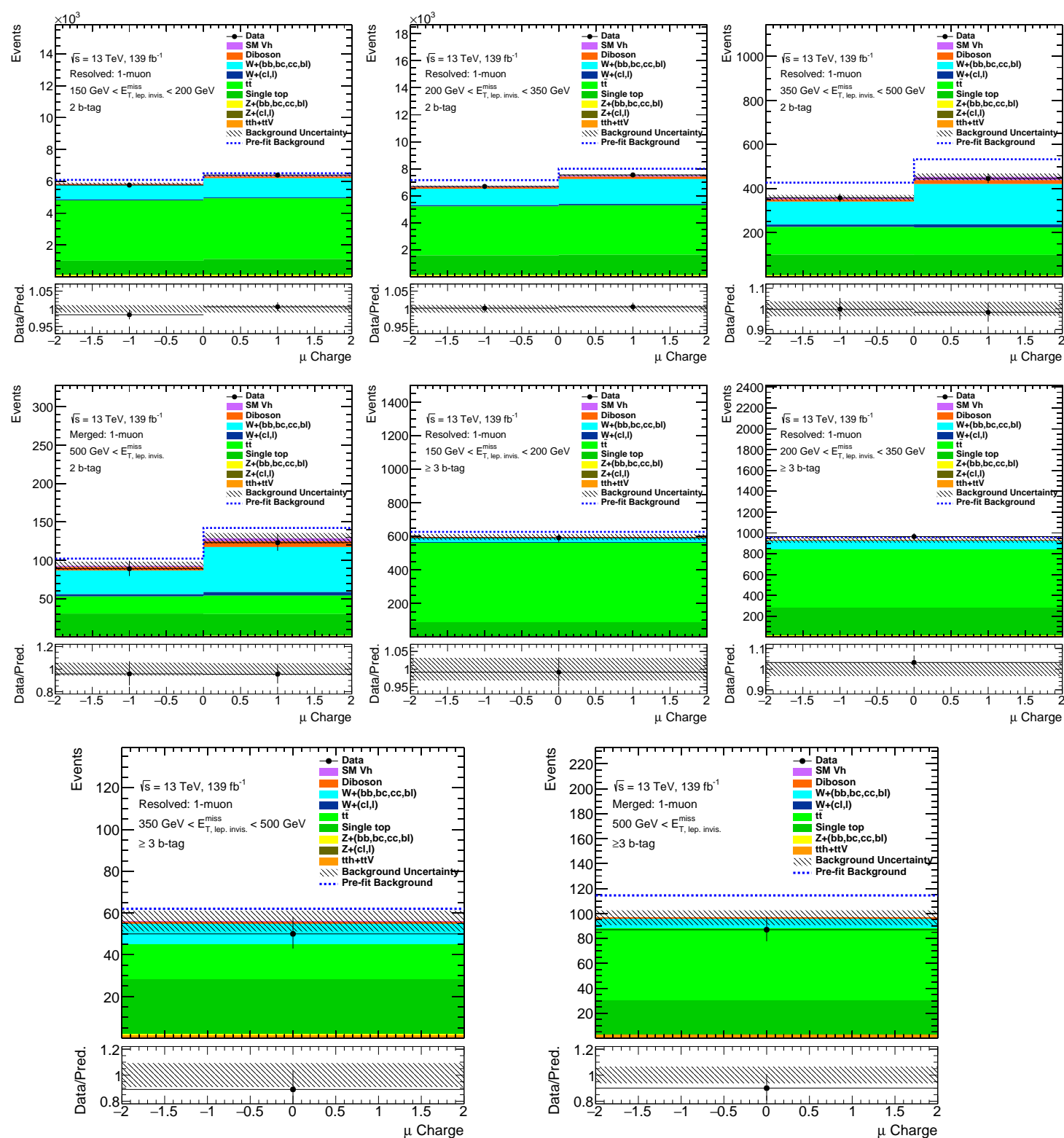

Figure 83: The $\mu$-charge distributions in the $1-\mu \mathrm{CR}$ across different $b$-tag categories and $E_{\mathrm{T}}^{\text {miss }}$ bins after the background-only fit. The blue dashed line represents the background prediction from MC before the simultaneous fit. The second canvas shows the ratio between the fitted background prediction and the observed data, and the dashed gray band represents the total MC uncertainty. 


\begin{tabular}{|l|c|c|c|c|}
\hline & $1 \mathrm{~L} 2 b$ & $1 \mathrm{~L} 2 b$ & $1 \mathrm{~L} 2 b$ & $1 \mathrm{~L} 2 b$ \\
& $150-200 \mathrm{GeV}$ & $200-350 \mathrm{GeV}$ & $350-500 \mathrm{GeV}$ & $>500 \mathrm{GeV}$ \\
\hline Observed data & 12152 & 14236 & 804 & 212 \\
\hline Pre-fit $Z+(b b, b c, c c, b l)$ & $157 \pm 13$ & $152 \pm 14$ & $7.6 \pm 1.1$ & $1.47 \pm 0.19$ \\
Pre-fit $Z+(c l, l)$ & $6.8 \pm 2.8$ & $6.1 \pm 2.2$ & $0.55 \pm 0.29$ & $0.15 \pm 0.11$ \\
Pre-fit $W+(b b, b c, c c, b l)$ & $2140 \pm 120$ & $3170 \pm 220$ & $312 \pm 27$ & $98 \pm 8$ \\
Pre-fit $W+(c l, l)$ & $190 \pm 80$ & $280 \pm 120$ & $42 \pm 16$ & $12 \pm 5$ \\
Pre-fit $t \bar{t}$ & $8200 \pm 1000$ & $8300 \pm 1000$ & $330 \pm 50$ & $58 \pm 6$ \\
Pre-fit Single top & $1600 \pm 700$ & $2800 \pm 1200$ & $220 \pm 110$ & $56 \pm 24$ \\
Pre-fit Diboson & $193 \pm 32$ & $300 \pm 50$ & $30 \pm 5$ & $12.7 \pm 2.3$ \\
Pre-fit SM $V h(b \bar{b})$ & $79 \pm 5$ & $132 \pm 9$ & $22.5 \pm 1.6$ & $5.9 \pm 0.6$ \\
Pre-fit $t t h, t t V$ & $14.1 \pm 0.9$ & $28.8 \pm 1.2$ & $2.18 \pm 0.23$ & $1.57 \pm 0.11$ \\
\hline Total Pre-fit SM Bkg & $12600 \pm 1300$ & $15200 \pm 1800$ & $960 \pm 130$ & $224 \pm 31$ \\
\hline \hline Post-fit $Z+(b b, b c, c c, b l)$ & $229 \pm 12$ & $219 \pm 10$ & $11.1 \pm 1.0$ & $1.79 \pm 0.20$ \\
Post-fit $Z+(c l, l)$ & $4.7 \pm 1.3$ & $5.7 \pm 1.6$ & $0.63 \pm 0.25$ & $0.08 \pm 0.05$ \\
Post-fit $W+(b b, b c, c c, b l)$ & $2090 \pm 260$ & $3000 \pm 400$ & $290 \pm 40$ & $90 \pm 12$ \\
Post-fit $W+(c l, l)$ & $110 \pm 50$ & $190 \pm 70$ & $25 \pm 9$ & $7.4 \pm 2.8$ \\
Post-fit $t \bar{t}$ & $7600 \pm 400$ & $7300 \pm 500$ & $253 \pm 31$ & $47 \pm 5$ \\
Post-fit Single top & $1900 \pm 500$ & $2900 \pm 700$ & $180 \pm 50$ & $58 \pm 13$ \\
Post-fit Diboson & $192 \pm 21$ & $295 \pm 32$ & $29 \pm 4$ & $12.2 \pm 1.4$ \\
Post-fit SM $V h(b \bar{b})$ & $80 \pm 5$ & $133 \pm 9$ & $22.3 \pm 1.6$ & $5.8 \pm 0.5$ \\
Post-fit $t t h, t t V$ & $13.8 \pm 0.6$ & $28.0 \pm 1.0$ & $2.08 \pm 0.16$ & $1.49 \pm 0.07$ \\
\hline Total Post-fit SM Bkg & $12210 \pm 110$ & $14180 \pm 120$ & $813 \pm 24$ & $224 \pm 10$ \\
\hline
\end{tabular}

Table 29: Pre-fit and Post-fit (background-only) yields for the $1-\mu \mathrm{CR}$ in the $2 b$-tag region across different $E_{\mathrm{T}}^{\mathrm{miss}}$ bins [234].

\begin{tabular}{|l|c|c|c|c|}
\hline & $1 \mathrm{~L} \geq 3 b$ & $1 \mathrm{~L} \geq 3 b$ & $1 \mathrm{~L} \geq 3 b$ & $1 \mathrm{~L} \geq 3 b$ \\
& $150-200 \mathrm{GeV}$ & $200-350 \mathrm{GeV}$ & $350-500 \mathrm{GeV}$ & $>500 \mathrm{GeV}$ \\
\hline Observed data & 591 & 965 & 50 & 87 \\
\hline Pre-fit $Z+(b b, b c, c c, b l)$ & $2.7 \pm 0.6$ & $5.3 \pm 0.5$ & $0.6 \pm 0.5$ & $0.197 \pm 0.026$ \\
Pre-fit $Z+(c l, l)$ & $0.020 \pm 0.010$ & $0.19 \pm 0.22$ & $0.024 \pm 0.008$ & - \\
Pre-fit $W+(b b, b c, c c, b l)$ & $30 \pm 5$ & $90 \pm 17$ & $12.2 \pm 2.5$ & $8.6 \pm 1.3$ \\
Pre-fit $W+(c l, l)$ & $3.5 \pm 2.3$ & $7 \pm 4$ & $0.06 \pm 0.04$ & $0.58 \pm 0.32$ \\
Pre-fit $t \bar{t}$ & $530 \pm 90$ & $640 \pm 140$ & $21 \pm 5$ & $74 \pm 9$ \\
Pre-fit Single top & $55 \pm 31$ & $200 \pm 110$ & $25 \pm 15$ & $27 \pm 12$ \\
Pre-fit Diboson & $2.1 \pm 0.6$ & $7.0 \pm 1.6$ & $1.22 \pm 0.34$ & $1.03 \pm 0.25$ \\
Pre-fit SM $V h(b \bar{b})$ & $0.40 \pm 0.08$ & $1.65 \pm 0.32$ & $0.34 \pm 0.06$ & $0.224 \pm 0.033$ \\
Pre-fit $t t h, t t V$ & $3.25 \pm 0.35$ & $9.8 \pm 0.4$ & $1.27 \pm 0.16$ & $2.49 \pm 0.20$ \\
\hline Total pre-fit SM Bkg & $630 \pm 100$ & $960 \pm 190$ & $62 \pm 17$ & $114 \pm 17$ \\
\hline \hline Post-fit $Z+(b b, b c, c c, b l)$ & $4.7 \pm 0.9$ & $9.0 \pm 1.1$ & $0.8 \pm 0.4$ & $0.34 \pm 0.05$ \\
Post-fit $Z+(c l, l)$ & $0.013 \pm 0.007$ & $0.05 \pm 0.07$ & $0.020 \pm 0.006$ & - \\
Post-fit $W+(b b, b c, c c, b l)$ & $30 \pm 6$ & $83 \pm 16$ & $9.9 \pm 2.0$ & $7.3 \pm 1.1$ \\
Post-fit $W+(c l, l)$ & $2.2 \pm 1.3$ & $4.4 \pm 2.1$ & $0.031 \pm 0.020$ & $0.32 \pm 0.16$ \\
Post-fit $t \bar{t}$ & $474 \pm 31$ & $560 \pm 50$ & $16.8 \pm 2.5$ & $59 \pm 6$ \\
Post-fit Single top & $80 \pm 23$ & $260 \pm 60$ & $26 \pm 6$ & $27 \pm 6$ \\
Post-fit Diboson & $1.8 \pm 0.4$ & $6.1 \pm 1.1$ & $1.04 \pm 0.23$ & $0.91 \pm 0.15$ \\
Post-fit SM $V h(b \bar{b})$ & $0.38 \pm 0.07$ & $1.58 \pm 0.28$ & $0.31 \pm 0.06$ & $0.203 \pm 0.022$ \\
Post-fit $t t h, t t V$ & $3.15 \pm 0.23$ & $9.5 \pm 0.4$ & $1.24 \pm 0.12$ & $2.39 \pm 0.15$ \\
\hline Total post-fit SM Bkg & $597 \pm 18$ & $935 \pm 29$ & $56 \pm 5$ & $97 \pm 6$ \\
\hline
\end{tabular}

Table 30: Pre-fit and Post-fit (background-only) yields for the $1-\mu \mathrm{CR}$ in the $\geq 3 b$-tag region across different $E_{\mathrm{T}}^{\mathrm{miss}}$ bins $[234]$. 

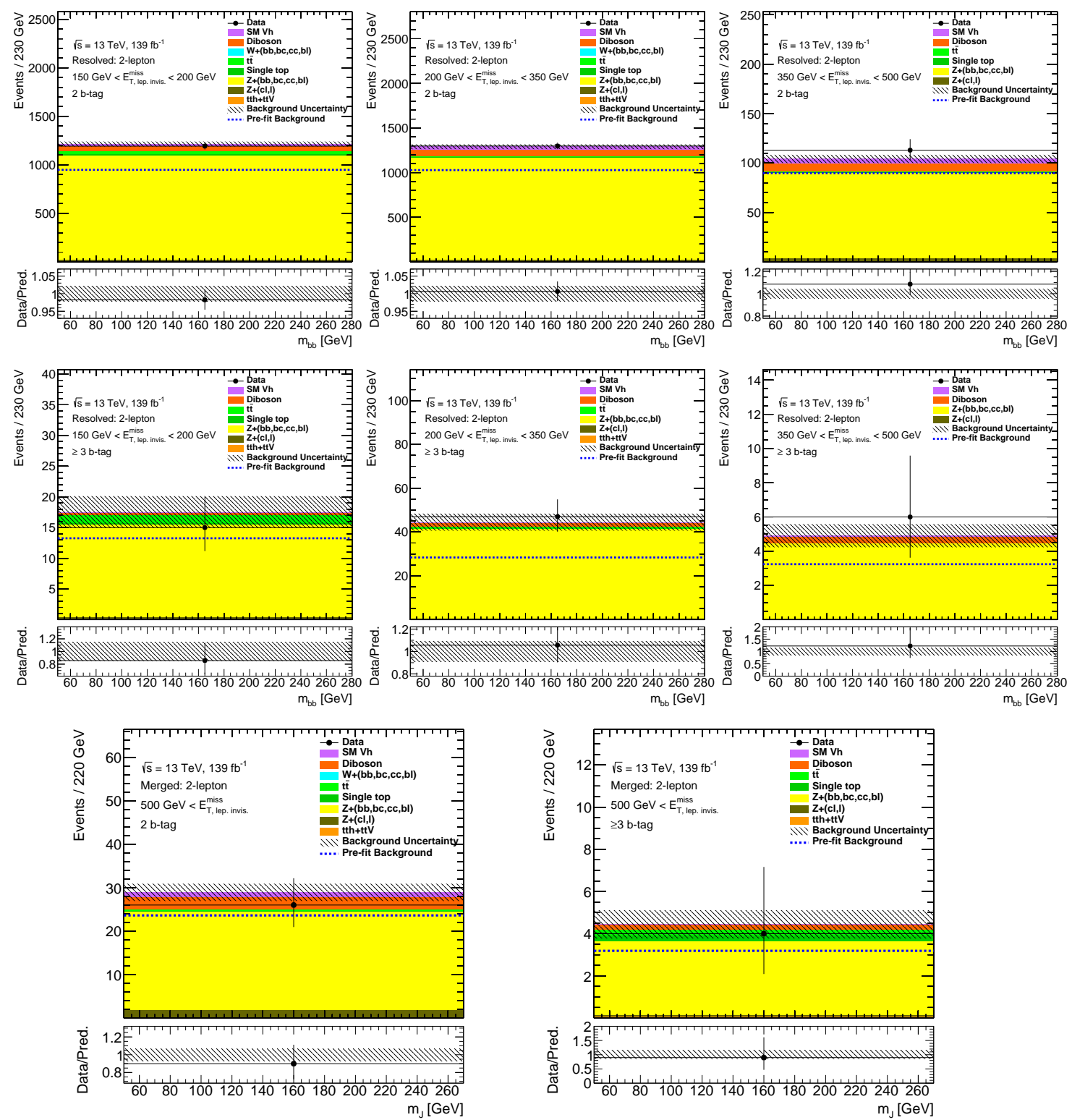

Figure 84: The total yield in the combined 2-lepton CR across different $b$-tag and $E_{\mathrm{T}}^{\text {miss }}$ regions after the background-only fit. The blue dashed line represents the background prediction from MC before the simultaneous fit. The second canvas shows the ratio between the fitted background prediction and the observed data, and the dashed gray band represents the total MC uncertainty. 


\begin{tabular}{|l|c|c|c|c|}
\hline & $2 \mathrm{~L} 2 b$ & $2 \mathrm{~L} 2 b$ & $2 \mathrm{~L} 2 b$ & $2 \mathrm{~L} 2 b$ \\
& $150-200 \mathrm{GeV}$ & $200-350 \mathrm{GeV}$ & $350-500 \mathrm{GeV}$ & $>500 \mathrm{GeV}$ \\
\hline Observed data & 1193 & 1298 & 113 & 26 \\
\hline Pre-fit $Z+(b b, b c, c c, b l)$ & $810 \pm 110$ & $870 \pm 120$ & $71 \pm 10$ & $16.7 \pm 1.4$ \\
Pre-fit $Z+(c l, l)$ & $22 \pm 8$ & $30 \pm 8$ & $4.2 \pm 1.5$ & $2.2 \pm 0.7$ \\
Pre-fit $W+(b b, b c, c c, b l)$ & $0.065 \pm 0.035$ & $0.28 \pm 0.16$ & - & $0.00056 \pm 0.00006$ \\
Pre-fit $W+(c l, l)$ & - & - & - & - \\
Pre-fit $t \bar{t}$ & $30 \pm 10$ & $7.0 \pm 2.5$ & $0.30 \pm 0.09$ & $0.25 \pm 0.09$ \\
Pre-fit Single top & $10 \pm 6$ & $5.3 \pm 3.5$ & $0.6 \pm 0.5$ & $0.28 \pm 0.26$ \\
Pre-fit Diboson & $53 \pm 9$ & $78 \pm 14$ & $9.0 \pm 1.6$ & $3.0 \pm 0.5$ \\
Pre-fit SM $V h(b \bar{b})$ & $23.7 \pm 1.9$ & $33.7 \pm 2.8$ & $4.7 \pm 0.4$ & $1.21 \pm 0.12$ \\
Pre-fit $t t h, t t V$ & $0.066 \pm 0.032$ & $0.06 \pm 0.05$ & - & $0.0025 \pm 0.0005$ \\
\hline Total pre-fit SM Bkg & $950 \pm 120$ & $1030 \pm 130$ & $90 \pm 11$ & $23.6 \pm 2.3$ \\
\hline \hline Post-fit $Z+(b b, b c, c c, b l)$ & $1079 \pm 28$ & $1144 \pm 29$ & $87 \pm 4$ & $23.0 \pm 1.6$ \\
Post-fit $Z+(c l, l)$ & $15 \pm 5$ & $23 \pm 5$ & $3.2 \pm 0.9$ & $1.7 \pm 0.5$ \\
Post-fit $W+(b b, b c, c c, b l)$ & $0.063 \pm 0.035$ & $0.26 \pm 0.15$ & - & $0.00053 \pm 0.00009$ \\
Post-fit $W+(c l, l)$ & - & - & - & - \\
Post-fit $t \bar{t}$ & $30 \pm 8$ & $5.2 \pm 1.5$ & $0.23 \pm 0.07$ & $0.26 \pm 0.07$ \\
Post-fit Single top & $12 \pm 6$ & $6.7 \pm 3.0$ & $0.62 \pm 0.31$ & $0.23 \pm 0.16$ \\
Post-fit Diboson & $54 \pm 6$ & $76 \pm 9$ & $8.7 \pm 1.1$ & $2.9 \pm 0.4$ \\
Post-fit SM $V h(b \bar{b})$ & $23.9 \pm 1.7$ & $33.9 \pm 2.4$ & $4.61 \pm 0.35$ & $1.18 \pm 0.10$ \\
Post-fit $t t h, t t V$ & $0.064 \pm 0.024$ & $0.043 \pm 0.023$ & - & $0.0022 \pm 0.0004$ \\
\hline Total post-fit SM Bkg & $1215 \pm 26$ & $1289 \pm 27$ & $104 \pm 4$ & $29.3 \pm 2.0$ \\
\hline
\end{tabular}

Table 31: Pre-fit and Post-fit (background-only) yields for the combined 2-lepton CR in the $2 b$ tag region across different $E_{\mathrm{T}}^{\text {miss }}$ bins [234].

\begin{tabular}{|l|c|c|c|c|}
\hline & $2 \mathrm{~L} \geq 3 b$ & $2 \mathrm{~L} \geq 3 b$ & $2 \mathrm{~L} \geq 3 b$ & $2 \mathrm{~L} \geq 3 b$ \\
& $150-200 \mathrm{GeV}$ & $200-350 \mathrm{GeV}$ & $350-500 \mathrm{GeV}$ & $>500 \mathrm{GeV}$ \\
\hline Observed data & 15 & 47 & 6 & 4 \\
\hline Pre-fit $Z+(b b, b c, c c, b l)$ & $10.0 \pm 2.4$ & $24 \pm 4$ & $2.7 \pm 0.5$ & $2.2 \pm 0.4$ \\
Pre-fit $Z+(c l, l)$ & $0.6 \pm 0.7$ & $0.32 \pm 0.28$ & $0.021 \pm 0.011$ & $0.12 \pm 0.04$ \\
Pre-fit $W+(b b, b c, c c, b l)$ & - & - & - & - \\
Pre-fit $W+(c l, l)$ & - & - & - & - \\
Pre-fit $t \bar{t}$ & $1.5 \pm 0.8$ & $1.3 \pm 1.0$ & - & $0.33 \pm 0.09$ \\
Pre-fit Single top & $0.4 \pm 0.6$ & - & - & $0.24 \pm 0.16$ \\
Pre-fit Diboson & $0.49 \pm 0.24$ & $1.8 \pm 0.4$ & $0.40 \pm 0.10$ & $0.24 \pm 0.05$ \\
Pre-fit SM $V h(b \bar{b})$ & $0.158 \pm 0.029$ & $0.50 \pm 0.10$ & $0.082 \pm 0.017$ & $0.049 \pm 0.007$ \\
Pre-fit $t t h, t t V$ & $0.0042 \pm 0.0004$ & $0.036 \pm 0.010$ & - & $0.041 \pm 0.005$ \\
\hline Total pre-fit SM Bkg & $13 \pm 4$ & $28 \pm 5$ & $3.2 \pm 0.5$ & $3.2 \pm 0.5$ \\
\hline \hline Post-fit $Z+(b b, b c, c c, b l)$ & $15.1 \pm 2.2$ & $41 \pm 4$ & $4.4 \pm 0.7$ & $3.5 \pm 0.6$ \\
Post-fit $Z+(c l, l)$ & $0.4 \pm 0.4$ & $0.42 \pm 0.29$ & $0.014 \pm 0.007$ & $0.075 \pm 0.021$ \\
Post-fit $W+(b b, b c, c c, b l)$ & - & - & - & - \\
Post-fit $W+(c l, l)$ & - & - & - & - \\
Post-fit $t \bar{t}$ & $1.2 \pm 0.5$ & $0.8 \pm 0.5$ & - & $0.27 \pm 0.08$ \\
Post-fit Single top & $0.27 \pm 0.29$ & - & - & $0.27 \pm 0.12$ \\
Post-fit Diboson & $0.39 \pm 0.13$ & $1.75 \pm 0.34$ & $0.38 \pm 0.08$ & $0.22 \pm 0.04$ \\
Post-fit SM $V h(b \bar{b})$ & $0.146 \pm 0.028$ & $0.49 \pm 0.09$ & $0.075 \pm 0.015$ & $0.045 \pm 0.007$ \\
Post-fit $t t h, t t V$ & $0.0040 \pm 0.0004$ & $0.030 \pm 0.005$ & - & $0.038 \pm 0.006$ \\
\hline Total post-fit SM Bkg & $17.5 \pm 2.6$ & $44 \pm 4$ & $4.9 \pm 0.7$ & $4.5 \pm 0.7$ \\
\hline
\end{tabular}

Table 32: Pre-fit and Post-fit (background-only) yields for the combined 2-lepton CR in the $\geq 3$ $b$-tag region across different $E_{\mathrm{T}}^{\text {miss }}$ bins [234]. 

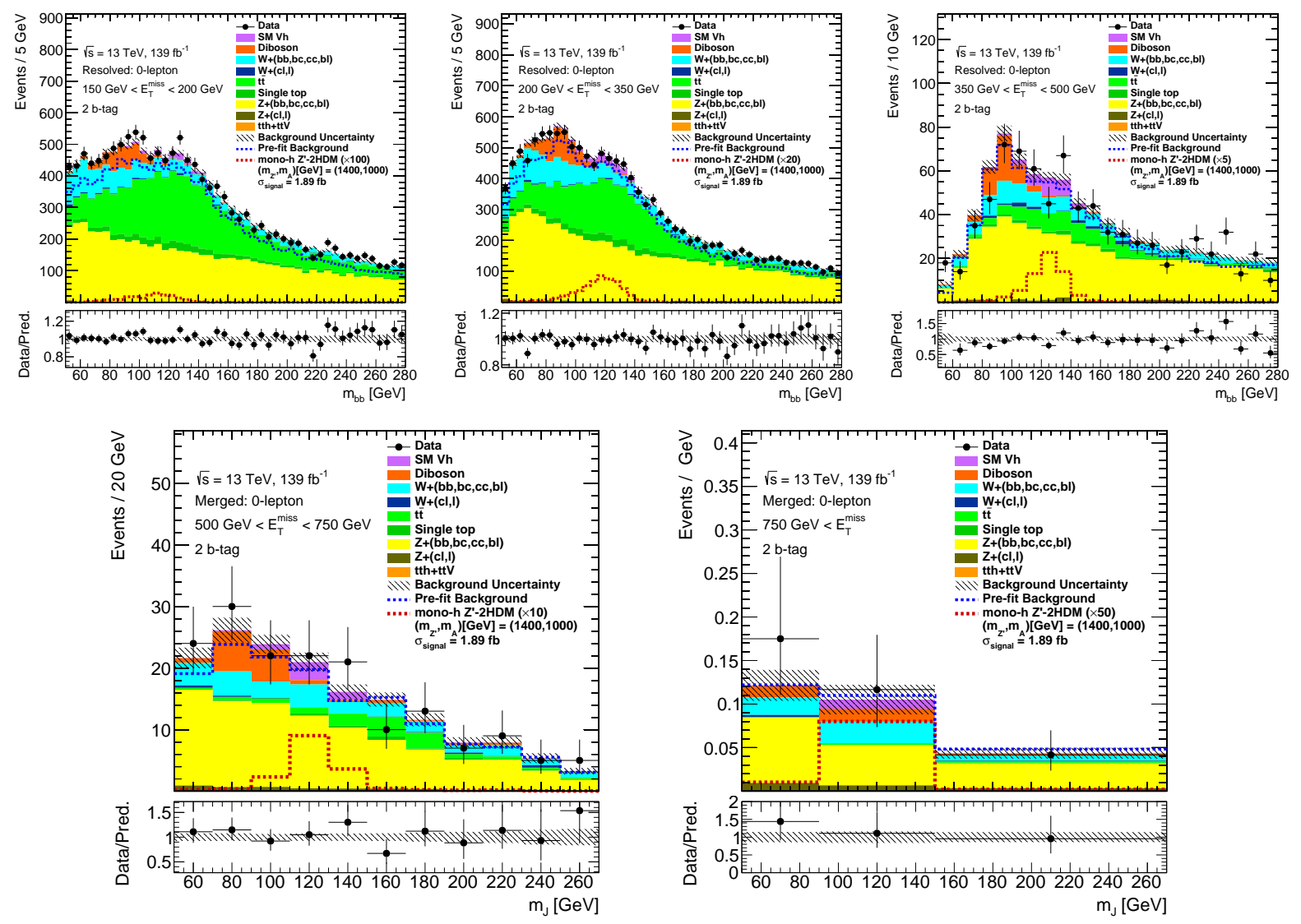

Figure 85: The Higgs candidate mass distribution in the 0-lepton SR for the $2 b$-tag category across different $E_{\mathrm{T}}^{\text {miss }}$ bins after the background-only fit. The blue dashed line represents the background prediction from MC before the simultaneous fit. The second canvas shows the ratio between the fitted background prediction and the observed data, and the dashed gray band represents the total MC uncertainty. The red dashed line shows an example signal point from the $Z^{\prime}$-2HDM model, which is scaled by a constant factor to make it visible [234]. 

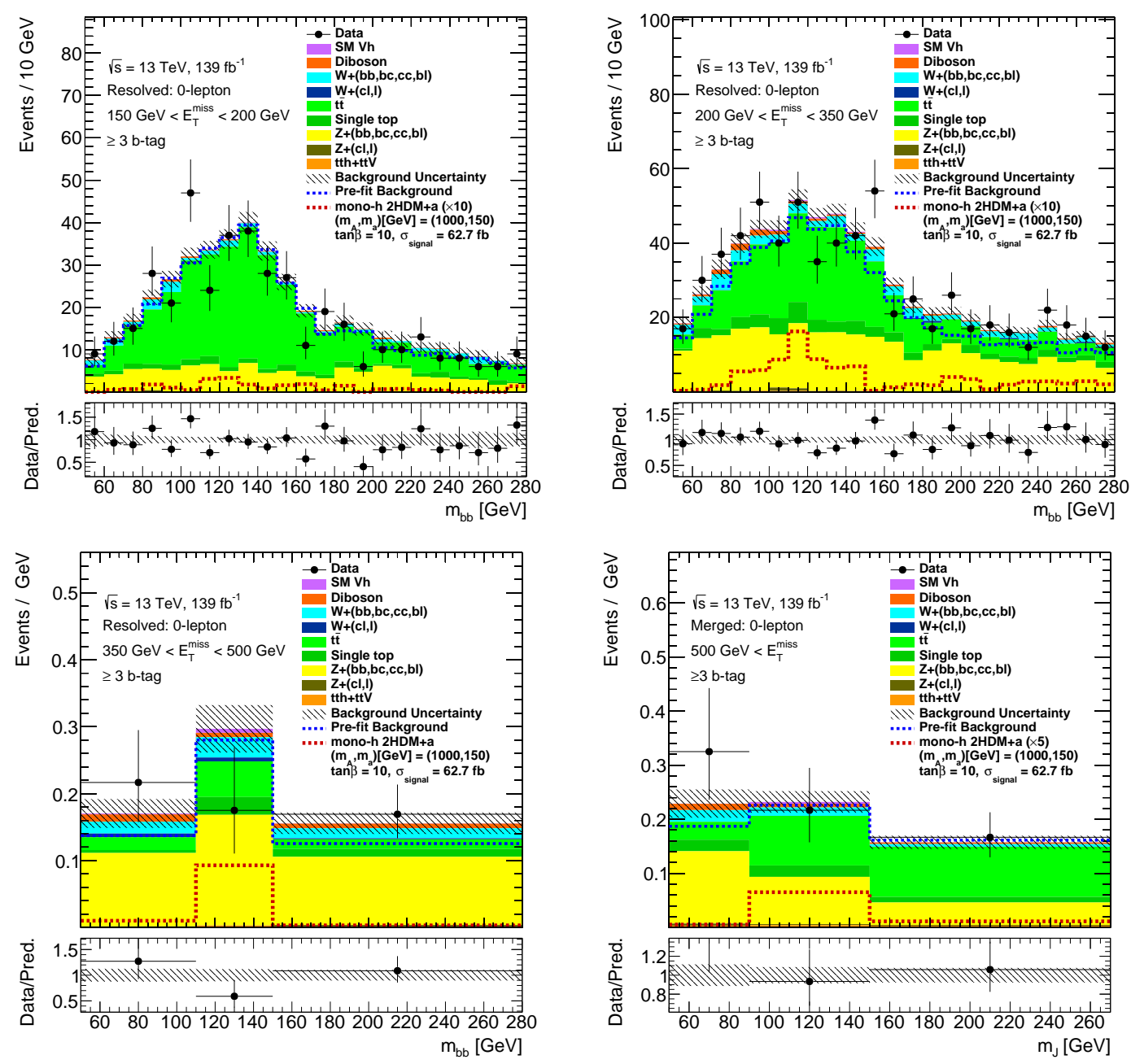

Figure 86: The Higgs candidate mass distribution in the 0-lepton SR for the $\geq 3 b$-tag category across different $E_{\mathrm{T}}^{\text {miss }}$ bins after the background-only fit. The blue dashed line represents the background prediction from MC before the simultaneous fit. The second canvas shows the ratio between the fitted background prediction and the observed data, and the dashed gray band represents the total MC uncertainty. The red dashed line shows an example signal point from the $2 \mathrm{HDM}+a b b$-induced model, which is scaled by a constant factor to make it visible [234]. 


\begin{tabular}{|l|c|c|c|c|c|}
\hline & $0 \mathrm{~L} 2 b$ & $0 \mathrm{~L} 2 b$ & $0 \mathrm{~L} 2 b$ & $0 \mathrm{~L} 2+0 b$ & $0 \mathrm{~L} 2 b$ \\
& $150-200 \mathrm{GeV}$ & $200-350 \mathrm{GeV}$ & $350-500 \mathrm{GeV}$ & $500-750 \mathrm{GeV}$ & $>750 \mathrm{GeV}$ \\
\hline Observed data & 14259 & 13724 & 799 & 168 & 19 \\
\hline Pre-fit $Z+(b b, b c, c c, b l)$ & $450 \pm 230$ & $5110 \pm 350$ & $377 \pm 24$ & $72 \pm 8$ & $8.3 \pm 2.1$ \\
Pre-fit $Z+(c l, l)$ & $91 \pm 23$ & $170 \pm 40$ & $26 \pm 8$ & $5.9 \pm 1.6$ & $1.5 \pm 0.5$ \\
Pre-fit $W+(b b, b c, c c, b l)$ & $1570 \pm 130$ & $1800 \pm 130$ & $116 \pm 10$ & $25.6 \pm 3.4$ & $3.7 \pm 0.7$ \\
Pre-fit $W+(c l, l)$ & $120 \pm 60$ & $140 \pm 60$ & $21 \pm 8$ & $2.6 \pm 1.1$ & $0.36 \pm 0.17$ \\
Pre-fit $t \bar{t}$ & $4800 \pm 600$ & $3600 \pm 500$ & $96 \pm 15$ & $14.3 \pm 2.4$ & $0.50 \pm 0.12$ \\
Pre-fit Single top & $600 \pm 400$ & $700 \pm 500$ & $33 \pm 24$ & $7 \pm 7$ & $0.5 \pm 0.7$ \\
Pre-fit Diboson & $440 \pm 70$ & $600 \pm 100$ & $60 \pm 11$ & $15.6 \pm 2.8$ & $1.68 \pm 0.34$ \\
Pre-fit SM $V h(b \bar{b})$ & $147 \pm 10$ & $200 \pm 14$ & $26.7 \pm 1.9$ & $5.8 \pm 0.6$ & $0.73 \pm 0.08$ \\
Pre-fit $t t h, t t V$ & $8.0 \pm 0.7$ & $12.3 \pm 0.7$ & $0.54 \pm 0.11$ & $0.32 \pm 0.04$ & $0.036 \pm 0.006$ \\
\hline Total pre-fit SM Bkg & $12300 \pm 1100$ & $12400 \pm 1100$ & $750 \pm 60$ & $149 \pm 15$ & $17.3 \pm 2.8$ \\
\hline \hline Post-fit $Z+(b b, b c, c c, b l)$ & $6470 \pm 310$ & $7200 \pm 310$ & $508 \pm 26$ & $94 \pm 7$ & $9.3 \pm 1.8$ \\
Post-fit $Z+(c l, l)$ & $70 \pm 14$ & $132 \pm 27$ & $18 \pm 4$ & $4.4 \pm 1.0$ & $1.18 \pm 0.31$ \\
Post-fit $W+(b b, b c, c c, b l)$ & $1580 \pm 210$ & $1750 \pm 230$ & $106 \pm 14$ & $25 \pm 4$ & $3.1 \pm 0.6$ \\
Post-fit $W+(c l, l)$ & $82 \pm 33$ & $89 \pm 34$ & $13 \pm 5$ & $1.6 \pm 0.6$ & $0.20 \pm 0.09$ \\
Post-fit $t \bar{t}$ & $4690 \pm 290$ & $3290 \pm 240$ & $77 \pm 9$ & $11.7 \pm 1.6$ & $0.40 \pm 0.08$ \\
Post-fit Single top & $570 \pm 260$ & $570 \pm 260$ & $21 \pm 10$ & $2.6 \pm 1.9$ & $0.11 \pm 0.17$ \\
Post-fit Diboson & $450 \pm 50$ & $600 \pm 60$ & $56 \pm 7$ & $15.3 \pm 1.9$ & $1.70 \pm 0.28$ \\
Post-fit SM $V h(b \bar{b})$ & $151 \pm 10$ & $202 \pm 13$ & $26.5 \pm 1.8$ & $5.7 \pm 0.5$ & $0.72 \pm 0.12$ \\
Post-fit $t t h, t t V$ & $7.6 \pm 0.4$ & $11.8 \pm 0.5$ & $0.45 \pm 0.06$ & $0.287 \pm 0.029$ & $0.037 \pm 0.006$ \\
\hline Total post-fit SM Bkg & $14070 \pm 110$ & $13860 \pm 100$ & $825 \pm 19$ & $160 \pm 8$ & $16.7 \pm 1.9$ \\
\hline
\end{tabular}

Table 33: Pre-fit and Post-fit (background-only) yields for the 0-lepton SR in the $2 b$-tag region across different $E_{\mathrm{T}}^{\mathrm{miss}}$ bins [234].

\begin{tabular}{|l|c|c|c|c|}
\hline & $\begin{array}{l}0 \mathrm{~L} \geq 3 b \\
150-200 \mathrm{GeV}\end{array}$ & $\begin{array}{c}0 \mathrm{~L} \geq 3 b \\
200-350 \mathrm{GeV}\end{array}$ & $\begin{array}{c}0 \mathrm{~L} \geq 3 b \\
350-500 \mathrm{GeV}\end{array}$ & $\begin{array}{c}0 \mathrm{~L} \geq 3 b \\
>500 \mathrm{GeV}\end{array}$ \\
\hline Observed data & 408 & 658 & 42 & 46 \\
\hline Pre-fit $Z+(b b, b c, c c, b l)$ & $54 \pm 11$ & $152 \pm 18$ & $15.3 \pm 2.3$ & $9.0 \pm 1.3$ \\
Pre-fit $Z+(c l, l)$ & $0.8 \pm 0.5$ & $3.9 \pm 1.2$ & $0.35 \pm 0.16$ & $0.65 \pm 0.20$ \\
Pre-fit $W+(b b, b c, c c, b l)$ & $23 \pm 4$ & $48 \pm 9$ & $5.3 \pm 1.3$ & $2.5 \pm 0.5$ \\
Pre-fit $W+(c l, l)$ & $0.08 \pm 0.14$ & $2.9 \pm 1.9$ & $1.5 \pm 0.7$ & $0.06 \pm 0.04$ \\
Pre-fit $t \bar{t}$ & $300 \pm 50$ & $280 \pm 60$ & $6.7 \pm 1.6$ & $21.3 \pm 2.9$ \\
Pre-fit Single top & $23 \pm 17$ & $50 \pm 40$ & $3.6 \pm 3.1$ & $4.4 \pm 3.1$ \\
Pre-fit Diboson & $4.4 \pm 1.7$ & $13.5 \pm 3.1$ & $2.2 \pm 0.6$ & $1.5 \pm 0.5$ \\
Pre-fit SM $V h(b \bar{b})$ & $0.8 \pm 0.5$ & $3.0 \pm 0.6$ & $0.44 \pm 0.08$ & $0.254 \pm 0.033$ \\
Pre-fit $t t h, t t V$ & $1.86 \pm 0.25$ & $4.3 \pm 0.4$ & $0.40 \pm 0.05$ & $0.78 \pm 0.12$ \\
\hline Total pre-fit SM Bkg & $410 \pm 70$ & $560 \pm 100$ & $36 \pm 6$ & $40 \pm 6$ \\
\hline \hline Post-fit $Z+(b b, b c, c c, b l)$ & $101 \pm 15$ & $277 \pm 28$ & $26.4 \pm 3.5$ & $15.6 \pm 2.0$ \\
Post-fit $Z+(c l, l)$ & $0.6 \pm 0.4$ & $2.8 \pm 0.7$ & $0.34 \pm 0.12$ & $0.44 \pm 0.11$ \\
Post-fit $W+(b b, b c, c c, b l)$ & $21 \pm 4$ & $47 \pm 9$ & $4.2 \pm 0.9$ & $2.4 \pm 0.4$ \\
Post-fit $W+(c l, l)$ & $0.01 \pm 0.04$ & $1.6 \pm 0.8$ & $0.7 \pm 0.4$ & $0.029 \pm 0.025$ \\
Post-fit $t \bar{t}$ & $277 \pm 19$ & $253 \pm 22$ & $5.2 \pm 0.7$ & $18.2 \pm 1.8$ \\
Post-fit Single top & $23 \pm 11$ & $54 \pm 25$ & $2.8 \pm 1.4$ & $3.4 \pm 1.7$ \\
Post-fit Diboson & $4.8 \pm 1.4$ & $12.8 \pm 2.2$ & $1.8 \pm 0.4$ & $1.26 \pm 0.31$ \\
Post-fit SM $V h(b \bar{b})$ & $0.65 \pm 0.28$ & $2.9 \pm 0.5$ & $0.40 \pm 0.07$ & $0.231 \pm 0.025$ \\
Post-fit $t t h, t t V$ & $1.78 \pm 0.17$ & $3.89 \pm 0.26$ & $0.371 \pm 0.035$ & $0.79 \pm 0.08$ \\
\hline Total post-fit SM Bkg & $430 \pm 15$ & $656 \pm 21$ & $42 \pm 4$ & $42.3 \pm 2.8$ \\
\hline
\end{tabular}

Table 34: Pre-fit and Post-fit (background-only) yields for the 0-lepton SR in the $\geq 3 b$-tag region across different $E_{\mathrm{T}}^{\mathrm{miss}}$ bins $[234]$. 
Figure 87 shows the $E_{\mathrm{T}}^{\text {miss }}$ distributions after the background-only fit across different lepton, $b$-tag and $E_{\mathrm{T}}^{\text {miss }}$ regions. Similar to the other post-fit plots shown above, there is good Data-MC agreement across all the regions.

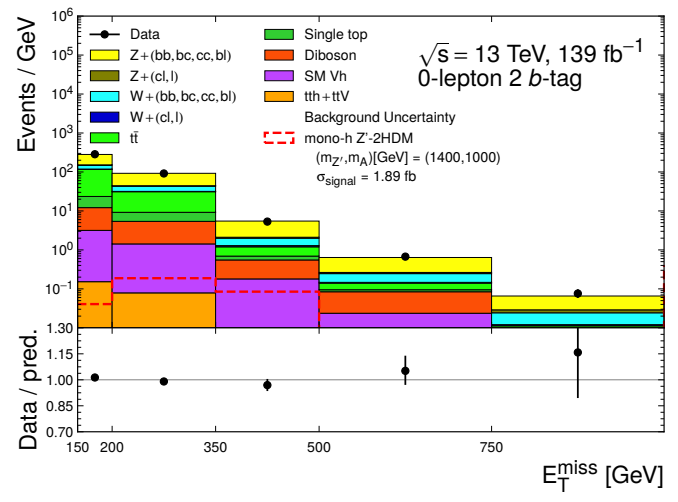

(a)

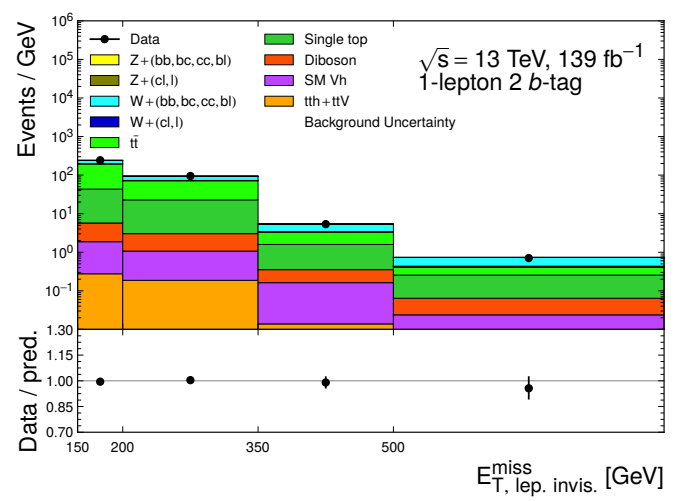

(c)

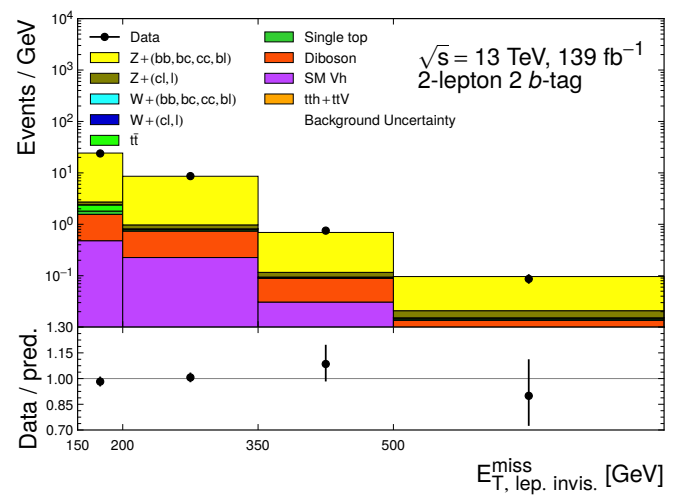

(e)

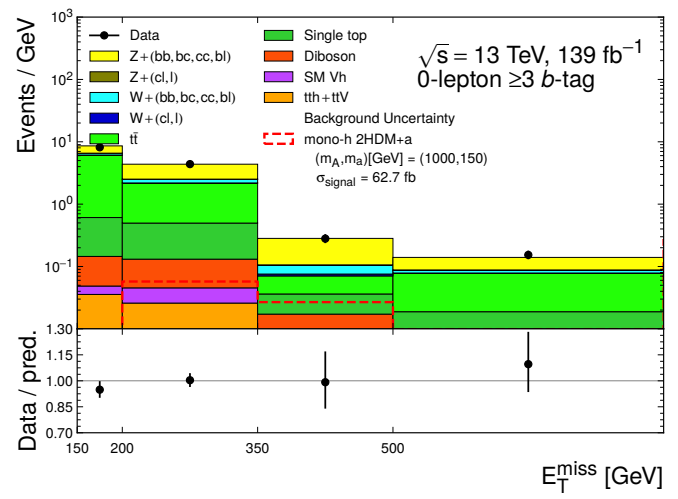

(b)

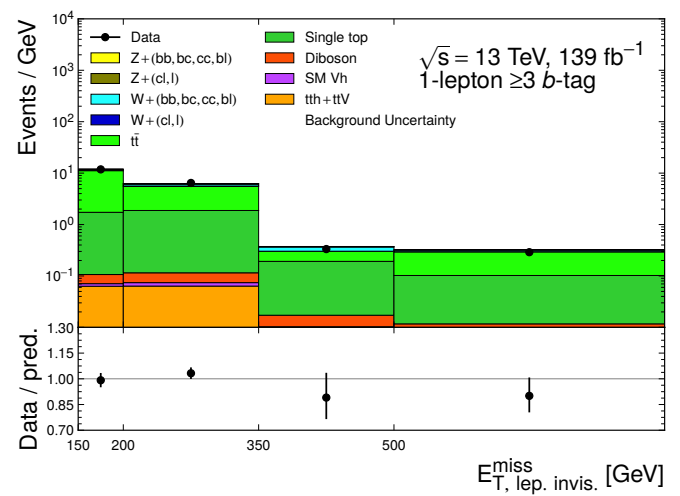

(d)

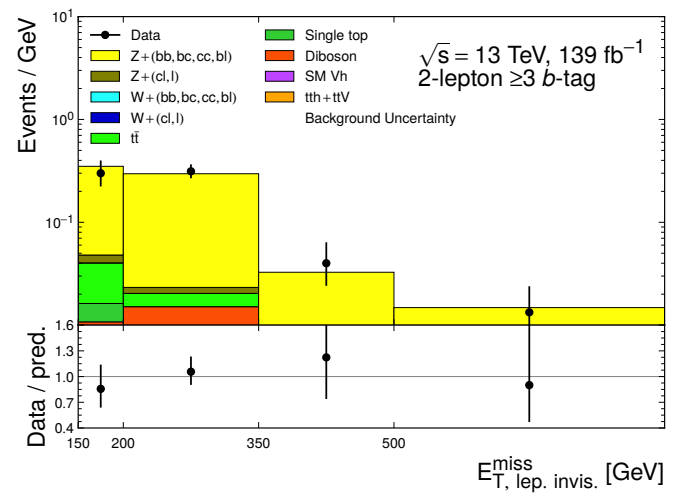

(f)

Figure 87: $E_{\mathrm{T}}^{\text {miss }}$ distributions in the 0-lepton (fig. 87a, 87b, 1- $\mu$ (fig. 87c, 87d), and 2-lepton (fig. 87e, 87f) regions for the 2 (left plots) and $\geq 3 \mathrm{~b}$-tag (right plots) categories across different $E_{\mathrm{T}}^{\mathrm{miss}}$ bins after the background-only fit. The second canvas shows the ratio between the fitted background prediction and the observed data (black dots). The dashed black band represents the total MC uncertainty. The red dashed line in the SR plots shows an example $Z^{\prime}$-2HDM signal point [234]. 


\section{- Observed significance:}

Figure 88 shows the observed significance against the background-only hypothesis for the $2 \mathrm{HDM}+a$ and $Z^{\prime}-2 \mathrm{HDM}$ signal points. No significant excess has been observed across the two signal models, hence exclusion limits are evaluated for both the signal models.

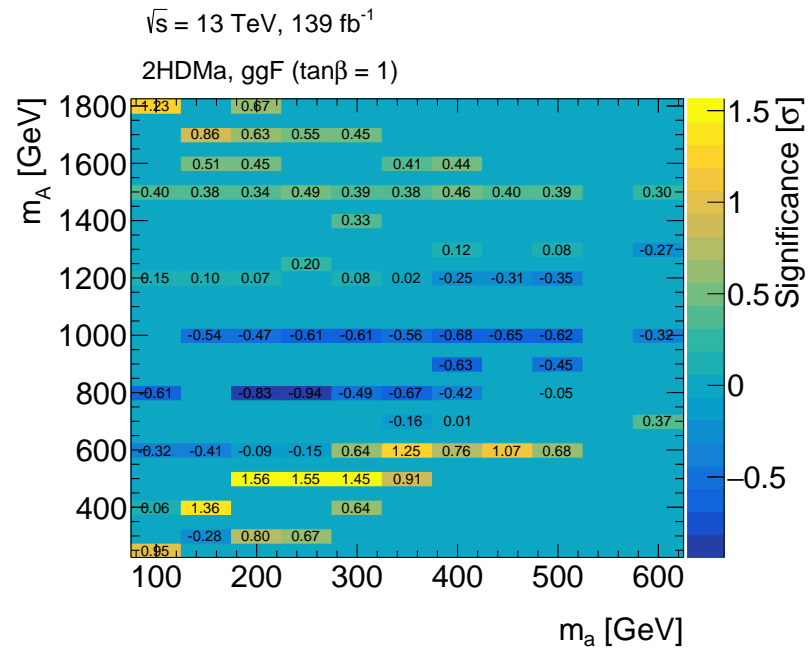

(a) $2 \mathrm{HDM}+a \mathrm{ggF}$

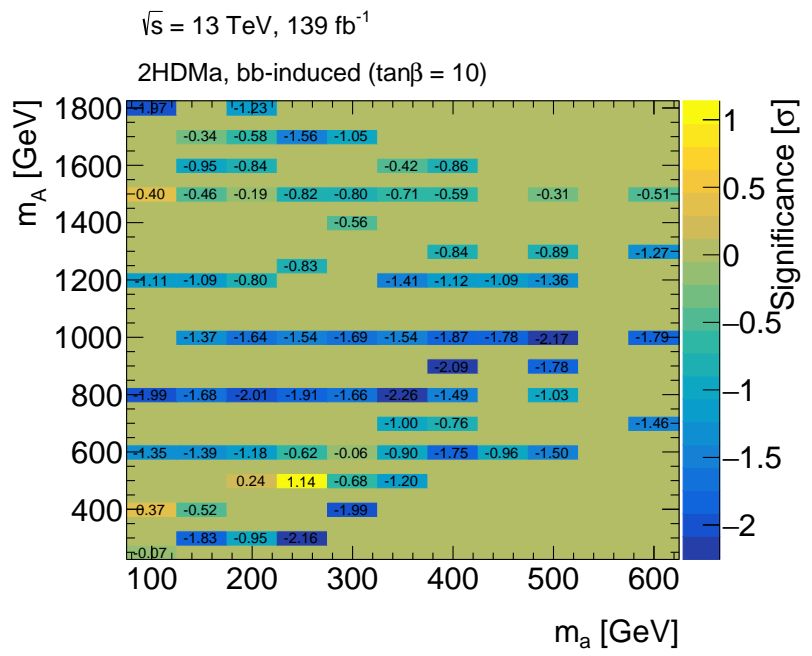

(b) $2 \mathrm{HDM}+a b b$

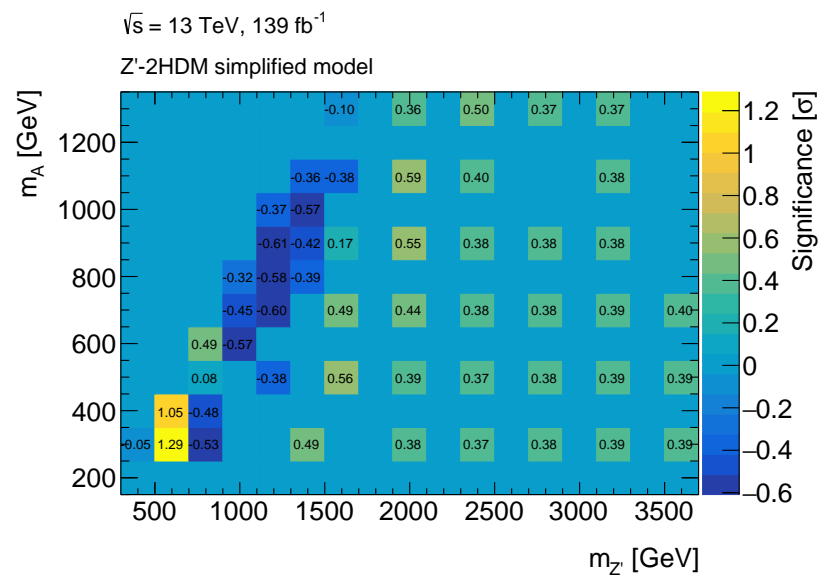

(c) $Z^{\prime}-2 \mathrm{HDM}$

Figure 88: Observed significance against the background-only hypothesis for the signal points of 2HDM+a ggF (fig. 88a), 2HDM+a bb-induced (fig. 88b), and $Z^{\prime}-2 \mathrm{HDM}$ (fig. 88c) [234].

\section{- Exclusion contours:}

Figures 89a, 89b, and $89 \mathrm{c}$ show the exclusion contours at a 95\% CL for the $2 \mathrm{HDM}+a$ ggF, 2HDM+a $b b$-induced, and $Z^{\prime}$-2HDM models respectively, where the signal points under the curves are excluded. In each plot the black solid line represents the observed limit, the black dashed 
line represent the expected limits, while the green and yellow bands represent the $\pm 1 \sigma$ and $\pm 2 \sigma$ uncertainties on the expected limits respectively. The gray dashed line represents the kinematically accessible regions i.e., $m_{A}=m_{a}+m_{h}$ and $m_{A}=m_{Z^{\prime}}-m_{h}$ for the $2 \mathrm{HDM}+a$ and $Z^{\prime}-2 \mathrm{HDM}$ models respectively.

For the $2 \mathrm{HDM}+a \mathrm{ggF}$ signal model the exclusion limit reaches up to $m_{A}=1.25 \mathrm{TeV}$ for $m_{a}=520 \mathrm{GeV}$. The gray area represents the exclusion contour results obtained from the analysis with $36 \mathrm{fb}^{-1}$ [62] ATLAS data, and as shown in Figure 89a the improvement for the exclusion contour is up to $180 \mathrm{GeV}$ for $m_{a}$. The exclusion limits go higher for low $m_{a}$, high $m_{A}$ regions due to the increase of $a \rightarrow a h$ cross section. Given the parameter choices of the analysis, for $m_{A} \gtrsim 1750 \mathrm{GeV}$ the aah coupling (c.f. fig. $17 \mathrm{c}$ ) becomes $>4 \pi$ and for $m_{A} \gtrsim 1250 \mathrm{GeV}$ [58, 247] the $2 \mathrm{HDM}+a$ scalar potential does not remain bounded-from-below. The width of the additional Higgs bosons becomes substantially higher for high $m_{A}$ values and the theoretical predictions are subject to additional theoretical uncertainties from the treatment of the width. Hence, exclusion limits for these regions with very large widths are not shown. Overall, there is good agreement between the expected and observed limits.

As shown in Figure $89 \mathrm{~b}$, the exclusion limit for the $2 \mathrm{HDM}+a b b$-induced model reaches up to $m_{A}=900 \mathrm{GeV}$ for $m_{a}=240 \mathrm{GeV}$. Due to the inclusion of the $\geq 3 b$-tag regions the analysis gained sensitivity for the $2 \mathrm{HDM}+a b b$-induced model (c.f appendix C), which was not the case in the previous analyses. The difference between the observed and expected limits is due to the data deficit observed in the 0-lepton $\geq 3 b$-tag regions for the $E_{\mathrm{T}}^{\text {miss }}$ regions of $350<E_{\mathrm{T}}^{\text {miss }} \leq$ $500 \mathrm{GeV}$ and $E_{\mathrm{T}}^{\text {miss }}>500 \mathrm{GeV}$, as shown in Figure 86 . This data deficit does not affect the other signal models since the $\geq 3 b$-tag regions are mostly sensitive to the $2 \mathrm{HDM}+a b b$-induced model. Between the $2 \mathrm{HDM}+a \mathrm{ggF}$ and $2 \mathrm{HDM}+a b b$-induced models, the later is sensitive to much smaller phase space in $\left(m_{A}, m_{a}\right)$ plane. This is because the 2HDM+a bb-induced model has much lower acceptance compared to the $2 \mathrm{HDM}+a$ ggF model and the number of signal events in the $\geq 3 b$ tag regions passing $E_{\mathrm{T}}^{\text {miss }}>150 \mathrm{GeV}$ selection is significantly fewer. Although the analysis is not sensitive to high $m_{A}$ regions of the $2 \mathrm{HDM}+a b b$-induced model, it should be noted that the 
bounded-from-below potential criterion breaks down for $m_{A} \gtrsim 2150 \mathrm{GeV}$.

Figure $89 \mathrm{c}$ shows the exclusion limit for the $Z^{\prime}-2 \mathrm{HDM}$ model where the exclusion limits reach up to $3 \mathrm{TeV}$ in $m_{Z^{\prime}}$ and $1 \mathrm{TeV}$ in $m_{A}$. The sharp drop near $m_{Z^{\prime}}=2800 \mathrm{GeV}$ is because the theoretical cross sections drop for $m_{A}>350 \mathrm{GeV}$ where the $A \rightarrow t \bar{t}$ decay modes have contributions. Overall, there is good agreement between the expected and observed limits.

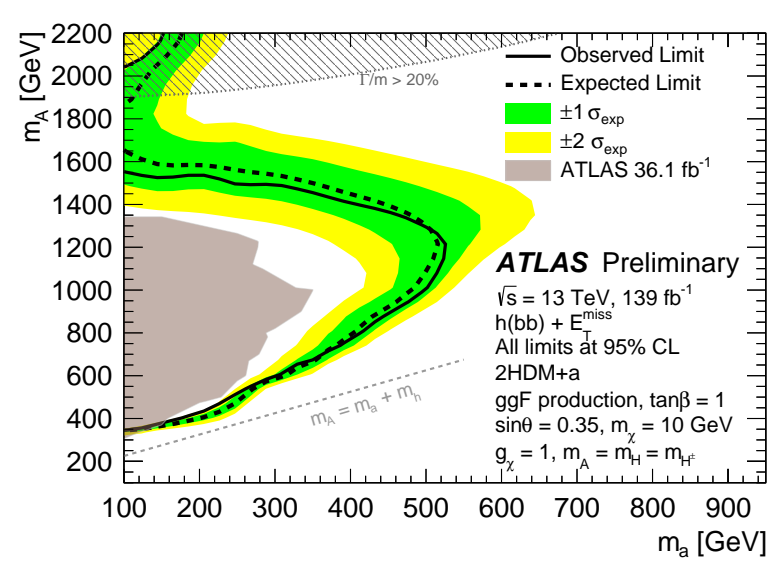

(a) $2 \mathrm{HDM}+a \mathrm{ggF}$

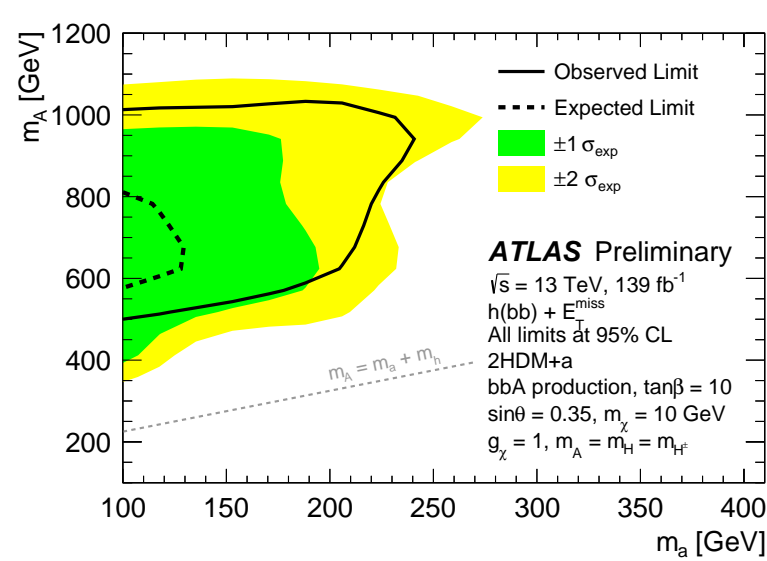

(b) $2 \mathrm{HDM}+a b b$

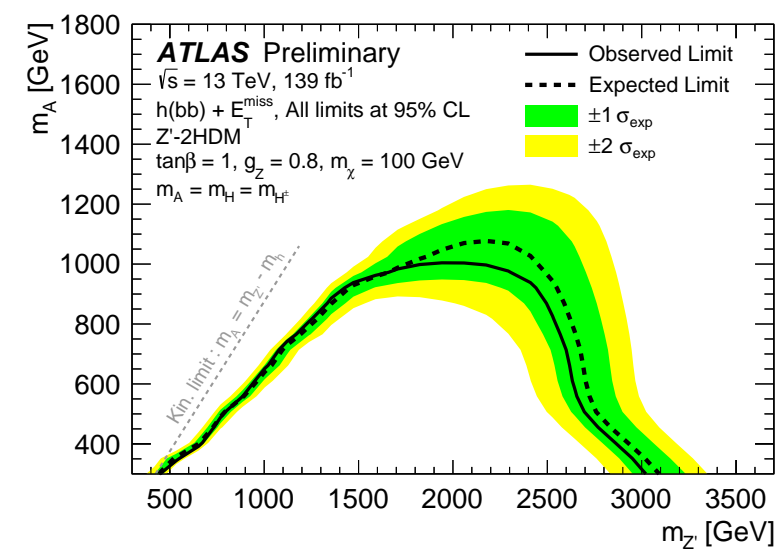

(c) $Z^{\prime}-2 \mathrm{HDM}$

Figure 89: Exclusion contours for the signal points of $2 \mathrm{HDM}+a$ ggF (Figure 89a), $2 \mathrm{HDM}+a b b$ induced (Figure 89b), and $Z^{\prime}-2 \mathrm{HDM}$ (Figure 89c) models [248]. The solid black line represents the observed limit at a 95\% CL, the dashed black line represents the expected limit, the green and yellow bands represent the $\pm 1 \sigma$ and $\pm 2 \sigma$ uncertainties on the expected limits respectively. The gray area in Figure 89a represents the exclusion contour obtained from the analysis with 36 $\mathrm{fb}^{-1}$ ATLAS dataset [62]. The shaded area in Figure 89a represents the region where the width of at least one of the Higgs bosons $\left(A, H\right.$ or $\left.H^{ \pm}\right)$or of the pseudoscalar $a$ is $>20 \%$ of its mass. In all three plots the parameter space enclosed by the curves are excluded: regions to the left of the curve for $2 \mathrm{HDM}+a$, and regions below the curve for $Z^{\prime}-2 \mathrm{HDM}$. 


\section{- Limit on the visible cross section:}

The above sections show the interpretations of the observed data in a $E_{\mathrm{T}}^{\text {miss }}+h \rightarrow b \bar{b}$ final state in terms of the $Z^{\prime}-2 \mathrm{HDM}$ and $2 \mathrm{HDM}+a \mathrm{DM}$ models. The results can also be interpreted with a minimal assumption that a $b \bar{b}$ resonance is produced with a mass close to the SM Higgs boson mass $m_{h}=125$ in association with a significant $E_{\mathrm{T}}^{\mathrm{miss}}$. This is also known as the modelindependent limit (MIL) and this section describes model-independent upper limits obtained on the visible cross section (effective cross section visible to the detector which is a fraction of the total cross section). The visible cross section is given as:

$$
\sigma_{\mathrm{vis}, h(b \bar{b})+\mathrm{DM}} \equiv \sigma_{h+\mathrm{DM}} \times \mathscr{B}(h \rightarrow b \bar{b}) \times \mathscr{A} \times \varepsilon
$$

where $\mathscr{A} \times \varepsilon$ represents the probability for an event to be reconstructed in a signal region with a kinematic acceptance of $\mathscr{A}$, and $\varepsilon$ represents the experimental efficiency. The upper limits are extracted with a similar likelihood analysis as described in section 5.1.11. However, the following modifications are made to make the limits model-independent:

- Instead of a binned histogram of $m_{h}$, the $m_{h}$ bins between $90-150 \mathrm{GeV}$ are merged into a single bin. As shown in appendix $\mathrm{D}$, the chosen bins ensure that majority of the signal events from the benchmark models fall in this range.

- A dummy signal with a cross section of $1 \mathrm{fb}$ is injected in the merged bin of $90-150 \mathrm{GeV}$ in each $E_{\mathrm{T}}^{\text {miss }}$ and $b$-tag signal regions. The signal strength acts as a floating normalization. All the signal regions are a part of the likelihood function but upper limits are set for one signal region at a time.

The expected and observed model-independent limits obtained at a 95\% CL for each individual signal regions are shown in Table 35 and Figure 90. 


\begin{tabular}{ccc}
\hline \hline Signal region & $\sigma_{\mathrm{vis}, h(b \bar{b})+\mathrm{DM}}^{\mathrm{obs}}[\mathrm{fb}]$ & $\sigma_{\mathrm{vis}, h(b \bar{b})+\mathrm{DM}}^{\exp }[\mathrm{fb}]$ \\
\hline $2 \mathrm{~b} E_{\mathrm{T}}^{\text {miss }} \in[150,200) \mathrm{GeV}$ & 3.26 & $2.07_{-0.58}^{+0.81}$ \\
$2 \mathrm{~b} E_{\mathrm{T}}^{\text {miss }} \in[300,350) \mathrm{GeV}$ & 1.47 & $1.99_{-0.56}^{+0.78}$ \\
$2 \mathrm{~b} E_{\mathrm{T}}^{\text {miss }} \in[350,500) \mathrm{GeV}$ & 0.33 & $0.33_{-0.09}^{+0.13}$ \\
$2 \mathrm{~b} E_{\mathrm{T}}^{\text {miss }} \in[500,750) \mathrm{GeV}$ & 0.15 & $0.13_{-0.04}^{+0.05}$ \\
$2 \mathrm{~b} E_{\mathrm{T}}^{\text {miss }} \in[750, \infty) \mathrm{GeV}$ & 0.05 & $0.05_{-0.01}^{+0.02}$ \\
$3+\mathrm{b} E_{\mathrm{T}}^{\text {miss }} \in[150,200) \mathrm{GeV}$ & 0.21 & $0.27_{-0.07}^{+0.10}$ \\
$3+\mathrm{b} E_{\mathrm{T}}^{\text {miss }} \in[200,350) \mathrm{GeV}$ & 0.18 & $0.32_{-0.09}^{+0.12}$ \\
$3+\mathrm{b} E_{\mathrm{T}}^{\text {miss }} \in[350,500) \mathrm{GeV}$ & 0.06 & $0.08_{-0.02}^{+0.03}$ \\
$3+\mathrm{b} E_{\mathrm{T}}^{\text {miss }} \in[500, \infty) \mathrm{GeV}$ & 0.06 & $0.06_{-0.02}^{+0.03}$ \\
\hline \hline
\end{tabular}

Table 35: Table summarizing the observed (obs) and expected (exp) upper limits at a 95\% CL on $\sigma_{\mathrm{vis}, h(b \bar{b})+\mathrm{DM}} \equiv \sigma_{h+\mathrm{DM}} \times \mathscr{B}(h \rightarrow b \bar{b}) \times \mathscr{A} \times \varepsilon$ across different analysis regions [234].

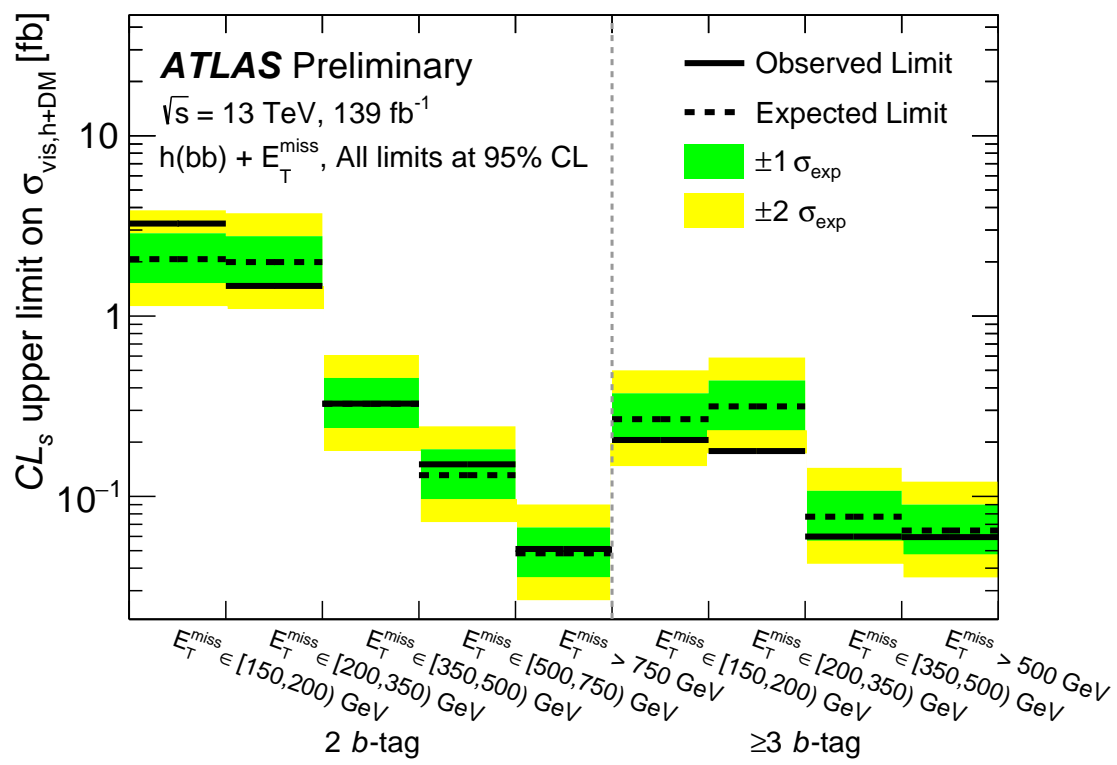

Figure 90: Figure shows the observed and expected upper limits at a 95\% CL on $\sigma_{\text {vis }, h(b \bar{b})+\mathrm{DM}} \equiv$ $\sigma_{h+\mathrm{DM}} \times \mathscr{B}(h \rightarrow b \bar{b}) \times \mathscr{A} \times \varepsilon$ across different analysis regions [248]. 


\subsection{Dark Energy analysis}

\subsubsection{Introduction}

So far, no DE search has been performed in collider experiments. A DE search at the LHC is based on the assumption that there is a non-zero coupling between the DE particles and SM fields. Since the LHC is sensitive to a wide range of signatures, if there are interactions between DE and SM particles, DE particles might be produced in collider experiments. At the LHC, the searches for DE can be performed in two ways: (i) EW-precision measurements which are sensitive to virtual $\mathrm{DE}$ processes, and (ii) the direct production of $\mathrm{DE}$ particles in $p p$ collisions. The $\mathrm{DE}$ search described in this thesis is based on the second procedure and is a reinterpretation of the search for top super-partners in the $t \bar{t}+E_{\mathrm{T}}^{\text {miss }}$ final state.

\subsubsection{Dark Energy model}

A limitation in developing DE models is that the microscopic dynamics of the DE interactions is unknown. This shortcoming can be circumvented by building a EFT based DE model where the microscopic dynamics of the DE interactions is irrelevant. Most DE models with scalar fields and second-order equations of motion are based on the framework of Horndeski theories [50], where one tries to find a generic way to parameterize the interactions between DE scalar and SM. The EFT based DE model [249] analyzed in this thesis is a part of the Horndeski theories and contains EFT operators invariant under a shift symmetry $\phi \rightarrow \phi+c$ where $\phi$ represents the DE scalar field, and $c$ is a constant factor. The model also assumes that DE is not a constant (c.f. cosmological constant problem [250]). It contains nine shift symmetry preserving operators:

$$
\mathcal{L}=\mathcal{L}_{S M}+\sum_{i=1}^{9} c_{i} \mathcal{L}_{i}=\mathcal{L}_{S M}+\sum_{i=1}^{9} \frac{c_{i}}{M_{i}^{(d-4)}} \mathcal{O}_{i}^{(d)}
$$

where $d$ is the dimension of the operator, $c_{i}$ 's are the Wilson coefficients, $M$ is the characteristic energy scale. As can be seen from equation 80 , each operator is suppressed by powers of $M$, where the power is the dimension of the operator. Different operators have specific physical interpre- 
tations, e.g., the operators $\mathcal{L}_{1}-\mathcal{L}_{5}$ correspond to interactions of the SM fields with the DE scalar field, $\mathcal{L}_{6}$ corresponds to a generalized kinematic term of the DE scalar, and $\mathcal{L}_{7}-\mathcal{L}_{9}$ correspond to non-trivial Galileon terms 5 , Of the nine possible operators, $\mathcal{L}_{1}$ and $\mathcal{L}_{2}$ are studied in the analysis:

$$
\mathcal{L}_{1}=\frac{\partial_{\mu} \phi \partial^{\mu} \phi}{M_{1}^{4}} T_{v}^{v} \quad \text { and } \quad \mathcal{L}_{2}=\frac{\partial_{\mu} \phi \partial_{\nu} \phi}{M_{2}^{4}} T^{\mu \nu}
$$

where $T^{\mu v}$ is the energy momentum tensor corresponding to all the SM fields. The motivations behind studying only $\mathcal{L}_{1}$ and $\mathcal{L}_{2}$ operators are: they provide shift-symmetric interactions to avoid Yukawa-like couplings between the DE scalar and SM fermions that are tightly constrained and these being the lowest dimension operators in the model, they should capture the leading BSM effects. Since for a Dirac field $\psi, T_{v}^{v}=m \psi \bar{\psi}$, the coupling between the DE scalar and SM fermions induced by the $\mathcal{L}_{1}$ operator is proportional to the mass of the SM fermions. On the other hand, the $\mathcal{L}_{2}$ operator contains derivatives of the SM fields and the coupling is proportional to their momenta. The couplings induced by the $\mathcal{L}_{1}$ and $\mathcal{L}_{2}$ operators are called conformal and disformal couplings respectively.

Since the $\mathcal{L}_{1}$ operator induces couplings proportional to the SM fermion masses, it is more sensitive to $t \bar{t}+\phi \phi$ final states. On the other hand, $\mathcal{L}_{1}$ is less sensitive to channels such as monojet (jet $+\phi \phi)$, since a significant contribution of the effective hadronic cross section comes from processes where the final state contains massless gluons and low-mass quarks. Since, the mono-jet channel has events with high momentum transfer, the $\mathcal{L}_{2}$ operator is more sensitive to the it. This thesis considers $\mathcal{L}_{1}$ and $\mathcal{L}_{2}$ operators only where the final state is $t \bar{t}+\phi \phi$ and the corresponding Feynman diagrams are shown in Figure 91.

\subsubsection{Dark Energy model implementation}

To consider the collider phenomenology of the two operators of interest one can write:

$$
\mathcal{L}_{B S M}=\mathcal{L}_{S M}+\sum_{i} c_{i} \mathcal{L}_{i}+\frac{1}{2} m_{\phi}^{2} \phi^{2} .
$$

\footnotetext{
${ }^{5}$ A type of kinematic term of the DE scalar containing multiple derivatives per field, e.g. $\mathcal{L}_{7} \sim \frac{\square \phi}{M^{3}}, \square=\partial^{\mu} \partial_{\mu}$.
} 


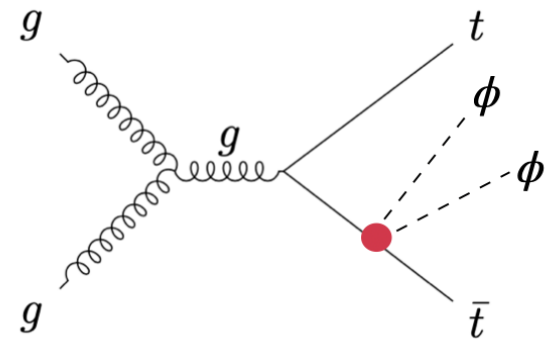

(a) $\mathcal{L}_{1}$ operator

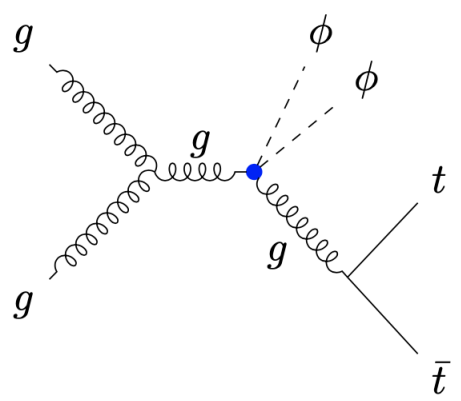

(b) $\mathcal{L}_{2}$ operator

Figure 91: Feynman diagrams corresponding to the production of a pair of DE particles $(\phi)$ along with $t \bar{t}$ for the $\mathcal{L}_{1}$ (fig. $91 \mathrm{a}$ with red vertex) and $\mathcal{L}_{2}$ (fig. $91 \mathrm{~b}$ with blue vertex) operators.

The DE analysis here considers the DE particle mass $m_{\phi}$ to be small compared to typical collider scales, hence $\frac{1}{2} m_{\phi}^{2} \phi^{2} \approx 0$. The production cross section for a particular multiplicity of $\phi$ depends on $\sim \frac{c_{i}^{2}}{M^{2 r}}$ where $r$ is the characteristic scaling of a given operator. To be able to derive constraints (e.g., upper limits on cross sections) which depend solely on $M$, the Wilson coefficients are set to $c_{i}=1$.

The $t \bar{t}+\phi \phi$ DE signal MC events are generated using the MADGRAPH5_AMC@NLO 2.5.5 generator [167]. The events corresponding to $\mathcal{L}_{1}$ and $\mathcal{L}_{2}$ operators are generated by setting $c_{1}=1$ and $c_{2}=1$ respectively, with all other $c_{i}=0$. The matrix elements are calculated at QCD LO, using a LO PDF set NNPDF 3.0 with $\alpha_{s}=0.13$, in the four-flavor scheme, and vetoing EW contributions. To ensure that the amplitude scales as $M^{-4}$ for any value of $M$, during the matrix element calculations either $\mathcal{L}_{1}$ or $\mathcal{L}_{2}$ operator is used. This ensures that while the cross section depends on $M$, the differential distributions of the signal process is independent of $M$. The parton shower and hadronization is performed by PYTHIA8.212 with the A14 set of tuned parameters, and the LO NNPDF 2.3 PDF set with $\alpha_{s}=0.13$. The renormalization and factorisation scales are set to $\mu=\frac{1}{2} \sum_{i} m_{T, i}$ where $i$ runs over all final state particles and $m_{T}$ is the transverse mass of a final state particle. The $t \bar{t}+\phi \phi$ samples are produced with the following particle level filters:

- 0 lepton: $E_{\mathrm{T}}^{\mathrm{miss}}>150 \mathrm{GeV}$.

- 1 lepton: at least 1 lepton with $p_{\mathrm{T}}>20 \mathrm{GeV},|\eta|<2.8$ and $E_{\mathrm{T}}^{\text {miss }}>60 \mathrm{GeV}$.

- 2 lepton: exactly 2 leptons with $p_{\mathrm{T}}>20 \mathrm{GeV},|\eta|<2.8$. 
To reproduce the correct equation of state for DE, the masses of the DE scalar fields should be very small $m_{\phi}=\mathcal{O}\left(H_{0}\right) \sim 10^{-42}$ [251]. Different values of $m_{\phi}$ were tested and as shown in Figure 92 the $t \bar{t}+\phi \phi$ production cross section and kinematics are independent of $m_{\phi}$. The DE scalar field mass is set at $m_{\phi}=0.1 \mathrm{GeV}[249]$ and the suppression scales are set at $M=400 \mathrm{GeV}$, and $M=600 \mathrm{GeV}$ for $\mathcal{L}_{1}$ and $\mathcal{L}_{2}$ respectively, which are rescaled as needed for other values of $M$. Table 36 shows the details of the MC signal samples $(t \bar{t}+\phi \phi)$ used.

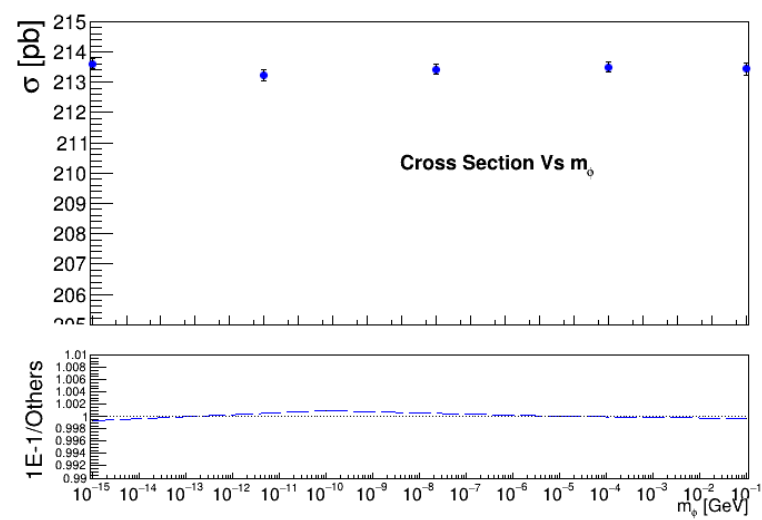

(a)

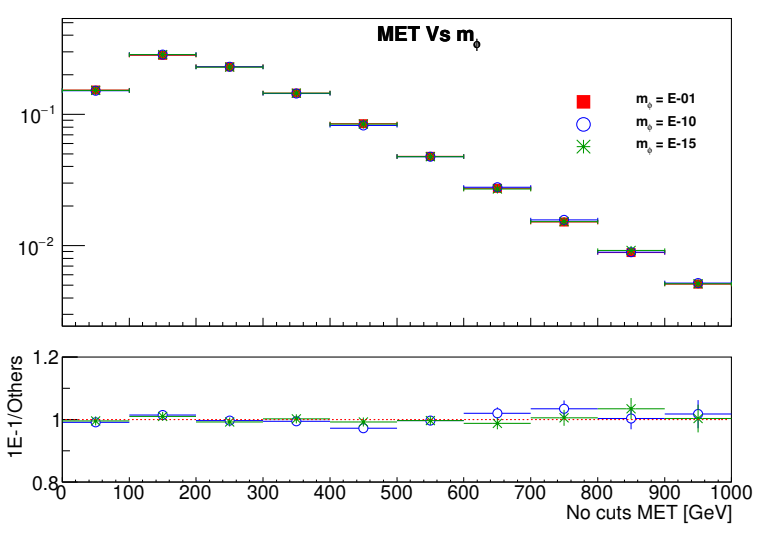

(b)

Figure 92: Figures show the dependency of $t \bar{t}+\phi \phi$ production cross section (figure 92a) and particle-level $E_{\mathrm{T}}^{\text {miss }}$ (figure $92 \mathrm{~b}$ on $m_{\phi}$ for the $\mathcal{L}_{1}$ operator without any generator filters [252].

\begin{tabular}{|c|c|c|c|}
\hline Operator & $M[\mathrm{GeV}]$ & $N_{\text {events }}$ & $\sigma[\mathrm{pb}]$ \\
\hline \hline $\mathcal{L}_{1}$ & 400 & 400000 & $3.2397 \times 10^{-3}$ \\
\hline $\mathcal{L}_{1}$ & 400 & 100000 & $3.2530 \times 10^{-3}$ \\
\hline $\mathcal{L}_{2}$ & 600 & 400000 & $2.1826 \times 10^{-2}$ \\
\hline $\mathcal{L}_{2}$ & 600 & 100000 & $2.2320 \times 10^{-2}$ \\
\hline
\end{tabular}

Table 36: Dark energy MC signal samples used for the two operators along with the production cross sections for the two operators [252].

\subsubsection{Signal regions and event selections}

The sensitivity estimation of the DE search in $t \bar{t}+E_{\mathrm{T}}^{\text {miss }}$ final state is performed using the selections from the search of DM produced in association with a $t \bar{t}$ pair [253] and from the search for SUSY partner of top quark [254-256]. Since the stop quarks analysis selections gave better exclusion limits, these are used in the analysis. Depending on the decay products of the $W^{ \pm}$-bosons 
coming from top-decays $(t \rightarrow W b)$, the $t \bar{t}+E_{\mathrm{T}}^{\text {miss }}$ final state can be categorized into three channels: 0-lepton where the $W^{ \pm}$-boson decays hadronically $\left(W \rightarrow q^{\prime} \bar{q}\right)$, 1-lepton where one of two $W^{ \pm}$. boson decays leptonically $\left(W^{+} \rightarrow \ell^{+} v\right.$ ), and 2-lepton where both $W^{ \pm}$-bosons decay leptonically. The selections used in this analysis are taken from: (i) 0-lepton channel: Signal Region A (SRA) selections from stop 0-lepton [254] analysis, (ii) 1-lepton: selections from tN_high [255] region where 'tN': top-neutralino and 'high' means SUSY top quark partner mass of $1 \mathrm{TeV}$, and (iii) 2lepton: Signal Region B (SRB) selections from the stop 2-lepton analysis [256]. The selections for the 0,1,2-lepton regions are described in Tables 37, 38, and 39 respectively.

\begin{tabular}{|c|c|c|c|}
\hline \multirow{2}{*}{ Variable } & \multicolumn{3}{|c|}{ Regions } \\
\cline { 2 - 4 } & SRA_TT & SRA_TW & SRA_T0 \\
\hline$m_{\text {jet }, R=1.2}^{0}$ & \multicolumn{3}{|c|}{$>120 \mathrm{GeV}$} \\
\hline$m_{j e t, R=1.2}^{1},>120 \mathrm{GeV}$ & {$[60,120] \mathrm{GeV}$} & $<60 \mathrm{GeV}$ \\
\hline$m_{T}^{b, \text { min }}$ & \multicolumn{3}{|c|}{$>200 \mathrm{GeV}$} \\
\hline$N_{b-\text { jet }} \geq 2$ \\
\hline$\tau$-veto & \multicolumn{3}{|c|}{ yes } \\
\hline $\mid \Delta \phi\left(\right.$ jet $\left.^{0,1,2}, p_{T}^{\text {miss }}\right)$ & \multicolumn{3}{|c|}{$>0.4$} \\
\hline$m_{j e t, R=0.8}^{0}$ & & \multicolumn{3}{|c|}{-} \\
\hline$\Delta R(b, b)$ & $>1$ & $\mathrm{GeV}$ \\
\hline$m_{T 2}^{\chi^{2}}$ & $>400 \mathrm{GeV}$ & $>400 \mathrm{GeV}$ & $>500 \mathrm{GeV}$ \\
\hline$E_{\mathrm{T}}^{\text {miss }}$ & $>400 \mathrm{GeV}$ & $>500 \mathrm{GeV}$ & $>550 \mathrm{GeV}$ \\
\hline
\end{tabular}

Table 37: Selections for 0-lepton channel, details about the variables are described in [254].

\begin{tabular}{|c|c|}
\hline Variable & tN high \\
\hline$N_{\text {leptons }}$ & exactly 1 loose lepton \\
\hline$p_{\mathrm{T}}$ (lepton) & $>25 \mathrm{GeV}$ \\
\hline$N_{\text {jets }} \& N_{b-\text { tags }}$ & $\geq 4 \& \geq 1$ \\
\hline Jet $p_{\mathrm{T}}[\mathrm{GeV}]$ & $>(100,80,50,30)$ \\
\hline$E_{\mathrm{T}}^{\text {miss }}$ & $>550 \mathrm{GeV}$ \\
\hline$m_{T}$ & $>160 \mathrm{GeV}$ \\
\hline$H_{T, \text { sig }}^{\text {miss }}$ & $>27$ \\
\hline$\alpha m_{T 2}$ & $>175 \mathrm{GeV}$ \\
\hline$m_{\text {top }}^{\text {reclustered }}$ & $>130 \mathrm{GeV}$ \\
\hline$\Delta R(b, l)$ & $<2$ \\
\hline $\mid \Delta \phi($ jet & $>0.4$ \\
\hline$m_{T 2}^{\tau}$ based $\tau$-veto & $>80 \mathrm{GeV}$ \\
\hline
\end{tabular}

Table 38: Selections for 1-lepton channel, details about the variables are described in [255]. 


\begin{tabular}{|c|c|c|c|c|}
\hline \multirow{2}{*}{ Variable } & \multicolumn{4}{|c|}{ Regions } \\
\cline { 2 - 5 } & $\mathrm{SRB}_{120} \mathrm{SF}$ & $\mathrm{SRB}_{140} \mathrm{SF}$ & $\mathrm{SRB}_{120} \mathrm{DF}$ & $\mathrm{SRB}_{140} \mathrm{DF}$ \\
\hline Lepton flavor & $\mathrm{SF}$ & $\mathrm{SF}$ & $\mathrm{DF}$ & $\mathrm{DF}$ \\
\hline$p_{\mathrm{T}}\left(\ell_{1}\right), p_{\mathrm{T}}\left(\ell_{2}\right)[\mathrm{GeV}]$ & \multicolumn{4}{|c|}{$>25,>20$} \\
\hline$m_{\ell \ell}[\mathrm{GeV}]$ & {$[20,71.2]$ or $>111.2$} & $>20$ \\
\hline$\Delta \phi_{\text {boost }}$ & \multicolumn{4}{|c|}{$<1.5$} \\
\hline$N_{\text {jets }} \& N_{b-\text { jets }}$ & \multicolumn{4}{|c|}{$\geq 2 \& \geq 1$} \\
\hline$m_{T 2}^{\ell \ell}[\mathrm{GeV}]$ & {$[120,140]$} & $>140$ & {$[120,140]$} & $>140$ \\
\hline
\end{tabular}

Table 39: Selections for 2-lepton channel, details about the variables are described in [256].

\subsubsection{Uncertainties}

Only the theoretical uncertainties related to the $\mathrm{DE}$ signal MC production is derived for this thesis, while the detector and SM uncertainties are taken from the stop quark search. The theory uncertainties can be classified into two categories: (i) cross section uncertainties that affect the signal normalization and are independent of the decay channel, and selections, and (ii) acceptance uncertainties that depend on the specific kinematic selections. These uncertainties arise from the choice of the renormalization and factorisation scale, PDF set, and strong coupling constant $\alpha_{s}$. The renormalization and factorisation scale uncertainties are calculated by varying the nominal values of renormalization and factorisation scales by a factor of two, and the combined PDF $+\alpha_{s}$ uncertainty is estimated using the PDF4LHC [242] prescription. The total uncertainty is calculated by adding the different uncertainties in quadrature. Tables 40 and 41 summarise the acceptance and cross section uncertainties respectively. Since the signal acceptance uncertainties are much smaller (5-12\%) than the data statistical uncertainty (20-35\%), and all other systematic uncertainties (15$35 \%)$, its impact on the fitted signal strength is small.

\begin{tabular}{|c|c|c|c|c|c|c|}
\hline Operator & \multicolumn{3}{|c|}{$\mathcal{L}_{1}$} & \multicolumn{3}{c|}{$\mathcal{L}_{2}$} \\
\hline Channel & 0-lepton & 1-lepton & 2-lepton & 0-lepton & 1-lepton & 2-lepton \\
\hline Scale & $1 \%$ & $1 \%$ & $1 \%$ & $1 \%$ & $1 \%$ & $1 \%$ \\
\hline PDF & $12 \%$ & $12 \%$ & $4 \%$ & $6 \%$ & $8 \%$ & $7 \%$ \\
\hline$\alpha_{s}$ & $0.3 \%$ & $0.8 \%$ & $2.7 \%$ & $1.7 \%$ & $1.5 \%$ & $3.7 \%$ \\
\hline Total & $12 \%$ & $12 \%$ & $5 \%$ & $6 \%$ & $8 \%$ & $8 \%$ \\
\hline
\end{tabular}

Table 40: Acceptance uncertainties for $\mathcal{L}_{1}$ and $\mathcal{L}_{2}$ operators [252]. 


\begin{tabular}{|c|c|c|c|c|}
\hline Operator & Scale & PDF & $\alpha_{s}$ & Total \\
\hline $\mathcal{L}_{1}$ & $+44 \%$ & $\pm 39 \%$ & $\pm 8 \%$ & $\begin{array}{l}+59 \% \\
-48 \%\end{array}$ \\
\hline $\mathcal{L}_{2}$ & $-28 \%$ & & & $+46 \%$ \\
& $-26 \%$ & $\pm 25 \%$ & $\pm 4 \%$ & $-36 \%$ \\
\hline
\end{tabular}

Table 41: Cross section uncertainties for $\mathcal{L}_{1}$ and $\mathcal{L}_{2}$ operators [252].

\subsubsection{Validity of EFT approximation}

The DE operators analysed in the thesis are derived by an expansion in the suppression scale $M$, hence are valid only in regions where the momentum transfer $\left(Q_{t r}\right)$ in the interaction is $Q_{t r} \ll M$. In practice the EFT approximation validity holds for events that satisfy $Q_{t r}<g_{\star} M^{6}$, and for the couplings to be in perturbative regime [257] $g_{\star}<4 \pi$ criterion must be satisfied. The momentum transfer is calculated using the partonic center-of-mass energy [258]:

$$
Q_{t r}=\sqrt{\hat{s}}=\sqrt{\left(\sum_{i} p_{i}\right)^{2}}<g_{\star} M, \text { where } i \in\{\text { partons in final state }\}
$$

For events that do not satisfy equation 83 , the EFT validity is preserved by rescaling the EFT limits by an iterative procedure [52]. To estimate the rescaling factor, the variable $R_{\text {valid, }}$, representing the fraction of events satisfying the EFT criterion is defined:

$$
R_{\mathrm{valid}}=\frac{N\left(Q<Q_{t r}^{\max }=g_{\star M}\right)}{N}
$$

where $Q_{t r}^{\max }$ represents the maximum momentum transferred in all events. The rescaled suppression scale $M_{\text {rescaled }}$ is obtained with the following procedure:

(i) Assume all the events satisfy EFT validity i.e., equation 84 becomes $R_{\text {valid }, i=1}=1$.

(ii) Calculate $Q_{t r}^{\max }(i)=g_{\star} M(i)=g_{\star} M(i-1)$.

(iii) Calculate $R_{\text {valid }, i}=\frac{N\left(Q<Q_{t r i, i}^{\max }\right)}{N\left(Q<Q_{t r, i-1}^{\max }\right)}$.

(iv) Evaluate $M(i)=R_{\text {valid }}^{1 / 8} M(i)$ and continue the iteration until $R_{\text {valid }, i}=0$ or $R_{\text {valid }, i}=1$.

\footnotetext{
${ }^{6} g_{\star}$ is a variable dependent on the ultra violet (UV) completion details of the EFT model. A UV complete theory is predictive at all high-energy scales.
} 
Since the DE production cross section $(\sigma)$ for $\mathcal{L}_{1}$ and $\mathcal{L}_{2}$ scales as $\sigma \propto M^{-1 / 8}$, the final rescaled suppression scale $M_{\text {rescaled }}$ is calculated as:

$$
M_{\text {rescaled }}=\prod_{i} R_{\mathrm{valid}, i}^{1 / 8} M
$$

\subsubsection{Results}

The presence of a DE signal is investigated by fitting the expected DE signal and the SM background yield to the observed data in the three lepton channels simultaneously, using a binned maximum-likelihood approach. Similar to the original analysis [254], no significant data excess is found compared to the SM backgrounds. Hence, upper limits are calculated on the production cross sections $(p p \rightarrow t \bar{t}+\phi \phi)$ for $\mathcal{L}_{1}$ and $\mathcal{L}_{2}$ operators, at a 95\% CL using the $\mathrm{CL}_{\mathrm{s}}$ method [259]. The upper limits on cross sections $(\sigma)$ are translated to the lower limits on $M$ since $\sigma \propto M^{-1 / 8}$. Table 42 shows the lower limit on $M$ across different channels. The 0-lepton channel has the highest sensitivity due to low data fluctuations and the corresponding exclusion plots are shown in Figure 93. For DE production, suppression scales up to $M<309 \mathrm{GeV}$ and $M<674 \mathrm{GeV}$ are excluded at $95 \% \mathrm{CL}$ for the $\mathcal{L}_{1}$ and $\mathcal{L}_{2}$ operators respectively. Figure 94 shows the exclusion limits on the $\left\{g_{*}, M\right\}$, after applying the EFT validity criterion (equation 83. The EFT scaling is performed using the $Q_{\mathrm{tr}}$ distribution without any selections. For DE production, suppression scales of $M<300 \mathrm{GeV}$ and $M<670 \mathrm{GeV}$ can be excluded at $95 \% \mathrm{CL}$ for the $\mathcal{L}_{1}$ and $\mathcal{L}_{2}$ operators respectively for $g_{\star} \geq 10$.

\begin{tabular}{|c|c|c|c|c|c|c|c|}
\hline Operator & Channel & Observed & Expected & $+2 \sigma$ & $+1 \sigma$ & $-1 \sigma$ & $-2 \sigma$ \\
\hline \multirow{3}{*}{$\mathcal{L}_{1}$} & 0-lepton & $309_{-24}^{+19}$ & 313 & 284 & 299 & 326 & 338 \\
\cline { 2 - 8 } & 1-lepton & $290_{-23}^{+17}$ & 312 & 282 & 297 & 326 & 338 \\
\cline { 2 - 8 } & 2-lepton & $266_{-21}^{+16}$ & 279 & 254 & 266 & 291 & 301 \\
\hline \multirow{3}{*}{$\mathcal{L}_{2}$} & 0-lepton & $674_{-37}^{+33}$ & 675 & 615 & 643 & 704 & 730 \\
\cline { 2 - 9 } & 1-lepton & $633_{-35}^{+30}$ & 680 & 617 & 648 & 709 & 735 \\
\cline { 2 - 9 } & 2-lepton & $536_{-29}^{+26}$ & 562 & 511 & 536 & 585 & 607 \\
\hline
\end{tabular}

Table 42: Expected and observed lower limits on the suppression scale $M$ (in GeV) before rescaling (EFT validity criterion not applied). The errors on the expected limits correspond to the uncertainty on the signal production cross section [252]. 

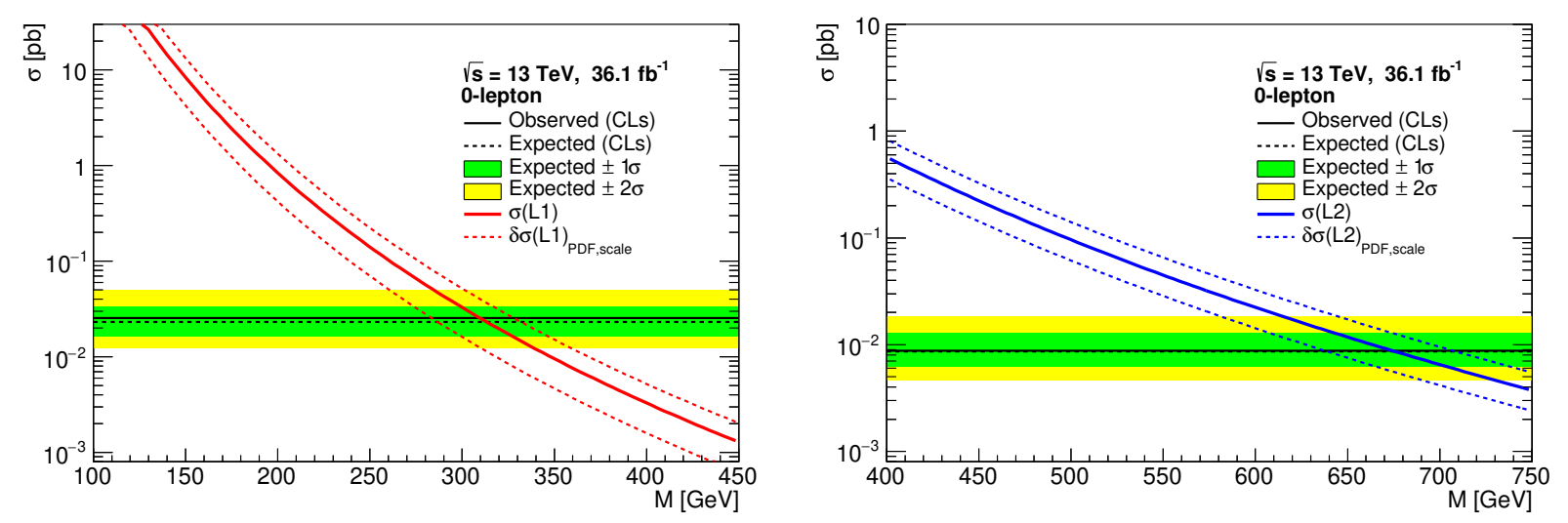

Figure 93: Exclusion plots for $\mathcal{L}_{1}$ (left) and $\mathcal{L}_{2}$ (right) operators in 0-lepton channel. The errors on the predicted cross sections correspond to the scale and PDF uncertainties. Suppression scale $(M)$ values to the left of the crossings of solid black-red $\left(\mathcal{L}_{1}\right)$ and solid black-blue $\left(\mathcal{L}_{2}\right)$ lines are excluded. Figure credits to Dr. Spyridon Argyropoulos.
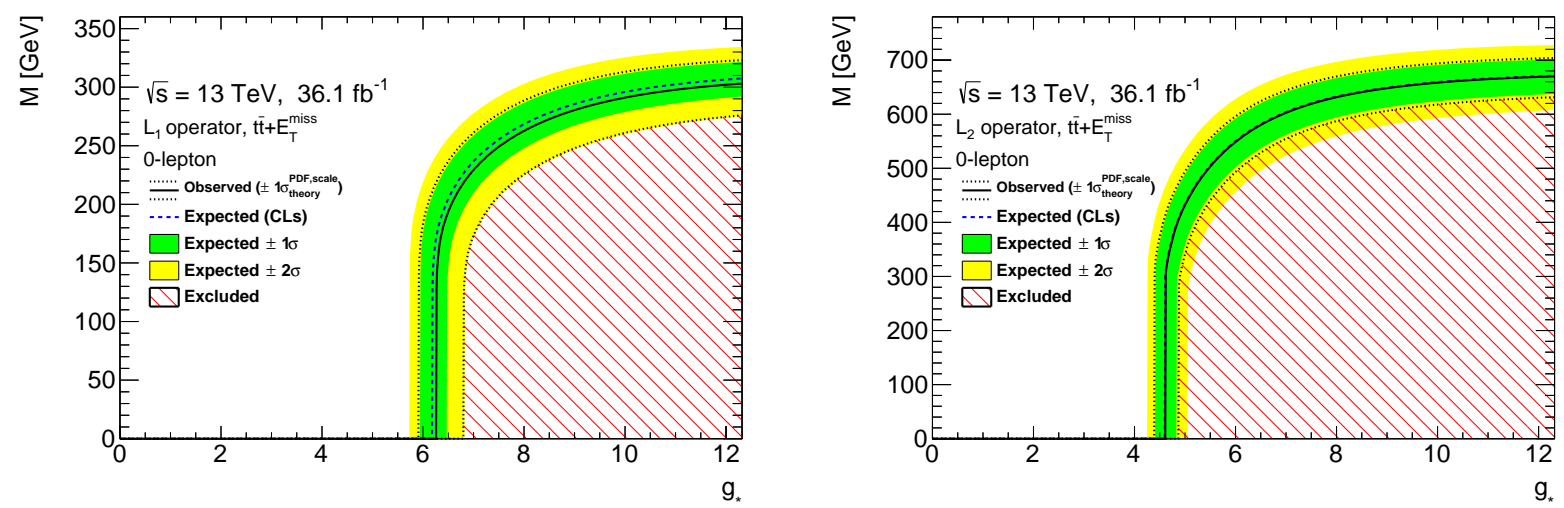

Figure 94: Exclusion contour in $\left\{g_{*}, M\right\}$ plane for $\mathcal{L}_{1}$ (left) and $\mathcal{L}_{2}$ (right) operators in 0-lepton channel, after taking into account the EFT validity criterion. Parameter points enclosed by the curve, shown by red hashed lines, are excluded. Figure credits to Dr. Spyridon Argyropoulos.

These are the first results of a direct DE search in a collider experiment. The $t \bar{t}+E_{\mathrm{T}}^{\mathrm{miss}}$ analysis provides the most stringent limits on the suppression scale $M$ from $\mathcal{L}_{1}$ operator. However, it is not yet sensitive to weakly coupled models. The most stringent limit from $\mathcal{L}_{2}$ operator is derived from the mono-jet search [260] (with one highly energetic jet) and is not a part of the thesis.

The EFT model of DE analysed here contains a DE light scalar field (and its associated quantum excitations, i.e. scalar particles) which can drive the accelerated expansion of the universe [261, 262]. If DE particles are produced at colliders large $E_{\mathrm{T}}^{\mathrm{miss}}$ is expected compared to the 
$\mathrm{SM}$ prediction. If deviations from the SM are found which are compatible with the predictions of the EFT DE model, further measurements will be needed to confirm the microscopic nature of the associated particles/interactions. The primary aim of DE searches at colliders is to provide additional constraints to the cosmological and astrophysical probes of DE. 


\section{SUMMARY, CONCLUSIONS AND FUTURE PROSPECTS}

This thesis describes searches for DM and DE using the dataset recorded by the ATLAS detector at the LHC. The thesis analyzes the missing mass prediction using the extensions of the 2HDM model for dark matter sector and a phenomenologically inspired effective field theory model for dark energy interacting with the Standard Model. This chapter summarizes the two above-mentioned analyses, results, and possible future works.

The main analysis described in this thesis is the search for DM in the mono- $h(b \bar{b})$ channel using the full Run 2 ATLAS dataset of $139 \mathrm{fb}^{-1}$. This analysis searches for DM particles produced in association with a SM Higgs boson decaying to two $b$-quarks. If the DM particles exist and are produced at the LHC, would very likely escape the detector undetected, there would be a significant amount of $E_{\mathrm{T}}^{\mathrm{miss}}$ in the final state. Consequently, the final state consists of $E_{\mathrm{T}}^{\text {miss }}$ and two $b$-jets. The results are interpreted in terms of two benchmark DM models, the $Z^{\prime}-2 \mathrm{HDM}$ and $2 \mathrm{HDM}+a$. Both these models are extensions of the 2HDM model and are related to the Higgs-portal models, where the DM particles interact only with the Higgs sector.

The $Z^{\prime}$-2HDM model predicts that the DM particles $(\chi)$ are produced through the physics process $p p \rightarrow Z^{\prime} \rightarrow h A \rightarrow(b \bar{b}) \chi \bar{\chi}$, where the $Z^{\prime}$-boson is a massive spin-1 mediator and $A$ is the CP-odd Higgs. The analysis investigates the $Z$-2HDM phenomenology for different masses of $Z^{\prime}$ and $A$. The $2 \mathrm{HDM}+a$ model has two production mechanisms to generate DM candidates, the ggF mechanism, and the $b b$-induced mechanism. The ggF and $b b$-induced mechanisms are dominant in the low $\tan \beta$ and high $\tan \beta$ regions respectively, where $\tan \beta$ is the ratio of the vacuum expectation values of the two Higgs doublets in the $2 \mathrm{HDM}$ model. In this work, fixed values of $\tan \beta=1$ and $\tan \beta=10$ are used for the $\mathrm{MC}$ simulation of the $2 \mathrm{HDM}+a \mathrm{ggF}$ and $b b$-induced signal samples, respectively. The masses of the two CP-odd particles $A$ and $a$ are also varied to investigate the $2 \mathrm{HDM}+a$ signal model.

In the SR of the mono- $h(b \bar{b})$ analysis, the leptons are vetoed, and since a significant amount of $E_{\mathrm{T}}^{\text {miss }}$ is expected in the final states, a $E_{\mathrm{T}}^{\text {miss }}>150 \mathrm{GeV}$ selection is applied. Depending on the 
$E_{\mathrm{T}}^{\mathrm{miss}}$, the phase space of the analysis is divided into two topologies: Resolved for $150<E_{\mathrm{T}}^{\text {miss }}$ $<500 \mathrm{GeV}$ and Merged for $E_{\mathrm{T}}^{\text {miss }}>500 \mathrm{GeV}$. Since the characteristics of the $E_{\mathrm{T}}^{\text {miss }}$ spectra depend on the mass parameters of the signal models $\left(m_{Z^{\prime}}, m_{A}, m_{a}\right)$, to achieve sensitivity to different signal points the $\mathrm{SR}$ is further divided into several $E_{\mathrm{T}}^{\mathrm{miss}}$ bins. Moreover, the analysis phase space is divided into different $b$-tag categories: $2 b$ and $\geq 3 b$-tag category. The $\geq 3 b$-tag category is introduced in this analysis to gain sensitivity to the $2 \mathrm{HDM}+a b b$-induced model.

The main SM backgrounds in the mono- $h(b \bar{b})$ analysis are the $W / Z+$ jets and $t \bar{t}$ processes, and to constrain these backgrounds, two separate CRs are designed. The $W+$ jets and $t \bar{t}$ backgrounds are constrained by using a $1-\mu \mathrm{CR}$, and the $Z+$ jets are constrained by two 2-lepton CRs, where the two leptons are either electrons or muons of opposite-sign.

The final results are interpreted by a binned profile likelihood fit of the observed data to the model containing the SM backgrounds, $Z^{\prime}-2 \mathrm{HDM}$, and $2 \mathrm{HDM}+a$ models. All analysis regions are fitted simultaneously. Since no significant excess is found in the observed data compared to the SM backgrounds, upper limits on the DM production cross-sections are obtained using the $C L_{s}$ method. These upper limits are used to derive exclusion limits on the $\left(m_{Z^{\prime}}, m_{A}\right)$ plane for the $Z^{\prime}$-2HDM model and on $\left(m_{a}, m_{A}\right)$ plane for the $2 \mathrm{HDM}+a$ model. Improvements on the exclusion limits for both the $Z^{\prime}-2 \mathrm{HDM}$ and $2 \mathrm{HDM}+a$ signal models are observed. The improvements come from both the increased data statistics and the analysis techniques. By including the $\geq 3 b$-tag regions, the analysis gained sensitivity to the $2 \mathrm{HDM}+a b b$-induced mechanism, which was not the case in previous iterations of the analysis. The $2 \mathrm{HDM}+a$ and $Z^{\prime}-2 \mathrm{HDM}$ models are recommended DM models to be studied at the LHC by the LHC DM working group. Although no DM particles are observed in the analysis, improved exclusion limits on the model parameter space are obtained.

Although the increase in data statistics and refined analysis techniques have improved the results of the mono- $h(b \bar{b})$ DM search, further optimizations could be implemented that could yield better results. At the end of Run 2, the amount of ATLAS data available for physics analysis is 139 $\mathrm{fb}^{-1}$, while at the end of Run 3 (planned for 2023) the total available data projection is $300 \mathrm{fb}^{-1}$. In addition to data statistics, analysis strategy improvements can also lead to better results. The lower 
bound for $E_{\mathrm{T}}^{\text {miss }}$ is set to $150 \mathrm{GeV}$, however, signal points such as low $m_{Z^{\prime}}$ points in the $Z^{\prime}$-2HDM model, the $b b$-induced $2 \mathrm{HDM}+a$ models, have lower $E_{\mathrm{T}}^{\text {miss }}$ values. Therefore, lowering the $E_{\mathrm{T}}^{\text {miss }}$ cut makes the analysis more sensitive to these signal points. The analysis also saw an increase in sensitivity by splitting the Merged regions into multiple $E_{\mathrm{T}}^{\text {miss }}$ bins. Although the Merged $E_{\mathrm{T}}^{\text {miss }}$ bins were optimized, the Resolved $E_{\mathrm{T}}^{\text {miss }}$ binnings were taken from the previous round of mono$h(b \bar{b})$ analysis, and optimized Resolved $E_{\mathrm{T}}^{\text {miss }}$ binning could be beneficial. Similarly, another area of optimization could be the choice of $m_{h}$ binning that goes into the simultaneous fit. As mentioned earlier, the $W^{ \pm}+$jets and $t \bar{t}$ backgrounds are estimated using the $1-\mu \mathrm{CR}$. Instead of a $1-\mu \mathrm{CR}$ one can design a dilepton $\mathrm{CR}$ with an electron and a muon of opposite-sign $\left(e^{+} \mu^{-}\right.$or $\left.e^{-} \mu^{+}\right)$to estimate the $t \bar{t}$ backgrounds. The choice of leptons makes the CR rich in $t \bar{t}$ events, since it cuts away the $Z+$ jets events. To estimate the $W^{ \pm}+$jets backgrounds, one can still use the $1-\mu \mathrm{CR}$ with an additional $W$-boson mass proxy cut to remove the $t \bar{t}$ events. In the analysis, a deep neural based $b$-tagger (DL1) was used to tag both the small-R jets and the VR track jets. Using the new $b$-tagger (DL1r), which has improved $b$-tagging performance for the boosted jets, could improve the results in the Merged category. For the $2 \mathrm{HDM}+a$ model exclusion limits are shown in the $m_{A}-m_{a}$ plane, however other parameter scans can also be studied, i.e. $\tan \beta, \sin \theta, m_{\chi}$. In direct and indirect detection experiments constraints are put on the WIMP-nucleon cross section and WIMP annihilation cross section respectively as a function of the WIMP mass. In the $2 \mathrm{HDM}+a$ model, it is possible to put constraint on WIMP production cross section as a function of WIMP mass, which would be aligned with the current non-collider DM results.

The search for DE described this thesis in a $t \bar{t}+E_{\mathrm{T}}^{\mathrm{miss}}$ final state and is a reinterpretation of the search for top super-partners in the $t \bar{t}+E_{\mathrm{T}}^{\text {miss }}$ final state. The analysis is based on a EFT based DE model in which the DE-SM interactions, kinematic properties of DE are characterized by nine shift symmetry preserving operators. Of the nine operators, two are analyzed in this thesis. One operator $\left(\mathcal{L}_{1}\right)$ induces a DE-SM coupling proportional to the mass of the SM fermions, while the coupling by the other operator $\left(\mathcal{L}_{2}\right)$ is proportional to their momenta. Since the coupling from the $\mathcal{L}_{1}$ operator is proportional to the fermion masses, it has the highest sensitivity to the $t \bar{t}+E_{\mathrm{T}}^{\text {miss }}$ 
final state. $\mathcal{L}_{2}$ is more sensitive to final states like mono-jet $+E_{\mathrm{T}}^{\text {miss }}$. Depending on the $t \bar{t}$ decay type the phase space of the analysis is divided into 0,1 , and 2-lepton regions. The presence of a DE signal is tested by fitting the expected DE signal and the SM background yield to the observed data in the three lepton regions simultaneously using a binned maximum-likelihood approach. Since no significant data excess is found compared to the SM backgrounds, upper limits on DE production cross-sections $(p p \rightarrow t \bar{t} \phi \phi)$ for $\mathcal{L}_{1}$ and $\mathcal{L}_{2}$ operators are calculated at a 95\% CL using the $\mathrm{CL}_{\mathrm{s}}$ method and are translated to the lower limits on the suppression scale $M$. Although the $\mathrm{DE}$ analysis is a reinterpretation of the search for top super-partners, it is the first result of a DE search in collider experiments. 


\section{REFERENCES}

[1] Colliders join the hunt for dark energy. URL: https://cerncourier.com/a/collidersjoin-the-hunt-for-dark-energy/.

[2] F. Halzen and Alan D. Martin. QUARKS AND LEPTONS: AN INTRODUCTORY COURSE IN MODERN PARTICLE PHYSICS. Jan. 1984. ISBN: 978-0-471-88741-6.

[3] Ian C. Brock. Physics at the terascale. Weinheim, Germany: Wiley-VCH, 2011. ISBN: 978-3-527-41001-9.

[4] M. Tanabashi et al. "Review of Particle Physics". In: Phys. Rev. D 98 (3 Aug. 2018), p. 030001. DOI: 10.1103/PhysRevD.98.030001. URL: https://link.aps.org/doi/ 10.1103/PhysRevD.98.030001.

[5] SM Particles chart. URL: https://upload.wikimedia.org/wikipedia/commons/a/ a3/Standard_Model_of_Elementary_Particles_Anti.svg.

[6] Murray Gell-Mann. "Symmetries of Baryons and Mesons". In: Phys. Rev. 125 (3 Feb. 1962), pp. 1067-1084. DOI: 10.1103/PhysRev .125.1067. URL: https://link .aps. org/doi/10.1103/PhysRev.125.1067.

[7] Abdus Salam. "Weak and Electromagnetic Interactions". In: Conf. Proc. C 680519 (1968), pp. 367-377. DOI: $10.1142 / 9789812795915 \backslash \_0034$.

[8] Steven Weinberg. “A Model of Leptons”. In: Phys. Rev. Lett. 19 (21 Nov. 1967), pp. 12641266. DOI: 10.1103/PhysRevLett.19.1264, URL: https://link.aps.org/doi/10. 1103/PhysRevLett.19.1264.

[9] S.L. Glashow. "Partial Symmetries of Weak Interactions". In: Nucl. Phys. 22 (1961), pp. 579588. DOI: 10.1016/0029-5582(61)90469-2.

[10] Antonio Pich. "The Standard Model of Electroweak Interactions". In: 2010 European School of High Energy Physics. Jan. 2012, pp. 1-50. arXiv: 1201.0537 [hep-ph]. 
[11] Kazuhiko Nishijima. "Charge Independence Theory of V Particles”. In: Prog. Theor. Phys. 13.3 (1955), pp. 285-304. DOI: 10.1143/PTP.13.285.

[12] M. Gell-Mann. "The interpretation of the new particles as displaced charge multiplets". In: Nuovo Cim. 4.S2 (1956), pp. 848-866. DOI: 10.1007/BF02748000.

[13] F. Englert and R. Brout. "Broken Symmetry and the Mass of Gauge Vector Mesons". In: Phys. Rev. Lett. 13 (9 Aug. 1964), pp. 321-323. DOI: 10.1103/PhysRevLett.13. 321. URL: https://link.aps.org/doi/10.1103/PhysRevLett.13.321.

[14] P.W. Higgs. "Broken symmetries, massless particles and gauge fields". In: Physics Letters 12.2 (1964), pp. 132-133. ISSN: 0031-9163. DOI: https ://doi .org/10.1016/00319163(64)91136-9. URL: http://www.sciencedirect.com/science/article/pii/ 0031916364911369 .

[15] Peter W. Higgs. "Broken Symmetries and the Masses of Gauge Bosons". In: Phys. Rev. Lett. 13 (16 Oct. 1964), pp. 508-509. DOI:10.1103/PhysRevLett.13.508. URL: https : //link.aps.org/doi/10.1103/PhysRevLett.13.508.

[16] M. Baak et al. "The global electroweak fit at NNLO and prospects for the LHC and ILC". In: Eur. Phys. J. C 74 (2014), p. 3046. DOI: 10.1140/epjc/s10052-014-3046-5 arXiv: $1407.3792[$ hep-ph].

[17] Standard Model Summary Plots Summer 2019. Tech. rep. ATL-PHYS-PUB-2019-024. Geneva: CERN, July 2019. URL: http://cds.cern.ch/record/2682186.

[18] Morad Aaboud et al. "Measurement of the $W$-boson mass in pp collisions at $\sqrt{s}=7 \mathrm{TeV}$ with the ATLAS detector". In: Eur. Phys. J. C 78.2 (2018). [Erratum: Eur.Phys.J.C 78, 898 (2018)], p. 110. DOI: 10 .1140/epjc/s10052-017-5475-4. arXiv: 1701.07240 $[\mathrm{hep}-\mathrm{ex}]$.

[19] Y. Fukuda et al. "Evidence for oscillation of atmospheric neutrinos". In: Phys. Rev. Lett. 81 (1998), pp. 1562-1567. DOI: 10 .1103/PhysRevLett . 81 . 1562, arXiv: hep-ex/ 9807003 . 
[20] F. Zwicky. "Die Rotverschiebung von extragalaktischen Nebeln". In: Helv. Phys. Acta 6 (1933). [Gen. Rel. Grav.41,207(2009)], pp. 110-127. DOI:10.1007/s10714-008-07074.

[21] C. L. Bennett et al. "Nine-Year Wilkinson Microwave Anisotropy Probe (WMAP) Observations: Final Maps and Results”. In: Astrophys. J. Suppl. 208 (2013), p. 20. DOI: 10 . 1088/0067-0049/208/2/20, arXiv: 1212.5225 [astro-ph.CO].

[22] R. Adam et al. "Planck 2015 results. I. Overview of products and scientific results". In: Astron. Astrophys. 594 (2016), A1. DOI: 10.1051/0004-6361/201527101. arXiv: 1502. 01582 [astro-ph.CO]

[23] Cosmic Microwave Background. URL: https : / / pdg . lbl . gov/ rev2019/2019/ reviews/rpp2019-rev-cosmic-microwave-background.pdf.

[24] Douglas Clowe et al. "A direct empirical proof of the existence of dark matter". In: Astrophys. J. 648 (2006), pp. L109-L113. DOI: 10.1086/508162, arXiv: astro-ph/0608407 [astro-ph].

[25] Lars Bergstrom. "Dark Matter Candidates". In: New J. Phys. 11 (2009), p. 105006. DOI: 10.1088/1367-2630/11/10/105006, arXiv: 0903.4849 [hep-ph].

[26] Graciela B. Gelmini. “The Hunt for Dark Matter”. In: Theoretical Advanced Study Institute in Elementary Particle Physics: Journeys Through the Precision Frontier: Amplitudes for Colliders. Feb. 2015. DOI: 10 .1142/9789814678766_0012, arXiv: 1502.01320 [hep-ph].

[27] Jihn E. Kim. "Weak Interaction Singlet and Strong CP Invariance". In: Phys. Rev. Lett. 43 (1979), p. 103. DOI: $10.1103 /$ PhysRevLett. 43.103

[28] Mikhail A. Shifman, A. I. Vainshtein, and Valentin I. Zakharov. "Can Confinement Ensure Natural CP Invariance of Strong Interactions?” In: Nucl. Phys. B 166 (1980), pp. 493-506. DOI: $10.1016 / 0550-3213(80) 90209-6$. 
[29] A. R. Zhitnitsky. "On Possible Suppression of the Axion Hadron Interactions. (In Russian)”. In: Sov. J. Nucl. Phys. 31 (1980), p. 260.

[30] R. D. Peccei and Helen R. Quinn. "Constraints Imposed by CP Conservation in the Presence of Instantons". In: Phys. Rev. D 16 (1977), pp. 1791-1797. DOI: 10.1103/PhysRevD. 16.1791 .

[31] A. Boyarsky et al. "Sterile neutrino Dark Matter". In: Prog. Part. Nucl. Phys. 104 (2019), pp. 1-45. DOI: $10.1016 /$ j.ppnp.2018.07.004. arXiv: 1807.07938 [hep-ph].

[32] Thomas Thummler. "Introduction to direct neutrino mass measurements and KATRIN". In: Nucl. Phys. Proc. Suppl. 229-232 (2012), pp. 146-151. DOI: 10.1016/j .nuclphysbps. 2012.09.024. arXiv: 1012.2282 [hep-ex].

[33] Peter Minkowski. " $\mu \rightarrow e \gamma$ at a Rate of One Out of $10^{9}$ Muon Decays?” In: Phys. Lett. B 67 (1977), pp. 421-428. DOI: 10.1016/0370-2693(77)90435-X.

[34] Tsutomu Yanagida. "Horizontal gauge symmetry and masses of neutrinos". In: Conf. Proc. C 7902131 (1979). Ed. by Osamu Sawada and Akio Sugamoto, pp. 95-99.

[35] Murray Gell-Mann, Pierre Ramond, and Richard Slansky. "Complex Spinors and Unified Theories”. In: Conf. Proc. C 790927 (1979), pp. 315-321. arXiv: 1306.4669 [hep-th].

[36] S. L. Glashow. "The Future of Elementary Particle Physics". In: NATO Sci. Ser. B 61 (1980), p. 687. DOI: $10.1007 / 978-1-4684-7197-7 \_15$.

[37] Joakim Edsjo and Paolo Gondolo. "Neutralino relic density including coannihilations". In: Phys. Rev. D 56 (1997), pp. 1879-1894. DOI: 10 . 1103/PhysRevD . 56 . 1879, arXiv: hep-ph/9704361.

[38] H. Baer et al. "Low energy supersymmetry phenomenology". In: (Mar. 1995), 74 p. DOI: 10.2172/72994. URL: https://cds . cern. ch/record/279512. 
[39] Howard E. Haber. "Introductory low-energy supersymmetry". In: Theoretical Advanced Study Institute (TASI 92): From Black Holes and Strings to Particles. Apr. 1993. arXiv: hep-ph/9306207.

[40] David H. Weinberg et al. "Cold dark matter: controversies on small scales". In: Proc. Nat. Acad. Sci. 112 (2015), pp. 12249-12255. DOI:10.1073/pnas.1308716112, arXiv: 1306. 0913 [astro-ph.C0].

[41] C. Amole et al. "Improved dark matter search results from PICO-2L Run 2". In: Phys. Rev. D 93.6 (2016), p. 061101. DOI: 10.1103/PhysRevD . 93.061101. arXiv: 1601.03729 [astro-ph.CO].

[42] C. Amole et al. "Dark Matter Search Results from the PICO-60 $\mathrm{C}_{3} \mathrm{~F}_{8}$ Bubble Chamber". In: Phys. Rev. Lett. 118.25 (2017), p. 251301. DOI: 10.1103/PhysRevLett.118.251301 arXiv: 1702.07666 [astro-ph.CO]

[43] D.S. Akerib et al. "Results on the Spin-Dependent Scattering of Weakly Interacting Massive Particles on Nucleons from the Run 3 Data of the LUX Experiment”. In: Phys. Rev. Lett. 116.16 (2016), p. 161302. DOI: 10.1103/PhysRevLett.116.161302. arXiv: 1602. 03489 [hep-ex].

[44] M. Ackermann et al. "Searching for Dark Matter Annihilation from Milky Way Dwarf Spheroidal Galaxies with Six Years of Fermi Large Area Telescope Data”. In: Phys. Rev. Lett. 115.23 (2015), p. 231301. DOI: 10.1103/PhysRevLett.115.231301. arXiv: 1503. 02641 [astro-ph.HE]

[45] Marc Schumann. "Direct Detection of WIMP Dark Matter: Concepts and Status". In: $J$. Phys. G46.10 (2019), p. 103003. DOI: 10.1088/1361-6471/ab2ea5 arXiv: 1903.03026 [astro-ph.CO].

[46] S. Perlmutter et al. "Measurements of $\Omega$ and $\Lambda$ from 42 high redshift supernovae". In: Astrophys. J. 517 (1999), pp. 565-586. DOI: 10.1086/307221. arXiv: astro-ph/9812133 [astro-ph]. 
[47] Adam G. Riess et al. "Observational evidence from supernovae for an accelerating universe and a cosmological constant”. In: Astron. J. 116 (1998), pp. 1009-1038. DOI: 10 . $1086 /$ 300499, arXiv: astro-ph/9805201 [astro-ph].

[48] Bharat Ratra and P.J.E. Peebles. "Cosmological Consequences of a Rolling Homogeneous Scalar Field”. In: Phys. Rev. D 37 (1988), p. 3406. DOI: 10.1103/PhysRevD.37.3406.

[49] Jaewon Yoo and Yuki Watanabe. "Theoretical Models of Dark Energy". In: Int. J. Mod. Phys. D 21 (2012), p. 1230002. DOI: 10.1142/S0218271812300029, arXiv: 1212.4726 [astro-ph.c0].

[50] Gregory Walter Horndeski. "Second-order scalar-tensor field equations in a four-dimensional space”. In: Int. J. Theor. Phys. 10 (1974), pp. 363-384. DOI: 10.1007/BF01807638

[51] Jalal Abdallah et al. "Simplified Models for Dark Matter Searches at the LHC". In: Phys. Dark Univ. 9-10 (2015), pp. 8-23. DOI: 10.1016/j.dark.2015.08.001 arXiv: 1506. $03116[$ hep-ph]

[52] Daniel Abercrombie et al. "Dark Matter Benchmark Models for Early LHC Run-2 Searches: Report of the ATLAS/CMS Dark Matter Forum”. In: Phys. Dark Univ. 27 (2020). Ed. by Antonio Boveia et al., p. 100371. DOI: 10 . 1016/j . dark . 2019 . 100371, arXiv: 1507.00966 [hep-ex]

[53] G. C. Branco et al. "Theory and phenomenology of two-Higgs-doublet models". In: Phys. Rept. 516 (2012), pp. 1-102. DOI: $10.1016 /$ j · physrep . 2012.02 .002. arXiv: 1106 . $0034[$ hep-ph].

[54] Emmanuel A. Paschos. "Diagonal neutral currents". In: Phys. Rev. D 15 (7 Apr. 1977), pp. 1966-1972. DOI: 10.1103/PhysRevD .15 .1966. URL: https : //link . aps .org/ doi/10.1103/PhysRevD.15.1966.

[55] Sheldon L. Glashow and Steven Weinberg. "Natural conservation laws for neutral currents". In: Phys. Rev. D 15 (7 Apr. 1977), pp. 1958-1965. DOI: 10.1103/PhysRevD.15. 1958. URL: https://link.aps.org/doi/10.1103/PhysRevD.15.1958. 
[56] Combined measurements of Higgs boson production and decay using up to $80 \mathrm{fb}^{-1}$ of proton-proton collision data at $\sqrt{s}=13 \mathrm{TeV}$ collected with the ATLAS experiment. Tech. rep. ATLAS-CONF-2018-031. Geneva: CERN, July 2018. URL: https ://cds . cern . ch/ record/2629412.

[57] M. Misiak et al. "Estimate of $\mathcal{B}\left(\bar{B} \rightarrow X_{s} \gamma\right)$ at $O\left(\alpha_{s}^{2}\right)$ ". In: Phys. Rev. Lett. 98 (2 Jan. 2007), p. 022002. DOI: 10.1103/PhysRevLett.98.022002, URL: https://link .aps .org/ doi/10.1103/PhysRevLett.98.022002.

[58] Tomohiro Abe et al. "LHC Dark Matter Working Group: Next-generation spin-0 dark matter models". In: Phys. Dark Univ. 27 (2020), p. 100351. DOI: 10 . 1016/ j . dark . 2019 . 100351. arXiv: 1810.09420 [hep-ex].

[59] Search for invisible Higgs boson decays with vector boson fusion signatures with the ATLAS detector using an integrated luminosity of $139 \mathrm{fb}^{-1}$. Tech. rep. ATLAS-CONF-2020008. Geneva: CERN, Apr. 2020. URL: http://cds . cern.ch/record/2715447.

[60] Albert M Sirunyan et al. "Search for narrow and broad dijet resonances in proton-proton collisions at $\sqrt{s}=13 \mathrm{TeV}$ and constraints on dark matter mediators and other new particles". In: JHEP 08 (2018), p. 130. DOI: 10.1007/JHEP08(2018)130, arXiv: 1806.00843 $[$ hep-ex].

[61] Morad Aaboud et al. "Search for new phenomena in dijet events using $37 \mathrm{fb}^{-1}$ of $p p$ collision data collected at $\sqrt{s}=13 \mathrm{TeV}$ with the ATLAS detector". In: Phys. Rev. D 96.5 (2017), p. 052004. DOI: 10.1103/PhysRevD.96.052004 arXiv: 1703.09127 [hep-ex].

[62] Morad Aaboud et al. "Constraints on mediator-based dark matter and scalar dark energy models using $\sqrt{s}=13 \mathrm{TeV} p p$ collision data collected by the ATLAS detector". In: JHEP 05 (2019), p. 142. DOI: 10.1007/JHEP05 (2019)142, arXiv: 1903.01400 [hep-ex].

[63] ATLAS: technical proposal for a general-purpose pp experiment at the Large Hadron Collider at CERN. LHC Tech. Proposal. Geneva: CERN, 1994. URL: http://cds . cern.ch/ record/290968. 
[64] Technical proposal. LHC Tech. Proposal. Cover title : CMS, the Compact Muon Solenoid : technical proposal. Geneva: CERN, 1994. URL: http://cds.cern.ch/record/290969

[65] LHCb : Technical Proposal. Tech. Proposal. Geneva: CERN, 1998. URL: http : //cds . cern.ch/record/622031.

[66] ALICE: Technical proposal for a Large Ion collider Experiment at the CERN LHC. LHC Tech. Proposal. Geneva: CERN, 1995. URL: http://cds . cern.ch/record/293391.

[67] Lyndon Evans and Philip Bryant. "LHC Machine". In: Journal of Instrumentation 3.08 (Aug. 2008), S08001-S08001. DOI: 10.1088/1748-0221/3/08/s08001. URL: https : //doi.org/10.1088\%2F1748-0221\%2F3\%2F08\%2Fs08001.

[68] LHC map at CERN. URL: https : //sciencesprings .files .wordpress . com/2015/ 08/cern-lhc-map.jpg.

[69] Oliver Sim Brüning et al. LHC Design Report. CERN Yellow Reports: Monographs. Geneva: CERN, 2004. DOI: 10 . 5170/CERN-2004-003-V-1. URL: http : / / cds . cern . ch / record/782076.

[70] Oliver Sim Brüning et al. LHC Design Report. CERN Yellow Reports: Monographs. Geneva: CERN, 2004. DOI: 10 . 5170/CERN-2004-003-V-2. URL: http : / / cds . cern . ch/ record/815187.

[71] Michael Benedikt et al. LHC Design Report. CERN Yellow Reports: Monographs. Geneva: CERN, 2004. DOI: 10 . 5170/CERN-2004-003-V-3. URL: http : / / cds . cern . ch / record/823808.

[72] Werner Herr and B Muratori. “Concept of luminosity”. In: (2006). DOI: 10.5170/CERN2006-002.361. URL: http://cds.cern.ch/record/941318.

[73] ATLAS Experiment at the HL-LHC. URL:https://https://livrepository. liverpool. ac.uk/, 
[74] Juan Casas et al. "Design concept and first experimental validation of the superfluid helium system for the large hadron collider (LHC) project at CERN". In: Cryogenics 32 (1992). Ed. by P. Komarek and C. Rizzuto, pp. 118-121. DOI: 10.1016/0011-2275(92)90122-Q.

[75] R Perin and J Vlogaert. Magnets for the Large Hadron Collider. Tech. rep. CERN-AT93-46-MA. LHC-NOTE-255. CERN-LHC-Note-255. Geneva: CERN, Feb. 1994. URL: https://cds.cern.ch/record/260440.

[76] G. Aad et al. "The ATLAS Experiment at the CERN Large Hadron Collider". In: JINST 3 (2008), S08003. DOI: 10.1088/1748-0221/3/08/S08003.

[77] Joao Pequenao. "Computer generated image of the whole ATLAS detector". Mar. 2008. URL: https://cds.cern.ch/record/1095924.

[78] ATLAS inner detector: Technical Design Report, 1. Technical Design Report ATLAS. Geneva: CERN, 1997. URL: https://cds.cern.ch/record/331063.

[79] Joao Pequenao. "Computer generated image of the ATLAS inner detector". Mar. 2008. URL: https://cds. cern.ch/record/1095926.

[80] Fabian Hugging. "Front end electronics and integration of ATLAS pixel modules". In: Nucl. Instrum. Meth. A 549 (2005). Ed. by S. J. Watts, pp. 157-164. DOI: 10.1016/j . nima.2005.04.045, arXiv: physics/0401106.

[81] R. Beccherle et al. "MCC: The Module Controller Chip for the ATLAS pixel detector". In: Nucl. Instrum. Meth. A 492 (2002), pp. 117-133. DOI:10.1016/S0168-9002(02)012792.

[82] F. Campabadal et al. "Design and performance of the ABCD3TA ASIC for readout of silicon strip detectors in the ATLAS semiconductor tracker". In: Nucl. Instrum. Meth. A 552 (2005), pp. 292-328. DOI: $10.1016 /$ j.nima.2005.07.002.

[83] A. Abdesselam et al. "The ATLAS SCT optoelectronics and the associated electrical services". In: (Jan. 2007). 
[84] EIC Calorimetry. URL: https://jleic-docdb.jlab.org/DocDB/0001/000154/003/ EIC_calorimetry_v5.2.pdf.

[85] Pablo Roldan and Paul Lecoq. "Quality control and preparation of the PWO crystals for the electromagnetic calorimeter of CMS”. In: (Jan. 2021).

[86] Kaoru Hagiwara et al. "Review of particle physics. Particle Data Group". In: Phys. Rev. D 66 (2002), p. 010001. DOI: 10.1103/PhysRevD.66.010001.

[87] Joao Pequenao. "Computer Generated image of the ATLAS calorimeter". Mar. 2008. URL: https://cds.cern. ch/record/1095927.

[88] ATLAS electromagnetic calorimeter. URL: https : //cds . cern . ch/record/2158117/ plots.

[89] ATLAS liquid-argon calorimeter: Technical Design Report. Technical Design Report ATLAS. Geneva: CERN, 1996. URL: http://cds . cern.ch/record/331061.

[90] “ATLAS tile calorimeter: Technical design report”. In: (Dec. 1996).

[91] Bernardo Sotto-Maior Peralva. Calibration and Performance of the ATLAS Tile Calorimeter. Tech. rep. arXiv:1305.0550. Comments: 4 pages, 7 figures, LISHEP 2013, 17-24 March 2013, Rio de Janeiro. May 2013. URL: https: //cds . cern. ch/record/1545496.

[92] D. M. Gingrich et al. "Construction, assembly and testing of the ATLAS hadronic end-cap calorimeter". In: JINST 2 (2007), P05005. DOI: 10.1088/1748-0221/2/05/P05005.

[93] James Pinfold. "Performance of the ATLAS liquid argon endcap calorimeter in the pseudorapidity region $2.5 i$ —eta_ 44.0 in beam tests”. In: Nuclear Instruments and Methods in Physics Research Section A, 324-342 (2008) (Jan. 2008).

[94] N. J. Buchanan et al. "Design and implementation of the Front End Board for the readout of the ATLAS liquid argon calorimeters". In: JINST 3 (2008), P03004. DOI: 10.1088/17480221/3/03/P03004 
[95] Joao Pequenao. “Computer generated image of the ATLAS Muons subsystem”. Mar. 2008. URL: https://cds.cern.ch/record/1095929.

[96] ATLAS magnet system: Technical Design Report, 1. Technical Design Report ATLAS. Geneva: CERN, 1997. URL: https://cds.cern.ch/record/338080.

[97] ATLAS Outreach. "ATLAS Fact Sheet : To raise awareness of the ATLAS detector and collaboration on the LHC". 2010. URL: https ://cds. cern. ch/record/1457044.

[98] A. Yamamoto et al. “The ATLAS central solenoid”. In: Nucl. Instrum. Meth. A 584 (2008), pp. 53-74. DOI: $10.1016 /$ j.nima.2007.09.047.

[99] J P Badiou et al. ATLAS barrel toroid: Technical Design Report. Technical Design Report ATLAS. Electronic version not available. Geneva: CERN, 1997. URL: https : / / cds . cern.ch/record/331065.

[100] ATLAS end-cap toroids: Technical Design Report. Technical Design Report ATLAS. Electronic version not available. Geneva: CERN, 1997. URL: https : / / cds . cern . ch / record/331066.

[101] Oleg Solovyanov. "Run 2 ATLAS Trigger and Detector Performance". In: (Jan. 2018). URL: https://cds.cern.ch/record/2301187.

[102] Rosa Simoniello. The ATLAS Level-1 Topological Processor: from design to routine usage in Run-2. Tech. rep. Geneva: CERN, Dec. 2018. DOI: 10.1109/NSSMIC . 2018.8824280 URL: https://cds.cern.ch/record/2649959.

[103] A. Sidoti. "Minimum Bias Trigger Scintillators in ATLAS Run II". In: JINST 9.10 (2014), p. C10020. DOI: 10.1088/1748-0221/9/10/C10020.

[104] G. Avoni et al. "The new LUCID-2 detector for luminosity measurement and monitoring in ATLAS”. In: JINST 13.07 (2018), P07017. DOI: 10.1088/1748-0221/13/07/P07017.

[105] Peter Jenni, Marzio Nessi, and Markus Nordberg. Zero Degree Calorimeters for ATLAS. Tech. rep. Geneva: CERN, Jan. 2007. URL: https://cds . cern.ch/record/1009649. 
[106] B.G. Taylor. "TTC distribution for LHC detectors". In: IEEE Transactions on Nuclear Science 45.3 (1998), pp. 821-828. DOI: $10.1109 / 23.682644$.

[107] B G Taylor. “Timing distribution at the LHC”. In: (2002). DOI: 10.5170/CERN-2002003.63. URL: https://cds. cern.ch/record/592719.

[108] LHC TTC system. URL: https://ttc.web.cern.ch/.

[109] WorldWide LHC computing grid. URL: https://wlcg.web.cern.ch/.

[110] L Adamczyk et al. Technical Design Report for the ATLAS Forward Proton Detector. Tech. rep. May 2015. URL: https ://cds.cern.ch/record/2017378.

[111] T Kawamoto et al. New Small Wheel Technical Design Report. Tech. rep. ATLAS New Small Wheel Technical Design Report. June 2013. URL: https : / / cds . cern . ch / record/1552862.

[112] M (CERN) Aleksa et al. ATLAS Liquid Argon Calorimeter Phase-I Upgrade: Technical Design Report. Tech. rep. Final version presented to December 2013 LHCC. Sept. 2013. URL: https://cds.cern.ch/record/1602230.

[113] Georges Aad et al. "Technical Design Report for the Phase-I Upgrade of the ATLAS TDAQ System”. In: (Sept. 2013).

[114] Apollinari G. et al. High-Luminosity Large Hadron Collider (HL-LHC): Technical Design Report V. 0.1. CERN Yellow Reports: Monographs. Geneva: CERN, 2017. DOI: 10 . 23731/CYRM-2017-004. URL: https://cds . cern.ch/record/2284929.

[115] Technical Design Report for the ATLAS Inner Tracker Pixel Detector. Tech. rep. CERNLHCC-2017-021. ATLAS-TDR-030. Geneva: CERN, Sept. 2017. URL: https : / / cds . cern.ch/record/2285585.

[116] Technical Design Report for the ATLAS Inner Tracker Strip Detector. Tech. rep. Geneva: CERN, Apr. 2017. URL: https://cds.cern.ch/record/2257755. 
[117] Technical Design Report for the Phase-II Upgrade of the ATLAS TDAQ System. Tech. rep. CERN-LHCC-2017-020. ATLAS-TDR-029. Geneva: CERN, Sept. 2017. URL: https : //cds.cern.ch/record/2285584.

[118] Technical Proposal: A High-Granularity Timing Detector for the ATLAS Phase-II Upgrade. Tech. rep. CERN-LHCC-2018-023. LHCC-P-012. Geneva: CERN, June 2018. URL: https://cds.cern.ch/record/2623663.

[119] Technical Design Report for the Phase-II Upgrade of the ATLAS Muon Spectrometer. Tech. rep. CERN-LHCC-2017-017. ATLAS-TDR-026. Geneva: CERN, Sept. 2017. URL: https://cds.cern.ch/record/2285580.

[120] "High-Luminosity Large Hadron Collider (HL-LHC): Technical Design Report V. 0.1”. In: 4/2017 (Nov. 2017). Ed. by G. Apollinari et al. DOI: 10.23731/CYRM-2017-004.

[121] G. Pellegrini et al. "Technology developments and first measurements of Low Gain Avalanche Detectors (LGAD) for high energy physics applications". In: Nucl. Instrum. Meth. A 765 (2014). Ed. by Yoshinobu Unno et al., pp. 12-16. DOI: 10.1016/j.nima.2014.06.008.

[122] T. Cornelissen et al. "Concepts, Design and Implementation of the ATLAS New Tracking (NEWT)”. In: (Mar. 2007). Ed. by A. Salzburger.

[123] “The Optimization of ATLAS Track Reconstruction in Dense Environments". In: (Mar. 2015).

[124] R. Fruhwirth. "Application of Kalman filtering to track and vertex fitting". In: Nucl. Instrum. Meth. A 262 (1987), pp. 444-450. DOI: 10.1016/0168-9002(87)90887-4.

[125] "Performance of primary vertex reconstruction in proton-proton collisions at $\sqrt{s}=7 \mathrm{TeV}$ in the ATLAS experiment". In: (July 2010).

[126] Morad Aaboud et al. "Reconstruction of primary vertices at the ATLAS experiment in Run 1 proton-proton collisions at the LHC". In: Eur. Phys. J. C 77.5 (2017), p. 332. DOI: 10.1140/epjc/s10052-017-4887-5, arXiv: 1611.10235 [physics.ins-det]. 
[127] E. Bouhova-Thacker et al. "Vertex reconstruction in the ATLAS experiment at the LHC". In: 2008 IEEE Nuclear Science Symposium and Medical Imaging Conference and 16th International Workshop on Room-Temperature Semiconductor X-Ray and Gamma-Ray Detectors. 2008, pp. 1720-1727. DOI: 10.1109/NSSMIC. 2008.4774734.

[128] Morad Aaboud et al. "Electron reconstruction and identification in the ATLAS experiment using the 2015 and 2016 LHC proton-proton collision data at $\sqrt{s}=13 \mathrm{TeV}$ ”. In: Eur. Phys. J. C 79.8 (2019), p. 639. DOI: 10.1140/epjc/s10052-019-7140-6. arXiv: 1902.04655 [physics.ins-det].

[129] W. Lampl et al. "Calorimeter clustering algorithms: Description and performance". In: (May 2008).

[130] Georges Aad et al. "Muon reconstruction performance of the ATLAS detector in protonproton collision data at $\sqrt{s}=13$ TeV". In: Eur. Phys. J. C 76.5 (2016), p. 292. DOI: 10 . 1140/epjc/s10052-016-4120-y, arXiv: 1603.05598 [hep-ex].

[131] Georges Aad et al. "Electron and photon performance measurements with the ATLAS detector using the 2015-2017 LHC proton-proton collision data". In: JINST 14.12 (2019), P12006. DOI: 10.1088/1748-0221/14/12/P12006 arXiv: 1908.00005 [hep-ex].

[132] Georges Aad et al. "Performance of electron and photon triggers in ATLAS during LHC Run 2". In: Eur. Phys. J. C 80.1 (2020), p. 47. DOI: 10.1140/epjc/s10052-019-7500-2. arXiv: 1909.00761 [hep-ex].

[133] Georges Aad et al. "Muon reconstruction and identification efficiency in ATLAS using the full Run $2 p p$ collision data set at $\sqrt{s}=13$ TeV”. In: (Dec. 2020). arXiv: 2012.00578 [hep-ex].

[134] Tancredi Carli, Klaus Rabbertz, and Steffen Schumann. "Studies of Quantum Chromodynamics at the LHC". In: The Large Hadron Collider: Harvest of Run 1. Ed. by Thomas Schörner-Sadenius. 2015, pp. 139-194. DOI: 10.1007/978-3-319-15001-7_5. arXiv: 1506.03239 [hep-ex] 
[135] Georges Aad et al. "Topological cell clustering in the ATLAS calorimeters and its performance in LHC Run 1”. In: Eur. Phys. J. C 77 (2017), p. 490. DOI: 10.1140/epjc/s10052017-5004-5, arXiv: 1603.02934 [hep-ex].

[136] Javier Montejo Berlingen. "Search for new physics in $t \bar{t}$ final states with additional heavyflavor jets with the ATLAS detector". PhD thesis. Barcelona, Autonoma U., Apr. 2015.

[137] Matteo Cacciari, Gavin P. Salam, and Gregory Soyez. "The anti- $k_{t}$ jet clustering algorithm”. In: JHEP 04 (2008), p. 063. DOI: 10.1088/1126-6708/2008/04/063, arXiv: 0802.1189 [hep-ph].

[138] M. Aaboud et al. "Jet energy scale measurements and their systematic uncertainties in proton-proton collisions at $\sqrt{s}=13 \mathrm{TeV}$ with the ATLAS detector". In: Phys. Rev. D 96.7 (2017), p. 072002. DOI: 10.1103/PhysRevD.96.072002 arXiv: 1703.09665 [hep-ex].

[139] Steven Schramm. "ATLAS Jet Reconstruction, Calibration, and Tagging of Lorentz-boosted Objects”. In: EPJ Web Conf. 182 (2018). Ed. by Y. Aharonov, L. Bravina, and S. Kabana, p. 02113. DOI: $10.1051 /$ epjconf/201818202113

[140] Matteo Cacciari, Gavin P. Salam, and Gregory Soyez. "The Catchment Area of Jets". In: JHEP 04 (2008), p. 005. DOI: 10.1088/1126-6708/2008/04/005 arXiv: 0802.1188 $[\mathrm{hep}-\mathrm{ph}]$.

[141] David Krohn, Jesse Thaler, and Lian-Tao Wang. "Jet Trimming”. In: JHEP 02 (2010), p. 084. DOI: 10.1007/JHEP02(2010)084. arXiv: 0912.1342 [hep-ph].

[142] Morad Aaboud et al. "Determination of jet calibration and energy resolution in protonproton collisions at $\sqrt{s}=8 \mathrm{TeV}$ using the ATLAS detector". In: (Oct. 2019). arXiv: 1910 . 04482 [hep-ex]

[143] "Data-driven determination of the energy scale and resolution of jets reconstructed in the ATLAS calorimeters using dijet and multijet events at $\sqrt{s}=8 \mathrm{TeV}$ '. In: (Apr. 2015). 
[144] Morad Aaboud et al. "In situ calibration of large-radius jet energy and mass in $13 \mathrm{TeV}$ proton-proton collisions with the ATLAS detector”. In: Eur. Phys. J. C 79.2 (2019), p. 135. DOI: 10.1140/epjc/s10052-019-6632-8, arXiv: 1807.09477 [hep-ex].

[145] "Measurement of the ATLAS Detector Jet Mass Response using Forward Folding with 80 $\mathrm{fb}^{-1}$ of $\sqrt{s}=13 \mathrm{TeV} p p$ data". In: (July 2020).

[146] Morad Aaboud et al. "Jet reconstruction and performance using particle flow with the ATLAS Detector". In: Eur. Phys. J. C 77.7 (2017), p. 466. DOI: 10.1140/epjc/s10052017-5031-2, arXiv: 1703.10485 [hep-ex].

[147] Morad Aaboud et al. "Jet reconstruction and performance using particle flow with the ATLAS Detector". In: Eur. Phys. J. C 77.7 (2017), p. 466. DOI: 10.1140/epjc/s10052017-5031-2, arXiv: 1703.10485 [hep-ex].

[148] David Krohn, Jesse Thaler, and Lian-Tao Wang. "Jets with Variable R". In: JHEP 06 (2009), p. 059. DOI: 10.1088/1126-6708/2009/06/059, arXiv:0903.0392 [hep-ph].

[149] "Variable Radius, Exclusive- $\mathrm{k}_{T}$, and Center-of-Mass Subjet Reconstruction for Higgs $(\rightarrow$ $b \bar{b}$ ) Tagging in ATLAS”. In: (June 2017).

[150] Georges Aad et al. "ATLAS b-jet identification performance and efficiency measurement with $t \bar{t}$ events in pp collisions at $\sqrt{s}=13$ TeV”. In: Eur. Phys. J. C 79.11 (2019), p. 970. DOI: 10.1140/epjc/s10052-019-7450-8, arXiv: 1907.05120 [hep-ex].

[151] "Optimisation and performance studies of the ATLAS $b$-tagging algorithms for the 201718 LHC run". In: (July 2017).

[152] 'Secondary vertex finding for jet flavour identification with the ATLAS detector". In: (June 2017).

[153] "Topological $b$-hadron decay reconstruction and identification of $b$-jets with the JetFitter package in the ATLAS experiment at the LHC'. In: (2018). 
[154] G Piacquadio and C Weiser. "A new inclusive secondary vertex algorithm for b-jet tagging in ATLAS”. In: Journal of Physics: Conference Series 119 (July 2008), p. 032032. DOI: $10.1088 / 1742-6596 / 119 / 3 / 032032$.

[155] The Theano Development Team et al. Theano: A Python framework for fast computation of mathematical expressions. 2016. arXiv: 1605.02688 [cs.SC].

[156] Diederik P. Kingma and Jimmy Ba. Adam: A Method for Stochastic Optimization. 2017. arXiv: 1412.6980 [cs.LG],

[157] Expected performance of the 2019 ATLAS PFlow b-taggers. URL: http://atlas . web. cern.ch/Atlas/GROUPS/PHYSICS/PLOTS/FTAG-2019-005.

[158] Morad Aaboud et al. "Performance of missing transverse momentum reconstruction with the ATLAS detector using proton-proton collisions at $\sqrt{s}=13 \mathrm{TeV}$ ". In: Eur. Phys. J. C 78.11 (2018), p. 903. DOI: $10.1140 / \mathrm{epjc} / \mathrm{s} 10052-018-6288-9$ arXiv: 1802.08168 [hep-ex].

[159] "Expected performance of missing transverse momentum reconstruction for the ATLAS detector at $\sqrt{s}=13 \mathrm{TeV}$ ". In: (July 2015).

[160] T. Gleisberg et al. "Event generation with SHERPA 1.1". In: JHEP 02 (2009), p. 007. DOI: 10.1088/1126-6708/2009/02/007, arXiv: 0811.4622 [hep-ph]

[161] Glen Cowan et al. "Asymptotic formulae for likelihood-based tests of new physics". In: Eur. Phys. J. C71 (2011). [Erratum: Eur. Phys. J.C73,2501(2013)], p. 1554. DOI:10.1140/ epjc/s10052-011-1554-0,10.1140/epjc/s10052-013-2501-z arXiv: 1007.1727 [physics.data-an].

[162] Daniel Joseph Antrim. "Sweet Little Nothings; or, Searching for a Pair of Stops, a Pair of Higgs Bosons, and a Pair of New Small Wheels for the Upgrade of the Forward Muon System of the ATLAS Detector at CERN". Presented 23 Oct 2019. 2019. URL: https: //cds.cern.ch/record/2699575. 
[163] Kyle Cranmer et al. HistFactory: A tool for creating statistical models for use with RooFit and RooStats. Tech. rep. CERN-OPEN-2012-016. New York: New York U., Jan. 2012. URL: https://cds.cern.ch/record/1456844.

[164] Jerzy Neyman and Egon Sharpe Pearson. "On the Problem of the Most Efficient Tests of Statistical Hypotheses”. In: Phil. Trans. Roy. Soc. Lond. A 231.694-706 (1933), pp. 289337. DOI: $10.1098 /$ rsta.1933.0009.

[165] Linda Carpenter et al. "Mono-Higgs-boson: A new collider probe of dark matter". In: Phys. Rev. D 89.7 (2014), p. 075017. DOI: 10.1103/PhysRevD.89.075017, arXiv: 1312.2592 [hep-ph].

[166] S. Dittmaier et al. "Handbook of LHC Higgs Cross Sections: 2. Differential Distributions". In: (Jan. 2012). DOI: 10.5170/CERN-2012-002, arXiv: 1201.3084 [hep-ph]

[167] J. Alwall et al. "The automated computation of tree-level and next-to-leading order differential cross sections, and their matching to parton shower simulations". In: JHEP 07 (2014), p. 079. DOI: 10.1007/JHEP07(2014)079. arXiv: 1405.0301 [hep-ph]

[168] Richard D. Ball et al. "Parton distributions for the LHC Run II". In: JHEP 04 (2015), p. 040. DOI: 10.1007/JHEP04(2015) 040, arXiv: 1410.8849 [hep-ph].

[169] Torbjörn Sjöstrand et al. “An introduction to PYTHIA 8.2”. In: Comput. Phys. Commun. 191 (2015), pp. 159-177. DOI: $10.1016 / \mathrm{j}$. cpc . 2015 .01 .024 arXiv: 1410.3012 [hep-ph].

[170] Richard D. Ball et al. "Impact of Heavy Quark Masses on Parton Distributions and LHC Phenomenology". In: Nucl. Phys. B 849 (2011), pp. 296-363. Dor:10.1016/j.nuclphyst. 2011.03.021. arXiv: 1101.1300 [hep-ph].

[171] “ATLAS Pythia 8 tunes to $7 \mathrm{TeV}$ data”. In: (Nov. 2014).

[172] Torbjorn Sjostrand, Stephen Mrenna, and Peter Z. Skands. "A Brief Introduction to PYTHIA 8.1”. In: Comput. Phys. Commun. 178 (2008), pp. 852-867. DOI: 10.1016/j.cpc. 2008. 01.036, arXiv: 0710.3820 [hep-ph] 
[173] Simone Alioli et al. "A general framework for implementing NLO calculations in shower Monte Carlo programs: the POWHEG BOX”. In: JHEP 06 (2010), p. 043. DOI: 10.1007/ JHEP06 (2010) 043, arXiv: 1002.2581 [hep-ph].

[174] Stefano Frixione, Paolo Nason, and Giovanni Ridolfi. “A Positive-weight next-to-leadingorder Monte Carlo for heavy flavour hadroproduction”. In: JHEP 09 (2007), p. 126. DOI: 10.1088/1126-6708/2007/09/126, arXiv: 0707.3088 [hep-ph].

[175] Paolo Nason. "A New method for combining NLO QCD with shower Monte Carlo algorithms". In: JHEP 11 (2004), p. 040. DOI: 10.1088/1126-6708/2004/11/040, arXiv: hep-ph/0409146.

[176] Stefano Frixione, Paolo Nason, and Carlo Oleari. "Matching NLO QCD computations with Parton Shower simulations: the POWHEG method". In: JHEP 11 (2007), p. 070. DOI: 10.1088/1126-6708/2007/11/070, arXiv: 0709.2092 [hep-ph].

[177] D.J. Lange. "The EvtGen particle decay simulation package". In: Nucl. Instrum. Meth. A 462 (2001). Ed. by S. Erhan, P. Schlein, and Y. Rozen, pp. 152-155. DOI: 10 . $1016 /$ S0168-9002(01)00089-4.

[178] M. Beneke et al. "Hadronic top-quark pair production with NNLL threshold resummation". In: Nucl. Phys. B 855 (2012), pp. 695-741. DOI: 10.1016/j .nuclphysb.2011.10.021. arXiv: 1109.1536 [hep-ph].

[179] Matteo Cacciari et al. "Top-pair production at hadron colliders with next-to-next-to-leading logarithmic soft-gluon resummation”. In: Phys. Lett. B 710 (2012), pp. 612-622. DOI: 10.1016/j.physletb.2012.03.013, arXiv: 1111.5869 [hep-ph].

[180] Peter Bärnreuther, Michal Czakon, and Alexander Mitov. "Percent Level Precision Physics at the Tevatron: First Genuine NNLO QCD Corrections to $q \bar{q} \rightarrow t \bar{t}+X ”$. In: Phys. Rev. Lett. 109 (2012), p. 132001. DOI: 10 . 1103/PhysRevLett.109.132001, arXiv: 1204.5201 [hep-ph]. 
[181] Michal Czakon and Alexander Mitov. "NNLO corrections to top-pair production at hadron colliders: the all-fermionic scattering channels". In: JHEP 12 (2012), p. 054. DOI: 10 . 1007/JHEP12(2012) 054 arXiv: 1207.0236 [hep-ph]

[182] Michal Czakon and Alexander Mitov. "NNLO corrections to top pair production at hadron colliders: the quark-gluon reaction”. In: JHEP 01 (2013), p. 080. DOI: 10.1007/JHEP01(2013) 080, arXiv: 1210.6832 [hep-ph].

[183] Michał Czakon, Paul Fiedler, and Alexander Mitov. "Total Top-Quark Pair-Production Cross Section at Hadron Colliders Through $O\left(\alpha_{S}^{4}\right)$ ". In: Phys. Rev. Lett. 110 (2013), p. 252004. DOI: 10.1103/PhysRevLett.110.252004 arXiv: 1303.6254 [hep-ph].

[184] Michal Czakon and Alexander Mitov. "Top++: A Program for the Calculation of the TopPair Cross-Section at Hadron Colliders”. In: Comput. Phys. Commun. 185 (2014), p. 2930. DOI: $10.1016 /$ j.cpc.2014.06.021, arXiv: 1112.5675 [hep-ph].

[185] Michiel Botje et al. “The PDF4LHC Working Group Interim Recommendations”. In: (Jan. 2011). arXiv: 1101.0538 [hep-ph].

[186] A.D. Martin et al. "Parton distributions for the LHC". In: Eur. Phys. J. C 63 (2009), pp. 189-285. DOI: 10.1140/epjc/s10052-009-1072-5, arXiv:0901.0002 [hep-ph].

[187] A.D. Martin et al. "Uncertainties on alpha(S) in global PDF analyses and implications for predicted hadronic cross sections". In: Eur. Phys. J. C 64 (2009), pp. 653-680. DOI: 10.1140/epjc/s10052-009-1164-2, arXiv: 0905.3531 [hep-ph].

[188] Hung-Liang Lai et al. "New parton distributions for collider physics". In: Phys. Rev. D 82 (2010), p. 074024. DOI: 10.1103/PhysRevD.82.074024. arXiv: 1007.2241 [hep-ph].

[189] Jun Gao et al. "CT10 next-to-next-to-leading order global analysis of QCD". In: Phys. Rev. D 89.3 (2014), p. 033009. DOI: 10.1103/PhysRevD.89.033009, arXiv: 1302.6246 $[\mathrm{hep}-\mathrm{ph}]$.

[190] Richard D. Ball et al. "Parton distributions with LHC data". In: Nucl. Phys. B 867 (2013), pp. 244-289. DOI: 10.1016/j.nuclphysb.2012.10.003 arXiv: 1207.1303 [hep-ph]. 
[191] Frank-Peter Schilling. "Top Quark Physics at the LHC: A Review of the First Two Years". In: Int. J. Mod. Phys. A 27 (2012), p. 1230016. DOI: 10.1142/S0217751X12300165 arXiv: 1206.4484 [hep-ex].

[192] Emanuele Re. "Single-top Wt-channel production matched with parton showers using the POWHEG method". In: Eur. Phys. J. C 71 (2011), p. 1547. DOI: 10.1140/epjc/s10052011-1547-z arXiv: 1009.2450 [hep-ph].

[193] Stefano Frixione et al. "Angular correlations of lepton pairs from vector boson and top quark decays in Monte Carlo simulations”. In: JHEP 04 (2007), p. 081. DOI: 10 . $1088 /$ 1126-6708/2007/04/081, arXiv: hep-ph/0702198.

[194] Pierre Artoisenet et al. "Automatic spin-entangled decays of heavy resonances in Monte Carlo simulations". In: JHEP 03 (2013), p. 015. DOI: 10.1007/JHEP03(2013)015, arXiv: 1212.3460 [hep-ph].

[195] M. Aliev et al. "HATHOR: HAdronic Top and Heavy quarks crOss section calculatoR". In: Comput. Phys. Commun. 182 (2011), pp. 1034-1046. DOI: $10.1016 /$ j . cpc . 2010. 12.040, arXiv: 1007.1327 [hep-ph]

[196] P. Kant et al. "HatHor for single top-quark production: Updated predictions and uncertainty estimates for single top-quark production in hadronic collisions”. In: Comput. Phys. Commun. 191 (2015), pp. 74-89. DOI: $10.1016 /$ j.cpc.2015.02.001 arXiv: 1406.4403 $[\mathrm{hep}-\mathrm{ph}]$.

[197] Simone Alioli et al. "NLO single-top production matched with shower in POWHEG: sand t-channel contributions". In: JHEP 09 (2009). [Erratum: JHEP 02, 011 (2010)], p. 111. DOI: 10.1088/1126-6708/2009/09/111. arXiv: 0907.4076 [hep-ph].

[198] Stefano Frixione et al. "Single-top hadroproduction in association with a W boson". In: JHEP 07 (2008), p. 029. DOI: 10.1088/1126-6708/2008/07/029, arXiv: 0805.3067 $[\mathrm{hep}-\mathrm{ph}]$.

[199] “Studies on top-quark Monte Carlo modelling for Top2016”. In: (Oct. 2016). 
[200] Enrico Bothmann et al. "Event Generation with Sherpa 2.2". In: SciPost Phys. 7.3 (2019), p. 034. DOI: 10.21468/SciPostPhys.7.3.034, arXiv: 1905.09127 [hep-ph].

[201] Tanju Gleisberg and Stefan Hoeche. "Comix, a new matrix element generator". In: JHEP 12 (2008), p. 039. DOI: 10 . 1088/1126-6708/2008/12/039, arXiv: 0808 . 3674 [hep-ph].

[202] Fabio Cascioli, Philipp Maierhofer, and Stefano Pozzorini. "Scattering Amplitudes with Open Loops". In: Phys. Rev. Lett. 108 (2012), p. 111601. DOI: 10.1103/PhysRevLett. 108.111601. arXiv: 1111.5206 [hep-ph].

[203] Ansgar Denner, Stefan Dittmaier, and Lars Hofer. "Collier: a fortran-based Complex OneLoop LIbrary in Extended Regularizations”. In: Comput. Phys. Commun. 212 (2017), pp. 220-238. DOI: 10.1016/j.cpc. 2016.10.013 arXiv: 1604.06792 [hep-ph].

[204] Steffen Schumann and Frank Krauss. "A Parton shower algorithm based on Catani-Seymour dipole factorisation". In: JHEP 03 (2008), p. 038. DOI: 10.1088/1126-6708/2008/03/ 038. arXiv: 0709.1027 [hep-ph]

[205] Stefan Hoeche et al. "A critical appraisal of NLO+PS matching methods". In: JHEP 09 (2012), p. 049. DOI: 10.1007/JHEP09 (2012)049, arXiv: 1111.1220 [hep-ph].

[206] Stefan Hoeche et al. "QCD matrix elements + parton showers: The NLO case". In: JHEP 04 (2013), p. 027. DOI: 10.1007/JHEP04(2013)027. arXiv: 1207.5030 [hep-ph].

[207] S. Catani et al. "QCD matrix elements + parton showers". In: JHEP 11 (2001), p. 063. DOI: 10.1088/1126-6708/2001/11/063 arXiv: hep-ph/0109231.

[208] Stefan Hoeche et al. "QCD matrix elements and truncated showers". In: JHEP 05 (2009), p. 053. DOI: 10.1088/1126-6708/2009/05/053, arXiv:0903.1219 [hep-ph].

[209] D. Levin. "Diboson Physics in ATLAS". In: Balk. Phys. Lett. 17 (2009). Ed. by Taylan Akdogan et al., pp. 102-107. 
[210] Keith Hamilton, Paolo Nason, and Giulia Zanderighi. "MINLO: Multi-Scale Improved NLO”. In: JHEP 10 (2012), p. 155. DOI: 10.1007/JHEP10(2012) 155, arXiv: 1206.3572 $[\mathrm{hep}-\mathrm{ph}]$.

[211] Gionata Luisoni et al. " $H W^{ \pm} / \mathrm{HZ}+0$ and 1 jet at NLO with the POWHEG BOX interfaced to GoSam and their merging within MiNLO”. In: JHEP 10 (2013), p. 083. DOI: 10.1007/ JHEP10 (2013)083, arXiv: 1306.2542 [hep-ph].

[212] Georges Aad et al. "Measurement of the $Z / \gamma^{*}$ boson transverse momentum distribution in $p p$ collisions at $\sqrt{s}=7 \mathrm{TeV}$ with the ATLAS detector". In: JHEP 09 (2014), p. 145. DOI: 10.1007/JHEP09(2014)145, arXiv: 1406.3660 [hep-ex].

[213] J. Pumplin et al. "New generation of parton distributions with uncertainties from global QCD analysis”. In: JHEP 07 (2002), p. 012. DOI: 10.1088/1126-6708/2002/07/012. arXiv: hep-ph/0201195.

[214] M.L. Ciccolini, S. Dittmaier, and M. Kramer. "Electroweak radiative corrections to associated WH and ZH production at hadron colliders". In: Phys. Rev. D 68 (2003), p. 073003. DOI: 10.1103/PhysRevD .68.073003, arXiv: hep-ph/0306234.

[215] Oliver Brein, Abdelhak Djouadi, and Robert Harlander. "NNLO QCD corrections to the Higgs-strahlung processes at hadron colliders”. In: Phys. Lett. B 579 (2004), pp. 149-156. DOI: $10.1016 / \mathrm{j} \cdot$ physletb.2003.10.112, arXiv: hep-ph/0307206.

[216] Giancarlo Ferrera, Massimiliano Grazzini, and Francesco Tramontano. "Associated WH production at hadron colliders: a fully exclusive QCD calculation at NNLO”. In: Phys. Rev. Lett. 107 (2011), p. 152003. DOI: 10.1103/PhysRevLett . 107 . 152003, arXiv: 1107.1164 [hep-ph].

[217] Giancarlo Ferrera, Massimiliano Grazzini, and Francesco Tramontano. "Higher-order QCD effects for associated WH production and decay at the LHC”. In: JHEP 04 (2014), p. 039. DOI: $10.1007 /$ JHEP04(2014)039 arXiv: 1312.1669 [hep-ph] 
[218] Giancarlo Ferrera, Massimiliano Grazzini, and Francesco Tramontano. "Associated ZH production at hadron colliders: the fully differential NNLO QCD calculation". In: Phys. Lett. B 740 (2015), pp. 51-55. DoI: 10.1016/ j . physletb . 2014 .11.040, arXiv: 1407.4747 [hep-ph].

[219] John M. Campbell, R. Keith Ellis, and Ciaran Williams. "Associated production of a Higgs boson at NNLO". In: JHEP 06 (2016), p. 179. DOI: 10.1007/JHEP06 (2016) 179, arXiv: 1601.00658 [hep-ph].

[220] Oliver Brein et al. "Top-Quark Mediated Effects in Hadronic Higgs-Strahlung”. In: Eur. Phys. J. C 72 (2012), p. 1868. DOI: $10.1140 /$ epjc/s10052-012-1868-6 arXiv: 1111.0761 [hep-ph].

[221] Lukas Altenkamp et al. "Gluon-induced Higgs-strahlung at next-to-leading order QCD". In: JHEP 02 (2013), p. 078. DOI: 10 . 1007 / JHEP02 (2013) 078 arXiv: 1211.5015 [hep-ph].

[222] Robert V. Harlander et al. "Soft gluon resummation for gluon-induced Higgs Strahlung". In: JHEP 11 (2014), p. 082. DOI: 10.1007 / JHEP11(2014) 082, arXiv: 1410.0217 [hep-ph].

[223] Oliver Brein, Robert V. Harlander, and Tom J.E. Zirke. "vh@nnlo - Higgs Strahlung at hadron colliders". In: Comput. Phys. Commun. 184 (2013), pp. 998-1003. DOI: 10.1016/ j.cpc. 2012.11.002, arXiv: 1210.5347 [hep-ph].

[224] D. de Florian et al. "Handbook of LHC Higgs Cross Sections: 4. Deciphering the Nature of the Higgs Sector". In: 2/2017 (Oct. 2016). DOI: 10 .23731/CYRM-2017-002, arXiv: $1610.07922[\mathrm{hep}-\mathrm{ph}]$

[225] ATLASPMG, $t t V N L O$ cross section, (2017). URL: https://twiki.cern.ch/twiki/ bin/viewauth/AtlasProtected/CrossSectionNLOttV. 
[226] Georges Aad et al. "Performance of the missing transverse momentum triggers for the ATLAS detector during Run-2 data taking”. In: JHEP 08 (2020), p. 080. DOI: 10 . $1007 /$ JHEP08(2020) 080, arXiv: 2005.09554 [hep-ex].

[227] Georges Aad et al. "Performance of the ATLAS muon triggers in Run 2". In: JINST 15.09 (2020), P09015. DOI: 10 . 1088/1748-0221/15/09/p09015. arXiv: 2004 . 13447 $[$ hep-ex].

[228] Morad Aaboud et al. "Performance of the ATLAS Trigger System in 2015". In: Eur. Phys. J. C 77.5 (2017), p. 317. DOI: 10.1140/epjc/s10052-017-4852-3. arXiv: 1611.09661 $[h e p-e x]$.

[229] "Search for Dark Matter Produced in Association with a Higgs Boson decaying to $b \bar{b}$ at $\sqrt{s}=13 \mathrm{TeV}$ with the ATLAS Detector using $79.8 \mathrm{fb}^{-1}$ of proton-proton collision data". In: (July 2018). URL: https://cds.cern.ch/record/2632344?ln=en.

[230] Matteo Cacciari and Gavin P. Salam. "Pileup subtraction using jet areas". In: Phys. Lett. B 659 (2008), pp. 119-126. DOI: 10.1016/j.physletb.2007.09.077 arXiv:0707.1378 [hep-ph].

[231] "Reconstruction, Energy Calibration, and Identification of Hadronically Decaying Tau Leptons in the ATLAS Experiment for Run-2 of the LHC”. In: (2015). URL: https : //cds.cern.ch/record/2064383.

[232] "Identification of hadronic tau lepton decays using neural networks in the ATLAS experiment". In: (2019). URL: https://cds.cern.ch/record/2688062.

[233] “Object-based missing transverse momentum significance in the ATLAS detector". In: (July 2018). URL: https : //cds . cern. ch/record/2630948?ln=en.

[234] Maria Ines Abreu Juliao Ochoa De Castro et al. JDM - Mono-H(bb) 2018 : ANA-EXOT2018-46. Tech. rep. ATL-COM-PHYS-2019-217. Geneva: CERN, Mar. 2000. URL: https: //cds.cern.ch/record/2667406. 


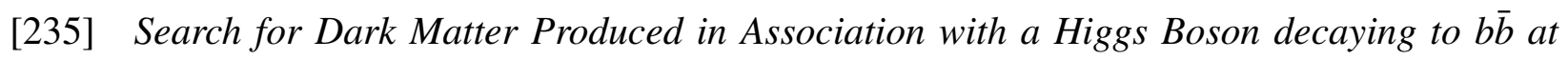
$\sqrt{s}=13 \mathrm{TeV}$ with the ATLAS Detector using $79.8 \mathrm{fb}^{-1}$ of proton-proton collision data. Tech. rep. ATLAS-CONF-2018-039. Geneva: CERN, July 2018. URL: https : / / cds . cern.ch/record/2632344.

[236] Spyridon Argyropoulos et al. Search for resonances decaying to a $W$ or $Z$ boson and a Higgs boson in the $v v b b, \ell v b b$ and $\ell \ell b b$ final states with $\sqrt{s}=13$ TeV ATLAS data in the context of models with 2 Higgs doublets or additional heavy vector triplets. Tech. rep. ATL-COM-PHYS-2016-479. Geneva: CERN, May 2016. URL: https ://cds . cern . ch/ record/2151842.

[237] Luminosity determination in pp collisions at $\sqrt{s}=13 \mathrm{TeV}$ using the ATLAS detector at the LHC. Tech. rep. ATLAS-CONF-2019-021. Geneva: CERN, June 2019. URL: http : //cds.cern.ch/record/2677054.

[238] Georges Aad et al. "Jet energy scale and resolution measured in proton-proton collisions at $\sqrt{s}=13 \mathrm{TeV}$ with the ATLAS detector". In: (July 2020). arXiv: 2007.02645 [hep-ex].

[239] In-situ measurements of the ATLAS large-radius jet response in $13 \mathrm{TeV}$ pp collisions. Tech. rep. ATLAS-CONF-2017-063. Geneva: CERN, July 2017. URL: http://cds . cern . ch/ record/2275655.

[240] Calibration of light-flavour b-jet mistagging rates using ATLAS proton-proton collision data at $\sqrt{s}=13 \mathrm{TeV}$. Tech. rep. ATLAS-CONF-2018-006. Geneva: CERN, Apr. 2018. URL: https://cds. cern. ch/record/2314418.

[241] Measurement of b-tagging Efficiency of c-jets in $t \bar{t}$ Events Using a Likelihood Approach with the ATLAS Detector. Tech. rep. ATLAS-CONF-2018-001. Geneva: CERN, Mar. 2018. URL: https://cds . cern.ch/record/2306649.

[242] Jon Butterworth et al. "PDF4LHC recommendations for LHC Run II". In: J. Phys. G 43 (2016), p. 023001. DOI: 10 . 1088/0954-3899/43/2/023001. arXiv: 1510.03865 $[\mathrm{hep}-\mathrm{ph}]$. 
[243] Stefan Hoeche and Marek Schonherr. "Uncertainties in next-to-leading order plus parton shower matched simulations of inclusive jet and dijet production". In: Phys. Rev. D 86 (2012), p. 094042. DOI: 10.1103/PhysRevD.86.094042. arXiv: 1208.2815 [hep-ph].

[244] M. Bahr et al. "Herwig++ Physics and Manual". In: Eur. Phys. J. C 58 (2008), pp. 639707. DOI: $10.1140 /$ epjc/s10052-008-0798-9, arXiv: 0803.0883 [hep-ph].

[245] Johannes Bellm et al. "Herwig 7.0/Herwig++ 3.0 release note". In: Eur. Phys. J. C 76.4 (2016), p. 196. DOI: 10 . 1140 / epjc / s10052-016-4018-8. arXiv: 1512.01178 $[\mathrm{hep}-\mathrm{ph}]$.

[246] Chun-Hay Kom and W.James Stirling. "Charge asymmetry in W + jets production at the LHC”. In: Eur. Phys. J. C 69 (2010), pp. 67-73. DOI: 10 . 1140/epjc/s10052-0101353-z, arXiv: 1004.3404 [hep-ph].

[247] Martin Bauer, Ulrich Haisch, and Felix Kahlhoefer. "Simplified dark matter models with two Higgs doublets: I. Pseudoscalar mediators”. In: JHEP 05 (2017), p. 138. DOI: 10 . 1007/JHEP05(2017)138, arXiv: 1701.07427 [hep-ph]

[248] Search for Dark Matter produced in association with a Standard Model Higgs boson decaying to b-quarks using the full Run 2 collision data with the ATLAS detector. Tech. rep. Figures https://atlas.web.cern.ch/Atlas/GROUPS/PHYSICS/CONFNOTES/ATLAS-CONF2021-006. Geneva: CERN, Mar. 2021. URL: http://cds . cern. ch/record/2759211.

[249] Philippe Brax et al. "LHC Signatures Of Scalar Dark Energy”. In: Phys. Rev. D 94.8 (2016), p. 084054. DOI: 10.1103/PhysRevD.94.084054 arXiv: 1604.04299 [hep-ph].

[250] Steven Weinberg. "The cosmological constant problem”. In: Rev. Mod. Phys. 61 (1 Jan. 1989), pp. 1-23. DOI: 10.1103/RevModPhys .61 .1. URL: https : //link . aps .org/ doi/10.1103/RevModPhys.61.1.

[251] Philippe Brax. "What makes the Universe accelerate? A review on what dark energy could be and how to test it". In: Rept. Prog. Phys. 81.1 (2018), p. 016902. DOI: 10.1088/1361$6633 / \mathrm{aa} 8 \mathrm{e} 64$. 
[252] Janna Katharina Behr et al. Searches for Dark Matter with the ATLAS Detector at the LHC. Tech. rep. ATL-COM-PHYS-2017-1031. Geneva: CERN, July 2017. URL: https : //cds.cern.ch/record/2273840.

[253] Morad Aaboud et al. "Search for dark matter produced in association with bottom or top quarks in $\sqrt{s}=13 \mathrm{TeV} p p$ collisions with the ATLAS detector". In: Eur. Phys. J. C 78.1 (2018), p. 18. DOI: 10.1140/epjc/s10052-017-5486-1. arXiv: 1710.11412 [hep-ex].

[254] Morad Aaboud et al. "Search for a scalar partner of the top quark in the jets plus missing transverse momentum final state at $\sqrt{s}=13 \mathrm{TeV}$ with the ATLAS detector". In: JHEP 12 (2017), p. 085. DOI: 10.1007/JHEP12(2017)085, arXiv: 1709.04183 [hep-ex].

[255] Morad Aaboud et al. "Search for top-squark pair production in final states with one lepton, jets, and missing transverse momentum using $36 \mathrm{fb}^{-1}$ of $\sqrt{s}=13 \mathrm{TeV} p p$ collision data with the ATLAS detector". In: JHEP 06 (2018), p. 108. DOI: 10.1007 / JHEP06 (2018) 108. arXiv: $1711.11520[$ hep-ex]

[256] M. Aaboud et al. "Search for direct top squark pair production in final states with two leptons in $\sqrt{s}=13 \mathrm{TeV} p p$ collisions with the ATLAS detector". In: Eur. Phys. J. C 77.12 (2017), p. 898. DOI: $10.1140 /$ epjc/s10052-017-5445-x arXiv: 1708.03247 $[$ hep-ex].

[257] Giorgio Busoni et al. "On the Validity of the Effective Field Theory for Dark Matter Searches at the LHC'. In: Phys. Lett. B 728 (2014), pp. 412-421. DOI: 10 . 1016 / j . physletb.2013.11.069, arXiv: 1307.2253 [hep-ph].

[258] Christoph Englert and Michael Spannowsky. "Effective Theories and Measurements at Colliders". In: Phys. Lett. B 740 (2015), pp. 8-15. DOI: 10.1016/j ·physletb.2014.11. 035, arXiv: 1408.5147 [hep-ph].

[259] Alexander L. Read. "Presentation of search results: The CL(s) technique". In: J. Phys. G 28 (2002). Ed. by M.R. Whalley and L. Lyons, pp. 2693-2704. DOI: 10 . 1088/0954$3899 / 28 / 10 / 313$ 
[260] Search for scalar dark energy in $t \bar{t}+E_{T}^{\text {miss }}$ and mono-jet final states with the ATLAS detector. Tech. rep. ATL-PHYS-PUB-2018-008. Geneva: CERN, June 2018. URL: http : //cds.cern.ch/record/2627837.

[261] Philippe Brax and Patrick Valageas. "Goldstone models of modified gravity”. In: Phys. Rev. D 95.4 (2017), p. 043515. DOI: 10.1103/PhysRevD . 95.043515 arXiv: 1611.08279 [astro-ph.CO].

[262] Eugeny Babichev et al. "Stability of Black Holes and the Speed of Gravitational Waves within Self-Tuning Cosmological Models”. In: Phys. Rev. Lett. 120.24 (2018), p. 241101. DOI: 10.1103/PhysRevLett.120.241101, arXiv: 1712.04398 [gr-qc].

[263] Asher Berlin, Tongyan Lin, and Lian-Tao Wang. "Mono-Higgs Detection of Dark Matter at the LHC”. In: JHEP 06 (2014), p. 078. DOI: 10 . 1007 / JHEP06 (2014 ) 078, arXiv: 1402.7074 [hep-ph].

[264] Morad Aaboud et al. "Observation of $H \rightarrow b \bar{b}$ decays and $V H$ production with the ATLAS detector". In: Phys. Lett. B786 (2018), pp. 59-86. DOI: 10.1016/j ·physletb.2018.09. 013 arXiv: 1808.08238 [hep-ex]

[265] Sean M. Carroll. “The Cosmological constant”. In: Living Rev. Rel. 4 (2001), p. 1. DOI: 10.12942/Irr-2001-1. arXiv: astro-ph/0004075.

[266] Peter M. Garnavich et al. "Constraints on cosmological models from Hubble Space Telescope observations of high z supernovae". In: Astrophys. J. 493 (1998), pp. L53-57. DOI: 10.1086/311140, arXiv: astro-ph/9710123.

[267] C.L. Bennett et al. "Four year COBE DMR cosmic microwave background observations: Maps and basic results”. In: Astrophys. J. Lett. 464 (1996), pp. L1-L4. DOI: 10 . $1086 /$ 310075, arXiv: astro-ph/9601067.

[268] Guido Walter Pettinari. "The intrinsic bispectrum of the Cosmic Microwave Background". PhD thesis. Portsmouth U., ICG, Sept. 2013. Dor: 10.1007/978-3-319-21882-3 arXiv: 1405.2280 [astro-ph.CO]. 
[269] J.B. Hartle. An introduction to Einstein's general relativity. 2003.

[270] Sean M. Carroll. "Lecture notes on general relativity”. In: (Dec. 1997). arXiv: gr-qc/ 9712019.

[271] Albert Einstein. "The Foundation of the General Theory of Relativity". In: Annalen Phys. 49.7 (1916), pp. 769-822. DOI: 10.1002/andp. 200590044.

[272] J.A. Peacock. Cosmological physics. Feb. 1999.

[273] Roy Maartens. "Is the Universe homogeneous?" In: Phil. Trans. Roy. Soc. Lond. A369 (2011), pp. 5115-5137. DOI: 10.1098/rsta.2011.0289 arXiv: 1104.1300 [astro-ph.CO].

[274] Daniela Saadeh et al. "How isotropic is the Universe?" In: Phys. Rev. Lett. 117.13 (2016), p. 131302. DOI: 10.1103/PhysRevLett.117.131302 arXiv: 1605.07178 [astro-ph.CO].

[275] Timothy Clifton, Chris Clarkson, and Philip Bull. "The isotropic blackbody CMB as evidence for a homogeneous universe". In: Phys. Rev. Lett. 109 (2012), p. 051303. DOI: 10.1103/PhysRevLett.109.051303, arXiv: 1111.3794 [gr-qc].

[276] C.A. Scharf et al. "The 2-10 kev xrb dipole and its cosmological implications". In: Astrophys. J. 544 (2000), p. 49. DOI: 10.1086/317174. arXiv: astro-ph/9908187.

[277] Dominik J. Schwarz et al. "Testing foundations of modern cosmology with SKA all-sky surveys". In: PoS AASKA14 (2015), p. 032. DOI: 10.22323/1.215.0032, arXiv: 1501. 03820 [astro-ph.CO]

[278] P.J.E. Peebles. Principles of physical cosmology. Sept. 1994.

[279] Zeljko Ivezic et al. "Optical and Radio Properties of Extragalactic Sources Observed by the First Survey and the Sloan Digital Sky Survey”. In: Astron. J. 124 (2002), p. 2364. DOI: $10.1086 / 344069$ arXiv: astro-ph/0202408.

[280] Vera C. Rubin and Jr. Ford W.Kent. "Rotation of the Andromeda Nebula from a Spectroscopic Survey of Emission Regions”. In: Astrophys. J. 159 (1970), pp. 379-403. DOI: $10.1086 / 150317$. 
[281] Vesto Slipher. "The radial velocity of the Andromeda Nebula". In: Lowell Observatory Bulletin 2 (Jan. 1912), pp. 56-57.

[282] Vesto Slipher. "Spectrographic Observations of Nebulae”. In: Popular Astronomy 23 (Jan. 1915), pp. 21-24.

[283] Edwin Hubble. "A relation between distance and radial velocity among extra-galactic nebulae". In: Proceedings of the National Academy of Sciences 15.3 (1929), pp. 168-173. ISSN: 0027-8424. DOI: $10.1073 /$ pnas .15.3.168 eprint: https : / /www . pnas .org/ content/15/3/168.full.pdf. URL: https://www .pnas.org/content/15/3/168.

[284] P.A.R. Ade et al. "Planck 2015 results. XIII. Cosmological parameters". In: Astron. Astrophys. 594 (2016), A13. DOI: 10.1051/0004-6361/201525830, arXiv: 1502.01589 [astro-ph.CO].

[285] A. Friedman. "Über die Krümmung des Raumes". In: Zeitschrift für Physik 10.1 (1922), pp. 377-386. DOI: 10 . 1007 / BF01332580. URL: https : / / doi . org / 10 . 1007 / BF01332580,

[286] Abbé G. Lemaître. “A Homogeneous Universe of Constant Mass and Increasing Radius accounting for the Radial Velocity of Extra-galactic Nebulæ". In: Monthly Notices of the Royal Astronomical Society 91.5 (Mar. 1931), pp. 483-490. ISSN: 0035-8711. DOI: 10 . 1093 /mnras / 91 .5 .483, eprint: https : / / academic . oup . com/mnras / articlepdf /91/5/483/3079971/mnras91-0483.pdf. URL: https : //doi .org/10 . 1093/ mnras/91.5.483.

[287] A. G. Walker. "On Milne's Theory of World-Structure”. In: Proceedings of the London Mathematical Society 42 (Jan. 1937), pp. 90-127. DOI: 10.1112/plms/s2-42.1.90.

[288] H. P. Robertson. "Kinematics and World-Structure III.” In: apj 83 (May 1936), p. 257. DOI: $10.1086 / 143726$.

[289] N. Aghanim et al. "Planck 2018 results. VI. Cosmological parameters". In: (July 2018). arXiv: 1807.06209 [astro-ph.CO]. 
[290] RD50 collaboration. URL: https://twiki.cern.ch/twiki/bin/view/AtlasPublic/ LuminosityPublicResultsRun2\#Luminosity_summary_plots_for_201.

[291] LEP design report. By the LEP Injector Study Group. Geneva: CERN, 1983. URL: https: //cds.cern.ch/record/98881.

[292] LEP design report. Copies shelved as reports in LEP, PS and SPS libraries. Geneva: CERN, 1984. URL: https://cds.cern.ch/record/102083.

[293] W Kienzle et al. TOTEM, Total Cross Section, Elastic Scattering and Diffraction Dissociation at the LHC: Technical Proposal. Tech. rep. CERN-LHCC-99-007. LHCC-P-5. Geneva: CERN, Mar. 1999. URL: http://cds.cern.ch/record/385483.

[294] J. Billan et al. "Magnetic Performance of the LEP Bending Magnets". In: Conf. Proc. C 8903201 (1989). Ed. by Floyd Bennett and Joyce Kopta, p. 1148.

[295] "Proceedings, ECFA-CERN Workshop on large hadron collider in the LEP tunnel: Lausanne and Geneva, Switzerland, March 21-27 March, 1984”. In: (Sept. 1984). Ed. by M. Jacob. DOI: 10.5170/CERN-1984-010-V-2.

[296] W.W. Armstrong et al. "ATLAS: Technical proposal for a general-purpose p p experiment at the Large Hadron Collider at CERN”. In: (Dec. 1994).

[297] Lucio Cerrito. "Electromagnetic and Hadronic Showers: Calorimeters". In: Radiation and Detectors: Introduction to the Physics of Radiation and Detection Devices. Cham: Springer International Publishing, 2017, pp. 171-185. ISBN: 978-3-319-53181-6. DOI: 10 .1007/ 978-3-319-53181-6_10. URL: https://doi .org/10 .1007/978-3-319-53181$6 \_10$.

[298] Performance of the ATLAS TDAQ system. URL: https : / cds . cern . ch / record / 2235584/files/content_tdaq_figures_tdaq-run2-schematic.png.

[299] Emma Ward. "LHC and HL-LHC timeline for ATLAS website". General Photo. Dec. 2018. URL: https://cds . cern.ch/record/2652466. 
[300] Top working group cross-section summary plots: Spring 2020. Tech. rep. ATL-PHYSPUB-2020-012. Geneva: CERN, May 2020. URL: http : / cds . cern . ch / record / 2718946 .

[301] S. Abachi et al. "Observation of the top quark". In: Phys. Rev. Lett. 74 (1995), pp. 2632 2637. DOI: $10.1103 /$ PhysRevLett.74.2632, arXiv: hep-ex/9503003.

[302] F. Abe et al. "Observation of top quark production in $\bar{p} p$ collisions". In: Phys. Rev. Lett. 74 (1995), pp. 2626-2631. DOI: 10.1103/PhysRevLett . 74 . 2626 arXiv: hep-ex/ 9503002 .

[303] Georges Aad et al. "Measurement of the $t \bar{t}$ production cross-section using $e \mu$ events with b-tagged jets in pp collisions at $\sqrt{s}=7$ and $8 \mathrm{TeV}$ with the ATLAS detector". In: Eur. Phys. J. C 74.10 (2014). [Addendum: Eur.Phys.J.C 76, 642 (2016)], p. 3109. DOI: 10 . 1140/epjc/s10052-016-4501-2, arXiv: 1406.5375 [hep-ex].

[304] Measurement of the top quark pair production cross-section with ATLAS in pp collisions at $\sqrt{s}=7 \mathrm{TeV}$ in the single-lepton channel using semileptonic $b$ decays. Tech. rep. ATLASCONF-2012-131. Geneva: CERN, Sept. 2012. URL: https://cds . cern.ch/record/ 1478370 .

[305] Morad Aaboud et al. "Measurement of the inclusive and fiducial $t \bar{t}$ production crosssections in the lepton+jets channel in $p p$ collisions at $\sqrt{s}=8 \mathrm{TeV}$ with the ATLAS detector". In: Eur. Phys. J. C 78 (2018), p. 487. DOI: 10.1140/epjc/s10052-018-5904-z. arXiv: 1712.06857 [hep-ex].

[306] Georges Aad et al. "Measurement of the $t \bar{t}$ production cross-section and lepton differential distributions in $e \mu$ dilepton events from $p p$ collisions at $\sqrt{s}=13 \mathrm{TeV}$ with the ATLAS detector". In: (Oct. 2019). arXiv: 1910.08819 [hep-ex].

[307] Measurement of the tz production cross-section in the lepton+jets channel at $\sqrt{s}=13 \mathrm{TeV}$ with the ATLAS experiment. Tech. rep. ATLAS-CONF-2019-044. Geneva: CERN, Sept. 2019. URL: https://cds.cern.ch/record/2690717. 
[308] Georges Aad et al. "Measurements of Higgs boson production and couplings in the fourlepton channel in pp collisions at center-of-mass energies of 7 and $8 \mathrm{TeV}$ with the ATLAS detector". In: Phys. Rev. D 91.1 (2015), p. 012006. DOI: 10.1103/PhysRevD.91.012006. arXiv: 1408.5191 [hep-ex].

[309] Observation of an excess of events in the search for the Standard Model Higgs boson in the gamma-gamma channel with the ATLAS detector. Tech. rep. ATLAS-CONF-2012-091. Geneva: CERN, July 2012. URL: https ://cds . cern.ch/record/1460410.

[310] Georges Aad et al. "Observation of a new particle in the search for the Standard Model Higgs boson with the ATLAS detector at the LHC”. In: Phys. Lett. B 716 (2012), pp. 1-29. DOI: $10.1016 / \mathrm{j} \cdot$ physletb.2012.08.020 arXiv: 1207.7214 [hep-ex].

[311] Serguei Chatrchyan et al. "Observation of a New Boson at a Mass of $125 \mathrm{GeV}$ with the CMS Experiment at the LHC”. In: Phys. Lett. B 716 (2012), pp. 30-61. DOI: 10.1016/j. physletb.2012.08.021, arXiv: 1207.7235 [hep-ex].

[312] Peter W. Higgs. "Broken Symmetries and the Masses of Gauge Bosons". In: Phys. Rev. Lett. 13 (1964). Ed. by J.C. Taylor, pp. 508-509. DOI: 10.1103/PhysRevLett.13.508.

[313] Measurements and interpretations of Higgs-boson fiducial cross sections in the diphoton decay channel using $139 \mathrm{fb}^{-1}$ of pp collision data at $\sqrt{\mathrm{s}}=13 \mathrm{TeV}$ with the ATLAS detector. Tech. rep. ATLAS-CONF-2019-029. Geneva: CERN, July 2019. URL: http : / / cds . cern.ch/record/2682800.

[314] Measurements of the Higgs boson inclusive, differential and production cross sections in the $4 \ell$ decay channel at $\sqrt{s}=13 \mathrm{TeV}$ with the ATLAS detector. Tech. rep. ATLAS-CONF2019-025. Geneva: CERN, July 2019. URL: http://cds . cern.ch/record/2682107.

[315] Ulrich Haisch and Giacomo Polesello. "Searching for heavy Higgs bosons in the $t \bar{t} Z$ and tbW final states". In: JHEP 09 (2018), p. 151. DOI: 10.1007/JHEP09 (2018) 151. arXiv: 1807.07734 [hep-ph] 
[316] Martin Bauer, Martin Klassen, and Valentin Tenorth. "Universal properties of pseudoscalar mediators in dark matter extensions of 2HDMs". In: JHEP 07 (2018), p. 107. DOI: 10 . 1007/JHEP07 (2018)107, arXiv: 1712.06597 [hep-ph].

[317] Felix Kahlhoefer. "Review of LHC Dark Matter Searches". In: Int. J. Mod. Phys. A 32.13 (2017), p. 1730006. DOI: 10.1142/S0217751X1730006X. arXiv: 1702.02430 [hep-ph].

[318] Dark matter summary plots. Tech. rep. ATL-PHYS-PUB-2019-030. Geneva: CERN, Aug. 2019. URL: https://cds.cern.ch/record/2684864.

[319] D.S. Akerib et al. "Results from a search for dark matter in the complete LUX exposure". In: Phys. Rev. Lett. 118.2 (2017), p. 021303. DoI: 10.1103/PhysRevLett.118.021303. arXiv: 1608.07648 [astro-ph.C0]

[320] Xiangyi Cui et al. "Dark Matter Results From 54-Ton-Day Exposure of PandaX-II Experiment”. In: Phys. Rev. Lett. 119.18 (2017), p. 181302. DOI: 10.1103/PhysRevLett.119. 181302, arXiv: 1708.06917 [astro-ph.CO].

[321] E. Aprile et al. "Dark Matter Search Results from a One Ton-Year Exposure of XENON1T". In: Phys. Rev. Lett. 121.11 (2018), p. 111302. DOI: 10.1103/PhysRevLett.121.111302. arXiv: 1805.12562 [astro-ph.CO].

[322] P. Agnes et al. "Low-Mass Dark Matter Search with the DarkSide-50 Experiment". In: Phys. Rev. Lett. 121.8 (2018), p. 081307. DOI: 10 . 1103/PhysRevLett . 121.081307. arXiv: 1802.06994 [astro-ph.HE]

[323] ATLAS muon spectrometer: Technical Design Report. Technical Design Report ATLAS. Geneva: CERN, 1997. URL: https://cds.cern.ch/record/331068.

[324] Shota Hayashida. The ATLAS Muon. Tech. rep. ATL-DAQ-PROC-2018-039. Geneva: CERN, Nov. 2018. URL: https://cds . cern.ch/record/2649074.

[325] “ATLAS central solenoid: Technical design report”. In: (Apr. 1997). 
[326] A. Airapetian et al. "ATLAS: Detector and physics performance technical design report. Volume 1". In: (May 1999).

[327] Stefanie Morgenstern. ATLAS LAr Calorimeter Performance in LHC Run-2. Tech. rep. ATL-LARG-PROC-2018-004. Geneva: CERN, June 2018. DOI: 10.1016/j .nima. 2018. 11.027. URL: http://cds.cern.ch/record/2627619.

[328] Pawel Klimek. Performance of the ATLAS Tile Calorimeter. Tech. rep. ATL-TILECALPROC-2020-002. Geneva: CERN, Jan. 2020. URL: https : / / cds . cern . ch/record/ 2707947.

[329] Marija Marjanovic. "ATLAS Tile calorimeter calibration and monitoring systems". In: IEEE Trans. Nucl. Sci. 66.arXiv:1806.09156. 7 (June 2018). 8 pages, 21 figures, IEEE Real Time 2018 proceedings, 1228-1235. 8 p. DOI: 10.1109/TNS . 2019.2921941, URL: http://cds.cern.ch/record/2629424.

[330] ATLAS tracking performance plots. URL: https : / / atlas . web . cern . ch / Atlas / GROUPS/PHYSICS/PLOTS/IDTR-2016-013.

[331] Peter W. Higgs. "Spontaneous Symmetry Breakdown without Massless Bosons”. In: Phys. Rev. 145 (4 May 1966), pp. 1156-1163. DOI:10.1103/PhysRev.145.1156. URL: https: //link.aps.org/doi/10.1103/PhysRev.145.1156.

[332] A combination of measurements of Higgs boson production and decay using up to 139 $\mathrm{fb}^{-1}$ of proton-proton collision data at $\sqrt{\mathrm{s}}=13 \mathrm{TeV}$ collected with the ATLAS experiment. Tech. rep. ATLAS-CONF-2020-027. Geneva: CERN, Aug. 2020. URL: http : / / cds . cern.ch/record/2725733.

[333] M. Misiak et al. "Updated NNLO QCD predictions for the weak radiative B-meson decays". In: Phys. Rev. Lett. 114.22 (2015), p. 221801. DOI: 10.1103/PhysRevLett . 114. 221801, arXiv: 1503.01789 [hep-ph]. 
[334] Vardan Khachatryan et al. "Searches for invisible decays of the Higgs boson in pp collisions at $\sqrt{s}=7,8$, and 13 TeV”. In: JHEP 02 (2017), p. 135. DOI: $10.1007 /$ JHEP02(2017) 135. arXiv: 1610.09218 [hep-ex]

[335] Georges Aad et al. "Constraints on new phenomena via Higgs boson couplings and invisible decays with the ATLAS detector". In: JHEP 11 (2015), p. 206. DOI: 10 . $1007 /$ JHEP11(2015) 206, arXiv: 1509.00672 [hep-ex].

[336] Joshua Ellis. “TikZ-Feynman: Feynman diagrams with TikZ”. In: Comput. Phys. Commun. 210 (2017), pp. 103-123. DOI: $10.1016 /$ j . cpc. 2016.08.019. arXiv: 1601.05437 $[\mathrm{hep}-\mathrm{ph}]$.

[337] Georges Aad et al. "Performance of Missing Transverse Momentum Reconstruction in Proton-Proton Collisions at $7 \mathrm{TeV}$ with ATLAS”. In: Eur. Phys. J. C 72 (2012), p. 1844. DOI: 10.1140/epjc/s10052-011-1844-6 arXiv: 1108.5602 [hep-ex]

[338] ATLAS Collaboration, JES Public Plots for Moriond 2017(2017). Mar. 2017. URL: https: //atlas.web.cern.ch/Atlas/GROUPS/PHYSICS/PLOTS/JETM-2017-003/.

[339] "Jet global sequential corrections with the ATLAS detector in proton-proton collisions at $\operatorname{sqrt}(\mathrm{s})=8 \mathrm{TeV}$ ". In: (Mar. 2015).

[340] Giulio Pellegrini et al. "Technology developments and first measurements of Low Gain Avalanche Detectors (LGAD) for high energy physics applications". In: Nuclear Instruments and Methods in Physics Research Section A Accelerators Spectrometers Detectors and Associated Equipment 765 (Nov. 2014), pp. 12-16. DOI: $10.1016 /$ j . nima. 2014 . 06.008

[341] Hartmut F.W. Sadrozinski, Abraham Seiden, and Nicolò Cartiglia. "4D tracking with ultrafast silicon detectors”. In: Rept. Prog. Phys. 81.2 (2018), p. 026101. DOI: 10.1088/13616633/aa94d3, arXiv: 1704.08666 [physics.ins-det]

[342] S. Xiao et al. "Beam test results of IHEP-NDL Low Gain Avalanche Detectors(LGAD)". In: (May 2020). arXiv: 2005.07323 [physics.ins-det]. 
[343] Andy Buckley et al. "General-purpose event generators for LHC physics". In: Phys. Rept. 504 (2011), pp. 145-233. DOI: 10.1016/j physrep.2011.03.005 arXiv: 1101.2599 $[\mathrm{hep}-\mathrm{ph}]$.

[344] ATLAS Pythia 8 tunes to $7 \mathrm{TeV}$ data. Tech. rep. ATL-PHYS-PUB-2014-021. Geneva: CERN, Nov. 2014. URL: https://cds . cern.ch/record/1966419.

[345] D.J. Kapner et al. "Tests of the gravitational inverse-square law below the dark-energy length scale”. In: Phys. Rev. Lett. 98 (2007), p. 021101. DOI: 10.1103/PhysRevLett. 98. 021101, arXiv: hep-ph/0611184.

[346] Paul Hamilton et al. "Atom-interferometry constraints on dark energy”. In: Science 349 (2015), pp. 849-851. DOI: 10.1126/science.aaa8883 arXiv: 1502.03888 [physics.atom-ph].

[347] John Butler et al. Search for Dark Matter Produced in Association with a Higgs Boson Decaying to $b \bar{b}$ at $\sqrt{s}=13 \mathrm{TeV}$ using $79.8 \mathrm{fb}^{-1}$ of pp collision data with the ATLAS Detector. Tech. rep. ATL-COM-PHYS-2018-042. Geneva: CERN, Jan. 2018. URL: https : //cds.cern.ch/record/2301321.

[348] Morad Aaboud et al. "Search for Dark Matter Produced in Association with a Higgs Boson Decaying to $b \bar{b}$ using $36 \mathrm{fb}^{-1}$ of $p p$ collisions at $\sqrt{s}=13 \mathrm{TeV}$ with the ATLAS Detector". In: Phys. Rev. Lett. 119.18 (2017), p. 181804. DOI: 10.1103/PhysRevLett.119.181804. arXiv: $1707.01302[$ hep-ex].

[349] Andrew Stuart Bell and Francesco Lo Sterzo. Signal and Background Modelling Studies for the Standard Model VH,H $\rightarrow b \bar{b}$ Analysis. Tech. rep. ATL-COM-PHYS-2018-505. Geneva: CERN, May 2018. URL: https://cds . cern.ch/record/2316951.

[350] M. Tanabashi et al. "Review of Particle Physics". In: Phys. Rev. D98.3 (2018), p. 030001. DOI: $10.1103 /$ PhysRevD.98.030001. 
APPENDICES 


\section{A. COMBINING DIFFERENT BACKGROUND SAMPLES}

Few of the important SM backgrounds in the mono- $h(b \bar{b})$ analysis are $t \bar{t}, Z+$ jets and Diboson (DB) backgrounds, as shown in Figure 73. In the previous iteration of the mono- $h(b \bar{b})$ analysis [235], only $E_{\mathrm{T}}^{\text {miss }}$ inclusive $t \bar{t}$, inclusive $\mathrm{DB}(Z \rightarrow q \bar{q})$ and $\mathrm{MAX}(\mathrm{HT}, \mathrm{PTV})$ sliced $Z(\rightarrow v v)+$ jets $\mathrm{MC}$ samples were used. In this iteration, in addition to the above-mentioned MC samples, MC samples produced with specific filters are also included such as, (i) $E_{\mathrm{T}}^{\text {miss }}$ filtered $t \bar{t}$ samples, (ii) B-filtered DB $V Z(Z \rightarrow b \bar{b})$, and (iii) $Z(\rightarrow v v)+$ jets PTV sliced samples. This section describes the methods of combining the filtered samples with the nominal ones, following the prescriptions described here [349], and how it affects the analysis. In general, the merging increases the MC statistics and reduces the MC statistical uncertainty. Each MC sample in ATLAS has a dataset identification number and is denoted as DSID in the following sections.

\section{- Combining inclusive and $E_{\mathrm{T}}^{\mathrm{miss}}$ filtered $t \bar{t}$ samples}

In the previous mono- $h(b \bar{b})$ iteration, the modelling of $t \bar{t}$ background was performed using only a $E_{\mathrm{T}}^{\text {miss }}$ inclusive non-all hadronic $t \bar{t} \mathrm{MC}$ sample. Since the sample is $E_{\mathrm{T}}^{\text {miss }}$ inclusive, the $E_{\mathrm{T}}^{\text {miss }}$ $>150 \mathrm{GeV}$ selection reduces a significant amount of statistics. There are two possible approaches to increase the statistical power and decrease the statistical uncertainties:

1. Generate the $E_{\mathrm{T}}^{\text {miss }}$ inclusive non-all hadronic $t \bar{t} \mathrm{MC}$ sample with more statistics, which requires significant CPU time.

2. Produce $E_{\mathrm{T}}^{\mathrm{miss}}$ filtered $t \bar{t} \mathrm{MC}$ samples targeting different phase spaces of the analysis, which consumes much less CPU time.

In the analysis described in this thesis, the second option has been implemented to increase the $t \bar{t}$ MC statistics and Table A.1 lists the nominal and different $E_{\mathrm{T}}^{\text {miss }}$ filtered $t \bar{t}$ MC samples used in the analysis. The $100<E_{\mathrm{T}}^{\text {miss }}<200 \mathrm{GeV}$ filtered $t \bar{t}$ sample is generated in such a way that there are no overlaps with the $E_{\mathrm{T}}^{\text {miss }}$ inclusive $t \bar{t}$ sample, while the $E_{\mathrm{T}}^{\text {miss }}>200 \mathrm{GeV}$ filtered $t \bar{t}$ samples can have overlaps. Different $t \bar{t}$ samples are merged together using the following reweighting strategy: 
1. For events with $E_{\mathrm{T}}^{\text {miss }}<100 \mathrm{GeV}$, only events from the $E_{\mathrm{T}}^{\text {miss }}$ inclusive $t \bar{t}$ sample are used with the original MC generator weight.

2. For events where $100<E_{\mathrm{T}}^{\text {miss }}<200 \mathrm{GeV}$, both the $E_{\mathrm{T}}^{\text {miss }}$ inclusive and $100<E_{\mathrm{T}}^{\text {miss }}<200$ GeV filtered $t \bar{t}$ (DSID:345935) sample events are used with half of the original MC generator weight.

3. For events with $E_{\mathrm{T}}^{\text {miss }}>200 \mathrm{GeV}$, only the $E_{\mathrm{T}}^{\text {miss }}$ filtered $t \bar{t}$ sample events are used with the original MC generator weight.

\begin{tabular}{|c|c|c|}
\hline DSID & Generator & Process \\
\hline \hline 410470 & PowhEG+PYTHiA8.230+EvtGen & non-allhad $t \bar{t}, E_{\mathrm{T}}^{\text {miss }}$ inclusive \\
\hline 345935 & PowhEG+PYTHiA8.230+EvtGen & non-allhad $t \bar{t}, 100<E_{\mathrm{T}}^{\text {miss }}<200 \mathrm{GeV}$ \\
\hline 407345 & PowhEG+PYTHIA8.230+EvtGen & non-allhad $t \bar{t}, 200<E_{\mathrm{T}}^{\text {miss }}<300 \mathrm{GeV}$ \\
\hline 407346 & PowhEG+PYTHIA8.230+EvtGen & non-allhad $t \bar{t}, 300<E_{\mathrm{T}}^{\text {miss }}<400 \mathrm{GeV}$ \\
\hline 407347 & PowhEG+PYTHIA8.230+EvtGen & non-allhad $t \bar{t}, E_{\mathrm{T}}^{\text {miss }}>400 \mathrm{GeV}$ \\
\hline
\end{tabular}

Table A.1: List of nominal and $E_{\mathrm{T}}^{\text {miss }}$ filtered $t \bar{t} \mathrm{MC}$ samples used in the mono- $h(b \bar{b})$ analysis.

The sample reweighting strategy is validated with the 0-lepton Resolved and Merged regions. Figure A.1 shows the $E_{\mathrm{T}}^{\text {miss }}$ spectrum comparison between the $E_{\mathrm{T}}^{\text {miss }}$ inclusive and $E_{\mathrm{T}}^{\text {miss }}$ filtered $t \bar{t}$ samples before and after the reweighting. The ratio plot corresponds to the ratio of $E_{\mathrm{T}}^{\text {miss }}$ inclusive $t \bar{t}$ before reweighting and the final $t \bar{t}$ sample used in the analysis. As shown in the plot,

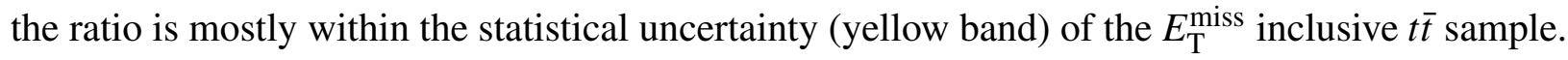

The increase in MC statistics decreases the MC statistical uncertainties and Figure A.2 shows the $m_{h}$ distribution comparison, before and after the sample extension in the 0-lepton SR for the $2 b$-tag category in $E_{\mathrm{T}}^{\text {miss }}$ inclusive Resolved (left plot) and Merged (right plot) regions. The ratio of the two is within the statistical uncertainty band (yellow) of the $E_{\mathrm{T}}^{\text {miss }}$ inclusive $t \bar{t}$ sample. For the Resolved and Merged $2 b$-tag regions the relative error on the area under the curve covered by the $t \bar{t}$ sample is reduced by $28 \%$ and $75 \%$ respectively, hence the sample extension increases the $t \bar{t}$ modelling precision. 


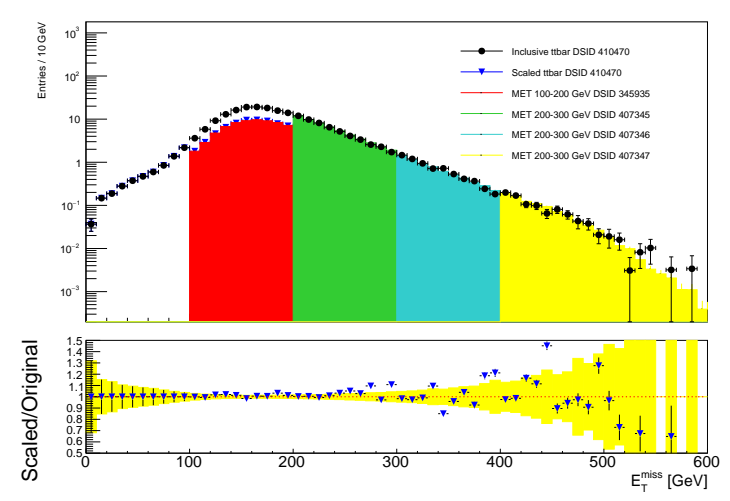

Figure A.1: $E_{\mathrm{T}}^{\text {miss }}$ spectrum for the nominal $t \bar{t}$ (black) and different $E_{\mathrm{T}}^{\text {miss }}$ filtered slices after reweighting.
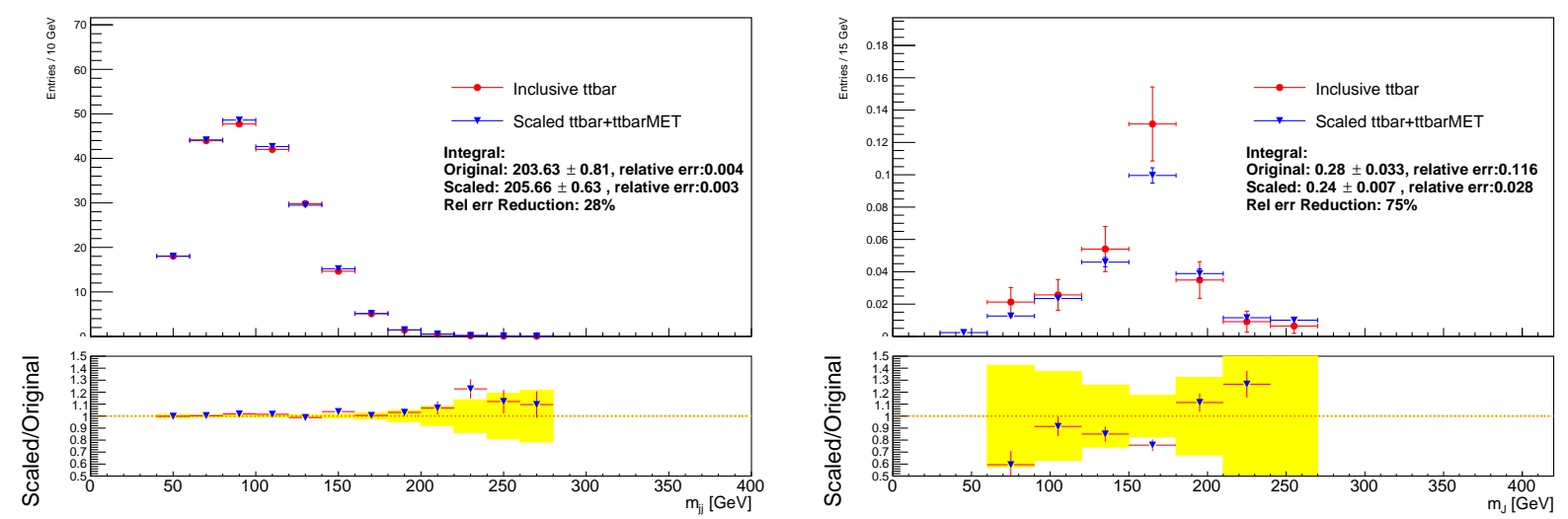

Figure A.2: Higgs candidate mass distribution $\left(m_{h}\right)$ comparison between the $E_{\mathrm{T}}^{\text {miss }}$ inclusive (red) and reweighted (blue) $t \bar{t}$ sample in Resolved (left plot) and Merged (right plot) regions. The yellow band represents the statistical uncertainty band for the $E_{\mathrm{T}}^{\text {miss }}$ inclusive $t \bar{t}$ sample.

\section{- Combining $Z v v+$ jets MAX(HT, PTV) with PTV sliced samples}

The $Z(\rightarrow v v)+$ jets are the irreducible SM backgrounds in the analysis. It contributes to $\sim 30 \%$ and $\sim 60 \%$ in the 0-lepton $2 b$-tagged Resolved $\left(150<E_{\mathrm{T}}^{\mathrm{miss}}<200 \mathrm{GeV}\right)$ and Merged regions respectively, to the total SM background. In the previous iteration of the mono- $h(b \bar{b})$ analysis only $Z(\rightarrow v v)+$ jets MAX (HT, PTV) $]^{7}$ samples were used. In this iteration, the MAX (HT, PTV) $Z(\rightarrow v v)+$ jets samples are merged together with the $B$-filtered ${ }^{7} \mathrm{PTV}$ sliced $Z(\rightarrow v v)+$ jets samples to increase the MC statistics. This section describes the method of combining the above-mentioned

\footnotetext{
${ }^{7}$ Selects events with either maximum $H_{T}$ or $p_{\mathrm{T}}(V)$. The PTV refers to the $p_{\mathrm{T}}$ of the $\mathrm{Z}$ boson $\left(p_{\mathrm{T}}(Z)\right)$ and $H_{T}=$ $\sum_{j e t s} p_{\mathrm{T}} . B$-filtered sample contains at least one $b$-jet which passes some selection criteria.
} 
types of $Z(\rightarrow v v)+$ jets samples and how it improves the statistical uncertainties. Table A.2 lists the $Z(\rightarrow v v)+$ jets MAX (HT,PTV) and PTV sliced $Z(\rightarrow v v)+$ jets $B$-filtered samples used in the analysis.

\begin{tabular}{|c|c|c|}
\hline DSID & Generator & Filter \\
\hline \multicolumn{3}{|c|}{$Z v v+$ jets MAX(HT, PTV) samples } \\
\hline 364144 & SHERPA v2.2.1 & MAX(HT, PTV) 0_70 \\
\hline 364147 & SHERPA v2.2.1 & MAX(HT, PTV) 70_140 \\
\hline 364150 & SHERPA v2.2.1 & MAX(HT, PTV) 140_280 \\
\hline 364153 & SHERPA v2.2.1 & MAX(HT, PTV) 280_500 \\
\hline 364154 & SHERPA v2.2.1 & MAX(HT, PTV) 500_1000 \\
\hline 364155 & SHERPA v2.2.1 & MAX(HT, PTV) 1000_E_CMS \\
\hline \multicolumn{3}{|c|}{$Z v v+$ jets PTV sliced $B$-filtered samples } \\
\hline 366010 & SHERPA v2.2.1 & PTV 70_100 \\
\hline 366011 & SHERPA v2.2.1 & PTV 100_140, $\mathrm{M}_{j j} 0 \_500$ \\
\hline 366012 & SHERPA v2.2.1 & PTV 100_140, $\mathrm{M}_{j j} 500 \_1000$ \\
\hline 366013 & SHERPA v2.2.1 & PTV 100_140, M ${ }_{j j}$ 1000_E_CMS \\
\hline 366014 & SHERPA v2.2.1 & PTV 140_280, $\mathrm{M}_{j j} 0 \_500$ \\
\hline 366015 & SHERPA v2.2.1 & PTV 140_280, $\mathrm{M}_{j j} 500 \_1000$ \\
\hline 366016 & SHERPA v2.2.1 & PTV 140_280, $\mathrm{M}_{j j}$ 1000_E_CMS \\
\hline 366017 & SHERPA v2.2.1 & PTV 280_500 \\
\hline 364222 & SHERPA v2.2.1 & PTV 500_1000 \\
\hline 364223 & SHERPA v2.2.1 & PTV 1000_E_CMS \\
\hline
\end{tabular}

Table A.2: List of the $Z(\rightarrow v v)+$ jets samples used in the analysis. Each sample filter name contains the filters applied it, e.g., MAX (HT, PTV) 70_140 represents $70<$ MAX (HT, PTV) $<140$ GeV. The E_CMS represents no upper bounds up to the Center Of Mass Scale (13 TeV).

Besides the $B$-filtered samples there are other PTV sliced samples available such as $C$ and light-filtered $Z(\rightarrow v v)+$ jets samples. However, as shown in Table A.3 the statistics of $C$ and lightfiltered samples in the 0 -lepton $2 b$-tag regions are negligible compared to the $B$-filtered samples. Hence, for simplicity purpose only $B$-filtered PTV sliced $Z(\rightarrow v v)+$ jets samples are merged with the MAX (HT, PTV) $Z(\rightarrow v v)+$ jets samples.

\begin{tabular}{|c|c|c|c|c|c|}
\hline Topology & $b$-tag & Total $Z v v+$ jets PTV yield & $B$-filter $C$-veto & $C$-filter $B$-veto & $B$-veto $C$-veto \\
\hline Merged & 2 & 0.758 & $97.6 \%$ & $1.4 \%$ & $1.0 \%$ \\
\hline Resolved & 2 & 31.67 & $89.6 \%$ & $8.2 \%$ & $2.2 \%$ \\
\hline
\end{tabular}

Table A.3: Yields comparison between different filters of PTV sliced $Z(\rightarrow v v)+$ jets samples. The total includes contributions from all three types of samples. 
The MAX (HT, PTV) and $B$-filtered PTV sliced $Z(\rightarrow v v)+$ jets samples are merged together with the MC generator weights halved for each event. Similar to $t \bar{t}$, the increase in MC statistics improves the statistical uncertainty for both Resolved and Merged regions. Figure A.3 shows the $m_{h}$ spectrum before and after the merging of the above-mentioned $Z(\rightarrow v v)+$ jets samples in the 0-lepton SR for the $2 b$-tagged Resolved and Merged regions. The MC statistical uncertainties decreased by $11 \%$ and $39 \%$ in Resolved and Merged region respectively. The ratio of MAX (HT, PTV) only and combined $Z(\rightarrow v v)+$ jets samples is within the statistical uncertainty of the MAX (HT, PTV) $Z(\rightarrow v v)+$ jets samples.
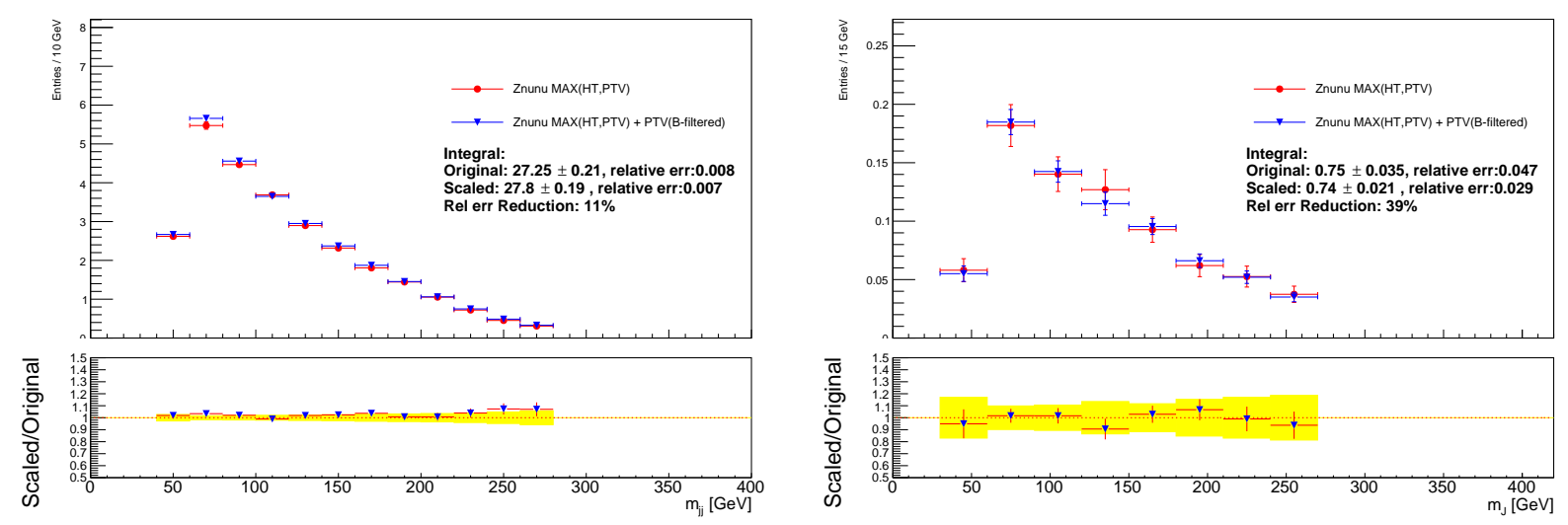

Figure A.3: The $m_{h}$ spectrum before (red) and after (blue) the merging of the $Z(\rightarrow v v)+$ jets samples in the 0-lepton SR for the $2 b$-tagged $E_{\mathrm{T}}^{\text {miss }}$ inclusive Resolved (left plot) and Merged (right plot) regions. The yellow band represents the statistical uncertainty band for the $v+$ jets MAX (HT, PTV) samples.

\section{- Combining inclusive and B-filtered diboson samples}

The other SM background sample that has been extended in the analysis is the diboson (DB) sample. In the previous iteration only inclusive DB samples $V Z(Z \rightarrow q \bar{q})$ were used, while in this iteration the $B$-filtered DB samples $V Z(Z \rightarrow b \bar{b})$ are also used to increase the MC statistics. The inclusive and $B$-filtered DB MC samples used in the analysis are listed in Table A.4.

Events from the two types of DB samples are reweighed and merged together, following the merging strategy described below:

1. All the events from the $B$-filtered DB $V Z(Z \rightarrow b \bar{b})$ samples are used with a reweighted MC generator weight. 


\begin{tabular}{|c|c|c|}
\hline DSID & Generator & Channel \\
\hline \hline \multicolumn{3}{|c|}{ Inclusive diboson $V Z(Z \rightarrow q \bar{q})$ samples } \\
\hline 363355 & SHERPA v2.2.1 & ZqqZ $v v$ \\
\hline 363356 & SHERPA v2.2.1 & ZqqZll \\
\hline 363489 & SHERPA v2.2.1 & ZqqWl $v$ \\
\hline$B$-filtered diboson $V Z(Z \rightarrow b \bar{b})$ samples \\
\hline 345043 & SHERPA v2.2.1 & ZbbZ $v v$ \\
\hline 345044 & SHERPA v2.2.1 & ZbbZll \\
\hline 345045 & SHERPA v2.2.1 & ZbbWl $v$ \\
\hline
\end{tabular}

Table A.4: List of inclusive and $B$-filtered diboson samples used in the analysis.

2. All the $Z \rightarrow b \bar{b}$ events from the inclusive DB $V Z(Z \rightarrow q \bar{q})$ sample are used with a reweighted MC generator weight.

3. All the non $Z \rightarrow b \bar{b}$ events from the inclusive $\mathrm{DB} V Z(Z \rightarrow q \bar{q})$ sample are used as it is.

The reweighting factors are evaluated such that it satisfies two conditions: (i) MC statistical uncertainty is minimised, and (ii) sum of the generator weights for a pair of reweighted events is 1 . The above-mentioned criterion are satisfied by the following equations:

$$
\frac{W_{b f}^{b b}}{W_{i n c}^{b b}}=\frac{N_{b f}^{b b}}{N_{i n c}^{b b}} \quad \text { and } \quad W_{b f}^{b b}+W_{i n c}^{b b}=1
$$

where $W_{b f}^{b b}$ is the MC generator event weight of a $B$-filtered event, $W_{i n c}^{b b}$ is the MC generator event weight of a $Z \rightarrow b \bar{b}$ event in the inclusive sample, $N_{b f}^{b b}$ is the number of events in the $B$-filtered sample, and $N_{i n c}^{b b}$ is the number of $Z \rightarrow b \bar{b}$ events in inclusive sample. From equation 86 one can write,

$$
W_{b f}^{b b}=\frac{N_{b f}^{b b}}{N_{b f}^{b b}+N_{i n c}^{b b}} \quad \text { and } \quad W_{i n c}^{b b}=\frac{N_{i n c}^{b b}}{N_{b f}^{b b}+N_{i n c}^{b b}}
$$

The number of events can be written as:

$$
N_{b f}^{b b}=N_{b b}^{\text {yield }} \quad \text { and } \quad N_{\text {inc }}^{b b}=N_{\text {inc }}^{\text {yield }} \times \frac{B R(Z \rightarrow b \bar{b})}{B R(Z \rightarrow q \bar{q})}
$$

where $\frac{B R(Z \rightarrow b \bar{b})}{B R(Z \rightarrow q \bar{q})}$ is $0.2161[350]$. From equations 87 and 88 , the reweighted MC generator event weights for the $Z \rightarrow b \bar{b}$ events in the inclusive sample $89 \mathrm{a}$ and for all the events in the $B$-filtered sample $(89 b)$ can be written as: 


$$
\begin{aligned}
& W_{b f}^{b b \_n e w}=W_{b f}^{b b} \times \frac{W_{b f}^{b b}}{W_{b f}^{b b}+\left(W_{i n c} \times \frac{B R(Z \rightarrow b \bar{b})}{B R(Z \rightarrow q \bar{q})}\right)} \\
& W_{\text {inc }}^{b b \_n e w}=W_{i n c}^{b b} \times \frac{W_{i n c} \times \frac{B R(Z \rightarrow b \bar{b})}{B R(Z \rightarrow q \bar{q})}}{W_{b f}^{b b}+\left(W_{i n c} \times \frac{B R(Z \rightarrow b \bar{b})}{B R(Z \rightarrow q \bar{q})}\right)}
\end{aligned}
$$

Similar to the previous samples, the DB sample extension reduces the MC statistical uncertainty. Figure A.4 shows the $m_{h}$ comparison between the inclusive only (red) and combined DB samples after reweighting (blue) in the 0-lepton channel for the $2 b$-tagged category in Resolved and Merged regions. The ratio between the two is within the statistical uncertainty of the inclusive diboson $V Z$ $(Z \rightarrow q \bar{q})$ sample. Due to the increased statistics, the MC statistical uncertainties were reduced by $47 \%$ and $46 \%$ for the Resolved and Merged regions, respectively.
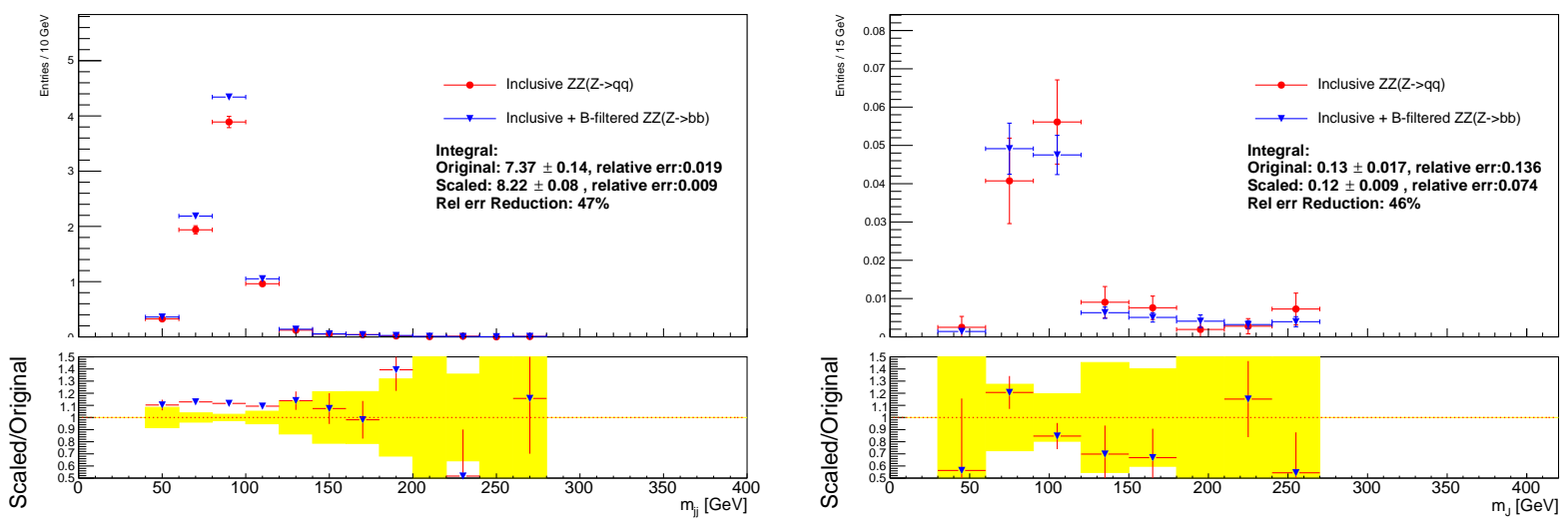

Figure A.4: $\mathrm{m}_{b b}$ distribution for the inclusive and combined diboson samples after reweighting in the 0-lepton SR for the $2 b$-tagged $E_{\mathrm{T}}^{\text {miss }}$ inclusive Resolved (left plot) and Merged (right plot) regions. The yellow band represents the statistical uncertainty band for the inclusive diboson samples. 


\section{B. MONO- $H(B \bar{B})$ PRE-FIT DISTRIBUTIONS}

This appendix shows the data and MC comparisons, before the simultaneous fit (pre-fit), for different variables used in the mono- $h(b \bar{b})$ analysis across different regions. Section B.1 shows the comparisons in the 0-lepton region, while section B.2 and B.3 shows the 1- $\mu$ CR and the combined 2-lepton CR respectively.

\section{B.1 0-lepton SR}

Figure B.1-B.9 show the Data-MC comparisons for different kinematic variables for the 0lepton SR across different $E_{\mathrm{T}}^{\text {miss }}$ and $b$-tag regions. In most of the regions the $\mathrm{MC}$ is underestimated compared to data, and this is due to the fact that the MC generators underestimate the $Z+\mathrm{HF}$ and $t \bar{t}$ event rates by $20-40 \%$ and $10 \%$ respectively. Overall, in all the regions the Data-MC comparisons are reasonable. 

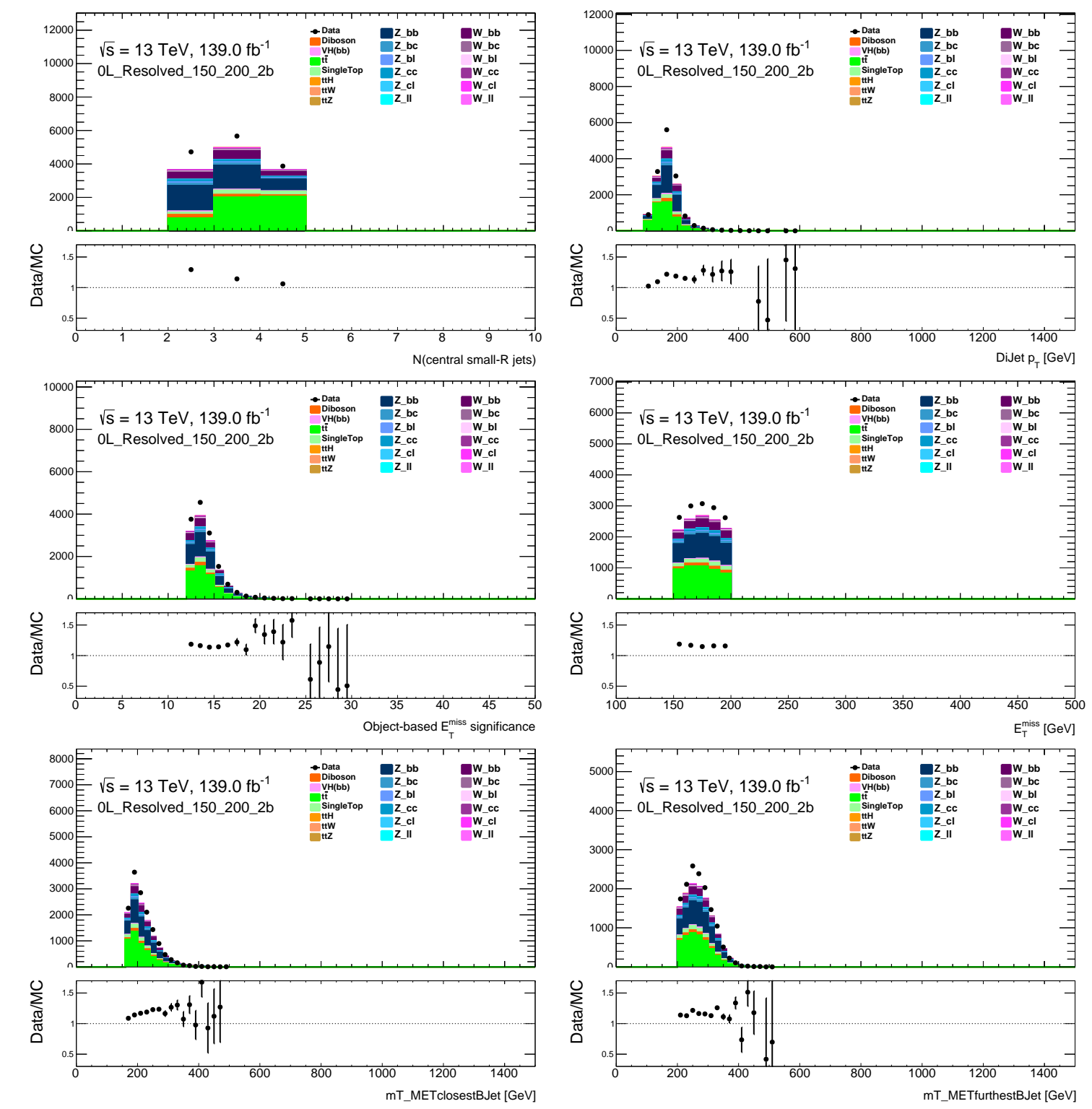

Figure B.1: Kinematic distributions in the 0-lepton region with $2 b$-tagged jets and in the $E_{\mathrm{T}}^{\text {miss }}$ region of $150<E_{\mathrm{T}}^{\text {miss }}<200 \mathrm{GeV}$. 

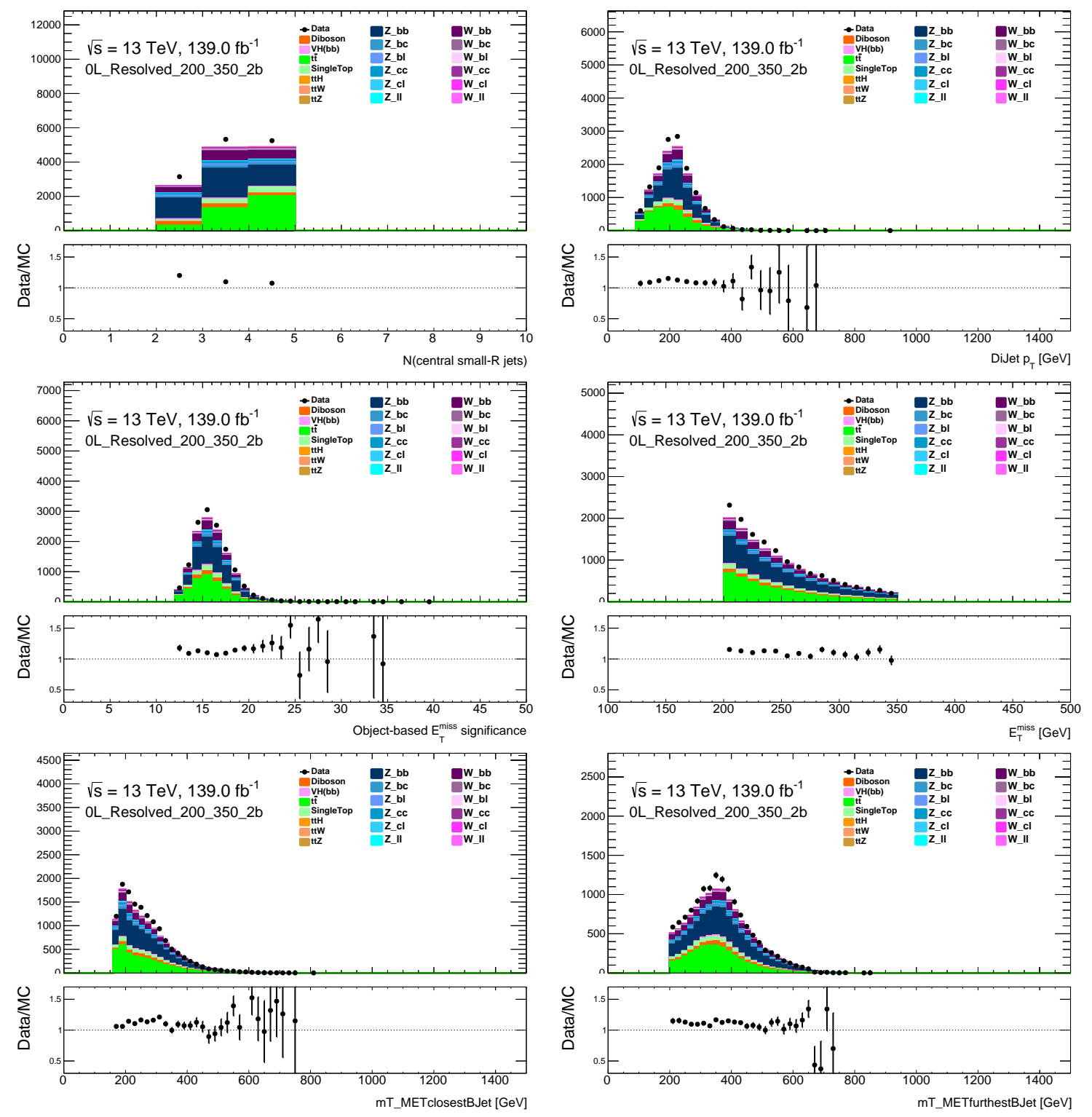

Figure B.2: Kinematic distributions in the 0-lepton region with $2 b$-tagged jets and in the $E_{\mathrm{T}}^{\text {miss }}$ region of $200<E_{\mathrm{T}}^{\text {miss }}<350 \mathrm{GeV}$. 

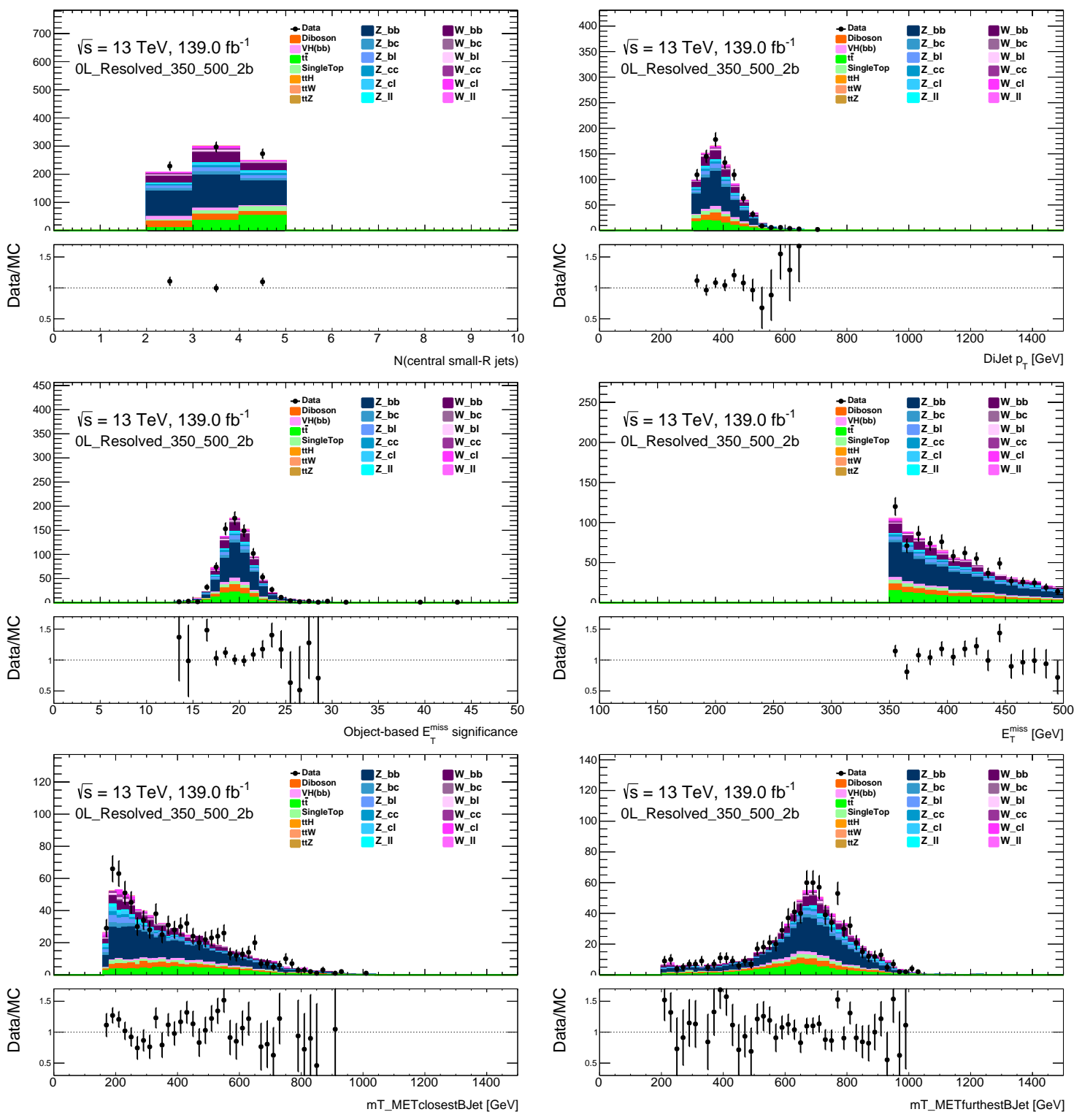

Figure B.3: Kinematic distributions in the 0-lepton region with $2 b$-tagged jets and in the $E_{\mathrm{T}}^{\text {miss }}$ region of $350<E_{\mathrm{T}}^{\text {miss }}<500 \mathrm{GeV}$. 

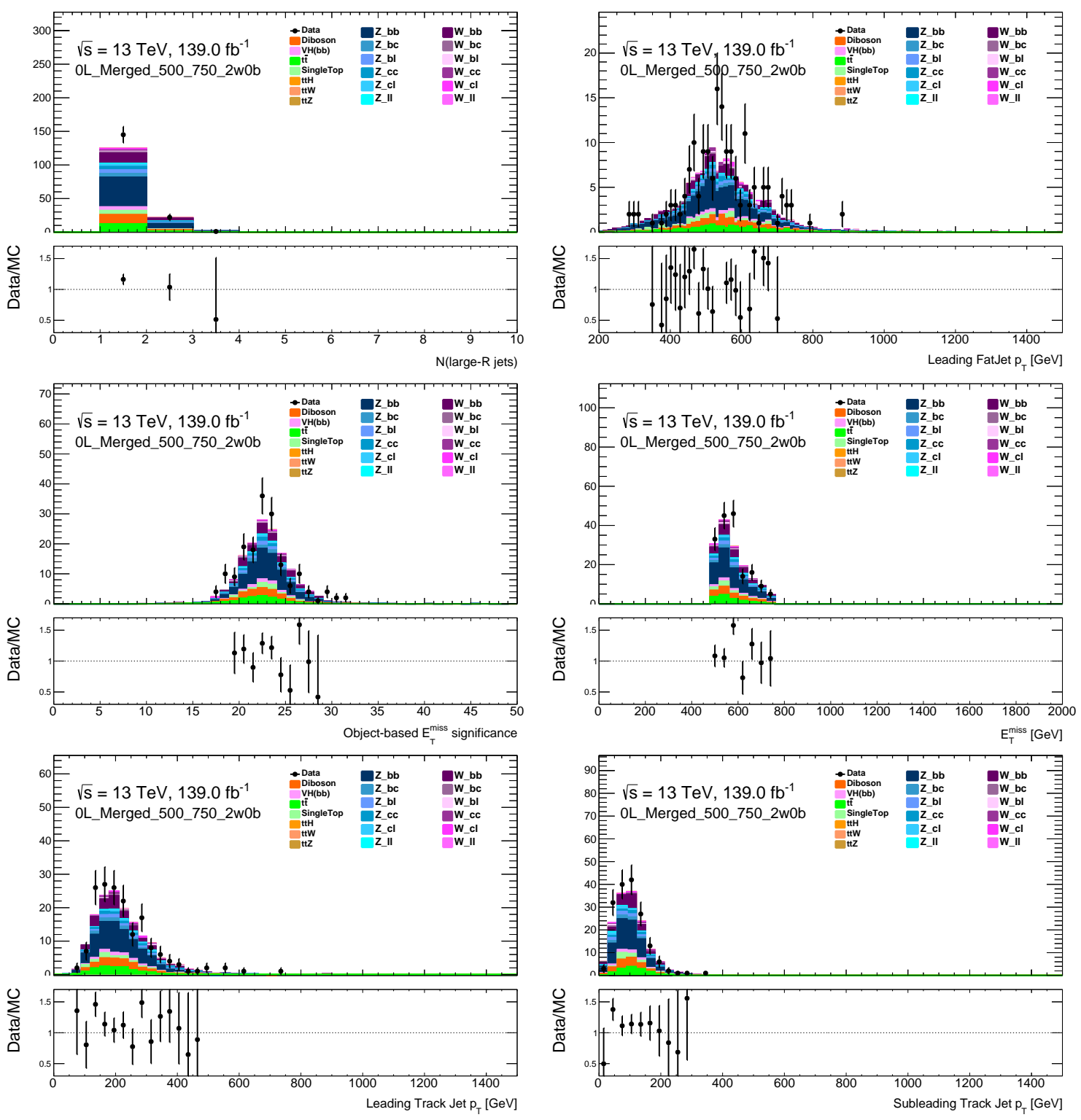

Figure B.4: Kinematic distributions in 0-lepton region with a $2 b$-tagged large-R jet and in the $E_{\mathrm{T}}^{\text {miss }}$ region of $500<E_{\mathrm{T}}^{\text {miss }}<750 \mathrm{GeV}$. 

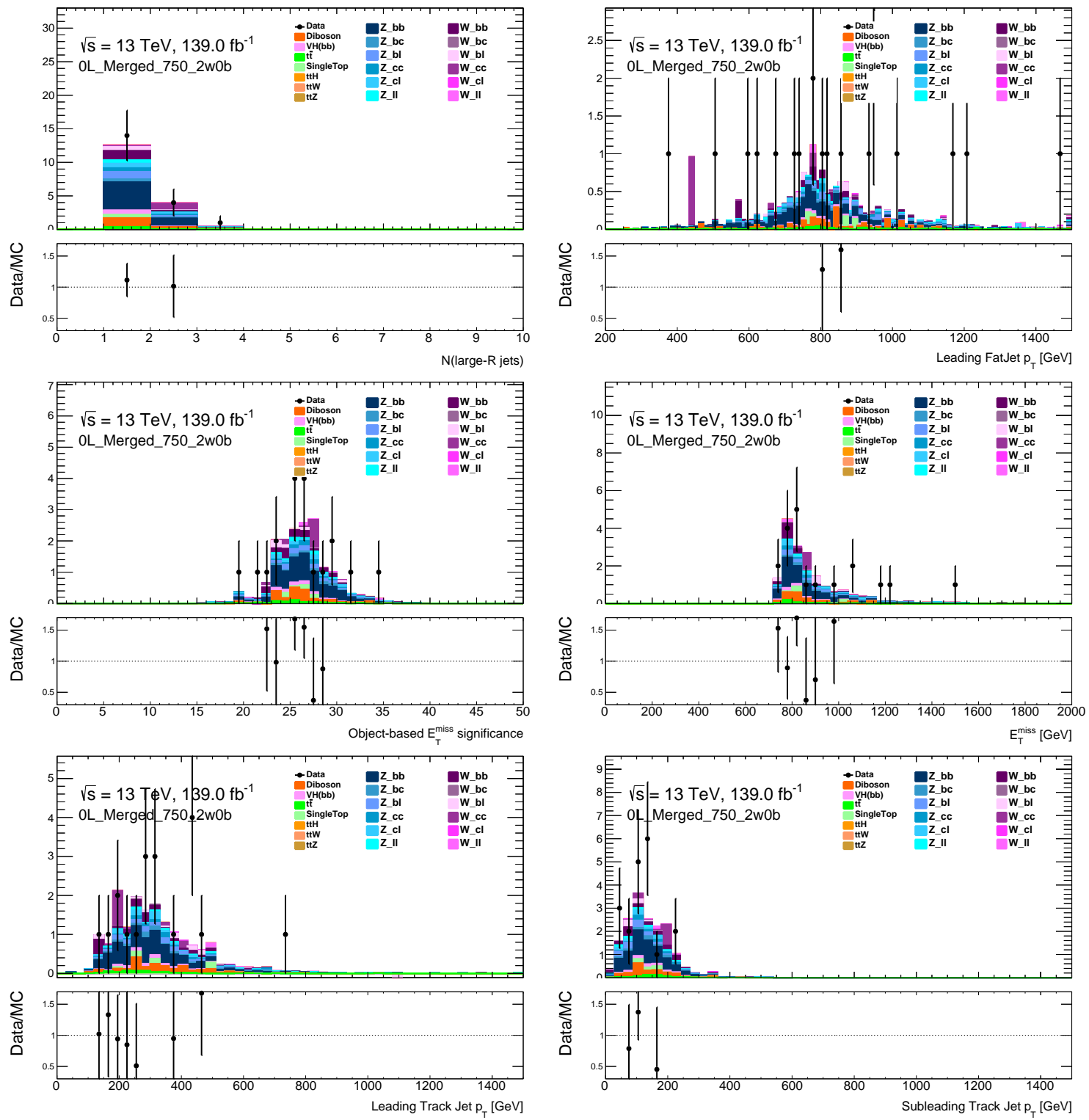

Figure B.5: Kinematic distributions in 0-lepton region with a $2 b$-tagged large-R jet and in the $E_{\mathrm{T}}^{\text {miss }}$ region of $E_{\mathrm{T}}^{\text {miss }}>750 \mathrm{GeV}$. 

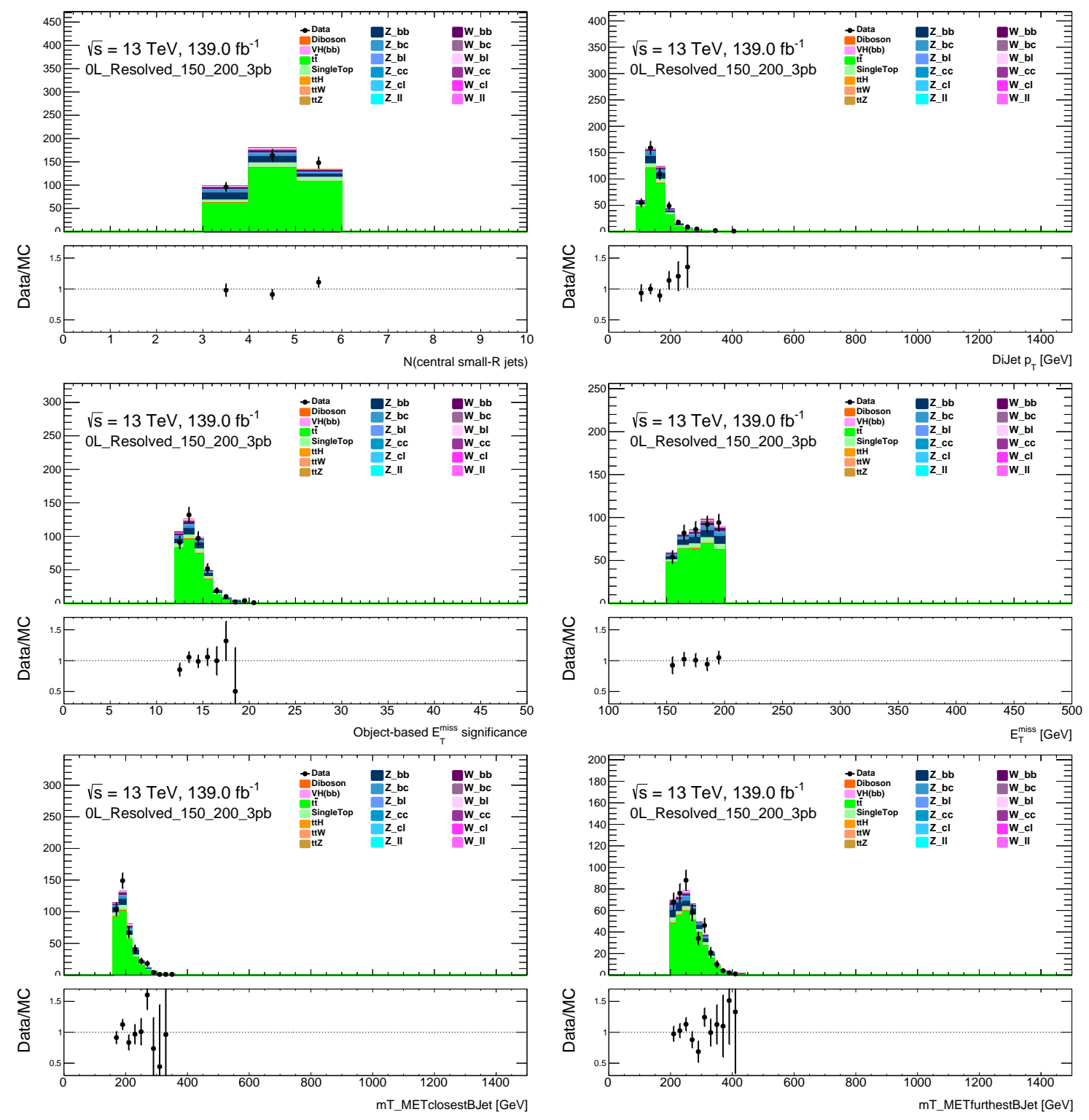

Figure B.6: Kinematic distributions in the 0-lepton region with $\geq 3 b$-tagged jets and in the $E_{\mathrm{T}}^{\text {miss }}$ region of $150<E_{\mathrm{T}}^{\text {miss }}<200 \mathrm{GeV}$. 

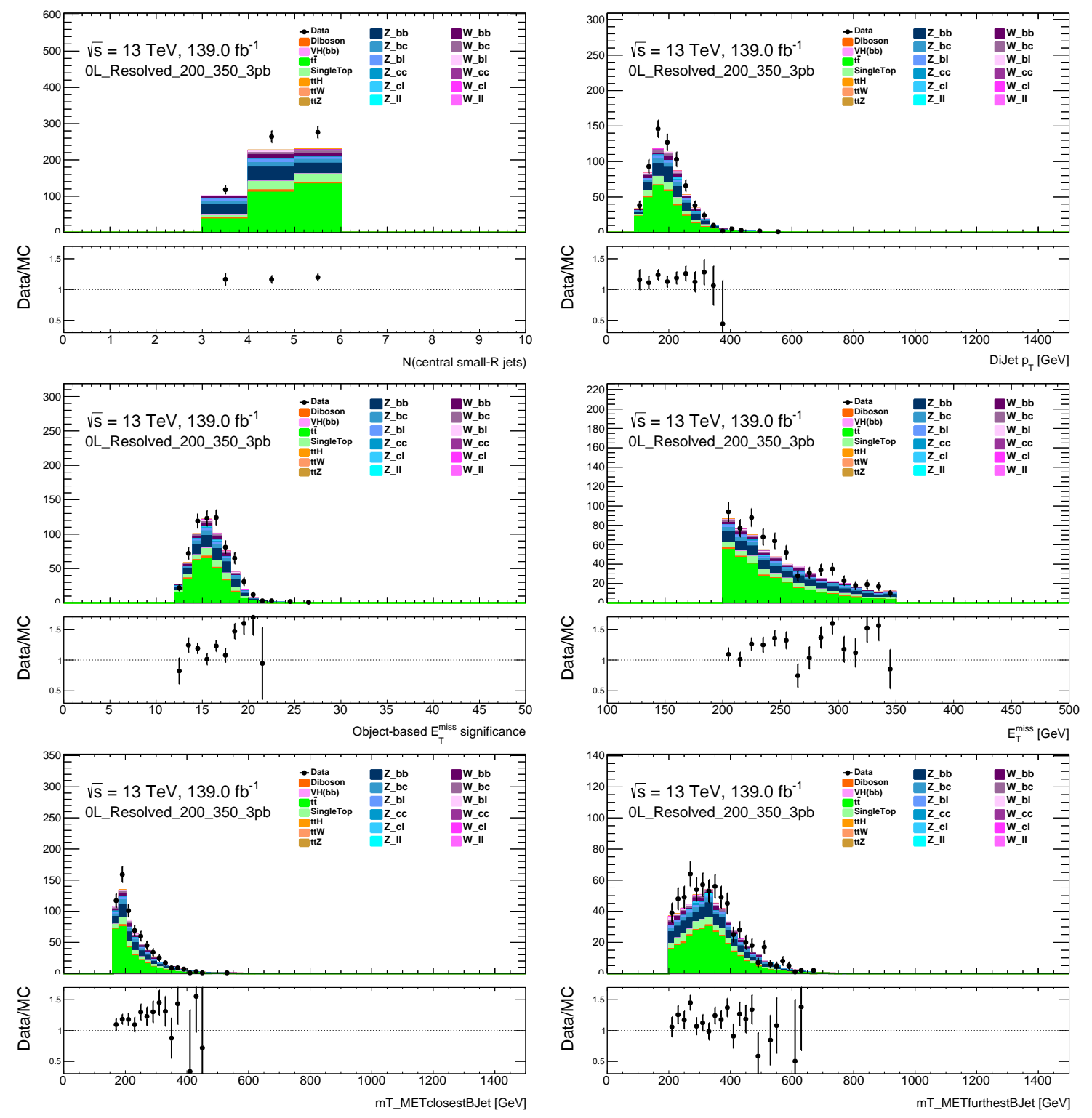

Figure B.7: Kinematic distributions in the 0-lepton region with $\geq 3 b$-tagged jets and in the $E_{\mathrm{T}}^{\text {miss }}$ region of $200<E_{\mathrm{T}}^{\text {miss }}<350 \mathrm{GeV}$. 

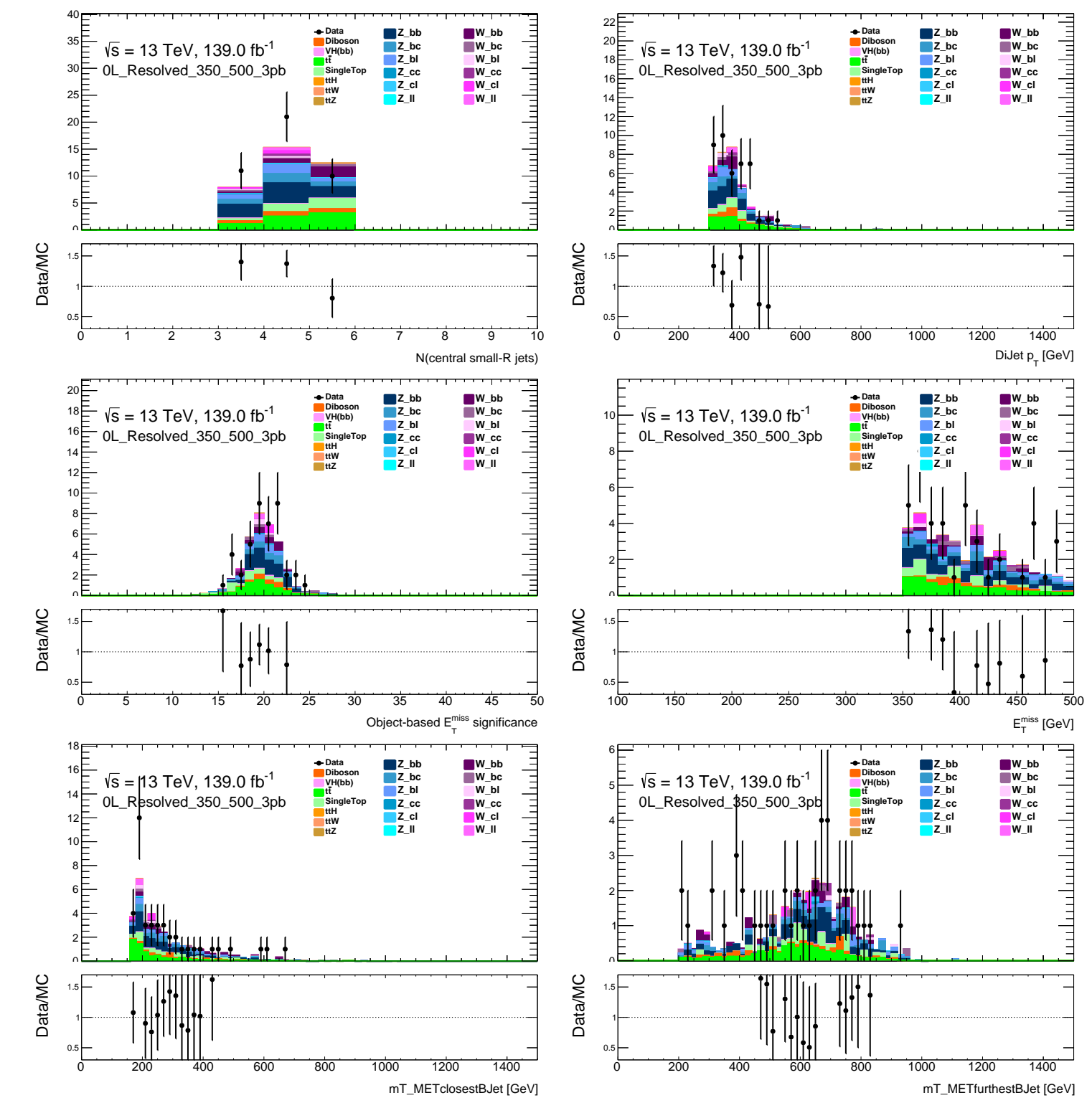

Figure B.8: Kinematic distributions in the 0-lepton region with $\geq 3 b$-tagged jets and in the $E_{\mathrm{T}}^{\text {miss }}$ region of $350<E_{\mathrm{T}}^{\text {miss }}<500 \mathrm{GeV}$. 

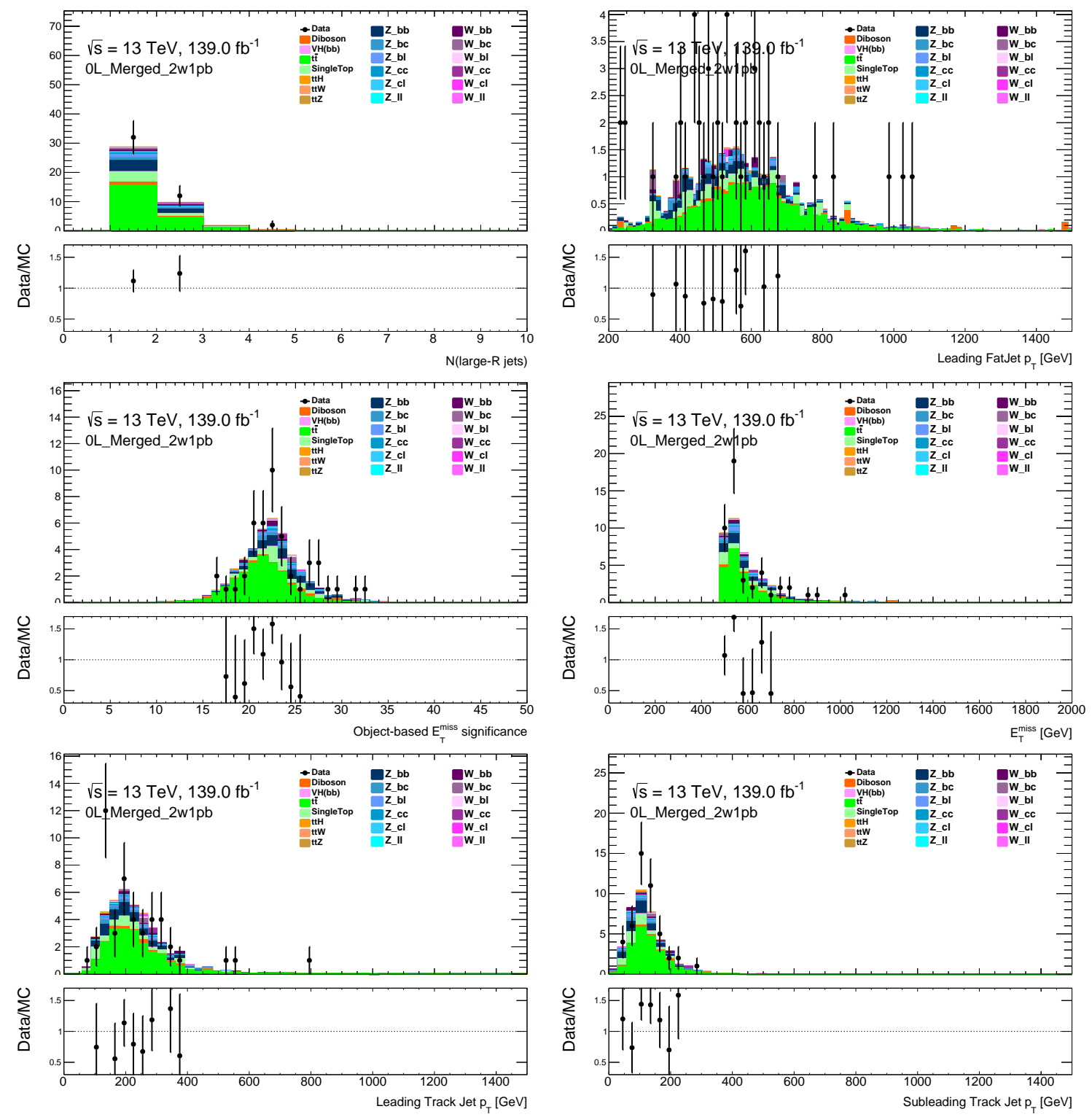

Figure B.9: Kinematic distributions in 0-lepton channel with 2 -tagged VR track jets associated to the large-R jet and $\geq 1 b$-tagged VR track jets not-associated to the large-R jet and in the $E_{\mathrm{T}}^{\text {miss }}$ region of $E_{\mathrm{T}}^{\mathrm{miss}}>500 \mathrm{GeV}$. 


\section{B.2 1-muon CR}

Figure B.10- B.17 show the Data-MC comparisons for different kinematic variables for the 1- $\mu \mathrm{CR}$ across different $E_{\mathrm{T}}^{\text {miss }}$ and $b$-tag regions. Overall, in all the regions the Data-MC comparisons are reasonable.
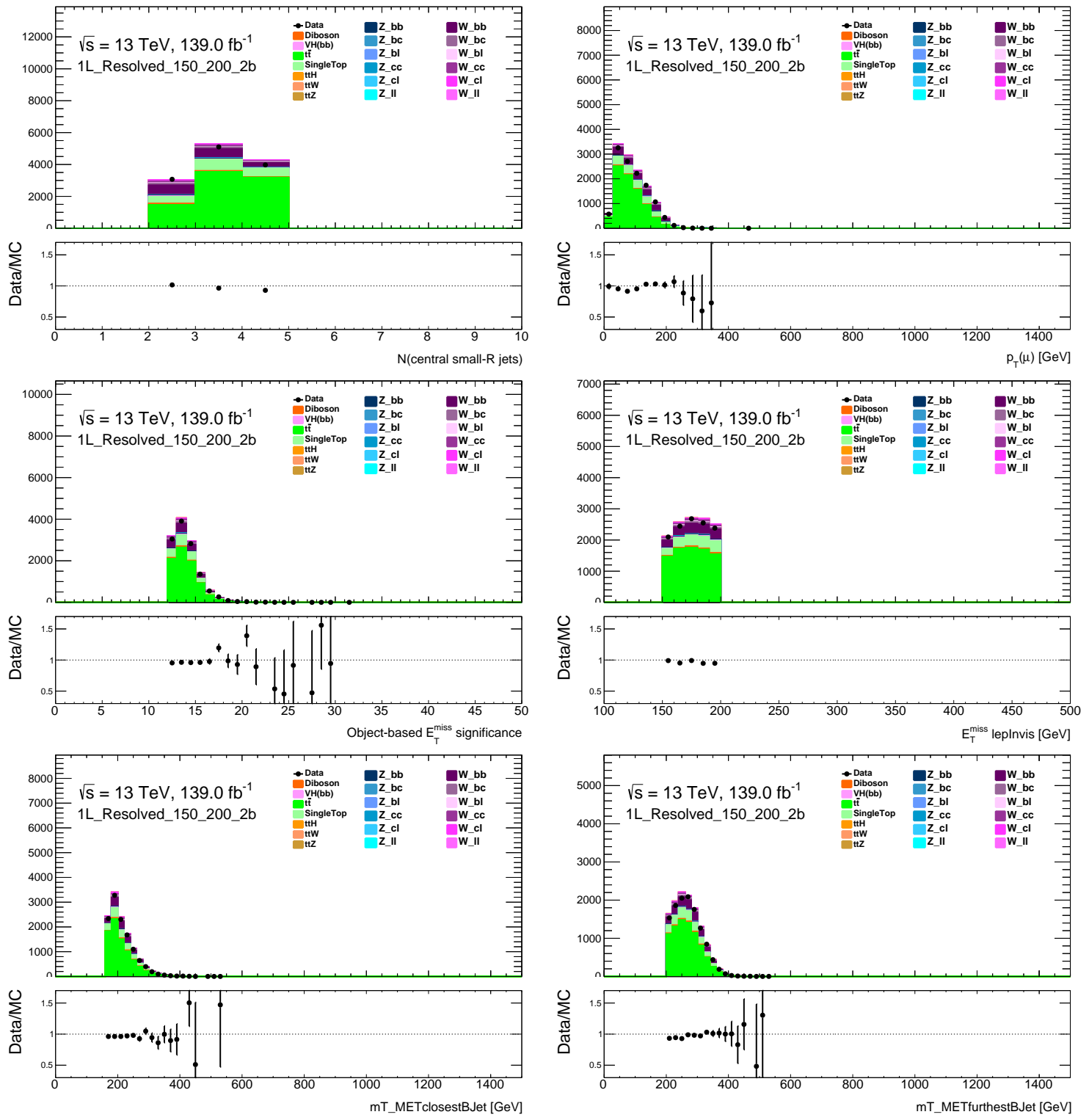

Figure B.10: Kinematic distributions in the $1-\mu \mathrm{CR}$ with $2 b$-tagged jets for the $E_{\mathrm{T}}^{\mathrm{miss}}$ region of $150<E_{\mathrm{T}}^{\text {miss }}<200 \mathrm{GeV}$. 

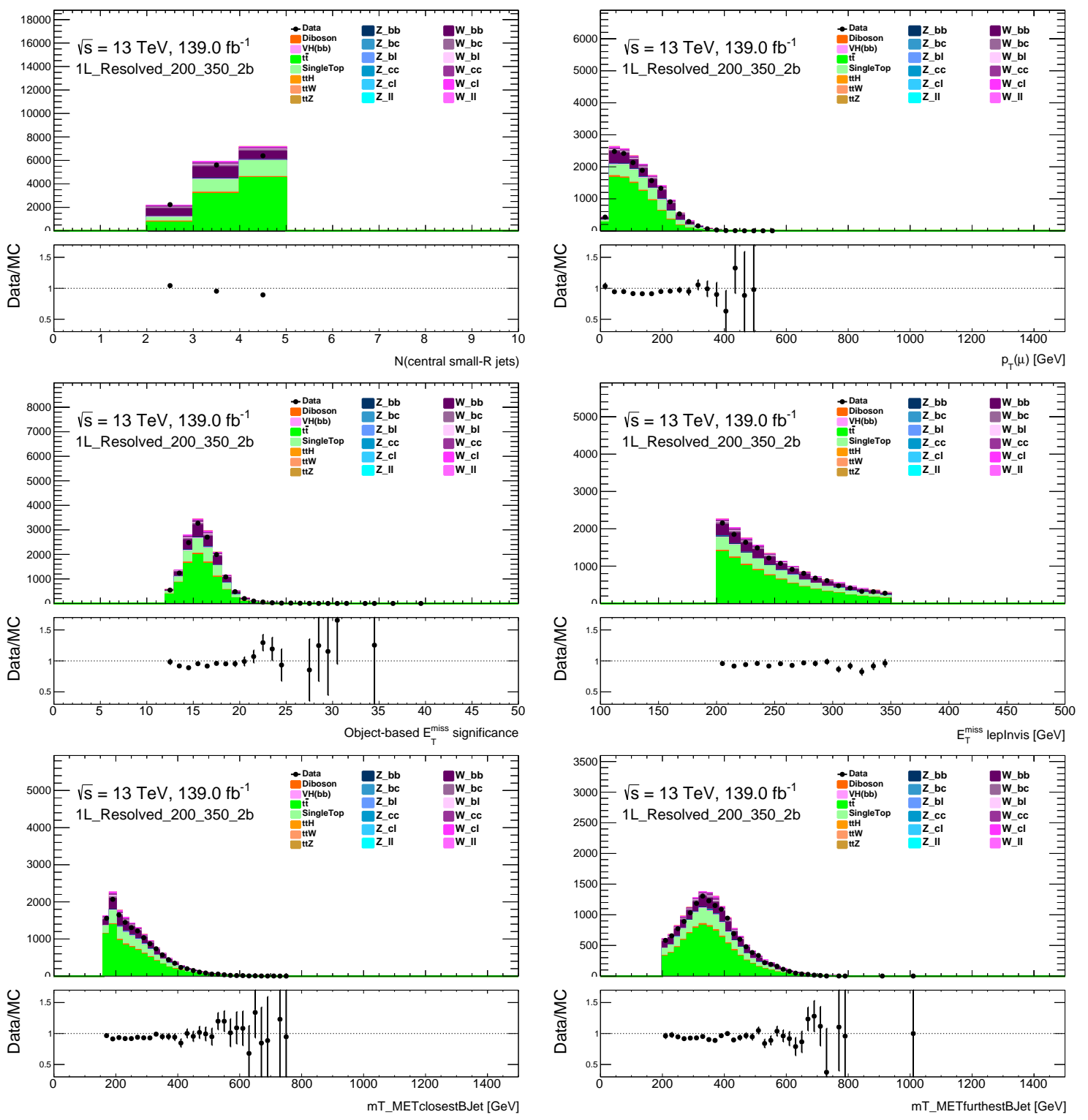

Figure B.11: Kinematic distributions in the $1-\mu \mathrm{CR}$ with $2 b$-tagged jets for the $E_{\mathrm{T}}^{\mathrm{miss}}$ region of $200<E_{\mathrm{T}}^{\text {miss }}<350 \mathrm{GeV}$. 

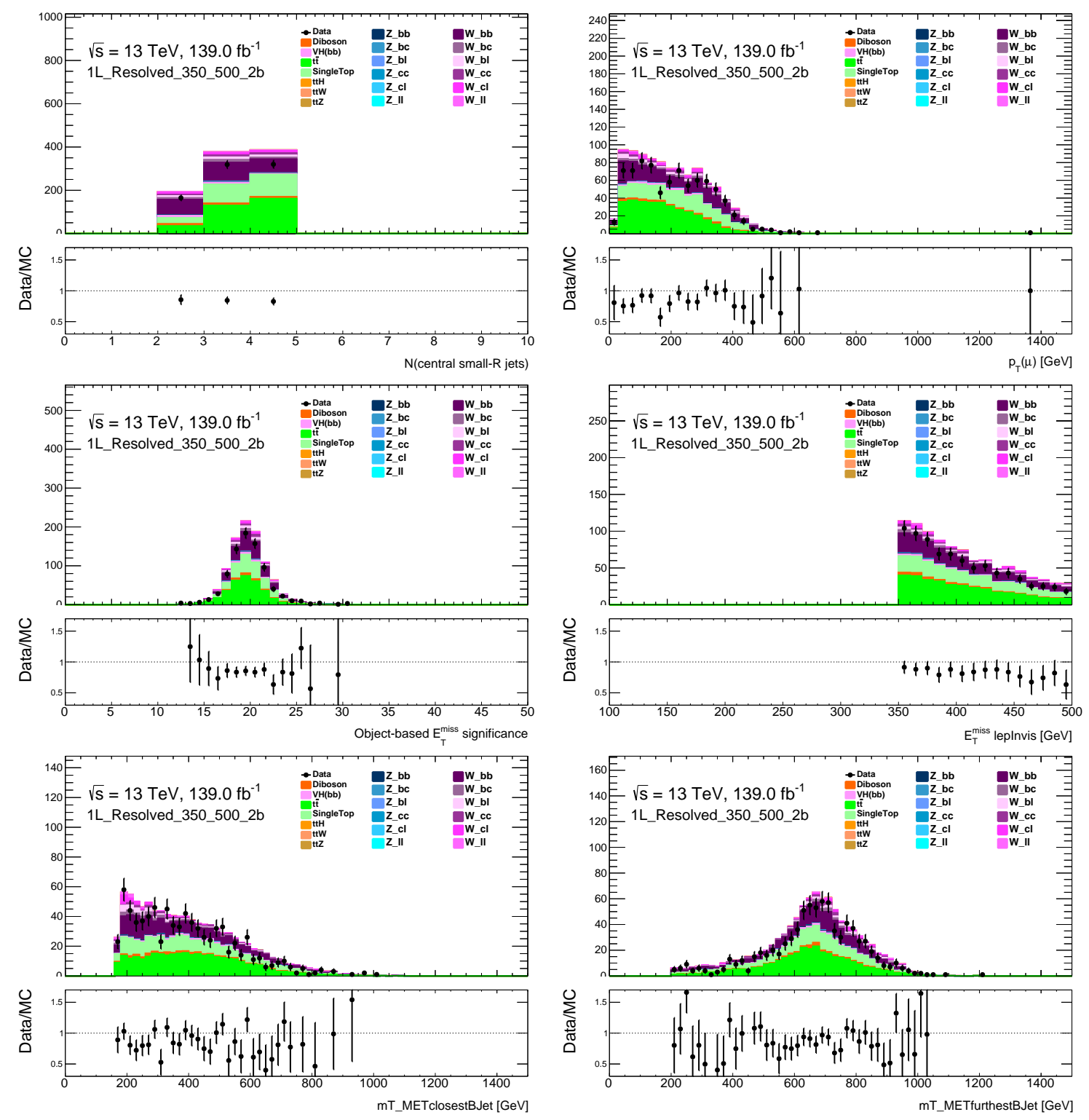

Figure B.12: Kinematic distributions in the $1-\mu \mathrm{CR}$ with $2 b$-tagged jets for the $E_{\mathrm{T}}^{\mathrm{miss}}$ region of $350<E_{\mathrm{T}}^{\text {miss }}<500 \mathrm{GeV}$. 

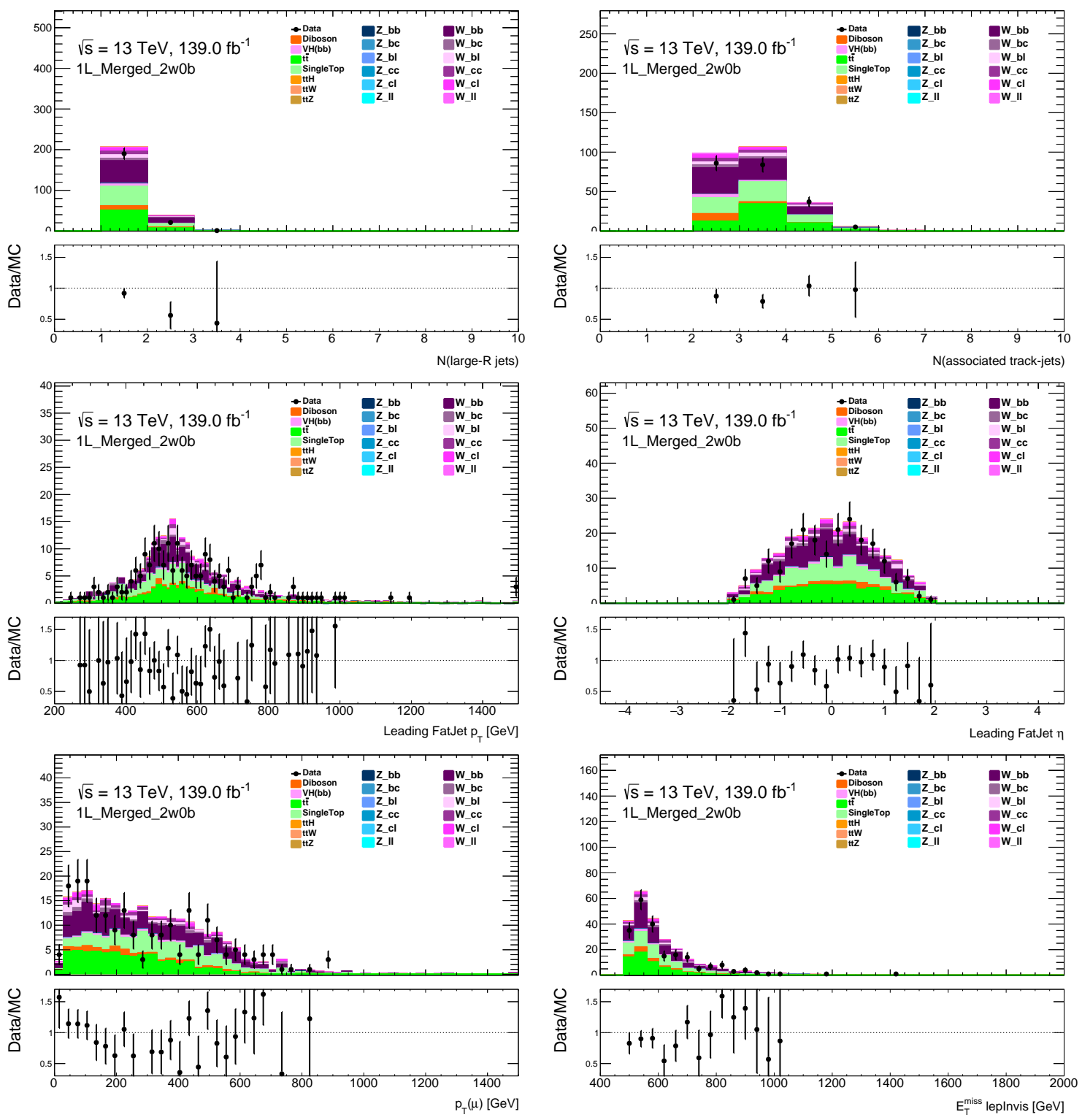

Figure B.13: Kinematic distributions in the $1-\mu$ region with $2 b$-tagged VR track jets associated to the large-R jet for the $E_{\mathrm{T}}^{\text {miss }}$ region of $E_{\mathrm{T}}^{\text {miss }}>500 \mathrm{GeV}$. 

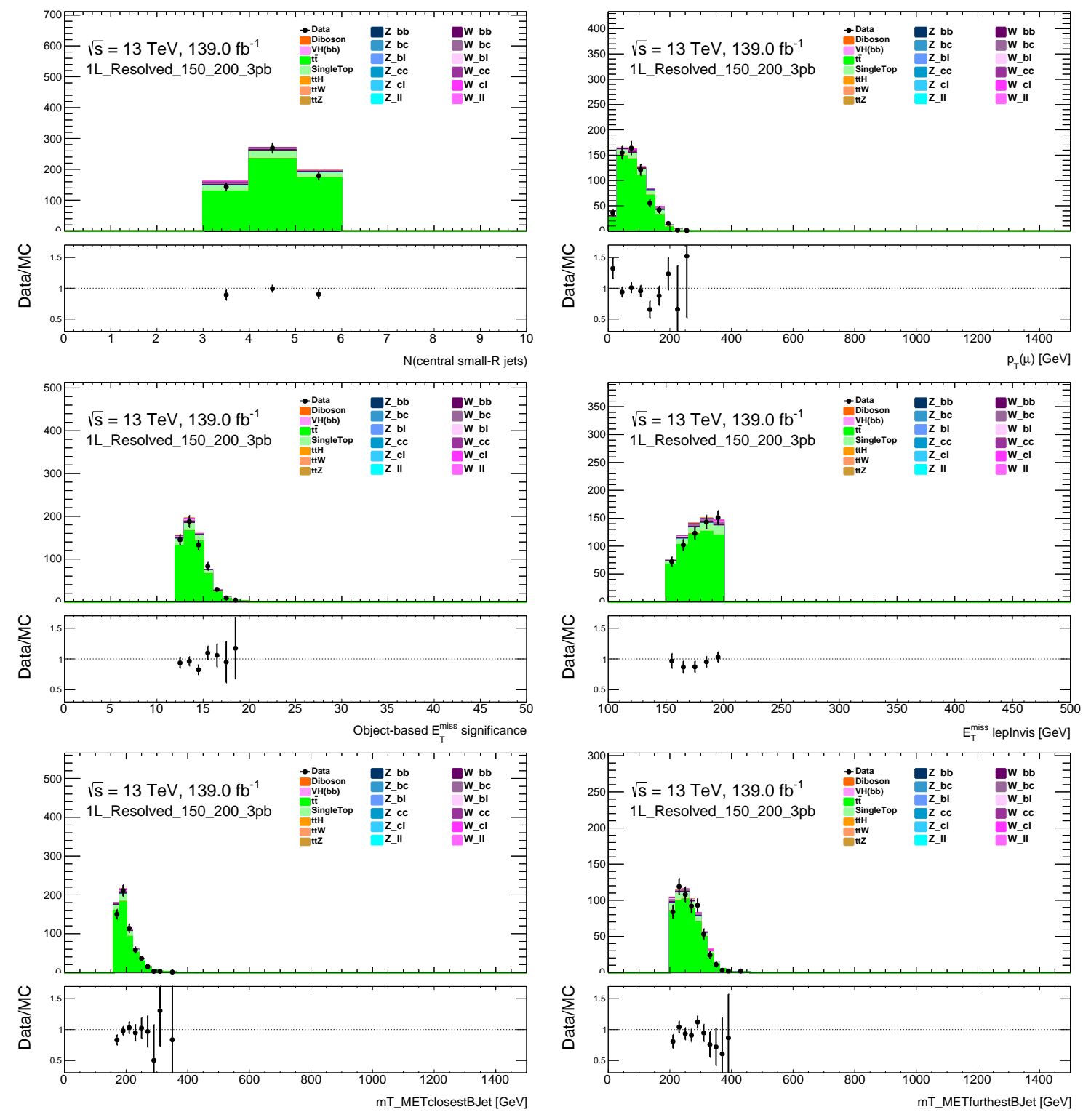

Figure B.14: Kinematic distributions in the $1-\mu \mathrm{CR}$ with $\geq 3 b$-tagged jets for the $E_{\mathrm{T}}^{\text {miss }}$ region of $150<E_{\mathrm{T}}^{\text {miss }}<200 \mathrm{GeV}$. 

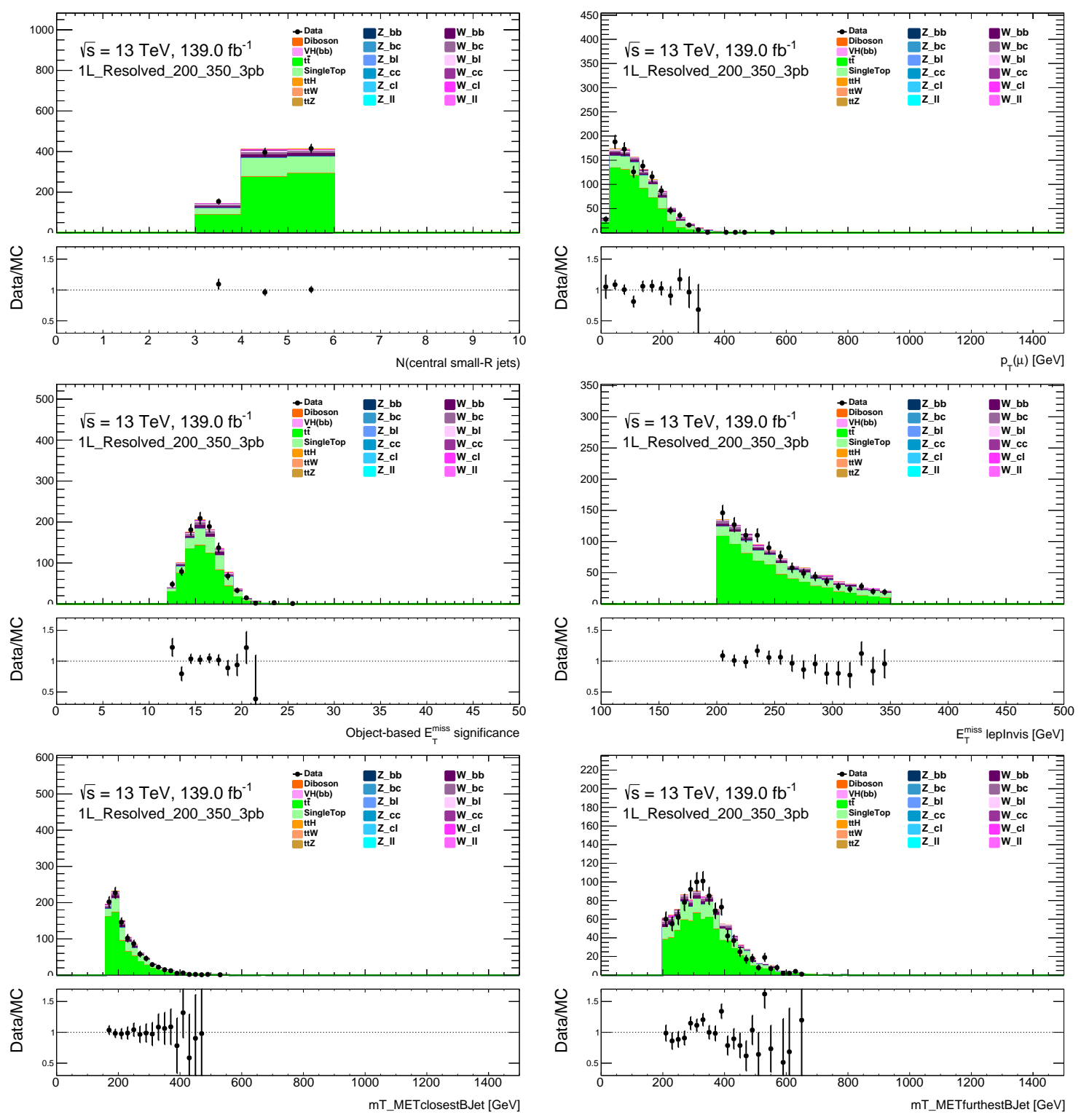

Figure B.15: Kinematic distributions in the $1-\mu \mathrm{CR}$ with $\geq 3 b$-tagged jets for the $E_{\mathrm{T}}^{\text {miss }}$ region of $200<E_{\mathrm{T}}^{\text {miss }}<350 \mathrm{GeV}$. 

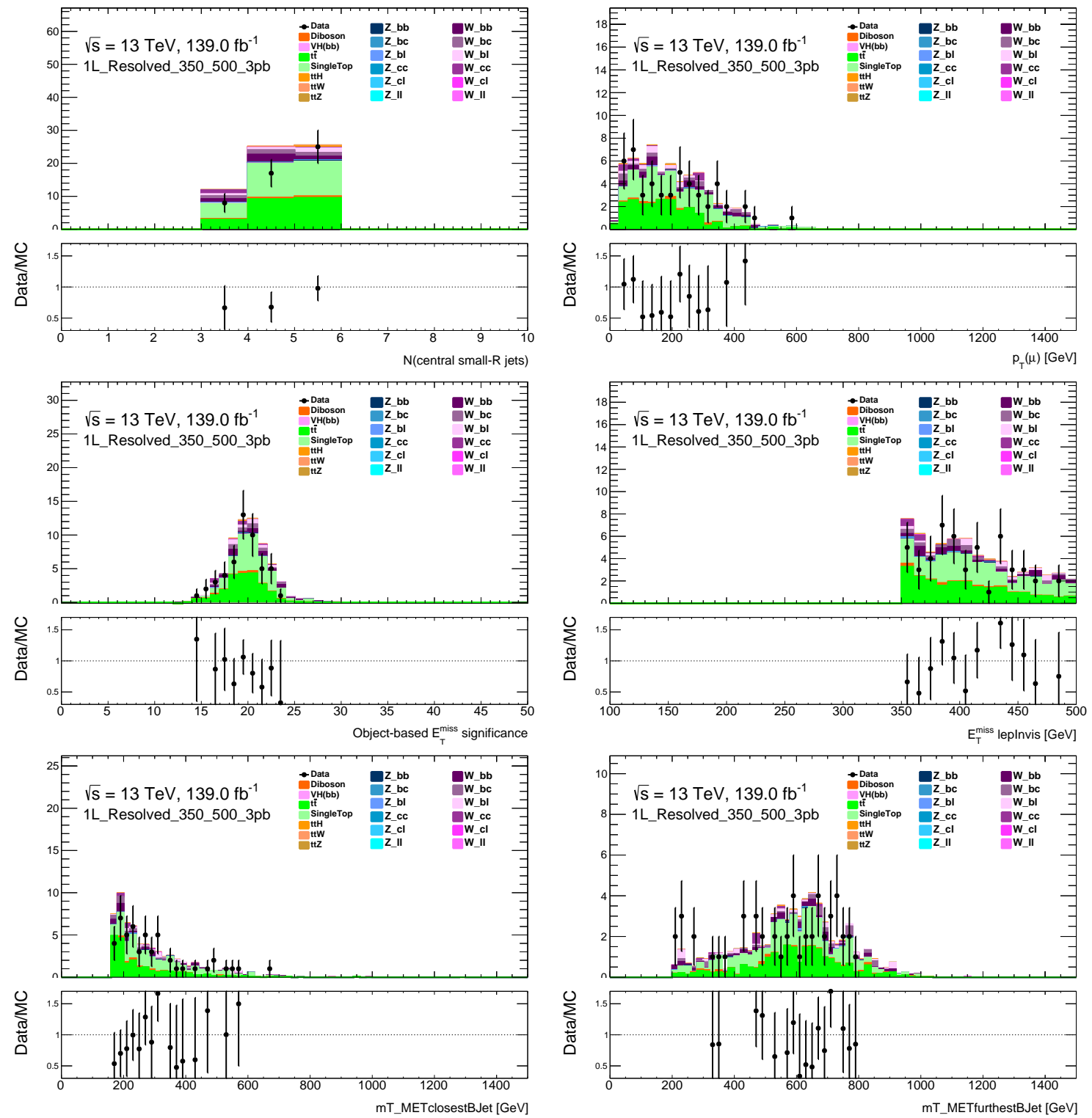

Figure B.16: Kinematic distributions in the $1-\mu \mathrm{CR}$ with $\geq 3 b$-tagged jets for the $E_{\mathrm{T}}^{\text {miss }}$ region of $350<E_{\mathrm{T}}^{\text {miss }}<500 \mathrm{GeV}$. 

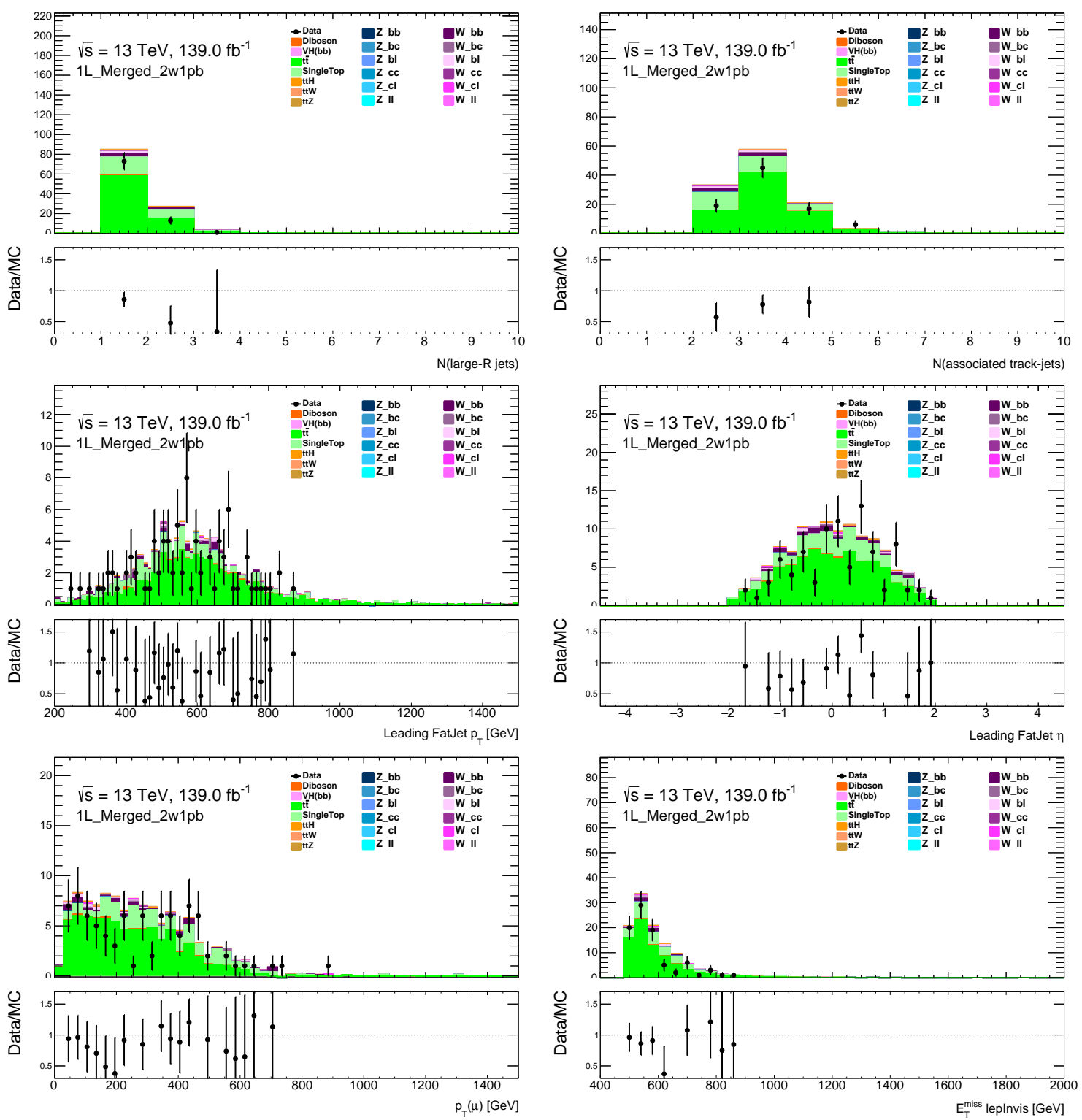

Figure B.17: Kinematic distributions in the $1-\mu \mathrm{CR}$ with $2 b$-tagged VR track jets associated to the large-R jet and $\geq 1 b$-tagged VR track jets not-associated to the large-R jet for the $E_{\mathrm{T}}^{\text {miss }}$ region of $E_{\mathrm{T}}^{\mathrm{miss}}>500 \mathrm{GeV}$.

\section{B.3 2-lepton CR}

Figure B.18. B.21 show the Data-MC comparisons for different kinematic variables for the combined 2-lepton $\mathrm{CR}(e e+\mu \mu)$ in the $2 b$-tagged region across different $E_{\mathrm{T}}^{\mathrm{miss}}$ regions. Here, the combined 2-lepton distributions are shown since in the simultaneous fit a combined yield from the $e e$ and $\mu \mu$ regions are used. Due to limited data statistics, the distributions for the $\geq 3 b$-jets 
regions cannot have proper Data-MC comparisons, hence are not shown. Overall, the Data-MC comparisons are reasonable across different regions.
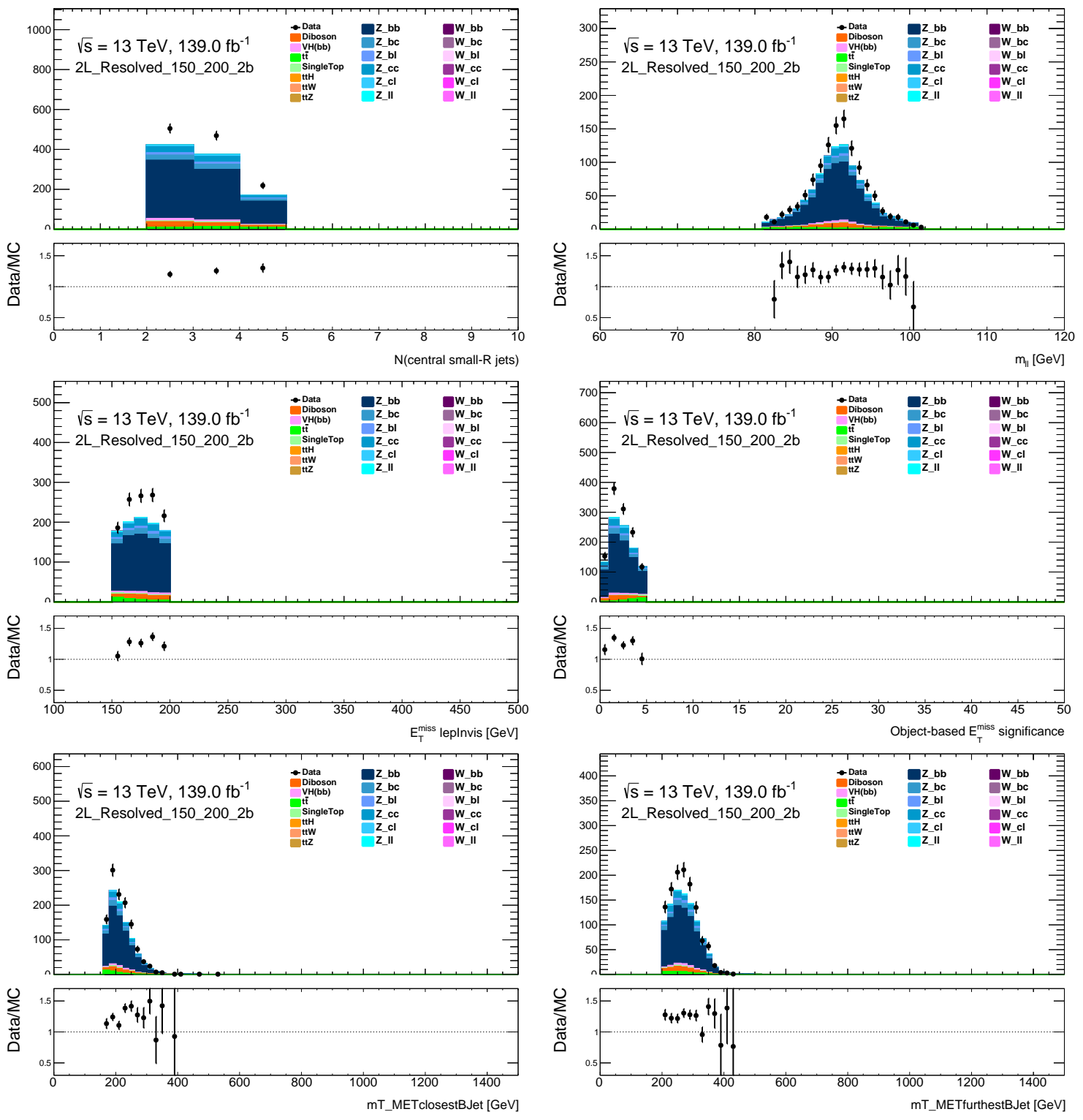

Figure B.18: Kinematic distributions in the combined 2-lepton CR with $2 b$-tagged jets for the $E_{\mathrm{T}}^{\mathrm{miss}}$ region of $150<E_{\mathrm{T}}^{\mathrm{miss}}<200 \mathrm{GeV}$. 

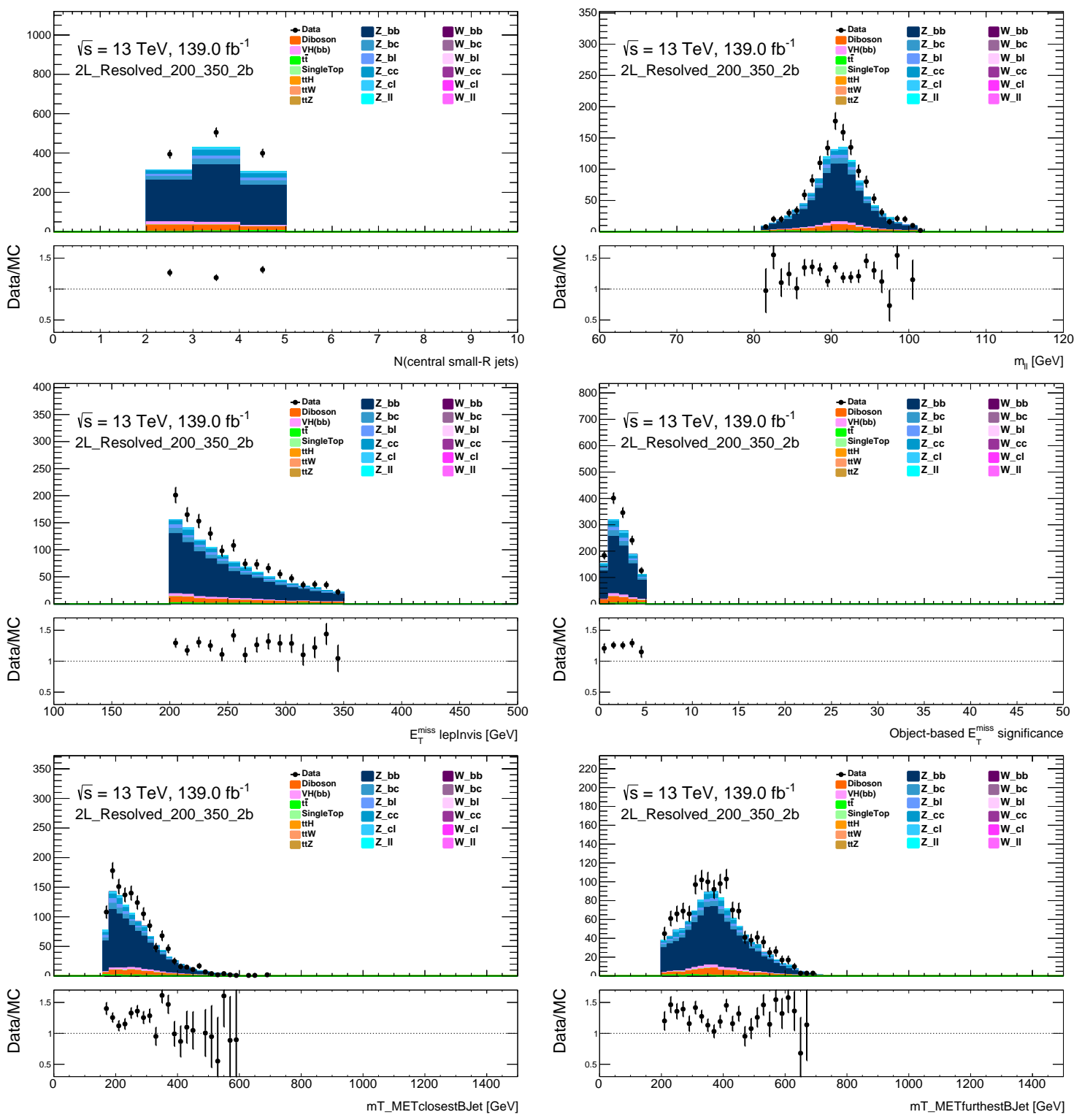

Figure B.19: Kinematic distributions in the combined 2-lepton CR with $2 b$-tagged jets for the $E_{\mathrm{T}}^{\text {miss }}$ region of $200<E_{\mathrm{T}}^{\text {miss }}<350 \mathrm{GeV}$. 

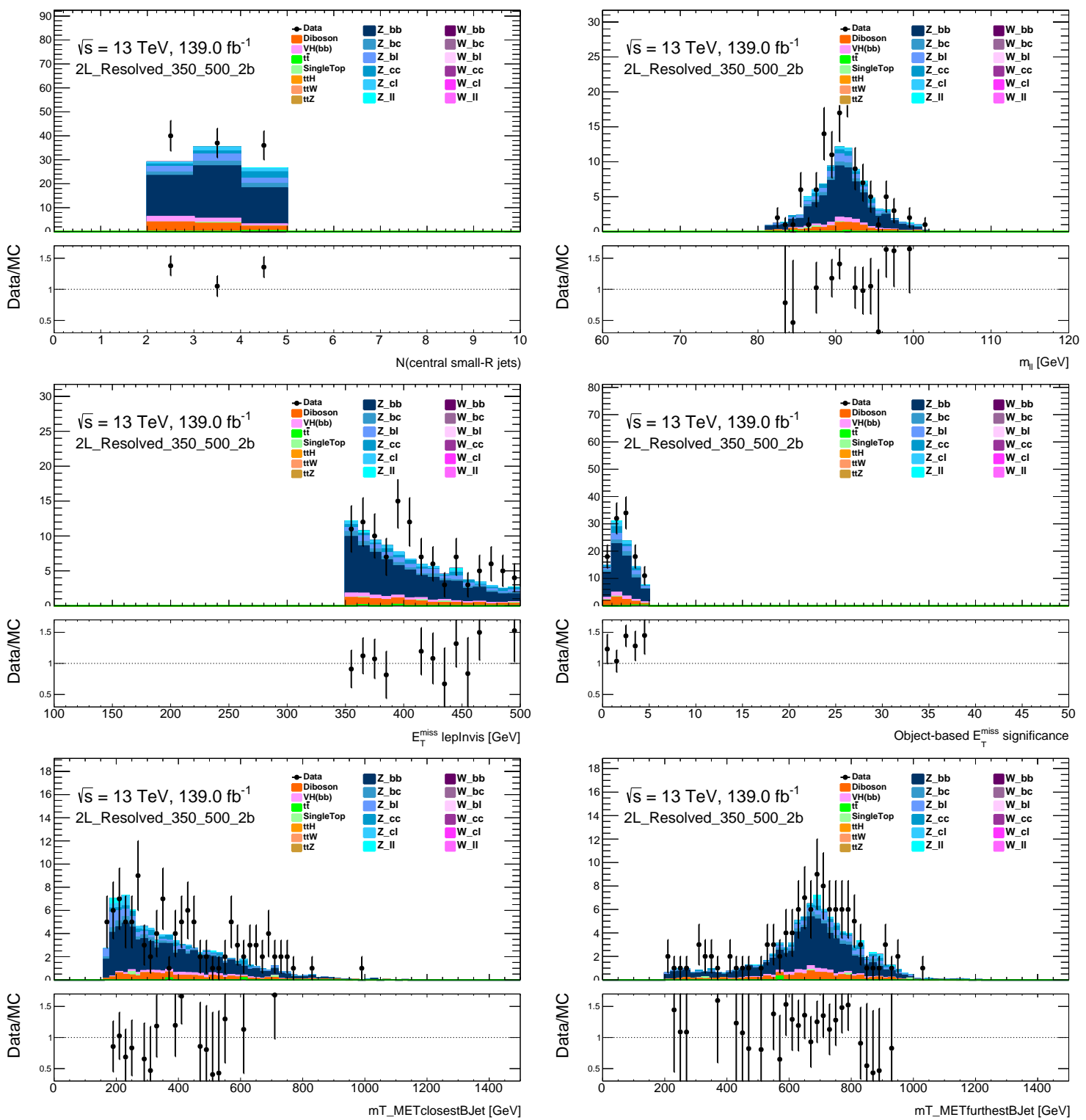

Figure B.20: Kinematic distributions in the combined 2-lepton CR with $2 b$-tagged jets for the $E_{\mathrm{T}}^{\text {miss }}$ region of $350<E_{\mathrm{T}}^{\text {miss }}<500 \mathrm{GeV}$. 

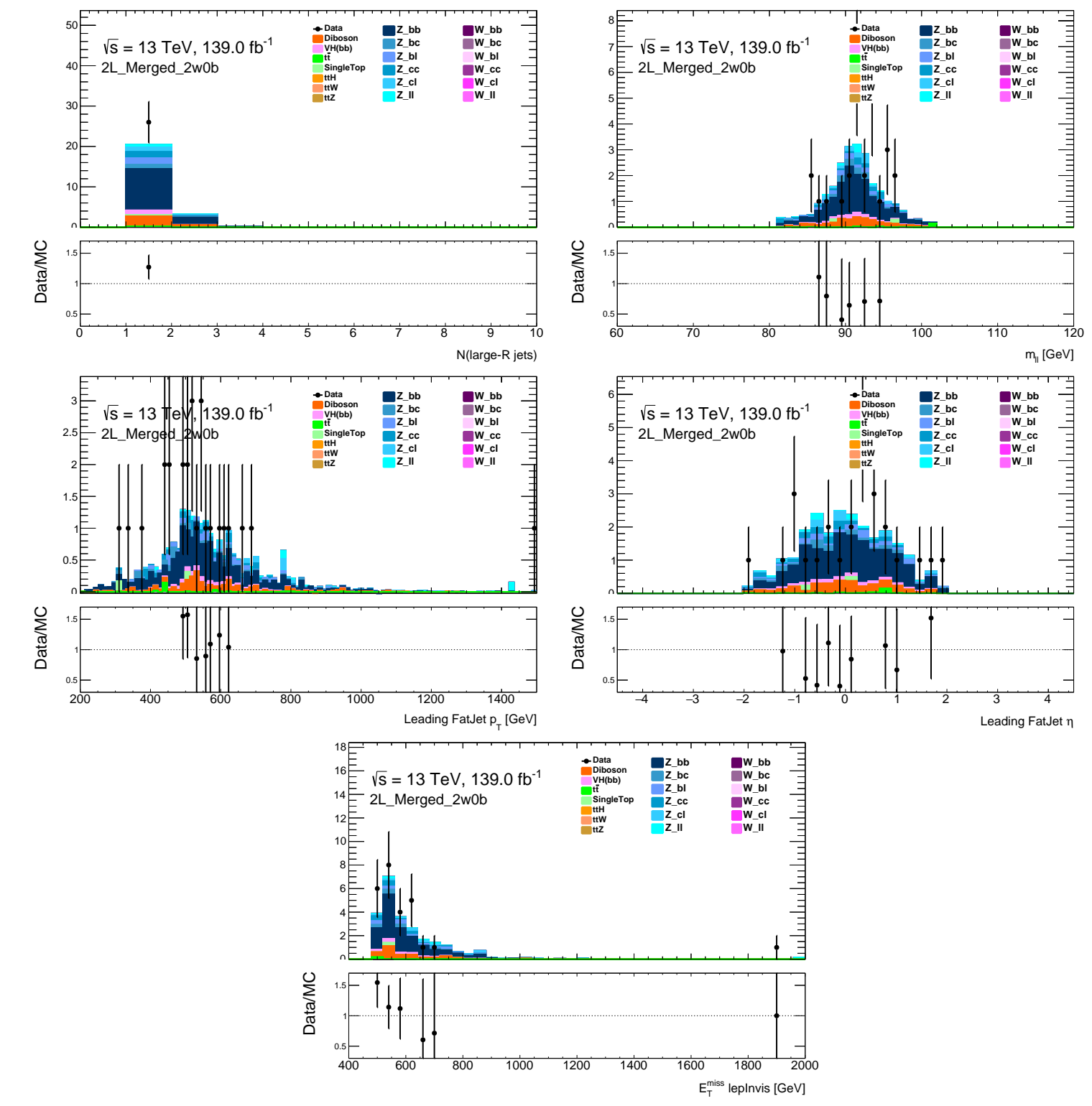

Figure B.21: Kinematic distributions in the combined 2-lepton CR with $2 b$-tagged VR track jets associated to the large-R jet for the $E_{\mathrm{T}}^{\text {miss }}$ region of $E_{\mathrm{T}}^{\text {miss }}>500 \mathrm{GeV}$. 


\section{SENSITIVITY IMPROVEMENTS WITH $\geq 3 B$-TAG REGIONS}

In this iteration of the mono- $h(b \bar{b})$ analysis the $\geq 3 b$-tag regions are included in the phasespace to increase the sensitivity for the $2 \mathrm{HDM}+\mathrm{a} b b$-induced model. The improvements from the inclusion $b$-tag category is shown in figure C.1 which shows the ratio of expected limits for the $2 \mathrm{HDM}+\mathrm{a} b b$-induced signal points, calculated with two different fit setups: (i) with only $2 b$-tag regions and (ii) with 2 and $\geq 3 b$-tag regions. The sensitivity improves by 5-63\% depending on the signal points across the grid.

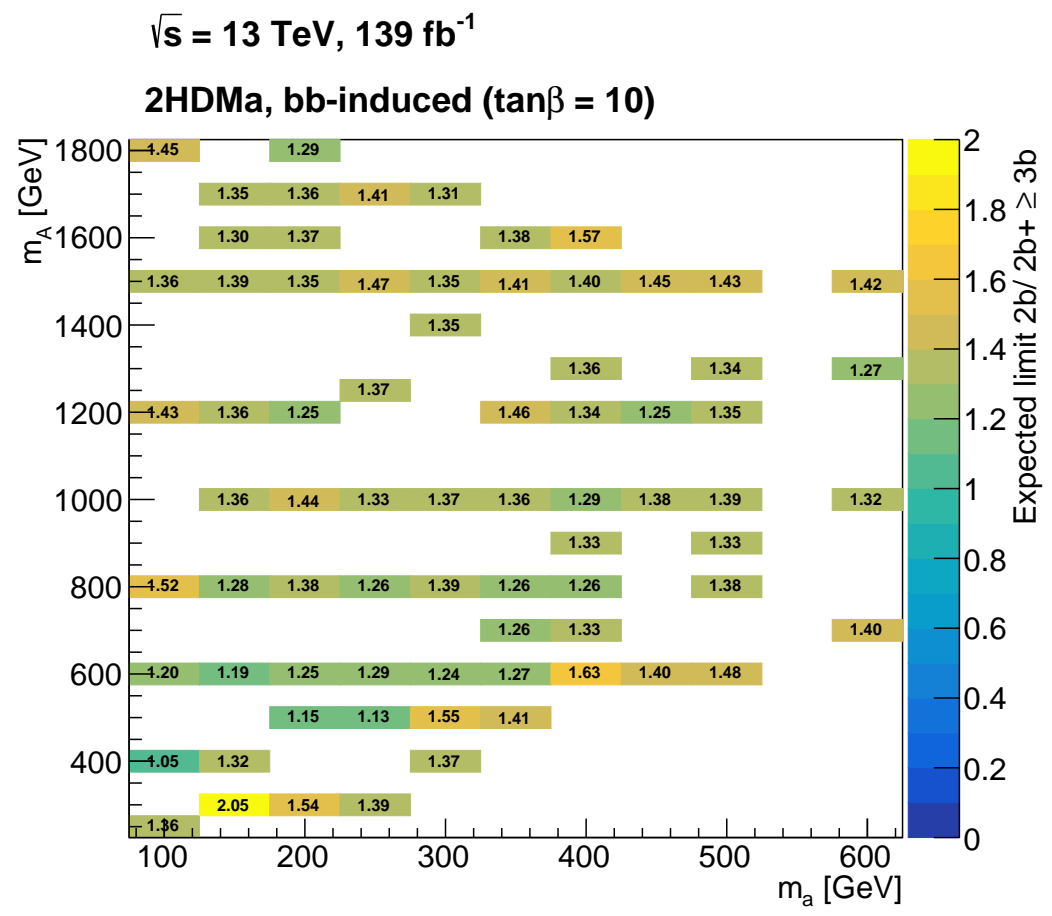

Figure C.1: Expected limit ratio 


\section{D. $M_{H}$ WINDOW FOR MONO-HIGGS MODEL INDEPENDENT LIMITS}

When extracting the model independent upper limits on the visible cross-section for the mono- $h(b \bar{b})$ analysis, the $m_{h}$ bins between $90-150 \mathrm{GeV}$ are merged into a single bin and a dummy signal is injected in that bin. As shown in figure D.1, the fraction of signal present in the $m_{h}$ window of $90-150 \mathrm{GeV}$ for the $Z^{\prime}-2 \mathrm{HDM}$ and $2 \mathrm{HDM}+\mathrm{a}$ signal models are $>70 \%$ and $>50 \%$ for the $2 b$ and $\geq 3 b$-tag regions respectively, i.e. majority of signal events fall in the $m_{h}$ bin of 90-150 $\mathrm{GeV}$.
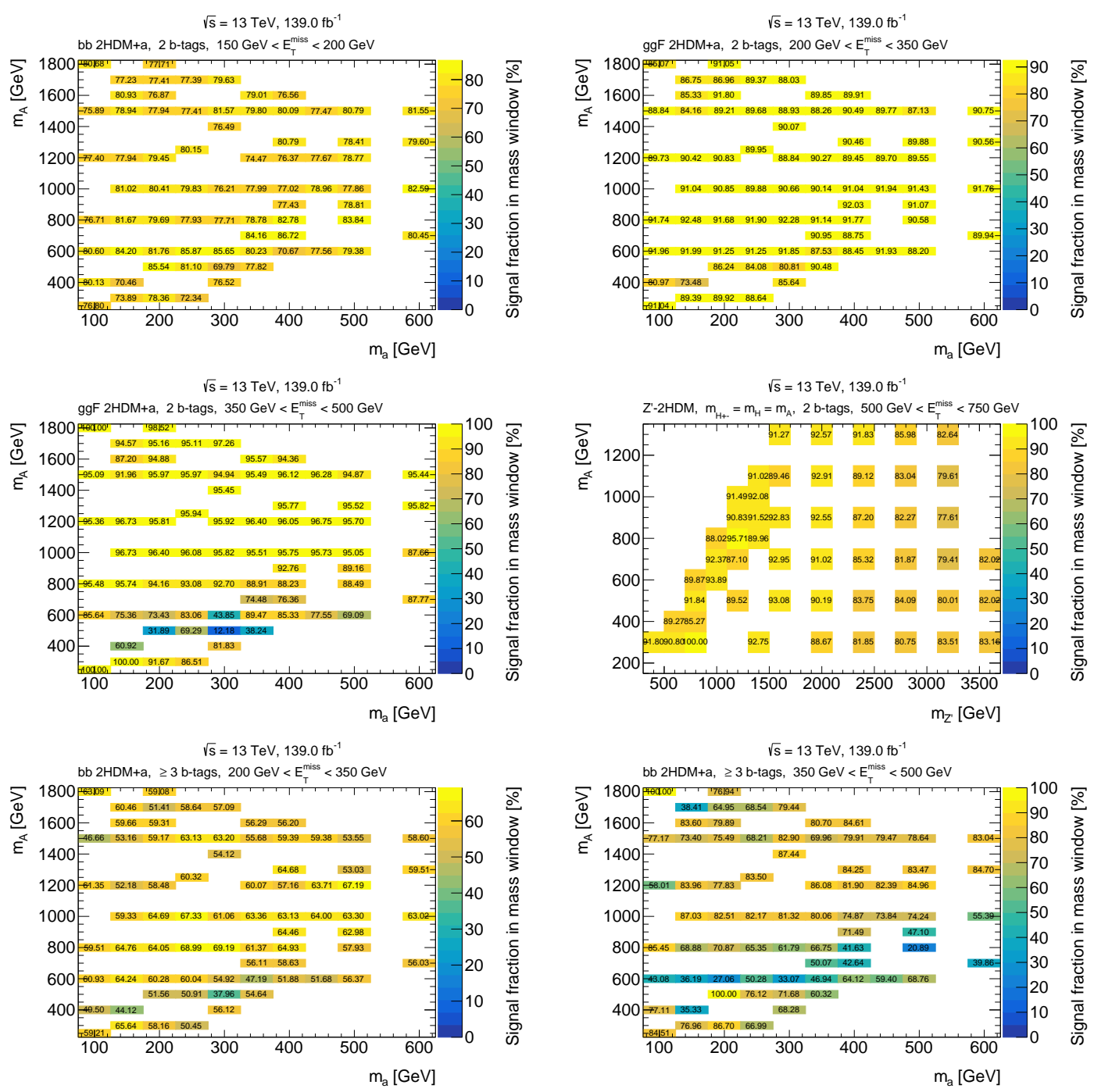

Figure D.1: Plots show the fraction of signal present in the $m_{h}$ window of 90-150 GeV for the $Z^{\prime}$-2HDM and 2HDM+a signal models across different $E_{\mathrm{T}}^{\text {miss }}$ and $b$-tag regions. 
Figure D.2 shows the $m_{h}$ distribution for some of the representative $Z^{\prime}-2 \mathrm{HDM}$ and $2 \mathrm{HDM}+\mathrm{a}$ signal points across different $E_{\mathrm{T}}^{\text {miss }}$ and $b$-tag regions.
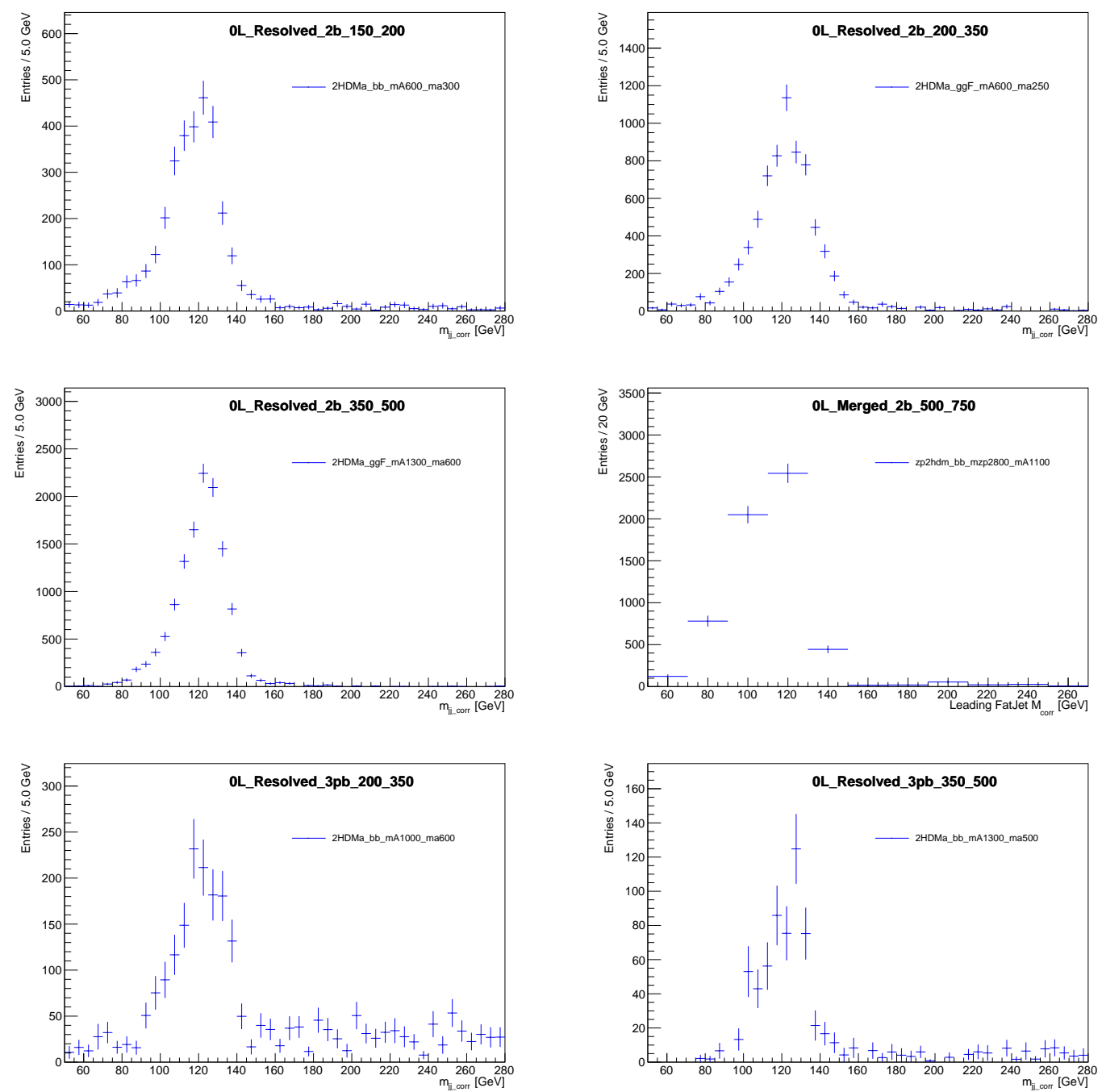

Figure D.2: Higgs candidate mass distributions for a few representative $Z^{\prime}-2 \mathrm{HDM}$ and $2 \mathrm{HDM}+\mathrm{a}$ signal points across different $E_{\mathrm{T}}^{\mathrm{miss}}$ and $b$-tag regions. As seen from the plots a Higgs mass window of 90-150 GeV covers majority of the signal events [234]. 